\title{
Modelos de mistura beta mistos sob abordagem bayesiana
}

Ana Paula Zerbeto

\author{
Tese Apresentada \\ $\mathrm{AO}$ \\ Instituto De Matemática e Estatística \\ DA \\ Universidade De SÃo PaUlo \\ PARA \\ OBTENÇÃO DO TÍTULO \\ $\mathrm{DE}$ \\ DOUTOR EM CIÊNCIAS \\ Programa: Estatística \\ Orientadora: Prof. Dra. Viviana Giampaoli
}

Durante o desenvolvimento deste trabalho o autor recebeu auxílio financeiro do CNPq/CAPES

São Paulo, fevereiro de 2018 


\section{Modelos de mistura beta mistos sob abordagem bayesiana}

Esta versão da tese contém as correções e alterações sugeridas pela Comissão Julgadora durante a defesa da versão original do trabalho, realizada em 14/12/2018. Uma cópia da versão original está disponível no

Instituto de Matemática e Estatística da Universidade de São Paulo.

Comissão Julgadora:

- $\operatorname{Prof}^{\mathrm{a}}$. Dr ${ }^{\mathrm{a}}$. Viviana Giampaoli (orientadora) - IME-USP

- Prof ${ }^{a}$. Dr ${ }^{\mathrm{a}}$. Marcia D’Elia Branco - IME-USP

- Prof ${ }^{a}$. Dr ${ }^{a}$. Denise Aparecida Botter - IME-USP

- Prof. Dr. Francisco José de Azevêdo Cysneiros - UFPE

- Prof. Dr. Celso Rômulo Barbosa Cabral - UFAM 


\section{Resumo}

ZERBETO, A. P. Modelos de mistura beta mistos sob abordagem bayesiana. 2018. Tese (Doutorado) - Instituto de Matemática e Estatística, Universidade de São Paulo, São Paulo, 2018.

Os modelos de mistura são muito eficazes para analisar dados compostos por diferentes subpopulações com alocações desconhecidas ou que apresentam assimetria, multimodalidade ou curtose. Esta tese propõe relacionar a distribuição de probabilidade beta e a técnica de ajuste de modelos mistos à metodologia de modelos de mistura para que sejam adequados na análise de dados que assumem valores em um intervalo restrito conhecido e que também são caracterizados por possuírem uma estrutura de agrupamento ou hierárquica. Foram especificados os modelos de mistura beta mistos linear, com dispersão constante e variável, e não linear. Foi considerada uma abordagem bayesiana com uso de métodos de Monte Carlo via Cadeias de Markov (MCMC). Estudos de simulação foram delineados para avaliar os resultados inferenciais destes modelos em relação à acurácia da estimação pontual dos parâmetros, ao desempenho de critérios de informação na seleção do número de elementos da mistura e ao diagnóstico de identificabilidade obtido com o algoritmo data cloning. O desempenho dos modelos foi muito promissor, principalmente pela boa acurácia da estimação pontual dos parâmetros e por não haver evidências de falta de identificabilidade. Três bancos de dados reais das áreas de saúde, marketing e educação foram estudados por meio das técnicas propostas. Tanto nos estudos de simulação quanto na aplicação a dados reais se obtiveram resultados muito satisfatórios que evidenciam tanto a utilidade dos modelos desenvolvidos aos objetivos tratados quanto a potencialidade de aplicação. Ressaltando que a metodologia apresentada também pode ser aplicada e estendida a outros modelos de mistura.

Palavras-chave: modelo de mistura, distribuição beta, modelo misto, inferência bayesiana, modelo não linear, efeitos aleatórios, data cloning. 


\section{Abstract}

ZERBETO, A. P. Mixture of beta mixed models: a Bayesian approach. 2018. Tese (Doutorado) - Instituto de Matemática e Estatística, Universidade de São Paulo, São Paulo, 2018.

Mixture models are very effective for analyzing data composed of different subpopulations with unknown allocations or with asymmetry, multimodality or kurtosis. This work proposes to link the beta probability distribution and the mixed models to the methodology of mixture models so that they are suitable to analyse data with values in a restricted and known interval and that also are characterized by having a grouping or hierarchical structure. There were specified the linear beta mixture models with random effects, with constant and varying dispersion, and also the nonlinear one with constant dispersion. It was considered a Bayesian approach using Markov Chain Monte Carlo (MCMC) methods. Simulation studies were designed to evaluate the inferential results of these models in relation to the accuracy of the parameter estimation, to the performance of information criteria in the selection of the number of elements of the mixture and to the diagnosis of identifiability obtained with the algorithm data cloning. The performance of the models was very promising, mainly due to the good accuracy of the point estimation of the parameters and because there was no evidence of lack of identifiability of the model. Three real databases of health, marketing and education were studied using the proposed techniques. In both the simulation studies and the application to real data had very satisfactory results that show both the usefulness of the models developed to the treated objectives and the potentiality of application. Note that the presented methodology can also be applied and extended to other mixing models.

Keywords: mixture model, beta distribution, mixed model, bayesian inference, nonlinear model, random effects, data cloning. 


\section{Sumário}

$\begin{array}{ll}\text { Lista de Abreviaturas } & \text { ix }\end{array}$

Lista de Símbolos $\quad$ xi

Lista de Figuras $\quad$ xiii

$\begin{array}{ll}\text { Lista de Tabelas } & \text { xxv }\end{array}$

1 Introdução $\quad 1$

1.1 Considerações Preliminares . . . . . . . . . . . . . . . . . . 1

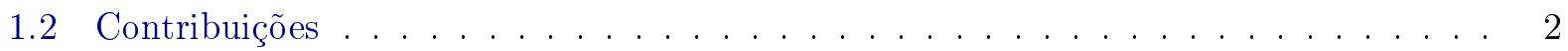

1.3 Estrutura do Trabalho . . . . . . . . . . . . . . . . . . . . . 2

1.4 Suporte computacional . . . . . . . . . . . . . . . . . . 3

2 Conceitos Preliminares $\quad 5$

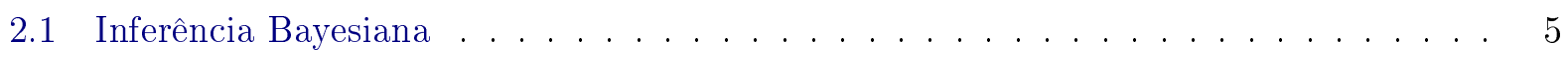

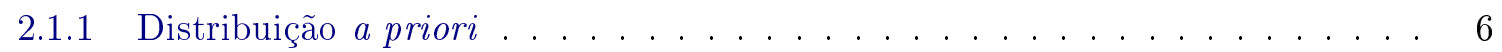

2.1 .2 Algoritmos MCMC . . . . . . . . . . . . . . . . . 7

2.1.3 Comparação e Seleção de Modelos . . . . . . . . . . . . . . . . . . . . . . 10

2.2 Modelo de Regressão Beta . . . . . . . . . . . . . . . . . . . . . 13

2.3 Modelo de Regressão Beta com Efeitos Aleatórios . . . . . . . . . . . . . . . . . 16

2.4 Modelo de Regressão Não Linear com Efeitos Aleatórios . . . . . . . . . . . . . . . 19

2.5 Mistura Finita de Distribuições . . . . . . . . . . . . . . . . . . . . 21

2.6 Modelo de Mistura Finita . . . . . . . . . . . . . . . . . . 26

2.7 Algoritmo Data Cloning para diagnóstico de identificabilidade . . . . . . . . . . 28

3 Modelo de Mistura Beta Linear com Efeitos Aleatórios 31

3.1 Introdução . . . . . . . . . . . . . . . . . . . . . . . . . 31

3.2 Modelo Estatístico . . . . . . . . . . . . . . . . . . . . . 33

3.3 Distribuições a priori e a posteriori . . . . . . . . . . . . . . . 36

3.4 Estudos de simulação . . . . . . . . . . . . . . . . . . . . . . 37

3.4.1 Acurácia das estimativas pontuais a posteriori . . . . . . . . . . . . . 40

3.4.2 Performance dos critérios de seleção de modelos . . . . . . . . . . . . . . . . . 42

3.4 .3 Diagnóstico de identificabilidade . . . . . . . . . . . . . . . . 44

3.5 Aplicações . . . . . . . . . . . . . . . . . . . . . . . . . . 45

3.5.1 Dados de pacientes diagnosticados com periodontite . . . . . . . . . 45 
3.5.2 Aglomeração das redes de fast food nas cidades brasileiras . . . . . . . . . . . 53

3.6 Considerações finais . . . . . . . . . . . . . . . . . . . 61 61

4 Modelo de Mistura Beta Misto Linear com Dispersão Variável 63

4.1 Introdução . . . . . . . . . . . . . . . . . . . . . . . . 6 63

4.2 Modelo Estatístico _ . . . . . . . . . . . . . . . . . . . . . 64

4.3 Distribuições a priori e a posteriori . . . . . . . . . . . . . . . . 67

4.4 Estudos de simulação . . . . . . . . . . . . . . . . . . . . . . 68

4.4.1 Acurácia das estimativas pontuais a posteriori . . . . . . . . . . . . 70

4.4 .2 Performance dos critérios de seleção de modelos . . . . . . . . . . . . . . . . 71

4.4 .3 Diagnóstico de identificabilidade . . . . . . . . . . . . . . . . . . 73

4.5 Aplicação a dados de pacientes diagnosticados com periodontite . . . . . . . . . . . . 74

4.6 Considerações finais . . . . . . . . . . . . . . . . . . . . . . . 79

5 Modelo de Mistura Beta Não Linear com Efeitos Aleatórios $\quad 81$

5.1 Introdução . . . . . . . . . . . . . . . . . . . . . . . . . 81

5.2 Modelo Estatístico . . . . . . . . . . . . . . . . . . . . . 82

5.3 Distribuições a priori e a posteriori . . . . . . . . . . . . . . . . 86

5.4 Estudos de simulação . . . . . . . . . . . . . . . . . . . . . 86

5.4 .1 Acurácia das estimativas pontuais a posteriori . . . . . . . . . . . 88

5.4 .2 Performance dos critérios de seleção de modelos . . . . . . . . . . . . . . . . 89

5.4 .3 Diagnóstico de identificabilidade . . . . . . . . . . . . . . . . . . . . 92

5.5 Aplicação a dados de desenvolvimento de habilidades de leitura no ensino básico . 93

5.6 Considerações finais . . . . . . . . . . . . . . . . . . . . . . . 99

6 Conclusão $r$

6.1 Considerações Finais . . . . . . . . . . . . . . . . . . . . . . . 101

6.2 Trabalhos futuros . . . . . . . . . . . . . . . . . . . . . . 102

A Tabelas com os Resultados de Medidas de Desempenho 105

A.1 Modelo linear com parâmetro de precisão constante . . . . . . . . . . . . . . . 105

A.2 Modelo linear com parâmetro de precisão constante - $\mathrm{N}=100, \mathrm{n}=5, \Sigma_{\beta^{s}}=40 I_{2} \quad \ldots 111$

A.3 Modelo linear com parâmetro de precisão constante - $\mathrm{N}=40, \mathrm{n}=5 \ldots \ldots$. . . . . 113

A.4 Modelo linear com dispersão variável . . . . . . . . . . . . . . . . . . . 116

A.5 Modelo não linear com parâmetro de precisão constante . . . . . . . . . . . . . 118

B Gráficos de Dispersão correspondentes aos Estudos de Simulação 121

B.1 Modelo linear com parâmetro de precisão constante - $\mathrm{N}=100, \mathrm{n}=5 \ldots \ldots$. . . . . . 121

B.2 Modelo linear com parâmetro de precisão constante - $\mathrm{N}=100, \mathrm{n}=10 \ldots \ldots$. . . . . . 128

B.3 Modelo linear com parâmetro de precisão constante - $\mathrm{N}=500, \mathrm{n}=5 \ldots \ldots$

B.4 Modelo linear com parâmetro de precisão constante - $\mathrm{N}=500, \mathrm{n}=10$. . . . . . . . . 142

B.5 Modelo linear com parâmetro de precisão constante - $\mathrm{N}=40, \mathrm{n}=5 \ldots \ldots$. . . . . . . 149

B.6 Modelo linear com parâmetro de precisão constante - $\mathrm{N}=100, \mathrm{n}=5, \Sigma_{\beta}=40 I_{2} \quad \ldots 155$

B.7 Modelo linear com dispersão variável . . . . . . . . . . . . . . . . . . . . . . 159

B.8 Modelo não linear com parâmetro de precisão constante . . . . . . . . . . . . . . 166 
Referências Bibliográficas 
viii SUMÁRIO 


\section{Lista de Abreviaturas}

$\begin{array}{ll}\text { ABF } & \text { Associação Brasileira de Franchising } \\ \text { AIC } & \text { Critério de Informação de Akaike } \\ \text { BIC } & \text { Critério de Informação Bayesiano } \\ \text { BUGS } & \text { Bayesian Inference Using Gibbs Sampling } \\ \text { CPO } & \text { Ordenada Preditiva Condicional } \\ \text { DIC } & \text { Critério de Informação do Desvio } \\ \text { EAIC } & \text { Critério de Informação de Akaike Esperado } \\ \text { EBIC } & \text { Critério de Informação Bayesiano Esperado } \\ \text { ECAIC } & \text { Critério de Informação de Akaike Esperado Corrigido } \\ \text { ECLS-K } & \text { Early Childhood Longitudinal Program, Kindergarten Class of 1998-99 } \\ \text { EM } & \text { Expectation Maximization } \\ \text { EAR } & \text { Erro Absoluto Relativo Médio } \\ \text { ER } & \text { Erro Relativo Médio } \\ \text { HPC } & \text { High Performance Computing } \\ \text { IC } & \text { Intervalo de Credibilidade } \\ \text { ICL } & \text { Integrated Classification Likelihood } \\ \text { iid } & \text { independentes e identicamente distribuídas } \\ \text { INLA } & \text { Integrated Nested Laplace Approximation } \\ \text { LML } & \text { Log-Verossimilhança Marginal } \\ \text { LPML } & \text { Logaritmo da Pseudo-Verossimilhança Marginal } \\ \text { MCMC } & \text { Monte Carlo via Cadeias de Markov } \\ \text { MV } & \text { Máxima Verossimilhança } \\ \text { PAT } & \text { Programa de Alimentação do Trabalhador } \\ \text { PIB } & \text { Rroduto Interno Bruto Erro Quadrático Médio } \\ \text { REQM } & \\ \text { VI } & \text { Raínal Inference } \\ & \end{array}$




\section{Lista de Símbolos}

$Y_{i j}$

K

$N$

$n_{i}$

$S_{i}$

$\pi_{i s}$

$\vartheta$

$g_{\mu}$

$h_{\mu}$

$\boldsymbol{\beta}^{s}=\left(\beta_{1}^{s}, \ldots, \beta_{p}^{s}\right)^{\top}$

$p$

$\boldsymbol{b}_{i}=\left(b_{i 1}, \ldots, b_{i d}\right)^{\top}$

$d$

$\Sigma_{b}$

$\boldsymbol{x}_{i j}=\left(x_{i j 1}, \ldots, x_{i j p}\right)^{\top}$

$\boldsymbol{z}_{i j}=\left(z_{i j 1}, \ldots, z_{i j d}\right)^{\top}$

$g_{\phi}$

$h_{\phi}$

$\boldsymbol{\delta}^{s}=\left(\delta_{1}^{s}, \ldots, \delta_{q}^{s}\right)^{\top}$

$q$

$\boldsymbol{u}_{i}=\left(u_{i 1}, \ldots, u_{i d^{*}}\right)^{\top}$ variável resposta

número de componentes da mistura

número de grupos da amostra

número de elementos do $i$-ésimo grupo, $i=1, \ldots, N$

variável indicadora discreta do componente da mistura associada ao $i$ ésimo grupo da amostra, $i=1, \ldots, N, S_{i} \in\{1, \ldots, K\}$

probabilidade do $i$-ésimo grupo estar associado ao $s$-ésimo componente da mistura, ou seja, $\pi_{i s}=P\left(S_{i}=s\right), 0<\pi_{i s}<1, \sum_{s=1}^{K} \pi_{i s}=1, i=$ $1, \ldots, N$ e $s=1, \ldots, K$

é o vetor de parâmetros do modelo

função de ligação, estritamente monótona e duplamente diferenciável, do modelo para a média

função sistemática não linear e conhecida do modelo para a média vetor $p$-dimensional de coeficientes fixos relacionado ao $s$-ésimo componente da mistura do modelo para a média, $s=1, \ldots, K$

número de efeitos fixos do modelo para a média

vetor de dimensão $d$ de efeitos aleatórios do modelo para a média do $i$-ésimo grupo, $i=1, \ldots, N$

número de efeitos aleatórios do modelo para a média

matriz de ordem $d \times d$ de variância-covariância dos efeitos aleatórios $\left(\boldsymbol{b}_{1}, \ldots, \boldsymbol{b}_{N}\right)$ do modelo para a média

vetor $p$-dimensional de covariáveis dos efeitos fixos do $j$-ésimo elemento do $i$-ésimo grupo do modelo para a média, $i=1, \ldots, N$ e $j=1, \ldots, n_{i}$ vetor $d$-dimensional de covariáveis dos efeitos aleatórios do $j$-ésimo elemento do $i$-ésimo grupo do modelo para a média, $i=1, \ldots, N$ e $j=1, \ldots, n_{i}$

função de ligação, estritamente monótona e duplamente diferenciável, do modelo para a precisão

função sistemática do modelo para a precisão

vetor $q$-dimensional de coeficientes fixos relacionado ao $s$-ésimo componente da mistura do modelo para a precisão, $s=1, \ldots, K$

número de efeitos fixos do modelo para a precisão

vetor de dimensão $d^{*}$ de efeitos aleatórios do modelo para a precisão do $i$-ésimo grupo, $i=1, \ldots, N$ 
$d^{*}$

$\boldsymbol{w}_{i j}=\left(w_{i j 1}, \ldots, w_{i j q}\right)^{\top}$

$\boldsymbol{r}_{i j}=\left(r_{i j 1}, \ldots, r_{i j d^{*}}\right)^{\top}$

$\Sigma_{u}$

$n_{i t}$

$n_{b}$

$n_{t}$

$n_{c}$ número de efeitos aleatórios do modelo para a precisão

vetor $q$-dimensional de covariáveis dos efeitos fixos do $j$-ésimo elemento do $i$-ésimo grupo do modelo para a precisão, $i=1, \ldots, N$ e $j=1, \ldots, n_{i}$ vetor $d^{*}$-dimensional de covariáveis dos efeitos aleatórios do $j$-ésimo elemento do $i$-ésimo grupo do modelo para a precisão, $i=1, \ldots, N$ e $j=1, \ldots, n_{i}$

matriz de ordem $d^{*} \times d^{*}$ de variância-covariância dos efeitos aleatórios $\left(\boldsymbol{u}_{1}, \ldots, \boldsymbol{u}_{N}\right)$ do modelo para a precisão número de iterações no algoritmo MCMC número de iterações iniciais a serem excluídas de forma a atingir a convergência desejada, denominada amostra de aquecimento (burn-in) número de elementos entre cada valor amostrado da cadeia de Markov gerada (após a amostra de aquecimento), denominado thinning número de cadeias de Markov geradas no algoritmo MCMC 


\section{Lista de Figuras}

2.1 Comparação entre as densidades da distribuição beta para diferentes valores dos

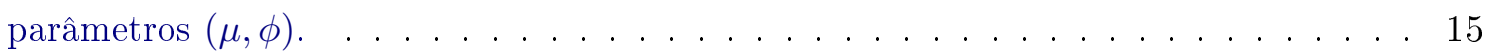

2.2 Comparação entre as densidades de distribuições mistura de normais para diferentes valores do parâmetro de média $\mu$ e de probabilidades $\pi$. . . . . . . . . . . . . . . 22

2.3 Comparação entre as densidades de distribuições mistura de beta para diferentes valores dos parâmetros $\mu, \phi$ e $\pi$. . . . . . . . . . . . . . . . . . . . . 25

3.1 Comparação entre as densidades da distribuição beta para diferentes valores dos parâmetros $\beta$ e $\phi$ nos cenários englobados pelos estudos de simulação. . . . . . . . . 38

3.2 Comparação entre as densidades da distribuição gama inversa, distribuição a priori de $\sigma_{b}$, para diferentes valores do parâmetro $\epsilon$. . . . . . . . . . . . . . . . . . . 40

3.3 Gráfico de linhas do inverso do número de clones (traço vermelho) e do maior autovalor padronizado da matriz de variâncias e covariâncias a posteriori dos parâmetros do modelo de mistura beta misto com cenários 1,2,3, 4, parâmetro de precisão assumindo valores $30(\triangle), 50(\bigcirc), 70(\times)$ e número de clones $1,3,5,9$. . . . . . . . . 44

3.4 Gráfico de perfis da variável anti-oxLDL nos dados de periodontite. . . . . . . . . . 47

3.5 Histograma da variável anti-oxLDL nos dados de periodontite. . . . . . . . . . . . . 47

3.6 Gráfico da função densidade de probabilidade estimada dos parâmetros $\boldsymbol{\beta}^{\boldsymbol{s}}, \boldsymbol{s}=1,2$, do modelo final ajustado para a variável anti-oxLDL dos dados de periodontite. . . 50

3.7 Gráfico da função densidade de probabilidade estimada dos parâmetros $\phi_{1}, \phi_{2}, \pi_{1}$ e $\sigma_{b}$ do modelo final ajustado para a variável anti-oxLDL dos dados de periodontite.

3.8 Gráfico de probabilidade normal dos resíduos quantílicos aleatorizados do modelo final ajustado aos dados de periodontite. . . . . . . . . . . . . . . . . . . . 51

3.9 Gráfico de linhas do maior autovalor padronizado da matriz de variâncias e covariâncias a posteriori dos parâmetros do modelo de mistura beta misto ajustado aos dados de periodontite. . . . . . . . . . . . . . . . . . . . 53

3.10 Gráfico de probabilidade normal dos resíduos quantílicos aleatorizados do modelo final ajustado aos dados de redes de fast food no Brasil. . . . . . . . . . . . . . . . . 58

3.11 Gráfico de linhas do maior autovalor padronizado da matriz de variâncias e covariâncias a posteriori dos parâmetros do modelo de mistura beta misto ajustado aos dados de redes de fast food no Brasil. . . . . . . . . . . . . . . . . . . . . . . . 58

3.12 Mapa das proporções de redes de fast food nacionais em cada estado brasileiro. $\quad$ . . 59 
3.13 Gráfico da função densidade de probabilidade estimada dos parâmetros $\boldsymbol{\beta}^{\boldsymbol{s}}, s=1,2$, do modelo final ajustado para a variável índice de Gini dos dados de redes de fast food no Brasil.

3.14 Gráfico da função densidade de probabilidade estimada dos parâmetros $\phi_{1}, \phi_{2}, \pi_{1}$ e $\sigma_{b}$ do modelo final ajustado para a variável índice de Gini dos dados de redes de fast food no Brasil.

4.1 Comparação entre as densidades da distribuição beta para diferentes valores dos parâmetros $\beta$ e $\delta$ nos cenários englobados pelos estudos de simulação. . . . . . . . . .

4.2 Gráfico de linhas do inverso do número de clones (traço vermelho) e do maior autovalor padronizado da matriz de variâncias e covariâncias a posteriori dos parâmetros do modelo de mistura beta misto linear com dispersão variável com cenários 1, 2, 3, 4 para os coeficientes fixos do modelo para a média e cenários $1(\triangle), 2(\bigcirc)$ e $3(\times)$ para os coeficientes fixos do modelo para o parâmetro de precisão. . . . . . . . . . . . .

4.3 Gráfico da função densidade de probabilidade estimada dos parâmetros $\boldsymbol{\beta}^{\boldsymbol{s}}, s=1,2$, do modelo final ajustado para a variável anti-oxLDL dos dados de periodontite. . .

4.4 Gráfico da função densidade de probabilidade estimada dos parâmetros $\boldsymbol{\delta}^{\boldsymbol{s}}, s=1,2$, $\pi_{1}, \pi_{2}, \sigma_{b}$ e $\sigma_{u}$ do modelo final ajustado para a variável anti-oxLDL dos dados de periodontite.

4.5 Gráfico de probabilidade normal dos resíduos quantílicos aleatorizados do modelo final ajustado aos dados de periodontite . . . . . . . . . . . . . .

5.1 Gráfico das observações geradas com $h_{\mu}(x, \boldsymbol{\beta})=\beta_{1}+x_{i j}^{\beta_{2}}$ e ligação logito para valores de $\left(\beta_{1} ; \beta_{2}\right)$ que compõem os cenários dos estudos de simulação.

5.2 Comparação entre as densidades da distribuição beta para diferentes valores dos parâmetros $\beta$ e $\phi$ nos cenários englobados pelos estudos de simulação.

5.3 Gráfico de linhas do inverso do número de clones (traço vermelho) e do maior autovalor padronizado da matriz de variâncias e covariâncias a posteriori dos parâmetros do modelo de mistura beta misto não linear com cenários 1, 2 e parâmetro de precisão assumindo valores $30(\triangle), 50(\bigcirc), 70(\times)$.

5.4 Gráfico de perfis do desenvolvimento da habilidade de leitura de 100 alunos participantes do estudo ECLS-K. . . . . . . . . . . . . . . . . . . . 9 94

5.5 Histograma do escore de habilidade de leitura de 100 alunos participantes do estudo ECLS-K nos diferentes momentos de aferição. . . . . . . . . . . . . . . . . . . . 94

5.6 Gráfico da função densidade de probabilidade estimada dos parâmetros $\boldsymbol{\beta}^{\boldsymbol{s}}$ e $\phi_{s}$, $s=1,2$, do modelo final ajustado para os dados de habilidade de leitura do estudo ECLS-K. . . . . . . . . . . . . . . . . . . . 97

5.7 Gráfico da função densidade de probabilidade estimada dos parâmetros $\pi_{1}$ e $\Sigma_{b}$ do modelo final ajustado para os dados de habilidade de leitura do estudo ECLS-K.

5.8 Gráfico de linhas do maior autovalor padronizado da matriz de variâncias e covariâncias a posteriori dos parâmetros do modelo de mistura beta misto não linear ajustado aos dados de habilidade de leitura do estudo ECLS-K. 
B.1 Gráfico de dispersão das estimativas pontuais - mediana a posteriori - dos estudos de simulação de $R=50$ bancos de dados do modelo de mistura beta linear com efeitos aleatórios para a média com cenário $1, \phi=30, N=100$ e $n=5$, em que $s=1(\bullet), 2(\boldsymbol{\bullet})$, e dos verdadeiros valores dos parâmetros (em vermelho). . . . . . . . 121

B.2 Gráfico de dispersão das estimativas pontuais - mediana a posteriori - dos estudos de simulação de $R=50$ bancos de dados do modelo de mistura beta linear com efeitos aleatórios para a média com cenário $1, \phi=50, N=100$ e $n=5$, em que $s=1(\bullet), 2(\boldsymbol{\bullet})$, e dos verdadeiros valores dos parâmetros (em vermelho). . . . . . . . .

B.3 Gráfico de dispersão das estimativas pontuais - mediana a posteriori - dos estudos de simulação de $R=50$ bancos de dados do modelo de mistura beta linear com efeitos aleatórios para a média com cenário $1, \phi=70, N=100$ e $n=5$, em que $s=1(\bullet), 2(\boldsymbol{\bullet})$, e dos verdadeiros valores dos parâmetros (em vermelho). . . . . . . . .

B.4 Gráfico de dispersão das estimativas pontuais - mediana a posteriori - dos estudos de simulação de $R=50$ bancos de dados do modelo de mistura beta linear com efeitos aleatórios para a média com cenário $2, \phi=30, N=100$ e $n=5$, em que $s=1(\bullet), 2(\boldsymbol{\bullet})$, e dos verdadeiros valores dos parâmetros (em vermelho). . . . . . . .

B.5 Gráfico de dispersão das estimativas pontuais - mediana a posteriori - dos estudos de simulação de $R=50$ bancos de dados do modelo de mistura beta linear com efeitos aleatórios para a média com cenário $2, \phi=50, N=100$ e $n=5$, em que $s=1(\bullet), 2(\boldsymbol{\bullet})$, e dos verdadeiros valores dos parâmetros (em vermelho). . . . . . . .

B.6 Gráfico de dispersão das estimativas pontuais - mediana a posteriori - dos estudos de simulação de $R=50$ bancos de dados do modelo de mistura beta linear com efeitos aleatórios para a média com cenário $2, \phi=70, N=100$ e $n=5$, em que $s=1(\bullet), 2(\boldsymbol{\bullet})$, e dos verdadeiros valores dos parâmetros (em vermelho). . . . . . . .

B.7 Gráfico de dispersão das estimativas pontuais - mediana a posteriori - dos estudos de simulação de $R=50$ bancos de dados do modelo de mistura beta linear com efeitos aleatórios para a média com cenário $3, \phi=30, N=100$ e $n=5$, em que $s=1(\bullet), 2(\boldsymbol{\bullet})$, e dos verdadeiros valores dos parâmetros (em vermelho). . . . . . . . .

B.8 Gráfico de dispersão das estimativas pontuais - mediana a posteriori - dos estudos de simulação de $R=50$ bancos de dados do modelo de mistura beta linear com efeitos aleatórios para a média com cenário $3, \phi=50, N=100$ e $n=5$, em que $s=1(\bullet), 2(\boldsymbol{\bullet})$, e dos verdadeiros valores dos parâmetros (em vermelho). . . . . . . .

B.9 Gráfico de dispersão das estimativas pontuais - mediana a posteriori - dos estudos de simulação de $R=50$ bancos de dados do modelo de mistura beta linear com efeitos aleatórios para a média com cenário $3, \phi=70, N=100$ e $n=5$, em que $s=1(\bullet), 2(\boldsymbol{\bullet})$, e dos verdadeiros valores dos parâmetros (em vermelho). . . . . . . .

B.10 Gráfico de dispersão das estimativas pontuais - mediana a posteriori - dos estudos de simulação de $R=50$ bancos de dados do modelo de mistura beta linear com efeitos aleatórios para a média com cenário 4, $\phi=30, N=100$ e $n=5$, em que $s=1(\bullet), 2(\mathbf{\bullet})$, e dos verdadeiros valores dos parâmetros (em vermelho). . . . . . . 126 
B.11 Gráfico de dispersão das estimativas pontuais - mediana a posteriori - dos estudos de simulação de $R=50$ bancos de dados do modelo de mistura beta linear com efeitos aleatórios para a média com cenário $4, \phi=50, N=100$ e $n=5$, em que $s=1(\bullet), 2(\bullet)$, e dos verdadeiros valores dos parâmetros (em vermelho). . . . . . . . 126

B.12 Gráfico de dispersão das estimativas pontuais - mediana a posteriori - dos estudos de simulação de $R=50$ bancos de dados do modelo de mistura beta linear com efeitos aleatórios para a média com cenário $4, \phi=70, N=100$ e $n=5$, em que $s=1(\bullet), 2(\bullet)$, e dos verdadeiros valores dos parâmetros (em vermelho) . . . . . . 127

B.13 Gráfico de dispersão das estimativas pontuais - mediana a posteriori - dos estudos de simulação de $R=50$ bancos de dados do modelo de mistura beta linear com efeitos aleatórios para a média com cenário $1, \phi=30, N=100$ e $n=10$, em que $s=1(\bullet), 2(\bullet)$, e dos verdadeiros valores dos parâmetros (em vermelho) . . . . . . . 128

B.14 Gráfico de dispersão das estimativas pontuais - mediana a posteriori - dos estudos de simulação de $R=50$ bancos de dados do modelo de mistura beta linear com efeitos aleatórios para a média com cenário $1, \phi=50, N=100$ e $n=10$, em que $s=1(\bullet), 2(\bullet)$, e dos verdadeiros valores dos parâmetros (em vermelho) . . . . . . . 12

B.15 Gráfico de dispersão das estimativas pontuais - mediana a posteriori - dos estudos de simulação de $R=50$ bancos de dados do modelo de mistura beta linear com efeitos aleatórios para a média com cenário $1, \phi=70, N=100$ e $n=10$, em que $s=1(\bullet), 2(\bullet)$, e dos verdadeiros valores dos parâmetros (em vermelho). . . . . . . 129

B.16 Gráfico de dispersão das estimativas pontuais - mediana a posteriori - dos estudos de simulação de $R=50$ bancos de dados do modelo de mistura beta linear com efeitos aleatórios para a média com cenário $2, \phi=30, N=100$ e $n=10$, em que $s=1(\bullet), 2(\bullet)$, e dos verdadeiros valores dos parâmetros (em vermelho) . . . . . . 130

B.17 Gráfico de dispersão das estimativas pontuais - mediana a posteriori - dos estudos de simulação de $R=50$ bancos de dados do modelo de mistura beta linear com efeitos aleatórios para a média com cenário $2, \phi=50, N=100$ e $n=10$, em que $s=1(\bullet), 2(\bullet)$, e dos verdadeiros valores dos parâmetros (em vermelho) . . . . . . 130

B.18 Gráfico de dispersão das estimativas pontuais - mediana a posteriori - dos estudos de simulação de $R=50$ bancos de dados do modelo de mistura beta linear com efeitos aleatórios para a média com cenário $2, \phi=70, N=100$ e $n=10$, em que $s=1(\bullet), 2(\bullet)$, e dos verdadeiros valores dos parâmetros (em vermelho) . . . . . . 131

B.19 Gráfico de dispersão das estimativas pontuais - mediana a posteriori - dos estudos de simulação de $R=50$ bancos de dados do modelo de mistura beta linear com efeitos aleatórios para a média com cenário $3, \phi=30, N=100$ e $n=10$, em que $s=1(\bullet), 2(\bullet)$, e dos verdadeiros valores dos parâmetros (em vermelho) . . . . . . 131

B.20 Gráfico de dispersão das estimativas pontuais - mediana a posteriori - dos estudos de simulação de $R=50$ bancos de dados do modelo de mistura beta linear com efeitos aleatórios para a média com cenário $3, \phi=50, N=100$ e $n=10$, em que $s=1(\bullet), 2(\bullet)$, e dos verdadeiros valores dos parâmetros (em vermelho) . . . . . . . 132 
B.21 Gráfico de dispersão das estimativas pontuais - mediana a posteriori - dos estudos de simulação de $R=50$ bancos de dados do modelo de mistura beta linear com efeitos aleatórios para a média com cenário 3, $\phi=70, N=100$ e $n=10$, em que $s=1(\bullet), 2(\mathbf{\bullet})$, e dos verdadeiros valores dos parâmetros (em vermelho). . . . . . . 132

B.22 Gráfico de dispersão das estimativas pontuais - mediana a posteriori - dos estudos de simulação de $R=50$ bancos de dados do modelo de mistura beta linear com efeitos aleatórios para a média com cenário 4, $\phi=30, N=100$ e $n=10$, em que $s=1(\bullet), 2(\mathbf{\bullet})$, e dos verdadeiros valores dos parâmetros (em vermelho) . . . . . . . 133

B.23 Gráfico de dispersão das estimativas pontuais - mediana a posteriori - dos estudos de simulação de $R=50$ bancos de dados do modelo de mistura beta linear com efeitos aleatórios para a média com cenário 4, $\phi=50, N=100$ e $n=10$, em que $s=1(\bullet), 2(\mathbf{\bullet})$, e dos verdadeiros valores dos parâmetros (em vermelho). . . . . . . . 133

B.24 Gráfico de dispersão das estimativas pontuais - mediana a posteriori - dos estudos de simulação de $R=50$ bancos de dados do modelo de mistura beta linear com efeitos aleatórios para a média com cenário 4, $\phi=70, N=100$ e $n=10$, em que $s=1(\bullet), 2(\mathbf{\bullet})$, e dos verdadeiros valores dos parâmetros (em vermelho). . . . . . . . .

B.25 Gráfico de dispersão das estimativas pontuais - mediana a posteriori - dos estudos de simulação de $R=25$ bancos de dados do modelo de mistura beta linear com efeitos aleatórios para a média com cenário $1, \phi=30, N=500$ e $n=5$, em que $s=1(\bullet), 2(\mathbf{\bullet})$, e dos verdadeiros valores dos parâmetros (em vermelho). . . . . . . 135

B.26 Gráfico de dispersão das estimativas pontuais - mediana a posteriori - dos estudos de simulação de $R=25$ bancos de dados do modelo de mistura beta linear com efeitos aleatórios para a média com cenário $1, \phi=50, N=500$ e $n=5$, em que $s=1(\bullet), 2(\mathbf{\bullet})$, e dos verdadeiros valores dos parâmetros (em vermelho). . . . . . . . 136

B.27 Gráfico de dispersão das estimativas pontuais - mediana a posteriori - dos estudos de simulação de $R=25$ bancos de dados do modelo de mistura beta linear com efeitos aleatórios para a média com cenário $1, \phi=70, N=500$ e $n=5$, em que $s=1(\bullet), 2(\mathbf{\bullet})$, e dos verdadeiros valores dos parâmetros (em vermelho). . . . . . . . 136

B.28 Gráfico de dispersão das estimativas pontuais - mediana a posteriori - dos estudos de simulação de $R=25$ bancos de dados do modelo de mistura beta linear com efeitos aleatórios para a média com cenário $2, \phi=30, N=500$ e $n=5$, em que $s=1(\bullet), 2(\bullet)$, e dos verdadeiros valores dos parâmetros (em vermelho). . . . . . . . 137

B.29 Gráfico de dispersão das estimativas pontuais - mediana a posteriori - dos estudos de simulação de $R=25$ bancos de dados do modelo de mistura beta linear com efeitos aleatórios para a média com cenário 2, $\phi=50, N=500$ e $n=5$, em que $s=1(\bullet), 2(\mathbf{\bullet})$, e dos verdadeiros valores dos parâmetros (em vermelho). . . . . . . . 137

B.30 Gráfico de dispersão das estimativas pontuais - mediana a posteriori - dos estudos de simulação de $R=25$ bancos de dados do modelo de mistura beta linear com efeitos aleatórios para a média com cenário 2, $\phi=70, N=500$ e $n=5$, em que $s=1(\bullet), 2(\mathbf{\bullet})$, e dos verdadeiros valores dos parâmetros (em vermelho). . . . . . . 138 
B.31 Gráfico de dispersão das estimativas pontuais - mediana a posteriori - dos estudos de simulação de $R=25$ bancos de dados do modelo de mistura beta linear com efeitos aleatórios para a média com cenário $3, \phi=30, N=500$ e $n=5$, em que $s=1(\bullet), 2(\bullet)$, e dos verdadeiros valores dos parâmetros (em vermelho) . . . . . . . 138

B.32 Gráfico de dispersão das estimativas pontuais - mediana a posteriori - dos estudos de simulação de $R=25$ bancos de dados do modelo de mistura beta linear com efeitos aleatórios para a média com cenário $3, \phi=50, N=500$ e $n=5$, em que $s=1(\bullet), 2(\bullet)$, e dos verdadeiros valores dos parâmetros (em vermelho) . . . . . . . 139

B.33 Gráfico de dispersão das estimativas pontuais - mediana a posteriori - dos estudos de simulação de $R=25$ bancos de dados do modelo de mistura beta linear com efeitos aleatórios para a média com cenário $3, \phi=70, N=500$ e $n=5$, em que $s=1(\bullet), 2(\mathbf{\bullet})$, e dos verdadeiros valores dos parâmetros (em vermelho) . . . . . . 139

B.34 Gráfico de dispersão das estimativas pontuais - mediana a posteriori - dos estudos de simulação de $R=25$ bancos de dados do modelo de mistura beta linear com efeitos aleatórios para a média com cenário $4, \phi=30, N=500$ e $n=5$, em que $s=1(\bullet), 2(\bullet)$, e dos verdadeiros valores dos parâmetros (em vermelho) . . . . . . . 140

B.35 Gráfico de dispersão das estimativas pontuais - mediana a posteriori - dos estudos de simulação de $R=25$ bancos de dados do modelo de mistura beta linear com efeitos aleatórios para a média com cenário $4, \phi=50, N=500$ e $n=5$, em que $s=1(\bullet), 2(\mathbf{\bullet})$, e dos verdadeiros valores dos parâmetros (em vermelho) . . . . . . . 140

B.36 Gráfico de dispersão das estimativas pontuais - mediana a posteriori - dos estudos de simulação de $R=25$ bancos de dados do modelo de mistura beta linear com efeitos aleatórios para a média com cenário $4, \phi=70, N=500$ e $n=5$, em que $s=1(\bullet), 2(\mathbf{\bullet})$, e dos verdadeiros valores dos parâmetros (em vermelho) . . . . . . . 141

B.37 Gráfico de dispersão das estimativas pontuais - mediana a posteriori - dos estudos de simulação de $R=25$ bancos de dados do modelo de mistura beta linear com efeitos aleatórios para a média com cenário $1, \phi=30, N=500$ e $n=10$, em que $s=1(\bullet), 2(\bullet)$, e dos verdadeiros valores dos parâmetros (em vermelho) . . . . . . . 142

B.38 Gráfico de dispersão das estimativas pontuais - mediana a posteriori - dos estudos de simulação de $R=25$ bancos de dados do modelo de mistura beta linear com efeitos aleatórios para a média com cenário $1, \phi=50, N=500$ e $n=10$, em que $s=1(\bullet), 2(\bullet)$, e dos verdadeiros valores dos parâmetros (em vermelho) . . . . . . 143

B.39 Gráfico de dispersão das estimativas pontuais - mediana a posteriori - dos estudos de simulação de $R=25$ bancos de dados do modelo de mistura beta linear com efeitos aleatórios para a média com cenário $1, \phi=70, N=500$ e $n=10$, em que $s=1(\bullet), 2(\bullet)$, e dos verdadeiros valores dos parâmetros (em vermelho) . . . . . . 143

B.40 Gráfico de dispersão das estimativas pontuais - mediana a posteriori - dos estudos de simulação de $R=25$ bancos de dados do modelo de mistura beta linear com efeitos aleatórios para a média com cenário $2, \phi=30, N=500$ e $n=10$, em que $s=1(\bullet), 2(\bullet)$, e dos verdadeiros valores dos parâmetros (em vermelho) . . . . . . . 144 
B.41 Gráfico de dispersão das estimativas pontuais - mediana a posteriori - dos estudos de simulação de $R=25$ bancos de dados do modelo de mistura beta linear com efeitos aleatórios para a média com cenário $2, \phi=50, N=500$ e $n=10$, em que $s=1(\bullet), 2(\bullet)$, e dos verdadeiros valores dos parâmetros (em vermelho). . . . . . . . 144

B.42 Gráfico de dispersão das estimativas pontuais - mediana a posteriori - dos estudos de simulação de $R=25$ bancos de dados do modelo de mistura beta linear com efeitos aleatórios para a média com cenário $2, \phi=70, N=500$ e $n=10$, em que $s=1(\bullet), 2(\bullet)$, e dos verdadeiros valores dos parâmetros (em vermelho) . . . . . . . 145

B.43 Gráfico de dispersão das estimativas pontuais - mediana a posteriori - dos estudos de simulação de $R=25$ bancos de dados do modelo de mistura beta linear com efeitos aleatórios para a média com cenário $3, \phi=30, N=500$ e $n=10$, em que $s=1(\bullet), 2(\bullet)$, e dos verdadeiros valores dos parâmetros (em vermelho). . . . . . . 145

B.44 Gráfico de dispersão das estimativas pontuais - mediana a posteriori - dos estudos de simulação de $R=25$ bancos de dados do modelo de mistura beta linear com efeitos aleatórios para a média com cenário $3, \phi=50, N=500$ e $n=10$, em que $s=1(\bullet), 2(\bullet)$, e dos verdadeiros valores dos parâmetros (em vermelho) . . . . . . 146

B.45 Gráfico de dispersão das estimativas pontuais - mediana a posteriori - dos estudos de simulação de $R=25$ bancos de dados do modelo de mistura beta linear com efeitos aleatórios para a média com cenário $3, \phi=70, N=500$ e $n=10$, em que $s=1(\bullet), 2(\bullet)$, e dos verdadeiros valores dos parâmetros (em vermelho). . . . . . 146

B.46 Gráfico de dispersão das estimativas pontuais - mediana a posteriori - dos estudos de simulação de $R=25$ bancos de dados do modelo de mistura beta linear com efeitos aleatórios para a média com cenário $4, \phi=30, N=500$ e $n=10$, em que $s=1(\bullet), 2(\boldsymbol{\bullet})$, e dos verdadeiros valores dos parâmetros (em vermelho) . . . . . . 147

B.47 Gráfico de dispersão das estimativas pontuais - mediana a posteriori - dos estudos de simulação de $R=25$ bancos de dados do modelo de mistura beta linear com efeitos aleatórios para a média com cenário $4, \phi=50, N=500$ e $n=10$, em que $s=1(\bullet), 2(\bullet)$, e dos verdadeiros valores dos parâmetros (em vermelho) . . . . . . 147

B.48 Gráfico de dispersão das estimativas pontuais - mediana a posteriori - dos estudos de simulação de $R=25$ bancos de dados do modelo de mistura beta linear com efeitos aleatórios para a média com cenário $4, \phi=70, N=500$ e $n=10$, em que $s=1(\bullet), 2(\bullet)$, e dos verdadeiros valores dos parâmetros (em vermelho) . . . . . . 148

B.49 Gráfico de dispersão das estimativas pontuais - mediana a posteriori - dos estudos de simulação de $R=50$ bancos de dados do modelo de mistura beta linear com efeitos aleatórios para a média com cenário $1, \phi=30, N=40$ e $n=5$, em que $s=1(\bullet), 2(\bullet)$, e dos verdadeiros valores dos parâmetros (em vermelho). . . . . . . 149

B.50 Gráfico de dispersão das estimativas pontuais - mediana a posteriori - dos estudos de simulação de $R=50$ bancos de dados do modelo de mistura beta linear com efeitos aleatórios para a média com cenário $1, \phi=50, N=40$ e $n=5$, em que $s=1(\bullet), 2(\mathbf{\bullet})$, e dos verdadeiros valores dos parâmetros (em vermelho). . . . . . 149 
B.51 Gráfico de dispersão das estimativas pontuais - mediana a posteriori - dos estudos de simulação de $R=50$ bancos de dados do modelo de mistura beta linear com efeitos aleatórios para a média com cenário $1, \phi=70, N=40$ e $n=5$, em que $s=1(\bullet), 2(\bullet)$, e dos verdadeiros valores dos parâmetros (em vermelho). . . . . . . . 150

B.52 Gráfico de dispersão das estimativas pontuais - mediana a posteriori - dos estudos de simulação de $R=50$ bancos de dados do modelo de mistura beta linear com efeitos aleatórios para a média com cenário $2, \phi=30, N=40$ e $n=5$, em que $s=1(\bullet), 2(\mathbf{\bullet})$, e dos verdadeiros valores dos parâmetros (em vermelho) . . . . . . . 150

B.53 Gráfico de dispersão das estimativas pontuais - mediana a posteriori - dos estudos de simulação de $R=50$ bancos de dados do modelo de mistura beta linear com efeitos aleatórios para a média com cenário $2, \phi=50, N=40$ e $n=5$, em que $s=1(\bullet), 2(\bullet)$, e dos verdadeiros valores dos parâmetros (em vermelho) . . . . . . 151

B.54 Gráfico de dispersão das estimativas pontuais - mediana a posteriori - dos estudos de simulação de $R=50$ bancos de dados do modelo de mistura beta linear com efeitos aleatórios para a média com cenário $2, \phi=70, N=40$ e $n=5$, em que $s=1(\bullet), 2(\bullet)$, e dos verdadeiros valores dos parâmetros (em vermelho) . . . . . . 151

B.55 Gráfico de dispersão das estimativas pontuais - mediana a posteriori - dos estudos de simulação de $R=50$ bancos de dados do modelo de mistura beta linear com efeitos aleatórios para a média com cenário $3, \phi=30, N=40$ e $n=5$, em que $s=1(\bullet), 2(\boldsymbol{\bullet})$, e dos verdadeiros valores dos parâmetros (em vermelho) . . . . . . . 152

B.56 Gráfico de dispersão das estimativas pontuais - mediana a posteriori - dos estudos de simulação de $R=50$ bancos de dados do modelo de mistura beta linear com efeitos aleatórios para a média com cenário $3, \phi=50, N=40$ e $n=5$, em que $s=1(\bullet), 2(\bullet)$, e dos verdadeiros valores dos parâmetros (em vermelho) . . . . . . . 152

B.57 Gráfico de dispersão das estimativas pontuais - mediana a posteriori - dos estudos de simulação de $R=50$ bancos de dados do modelo de mistura beta linear com efeitos aleatórios para a média com cenário $3, \phi=70, N=40$ e $n=5$, em que $s=1(\bullet), 2(\bullet)$, e dos verdadeiros valores dos parâmetros (em vermelho) . . . . . . . 153

B.58 Gráfico de dispersão das estimativas pontuais - mediana a posteriori - dos estudos de simulação de $R=50$ bancos de dados do modelo de mistura beta linear com efeitos aleatórios para a média com cenário $4, \phi=30, N=40$ e $n=5$, em que $s=1(\bullet), 2(\bullet)$, e dos verdadeiros valores dos parâmetros (em vermelho) . . . . . . 153

B.59 Gráfico de dispersão das estimativas pontuais - mediana a posteriori - dos estudos de simulação de $R=50$ bancos de dados do modelo de mistura beta linear com efeitos aleatórios para a média com cenário $4, \phi=50, N=40$ e $n=5$, em que $s=1(\bullet), 2(\bullet)$, e dos verdadeiros valores dos parâmetros (em vermelho) . . . . . . . 154

B.60 Gráfico de dispersão das estimativas pontuais - mediana a posteriori - dos estudos de simulação de $R=50$ bancos de dados do modelo de mistura beta linear com efeitos aleatórios para a média com cenário $4, \phi=70, N=40$ e $n=5$, em que $s=1(\bullet), 2(\bullet)$, e dos verdadeiros valores dos parâmetros (em vermelho) . . . . . . 154 
B.61 Gráfico de dispersão das estimativas pontuais - mediana a posteriori - dos estudos de simulação de $R=50$ bancos de dados do modelo de mistura beta linear com efeitos aleatórios para a média com cenário $1, \phi=30, N=100, n=5$ e $\Sigma_{\beta^{s}}=40 I_{2}$, em que $s=1(\bullet), 2(\bullet)$, e dos verdadeiros valores dos parâmetros (em vermelho). . . . . 155

B.62 Gráfico de dispersão das estimativas pontuais - mediana a posteriori - dos estudos de simulação de $R=50$ bancos de dados do modelo de mistura beta linear com efeitos aleatórios para a média com cenário $1, \phi=50, N=100, n=5$ e $\Sigma_{\beta^{s}}=40 I_{2}$, em que $s=1(\bullet), 2(\mathbf{\bullet})$, e dos verdadeiros valores dos parâmetros (em vermelho). . . . 156

B.63 Gráfico de dispersão das estimativas pontuais - mediana a posteriori - dos estudos de simulação de $R=50$ bancos de dados do modelo de mistura beta linear com efeitos aleatórios para a média com cenário $1, \phi=70, N=100, n=5$ e $\Sigma_{\beta^{s}}=40 I_{2}$, em que $s=1(\bullet), 2(\boldsymbol{\bullet})$, e dos verdadeiros valores dos parâmetros (em vermelho). . . . . 156

B.64 Gráfico de dispersão das estimativas pontuais - mediana a posteriori - dos estudos de simulação de $R=50$ bancos de dados do modelo de mistura beta linear com efeitos aleatórios para a média com cenário $2, \phi=30, N=100, n=5$ e $\Sigma_{\beta^{s}}=40 I_{2}$, em que $s=1(\bullet), 2(\bullet)$, e dos verdadeiros valores dos parâmetros (em vermelho). . . . . 157

B.65 Gráfico de dispersão das estimativas pontuais - mediana a posteriori - dos estudos de simulação de $R=50$ bancos de dados do modelo de mistura beta linear com efeitos aleatórios para a média com cenário $2, \phi=50, N=100, n=5$ e $\Sigma_{\beta^{s}}=40 I_{2}$, em que $s=1(\bullet), 2(\boldsymbol{\bullet})$, e dos verdadeiros valores dos parâmetros (em vermelho). . . . . 157

B.66 Gráfico de dispersão das estimativas pontuais - mediana a posteriori - dos estudos de simulação de $R=50$ bancos de dados do modelo de mistura beta linear com efeitos aleatórios para a média com cenário $2, \phi=70, N=100, n=5$ e $\Sigma_{\beta^{s}}=40 I_{2}$, em que $s=1(\bullet), 2(\bullet)$, e dos verdadeiros valores dos parâmetros (em vermelho). . . . . 158

B.67 Gráfico de dispersão das estimativas pontuais - mediana a posteriori - dos estudos de simulação de $R=50$ bancos de dados do modelo de mistura beta linear com efeitos aleatórios para a média e precisão com respectivos cenários para os parâmetros fixos: 1 e 1 , em que $s=1(\bullet), 2(\bullet)$, e dos verdadeiros valores dos parâmetros (em vermelho).159

B.68 Gráfico de dispersão das estimativas pontuais - mediana a posteriori - dos estudos de simulação de $R=50$ bancos de dados do modelo de mistura beta linear com efeitos aleatórios para a média e precisão com respectivos cenários para os parâmetros fixos: 1 e 2 , em que $s=1(\bullet), 2(\bullet)$, e dos verdadeiros valores dos parâmetros (em vermelho). 160

B.69 Gráfico de dispersão das estimativas pontuais - mediana a posteriori - dos estudos de simulação de $R=50$ bancos de dados do modelo de mistura beta linear com efeitos aleatórios para a média e precisão com respectivos cenários para os parâmetros fixos: 1 e 3, em que $s=1(\bullet), 2(\bullet)$, e dos verdadeiros valores dos parâmetros (em vermelho).160

B.70 Gráfico de dispersão das estimativas pontuais - mediana a posteriori - dos estudos de simulação de $R=50$ bancos de dados do modelo de mistura beta linear com efeitos aleatórios para a média e precisão com respectivos cenários para os parâmetros fixos: 2 e 1 , em que $s=1(\bullet), 2(\bullet)$, e dos verdadeiros valores dos parâmetros (em vermelho).161 
B.71 Gráfico de dispersão das estimativas pontuais - mediana a posteriori - dos estudos de simulação de $R=50$ bancos de dados do modelo de mistura beta linear com efeitos aleatórios para a média e precisão com respectivos cenários para os parâmetros fixos: 2 e 2 , em que $s=1(\bullet), 2(\bullet)$, e dos verdadeiros valores dos parâmetros (em vermelho).161

B.72 Gráfico de dispersão das estimativas pontuais - mediana a posteriori - dos estudos de simulação de $R=50$ bancos de dados do modelo de mistura beta linear com efeitos aleatórios para a média e precisão com respectivos cenários para os parâmetros fixos: 2 e 3, em que $s=1(\bullet), 2(\boldsymbol{\bullet})$, e dos verdadeiros valores dos parâmetros (em vermelho).162

B.73 Gráfico de dispersão das estimativas pontuais - mediana a posteriori - dos estudos de simulação de $R=50$ bancos de dados do modelo de mistura beta linear com efeitos aleatórios para a média e precisão com respectivos cenários para os parâmetros fixos: 3 e 1 , em que $s=1(\bullet), 2(\bullet)$, e dos verdadeiros valores dos parâmetros (em vermelho).162

B.74 Gráfico de dispersão das estimativas pontuais - mediana a posteriori - dos estudos de simulação de $R=50$ bancos de dados do modelo de mistura beta linear com efeitos aleatórios para a média e precisão com respectivos cenários para os parâmetros fixos: 3 e 2 , em que $s=1(\bullet), 2(\bullet)$, e dos verdadeiros valores dos parâmetros (em vermelho).163

B.75 Gráfico de dispersão das estimativas pontuais - mediana a posteriori - dos estudos de simulação de $R=50$ bancos de dados do modelo de mistura beta linear com efeitos aleatórios para a média e precisão com respectivos cenários para os parâmetros fixos: 3 e 3, em que $s=1(\bullet), 2(\bullet)$, e dos verdadeiros valores dos parâmetros (em vermelho).163

B.76 Gráfico de dispersão das estimativas pontuais - mediana a posteriori - dos estudos de simulação de $R=50$ bancos de dados do modelo de mistura beta linear com efeitos aleatórios para a média e precisão com respectivos cenários para os parâmetros fixos: 4 e 1 , em que $s=1(\bullet), 2(\bullet)$, e dos verdadeiros valores dos parâmetros (em vermelho).164

B.77 Gráfico de dispersão das estimativas pontuais - mediana a posteriori - dos estudos de simulação de $R=50$ bancos de dados do modelo de mistura beta linear com efeitos aleatórios para a média e precisão com respectivos cenários para os parâmetros fixos: 4 e 2 , em que $s=1(\bullet), 2(\bullet)$, e dos verdadeiros valores dos parâmetros (em vermelho).164

B.78 Gráfico de dispersão das estimativas pontuais - mediana a posteriori - dos estudos de simulação de $R=50$ bancos de dados do modelo de mistura beta linear com efeitos aleatórios para a média e precisão com respectivos cenários para os parâmetros fixos: 4 e 3, em que $s=1(\bullet), 2(\bullet)$, e dos verdadeiros valores dos parâmetros (em vermelho).165

B.79 Gráfico de dispersão das estimativas pontuais - mediana a posteriori - dos estudos de simulação de $R=50$ bancos de dados do modelo de mistura beta não linear com efeitos aleatórios para a média com cenário $1, \phi=30, N=100$ e $n=5$, em que $s=1(\bullet), 2(\bullet)$, e dos verdadeiros valores dos parâmetros (em vermelho) . . . . . 166

B.80 Gráfico de dispersão das estimativas pontuais - mediana a posteriori - dos estudos de simulação de $R=50$ bancos de dados do modelo de mistura beta não linear com efeitos aleatórios para a média com cenário $1, \phi=50, N=100$ e $n=5$, em que $s=1(\bullet), 2(\bullet)$, e dos verdadeiros valores dos parâmetros (em vermelho) . . . . . 167 
B.81 Gráfico de dispersão das estimativas pontuais - mediana a posteriori - dos estudos de simulação de $R=50$ bancos de dados do modelo de mistura beta não linear com efeitos aleatórios para a média com cenário $1, \phi=70, N=100$ e $n=5$, em que $s=1(\bullet), 2(\bullet)$, e dos verdadeiros valores dos parâmetros (em vermelho) . . . . . . 167

B.82 Gráfico de dispersão das estimativas pontuais - mediana a posteriori - dos estudos de simulação de $R=50$ bancos de dados do modelo de mistura beta não linear com efeitos aleatórios para a média com cenário $2, \phi=30, N=100$ e $n=5$, em que $s=1(\bullet), 2(\bullet)$, e dos verdadeiros valores dos parâmetros (em vermelho). . . . . . 168

B.83 Gráfico de dispersão das estimativas pontuais - mediana a posteriori - dos estudos de simulação de $R=50$ bancos de dados do modelo de mistura beta não linear com efeitos aleatórios para a média com cenário $2, \phi=50, N=100$ e $n=5$, em que $s=1(\bullet), 2(\bullet)$, e dos verdadeiros valores dos parâmetros (em vermelho) . . . . . . 168

B.84 Gráfico de dispersão das estimativas pontuais - mediana a posteriori - dos estudos de simulação de $R=50$ bancos de dados do modelo de mistura beta não linear com efeitos aleatórios para a média com cenário $2, \phi=70, N=100$ e $n=5$, em que

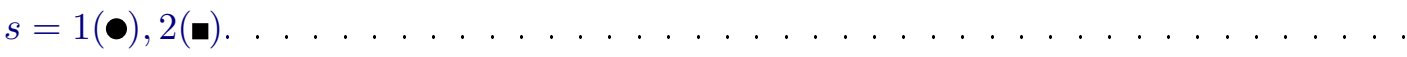

B.85 Gráfico de dispersão das estimativas pontuais - mediana a posteriori - dos parâmetros $\beta_{i_{p}}^{s}, i_{p}=1,2,3 s=1,2$, dos estudos de simulação de $R=25$ bancos de dados do modelo de mistura beta não linear com efeitos aleatórios para a média com cenário 3.169

B.86 Gráfico de dispersão das estimativas pontuais - mediana a posteriori - dos parâmetros $\sigma_{b, i_{d 1} i_{d 2}}, i_{d 1}, i_{d 2} \in\{1,2,3\}$ dos estudos de simulação de $R=25$ bancos de dados do modelo de mistura beta não linear com efeitos aleatórios para a média com cenário 3.170

B.87 Gráfico de dispersão das estimativas pontuais - mediana a posteriori - dos parâmetros $\phi_{1}, \phi_{2}$ e $\pi_{1}$ dos estudos de simulação de $R=25$ bancos de dados do modelo de mistura beta não linear com efeitos aleatórios para a média com cenário 3. . . . . . . . . . 170 


\section{Lista de Tabelas}

3.1 Parâmetros e distribuições a priori indicadas para ajuste do modelo de mistura beta linear com efeitos aleatórios para a média, $s=1, \ldots, K$ e $i=1, \ldots, N \ldots \ldots 36$

3.2 Definição dos cenários estudados por simulação para os parâmetros $\boldsymbol{\beta}^{s}, s=1,2$. . . 38

3.3 Porcentagem das 10 réplicas simuladas do modelo de mistura beta linear com efeitos aleatórios para a média cujos critérios EAIC, ECAIC, EBIC, $D I C_{3}$ e LPML selecionaram cada um dos 5 possíveis valores de números de componentes da mistura $(K)$

3.4 Estimativas pontuais (média a posteriori) dos parâmetros, desvis padrões e intervalos de credibilidade de $95 \%$ do modelo final com $K=1$ ajustado para a variável antioxLDL dos dados de periodontite. . . . . . . . . . . . . . . . . 49

3.5 Valores dos critérios de informação EAIC, ECAIC, EBIC, $D I C_{3}$ e LPML para o modelo (3.11) com $K=1,2,3$ para os dados de periodontite. . . . . . . . . 49

3.6 Estimativas pontuais (média a posteriori) dos parâmetros, desvios padrões e intervalos de credibilidade de $95 \%$ do modelo final ajustado para a variável anti-oxLDL dos dados de periodontite. . . . . . . . . . . . . . . . . . . 50 50

3.7 Valores dos critérios de informação EAIC, ECAIC, EBIC, $D I C_{3}$ e LPML para o ajuste do modelo (3.13) com $K=1,2,3$ da variável índice de Gini dos dados de redes de fast food no Brasil. . . . . . . . . . . . . . . . . . . . . 56

3.8 Estimativas pontuais (mediana a posteriori) dos parâmetros, desvios padrões e intervalos de credibilidade de $90 \%$ do modelo final ajustado para a variável índice de Gini dos dados de redes de fast food no Brasil. . . . . . . . . . . . . . . . . . 5

4.1 Parâmetros e distribuições a priori indicadas para ajuste simultâneo do modelo de mistura beta linear com efeitos aleatórios para a média e precisão, $s=1, \ldots, K$ e

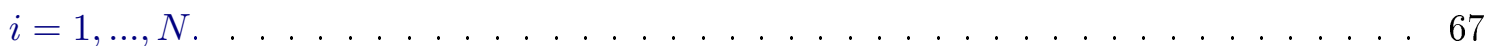

4.2 Definição dos cenários estudados por simulação para os parâmetros $\boldsymbol{\beta}^{s}, s=1,2$. . . 69

4.3 Definição dos cenários estudados por simulação para os parâmetros $\delta$. . . . . . . 69

4.4 Porcentagem das 10 réplicas simuladas do modelo de mistura beta linear com efeitos aleatórios para a média e precisão cujos critérios EAIC, ECAIC, EBIC, $D I C_{3}$ e LPML selecionaram cada um dos 5 possíveis valores de números de componentes da

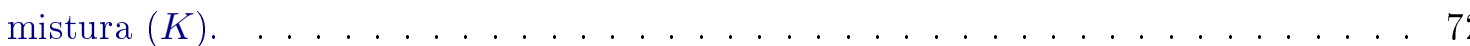

4.5 Estimativas pontuais (mediana a posteriori) dos parâmetros, desvios padrões e intervalos de credibilidade (IC) de $95 \%$ do modelo final com $K=1$ ajustado para a variável anti-oxLDL dos dados de periodontite. . . . . . . . . . . . . 75 
4.6 Valores dos critérios de informação EAIC, ECAIC, EBIC, $D I C_{3}$ e LPML para o ajuste do modelo (4.10) com $K=1,2$ para os dados de periodontite. . . . . . . . 76

4.7 Estimativas pontuais (média a posteriori) dos parâmetros, desvios padrões e intervalos de credibilidade de $95 \%$ do modelo final ajustado para a variável anti-oxLDL dos dados de periodontite. . . . . . . . . . . . . . . . . 77

5.1 Parâmetros e distribuições a priori indicadas para ajuste do modelo de mistura beta não linear com efeitos aleatórios para a média, $s=1, \ldots, K$ e $i=1, \ldots, N \ldots \ldots$. . . 86

5.2 Definição dos cenários estudados por simulação para os parâmetros $\boldsymbol{\beta}^{s}, s=1,2 \ldots$. . 87

5.3 Porcentagem das 10 réplicas simuladas do modelo de mistura beta não linear com efeitos aleatórios para a média cujos critérios EAIC, ECAIC, EBIC, $D I C_{3}$ e LPML selecionaram cada um dos 5 possíveis valores de números de componentes da mistura $(K)$

5.4 Valores dos critérios de informação EAIC, ECAIC, EBIC para o ajuste do modelo (5.9) com $K=1,2,3$ para os dados de habilidade de leitura do estudo ECLS-K. . . . 96

5.5 Estimativas pontuais (mediana a posteriori) dos parâmetros, desvios padrões e intervalos de credibilidade (IC) de $95 \%$ do modelo final ajustado para os dados de habilidade de leitura do estudo ECLS-K. . . . . . . . . . . . . . . .

A.1 Resultados descritivos: ER, EAR e REQM, da mediana a posteriori dos parâmetros do modelo de mistura beta linear com efeitos aleatórios para a média dos estudos de simulação com cenário 1 e $\phi=30$ de $R$ bancos de dados com tamanhos amostrais

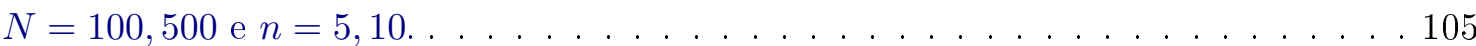

A.2 Resultados descritivos: ER, EAR e REQM, da mediana a posteriori dos parâmetros do modelo de mistura beta linear com efeitos aleatórios para a média dos estudos de simulação com cenário 1 e $\phi=50$ de $R$ bancos de dados com tamanhos amostrais

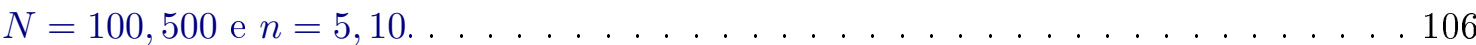

A.3 Resultados descritivos: ER, EAR e REQM, da mediana a posteriori dos parâmetros do modelo de mistura beta linear com efeitos aleatórios para a média dos estudos de simulação com cenário 1 e $\phi=70$ de $R$ bancos de dados com tamanhos amostrais

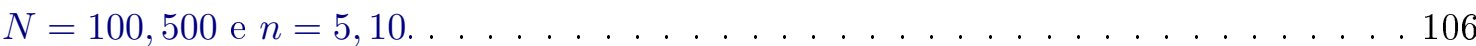

A.4 Resultados descritivos: ER, EAR e REQM, da mediana a posteriori dos parâmetros do modelo de mistura beta linear com efeitos aleatórios para a média dos estudos de simulação com cenário 2 e $\phi=30$ de $R$ bancos de dados com tamanhos amostrais

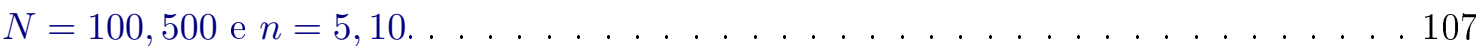

A.5 Resultados descritivos: ER, EAR e REQM, da mediana a posteriori dos parâmetros do modelo de mistura beta linear com efeitos aleatórios para a média dos estudos de simulação com cenário 2 e $\phi=50$ de $R$ bancos de dados com tamanhos amostrais

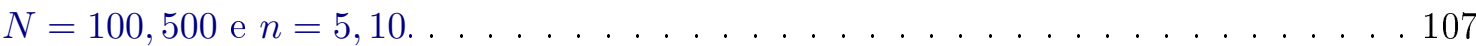

A.6 Resultados descritivos: ER, EAR e REQM, da mediana a posteriori dos parâmetros do modelo de mistura beta linear com efeitos aleatórios para a média dos estudos de simulação com cenário 2 e $\phi=70$ de $R$ bancos de dados com tamanhos amostrais

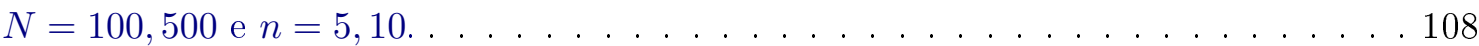


A.7 Resultados descritivos: ER, EAR e REQM, da mediana a posteriori dos parâmetros do modelo de mistura beta linear com efeitos aleatórios para a média dos estudos de simulação com cenário 3 e $\phi=30$ de $R$ bancos de dados com tamanhos amostrais

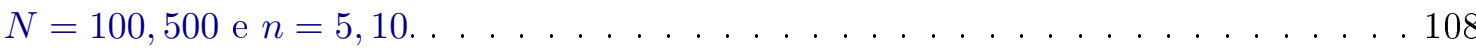

A.8 Resultados descritivos: ER, EAR e REQM, da mediana a posteriori dos parâmetros do modelo de mistura beta linear com efeitos aleatórios para a média dos estudos de simulação com cenário 3 e $\phi=50$ de $R$ bancos de dados com tamanhos amostrais

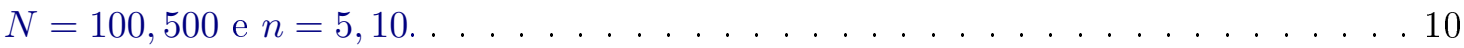

A.9 Resultados descritivos: ER, EAR e REQM, da mediana a posteriori dos parâmetros do modelo de mistura beta linear com efeitos aleatórios para a média dos estudos de simulação com cenário 3 e $\phi=70$ de $R$ bancos de dados com tamanhos amostrais

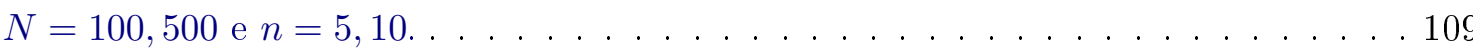

A.10 Resultados descritivos: ER, EAR e REQM, da mediana a posteriori dos parâmetros do modelo de mistura beta linear com efeitos aleatórios para a média dos estudos de simulação com cenário 4 e $\phi=30$ de $R$ bancos de dados com tamanhos amostrais $N=100,500$ e $n=5,10$.

A.11 Resultados descritivos: ER, EAR e REQM, da mediana a posteriori dos parâmetros do modelo de mistura beta linear com efeitos aleatórios para a média dos estudos de simulação com cenário 4 e $\phi=50$ de $R$ bancos de dados com tamanhos amostrais $N=100,500$ e $n=5,10$.

A.12 Resultados descritivos: ER, EAR e REQM, da mediana a posteriori dos parâmetros do modelo de mistura beta linear com efeitos aleatórios para a média dos estudos de simulação com cenário 4 e $\phi=70$ de $R$ bancos de dados com tamanhos amostrais $N=100,500$ e $n=5,10$.

A.13 Resultados descritivos: ER, EAR e REQM, da mediana a posteriori dos parâmetros do modelo de mistura beta linear com efeitos aleatórios para a média dos estudos de simulação com cenário 1 e $\phi=30$ de $R=50$ bancos de dados e com $N=100, n=5$ e $\Sigma_{\beta^{s}}=40 I_{2} \ldots \ldots \ldots \ldots \ldots \ldots \ldots \ldots \ldots \ldots \ldots \ldots \ldots \ldots \ldots \ldots \ldots \ldots$

A.14 Resultados descritivos: ER, EAR e REQM, da mediana a posteriori dos parâmetros do modelo de mistura beta linear com efeitos aleatórios para a média dos estudos de simulação com cenário 1 e $\phi=50$ de $R=50$ bancos de dados e com $N=100, n=5$ e $\Sigma_{\beta^{s}}=40 I_{2} \ldots \ldots \ldots \ldots \ldots \ldots \ldots \ldots \ldots \ldots \ldots \ldots \ldots \ldots \ldots \ldots \ldots \ldots \ldots \ldots$

A.15 Resultados descritivos: ER, EAR e REQM, da mediana a posteriori dos parâmetros do modelo de mistura beta linear com efeitos aleatórios para a média dos estudos de simulação com cenário 1 e $\phi=70$ de $R=50$ bancos de dados e com $N=100, n=5$ e $\Sigma_{\beta^{s}}=40 I_{2} \ldots \ldots \ldots \ldots \ldots \ldots \ldots \ldots \ldots \ldots \ldots \ldots \ldots \ldots \ldots \ldots \ldots \ldots \ldots \ldots$

A.16 Resultados descritivos: ER, EAR e REQM, da mediana a posteriori dos parâmetros do modelo de mistura beta linear com efeitos aleatórios para a média dos estudos de simulação com cenário 2 e $\phi=30$ de $R=50$ bancos de dados e com $N=100, n=5$

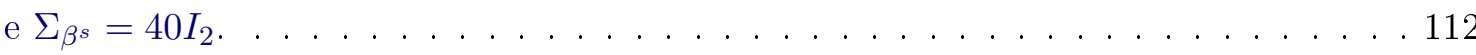


A.17 Resultados descritivos: ER, EAR e REQM, da mediana a posteriori dos parâmetros do modelo de mistura beta linear com efeitos aleatórios para a média dos estudos de simulação com cenário 2 e $\phi=50$ de $R=50$ bancos de dados e com $N=100, n=5$

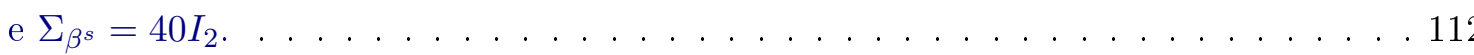

A.18 Resultados descritivos: ER, EAR e REQM, da mediana a posteriori dos parâmetros do modelo de mistura beta linear com efeitos aleatórios para a média dos estudos de simulação com cenário 2 e $\phi=70$ de $R=50$ bancos de dados e com $N=100, n=5$

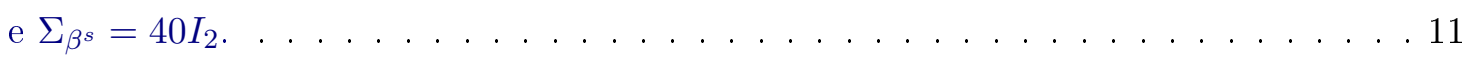

A.19 Resultados descritivos: ER, EAR e REQM, da mediana a posteriori dos parâmetros do modelo de mistura beta linear com efeitos aleatórios para a média dos estudos de simulação com cenário 1 e $\phi=30$ de $R=50$ bancos de dados e com $N=40, n=5$.

A.20 Resultados descritivos: ER, EAR e REQM, da mediana a posteriori dos parâmetros do modelo de mistura beta linear com efeitos aleatórios para a média dos estudos de simulação com cenário 1 e $\phi=50$ de $R=50$ bancos de dados e com $N=40, n=5$.

A.21 Resultados descritivos: ER, EAR e REQM, da mediana a posteriori dos parâmetros do modelo de mistura beta linear com efeitos aleatórios para a média dos estudos de simulação com cenário 1 e $\phi=70$ de $R=50$ bancos de dados e com $N=40, n=5$.

A.22 Resultados descritivos: ER, EAR e REQM, da mediana a posteriori dos parâmetros do modelo de mistura beta linear com efeitos aleatórios para a média dos estudos de simulação com cenário 2 e $\phi=30$ de $R=50$ bancos de dados e com $N=40, n=5$.

A.23 Resultados descritivos: ER, EAR e REQM, da mediana a posteriori dos parâmetros do modelo de mistura beta linear com efeitos aleatórios para a média dos estudos de simulação com cenário 2 e $\phi=50$ de $R=50$ bancos de dados e com $N=40, n=5$.

A.24 Resultados descritivos: ER, EAR e REQM, da mediana a posteriori dos parâmetros do modelo de mistura beta linear com efeitos aleatórios para a média dos estudos de simulação com cenário 2 e $\phi=70$ de $R=50$ bancos de dados e com $N=40, n=5$.

A.25 Resultados descritivos: ER, EAR e REQM, da mediana a posteriori dos parâmetros do modelo de mistura beta linear com efeitos aleatórios para a média dos estudos de simulação com cenário 3 e $\phi=30$ de $R=50$ bancos de dados e com $N=40, n=5$.

A.26 Resultados descritivos: ER, EAR e REQM, da mediana a posteriori dos parâmetros do modelo de mistura beta linear com efeitos aleatórios para a média dos estudos de simulação com cenário 3 e $\phi=50$ de $R=50$ bancos de dados e com $N=40, n=5$.

A.27 Resultados descritivos: ER, EAR e REQM, da mediana a posteriori dos parâmetros do modelo de mistura beta linear com efeitos aleatórios para a média dos estudos de simulação com cenário 3 e $\phi=70$ de $R=50$ bancos de dados e com $N=40, n=5$.

A.28 Resultados descritivos: ER, EAR e REQM, da mediana a posteriori dos parâmetros do modelo de mistura beta linear com efeitos aleatórios para a média dos estudos de simulação com cenário 4 e $\phi=30$ de $R=50$ bancos de dados e com $N=40, n=5$.

A.29 Resultados descritivos: ER, EAR e REQM, da mediana a posteriori dos parâmetros do modelo de mistura beta linear com efeitos aleatórios para a média dos estudos de simulação com cenário 4 e $\phi=50$ de $R=50$ bancos de dados e com $N=40, n=5$. 
A.30 Resultados descritivos: ER, EAR e REQM, da mediana a posteriori dos parâmetros do modelo de mistura beta linear com efeitos aleatórios para a média dos estudos de simulação com cenário 4 e $\phi=70$ de $R=50$ bancos de dados e com $N=40, n=5$.

A.31 Resultados descritivos: ER, EAR e REQM, da mediana a posteriori dos parâmetros do modelo de mistura beta linear com efeitos aleatórios para a média e precisão dos estudos de simulação com respectivos cenários para os parâmetros fixos: 1 e 1 de $R=50$ bancos de dados.

A.32 Resultados descritivos: ER, EAR e REQM, da mediana a posteriori dos parâmetros do modelo de mistura beta linear com efeitos aleatórios para a média e precisão dos estudos de simulação com respectivos cenários para os parâmetros fixos: 1 e 2 de $R=50$ bancos de dados.

A.33 Resultados descritivos: ER, EAR e REQM, da mediana a posteriori dos parâmetros do modelo de mistura beta linear com efeitos aleatórios para a média e precisão dos estudos de simulação com respectivos cenários para os parâmetros fixos: 1 e 3 de $R=50$ bancos de dados

A.34 Resultados descritivos: ER, EAR e REQM, da mediana a posteriori dos parâmetros do modelo de mistura beta linear com efeitos aleatórios para a média e precisão dos estudos de simulação com respectivos cenários para os parâmetros fixos: 2 e 1 de $R=50$ bancos de dados

A.35 Resultados descritivos: ER, EAR e REQM, da mediana a posteriori dos parâmetros do modelo de mistura beta linear com efeitos aleatórios para a média e precisão dos estudos de simulação com respectivos cenários para os parâmetros fixos: 2 e 2 de $R=50$ bancos de dados

A.36 Resultados descritivos: ER, EAR e REQM, da mediana a posteriori dos parâmetros do modelo de mistura beta linear com efeitos aleatórios para a média e precisão dos estudos de simulação com respectivos cenários para os parâmetros fixos: 3 e 1 de $R=50$ bancos de dados

A.37 Resultados descritivos: ER, EAR e REQM, da mediana a posteriori dos parâmetros do modelo de mistura beta linear com efeitos aleatórios para a média e precisão dos estudos de simulação com respectivos cenários para os parâmetros fixos: 3 e 2 de $R=50$ bancos de dados

A.38 Resultados descritivos: ER, EAR e REQM, da mediana a posteriori dos parâmetros do modelo de mistura beta linear com efeitos aleatórios para a média e precisão dos estudos de simulação com respectivos cenários para os parâmetros fixos: 4 e 1 de $R=50$ bancos de dados

A.39 Resultados descritivos: ER, EAR e REQM, da mediana a posteriori dos parâmetros do modelo de mistura beta linear com efeitos aleatórios para a média e precisão dos estudos de simulação com respectivos cenários para os parâmetros fixos: 4 e 2 de $R=50$ bancos de dados

A.40 Resultados descritivos: ER, EAR e REQM, da mediana a posteriori dos parâmetros do modelo de mistura beta linear com efeitos aleatórios para a média e precisão dos estudos de simulação com respectivos cenários para os parâmetros fixos: 4 e 3 de $R=50$ bancos de dados. . . . . . . . . . . . . . . . . . . . . . 
A.41 Resultados descritivos: ER, EAR e REQM, da mediana a posteriori dos parâmetros do modelo de mistura beta não linear com efeitos aleatórios para a média dos estudos de simulação com cenário 1 e $\phi=30$ de $R=50$ bancos de dados e com $N=100$, $n=5$.

A.42 Resultados descritivos: ER, EAR e REQM, da mediana a posteriori dos parâmetros do modelo de mistura beta não linear com efeitos aleatórios para a média dos estudos de simulação com cenário 1 e $\phi=50$ de $R=50$ bancos de dados e com $N=100$, $n=5$.

A.43 Resultados descritivos: ER, EAR e REQM, da mediana a posteriori dos parâmetros do modelo de mistura beta não linear com efeitos aleatórios para a média dos estudos de simulação com cenário 1 e $\phi=70$ de $R=50$ bancos de dados e com $N=100$, $n=5$.

A.44 Resultados descritivos: ER, EAR e REQM, da mediana a posteriori dos parâmetros do modelo de mistura beta não linear com efeitos aleatórios para a média dos estudos de simulação com cenário 2 e $\phi=30$ de $R=50$ bancos de dados e com $N=100$, $n=5$.

A.45 Resultados descritivos: ER, EAR e REQM, da mediana a posteriori dos parâmetros do modelo de mistura beta não linear com efeitos aleatórios para a média dos estudos de simulação com cenário 2 e $\phi=50$ de $R=50$ bancos de dados e com $N=100$, $n=5$.

A.46 Resultados descritivos: ER, EAR e REQM, da mediana a posteriori dos parâmetros do modelo de mistura beta não linear com efeitos aleatórios para a média dos estudos de simulação com cenário 2 e $\phi=70$ de $R=50$ bancos de dados e com $N=100$, $n=5$

A.47 Resultados descritivos: ER, EAR e REQM, da mediana a posteriori dos parâmetros $\beta_{i_{p}}^{s}, \phi_{s}, i_{p}=1,2,3, s=1,2$ e $\pi_{1}$ do modelo de mistura beta não linear com efeitos aleatórios para a média dos estudos de simulação com cenário 3 de $R=25$ bancos de dados.

A.48 Resultados descritivos: ER, EAR e REQM, da mediana a posteriori dos parâmetros $\sigma_{b, i_{d 1} i_{d 2}}, i_{d 1}, i_{d 2} \in\{1,2,3\}$ do modelo de mistura beta não linear com efeitos aleatórios para a média dos estudos de simulação com cenário 3 de $R=25$ bancos de dados. . 120 


\section{Capítulo 1}

\section{Introdução}

\subsection{Considerações Preliminares}

É muito recorrente em pesquisas de distintos campos a existência de dados compostos por diferentes subpopulações com alocações desconhecidas, ou seja, apresentam heterogeneidade não observada. Os modelos de mistura são adequados para serem aplicados nessas situações e as inferências são obtidas com base na atribuição de uma distribuição de probabilidade para cada uma dessas subpopulações. Por causa dessa flexibilidade são muito eficazes para analisar dados que apresentam multimodalidade, curtose, assimetria, hetoregeineidade e que envolvem variáveis latentes.

$\mathrm{Na}$ área de genética, por exemplo, há interesse em agrupar genes com expressões afins (Ji et al. , 2005) e na área de psiquiatria pode ser interessante identificar grupos de pacientes com reações similares aos tratamentos para compreender os padrões observados (Xu e Hedeker, 2001).

Nos últimos anos os estudos referentes a esses modelos têm se propagado devido à facilidade de acesso a recursos computacionais, já que sua análise inferencial apresenta grande complexidade. Adicionalmente, o desenvolvimento de tecnologias de armazenamento e processamento mais eficentes teve como consequência o crescimento de estudos com dados agrupados ou hierárquicos.

Como exemplo de dados agrupados podem ser citados dados de medidas repetidas em que as informações de uma mesma unidade amostral são coletadas ao longo de um período, como aferições de características clínicas de pacientes, medições de desempenho escolar de alunos, informações de compras de clientes. Nesses casos não é adequado assumir que os dados de mesma unidade amostral são independentes.

O modelo de regressão beta com efeitos aleatórios é extremamente recomendado para analisar dados correlacionados que assumem valores num intervalo restrito conhecido. Estes modelos são teoricamente estruturados para ajustar variáveis resposta com valores limitados e uma possível estrutura de correlação presente nos dados, possibilitando a obtenção de resultados mais robustos do que métodos clássicos adaptados como transformações de variáveis e modelos de efeitos fixos.

Assim, o objetivo geral desta tese é relacionar os conceitos de distribuição de probabilidade beta e de mistura de distribuições à técnica de ajuste de modelos de regressão com efeitos aleatórios propondo os modelos de mistura beta mistos sob uma abordagem bayesiana.

Os modelos de mistura beta mistos são apropriados para analisar dados que possuem ambas características: falta de independência e são formados por subgrupos homogêneos com relação a características não observadas. Dados de notas de alunos ao longo de um período é um exemplo no qual a aplicação destes modelos é de muita utilidade pois as notas de um mesmo aluno são 
correlacionadas e as subpopulações são compostas por alunos com padrões de desenvolvimento semelhantes no período.

No presente trabalho, tanto o caso em que o parâmetro de precisão é constante, quanto o caso de estimação simultânea de ajustes para a média e para a dispersão são abordados de forma inédita para modelos de mistura beta mistos lineares. Ademais, também de forma inédita, é formulado o modelo de mistura beta não linear com efeitos aleatórios e dispersão constante.

Todos os principais aspectos inferenciais destes modelos são tratados originalmente, como o desempenho da estimação dos parâmetros sob abordagem bayesiana, a performance de métodos de determinação do número de componentes da mistura e a identificabilidade dos modelos. Em função da complexidade dessa classe de modelos, esses tópicos foram estudados por meio de estudos de simulação.

O trabalho teve com foco principal estes modelos, porém pode-se considerar que uma metodologia geral foi proposta após uma revisão profunda da literatura e que pode ser amplicada a outros modelos de mistura que envolvem outros tipos de distribuições.

\subsection{Contribuições}

Em síntese, as contribuições desta tese são:

- especificação dos modelos de regressão de mistura de distribuições beta com efeitos aleatórios, cuja variável resposta possui espaço amostral restrito conhecido, além de poder apresentar estrutura de correlação, assimetria, curtose, multimodalidade e heterogeneidade em relação à variáveis não observadas;

- avaliação do desempenho da estimação dos parâmetros dos modelos em estudo sob a perspectiva da inferência bayesiana;

- estudo do desempenho de critérios de informação para determinação do número de componentes da mistura de distribuições;

- análise da identificabilidade dos modelos estudados;

- ilustração da metodologia proposta por meio de aplicações em dados reais.

\subsection{Estrutura do Trabalho}

No Capítulo 2 são apresentados e contextualizados aspectos presentes na literatura que são fundamentais à compreensão do conteúdo desenvolvido nos demais capítulos desta tese.

Os Capítulos 3, 4 e 5 especificam e discutem, respectivamente: o modelo de mistura beta linear com efeitos aleatórios e dispersão constante, o modelo de mistura beta linear com efeitos aleatórios e dispersão variável e o modelo de mistura beta não linear com efeitos aleatórios. A inferência de seus parâmetros, a seleção do número de componentes da mistura e o estudo de identificabilidade também são analisados por meio de estudos de simulação e em todos os casos são efetuadas aplicações em dados reais.

As considerações finais e propostas de estudos futuros estão expostos no Capítulo 6. 
Por fim, os trabalhos auxiliares consultados para desenvolvimento desta tese e que foram citados no texto, como livros, artigos e demais publicações, encontram-se listados nas referências bibliográficas.

\subsection{Suporte computacional}

Dentre o conteúdo desenvolvido nesta tese, o texto foi escrito através do sistema de composição tipográfica LaTeX e processado no editor TeXstudio, cujas informações podem ser encontradas nos respectivos sites http://www.latex-project.org/ e http://www.texstudio.org/.

Os modelos foram todos ajustados por meio do software OpenBUGS (Spiegelhalter et al., 1999) através do ambiente integrado RStudio (https://www.rstudio.com/) da linguagem de programação R versão 3.2.3 (R Core Team, 2016).

Os estudos de simulação foram efetuados com o auxílio dos recursos de computação de alto desempenho ou HPC (do inglês High Performance Computing) disponibilizados pela Superintendência de Tecnologia da Informação da Universidade de São Paulo (http://www.usp.br/hpc/).

Todas as rotinas computacionais encontram-se disponíveis na plataforma GitHub que pode ser acessada por meio do link https://github.com/apzerbeto/tese_2018_modelos_de_mistura_beta_ mistos. 


\section{Capítulo 2}

\section{Conceitos Preliminares}

Este capítulo apresenta e discute aspectos de conceitos fundamentais à compreensão do desenvolvimento deste trabalho, assim como referências bibliográficas indicadas aos leitores que desejem se aprofundar em algum conteúdo abordado; sua leitura não é imprescindível para entendimento da proposta desenvolvida nesta tese para leitores familiarizados com modelos de regressão e inferência bayesiana, sendo também possível de ser consultado a qualquer dúvida relativa aos tópicos abordados.

Primeiramente, a Seção 2.1 consiste de explicações da metodologia bayesiana e dos aspectos práticos e computacionais da inferência; em seguida, as Seções 2.2 e 2.3 contextualizam os modelos de regressão beta em suas formas linear com efeitos fixos e linear com efeitos aleatórios. Por fim, em 2.5 e 2.6 elucida-se a respeito das distribuições e modelos de misturas finitas.

\subsection{Inferência Bayesiana}

Esta seção tem o objetivo de apresentar os conceitos básicos da inferência bayesiana e dos métodos de Monte Carlo via Cadeias de Markov (MCMC) de forma a proporcionar uma compreensão da escolha desta abordagem e dos resultados obtidos neste trabalho. A literatura especializada nessa área é extensiva e abrange inúmeros tópicos teóricos e práticos que complementam e acrescentam o que é apresentado nessa seção, dentre elas destacam-se: Bernardo e Smith (1994), Chen et al. (2000), Robert e Casella (2004), Gamerman e Lopes (2006), Albert (2009) e Brooks et al. (2011).

A essência da inferência estatística paramétrica é obter informações a respeito de um vetor de parâmetros $\boldsymbol{\theta}$ não observável baseando-se nos dados observados $(\boldsymbol{y})$. O diferencial da abordagem bayesiana é a incorporação de modelos probabilísticos para $\boldsymbol{\theta}: p(\boldsymbol{\theta})$, denominada distribuição $a$ priori, possibilitando a atribuição de incerteza para os parâmetros, diferentemente da abordagem clássica, na qual os parâmetros são considerados quantidades também desconhecidas porém fixas.

Em linguagem informal pode-se dizer que a inferência bayesiana, por meio do teorema de Bayes, atualiza a informação existente sobre $\boldsymbol{\theta}$-expressada probabilisticamente por $p(\boldsymbol{\theta})$ - utilizando-se da informação disponível nos dados observados (expressada pela função de verossimilhança $L(\boldsymbol{y} \mid \boldsymbol{\theta})$ ), resultando na distribuição a posteriori: $p(\boldsymbol{\theta} \mid \boldsymbol{y})$. Em notação matemática, sejam:

- $p(\boldsymbol{\theta})$ a distribuição a priori, ou seja, o modelo probabilístico que expressa o conhecimento disponível sobre o vetor de parâmetros antes da observação dos dados; 
- $L(\boldsymbol{y} \mid \boldsymbol{\theta})$ a função de verossimilhança, ou seja, a distribuição dos dados observados condicionada ao vetor de parâmetros, que expressa quão provável é observar a amostra $\boldsymbol{y}$ para cada possível valor de $\boldsymbol{\theta}$;

- $p(\boldsymbol{\theta} \mid \boldsymbol{y})$ a distribuição a posteriori, a qual representa a informação de $\boldsymbol{\theta}$ após a incorporação do conhecimento da análise dos dados observados.

O teorema de Bayes expressa o cálculo de $p(\boldsymbol{\theta} \mid \boldsymbol{y})$ por meio da Expressão (2.1):

$$
p(\boldsymbol{\theta} \mid \boldsymbol{y})=\frac{L(\boldsymbol{y} \mid \boldsymbol{\theta}) p(\boldsymbol{\theta})}{\int_{\boldsymbol{\theta} \in \Theta} L(\boldsymbol{y} \mid \boldsymbol{\theta}) p(\boldsymbol{\theta}) d \boldsymbol{\theta}} \propto L(\boldsymbol{y} \mid \boldsymbol{\theta}) p(\boldsymbol{\theta}) .
$$

Se a expressão analítica da distribuição a posteriori é possível de ser calculada, a inferência sobre $\boldsymbol{\theta}$ após a observação de dados pode ser efetuada por meio de medidas resumo, gráficos, intervalos de credibilidade (IC), testes de hipóteses, dentre outras técnicas de análises possíveis de serem desenvolvidas a partir de densidades de probabilidades.

Porém, um dos grandes contribuintes da popularização da inferência bayesiana nas últimas duas décadas foi a facilidade de implementação e simulação de amostras das distribuições a posteriori através de algoritmos MCMC (para mais detalhes ver Subseção 2.1.2); nesses casos não se conhece a expressão analítica da distribuição a posteriori, porém, por meio dos algoritmos, se obtém amostras dessa distribuição e, com essas amostras, se faz possível analisar inferencialmente $\boldsymbol{\theta}$ após a observação de dados com o uso de medidas resumo amostrais -momentos e quartis amostrais-, intervalos de credibilidade (seção 2.2.2 de Gamerman e Lopes (2006)), gráficos da densidade estimada, dentre outros recursos.

\subsubsection{Distribuição a priori}

A noção de atribuição da incerteza a respeito do vetor de parâmetros $\boldsymbol{\theta}$, ou seja, a definição da distribuição a priori $p(\boldsymbol{\theta})$ pode ser feita de acordo com diversos critérios, desde os mais subjetivos até os que objetivam a obtenção de propriedades desejáveis.

Teoricamente, o ideal seria o estatístico especificar $p(\boldsymbol{\theta})$ baseando-se em informações coletadas do conhecimento prévio de especialistas da área de aplicação, como por exemplo: mínimo, máximo, quartis, correlação, mediana, dentre outras. Esse processo de coletar as informações subjetivas fornecidas pelos especialistas e expressá-las em um modelo probabilístico, apesar de apresentar ganho na análise, pode ser um fator que dificulte a utilização da inferência bayesiana por ser um processo individualizado que requer tempo e recursos específicos.

Assim sendo, técnicas de atribuição de distribuições a priori alternativas que não necessariamente expressam conhecimento sobre $\boldsymbol{\theta}$ ou são atribuídas antes da observação dos dados são amplamente estudadas e aplicadas, apesar da discussão constante acerca deste tópico.

Uma destas técnicas é a escolha de distribuições a priori não informativas ou pouco informativas, que são distribuições que associam probabilidades iguais a todos os possíveis valores de $\boldsymbol{\theta}$ ou a algum intervalo desse espaço paramétrico, e que podem ser úteis em cenários de desconhecimento prévio sobre o vetor de parâmetros. Porém, ao aplicar essa técnica pode-se obter distribuições a priori cuja massa de probabilidade no espaço amostral é diferente de um, caso este em que a distribuição é denominada imprópria; quando isso acontece, algumas vezes é possível que a distribuição a posteriori seja própria (sua massa de probabilidade no espaço amostral é igual a um), mas essa verificação 
não é trivial em muitas ocasiões. Gelman (2009) apresenta e discute a performance de opções de distribuições a priori não informativas fundamentadas teoricamente com resultados demonstrados interessantes como distribuição a priori de Jeffreys e de Bernardo.

Outra forma usual é a escolha de distribuições a priori de forma a obter famílias de distribuições conjugadas, ou seja, a família paramétrica da distribuição a priori, $p(\boldsymbol{\theta})$, e a posteriori, $p(\boldsymbol{\theta} \mid \boldsymbol{y})$, é a mesma e, o que são atualizados são os parâmetros das distribuições dessa família, denominados hiperparâmetros. As vantagens das famílias conjugadas estão relacionadas às propriedades teóricas resultantes, o que proporciona maior facilidade para cálculos analíticos como o de medidas resumo da distribuição a posteriori e para implementação de algoritmos como os apresentados na Seção 2.1.2.

Alternativamente, a atribuição de distribuições a priori informativas pode ser efetuada através do método de Bayes empírico. A ideia central deste método é definir os hiperparâmetros baseandose nas análises, paramétricas e não paramétricas, dos dados (Robbins, 1956; Carlin e Louis, 2000). Convém salientar que, mesmo sendo esse um método não filosoficamente compatível com os métodos bayesianos de atribuição de distribuições a priori sem a observação dos dados, cabe ao profissional que esteja analisando os dados optar pelo método de atribuição da distribuição a priori que mais lhe seja conveniente. Um exemplo de utilização de Bayes empírico é apresentado em Johnson et al. (2007), no qual o autor fez uso de prioris paramétricas empíricas ao estimar seus hiperparâmetros através do método dos momentos.

Durante um longo período a escolha de distribuições a priori em famílias conjugadas se deu em função da facilidade de implementação e de cálculo, porém, com o crescimento da acessibilidade a recursos computacionais cada vez mais eficientes, os algoritmos MCMC se tornaram populares e essa necessidade foi restrita a modelos extremamente complexos ou que apresentam propriedades muito desejadas.

\subsubsection{Algoritmos MCMC}

Nos casos em que as quantidades de interesse a respeito da distribuição a posteriori são de difícil obtenção, tanto analiticamente quanto por amostras simuladas diretamente da distribuição teórica $p(\boldsymbol{\theta} \mid \boldsymbol{y})$, um método frequentemente utilizado é a simulação de amostras de $p(\boldsymbol{\theta} \mid \boldsymbol{y})$ por meio de algoritmos MCMC. Inclusive por, em muitos casos, este método poder ser implementado apenas conhecendo-se a verossimilhança $(L(\boldsymbol{y} \mid \boldsymbol{\theta}))$ e a distribuição a priori $(p(\boldsymbol{\theta}))$.

Os algoritmos MCMC baseiam-se teoricamente em propriedades de cadeias de Markov para aproximar a distribuição dos valores da cadeia gerada pelos algoritmos à distribuição de interesse. Esses valores são simulados sequencialmente e a acurácia da convergência está diretamente relacionada ao número de iterações.

O amostrador de Gibbs aborda a simulação de vetores $(\boldsymbol{\theta})$ por meio de sua partição em $\left(\boldsymbol{\theta}_{1}, \ldots, \boldsymbol{\theta}_{m}\right)$ de forma que as distribuições condicionais completas $p\left(\boldsymbol{\theta}_{1} \mid \boldsymbol{\theta}_{2}, \ldots, \boldsymbol{\theta}_{m}, \boldsymbol{y}\right), p\left(\boldsymbol{\theta}_{2} \mid \boldsymbol{\theta}_{1}, \boldsymbol{\theta}_{3}, \ldots\right.$, $\left.\boldsymbol{\theta}_{m}, \boldsymbol{y}\right), \ldots, p\left(\boldsymbol{\theta}_{m} \mid \boldsymbol{\theta}_{1}, \ldots, \boldsymbol{\theta}_{m-1}, \boldsymbol{y}\right)$ sejam conhecidas. Note que ao conhecer essas distribuições condicionais completas é possível obter a distribuição conjunta.

Seja $\boldsymbol{\theta}^{(t)}=\left(\boldsymbol{\theta}_{1}^{(t)}, \ldots, \boldsymbol{\theta}_{m}^{(t)}\right)$ o valor atribuído a $\boldsymbol{\theta}$ na $t$-ésima iteração, o algoritmo amostrador de Gibbs pode ser esquematizado como:

1. atribuir um valor inicial $\boldsymbol{\theta}^{(0)}$; 
2. para $t=1, \ldots, G$, gerar valores de $\boldsymbol{\theta}^{(t)}$ por meio das distribuições condicionais completas:

$$
\begin{aligned}
& \text { - } \boldsymbol{\theta}_{1}^{(t)} \sim p\left(\boldsymbol{\theta}_{1} \mid \boldsymbol{\theta}_{2}^{(t-1)}, \ldots, \boldsymbol{\theta}_{m}^{(t-1)}, \boldsymbol{y}\right) \\
& \text { - } \boldsymbol{\theta}_{2}^{(t)} \sim p\left(\boldsymbol{\theta}_{2} \mid \boldsymbol{\theta}_{1}^{(t)}, \boldsymbol{\theta}_{3}^{(t-1)}, \ldots, \boldsymbol{\theta}_{m}^{(t-1)}, \boldsymbol{y}\right) \\
& \quad \vdots \\
& \text { - } \boldsymbol{\theta}_{m}^{(t)} \sim p\left(\boldsymbol{\theta}_{m} \mid \boldsymbol{\theta}_{1}^{(t)}, \ldots, \boldsymbol{\theta}_{m-1}^{(t)}, \boldsymbol{y}\right) .
\end{aligned}
$$

São referências complementares e que abordam tópicos não apresentados nesta seção sobre o amostrador de Gibbs: Casella e George (1992), o Capítulo 7 de Robert e Casella (2004) e o Capítulo 5 de Gamerman e Lopes (2006).

Outro algoritmo vastamente utilizado é o de Metropolis-Hastings (M-H), o qual, juntamente com suas extensões, pode ser útil na grande maioria das análises bayesianas de dados e essa versatilidade se dá em função da construção do método que se baseia em um algoritmo de rejeição. Na impossibilidade de gerar valores da distribuição desejada, os algoritmos de rejeição propõem gerar valores de uma distribuição auxiliar menos complexa, selecionada conforme critérios de interesse e, por fim, decidir por incorporar ou não esses valores à amostra da distribuição alvo conforme determinada regra de aceitação/rejeição. Um ponto extremamente importante desses algoritmos é justamente a escolha da distribuição de cujos valores candidatos são gerados; resumidamente, indica-se que essa escolha baseia-se na facilidade da geração de valores pertencentes ao suporte da distribuição alvo ou em algum método desenvolvido para essa finalidade como, por exemplo, densidade do passeio aleatório.

A primeira menção ao algoritmo de Metropolis-Hastings que se tem registro é a sua utilização na área da física em Metropolis et al. (1953); em seguida, Hastings (1970) formalizou o método ao generalizá-lo.

Sejam $\boldsymbol{\theta}$ o vetor de parâmetros de interesse e $q(\cdot, \cdot)$ a densidade auxiliar proposta, possuindo o mesmo suporte que a distribuição a posteriori $p(\boldsymbol{\theta} \mid \boldsymbol{y})$, escolhida de forma a gerar valores de forma fácil, objetivando a amostragem de $p(\boldsymbol{\theta} \mid \boldsymbol{y})$, o algoritmo de Metropolis-Hastings é dado pelo algoritmo que segue:

1. atribuir um valor inicial $\boldsymbol{\theta}^{(0)}$;

Para $t=1,2, \ldots, G$, em que $G$ seja suficiente para alcançar a convergência estipulada:

2. gerar $\boldsymbol{\theta}^{(*)}$ através de $q\left(\boldsymbol{\theta}^{(t-1)}, \boldsymbol{\theta}^{(*)}\right)$ e $u \sim U(0,1)$;

3. calcular $\alpha\left(\boldsymbol{\theta}^{(t-1)}, \boldsymbol{\theta}^{(*)}\right)=\min \left\{1, \frac{p\left(\boldsymbol{\theta}^{(*)}\right) q\left(\boldsymbol{\theta}^{(*)}, \boldsymbol{\theta}^{(t-1)}\right)}{p\left(\boldsymbol{\theta}^{(t-1)}\right) q\left(\boldsymbol{\theta}^{(t-1)}, \boldsymbol{\theta}^{(*)}\right)}\right\}$;

4. se $u<\alpha\left(\boldsymbol{\theta}^{(t-1)}, \boldsymbol{\theta}^{(*)}\right): \boldsymbol{\theta}^{(t)}=\boldsymbol{\theta}^{(*)}$, caso contrário retornar ao passo 2 .

O diagnóstico da convergência pode ser efetuado por meio de medidas resumo amostrais, da taxa de aceitação/rejeição, da autocorrelação da amostra resultante e de medidas de diagnóstico. Inúmeras referências apresentam explicações claras e completas sobre a infinidade de tópicos relacionados aos algoritmos de Metropolis-Hastings, dentre elas Robert e Casella (2004) e Robert e Casella (2009). 
Devido ao maior número de etapas no algoritmo, Metropolis-Hastings é computacionalmente mais custoso que o amostrador de Gibbs na grande maioria das vezes, ao mesmo tempo que aquele pode ser aplicado em situações que esse não se aplica.

As análises bayesianas efetuadas neste trabalho são baseadas nas amostras das distribuições $a$ posteriori obtidas através do software OpenBUGS. Este é um software livre resultante do projeto BUGS (Bayesian Inference using Gibbs Sampling) (Spiegelhalter et al., 1999), que é um projeto que possibilita análises através da inferência bayesiana de forma automatizada e mais rápida e prática por necessitar apenas das distribuições a priori, da verossimilhança e dos dados.

Segundo Lunn et al. (2012), o software possui um sistema específico implementado para gerenciar as atribuições dos possíveis algoritmos MCMC a cada um dos parâmetros a serem amostrados a posteriori: primeiramente, classifica-se as distribuições condicionais completas em suas distribuições (normal, gama, etc.) quando elas possuem formas fechadas ou, quando elas não possuem forma fechada, identifica-se suas propriedades como restrição do espaço paramétrico e concavidade. Listados, então, por ordem de generalidade, os algoritmos são alocados aos parâmetros cujas propriedades classificadas são compatíveis. Dentre os inúmeros algoritmos implementados no OpenBUGS estão a amostragem direta das distribuições condicionais completas, Metropolis-Hastings adaptativo, Metropolis-Hastings multivariado, esquema híbrido para amostragem, amostragem por corte (Slice sampling), evolução diferencial (differential evolution), dentre outros. Nas análises deste trabalho o software OpenBUGS foi utilizado através do pacote R2OpenBUGS (Sturtz et al., 2005) do software R.

Note que essa facilidade de implementação não descarta a importância do conhecimento do procedimento do software e de análises de consistência dos resultados. Em Lunn et al. (2009) são discutidos os pontos favoráveis e críticos da utilização de softwares que se baseiam na técnica desenvolvida nesse projeto.

Para compor a amostra é necessário estabelecer:

- $n_{b}$ : o número de iterações iniciais a serem excluídas de forma a atingir a convergência desejada, denominada amostra de aquecimento (burn-in);

- $n_{t}$ : o número de elementos entre cada valor amostrado da cadeia gerada (após a amostra de aquecimento), denominado thinning.

Estes valores são geralmente estabelecidos através da análise visual dos gráficos das trajetórias da cadeia $\left(\boldsymbol{\theta}^{(1)}, \ldots, \boldsymbol{\theta}^{(G)}\right)$ e são necessários pois, apesar dos resultados teóricos comprovarem a convergência desejada, eles não são de fácil verificação; ao estabelecê-los a intenção é obter uma amostra aleatória da distribuição de interesse (uma explicação mais detalhada pode ser encontrada no Capítulo 6 de Brooks et al. (2011)).

Na utilização dos algoritmos MCMC o diagnóstico da convergência da cadeia se faz necessário pela dificuldade de em alguns casos verificar as condições necessárias para que esta ocorra. Brooks et al. (2011) e Gelman et al. (2013) apresentam, explicam e discutem este tópico de forma detalhada, mas, resumidamente, este diagnóstico pode ser efetuado através de análise gráfica e de medidas que avaliam as variabilidades, valores médios e correlação intra e entre as cadeias, estabilização da média ou mediana da amostra ao longo das iterações, dentre outros. Por meio do pacote coda (Plummer et al., 2006) do software R, grande parte dessa análise pode ser efetuada. 
Depois de apresentar brevemente a teoria em que se baseiam os algoritmos MCMC, é interessante mencionar que, mesmo com a disponibilidade de recursos computacionais cada vez mais eficientes, o processo de estimação por meio desses algoritmos pode ter tempos de execução extremamente longos, principalmente em casos de modelagem mais complexas. Nessas situações, uma alternativa possível é calcular as quantidades de interesse fazendo uso da técnica baseada na aproximação de Laplace denominada INLA (Integrated Nested Laplace Approximation) (Rue et al., 2009); este método está implementado no pacote R-INLA (Martins et al., 2013) do software R e, por não ser um método iterativo, seu tempo computacional é consideravelmente mais reduzido que os algoritmos MCMC.

\subsubsection{Comparação e Seleção de Modelos}

Modelos de regressão são ajustados com o interesse de representar a relação entre as variáveis explicativas e a(s) variável(s) resposta(s) objetivando análises como identificação de variáveis significantes, e consequentemente estimação dos seus respectivos parâmetros, predição da(s) variável(s) resposta(s) para valores das variáveis explicativas não observadas na amostra, dentre outros. Dependendo do objetivo desejado, as medidas de avaliação da qualidade do ajuste adequadas são diferentes. Nesta subseção primeiramente são abordados os critérios de informação e, em seguida, a avaliação preditiva a posteriori como métodos de comparação e seleção de modelos.

\section{Critérios de Informação}

Os critérios de informação são ferramentas extremamente úteis para comparação de modelos de forma a auxiliar na seleção de um ajuste para análise. Estes critérios, em sua maioria, assimilam um valor de adequabilidade para cada ajuste composto por uma medida de qualidade do ajuste penalizada pela complexidade do modelo, no sentido do número de parâmetros do modelo.

Dado um banco de dados de interesse e diversos modelos ajustados através de métodos frequentistas para esses dados, claramente os ajustes com maior número de parâmetros apresentam maior acurácia; assim, os critérios de informação de Akaike (AIC) e Bayesiano (BIC), também conhecido por critério de Schwarz, baseiam-se na verossimilhança avaliada nos parâmetros estimados penalizada por uma função do número de parâmetros e, no caso do BIC, também do tamanho da amostra.

Sejam $l(\widehat{\boldsymbol{\theta}})$ a log-verosssimilhança do modelo ajustado, $d$ o número de parâmetros - dimensão de $\boldsymbol{\theta}$ - e $n$ o tamanho da amostra, o AIC e BIC são definidos, respectivamente, pelas seguintes equações:

$$
\begin{gathered}
A I C=-2 l(\widehat{\boldsymbol{\theta}})+2 d, \\
B I C=-2 l(\widehat{\boldsymbol{\theta}})+d \cdot \ln (n) .
\end{gathered}
$$

Em termos práticos, calcula-se o critério de informação para cada modelo ajustado à amostra e opta-se pelo modelo que apresentar menor valor do critério.

No contexto bayesiano a função alvo em que os métodos objetivam estudar é a distribuição a posteriori dos parâmetros, consequentemente os critérios de informação propostos para estes modelos são baseados na densidade dessa distribuição a posteriori. 
Defina-se:

- $l(\boldsymbol{\theta})=\ln (L(\boldsymbol{y} \mid \boldsymbol{\theta}))$ a log-verossimilhança do modelo;

- $D(\boldsymbol{\theta})=-2 l(\boldsymbol{\theta})$ o desvio (deviance);

- $\boldsymbol{\theta}^{(g)}$ o valor do vetor de parâmetros na $g$-ésima amostra da distribuição a posteriori, $g=$ $1, \ldots, G$;

- $\bar{D}(\boldsymbol{\theta})=E_{\boldsymbol{\theta} \mid \boldsymbol{y}}(D(\boldsymbol{\theta}))$ a esperança a posteriori do desvio $D(\boldsymbol{\theta})$;

- $\widehat{\boldsymbol{\theta}}$ uma estimativa pontual do vetor de parâmetros $\boldsymbol{\theta}$ baseada na amostra da distribuição $a$ posteriori (por exemplo média, mediana e moda);

- $\rho_{D}=\bar{D}(\boldsymbol{\theta})-D(\widehat{\boldsymbol{\theta}})$ o número efetivo de parâmetros;

O critério de informação do desvio (DIC) desenvolvido por Spiegelhalter et al. (2002) pode ser considerado uma generalização do AIC no sentido de ser composto por uma medida de qualidade do ajuste penalizada pela complexidade do modelo, como está definido a seguir:

$$
D I C=D(\widehat{\boldsymbol{\theta}})+2 \rho_{D}=2 \bar{D}(\boldsymbol{\theta})-D(\widehat{\boldsymbol{\theta}}),
$$

sendo o modelo com menor valor de DIC o selecionado. Outra referência interessante sobre esse critério é Spiegelhalter et al. (2014).

O número efetivo de parâmetros $\left(\rho_{D}\right)$ é invariante sob transformação dos parâmetros, isso pode influenciar na eficiência desse critério por não considerar a complexidade do modelo de forma coerente. Nos casos em que o cálculo de $\bar{D}(\boldsymbol{\theta})$ é muito complexo ou a inferência bayesiana é efetuada através de simulação por algoritmos MCMC e não se conhece a expressão fechada da distribuição a posteriori, mas sim uma amostra dela, pode-se considerar a aproximação (2.5).

$$
\widehat{D I C}=2 \widehat{\bar{D}}(\boldsymbol{\theta})-D(\widehat{\boldsymbol{\theta}})
$$

em que $\bar{D}(\boldsymbol{\theta}) \approx \widehat{\bar{D}}(\boldsymbol{\theta})=\frac{1}{G} \sum_{g=1}^{G} D\left(\boldsymbol{\theta}^{(g)}\right)=\frac{-2}{G} \sum_{g=1}^{G} l\left(\boldsymbol{\theta}^{(g)}\right)$.

Sob determinadas condições como os modelos de mistura, forte assimetria, modelos mistos, dentre outros, a utilização do DIC não é indicada (DeIorio e Robert, 2002). Celeux et al. (2006) explica detalhadamente as inconsistências da utilização do DIC para avaliação de modelos de mistura e de modelos com efeitos aleatórios e, apresenta oito extensões deste critério, as indicando cada uma para um caso dos modelos em que o uso do DIC não é recomendado. Em específico, para os modelos de mistura como os estudados nesta tese, Celeux et al. (2006) sugere utilizar o critério $D I C_{3}$, definido na Equação (2.6), para seleção do número de componentes da mistura.

$$
\begin{aligned}
D I C_{3} & =-4 E\{\ln [L(\boldsymbol{y} \mid \boldsymbol{\theta})] \mid \boldsymbol{y}\}+2 \ln (E[L(\boldsymbol{y} \mid \boldsymbol{\theta}) \mid \boldsymbol{y}]) \\
& \approx-4\left\{\frac{1}{G} \sum_{g=1}^{G} \sum_{i=1}^{N} \ln \left[L\left(y_{i} \mid \boldsymbol{\theta}^{(g)}\right)\right]\right\}+2 \sum_{i=1}^{N} \ln \left[\frac{1}{G} \sum_{g=1}^{G} L\left(y_{i} \mid \boldsymbol{\theta}^{(g)}\right)\right] .
\end{aligned}
$$


A literatura também indica a utilização dos critérios de informação esperados de Akaike (EAIC), de Akaike corrigido (ECAIC) e Bayesiano (EBIC) apresentados, respectivamente, em Brooks (2002), Liang e Zou (2008) e Carlin e Louis (2000) e definidos nas Equações (2.7), (2.8) e (2.9).

$$
\begin{gathered}
E A I C=\bar{D}(\boldsymbol{\theta})+2 d . \\
E C A I C=E A I C+\frac{2(d+2)(d+3)}{n-d-3} . \\
E B I C=\bar{D}(\boldsymbol{\theta})+d \cdot \ln (n) .
\end{gathered}
$$

Da mesma forma que os critérios anteriormente apresentados, quanto menor os valores de EAIC, ECAIC, EBIC e $\mathrm{DIC}_{3}$, mais adequado é o ajuste comparado aos outros modelos ajustados e estes critérios podem ser calculados estimando $\bar{D}(\boldsymbol{\theta})$ através de $\widehat{\bar{D}}(\boldsymbol{\theta})$.

A estatística ordenada preditiva condicional (CPO) é uma das quantidades com melhor eficácia na avaliação de desempenho dos modelos beta mistos e modelos de mistura beta (Verkuilen e Smithson, 2012).

Essa estatística é a probabilidade preditiva de cada observação condicionada às demais observações da amostra e, denotando por $y_{(-i)}$ a amostra com a exclusão dos dados da $i$-ésima observação, é expressa na Equação (2.10).

$$
C P O_{i}=f\left(y_{i} \mid y_{(-i)}\right)=\int L\left(y_{i} \mid \boldsymbol{\theta}\right) \pi\left(\boldsymbol{\theta} \mid y_{(-i)}\right) d \boldsymbol{\theta},
$$

em que $i=1, \ldots, N, \boldsymbol{\theta}$ é o vetor de parâmetros desconhecido, $f\left(y_{i} \mid \boldsymbol{\theta}\right)$ é a verossimilhança condicional da $i$-ésima observação dado o vetor de parâmetros e $\pi\left(\boldsymbol{\theta} \mid y_{(-i)}\right)$ é a distribuição a posteriori considerando como dados a amostra sem a $i$-ésima observação.

Para as observações cuja CPO apresenta valores baixos há indícios que sejam discrepantes sob o modelo em avaliação. Dessa forma, a estatística ordenada preditiva condicional é útil tanto para identificação de observações discrepantes quanto para seleção de modelos mais adequados aos dados.

Quando a estimação dos parâmetros é efetuada por meio de algoritmos MCMC, é indicada a aproximação expressa na Equação (2.11).

$$
\widehat{C P O}_{i}=\left[\frac{1}{G} \sum_{g=1}^{G} \frac{1}{L\left(y_{i} \mid \boldsymbol{\theta}^{(g)}\right)}\right]^{-1} .
$$

O logaritmo da pseudo-verossimilhança marginal (LPML) é uma medida que resume a CPO de todas as observações da amostra conforme definido na Equação (2.12) (Geisser e Eddy, 1979) e, diferentemente dos outros critérios apresentados, maiores valores indicam ajustes mais adequados.

$$
L P M L=\sum_{i=1}^{N} \log \left(C P O_{i}\right) .
$$

\section{Avaliação Preditiva a posteriori}

A avaliação preditiva a posteriori averigua a adequabilidade de modelos ajustados analisando quão consistentes são estes modelos com os dados observados. Esta metodologia propõe comparar 
as observações da amostra observada com amostras simuladas da distribuição preditiva a posteriori (dados replicados: $y^{r e p}$ ) em relação a determinada medida de discrepância $T$.

Primeiramente, determina-se $T$ de forma a resumir as características que se tem interesse em comparar entre os dados observados e os dados replicados, ou seja, características que expressem diferença nesses dados caso haja inconsistências no modelo. Essa medida é uma função dos dados e, em alguns casos, também dos parâmetros.

Em seguida, fixando-se o tamanho amostral e os valores das variáveis explicativas iguais aos observados nos dados ajustados, gera-se os valores $y^{r e p}$ da distribiução preditiva a posteriori. Quando essa distribuição é complexa ou de difícil identificação e os parâmetros do modelo são estimados através de algoritmos MCMC, Gelman et al. (2013) indica no Apêndice C que $y^{\text {rep }}$ seja gerado utilizando-se os valores da amostra a posteriori dos parâmetros.

Por fim, calcula-se a medida de discrepância para os dados observados e replicados. Esses valores podem ser analisados por meio de gráficos ou utilizados para estimar o p-valor preditivo a posteriori através do cálculo da proporção das réplicas cuja medida de discrepância é maior que $T(y)$.

Sejam $N_{\text {rep }}$ o número de réplicas simuladas da distribiução preditiva a posteriori, $y_{i}^{\text {rep }}$ a $i$-ésima réplica e $\mathbf{I}_{\{A\}}$ a função indicadora que assume valor um quando o evento A ocorre, formalmente, o p-valor preditivo a posteriori é definido tal qual expresso na Equação (2.13).

$$
\text { p-valor preditivo a posteriori }=\frac{1}{N_{r e p}} \sum_{i=1}^{N_{r e p}} \mathbf{I}_{\left\{T\left(y_{i}^{r e p}\right)>T(y)\right\}} .
$$

Se o modelo ajustado for adequado, espera-se que o p-valor preditivo a posteriori assuma valores intermediários no intervalo unitário pois valores extremos dessa medida indicam que as especificações do modelo não condizem com os dados observados.

\subsection{Modelo de Regressão Beta}

A distribuição de probabilidades beta é muito conhecida na área estatística pela sua flexibilidade e, por esta razão, abrange um vasto ramo de aplicações em muitas áreas de análise de dados. Esta distribuição possui diversas parametrizações para sua função densidade de probabilidade e, em função de ela ser aplicada neste trabalho como distribuição da variável resposta no ajuste de modelos de regressão, foi adotada a parametrização apresentada em Ferrari e Cribari-Neto (2004):

$$
f(y ; \mu, \phi)=\frac{\Gamma(\phi)}{\Gamma(\phi \mu) \Gamma(\phi(1-\mu))} y^{(\mu \phi-1)}(1-y)^{[(1-\mu) \phi-1]},
$$

em que:

- $0<\mathrm{y}<1$;

- $0<\mu<1$;

- $\phi>0$;

- $\mathrm{E}(\mathrm{Y})=\mu$;

- $\operatorname{Var}(\mathrm{Y})=\frac{\mu(1-\mu)}{1+\phi}=\frac{V(\mu)}{1+\phi}$, em que $V(\mu)=\mu(1-\mu)$. 
Nesta parametrização, $\mu$ e $\phi$ são, respectivamente, parâmetros de média e precisão, fato esse que proporciona maior facilidade de interpretação dos resultados do ajuste do modelo, inclusive por estas quantidades serem independentes. Define-se $\phi$ como parâmetro de precisão no sentido de que à medida que o valor de $\phi$ aumenta, a variância $\frac{\mu(1-\mu)}{1+\phi}$ diminui, diferentemente do uso convencional desse termo como inverso da variância.

Analogamente, a parametrização apresentada em Cribari-Neto e Souza (2012) difere da apresentada anteriormente ao utilizar o parâmetro de dispersão $\sigma$ ao invés do parâmetro de precisão $\phi$; assim, a função densidade de probabilidade dada em termos do parâmetro de dispersão $\sigma$ pode ser expressa como:

$$
f(y ; \mu, \sigma)=\frac{\Gamma\left(\left(1-\sigma^{2}\right) / \sigma^{2}\right)}{\Gamma\left(\mu\left(\left(1-\sigma^{2}\right) / \sigma^{2}\right)\right) \Gamma\left((1-\mu)\left(\left(1-\sigma^{2}\right) / \sigma^{2}\right)\right)} y^{\left(\mu\left(\left(1-\sigma^{2}\right) / \sigma^{2}\right)-1\right)}(1-y)^{\left[(1-\mu)\left(\left(1-\sigma^{2}\right) / \sigma^{2}\right)-1\right]},
$$

em que:

- $0<\mathrm{y}<1$

- $0<\mu<1$;

- $0<\sigma<1$;

- $\mathrm{E}(\mathrm{Y})=\mu$;

- $\operatorname{Var}(\mathrm{Y})=\sigma^{2} \mu(1-\mu)=\sigma^{2} \mathrm{~V}(\mu)$.

A relação bijetora entre os parâmetros $\sigma$ e $\phi$, respectivamente de dispersão e precisão, ocorre segundo a Expressão (2.16).

$$
\sigma^{2}=\frac{1}{1+\phi}
$$

Na Figura 2.1 são exibidas as formas da função densidade de probabilidade da distribuição beta para alguns exemplos de combinações dos parâmetros $(\mu, \phi)$. 
Função densidade de probabilidade da distribuição beta
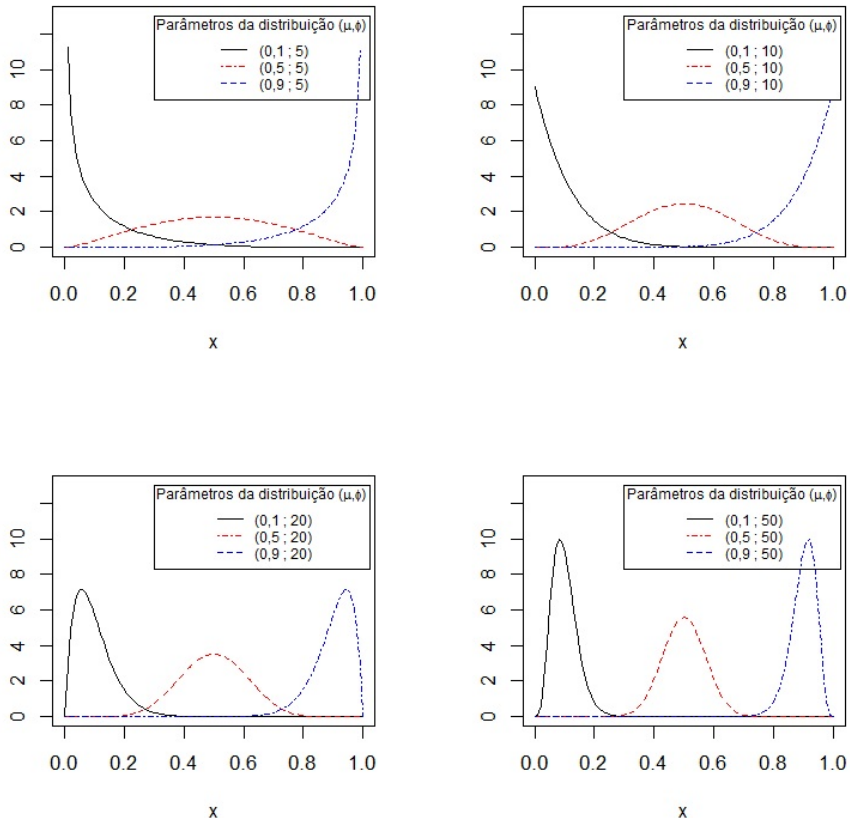

Figura 2.1: Comparação entre as densidades da distribuição beta para diferentes valores dos parâmetros $(\mu, \phi)$.

Os modelos de regressão beta têm por finalidade ajustar variáveis respostas contínuas, cujas observações estejam em um intervalo restrito conhecido $(\mathrm{a}, \mathrm{b})$; como exemplos de dados que apresentam estas características podem ser citados taxas, proporções, frações, dentre outras.

Em diversas situações, para que seja possível o ajuste de modelos de regressão normal, aplica-se uma transformação nos dados, o que pode resultar em dificuldades de interpretação dos resultados do ajuste, diferente de quando é possível considerar que a varíavel resposta do modelo de regressão possui distribuição beta, caso este em que obtém-se a vantagem de interpretar mais facilmente os resultados do ajuste. Um exemplo dessa situação é a aplicação destes modelos em dados assimétricos, e o ganho se dá em virtude da flexibilidade da distribuição beta (Figura 2.1). Exemplos de pesquisas relacionadas a estes modelos podem ser encontrados por exemplo em Paolino (2001), Kieschnick e McCullough (2003), Ferrari e Cribari-Neto (2004) e Simas et al. (2010), entre outras.

Os modelos de regressão beta de efeitos fixos propostos por Ferrari e Cribari-Neto (2004), os quais utilizam-se da parametrização (2.14) e consideram o ajuste da média da distribuição beta com parâmetro de precisão constante, foram pioneiros.

Sejam $Y_{1}, \ldots, Y_{n}$ variáveis aleatórias independentes tais que, para $\mathrm{t}=1, \ldots, \mathrm{n}, Y_{t}$ tem distribuição $\operatorname{Beta}\left(\mu_{t}, \phi\right)$, o modelo proposto por Ferrari e Cribari-Neto (2004) pode ser representado pela Equação (2.17).

$$
g\left(\mu_{t}\right)=\beta_{0}+\sum_{i=1}^{p} x_{t i} \beta_{i}=\eta_{t}
$$

em que:

- $\boldsymbol{\beta}=\left(\beta_{0}, \beta_{1}, \ldots, \beta_{p}\right)^{\top}$ é o vetor de tamanho $(p+1)$ de parâmetros desconhecidos da regressão;

- $x_{t 1}, \ldots, x_{t p}$ são os valores conhecidos das $p$ variáveis explicativas referentes a $t$-ésima observa- 
ção;

- $g(\cdot)$ é a função de ligação estritamente monótona e duplamente diferenciável.

O modelo (2.17) também pode ser ajustado em termos da parametrização (2.15); note que, se considerados constantes, os parâmetros de precisão e dispersão seguem a relação (2.16). Existe também a possibilidade de ajustar simultaneamente modelos de regressão para $\mu_{t}$ e para o parâmetro de dispersão (Rigby e Stasinopoulos, 2005), como efetuado em Smithson e Verkuilen (2006), que estuda esse ajuste simultâneo sob abordagem clássica.

O primeiro trabalho a tratar da inferência destes modelos sob abordagem bayesiana foi CepedaCuervo (2001); atualmente, essa inferência pode ser efetuada com o uso do pacote Bayesianbetareg (Cepeda-Cuervo et al., 2016) do software $R$, o qual baseia-se no algoritmo Metropolis-Hastings.

\subsection{Modelo de Regressão Beta com Efeitos Aleatórios}

A análise de dados por meio do ajuste de modelos de regressão de efeitos fixos é eficiente e satisfatória em uma diversa gama de estudos; porém, ao assumir a suposição de independência dos dados a serem ajustados, esta técnica não se apresenta adequada quando a estrutura do banco de dados não satisfaz essa suposição.

Pesquisadores de diversas áreas como educação, saúde, marketing, ciências sociais, dentre outras, se interessam em estudar dados que apresentam relações entre si, como por exemplo: desempenho escolar dos alunos de diferentes distritos de uma cidade, resposta ao tratamento de uma doença em pacientes de diferentes unidades hospitalares, dados de pagamento de faturas de cartão de crédito de um mesmo cliente, entre outros exemplos. Nestes casos a suposição de independência dos dados não é satisfeita, pois alunos que possuem mesma professora e/ou escola possuem desempenhos de alguma maneira associados, assim como doentes que receberam o tratamento na mesma unidade hospitalar. De forma geral, as unidades de mesmo grupo compartilham características semelhantes e ambientes comuns e esta característica deve ser levada em consideração ao analisar estes dados.

De forma geral, dados correlacionados é um termo utilizado para designar dados espacialmente correlacionados, dados agrupados, amostras multivariadas, dados longitudinais, dentre outros. Por dados longitudinais entende-se que as mesmas unidades amostrais foram observadas ao longo de um período de tempo repetidamente.

Bancos de dados correlacionados são usualmente estudados através de modelos mistos. Esta classe de modelos adiciona componentes aleatórios aos modelos com efeitos fixos, possibilitando a incorporação da existência de correlação entre as observações.

Uma classe de dados comum em diversas áreas de aplicação são os dados longitudinais; estes são compostos por uma mesma medida observada repetidamente ao longo do tempo. Como exemplos de dados longitudinais tem-se: aferição da pressão arterial sistólica de um mesmo paciente observada em diferentes momentos e umidade do ar nos dias de inverno em uma mesma localidade.

Para o caso específico em que a variável resposta do modelo misto possui distribuição beta, Verkuilen e Smithson (2012), Figueroa-Zúñiga et al. (2013) e Usuga (2013) estudaram as características deste modelo.

Assim, os modelos de regressão beta com efeitos aleatórios, também denominados modelos beta mistos, com precisão constante e efeitos aleatórios normalmente distribuídos são descritos pela 
Equação (2.18).

$$
\eta_{i j}=g\left(\mu_{i j}\right)=g\left(E\left(Y_{i j} \mid \boldsymbol{b}_{i}\right)\right)=\boldsymbol{x}_{i j}^{\top} \boldsymbol{\beta}+\boldsymbol{z}_{i j}^{\top} \boldsymbol{b}_{i},
$$

em que:

- $y_{i j}$ é a $j$-ésima observação do $i$-ésimo grupo, $i=1, \ldots, N, j=1, \ldots, n_{i}$;

- $\boldsymbol{y}_{i}=\left(y_{i 1}, \ldots, y_{i n_{i}}\right)^{\top}$ é o vetor com as $n_{i}$ observações do $i$-ésimo grupo;

- $Y_{i j} \mid \boldsymbol{b}_{i}$ são v.a. independentes com distribuição $\operatorname{Beta}\left(\mu_{i j}, \phi\right)$ ou $\operatorname{Beta}\left(\mu_{i j}, \sigma\right)$;

- $\boldsymbol{b}_{i}=\left(b_{i 1}, \ldots, b_{i d}\right)^{\top}$ é o vetor $d$-dimensional de efeitos aleatórios associado ao $i$-ésimo grupo;

- $\boldsymbol{b}_{1}, \ldots, \boldsymbol{b}_{N}$ são v.a. independentes com distribuição $N_{d}\left(\mathbf{0}, \Sigma_{b}\right)$.

- $\boldsymbol{x}_{i j}=\left(x_{i j 1}, \ldots, x_{i j p}\right)^{\top}$ é o vetor de dimensão $p$ com os valores conhecidos das variáveis explicativas associadas aos efeitos fixos para a $j$-ésima observação do $i$-ésimo grupo;

- $\boldsymbol{\beta}=\left(\beta_{1}, \ldots, \beta_{p}\right)^{\top}$ é o vetor de tamanho $p$ de parâmetros fixos da regressão;

- $\boldsymbol{z}_{i j}=\left(z_{i j 1}, \ldots, z_{i j d}\right)^{\top}$ é o vetor de dimensão $d$ com os valores conhecidos das variáveis explicativas associadas aos efeitos aleatórios para a $j$-ésima observação do $i$-ésimo grupo;

- $g(\cdot)$ é a função de ligação estritamente monótona e duplamente diferenciável.

Em Bell et al. (2016) pode ser encontrado um glossário repleto de explicações sobre termos relacionados a estes modelos.

Os estimadores dos parâmetros $\boldsymbol{\beta}, \phi$ e $\Sigma_{b}$ são denotados por $\widehat{\boldsymbol{\beta}}, \widehat{\phi}$ e $\widehat{\Sigma}_{b}$ e podem ser obtidos pelo método de máxima verossimilhança mesmo não possuindo expressões analíticas, por meio de métodos numéricos, como por exemplo a quadratura de Gauss-Hermite (Liu e Pierce, 1994; Hernández et al., submetido a) que é sugerida em Usuga (2013) para calcular os estimadores em questão. Para o ajuste e análise de diagnóstico dos modelos beta mistos de forma frequentista indica-se o uso do software estatístico $R$, cuja linguagem de programação encontra-se explicada em R Core Team (2016). Dentre os pacotes que disponibilizam estes procedimentos estão o gamlss (Generalised Additive Models for Location Scale and Shape) e o blmm. Informações a respeito destes dois pacotes podem ser encontradas, respectivamente, em Stasinopoulos et al. (2008) e em Usuga (2013).

Muitas publicações como Sorensen (1996) e Resende et al. (2001) estudaram as diferenças de performances de metodologias bayesianas e frequentistas na inferência de modelos mistos e os resultados indicaram que a abordagem bayesiana apresenta vantagens, inclusive pela utilidade dos intervalos de credibilidade dos componentes de variância. Por meio de estudos de simulação, Liu e Eugenio (2016) mostraram que, em geral, apesar da metodologia frequentista desenvolvida ser computacionalmente mais rápida que os algoritmos MCMC, ela apresenta viés significativo, além de problemas de convergência e de sensibilidade aos valores iniciais do algoritmo.

Figueroa-Zúñiga et al. (2013) apresentam e analisam os modelos beta mistos (2.18) sob a abordagem bayesiana por meio do software WinBUGS, o qual também provém do projeto BUGS (como é o OpenBUGS, anteriormente citado na Subseção 2.1.2); nesse caso os autores consideram 
as distribuições normal e $t$ de Student para os efeitos aleatórios. Outras referências indicadas são Bonat et al. (2015) e Gheorghe et al. (2016), que também estudam esses modelos sob abordagem bayesiana; sendo que a primeira utiliza também o método computacional data cloning (Lele et al. , 2007) para calcular estimadores de máxima verossimilhança e averiguar identificabilidade através de algoritmos MCMC.

No que se refere à característica da abordagem bayesiana de atribuir incerteza aos parâmetros, considerados aleatórios, existem muitos estudos de simulação e análises sobre a influência que a distribuição a priori atribuída ao parâmetro de precisão $(\phi)$ exerce sobre o resultado das estimações; isso porque, como afirmado em Verkuilen e Smithson (2012), Bonat et al. (2014) e outras muitas referências, a implementação do algoritmo MCMC para os modelos beta mistos, apesar de simples, pode apresentar dificuldades na atribuição da distribuição a priori para o parâmetro da variância $(\sigma)$ e para os componentes de variância associados aos efeitos aleatórios, pois sua estimação é comprovadamente sensível a essa escolha.

Gelman (2006) recomenda iniciar a análise com uma distribuição uniforme não informativa, já que distribuições a priori não informativas permitem a inferência bayesiana dos parâmetros que não se tem conhecimento prévio, porém esta distribuição a priori tende a superestimar $\phi$; e, nos casos em que se deseja mais informação a priori, Gelman (2006) recomenda a utilização de uma distribuição da família half-t por ela possuir melhor desempenho na vizinhança de zero comparada à distribuição gama inversa (que possui conjugância condicional). Lunn et al. (2009) explica que a escolha da distribuição Gama $(\epsilon, \epsilon)(\epsilon>0)$ como distribuição a priori para os parâmetros de precisão se dá em função de ela ser uma aproximação à distribuição a priori de Jeffreys, assim como o é a distribuição uniforme para o log da variância. Considerando estes aspectos, Figueroa-Zúñiga et al. (2013) considera $\phi \sim(a B)^{2}$, em que $B \sim \operatorname{Beta}(1+\epsilon, 1+\epsilon), a>0$ e $\epsilon>0$, por ser uma distribuição a priori pouco informativa e flexível.

Com relação à atribuição da distribuição a priori para o desvio-padrão dos efeitos aleatórios (é o único elemento de $\Sigma_{b}$ no caso $d=1$ ), Gelman (2006) recomenda iniciar a modelagem com uma distribuição uniforme não informativa (dado que ela costuma apresentar resultados satisfatórios em casos de $N>5$ ) e, para $N<5$ esta distribuição a priori tende a superestimar. Apesar de $\Sigma_{b}^{-1}$ ser um parâmetro de precisão, Lunn et al. (2009) não indica a utilização da distribuição Gama $(\epsilon, \epsilon), \epsilon>0$ ou Wishart como distribuição a priori.

A respeito aos demais parâmetros, distribuições a priori pouco informativas são especificadas por Figueroa-Zúñiga et al. (2013): as distribuições normal multivariada ou $t$ de Student são atribuídas à $\boldsymbol{\beta}$ e para sua variância $\left(\Sigma_{\beta}\right)$ foi escolhida a distribuição Wishart inversa pela simplicidade computacional.

Smithson et al. (2011) estuda os modelos de mistura beta de efeitos fixos com ajuste simultâneo para os parâmetros de média e de precisão, enquanto que em Verkuilen e Smithson (2012) e em Figueroa-Zúñiga et al. (2013) é abordado o ajuste do modelo beta com efeitos aleatórios para o parâmetro de precisão (2.19) (conforme notação da referência) simultaneamente se considerou (2.18).

$$
\tau_{i j}=\log \left(\phi_{i j}\right)=\boldsymbol{w}_{i j}^{\top} \boldsymbol{\delta}+\boldsymbol{r}_{i j}^{\top} \boldsymbol{u}_{i}
$$

em que: 
- $\boldsymbol{w}_{i j}=\left(w_{i j 1}, \ldots, w_{i j q}\right)^{\top}$ é o vetor de dimensão $q$ com os valores conhecidos das variáveis explicativas associadas aos efeitos fixos para a $j$-ésima observação do $i$-ésimo grupo;

- $\boldsymbol{\delta}=\left(\delta_{1}, \ldots, \delta_{q}\right)^{\top}$ é o vetor de tamanho $q$ de parâmetros fixos da regressão;

- $\boldsymbol{r}_{i j}=\left(r_{i j 1}, \ldots, r_{i j d^{*}}\right)^{\top}$ é o vetor de dimensão $d^{*}$ com os valores conhecidos das variáveis explicativas associadas aos efeitos aleatórios para a $j$-ésima observação do $i$-ésimo grupo;

- $\boldsymbol{u}_{i}=\left(u_{i 1}, \ldots, u_{i d^{*}}\right)^{\top}$ é o vetor $d^{*}$-dimensional de efeitos aleatórios associado ao $i$-ésimo grupo;

- $\boldsymbol{u}_{i} \mid \Sigma_{u} \stackrel{i n d}{\sim} N_{d^{*}}\left(\mathbf{0}, \Sigma_{u}\right)$ ou $\boldsymbol{u}_{i} \mid \Sigma_{u}, \nu \stackrel{i n d}{\sim} t_{d^{*}}\left(\nu_{u}, \mathbf{0}, \Sigma_{u}\right)$.

Analogamente, completando as atribuições de distribuições a priori aos parâmetros para efetuar a análise bayesiana, Figueroa-Zúñiga et al. (2013) consideram $\boldsymbol{\delta} \stackrel{i n d}{\sim} N_{q}\left(\boldsymbol{\mu}_{\delta}, \Sigma_{\delta}\right)$ e $\Sigma_{u} \sim I W_{d^{*}}(\cdot, \cdot)$.

Como alternativa aos algoritmos MCMC nos quais se baseiam os softwares resultantes do projeto BUGS, Bonat et al. (2014) analisa os modelos beta mistos por meio do algoritmo INLA da inferência por abordagem bayesiana, inclusive o comparando com um algoritmo MCMC e com a estimação por máxima verossimilhança por meio dos critérios de informação: LML (Log-Verossimilhança Marginal), DIC e CPO; os resultados mostraram que os algoritmos INLA e MCMC se mostraram similares e compatíveis com a análise por verossimilhança. Também foi desenvolvido em Carroll et al. (2015) um estudo de simulação comparativo entre o software OpenBUGS e a metodologia INLA e, os resultados indicaram que a performance inferencial do OpenBUGS dos parâmetros relacionados aos efeitos aleatórios foram consideravelmente melhores, além deste possibilitar facilmente a implementação de distribuições a priori, o que não é possível ainda no outro método. Por estes motivos que neste trabalho o software OpenBUGS foi considerado para a execução dos algoritmos MCMC.

\subsection{Modelo de Regressão Não Linear com Efeitos Aleatórios}

Os modelos de regressão não lineares utilizam-se de funções não lineares em sua especificação para analisar dados de diversas áreas que não são adequadamente ajustados por modelos lineares como, por exemplo, dados de crescimento corporal de animais, dados provenientes de pesquisas de farmacologia, assim como dados de estudos de aprendizado ao longo da educação básica, dentre outros.

Muitas dessas aplicações são estudos longitudinais ou trabalham com dados correlacionados, características essas que tornam inapropriada a utilização de modelos de regressão de efeitos fixos por haver a suposição de independência dos dados em sua inferência. Para esses e outros casos, Sheiner e Beal (1983), Liang e Zeger (1986), Zeger et al. (1988), Lindstrom e Bates (1990), Vonesh e Carter (1992), Davidian e Giltinan (1993), Vonesh e Chinchilli (1997) e Pinheiro e Bates (2000) propuseram os modelos de regressão não lineares mistos. Porém, nenhum destes trabalhos considerou variáveis resposta com distribuição beta.

No caso dos modelos não lineares de efeitos fixos com distribuição beta, Simas et al. (2010) foram pioneiros apresentando e analisando um estimador de máxima verossimilhança corrigido, já que estes estimadores são viesados nestes modelos. 
A equação a seguir apresenta os modelos beta mistos não lineares.

$$
\eta_{i j}=g\left(\mu_{i j}\right)=g\left(E\left(Y_{i j} \mid \boldsymbol{b}_{i}\right)\right)=h\left(\boldsymbol{x}_{i j}, \boldsymbol{\beta}\right)+\boldsymbol{z}_{i j}^{\top} \boldsymbol{b}_{i},
$$

em que:

- $y_{i j}$ é a $j$-ésima observação do $i$-ésimo grupo, $i=1, \ldots, N, j=1, \ldots, n_{i}$;

- $\boldsymbol{y}_{i}=\left(y_{i 1}, \ldots, y_{i n_{i}}\right)^{\top}$ é o vetor com as $n_{i}$ observações do $i$-ésimo grupo;

- $Y_{i j} \mid \boldsymbol{b}_{i}$ são v.a.'s independentes com distribuição $\operatorname{Beta}\left(\mu_{i j}, \phi\right)$ ou $\operatorname{Beta}\left(\mu_{i j}, \sigma\right)$;

- $\boldsymbol{b}_{i}=\left(b_{i 1}, \ldots, b_{i d}\right)^{\top}$ é o vetor $d$-dimensional de efeitos aleatórios associado ao $i$-ésimo grupo;

- $\boldsymbol{b}_{1}, \ldots, \boldsymbol{b}_{N}$ são v.a.'s independentes com distribuição $N_{d}\left(\mathbf{0}, \Sigma_{b}\right)$.

- $\boldsymbol{x}_{i j}=\left(x_{i j 1}, \ldots, x_{i j p}\right)^{\top}$ é o vetor de dimensão $p$ com os valores conhecidos das variáveis explicativas associadas aos efeitos fixos para a $j$-ésima observação do $i$-ésimo grupo;

- $\boldsymbol{\beta}=\left(\beta_{1}, \ldots, \beta_{p}\right)^{\top}$ é o vetor de tamanho $p$ de parâmetros fixos da regressão;

- $\boldsymbol{z}_{i j}=\left(z_{i j 1}, \ldots, z_{i j d}\right)^{\top}$ é o vetor de dimensão $d$ com os valores conhecidos das variáveis explicativas associadas aos efeitos aleatórios para a $j$-ésima observação do $i$-ésimo grupo;

- $g(\cdot)$ é a função de ligação estritamente monótona e duplamente diferenciável;

- $h(\cdot)$ é a função sistemática não linear e conhecida do modelo.

Nestes modelos, o valor esperado da variável resposta de um determinado grupo da amostra é explicado por uma função não linear especificada de forma a descrever, através dos parâmetros, como ocorre o processo de variabilidade dentro do grupo.

Da forma como especificado, este modelo apresenta não linearidade apenas nos parâmetros fixos $(\boldsymbol{\beta})$ e, por isso, muitas vezes ele pode ser chamado também de modelo misto de efeitos fixos não lineares.

Com relação à estimação dos parâmetros dos modelos de regressão mistos de efeitos fixos não lineares, em Lindstrom e Bates (1990) é proposta e analisada a estimação dos efeitos fixos por meio do estimador de mínimos quadrados e dos efeitos aleatórios utilizando o estimador de máxima verossimilhança.

A determinação da função sistemática $h(\cdot)$ deve ser efetuada com base na análise exploratória dos dados e no conhecimento de especialistas da área pesquisada, de forma a obter um modelo que represente a associação das variáveis explicativas com a variável resposta e com potencial de interpretação.

Atualmente o ajuste destes modelos pode ser efetuado sob abordagem clássica por meio das funções gnlmix e gnlmm do pacote repeated (Swihart e Lindsey, 2017) do software R, respectivamente, para modelos que contêm apenas intercepto aleatório e modelos com efeitos aleatórios normalmente distribuídos. Sob abordagem bayesiana, este mesmo software disponibiliza algumas opções de pacotes como R20penBUGS, R2WinBUGS (Sturtz et al., 2005), R2Jags (Su e Yajima, 2015), dentre outros. No software SAS existe a opção de utilizar o pacote Proc GLIMMIX. 


\subsection{Mistura Finita de Distribuições}

As distribuições que surgem como uma mistura finita de distribuições são indicadas para análise de dados cuja população de interesse é composta por subgrupos homogêneos, em relação a determinada característica, porém eles não são observáveis, ou seja, apresentam heterogeneidade não observada. Ademais, também são amplamente aplicadas em dados que apresentam assimetria, multimodalidade ou curtose.

Um dos primeiros estudos publicados envolvendo o conceito de mistura de distribuições foi Pearson (1894) ao analisar medidas corporais de 1000 caranguejos por meio da mistura de duas distribuições normais, já que os dados apresentavam assimetria e, após as análises, chegou-se a conclusão de que a amostra contemplava dados de duas subespécies; Feller (1943) é outra referência clássica que foi pioneira no estudo das propriedades de mistura de distribuições. Ao longo do tempo, publicações como Everitt e Hand (1981), Titterington et al. (1985), McLachlan e Basford (1988), Titterington (1990), McLachlan e Peel (2000) e Frühwirth-Schnatter (2006) desenvolveram extensamente os métodos estatísticos baseados em mistura de distribuições e suas aplicações (Xu e Hedeker, 2001). A quem interessar conhecer mais detalhes sobre o desenvolvimento das técnicas de aplicação de mistura de distribuições em análise de dados, a leitura do Capítulo 1 de McLachlan e Peel (2000) é indicada.

Para os fins deste trabalho, as misturas de distribuições são utilizadas em variáveis contínuas, sendo assim, será abordado apenas este caso.

$\mathrm{Na}$ quase totalidade das publicações de análises por meio de misturas de distribuições todos os elementos das misturas pertencem à mesma família paramétrica e os elementos variantes são os parâmetros, por exemplo, os elementos são distribuições de probabilidade normais com médias e/ou variâncias diferentes. No presente estudo será considerado esse caso, sem perda de generalidade para a abordagem bayesiana.

A seguir são apresentadas as principais definições relacionadas a este tema que serão utilizadas neste trabalho.

A variável aleatória contínua $Y$ é probabilisticamente distribuída por uma mistura de distribuições se e somente se sua função densidade de probabilidade pode ser expressa por:

$$
f(y)=\sum_{s=1}^{K} \pi_{s} f\left(y \mid \boldsymbol{\theta}_{s}\right),
$$

em que:

- $K$ o número de componentes da mistura;

- $\pi_{s}$ a probabilidade de $Y_{i}$ ser distribuído de acordo com o s-ésimo componente da mistura, $i=1, \ldots, N, s=1, \ldots, K ;$ em que $0<\pi_{s}<1$ e $\sum_{s=1}^{K} \pi_{s}=1 ;$

- $f(\cdot \mid \cdot)$ a função densidade de probabilidade da família paramétrica em estudo;

- $\boldsymbol{\theta}_{s}$ o vetor de parâmetros relacionado ao $s$-ésimo componente da mistura, $s=1, \ldots, K$;

- $f\left(\cdot \mid \boldsymbol{\theta}_{s}\right)$ a função densidade de probabilidade que define o $s$-ésimo componente da mistura, $s=1, \ldots, K$. 
A Figura 2.2 é composta por quatro gráficos que exemplificam a distribuição mistura de normais com $K=2$ para diferentes valores de média e probabilidade para cada componente da mistura.

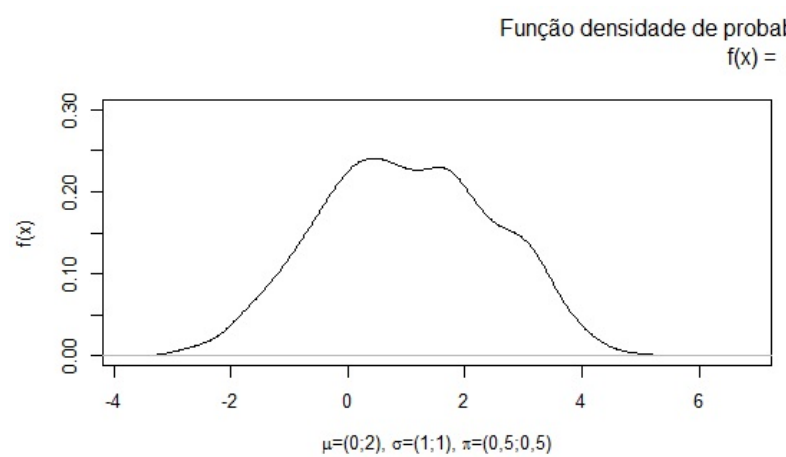

distribuições mistura de normais $=\pi_{1} f\left(x \mid \mu_{1}, \sigma_{1}\right)+\pi_{2} f\left(x \mid \mu_{2}, \sigma_{2}\right)$
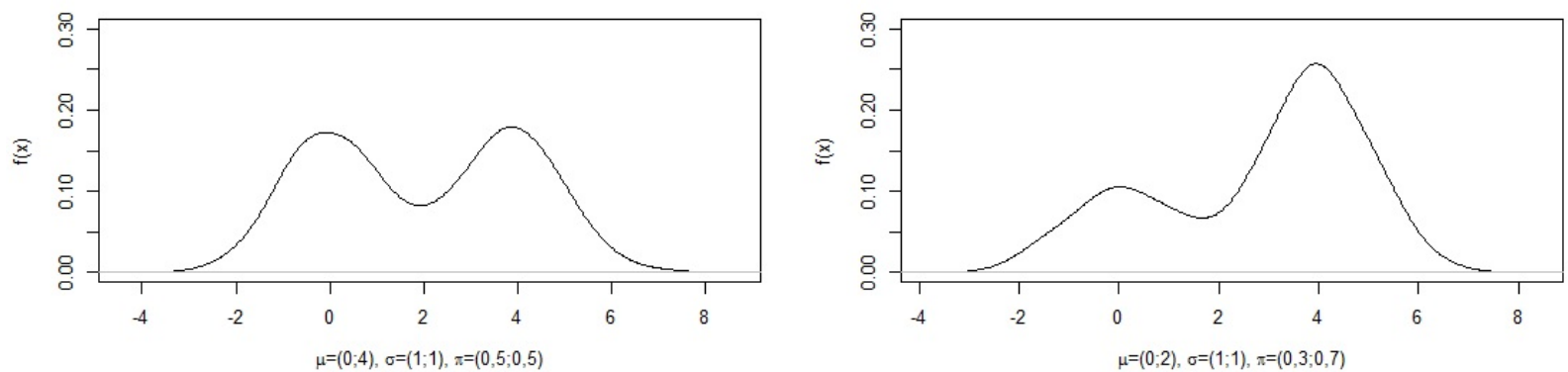

Figura 2.2: Comparação entre as densidades de distribuições mistura de normais para diferentes valores do parâmetro de média $\mu$ e de probabilidades $\pi$.

Para estimar os parâmetros $\boldsymbol{\theta}_{1}, \ldots, \boldsymbol{\theta}_{K}$, pode-se utilizar os métodos bayesianos, de máxima verossimilhança, dos momentos, dentre outros.

No processo inferencial destas misturas de distribuições, o número de componentes da mistura, $K$, pode ser visto sob dois enfoques. O primeiro e mais frequente é considerá-lo fixo, estimar os parâmetros e escolher $K$ de acordo com a adequabilidade dos resultados obtidos, a qual pode ser avaliada por meio de critérios de informação (como os apresentados na Subseção 2.1.3), medidas de adequabilidade e diagnóstico do ajuste, dentre outros. No outro enfoque, $K$ é considerado parâmetro e seu valor é determinado no processo inferencial. Em inferência bayesiana, o algoritmo MCMC com saltos reversíveis (do inglês Reversible Jump MCMC) (Richardson e Green, 1997) é um exemplo de metodologia que possibilita a variação da dimensão paramétrica e se baseia no mecanismo de aceitação/rejeição aos moldes do algoritmo M-H. Devido ao custo computacional e para evitar problemas relacionados à convergência, neste trabalho é utilizado o primeiro enfoque.

Um tópico muito importante de ser discutido a respeito do processo de estimação é identificabilidade: de forma geral, identificabilidade pode ser definida como a existência de um único conjunto de parâmetros para caracterizar o modelo estatístico, ou seja, uma distribuição não identificável é aquela em que existem no mínimo dois possíveis valores de $\boldsymbol{\theta}_{1}, \ldots, \boldsymbol{\theta}_{K}, \boldsymbol{\pi}$ em que os valores das densidades marginais são iguais. Note que nesse caso podem existir conflitos nas estimações, prejudicando principalmente a inferência a respeito dos parâmetros. Teicher (1963) foi uma das primeiras referências a abordar a questão da falta de identificabilidade em mistura de distribuições e, atualmente ela é estudada de acordo com os três possíveis tipos que a caracterizam: superparametrização, invariância à permutação dos componentes da mistura (label switching) e não identificabilidade característica 
de determinadas distribuições de probabilidade (Seção 1.3 de Frühwirth-Schnatter (2006)).

A não identificabilidade proveniente de uma possível superparametrização é de fácil compreensão quando, por exemplo, observa-se que o valor da função densidade de probabilidade definida na Equação (2.21) permanece o mesmo ao adicionar à ela mais um elemento cuja probabilidade associada é nula, ou seja, $\pi_{K+1}=0$ e valor arbitrário de $\boldsymbol{\theta}_{K+1}$ ou com $\pi_{K+1}$ assumindo parte do valor de $\pi_{s}$ para algum $s=1, \ldots, K$ e $\boldsymbol{\theta}_{K+1}=\boldsymbol{\theta}_{s}$. Para contornar essas questões é usual assumir $\pi_{s}>0, \forall s=1, \ldots, K$ e restrições no espaço paramétrico.

Em contrapartida, a invariância dessa classe de distribuições à permutação dos componentes da mistura só necessita ser avaliada nos casos em que os elementos da mistura representam grupos pré definidos da população (Titterington et al., 1985) ou, sob a abordagem bayesiana, distribuições $a$ priori são especificamente atribuídas para cada um desses elementos. Nesses casos, Aitkin e Rubin (1985) propõem que seja imposta a suposição $\pi_{1}>\pi_{2}>\ldots>\pi_{K}$ ou $\pi_{1}<\pi_{2}<\ldots<\pi_{K}$ e Jasra et al. (2005) discutem de que forma a escolha das distribuições a priori e seus parâmetros podem interferir nessa questão. Contudo, Stephens (2000) constata que restrições desse tipo não são suficientes para resolução desse tipo de falta de identificabilidade e apresentam uma técnica alternativa para contornar essa questão. Porém, em geral, indica-se que a interpretação das estimativas de cada parâmetro não seja efetuada isoladamente; o correto é fazer a análise considerando todo o contexto dessas distribuições (Green, 2017).

Por fim, Yakowitz e Spragins (1968) apresentam um resultado que generaliza as condições para estudo da não identificabilidade característica de determinadas distribuições de probabilidade.Titterington et al. (1985), McLachlan e Peel (2000) e Holzmann et al. (2006) estudaram a identificabilidade de algumas distribuições de probabilidade. Em geral os autores concluíram que as distribuições normal, gama, exponencial, Cauchy e Poisson são sempre identificáveis, as misturas das distribuições binomial ou multinomial são identificáveis se e somente se o tamanho de amostra é maior ou igual a $2 K-1$ e; as misturas da distribuição uniforme, tanto contínua como discreta, não são identificáveis.

Especificamente para a distribuição de probabilidades beta, ao definir $S$ como uma variável indicadora discreta do componente da mistura, uma variável aleatória $Y$ tem distribuição mistura finita de betas denotada como $Y \sim \sum_{s=1}^{K} \pi_{s} \operatorname{Beta}\left(\mu_{s}, \phi_{s}\right)$, se e somente se sua função densidade de probabilidade condicional é expressa por

$$
f(y \mid S=s)=f\left(y \mid \boldsymbol{\theta}_{s}\right)=f\left(y \mid \mu_{s}, \phi_{s}\right)=\frac{\gamma\left(\phi_{s}\right)}{\gamma\left(\mu_{s} \phi_{s}\right) \gamma\left(\left(1-\mu_{s}\right) \phi_{s}\right)} y^{\mu_{s} \phi_{s}-1}(1-y)^{\left(1-\mu_{s}\right) \phi_{s}-1}
$$

e, denotando $\pi_{s}=P(S=s)$, sua função densidade de probabilidade marginal (2.21) é dada por

$$
f(y)=\sum_{s=1}^{K} P(S=s) f\left(y \mid \mu_{s}, \phi_{s}\right)=\sum_{s=1}^{K} \pi_{s} \frac{\gamma\left(\phi_{s}\right)}{\gamma\left(\mu_{s} \phi_{s}\right) \gamma\left(\left(1-\mu_{s}\right) \phi_{s}\right)} y^{\mu_{s} \phi_{s}-1}(1-y)^{\left(1-\mu_{s}\right) \phi_{s}-1} .
$$

Propriedade 2.5.1. Se a variável aleatória $Y$ tem distribuição mistura finita de betas: $Y \sim$ 
$\sum_{s=1}^{K} \pi_{s} \operatorname{Beta}\left(\mu_{s}, \phi_{s}\right)$, sua esperança e variância condicionais são dadas por:

$$
E(Y \mid S=s)=\mu_{s}
$$

e

$$
\operatorname{Var}(Y \mid S=s)=\frac{\mu_{s}\left(1-\mu_{s}\right)}{1+\phi_{s}} .
$$

Demonstração. Defina $Y_{s} \sim \operatorname{Beta}\left(\mu_{s}, \phi_{s}\right), s=1, \ldots, K$.

$$
\begin{aligned}
E(Y \mid S=s) & =E\left(Y_{s}\right) \\
& =\mu_{s}, \\
\operatorname{Var}(Y \mid S=s) & =\operatorname{Var}\left(Y_{s}\right) \\
& =\frac{\mu_{s}\left(1-\mu_{s}\right)}{1+\phi_{s}} .
\end{aligned}
$$

Propriedade 2.5.2. Se a variável aleatória $Y$ tem distribuição mistura finita de betas: $Y \sim$ $\sum_{s=1}^{K} \pi_{s} \operatorname{Beta}\left(\mu_{s}, \phi_{s}\right)$, sua esperança e variância marginais são dadas por:

$$
E(Y)=\sum_{s=1}^{K} \pi_{s} \mu_{s}
$$

e

$$
\begin{aligned}
\operatorname{Var}(Y) & =\sum_{s=1}^{K} \pi_{s} \frac{\mu_{s}\left(1-\mu_{s}\right)}{1+\phi_{s}}+\sum_{s=1}^{K} \pi_{s}\left[\mu_{s}-E(Y)\right]^{2} \\
& =\sum_{s=1}^{K} \pi_{s} \frac{\mu_{s}\left(1-\mu_{s}\right)}{1+\phi_{s}}+\sum_{s=1}^{K} \pi_{s}\left[\mu_{s}-\sum_{s=1}^{K} \pi_{s} \mu_{s}\right]^{2}
\end{aligned}
$$

Demonstração.

$$
\begin{aligned}
E(Y) & =E(E(Y \mid S)) \\
& =\sum_{s=1}^{K} E(Y \mid S=s) P(S=s) \\
& =\sum_{s=1}^{K} \pi_{s} \mu_{s},
\end{aligned}
$$




$$
\begin{aligned}
\operatorname{Var}(Y) & =E(\operatorname{Var}(Y \mid S))+\operatorname{Var}(E(Y \mid S)) \\
& =\sum_{s=1}^{K} \operatorname{Var}(Y \mid S=s) P(S=s)+\sum_{s=1}^{K}[E(Y \mid S=s)-E(E(Y \mid S))]^{2} P(S=s) \\
& =\sum_{s=1}^{K} \pi_{s} \frac{\mu_{s}\left(1-\mu_{s}\right)}{1+\phi_{s}}+\sum_{s=1}^{K} \pi_{s}\left[\mu_{s}-E(Y)\right]^{2} .
\end{aligned}
$$

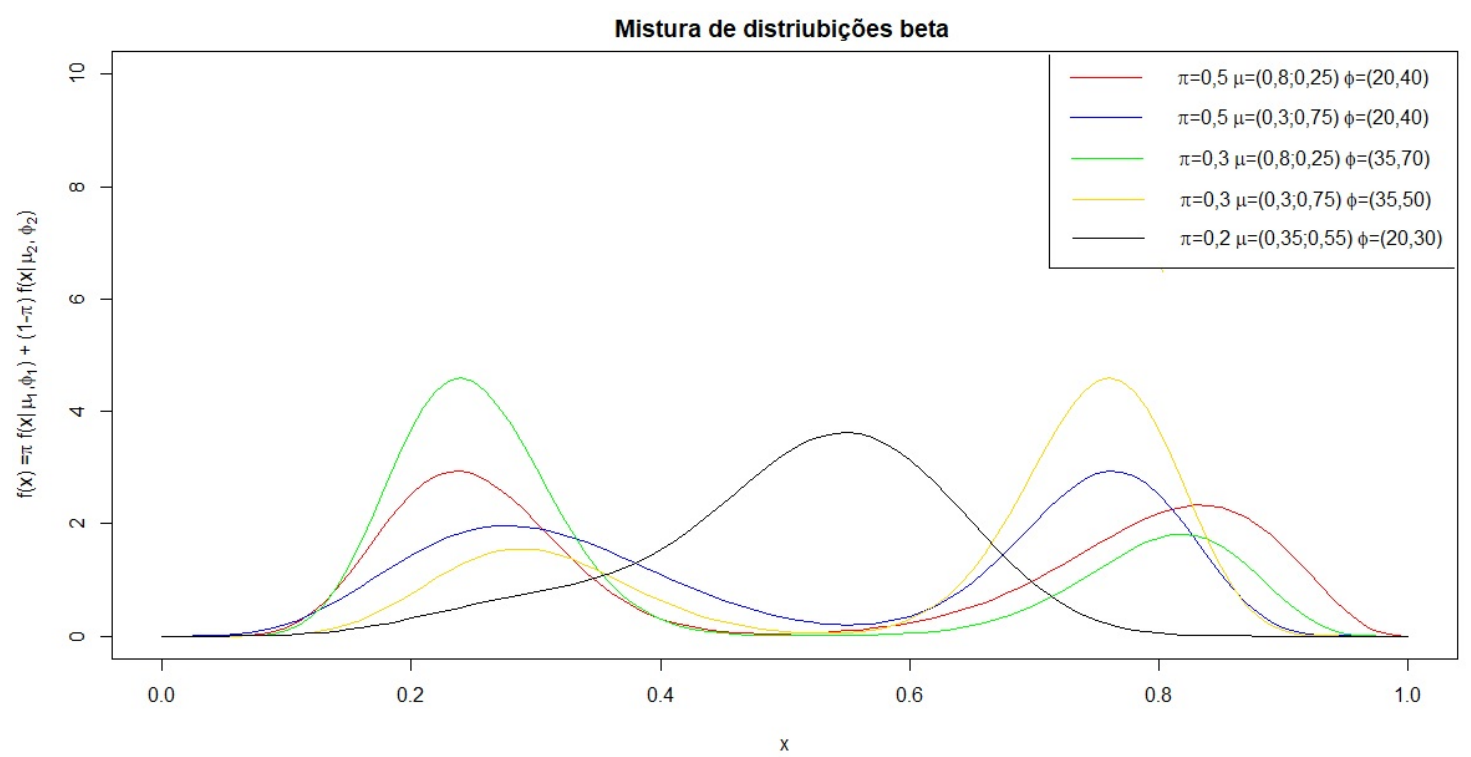

Figura 2.3: Comparação entre as densidades de distribuições mistura de beta para diferentes valores dos parâmetros $\mu$, $\phi$ e $\pi$.

Para fins de ilustração, a Figura 2.3 contém o gráfico da função densidade de probabilidade das distribuições de mistura de betas com $K=2$ para diferentes valores de média e parâmetro de precisão para cada componente da mistura.

Já existe um número significativo de trabalhos que utilizam-se da mistura finita de distribuições beta para análise de dados ou desenvolvimento de técnicas estatísticas, porém muitos deles apenas comentam a questão da identificabilidade nessa metodologia nos textos introdutórios. Alguns chegam a fazer somente restrições nos parâmetros $\pi_{1}, \ldots, \pi_{K}$, entretanto não apresentam justificativas, explicações ou demonstrações sobre essa restrição ser por si só suficiente para obter modelos identificáveis. Exemplos de trabalhos que fazem parte da limitadíssima parcela de publicações que abordam de forma mais específica esse tópico são: Fu et al. (2011) e Qi e Charnigo (2017).

Em Fu et al. (2011) os autores fazem uso da mistura finita de distribuições beta para analisar dados de genética, sendo que para as finalidades desejadas o parâmetro de média foi considerado fixo para todos os componentes da mistura. Como forma de garantir a identificabilidade a seguinte restrição acerca do parâmetro de precisão é assumida sem nenhuma justificativa: $\phi_{1}>\ldots>\phi_{K}$.

Para o caso mais geral, definido nas Equações (2.22) e (2.23), Smithson et al. (2011) mencionam não ser possível encontrar um resultado definitivo sobre a identificabilidade para a mistura de 
distribuições beta parametrizadas com parâmetros $\alpha=\mu \phi$ e $\beta=(1-\mu) \phi$, porém, os autores estudam esse tópico através de estudos de simulação com $K=2$. Foram simuladas 5000 réplicas de cada uma das três condições consideradas - mistura de duas distribuições razoavelmente precisas, de duas distribuições razoavelmente imprecisas e de uma distribuição razoavelmente imprecisa e uma distribuição precisa - e concluiu-se que existem indícios de identificabilidade sob condições realistas na grande parte das vezes pois o posto da matriz Jacobiana foi completo em $98,4 \%$ das réplicas. Porém os autores ressaltam que esse resultado não exclui a necessidade de verificação para casos específicos como aplicações, por exemplo. Segundo Qi e Charnigo (2017) as condições necessárias para que a mistura de $K=3$ distribuições beta com essa mesma parametrização seja identificável são: $\alpha_{1}<\alpha_{2}<\alpha_{3}$ e $\beta_{1}<\beta_{2}<\beta_{3}$.

Por fim, é importante esclarecer que todas as restrições mencionadas anteriormente limitam o espaço paramétrico de forma artificial, o que segundo Jasra et al. (2005) não é indicado a menos que seja necessária a interpretação dos parâmetros individualmente. Outra alternativa sugerida por estes autores é que essas restrições sejam definidas de acordo com cada estudo, ou seja, que elas se baseiem em conhecimentos específicos de especialistas da área da pesquisa, o que adiciona a complexidade extra de conciliar a adequação das restrições ao estudo com a garantia da identificabilidade.

\subsection{Modelo de Mistura Finita}

As propriedades que caracterizam as misturas finitas de distribuições podem ser desejáveis também quando existe interesse em analisar como ocorre a relação entre determinadas variáveis, denominadas explicativas, e uma ou mais variáveis resposta. Uma metodologia adequada para essa finalidade são os modelos de mistura finita, cuja variável resposta, no caso contínuo, é marginalmente distribuída por uma combinação linear de funções densidade de probabilidade (conforme definido na Equação (2.21)).

Em relação à mistura finita de distribuições, o modelo de mistura finita se diferencia por possibilitar agregar informação de covariáveis com o ajuste de modelos de regressão tanto para os parâmetros $\pi_{1}, \ldots, \pi_{K}$ quanto para os parâmetros $\boldsymbol{\theta}_{1}, \ldots, \boldsymbol{\theta}_{K}$. Assim, tem-se ideia da grande gama de dados que contém padrões em comum eletivos a serem estudados por meio destes modelos, como por exemplo a identificação de períodos de crise no mercado financeiro para melhor planejamento das ações e a classificação de imóveis em zonas para precificação e direcionamento dos clientes.

Quandt (1972), David Jr (1974) e Quandt e Ramsey (1978) iniciaram os estudos desses modelos com distribuição normal para $K=2$ e DeSarbo e Cron (1988) os generalizaram ao considerarem mais de dois elementos na mistura. Posteriormente, grande número de trabalhos foram publicados estendendo a metodologia para diversas outras distribuições, como detalha a revisão bibliográfica de Wedel e DeSarbo (1994).

Em Jansen e Nijs (1993) os autores utilizam uma mistura de duas distribuições normais num modelo de efeitos fixos para estudar o tamanho de grãos de pólen classificando-os em haploides e diploides; tal qual Xu e Hedeker (2001), que considera esta classe de modelos afim de classificar a reação de pacientes em estudos psiquiátricos longitudinais para tratamentos de depressão e esquizofrenia. Para o grande volume de dados que os estudos em genética estão disponibilizando à ciência, Ji et al. (2005) propõe uma sugestão de abordagem no estudo de correlação de expressão gênica ao considerar que cada componente da mistura agrupa genes populacionais cujos coeficientes 
de correlação possuem comportamentos similares.

Tanto em Schlattmann (2009) quanto em Theobald et al. (2012) os dados estudados são provenientes de estudos clínicos, respectivamente, de tratamentos diversos e de rotinas alimentares; os autores utilizam-se desta metodologia de análise pois, como a resposta dos pacientes aos tratamentos depende de variáveis não observáveis ou de difícil coleta, os modelos de mistura permitem classificar estes pacientes em grupos, por padrão de resposta ao tratamento, para então estudar o efeito dos tratamentos em cada um destes grupos.

Claramente a inferência nestes modelos não é trivial em função do número de parâmetros que os compõem, da escolha do número de componentes da mistura $(K)$, da não identificabilidade, da complexidade de estimação, dentre outros.

Nos casos em que $K$ é considerado fixo, a literatura especializada sugere fortemente que a escolha de seu valor seja efetuada baseando-se não somente em critérios de informação, como os apresentados na Subseção 2.1.3, mas também levando-se em consideração as características particulares da aplicação e a análise dos modelos ajustados com esses valores. Essa recomendação se faz necessária pelo fato desses critérios serem conhecidamente viesados para essa escolha. Os estudos de simulação efetuados em Sarstedt (2008) para $K=2$, por exemplo, mostraram que o AIC só seleciona o verdadeiro valor de $K$ quando $\pi_{1}$ é consideravelmente menor que $\pi_{2}$ (ou vice-versa) e, que o desempenho do BIC é consideravelmente independente dos valores de $\pi_{1}$ e $\pi_{2}$.

A identificabilidade dessas misturas de modelos de regressão de efeitos fixos é um assunto de grande necessidade de estudo principalmente quando há interesse na interpretação condicional dos parâmetros. Contudo, a complexidade de estudo desse tópico aumenta à medida que o número de parâmetros do modelo cresce e isso dificulta a análise desse tópico em muitos casos.

Uma condição necessária mas não sempre suficiente para identificabilidade é que a matriz de variáveis explicativas possua posto completo, ou seja, os vetores com valores das covariáveis sejam linearmente independentes.

Segundo Hennig (2000), os modelos de mistura lineares normais são identificáveis se $K$ for menor que o número mínimo de hiperplanos necessários para abranger os valores das variáveis explicativas. Para a mistura de modelos lineares generalizados, são necessárias condições adicionais às duas já mencionadas que dependem especificamente da distribuição dos modelos, como estudado em Grün e Leisch (2008) para os modelos multinomiais.

Com relação aos métodos apresentados na literatura para inferência dos modelos de mistura, Diebolt e Robert (1990) e Diebolt e Robert (1994) tiveram um papel importantíssimo na disseminação da abordagem bayesiana, já que os esforços no estudo dos tópicos relacionados a este tema cresceram consideravelmente a partir da publicação destes trabalhos. Eles popularizaram métodos de estimação e inferência nestes modelos através de Monte Carlo via Cadeias de Markov (MCMC), em sua maioria com o amostrador de Gibbs (Diebolt e Robert, 1994; Escobar e West, 1995; Bouguila et al., 2006). Outros precursores deste tema são Gelman e King (1990), Verdinelli e Wasserman (1991), Evans et al. (1992) e Lavine e West (1992).

Outra técnica de inferência bayesiana é abordada em Astuti et al. (2016) que considera a metodologia Bayesian mixture model averaging (BMMA) na análise de dados longitudinais de pacientes com diabetes; essa é uma metodologia indicada para estimação bayesiana de modelos de mistura em que o número de componentes $(K)$ é estipulado de acordo com a distribuição a posteriori dos possíveis modelos. 
Acerca da análise de diagnóstico, Smithson et al. (2011) relatam que nos modelos lineares generalizados de mistura finita esta analisa, em geral, a qualidade das estruturas de média e variância por meio da reprodutibilidade da simulação da densidade preditiva posteriori.

O modelo de mistura beta de efeitos fixos foi aplicado por exemplo por Heisterkamp e Pennings (2004) e Laurila et al. (2011) para analisar dados genéticos (estimando com algoritmo EM), sendo que em Laurila et al. (2011) os elementos da mistura caracterizam três categorias de reações químicas específicas do DNA, por Smithson et al. (2011) para analisar dados judiciais, por Adhikari et al. (2016) que propõem um método de análise de dados heterogêneos composto por três passos: categorização por meio de modelos de mistura, compreensão dos padrões de categorização e, representação dos resultados por meio de uma técnica denominada banded matrix e, por fim, por Anuradha e Satyanarayana (2017) que o utilizou para identificar tumores cerebrais em exames de imagem.

Sobre a inferência nos modelos de mistura beta, Verkuilen e Smithson (2012) comparam a estimação dos parâmetros dos modelos de regressão beta mistos e dos modelos de mistura beta de efeitos fixos pelos métodos de máxima verossimilhança, de quasi-verossimilhança e MCMC; este último se mostrou mais eficiente já que, geralmente, possui desempenho melhor na estimação em modelos complexos; porém, o MCMC pode ser de difícil análise da convergência e de atribuição de distribuição a priori dos parâmetros da variância.

Bouguila et al. (2006) exemplificam o uso do amostrador de Gibbs na estimação dos parâmetros do modelo de mistura beta quando o número de elementos da mistura $(K)$ é conhecido, identificando dificuldades com o tempo computacional e com a verificação da convergência do algoritmo. Outra vertente possível da abordagem bayesiana é a aproximação fatorizada para aproximar as distribuições a priori e a posteriori no contexto de variational inference (VI), como apresenta Ma e Leijon (2011); essa abordagem pode evitar o problema de superestimação nos modelos de mistura beta, porém também pode trazer erros desconhecidos, o que não ocorre em algoritmos MCMC.

Sobre a identificabilidade dos modelos de mistura beta, Smithson et al. (2011) declaram não ser possível estabelecer condições teóricas que garantam a presença de identificabilidade nesses modelos e sugerem que essa verificação seja efetuada por meio de estudos de simulação que variem os valores dos parâmetros: quanto maior a proporção de matrizes jacobianas com posto completo maiores são as evidências de que o modelo é identificável.

\subsection{Algoritmo Data Cloning para diagnóstico de identificabilidade}

Além da complexidade da estimação dos parâmetros, a dificuldade de se verificar possíveis problemas de identificabilidade pode dificultar e desencorajar o uso de modelos de mistura. Alternativamente às definições de identificabilidade previamente apresentadas, pode-se dizer que a não identificabilidade de um conjunto de parâmetros ocorre quando a função de verossimilhança conjunta para os dados observados possui mais de um valor máximo (Lele et al., 2010).

Dentre as consequências da falta de identificabilidade de um modelo estatístico está a invalidação da interpretação dos resultados inferenciais dos parâmetros deste modelo de forma isolada. Por exemplo, quando as estimativas de máxima verossimilhança dos parâmetros de um modelo de regressão simples não são únicas, pois mais de um conjunto de valores maximizam a verossimilhança do modelo, é incorreto analisar a estimativa do intercepto desse modelo. Outra contraindicação 
importante é a respeito do uso das propriedades assintóticas dos estimadores de máxima verossimilhança, principalmente quando utilizadas para cálculo da variância dos estimadores, através do inverso da matriz de informação de Fisher, e tomada de decisões baseada em testes de hipóteses.

Introduzido em Lele et al. (2007), o algoritmo, do inglês denominado, data cloning é um método computacional que se baseia em técnicas computacionais bayesianas, principalmente algoritmos MCMC, para calcular estimadores de máxima verossimilhança e suas variâncias, assim como intervalos de predição para componentes aleatórios do modelo.

Como seu próprio nome expressa, o algoritmo consiste em primeiramente construir um banco de dados fazendo $n_{\text {clone }}$ repetições dos dados originais, em que $n_{\text {clone }}$ denota o número de clones. Para dados correlacionados como os tratados nesta tese, por exemplo, a base de dados da variável resposta utilizada é constituída por $\boldsymbol{y}^{n_{\text {clone }}}=\left\{\boldsymbol{y}^{(N)}, \boldsymbol{y}^{(N)}, \ldots, \boldsymbol{y}^{(N)}\right\}$, em que $\boldsymbol{y}^{(N)}$ são os dados dos $N$ grupos $\left(n_{i}\right.$ observações por grupo, $\left.i=1, \ldots, N\right)$, assim os dados clonados são compostos por $N \times n_{\text {clone }}$ grupos com as mesmas $n_{i}$ observações por grupo.

Segundo Lele et al. (2010), como a função de verossimilhança dos dados clonados é igual a $\left[L\left(\boldsymbol{\theta} ; \boldsymbol{y}^{(N)}\right)\right]^{n_{\text {clone }}}$, em que $L\left(\boldsymbol{\theta} ; \boldsymbol{y}^{(N)}\right)$ é a função de verossimilhança dos dados originais, tem-se que os pontos de máximo de ambas são iguais e que a matriz de informação de Fisher da primeira é $n_{\text {clone vezes a da segunda. }}$

Em seguida, atribui-se distribuições a priori para os parâmetros do modelo, executam-se algoritmos MCMC e obtém-se amostras a posteriori destes parâmetros. Como consequência do aumento de $n_{\text {clone }}$ ocorre o aumento do tamanho dos dados, porém se mantém as mesmas informações da amostra original, portanto quanto maior o número de clones, menor é a influência das distribuições a priori no processo.

Garantida a convergência dos algoritmos por meio da estatística de Gelman-Rubin (Sólymos , 2010), tem-se que a medida que o valor $n_{\text {clone }}$ cresce, além da média das amostras a posteriori convergir para a estimativa de máxima verossimilhança, a variância a posteriori multiplicada por $n_{\text {clone }}$ converge para a variância assintótica destas estimativas.

Adicionalmente a essas finalidades, Lele et al. (2010) destacam que a mais importante das utilidades do método é a detecção de problemas de identificabilidade em modelos hierárquicos. Os autores demonstram que uma consequência da não identificabilidade dos parâmetros é que o maior autovalor padronizado da matriz de variâncias e covariâncias a posteriori não converge para o valor zero a medida que o número de clones aumenta, enfatizando que esse resultado é valioso na verificação de falhas de identificabilidade em modelos. Essa padronização é a divisão dos autovalores pelo autovalor obtido na aplicação do método com $n_{\text {clone }}=1$. Com relação à convergência, quanto mais próxima do inverso do número de clones ela ocorrer, mais evidências existem de que não há problemas de identificabilidade com os parâmetros do modelo.

Outra referência que preconiza a utilização do método como ferramenta precisa de diagnóstico de não identificabilidade ao invés da sua aplicação para fins inferenciais, como inicialmente apresentado, é Ponciano et al. (2012), que estuda a identificabilidade de modelos filogenéticos.

Dentre os diversos métodos com diferentes enfoques para explorar a identificabilidade nos modelos apresentados neste trabalho, o que apresentou maior viabilidade considerando o custo computacional e a tratabilidade da teoria envolvida foi o método data cloning.

Vale ressaltar que a questão da identificabilidade em modelos complexos, com exceção de alguns casos específicos, é estudada através do diagnóstico de não identificabilidade, assim, os resultados das 
análises indicam que não há evidências de problemas com identificabilidade considererando os dados ajustados. Não é indicado afirmar a existência de identificabilidade como conclusão, principalmente sem um estudo robusto com diversos valores de parâmetros no caso de modelos teóricos; por isso, recomenda-se que em aplicações nas quais essa propriedade seja muito importante ao pesquisador, a avaliação da convergência dos autovalores padronizados seja efetuada para os dados do estudo.

Por tudo o que foi apontado sobre o data cloning, principalmente pela sua praticabilidade e resultados satisfatórios em modelos complexos, essa foi a opção considerada como a mais adequada para abordar o tópico de identificabilidade nos modelos apresentados neste trabalho. Para tal, o método foi aplicado através do pacote dclone (Sólymos, 2010) do software R, o qual disponibiliza como opções de softwares bayesianos tanto o OpenBUGS como o WinBUGS e o JAGS. 


\section{Capítulo 3}

\section{Modelo de Mistura Beta Linear com Efeitos Aleatórios}

Este capítulo é dedicado à apresentação do modelo de mistura beta com efeitos aleatórios com parâmetro de precisão constante em todos os seus aspectos. Alguns tópicos que já foram citados no capítulo anterior são retomados para uma melhor contextualização. Após a especificação do modelo (Seção 3.2), são sugeridas distribuições a priori para os parâmetros (Seção 3.3) e analisada por meio de estudos de simulação a performance inferencial da metodologia bayesiana utilizada (Seção 3.4). Por fim, na Seção 3.5, dois bancos de dados são analisados através da técnica estatística proposta ao longo do capítulo.

\subsection{Introdução}

Para a análise de bancos de dados formados por subgrupos homogêneos em relação a características não observáveis ou de difícil aferição, os modelos de mistura são fortemente indicados. Porém, quando, nesses dados, além de existir essa heterogeneidade não observada, também existe heterogeneidade nas observações dessas subpopulações, a não incorporação de efeitos aleatórios no modelo pode resultar em imprecisões inferenciais como superparametrização tanto na estimação do número de componentes da mistura $(K)$ quanto no número de covariáveis selecionadas.

Quando se estuda o comportamento de dados clínicos de pacientes aferidos em diferentes momentos de um intervalo de tempo, denominados dados longitudinais, é necessário entender que, além da correlação existente entre observações de cada paciente, também é possível haver subgrupos de pacientes cuja evolução das medidas seguem padrões semelhantes devido a particularidades não observadas como hábitos alimentares, herança genética, sedentarismo, complicações de saúde não diagnosticadas, dentre outras. Esses subgrupos de pacientes têm suas especificidades representadas ou levadas em conta pelos componentes da mistura e os efeitos aleatórios retratam a dependência entre as observações de cada paciente.

Outro exemplo de dados com essa estrutura é a aglomeração de lojas de franquias alimentícias nas cidades brasileiras: as observações de unidades de lojas e redes instaladas em um mesmo município não são independentes, assim como existem diferentes padrões de aglomeração das redes de acordo com particularidades não observáveis. Estas duas características justificam a adequação da presença de efeitos aleatórios associados às cidades e componentes da mistura no modelo ajustado 
ao banco de dados.

Theobald et al. (2012) ilustram a teoria de forma clara ao utilizarem o modelo de mistura com efeitos aleatórios para analisar dados longitudinais de dieta alimentar de indivíduos. Neste caso, a presença dos efeitos aleatórios e dos componentes da mistura no modelo é apropriada devido à existência, respectivamente, de correlação entre as observações de cada paciente e de heterogeneidade decorrente da mudança dos hábitos alimentares entre os dias úteis e os demais dias da semana.

Outro exemplo de aplicação é apresentado em Wang et al. (2002), no qual foram analisados os tempos de internação de pacientes gestantes do setor obstétrico de diferentes hospitais para estudar de que forma as características hospitalares influenciam na variável resposta através dos efeitos aleatórios, dado que as variações provenientes das gestações estão adequadamente representadas no modelo através dos componentes da mistura do modelo hierárquico Poisson.

Os pioneiros na inclusão de efeitos aleatórios nos modelos de mistura para analisar dados correlacionados foram Belin e Rubin (1995), os quais engajaram o crescimento dos estudos nessa área. Uma boa referência sobre o assunto é Frühwirth-Schnatter (2006), na qual grande parte dos aspectos essenciais desses modelos são abordados de forma clara.

No que concerne aos diferentes métodos de estimação do modelo de mistura para dados correlacionados, dentre as publicações que examinam suas performances, Hall e Wang (2005) concluem através de estudos de simulação e de resultados teóricos que o desempenho da estimação por máxima verossimilhança é fraco em relação aos que utilizam-se de técnicas bayesianas. Em Theobald et al. (2012), os pesquisadores obtiveram resultados satisfatórios, principalmente na questão preditiva, ajustando os modelos também sob essa abordagem através do software WinBUGS.

Vermunt (2008) faz uma breve discussão a respeito dos processos de estimação ao considerar o método de MV que pode ser efetuado por meio do algoritmo EM. Segundo o autor, a estimação Bayesiana pode ser complicada em função da possibilidade de ocorrência da invariância à permutação dos componentes da mistura (do inglês denominada label-switching), fenômeno esse que pode ser evitado com o uso de restrições tal qual as apresentadas na Seção 2.5. Esse método de estimação também foi utilizado em Wang et al. (2002) e Yu e Yau (2013) para modelos de mistura Poisson com efeitos aleatórios.

Dentre os escassos trabalhos que mencionam o tópico de identificabilidade nestes modelos estão Ng et al. (2006) e Tutz e Oelker (2017). O primeiro apenas cita uma situação que pode ocasionar problemas de identificabilidade: quando as matrizes de delineamento dos efeitos fixos e de um subconjunto dos efeitos aleatórios são iguais no modelo de mistura misto normal utilizado para aplicação em genética. A segunda referência somente declara não considerar modelos cuja matriz de delineamento dos efeitos aleatórios seja um subconjunto da matriz de covariáveis dos efeitos fixos para evitar problemas de falta de identificabilidade.

Referindo-se especificamente à distribuição beta, Smithson et al. (2011) consideram o modelo de mistura beta sem efeitos aleatórios para dados que apresentam bimodalidade, assimetrias, heteroscedasticidade, já que os modelos normais são inadequados para ajustar dados limitados. Em Ji et al. (2005) este modelo é utilizado para analisar dados de genética, utilizando algoritmo EM, com o objetivo de classificar genes com coeficiente de correlação semelhantes.

Em Verkuilen e Smithson (2012) são abordados os modelos beta mistos e os modelos de mistura beta de efeitos fixos, inclusive mencionando se tratarem ambos, essencialmente, da mesma metodologia pois nos dois casos as variáveis latentes caracterizam dependência entre observações de um 
mesmo grupo; porém, em nenhuma parte da publicação os autores fazem menção aos modelos de regressão beta com ambas características (presença de componentes de mistura e efeitos aleatórios).

Portanto, neste capítulo é especificado e estudado o novo modelo de mistura beta linear com efeitos aleatórios.

\subsection{Modelo Estatístico}

O modelo proposto nesta seção é indicado para análise de dados que apresentam heterogeneidade intra e entre subpopulações componentes da mistura, como contextualizado no texto até aqui apresentado. Esse modelo foi desenvolvido assumindo-se que todas as observações de uma mesma unidade amostral $\left(y_{i 1}, \ldots, y_{i n_{i}}\right), i=1, \ldots N$, se associam a um mesmo componente da mistura e que a estimação dos parâmetros é efetuada inicialmente fixando-se o número de componentes da mistura $K$, o qual será definido posteriormente com a análise das estimativas e dos critérios de informação.

Na Seção 3.4 estudos de simulação são apresentados com o intuito de analisar mais detalhadamente o desempenho da estimação dos respectivos parâmetros e demais aspectos inferenciais.

Sejam $\boldsymbol{y}_{1}, \ldots, \boldsymbol{y}_{N}$ as observações da variável resposta tais que cada $i$-ésima unidade amostral $(i=1, \ldots, N)$ é composta pelas suas $n_{i}$ aferições, ou seja, $\boldsymbol{y}_{i}=\left(y_{i 1}, \ldots, y_{i n_{i}}\right)^{\top}, S_{i}$ a variável discreta que indica o componente da mistura associado ao $i$-ésimo grupo da amostra, tal que os valores de $S_{i}$ pertencem ao conjunto $\{1, \ldots, K\}$ e, assumindo que

$$
Y_{i j} \mid \boldsymbol{b}_{i} \sim \sum_{S_{i}=1}^{K} \pi_{i S_{i}} \operatorname{Beta}\left(\mu_{S_{i}, i j}, \phi_{S_{i}}\right)
$$

$\mathrm{e}$

$$
\mu_{S_{i}, i j}=E\left(Y_{i j} \mid \boldsymbol{b}_{i}, S_{i}\right)
$$

o modelo de mistura beta linear com efeitos aleatórios para a média tem sua parte sistemática dada por

$$
\eta_{S_{i}, i j}=g_{\mu}\left(\mu_{S_{i}, i j}\right)=\boldsymbol{x}_{i j}^{\top} \boldsymbol{\beta}^{S_{i}}+\boldsymbol{z}_{i j}^{\top} \boldsymbol{b}_{i}
$$

em que:

- $Y_{i j}$ é a variável resposta;

- $K$ é o número de componentes da mistura;

- $N$ é o número de grupos da amostra;

- $n_{i}$ é o número de elementos do $i$-ésimo grupo, $i=1, \ldots, N$;

- $\phi=\left(\phi_{1}, \ldots, \phi_{K}\right)$;

- $\boldsymbol{\pi}_{i}=\left(\pi_{i 1}, \ldots, \pi_{i K}\right)^{\top}$, em que $\pi_{i s}$ é a probabilidade do $i$-ésimo grupo estar associado ao $s$-ésimo componente da mistura, ou seja, $\pi_{i s}=P\left(S_{i}=s\right), 0<\pi_{i s}<1, \sum_{s=1}^{K} \pi_{i s}=1, i=1, \ldots, N$ e $s=1, \ldots, K$ 
- $\boldsymbol{\pi}=\left(\boldsymbol{\pi}_{1}, \ldots, \boldsymbol{\pi}_{N}\right)$

- $g_{\mu}$ é a função de ligação, estritamente monótona e duplamente diferenciável, do modelo para a média;

- $\boldsymbol{\beta}^{s}=\left(\beta_{1}^{s}, \ldots, \beta_{p}^{s}\right)^{\top}$ é o vetor $p$-dimensional de coeficientes fixos relacionado ao $s$-ésimo componente da mistura associada ao modelo para a média, $s=1, \ldots, K$;

- $\boldsymbol{\beta}=\left(\boldsymbol{\beta}^{1}, \ldots, \boldsymbol{\beta}^{K}\right)$ é a matriz de ordem $p \times K$ de coeficientes fixos do modelo para a média;

- $p$ é o número de efeitos fixos do modelo para a média;

- $\boldsymbol{b}_{i}=\left(b_{i 1}, \ldots, b_{i d}\right)^{\top}$ é o vetor de dimensão $d$ de efeitos aleatórios do $i$-ésimo grupo do modelo para a média;

- $d$ é o número de efeitos aleatórios do modelo para a média;

- $\boldsymbol{b}_{i} \mid \boldsymbol{\Sigma}_{b} \stackrel{i i d}{\sim} N_{d}\left(\mathbf{0}, \boldsymbol{\Sigma}_{b}\right)$

- $\boldsymbol{\Sigma}_{b}$ é a matriz de ordem $d \times d$ de variância-covariância dos efeitos aleatórios $\left(\boldsymbol{b}_{1}, \ldots, \boldsymbol{b}_{N}\right)$ do modelo para a média;

- $\boldsymbol{x}_{i j}=\left(x_{i j 1}, \ldots, x_{i j p}\right)^{\top}$ é o vetor $p$-dimensional de covariáveis dos efeitos fixos do $j$-ésimo elemento do $i$-ésimo grupo do modelo para a média, $i=1, \ldots, N$ e $j=1, \ldots, n_{i}$;

- $\boldsymbol{z}_{i j}=\left(z_{i j 1}, \ldots, z_{i j d}\right)^{\top}$ é o vetor $d$-dimensional de covariáveis dos efeitos aleatórios do $j$-ésimo elemento do $i$-ésimo grupo do modelo para a média, $i=1, \ldots, N$ e $j=1, \ldots, n_{i}$;

- $\boldsymbol{\vartheta}=\left\{\boldsymbol{\beta}^{1^{\top}}, \ldots, \boldsymbol{\beta}^{K^{\top}}, \operatorname{vech}^{\top}\left(\boldsymbol{\Sigma}_{b}\right), \boldsymbol{\pi}_{1}^{\top}, \ldots, \boldsymbol{\pi}_{N}^{\top}, \boldsymbol{\phi}\right\}$ é o vetor de parâmetros do modelo, lembrando que vech $(A)$ é o vetor composto pelos $\frac{K(K+1)}{2}$ elementos únicos da matriz simétrica $A_{K \times K}$.

Qualquer dúvida que possa surgir a respeito da notação pode-se verificar na lista de símbolos que se encontra no início do texto.

Alternativamente, pode-se especificar esse modelo de forma hierárquica como:

$$
\begin{array}{r}
Y_{i j} \mid \boldsymbol{b}_{i}, S_{i}=s \sim \operatorname{Beta}\left(g_{\mu}^{-1}\left(\boldsymbol{x}_{i j}^{\top} \boldsymbol{\beta}^{s}+\boldsymbol{z}_{i j}^{\top} \boldsymbol{b}_{i}\right), \phi_{s}\right) \\
\boldsymbol{b}_{i} \mid \boldsymbol{\Sigma}_{b} \stackrel{i i d}{\sim} N_{d}\left(\mathbf{0}, \boldsymbol{\Sigma}_{b}\right) \\
P\left(S_{i}=s\right)=\pi_{s},
\end{array}
$$

isto é: $Y_{i j} \mid \boldsymbol{b}_{i} \sim \sum_{s=1}^{K} \pi_{i s} \operatorname{Beta}\left(g_{\mu}^{-1}\left(\boldsymbol{x}_{i j}^{\top} \boldsymbol{\beta}^{s}+\boldsymbol{z}_{i j}^{\top} \boldsymbol{b}_{i}\right), \phi_{s}\right)$.

Apesar da suposição de normalidade dos efeitos aleatórios na especificação do modelo, pode-se considerar outras distribuições para os efeitos aleatórios que sejam mais adequadas à analise em desenvolvimento, como por exemplo a distribuição $t$ de Student. Com o uso de outras distribuições para os efeitos aleatórios, toda metodologia apresentada permanece válida e apenas haverá a necessidade da atribuição de distribuições a priori para os hiperparâmetros dos efeitos aleatórios. Indicações de referências que explanam sobre essa atribuição e outras considerações são apresentadas na Seção 2.3. 
Também é possível considerar o caso particular em que o parâmetro de precisão é o mesmo para todos os componentes da mistura, ou seja, $\phi_{1}=\phi_{2}=\ldots=\phi_{K}=\phi$; caso esse que simplifica o processo de inferência do modelo por reduzir o número de parâmetros (dimensão de $\boldsymbol{\vartheta}$ ) e, consecutivamente, o tempo computacional.

Considerando o modelo (3.3) e a condicionalidade nos efeitos aleatórios, as Equações (3.5) e (3.6) expressam, respectivamente, as verossimilhanças condicional e marginal conjuntas.

$$
\begin{aligned}
L(\boldsymbol{y} \mid \boldsymbol{\vartheta}, \boldsymbol{X}, \boldsymbol{Z}, \boldsymbol{b}, \boldsymbol{S}) & =\prod_{i=1}^{N} \prod_{j=1}^{n_{i}}\left[L_{i j}\left(y_{i j} \mid \boldsymbol{\vartheta}, \boldsymbol{x}_{i j}, \boldsymbol{z}_{i j}, \boldsymbol{b}_{i}, S_{i}\right)\right] \\
& =\prod_{i=1}^{N} \prod_{j=1}^{n_{i}} \frac{\Gamma\left(\phi_{S_{i}}\right) y_{i j}^{g_{\mu}^{-1}\left(\eta_{i j}\right) \phi_{S_{i}}-1}\left(1-y_{i j}\right)^{\left(1-g_{\mu}^{-1}\left(\eta_{i j}\right)\right) \phi_{S_{i}}-1}}{\Gamma\left(g_{\mu}^{-1}\left(\eta_{i j}\right) \phi_{S_{i}}\right) \Gamma\left(\left(1-g_{\mu}^{-1}\left(\eta_{i j}\right)\right) \phi_{S_{i}}\right)} \\
& =\prod_{i=1}^{N} \prod_{j=1}^{n_{i}} \frac{\Gamma\left(\phi_{S_{i}}\right) y_{i j}^{g_{\mu}^{-1}\left(\boldsymbol{x}_{i j}^{\top} \boldsymbol{\beta}^{S_{i}}+\boldsymbol{z}_{i j}^{\top} \boldsymbol{b}_{i}\right) \phi_{S_{i}}-1}\left(1-y_{i j}\right)^{\left(1-g_{\mu}^{-1}\left(\boldsymbol{x}_{i j}^{\top} \boldsymbol{\beta}^{S_{i}}+\boldsymbol{z}_{i j}^{\top} \boldsymbol{b}_{i}\right)\right) \phi_{S_{i}}-1}}{\Gamma\left(g_{\mu}^{-1}\left(\boldsymbol{x}_{i j}^{\top} \boldsymbol{\beta}^{S_{i}}+\boldsymbol{z}_{i j}^{\top} \boldsymbol{b}_{i}\right) \phi_{S_{i}}\right) \Gamma\left(\left(1-g_{\mu}^{-1}\left(\boldsymbol{x}_{i j}^{\top} \boldsymbol{\beta}^{S_{i}}+\boldsymbol{z}_{i j}^{\top} \boldsymbol{b}_{i}\right)\right) \phi_{S_{i}}\right)} . \\
L(\boldsymbol{y} \mid \boldsymbol{\vartheta}, \boldsymbol{X}, \boldsymbol{Z}, \boldsymbol{b}) & =\prod_{i=1}^{N} \prod_{j=1}^{n_{i}}\left[L_{i j}\left(y_{i j} \mid \boldsymbol{\vartheta}, \boldsymbol{x}_{i j}, \boldsymbol{z}_{i j}, \boldsymbol{b}_{i}\right)\right] \\
& =\prod_{i=1}^{N} \prod_{j=1}^{n_{i}} \sum_{S_{i}=1}^{K} \pi_{i S_{i}}\left[L_{i j}\left(y_{i j} \mid \boldsymbol{\vartheta}, \boldsymbol{x}_{i j}, \boldsymbol{z}_{i j}, \boldsymbol{b}_{i}, S_{i}\right)\right] \\
& =\prod_{i=1}^{N} \sum_{S_{i}=1}^{K} \pi_{i S_{i}} \prod_{j=1}^{n_{i}}\left[L_{i j}\left(y_{i j} \mid \boldsymbol{\vartheta}, \boldsymbol{x}_{i j}, \boldsymbol{z}_{i j}, \boldsymbol{b}_{i}, S_{i}\right)\right]
\end{aligned}
$$

em que

$$
L_{i j}\left(y_{i j} \mid \boldsymbol{\vartheta}, \boldsymbol{x}_{i j}, \boldsymbol{z}_{i j}, \boldsymbol{b}_{i}, S_{i}\right)=\frac{\Gamma\left(\phi_{S_{i}}\right) y_{i j}^{g_{\mu}^{-1}\left(\boldsymbol{x}_{i j}^{\top} \boldsymbol{\beta}^{S_{i}}+\boldsymbol{z}_{i j}^{\top} \boldsymbol{b}_{i}\right) \phi_{S_{i}}-1}\left(1-y_{i j}\right)^{\left(1-g_{\mu}^{-1}\left(\boldsymbol{x}_{i j}^{\top} \boldsymbol{\beta}^{S_{i}}+\boldsymbol{z}_{i j}^{\top} \boldsymbol{b}_{i}\right)\right) \phi_{S_{i}}-1}}{\Gamma\left(g_{\mu}^{-1}\left(\boldsymbol{x}_{i j}^{\top} \boldsymbol{\beta}^{S_{i}}+\boldsymbol{z}_{i j}^{\top} \boldsymbol{b}_{i}\right) \phi_{S_{i}}\right) \Gamma\left(\left(1-g_{\mu}^{-1}\left(\boldsymbol{x}_{i j}^{\top} \boldsymbol{\beta}^{S_{i}}+\boldsymbol{z}_{i j}^{\top} \boldsymbol{b}_{i}\right)\right) \phi_{S_{i}}\right)} .
$$

Seja $p_{b}(\cdot)$ a distribuição de $\boldsymbol{b}_{i}$, incorporando a integração dos efeitos aleatórios, as verossimilhanças condicional e marginal conjuntas sâo expostas nas Equações (3.8) e (3.9).

$$
L(\boldsymbol{y} \mid \boldsymbol{\vartheta}, \boldsymbol{X}, \boldsymbol{Z}, \boldsymbol{S})=\prod_{i=1}^{N} \prod_{j=1}^{n_{i}}\left[\int L_{i j}\left(y_{i j} \mid \boldsymbol{\vartheta}, \boldsymbol{x}_{i j}, \boldsymbol{z}_{i j}, \boldsymbol{b}_{i}, S_{i}\right) p_{b}\left(\boldsymbol{b}_{i}\right) d \boldsymbol{b}_{i}\right] .
$$




$$
\begin{aligned}
L(\boldsymbol{y} \mid \boldsymbol{\vartheta}, \boldsymbol{X}, \boldsymbol{Z}) & =\prod_{i=1}^{N} \prod_{j=1}^{n_{i}}\left[\int L_{i j}\left(y_{i j} \mid \boldsymbol{\vartheta}, \boldsymbol{x}_{i j}, \boldsymbol{z}_{i j}, \boldsymbol{b}_{i}\right) p_{b}\left(\boldsymbol{b}_{i}\right) d \boldsymbol{b}_{i}\right] \\
& =\prod_{i=1}^{N} \prod_{j=1}^{n_{i}} \sum_{S_{i}=1}^{K} \pi_{i S_{i}}\left[\int L_{i j}\left(y_{i j} \mid \boldsymbol{\vartheta}, \boldsymbol{x}_{i j}, \boldsymbol{z}_{i j}, \boldsymbol{b}_{i}, S_{i}\right) p_{b}\left(\boldsymbol{b}_{i}\right) d \boldsymbol{b}_{i}\right] \\
& =\prod_{i=1}^{N} \sum_{S_{i}=1}^{K} \pi_{i S_{i}} \prod_{j=1}^{n_{i}}\left[\int L_{i j}\left(y_{i j} \mid \boldsymbol{\vartheta}, \boldsymbol{x}_{i j}, \boldsymbol{z}_{i j}, \boldsymbol{b}_{i}, S_{i}\right) p_{b}\left(\boldsymbol{b}_{i}\right) d \boldsymbol{b}_{i}\right],
\end{aligned}
$$

em que $L_{i j}\left(y_{i j} \mid \boldsymbol{\vartheta}, \boldsymbol{x}_{i j}, \boldsymbol{z}_{i j}, \boldsymbol{b}_{i}, S_{i}\right)$ é expresso na Equação (3.7).

Sob abordagem frequentista, a estimação dos parâmetros dos modelos de mistura são em sua maioria efetuadas por meio do algoritmo EM (Frühwirth-Schnatter, 2006); isso ocorre em função de este ser um processo iterativo mais facilmente implementável do que expressões fechadas para um modelo tão complexo quanto o apresentado. Bouguila et al. (2006) relatam que o alto custo computacional do passo M (maximization) e superparametrização são duas desvantagens do uso dessa técnica para fazer inferência em modelos de mistura beta.

Baseando-se nos relatos e recomendações da literatura especializada e nas experiências próprias, foi escolhida a abordagem bayesiana nos estudos de simulação e nas aplicações deste trabalho.

\subsection{Distribuições a priori e a posteriori}

Estabelecer as distribuições a priori para os parâmetros que compõem $\boldsymbol{\vartheta}$ é o primeiro passo para ajustar o modelo proposto; essas distribuições são respectivamente: $p_{\beta}\left(\boldsymbol{\beta}^{s}\right), p_{\phi}\left(\phi_{s}\right), p_{\pi}\left(\boldsymbol{\pi}_{i}\right)$, $p_{\Sigma_{b}}\left(\boldsymbol{\Sigma}_{b}\right), i=1, \ldots, N$ e $s=1, \ldots, K$.

Considerando o conteúdo apresentado sobre atribuição de distribuições a priori no capítulo de contextualizações (2.1.1) e as indicações das referências estudadas, a Tabela 3.1 traz as sugestões das referentes distribuições a priori, enfatizando que são sugestões e a não aderência não prejudica necessariamente a acurácia dos resultados e nem a generalidade da metodologia.

Para não sobrecarregar a linguagem e nem a notação, tanto os vetores de parâmetros quanto os componentes de matrizes de parâmetros serão referenciados apenas pelo termo parâmetro e de maneira similar se fará a menção aos hiperparâmetros.

\begin{tabular}{c|c|c}
\hline Parâmetro & Notação da distribuição a priori & Distribuição a priori \\
\hline $\boldsymbol{\beta}^{s}$ & $p_{\beta}\left(\boldsymbol{\beta}^{s}\right)$ & $N_{p}\left(\boldsymbol{\mu}_{\boldsymbol{\beta}^{s}}, a_{\beta} \boldsymbol{I}_{p}\right)$ \\
$\boldsymbol{\pi}_{i}$ & $p_{\pi}\left(\boldsymbol{\pi}_{i}\right)$ & $\operatorname{Dir}(\boldsymbol{\alpha})$ \\
$\phi_{s}$ & $p_{\phi}\left(\phi_{s}\right)$ & $\operatorname{Unif}(a, b)$ \\
$\boldsymbol{\Sigma}_{b}$ & $p_{\Sigma_{b}}\left(\boldsymbol{\Sigma}_{b}\right)$ & $I W(\boldsymbol{\Psi}, \boldsymbol{C})$ \\
\hline
\end{tabular}

Tabela 3.1: Parâmetros e distribuições a priori indicadas para ajuste do modelo de mistura beta linear com efeitos aleatórios para a média, $s=1, \ldots, K$ e $i=1, \ldots, N$.

Com relação à escolha dos hiperparâmetros $\boldsymbol{\mu}_{\boldsymbol{\beta}^{s}}, a_{\beta}, \boldsymbol{\alpha}, a, b, \boldsymbol{\Psi}$ e $\boldsymbol{C}$, ela pode ser efetuada de forma a obter distribuições a priori não informativas ou distribuições a priori que agregam informações prévias; inclusive podendo-se considerar modelos hierárquicos, os quais atribuem distribuições 
para os hiperparâmetros. Nos estudos de simulação são fixados alguns valores de hiperparâmetros e seus respectivos desempenhos são analisados.

Sejam $L(\boldsymbol{y} \mid \boldsymbol{\vartheta}, \boldsymbol{X}, \boldsymbol{Z}, \boldsymbol{b})$ a função de verossimilhança (3.6), $p_{b}(\boldsymbol{b} \mid \boldsymbol{\vartheta})$ a função densidade de probabilidade dos efeitos aleatórios e $p(\boldsymbol{\vartheta})$ a distribuição a priori do vetor de parâmetros, aplicando o teorema de Bayes (2.1), a distribuição a posteriori de $\boldsymbol{\vartheta}, p(\boldsymbol{\vartheta}, \boldsymbol{b} \mid \boldsymbol{y}, \boldsymbol{X}, \boldsymbol{Z})$, é especificada na Equação (3.10).

$$
\begin{aligned}
p(\boldsymbol{\vartheta}, \boldsymbol{b} \mid \boldsymbol{y}, \boldsymbol{X}, \boldsymbol{Z}) & \propto L(\boldsymbol{y} \mid \boldsymbol{\vartheta}, \boldsymbol{X}, \boldsymbol{Z}, \boldsymbol{b}) p_{b}(\boldsymbol{b} \mid \boldsymbol{\vartheta}) p(\boldsymbol{\vartheta}) \\
& =p_{b}\left(\boldsymbol{b} \mid \boldsymbol{\Sigma}_{b}\right) p_{\Sigma_{b}}\left(\boldsymbol{\Sigma}_{b}\right) \prod_{i=1}^{N} \sum_{s=1}^{K} \pi_{i s} p_{\pi}\left(\boldsymbol{\pi}_{i}\right) p_{\phi}\left(\phi_{s}\right) p_{\beta}\left(\boldsymbol{\beta}^{s}\right) \prod_{j=1}^{n_{i}}\left[L_{i j}\left(y_{i j} \mid \boldsymbol{\vartheta}, \boldsymbol{x}_{i j}, \boldsymbol{z}_{i j}, \boldsymbol{b}_{i}, s\right)\right] .
\end{aligned}
$$

\subsection{Estudos de simulação}

Os estudos de simulação efetuados nesta seção foram planejados objetivando explorar a performance da inferência dos modelos de mistura beta lineares com interceptos aleatórios $(d=1)$, parâmetro de precisão constante $\phi_{1}=\phi_{2}=\ldots=\phi_{K}=\phi$, uma variável explicativa $x_{i j} \sim \operatorname{Unif}(0,1)$ e com função de ligação logito, $g_{\mu}\left(\mu_{i j}\right)=\log \left(\frac{\mu_{i j}}{1-\mu_{i j}}\right)$, a respeito dos seguintes aspectos:

1. acurácia das estimativas pontuais a posteriori obtidas pela média e mediana da distribuição a posteriori;

2. performance das medidas de seleção de modelos para determinação de $K$ e,

3. averiguação de não identificabilidade.

Para que os estudos de simulação sejam efetivos é necessário que explorem cenários que representem a diversidade de possibilidades da técnica analisada. O conteúdo desta intrudução da seção tem o intuito de definir os valores dos parâmetros dos cenários dos estudos de simulação e suas distribuições a priori e hiperparâmetros.

Em seguida, nas Subseções 3.4.1, 3.4.2 e 3.4.3 são explicadas as diretrizes e apresentados os resultados dos estudos de simulação para os aspectos anteriormente apontados.

Inicialmente, para definir os valores dos parâmetros dos cenários abordados nos estudos de simulação com réplicas, foi efetuada uma análise exploratória do diagnóstico de convergência e do desempenho da estimação de parâmetros de modelos para diferentes possíveis valores. Essa análise inicial é importante pois o tempo computacional da execução dos algoritmos é alto e isso dificulta a seleção de cenários que englobem diversificação com base na execução com réplicas para um grande número de cenários. Os valores considerados nessa análise inicial foram: $N=50,100,150,200,300,400,500$, $n_{i}=5,10, K=2,3, \boldsymbol{\pi}_{i}^{\top}=(0,5 ; 0,5),(0,7 ; 0,3),(0,4 ; 0,3 ; 0,3),(0,6 ; 0,3 ; 0,1), \boldsymbol{\Sigma}_{b}=\sigma_{b}=1, \phi=$ $25,30,50,70,75,(25,45),(30,60),(35,50),(40,65),(60,75),\left\{\boldsymbol{\beta}_{1}^{\top}, \ldots, \boldsymbol{\beta}_{K}^{\top}\right\}=\{(-1,5 ; 1),(-0,5 ; 3)\}$, $\{(-2 ; 0,5),(-0,5 ; 3)\},\{(-2 ; 1),(-0,5 ; 1,5)\},\{(-0,75 ; 2),(-1,5 ; 0,5)\},\{(-0,75 ; 2),(-1,5 ; 1)\},\{(-1$; $3),(-3 ; 1)\},\{(-0,75 ; 2),(-1,5 ; 0,5),(-2,5 ; 1)\},\{(-0,75 ; 2),(-1,5 ; 0,5),(-3 ; 2,5)\},\{(-2 ; 0,5),(-1$, $5 ; 1),(-0,5 ; 3)\}, n_{i t}=30000,50000,70000,90000,100000, n_{b}=10000,20000,50000, n_{t}=5,10 \mathrm{e}$ $n_{c}=1,2$. 
Por não haver réplicas nessa fase exploratória do estudo de simulação, foi possível efetuar o diagnóstico de convergência por meio da avaliação dos gráficos da trajetória das cadeias, de autocorrelação, da mediana ao longo das iterações e histograma das amostras resultantes e das estatísticas de Geweke (Geweke, 1992), de Gelman-Rubin (Gelman e Rubin, 1992) e de Raftery-Lewis (Raftery e Lewis, 1992). O desempenho da estimação dos parâmetros, nesse caso, foi avaliado através de gráficos da amostra resultante, estimativas pontuais e intervalos de credibilidade.

Seja $\pi_{i s}=P\left(S_{i}=s\right), s=1, \ldots, K$, a probabilidade da $i$-ésima unidade amostral estar associada ao $s$-ésimo componente da mistura, $S_{1}, S_{2}, \ldots, S_{N}$ são variáveis aleatórias independentes. Assim, sendo $\boldsymbol{\pi}_{i}^{\top}=\left(\pi_{i 1} ; \pi_{i 2}\right) \sim \operatorname{Dir}(\boldsymbol{\alpha})$, estabeleceu-se $\boldsymbol{\pi}_{i}^{\top}=(0,7 ; 0,3)$. Os valores de $\boldsymbol{\alpha}$ primeiramente estudados foram $(160 ; 40),(50 ; 50)$ e $\boldsymbol{\alpha} \sim \operatorname{Gama}(1 ; 0,001)$ e, então, optou-se por utilizar $\boldsymbol{\alpha}=$ $(160 ; 40)$ para os estudos com réplicas.

\begin{tabular}{c|c}
\hline Cenário & $\left\{\boldsymbol{\beta}^{\mathbf{1}^{\top}} ; \boldsymbol{\beta}^{\boldsymbol{2}^{\top}}\right\}$ \\
\hline 1 & $\{(-0,75 ; 2) ;(-1,5 ; 0,5)\}$ \\
2 & $\{(-1,5 ; 1) ;(-0,5 ; 3)\}$ \\
3 & $\{(-2 ; 0,5) ;(-0,5 ; 3)\}$ \\
4 & $\{(-1 ; 3) ;(-3 ; 1)\}$ \\
\hline
\end{tabular}

Tabela 3.2: Definição dos cenários estudados por simulação para os parâmetros $\boldsymbol{\beta}^{s}, s=1,2$.
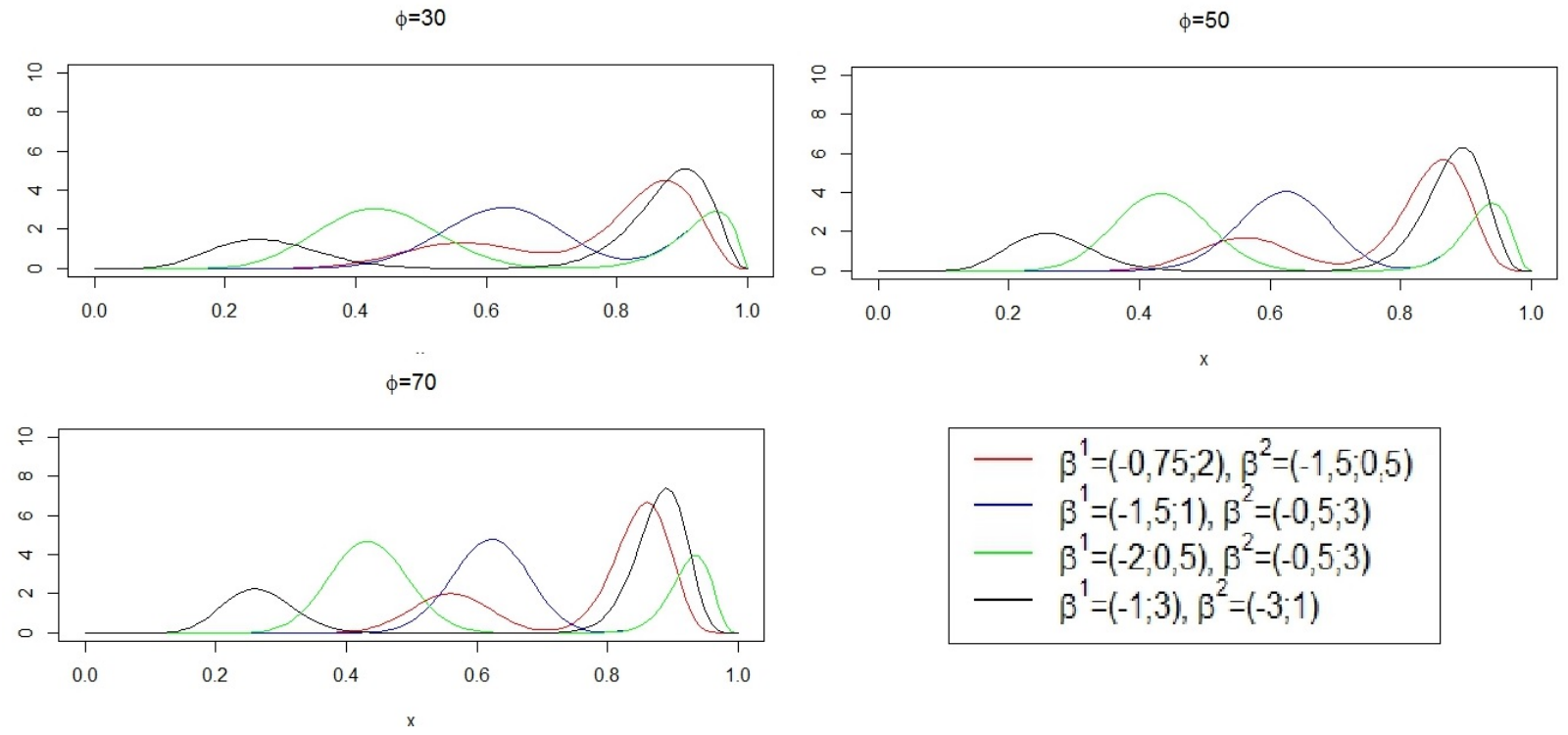

Figura 3.1: Comparação entre as densidades da distribuição beta para diferentes valores dos parâmetros $\beta$ e $\phi$ nos cenários englobados pelos estudos de simulação.

A Tabela 3.2 detalha os valores dos parâmetros $\boldsymbol{\beta}^{s}, s=1, \ldots, K$ analisados neste estudo de simulação e a Figura 3.1 esboça a função densidade de probabilidade gerada para cada um desses valores. Nesses gráficos observa-se que, à medida que $\phi$ aumenta, a massa de probabilidade se concentra ao redor da(s) moda(s), sendo que nos três cenários que apresentam bimodalidade $(1,3 \mathrm{e}$ 4) é possível distinguir claramente entre os elementos da mistura, sendo o cenário 4 o que apresenta maior distância entre as modas; o cenário 1 também apresenta essa distinção entre os elementos, porém com intervalos de confundimento, principalmente para $\phi=30$, e com maior concentração da massa de probabilidade ao redor de uma das duas modas. 
Para a distribuição a priori de $\boldsymbol{\beta}^{s}, s=1, \ldots, K$, optou-se primeiramente pela normal $N_{2}\left(\mathbf{0}, \alpha_{\beta} \boldsymbol{I}_{2}\right)$, $\alpha_{\beta} \in\{10,20\}$ e, nesse caso, o custo computacional foi grande sem que os resultados fossem mais satisfatórios do que os obtidos com a distribuição a priori $N_{2}\left(\boldsymbol{\mu}_{\boldsymbol{\beta}^{s}}, 10 \boldsymbol{I}_{2}\right)$. Os valores de $\boldsymbol{\mu}_{\boldsymbol{\beta}^{s}}$, nos estudos apresentados, foram definidos em dois passos:

1. categorizar as unidades amostrais em $K$ grupos por meio do método de agrupamento Kmédias (K-means) para dados longitudinais implementado no pacote kml (Genolini et al. , 2015) do software $R$;

2. ajustar um modelo beta misto com parte sistemática (2.18) para cada um dos $K$ grupos compostos no passo 1 através do pacote gamlss do software R, obtendo as respectivas estimativas dos coeficientes dos efeitos fixos $\widehat{\boldsymbol{\beta}}^{1}, \ldots, \widehat{\boldsymbol{\beta}}^{K}$;

3. por fim, estabelecer $\boldsymbol{\mu}_{\boldsymbol{\beta}^{s}}=\widehat{\boldsymbol{\beta}}^{s}, s=1, \ldots, K$.

Sabe-se que, em geral, defensores da inferência bayesiana aos moldes de seu desenvolvimento defendem a atribuição da distribuição a priori apenas com o conhecimento prévio à observação dos dados, sem que haja nenhuma influência dos dados observados. Contudo, também existem pesquisadores que utilizam-se de técnicas de atribuição de distribuições a priori informativas que baseiam-se nas análises visual e/ou analítica de parte ou totalidade dos dados. Assim sendo, apesar da opção pelo método anteriormente descrito, fica a critério do leitor atribuir as distribuições $a$ priori que lhe sejam convenientes sem perda de generalidade da metodologia proposta.

Com relação ao parâmetro $\phi$, primeiramente foi considerado $\phi=50$, aos moldes dos estudos de simulação apresentados em Figueroa-Zúñiga et al. (2013), para gerar os dados para os quais foram ajustados modelos considerando as distribuições a priori não informativas: Unif $(a, b)$, para $(a, b) \in\{(0 ; 50),(0 ; 100),(0 ; 200)\}, \operatorname{Gama}(0,1 ; 0,001)$ e $[50 * \operatorname{Beta}(\epsilon ; \epsilon)]^{2}$, em que $\epsilon \in\{1,1 ; 1,5\}$; e as distibuições informativas $\operatorname{Unif}(a ; b)$, em que $(a ; b)$ foram determinados de forma simétrica com $b-a=40$. Dessa forma, os estudos aqui desenvolvidos consideram $\phi \in\{30,50,70\}$ e, com base nos resultados obtidos para o caso $\phi=50, \phi \sim \operatorname{Unif}(a ; b) \operatorname{com} b-a=40$.

Para a única componente de $\boldsymbol{\Sigma}_{b}, \sigma_{b}$ pois $d=1$, foram estudadas distribuições a priori Gama $(\epsilon ; \epsilon)$, $\epsilon \in\{0,05 ; 0,1 ; 0,3 ; 0,5 ; 1 ; 3 ; 5 ; 7\}$, para $\psi=\frac{1}{\sigma_{b}}$ e optou-se por $\epsilon=1$. Essa quantidade de opções foram testadas para minimizar a subestimação de $\sigma_{b}$ que é frequentemente observada na literatura (Gelman, 2006) e suas respectivas funções densidade de probabilidade encontram-se graficadas na Figura 3.2 . 


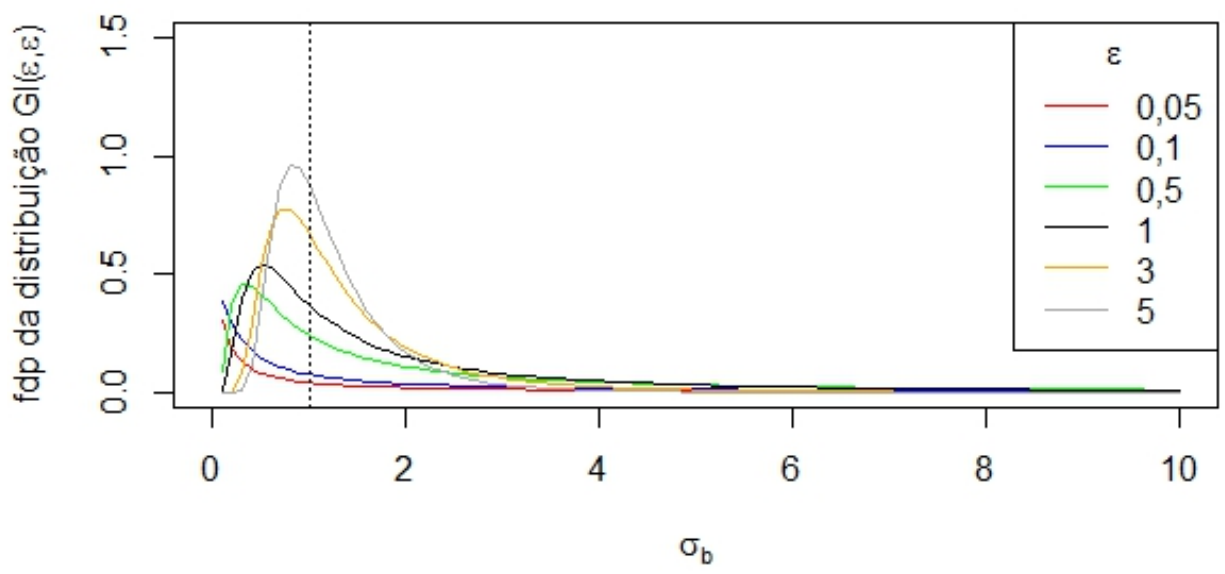

Figura 3.2: Comparação entre as densidades da distribuição gama inversa, distribuição a priori de $\sigma_{b}$, para diferentes valores do parâmetro $\epsilon$.

\subsubsection{Acurácia das estimativas pontuais a posteriori}

Cada banco de dados gerado foi composto por observações de $N=40,100,500$ grupos com $n=n_{1}=\ldots=n_{N} \in\{5,10\}$ observações por grupo. O número de banco de dados (réplicas) simulados para ajuste dos modelos foi $R=50$ para $N=100$ e $R=25$ para $N=500$. Estas quantidades de réplicas inviabilizam análises mais completas das amostras das distribuições a posteriori, dessa forma, a análise dos resultados dos estudos foi efetuada com relação a estimativas pontuais dos parâmetros. As estimativas consideradas foram a média e a mediana amostrais a posteriori, contudo, elas apresentaram valores extremamente semelhantes e, dado isso, para que a exposição dos resultados seja mais sucinta, serão apresentados e analisados apenas os resultados da mediana amostral a posteriori.

Como medidas de avaliação da acurácia dessas estimativas pontuais considerou-se o erro relativo médio (ER), o erro absoluto relativo médio (EAR) e a raíz do erro quadrático médio (REQM).

Outra medida contabilizada foi a probabilidade de cobertura frequentista dos intervalos de credibilidade de $95 \%$, ou seja, calculou-se a porcentagem das réplicas nas quais o verdadeiro valor dos parâmetros está contido no seu respectivo intervalo de credibilidade de $95 \%$.

Os valores definidos para a análise via algoritmos MCMC foram: $n_{i t}=50000$ iterações, $n_{b}=$ $10000, n_{t}=10$ e $n_{c}=1$, totalizando amostras de tamanho 4000 da distribuição a posteriori de cada conjunto de dados.

Em um computador com processador Intel Core i7-6500U 2.50GHz, 8 GB de memória RAM e sistema operacional de 64 bits, o tempo computacional de cada réplica foi, em média, de 12 horas para os bancos de dados com $N=100$ grupos. Esse tempo foi similar a estudos de simulação de modelos de regressão com complexidade e métodos de estimação compatíveis, como por exemplo Liu e Li (2014).

As probabilidades de cobertura frequentistas dos intervalos de credibilidade de $95 \%$ foram todas iguais a $100 \%$, ou seja, em todas as réplicas os verdadeiros valores dos parâmetros estão inclusos aos seus respectivos intervalos de credibilidade de $95 \%$, resultado esse muito satisfatório e promissor 
dada a complexidade dos modelos de regressão em estudo.

Os resultados do estudo planejado para os cenários da Tabela 3.2 e valores de $\phi \in\{30 ; 50 ; 70\}$ encontram-se dispostos nas tabelas das Seções A.1, A.2 e A.3 do Apêndice A e nos gráficos das estimativas pontuais dos parâmetros ao longo das réplicas apresentados nas Seções B.1, B.2, B.3, B.4, B.5 e B.6 do Apêndice B, nos quais os valores verdadeiros são destacados com outros símbolos em vermelho. Em função da grande quantidade de dados resultantes, a interpretação destes é efetuada a seguir primeiramente com uma análise geral e, em seguida, através das comparações de mudanças observadas com a variação dos cenários e dos valores de $n, N, \phi$ e $\Sigma_{\beta^{s}}$. Adicionalmente, um exemplo de estudo mais detalhado é retratado no final desta subseção.

De forma geral, os parâmetros $\boldsymbol{\pi}$ e $\sigma_{b}$ obtiveram resultados muito satisfatórios quando estimados pontualmente pela mediana a posteriori, enquanto a performance das estimativas do parâmetro de precisão $(\phi)$ e dos coeficientes fixos $\left(\boldsymbol{\beta}^{1}\right.$ e $\left.\boldsymbol{\beta}^{2}\right)$ sofreu variação de acordo com as situações analisadas.

Tal qual observado na Figura 3.1, o cenário 4 é o que permite a maior distinção entre os componentes da mistura, o que acarretou em estimativas mais precisas dos parâmetros do modelo do que nos outros cenários.

Contrapondo as tabelas e gráficos com $n=5$ e $n=10$, nota-se que as estimativas de $\sigma_{b}$ tendem a ser maiores com o aumento do número de observações por grupo, o que para a grande maioria dos casos dos cenários 1 e 2 ocasionou o aumento da acurácia da estimação pois este parâmetro estava sendo subestimado.

Com o aumento do número de grupos $(N)$ de quarenta para cem e de cem para quinhentos, observa-se um grande aumento de precisão das estimativas de praticamente todos os parâmetros dos cenários 1 e 4. Isso aconteceu pois a dispersão das estimativas pontuais das réplicas diminui consideravelmente com esse crescimento amostral. Isto também foi observado no geral porém com uma intensidade bem menor, nos demais cenários.

Apesar de esperar resultados com maior acurácia com o aumento do valor do parâmetro de precisão, esse fato não foi observado da forma como era esperado pois as estimativas pontuais de $\phi$ apresentaram superestimação e subestimação, o que fez com que esse efeito não fosse verificado.

Por fim, comparando os resultados obtidos com $N=100$ e $n=5$ entre $\Sigma_{\beta}$ igual a $10 I_{2}$ e $40 I_{2}$, observou-se o aumento da precisão das estimativas pontuais dos parâmetros $\phi$ e $\boldsymbol{\pi}$ em todos os cenários.

A título de exemplificação, estudando mais detalhadamente as tabelas e gráficos do cenário 2, nota-se que as medidas de avaliação consideradas diminuiram de valor com o aumento do valor de $\phi$ com $N=100$ para as estimativas dos parâmetros $\beta_{1}^{2}, \phi$ e $\pi_{1}$ e, o oposto ocorreu para os parâmetros $\beta_{1}^{1}$ e $\beta_{2}^{2}$. Para $\sigma_{b}$ os resultados foram muito próximos para todos os valores de $\phi$ simulados nesse cenário. Analisando os gráficos nota-se imprecisão e subestimação de $\beta_{1}^{2}$ e estimativas tendenciosas do parâmetro de precisão para todos os valores simulados de $\phi$; sendo que para $\phi=50$ e $\phi=70$ também é possível observar menos precisão nas estimativas de $\sigma_{b}$.

Parcimoniamente, os custos experimental e computacional de aumentar o número de observações por grupo e o número de grupos podem não ser vantajosos quando visa-se apenas o ganho na acurácia da estimação dos parâmetros; inclusive pelo fato dos resultados obtidos com $N=100$ e $n=5$ terem sido satisfatórios, o que não foi observado em Lenk e DeSarbo (2000) para os modelos de mistura normais com efeitos aleatórios. 


\subsubsection{Performance dos critérios de seleção de modelos}

Objetivando analisar como os critérios de informação EAIC, ECAIC, EBIC, $D I C_{3}$ e LPML, definidos na Subseção 2.1.3, se comportam quando utilizados para seleção dos modelos propostos, novos estudos de simulação foram realizados. O procedimento adotado foi ajustar modelos com diferentes números de componentes da mistura para os dados simulados de acordo com cenários estabelecidos, calcular os valores desses critérios de informação para os modelos ajustados e contabilizar a proporção de vezes que os critérios, por si só, selecionam o modelo que gerou os dados.

Considerou-se os valores dos parâmetros $\boldsymbol{\beta}^{s}, s=1,2$, apresentados na Tabela $3.2, \phi \in\{30 ; 50 ; 70\}$, $K=2$ e $\pi_{1}=0,7$, assim como apresentados na introdução desta seção (3.4), tal qual suas respectivas distribuições a priori e métodos de estimação. Nesse caso, utilizou-se $N=100$ grupos com $n=5$ observações por grupo, $n_{i t}=30000, n_{b}=10000, n_{t}=10$ e $n_{c}=1$, totalizando 2000 amostras da distribuição a posteriori.

Em função da complexidade computacional foram analisados $R=10$ bancos de dados (réplicas) simulados de cada cenário e, a estes dados foram ajustados modelos de mistura beta misto com $K=1,2, \ldots, 5$ componentes na mistura. Em seguida, os valores dos critérios de informação foram calculados para estes ajustes e analisando unicamente estes valores foi selecionado o valor de $K$ para cada uma das réplicas de cada um dos cenários. Estes resultados foram resumidos na porcentagem de réplicas em que determinado valor de $K$ foi selecionado em cada cenário e estão disponibilizados na Tabela 3.3. Como os dados foram gerados com $K=2$, quanto maior a porcentagem para este valor, melhor é o desempenho do critério para seleção dos modelos; se o critério na maioria das réplicas seleciona valores mais altos de $K$, isso é um indício de que este critério superestima o número de componentes quando utilizado como único método de seleção do modelo, o que pode ocasionar problemas inferenciais e interpretações errôneas. 


\begin{tabular}{|c|c|c|c|c|c|c|c|c|c|c|c|c|c|}
\hline \multirow[b]{3}{*}{ Critério } & \multirow[b]{3}{*}{$K$} & \multicolumn{3}{|c|}{ Cenário 1} & \multicolumn{3}{|c|}{ Cenário 2} & \multicolumn{3}{|c|}{ Cenário 3} & \multicolumn{3}{|c|}{ Cenário 4} \\
\hline & & & & & & & $\phi$ & & & & & & \\
\hline & & 30 & 50 & 70 & 30 & 50 & 70 & 30 & 50 & 70 & 30 & 50 & 70 \\
\hline \multirow[t]{5}{*}{ EAIC } & 1 & 0 & 0 & 0 & 0 & 0 & 0 & 0 & 0 & 0 & 0 & 0 & 0 \\
\hline & 2 & 30 & 0 & 10 & 10 & 0 & 0 & 0 & 0 & 0 & 0 & 0 & 0 \\
\hline & 3 & 20 & 40 & 40 & 70 & 20 & 20 & 30 & 20 & 0 & 0 & 0 & 0 \\
\hline & 4 & 30 & 50 & 50 & 0 & 70 & 30 & 40 & 30 & 30 & 40 & 40 & 20 \\
\hline & 5 & 20 & 10 & 0 & 20 & 10 & 50 & 30 & 50 & 70 & 60 & 60 & 80 \\
\hline \multirow[t]{5}{*}{ ECAIC } & 1 & 0 & 0 & 0 & 0 & 0 & 0 & 0 & 0 & 0 & 0 & 0 & 0 \\
\hline & 2 & 30 & 0 & 10 & 10 & 0 & 0 & 0 & 0 & 0 & 0 & 0 & 0 \\
\hline & 3 & 20 & 40 & 40 & 70 & 20 & 20 & 30 & 20 & 0 & 0 & 0 & 0 \\
\hline & 4 & 40 & 50 & 50 & 0 & 70 & 30 & 40 & 30 & 30 & 40 & 40 & 20 \\
\hline & 5 & 10 & 10 & 0 & 20 & 10 & 50 & 30 & 50 & 70 & 60 & 60 & 80 \\
\hline \multirow[t]{5}{*}{ EBIC } & 1 & 10 & 0 & 10 & 0 & 0 & 0 & 0 & 0 & 0 & 20 & 20 & 10 \\
\hline & 2 & 70 & 10 & 20 & 20 & 0 & 0 & 0 & 0 & 0 & 0 & 0 & 10 \\
\hline & 3 & 20 & 80 & 50 & 70 & 30 & 40 & 50 & 20 & 10 & 0 & 0 & 0 \\
\hline & 4 & 0 & 10 & 20 & 10 & 70 & 40 & 40 & 70 & 40 & 50 & 50 & 20 \\
\hline & 5 & 0 & 0 & 0 & 0 & 0 & 20 & 10 & 10 & 50 & 30 & 30 & 60 \\
\hline \multirow[t]{5}{*}{$D I C_{3}$} & 1 & 0 & 0 & 0 & 0 & 0 & 0 & 0 & 0 & 0 & 0 & 0 & 0 \\
\hline & 2 & 80 & 100 & 90 & 60 & 60 & 60 & 60 & 80 & 60 & 100 & 80 & 100 \\
\hline & 3 & 20 & 0 & 10 & 40 & 30 & 20 & 20 & 0 & 0 & 0 & 20 & 0 \\
\hline & 4 & 0 & 0 & 0 & 0 & 10 & 0 & 0 & 20 & 40 & 0 & 0 & 0 \\
\hline & 5 & 0 & 0 & 0 & 0 & 0 & 20 & 20 & 0 & 0 & 0 & 0 & 0 \\
\hline \multirow[t]{5}{*}{$L P M L$} & 1 & 0 & 0 & 0 & 0 & 10 & 0 & 0 & 0 & 0 & 0 & 0 & 0 \\
\hline & 2 & 0 & 0 & 0 & 0 & 0 & 0 & 0 & 10 & 10 & 0 & 0 & 0 \\
\hline & 3 & 0 & 0 & 0 & 0 & 0 & 10 & 0 & 0 & 0 & 0 & 0 & 0 \\
\hline & 4 & 0 & 0 & 0 & 0 & 0 & 20 & 20 & 20 & 0 & 10 & 0 & 10 \\
\hline & 5 & 100 & 100 & 100 & 100 & 90 & 70 & 80 & 70 & 90 & 90 & 100 & 90 \\
\hline
\end{tabular}

Tabela 3.3: Porcentagem das 10 réplicas simuladas do modelo de mistura beta linear com efeitos aleatórios para a média cujos critérios EAIC, ECAIC, EBIC, DIC 3 e LPML selecionaram cada um dos 5 possiveis valores de números de componentes da mistura $(K)$.

Os resultados obtidos indicam claramente que o critério $D I C_{3}$ foi o que obteve melhor desempenho visto que selecionou o modelo correto em no mínimo $80 \%$ das réplicas nos cenários 1 e 4 , porcentagem essa que chega a $100 \%$ no cenário $1 \operatorname{com} \phi=50$ e no cenário 4 com $\phi=30,70$. Nos outros casos obteve-se acerto em $80 \%$ das réplicas do cenário 3 com $\phi=50$ e em $60 \%$ das réplicas dos demais cenários.

Com exceção do cenário $1 \operatorname{com} \phi=30$, os critérios EAIC e ECAIC tiveram resultados exatamente iguais: no geral houve superestimação de $K$, principalmente no cenário 4 e no cenário 3 com $\phi=50,70$. Comparando esses desempenhos com a performance do EBIC, conclui-se que a superestimação observada pelo segundo é menos frequente, sendo que para o cenário 1 com $\phi=30$ a porcentagem de réplicas em que houve seleção do modelo correto foi de $70 \%$.

Em contrapartida, o desempenho do critério LPML foi caracterizado por superestimar o valor de 
$K$, já que em todos os cenários selecionou $K=5$ em mais de $70 \%$ das réplicas. Esse resultado pode ser explicado devido ao fato desse critério ser baseado na função de verossimilhança sem penalizar o número de parâmetros.

Dado isso, conclui-se que o $\mathrm{DIC}_{3}$ é uma eficiente ferramenta auxiliar na determinação de $K$.

\subsubsection{Diagnóstico de identificabilidade}

A verificação da identificabilidade dos modelos com parâmetros, distribuições a priori e métodos de estimação previamente definidos na introdução desta seção (3.4) foi efetuada com base no método data cloning, tal qual apresentado e explicado na Seção 2.7. Foram considerados os modelos de mistura beta mistos com valores dos parâmetros $\boldsymbol{\beta}^{s}, s=1, \ldots, K$, apresentados na Tabela 3.2, $\phi \in\{30 ; 50 ; 70\}, K=2, \pi_{1}=0,7, N=100$ e $n=5$.

Os valores utilizados para os números de clones são 1,3,5 e 9. Estes valores foram selecionados pois foram os que convergiram para todos os cenários, já que ao longo do processo de aplicação do método houve alguns casos com problemas de convergência das cadeias MCMC, fato esse que Lele et al. (2010) relatam ser possível de ser observado e descrevem algumas possíveis causas sem apresentar soluções ou alternativas.

Na Figura 3.3 encontram-se os valores do maior autovalor padronizado da matriz de variâncias e covariâncias a posteriori dos parâmetros para os números de clones considerados. De acordo com o método, ao observar a convergência desses autovalores para o limite zero a medida que cresce o número de clones, pode-se concluir que não existem evidências de falta de identificabilidade, principalmente se essa taxa de convergência for próxima a observada no inverso do número de clones.

Cenário 1

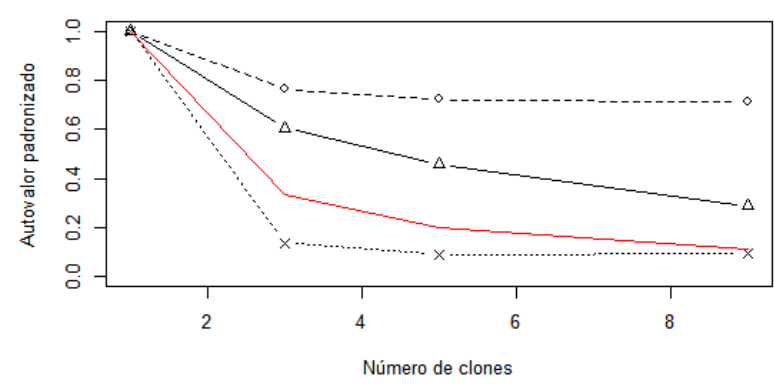

Cenário 3

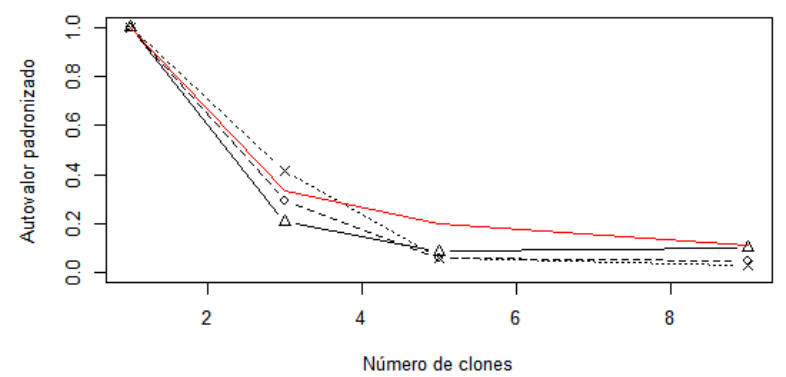

Cenário 2

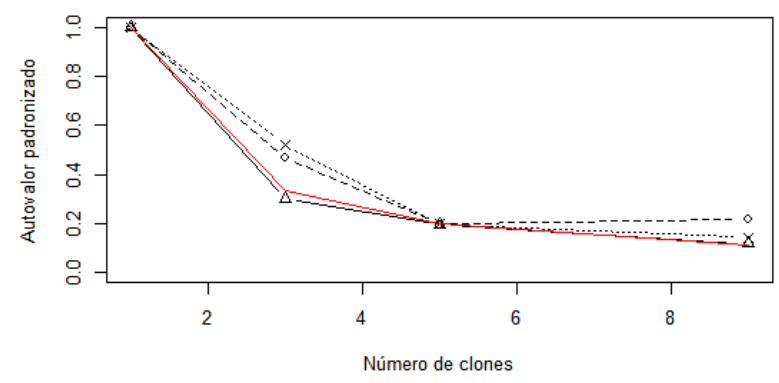

Cenário 4

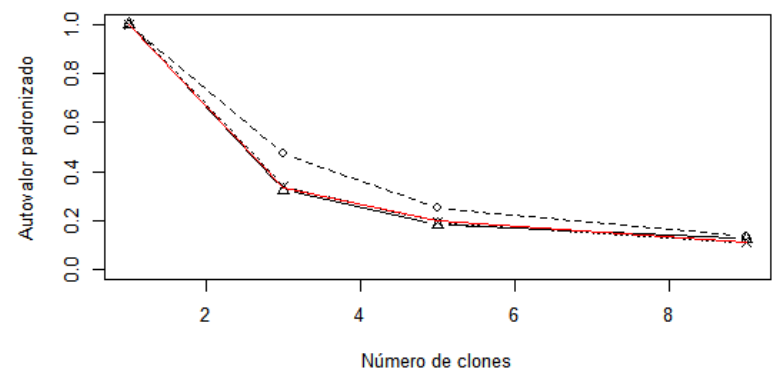

Figura 3.3: Gráfico de linhas do inverso do número de clones (traço vermelho) e do maior autovalor padronizado da matriz de variâncias e covariâncias a posteriori dos parâmetros do modelo de mistura beta misto com cenários $1,2,3,4$, parâmetro de precisão assumindo valores $30(\triangle), 50(\bigcirc), 70(\times)$ e número de clones $1,3,5,9$. 
Os resultados expostos nos gráficos apresentam decrescimento em todos os casos analisados. Com relação à similaridade deste declínio com a função inversa do número de clones, nota-se claramente que os resultados que mais se assemelham são os do cenário 4, fato esse que reflete a influência do distanciamento entre os componentes da mistura, ilustrado na Figura 3.1, no processo inferencial destes modelos.

Complementarmente, com exceção dos dados obtidos para o cenário 1 e $\phi=30,50$, nos demais casos também se observa uma semelhança com a curva de referência. Nessas duas situações particulares não há redução numérica tão significativa dos autovalores como a da curva de referência em decorrência da presença dos intervalos de confundimento entre os componentes da mistura ilustrados na Figura 3.1.

Dado isso, baseado na verificação de identificabilidade através do método data cloning, pode-se concluir que sob as condições analisados não há evidências de falta de identificabilidade dos modelos considerados e que os resultados indicam mais fortemetente essa conclusão quanto mais claramente distinguíveis são os elementos da mistura e é justamente onde os modelos de mistura podem ser os mais recomendados.

\subsection{Aplicações}

Com o intuito de ilustrar a aplicação do método desenvolvido neste capítulo, nesta seção são analisados dois bancos de dados por meio dos modelos de mistura beta mistos lineares com parâmetro de precisão constante. Essas aplicações são de grande valia para auxiliar no entendimento da teoria apresentada e na assimilação de estudos em que seja apropriada a aplicação da metodologia.

A primeira aplicação é um estudo longitudinal na área odontológica, no qual foram monitoradas características clínicas de pacientes diagnosticados com periodontite ao longo de 12 meses após o término do tratamento. O interesse nesse caso é compreender a eficácia do tratamento, quais variáveis clínicas reagem a ele e como ocorrem essas reações no período analisado.

A segunda pesquisa é da área de marketing e sua motivação é estudar de que forma as peculiaridades municipais influenciam na dispersão geográfica (aglomeração) de redes de fast food nacionais e estrangeiras nas cidades brasileiras.

Em ambas as aplicações os modelos foram ajustados de acordo com o protocolo seguido nos estudos de simulação, ou seja, as amostras a posteriori foram geradas por meio de algoritmos MCMC no software OpenBUGS e as distribuições a priori foram atribuídas aos parâmetros de acordo com as indicações da Seção 3.3.

\subsubsection{Dados de pacientes diagnosticados com periodontite}

Em vista do aumento da incidência de doenças cardiovasculares, que em 2011 acarretaram mais de 21,88\% dos óbitos a nível mundial segundo a organização mundial da saúde (World Health Organization, WHO and World Heart Federation and World Stroke Organization, 2011), há interesse em estudar os seus fatores agravantes ou de risco para que em posse desse conhecimento haja um melhor planejamento de projetos na área da saúde.

Uma moléstia que vem sendo constantemente estudada na área de saúde pública é a periodontite: doença inflamatória que ocorre nos tecidos responsáveis pela sustentação do dente (periodonto) e que causa desde mobibilidade e perda dos dentes até agravamento ou desenvolvimento de outras 
doenças em outras partes do corpo e órgãos. Muitos estudos, como os apresentados em Buhlin et al. (2003) e Cairo et al. (2008), investigam a contribuição da periodontite em doenças cardiovasculares.

Em estudos deste escopo costuma-se considerar como indicadores de risco de problemas cardiovasculares os perfis dos componentes hematológicos e dos lipídios. Um dos indicadores lipídicos é a lipoproteína de baixa densidade oxidada (LDL oxidada), a qual é uma medida de distinção de pacientes com risco de doenças cardiovasculares muito útil na fase de avaliação. A LDL oxidada é aferida em exames clínicos através da concentração de anticorpos anti-LDL oxidada (anti-oxLDL).

Nesta aplicação tem-se o propósito de estudar o comportamento de anti-oxLDL e de outros indicadores de doenças cardiovasculares de um amostra de pacientes diagnosticados com periodontite ao longo de um ano após o tratamento. Dessa forma, pode-se elucidar a respeito da influência da periodontite no desenvolvimento ou agravamento de doenças cardiovasculares e assim avaliar o sucesso do tratamento em outros aspectos além dos odontológicos.

O banco de dados é composto pelos resultados de exames clínicos de 40 pacientes que passaram pelo tratamento de periodontite e as aferições foram coletadas em quatro momentos distintos: antes do início do tratamento e 3,6 e 12 meses após o início do tratamento.

Detalhes dos meios de seleção dos pacientes e dos procedimentos de coleta de dados podem ser encontrados em Monteiro et al. (2012), assim como os resultados da análise dos dados transformados através de modelos mistos normais efetuada pelos autores.

Como alternativa a essa forma de análise, Zerbeto (2014) optou por fazer uso dos modelos de mistura beta mistos para não trabalhar com os dados transformados e, assim, facilitar a interpretação dos resultados. Outras pesquisas sobre o risco de doenças cardiovasculares em função do LDL sob diferentes enfoques já foram analisadas com modelos de regressão beta, como por exemplo em Carrasco et al. (2014) que fazem uso dos modelos de regressão beta com erro nas variáveis.

Originalmente, a variável resposta desta aplicação (anti-oxLDL) assume valores positivos e restritos. Para que os resultados sejam comparáveis aos de outras populações, uma padronização da variável resposta original foi realizada de acordo com os valores máximo e mínimo observados, transformando os valores da resposta original para o intervalo $(0,1)$.

As variáveis da base de dados selecionadas para a aplicação são:

1. anti-oxLDL : unidades de anticorpos anti-LDL oxidada;

2. sexo: sexo do paciente;

3. idade : idade do paciente (em anos);

4. raça : raça declarada pelo próprio paciente;

5. imc : índice de massa corporal do paciente (IMC);

6. HDL : concentração de HDL $(\mathrm{mg} / \mathrm{dL})$;

7. $L D L$ : concentração de LDL $(\mathrm{mg} / \mathrm{dL})$;

8. $P C R$ : concentração de proteína C-reativa $(\mathrm{em} \mathrm{mg} / \mathrm{mL})$;

9. $P B>4 \mathrm{~mm}$ : porcentagem de bolsas periodontais com profundidade superior a $4 \mathrm{~mm}$. 
Iniciando a análise exploratória dos dados, a Figura 3.4 apresenta o gráfico de perfis da variável anti-oxLDL.

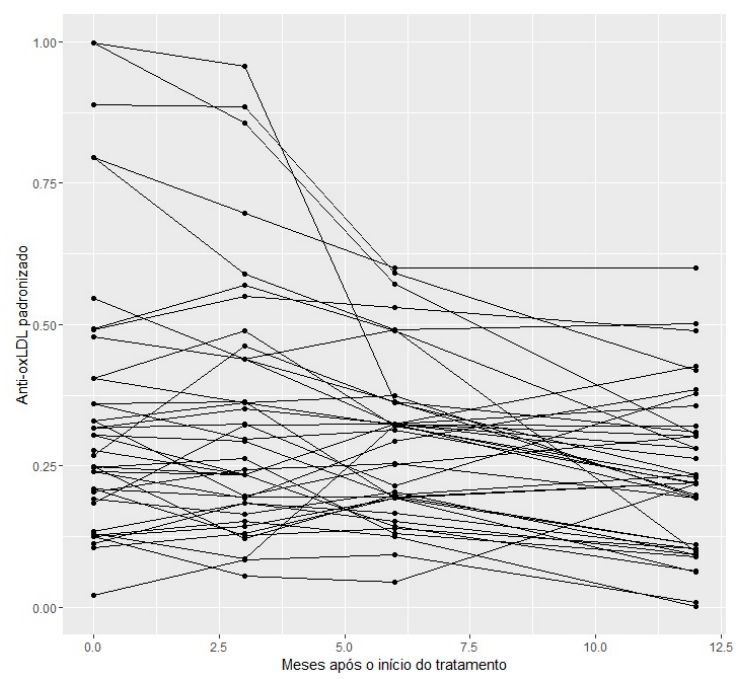

Figura 3.4: Gráfico de perfis da variável anti-oxLDL nos dados de periodontite.

Alguns pacientes apresentam valores de anti-oxLDL aparentemente atípicos antes de passar pelo tratamento de periodontite (se comparado com a mesma medida em outros pacientes). Note também que o comportamento decrescente de anti-oxLDL a medida que o tempo decorrente do início do tratamento aumenta é predominante, indicando o sucesso do tratamento com relação a esta variável. A utilização é também para incorporar a correlação pelo caráter longitudinal. Porém, a intensidade deste declínio varia muito entre os pacientes, o que sugeri que existem grupos de pacientes que respondem de forma diferente ao tratamento e indica a adequação de análise por meio de modelos de mistura.
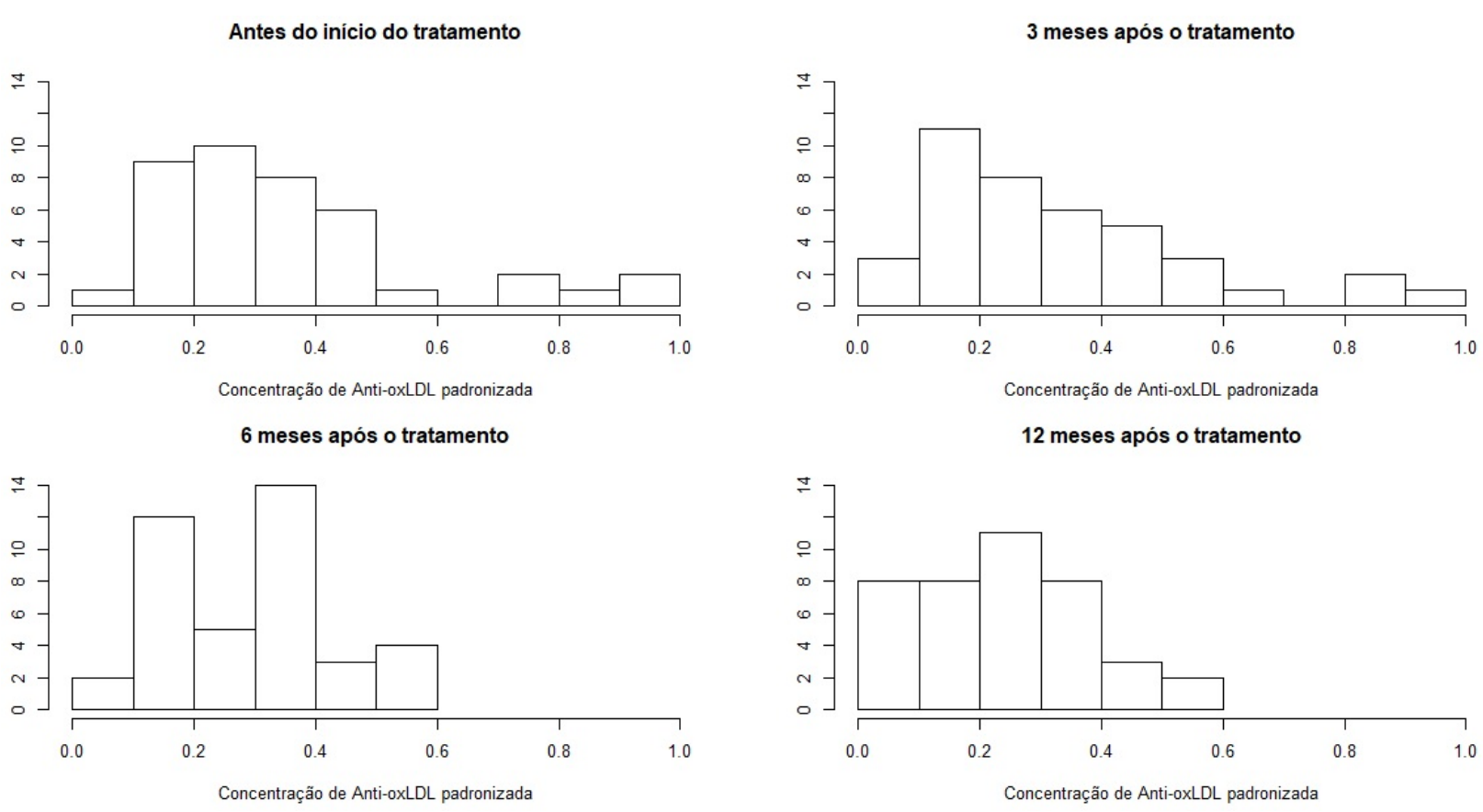

Figura 3.5: Histograma da variável anti-oxLDL nos dados de periodontite.

A Figura 3.5 expõe o histograma da variável anti-oxLDL nos diferentes momentos de medi- 
ção; neles, verifica-se que os dados se concentram no intervalo $(0,1 ; 0,4)$, mas continuam tendo incidência no intervalo $(0,4 ; 0,5)$. Este comportamento assimétrico indica a adequabilidade de se considerar a distribuição de probabilidade beta para a variável em questão. Além disso, a diferença da distribuição dos valores nos tempos de medição indica que o ajuste de modelos mistos para a variável anti-oxLDL é adequado.

Seguindo os roteiros sugeridos para análises de dados longitudinais por Cnaan et al. (1997), McCulloch et al. (2008) e Lin e Lee (2008), prosseguiu-se a aplicação com o ajuste do modelo de regressão que a análise exploratória dos dados indicou como sendo adequado.

Todos os modelos foram ajustados através do software OpenBUGS com $n_{i t}=60000, n_{b}=15000$, $n_{t}=20$ e $n_{c}=1$.

O modelo inicialmente ajustado para a variável anti-oxLDL foi considerando o modelo beta misto $(K=1)$ e as variáveis explicativas $2, \ldots, 9$, a partir da seleção destas com base no AIC e nos conhecimentos de especialistas do assunto estudado se determinou quais foram as variáveis incluídas no modelo de mistura apresentado a seguir.

Sejam $\boldsymbol{y}_{\boldsymbol{i}}=\left(y_{i 1}, \ldots, y_{i n_{i}}\right)^{\top}$ e $S_{i}$, respectivamente, o vetor de observações da variável resposta e a variável indicadora discreta do componente da mistura associados ao $i$-ésimo paciente, $i=1, \ldots, 40$, e, considerando $Y_{i j} \mid b_{i} \sim \sum_{S_{i}=1}^{K} \pi_{i S_{i}} \operatorname{Beta}\left(\mu_{S_{i}, i j}, \phi_{S_{i}}\right)$ e $\mu_{S_{i}, i j}=E\left(Y_{i j} \mid b_{i}, S_{i}\right)$, este modelo é apresentado na Equação (3.11).

$$
\log \left(\frac{\mu_{S_{i}, i j}}{1-\mu_{S_{i}, i j}}\right)=\beta_{1}^{S_{i}}+\beta_{2}^{S_{i}} m e s .3_{i j}+\beta_{3}^{S_{i}} m e s .6_{i j}+\beta_{4}^{S_{i}} m e s .12_{i j}+\beta_{5}^{S_{i}} H D L_{i j}+\beta_{6}^{S_{i}} L D L_{i j}+b_{i}
$$

em que:

- $i$ indica o paciente em questão;

- $j$ indica o número de meses decorridos pós-tratamento:

- $j=0$ indica que as aferições ocorreram antes do início do tratamento;

- $j=3$ indica que as aferições ocorreram 3 meses após o início do tratamento;

- j=6 indica que as aferições ocorreram 6 meses após o início do tratamento;

- j=12 indica que as aferições ocorreram 12 meses após o início do tratamento.

- $y_{i j}$ é o número de anticorpos contra LDL oxidada do $i$-ésimo paciente no $j$-ésimo mês póstratamento;

- mes. $3_{i j}$ é a variável indicadora que assume valor 1 se $j=3$ e assume valor 0 caso contrário.

Da mesma forma foram construídas as variáveis mes. $6_{i j}$ e mes.12 ${ }_{i j}$;

- $b_{i}$ é o intercepto aleatório associado ao $i$-ésimo paciente;

- $b_{1}, b_{2}, \ldots, b_{40}$ são independentes e identicamente distribuídos $N\left(0, \sigma_{b}^{2}\right)$;

- $\beta_{1}^{s}, \beta_{2}^{s}, \beta_{3}^{s}, \beta_{4}^{s}, \beta_{5}^{s}, \beta_{6}^{s}, \phi_{s}, \pi_{s}$ e $\sigma_{b}, s=1, \ldots, K$, são parâmetros do modelo. 
Aos fins de comparação, as estimativas do ajuste do modelo (3.11) para $K=1$ encontram-se na Tabela 3.4.

\begin{tabular}{c|c|c|c|c}
\hline Parâmetro & Covariável associada & Estimativa pontual & Desvio padrão & IC 95\% \\
\hline$\beta_{1}^{1}$ & - & $-1,815$ & 0,418 & $(-2,534 ;-1,162)$ \\
$\beta_{2}^{1}$ & mes.3 & $-0,135$ & 0,123 & $(-0,391 ; 0,095)$ \\
$\beta_{3}^{1}$ & mes.6 & $-0,355$ & 0,146 & $(-0,680 ;-0,087)$ \\
$\beta_{4}^{1}$ & mes.12 & $-0,724$ & 0,131 & $(-0,974 ;-0,455)$ \\
$\beta_{5}^{1}$ & HDL & 0,021 & 0,011 & $(-0,001 ; 0,042)$ \\
$\beta_{6}^{1}$ & LDL & 0,002 & 0,003 & $(-0,003 ; 0,007)$ \\
$\phi_{1}$ & - & 11,630 & 1,422 & $(9 ; 14,580)$ \\
$\sigma_{b}$ & - & 0,765 & 0,213 & $(0,439 ; 1,258)$ \\
\hline
\end{tabular}

Tabela 3.4: Estimativas pontuais (média a posteriori) dos parâmetros, desvis padrões e intervalos de credibilidade de $95 \%$ do modelo final com $K=1$ ajustado para a variável anti-oxLDL dos dados de periodontite.

Em seguida, ajustou-se o modelo (3.11) para outros valores de $K$ definidos com base no contexto da aplicação, $K=2,3$, e calculados os valores dos critérios EAIC, ECAIC, EBIC, $D I C_{3}$ e LPML, conforme expostos na Tabela 3.5.

\begin{tabular}{c|ccc}
\hline & \multicolumn{3}{|c}{$K$} \\
\hline Critério & 1 & 2 & 3 \\
\hline EAIC & $-216,44$ & $-389,45$ & $-338,57$ \\
ECAIC & $-214,93$ & $-384,45$ & $-327,69$ \\
EBIC & $-192,05$ & $-340,65$ & $-265,37$ \\
$D I C_{3}$ & $-1380,59$ & $-2402,90$ & $-1642,89$ \\
LPML & $-16744,74$ & $-20778,26$ & $-15207,18$ \\
\hline
\end{tabular}

Tabela 3.5: Valores dos critérios de informação EAIC, ECAIC, EBIC, DIC $C_{3}$ e LPML para o modelo (3.11) com $K=1,2,3$ para os dados de periodontite.

Por fim, quatro dos cinco critérios calculados indicaram que o modelo com dois componentes na mistura $(K=2)$ apresentou maior adequabilidade. O critério LPML selecionou o modelo com $K=3$ e ele também apresentou essa tendência de superestimação de $K$ nos estudos de simulação conforme exposto na Tabela 3.3. Na Tabela 3.6 encontram-se as informações relacionadas aos parâmetros do modelo final (3.11) e as Figuras 3.6 e 3.7 apresentam as funções densidade de probabilidade estimadas desses parâmetros a posteriori. 


\begin{tabular}{c|c|c|c|c}
\hline Parâmetro & Covariável associada & Estimativa pontual & Desvio padrão & IC 95\% \\
\hline$\beta_{1}^{1}$ & - & $-1,192$ & 0,294 & $(-1,602 ;-0,705)$ \\
$\beta_{2}^{1}$ & mes.3 & 0,060 & 0,145 & $(-0,200 ; 0,326)$ \\
$\beta_{3}^{1}$ & mes.6 & $-0,157$ & 0,149 & $(-0,414 ; 0,108)$ \\
$\beta_{4}^{1}$ & mes.12 & $-0,559$ & 0,161 & $(-0,818 ;-0,254)$ \\
$\beta_{5}^{1}$ & HDL & $-0,0003$ & 0,016 & $(-0,037 ; 0,032)$ \\
$\beta_{6}^{1}$ & LDL & 0,007 & 0,007 & $(-0,013 ; 0,029)$ \\
$\phi_{1}$ & - & 37,35 & 4,400 & $(26,01 ; 42,390)$ \\
$\beta_{1}^{2}$ & - & 0,175 & 0,502 & $(-0,801 ; 1,172)$ \\
$\beta_{2}^{2}$ & mes.3 & $-0,896$ & 0,516 & $(-1,879 ; 0,099)$ \\
$\beta_{3}^{2}$ & mes.6 & $-1,660$ & 0,499 & $(-2,605 ;-0,710)$ \\
$\beta_{4}^{2}$ & mes.12 & $-2,197$ & 0,489 & $(-3,155 ;-1,230)$ \\
$\beta_{5}^{2}$ & HDL & $-0,224$ & 0,440 & $(-1,128 ; 0,627)$ \\
$\beta_{6}^{2}$ & LDL & $-0,507$ & 0,312 & $(-1,212 ;-0,001)$ \\
$\phi_{2}$ & - & 1,245 & 0,019 & $(1,214 ; 1,301)$ \\
$\pi_{1}$ & - & 0,721 & 0,031 & $(0,655 ; 0,779)$ \\
$\sigma_{b}$ & - & 0,538 & 0,338 & $(0,204 ; 1,308)$ \\
\hline
\end{tabular}

Tabela 3.6: Estimativas pontuais (média a posteriori) dos parâmetros, desvios padrões e intervalos de credibilidade de $95 \%$ do modelo final ajustado para a variável anti-oxLDL dos dados de periodontite.
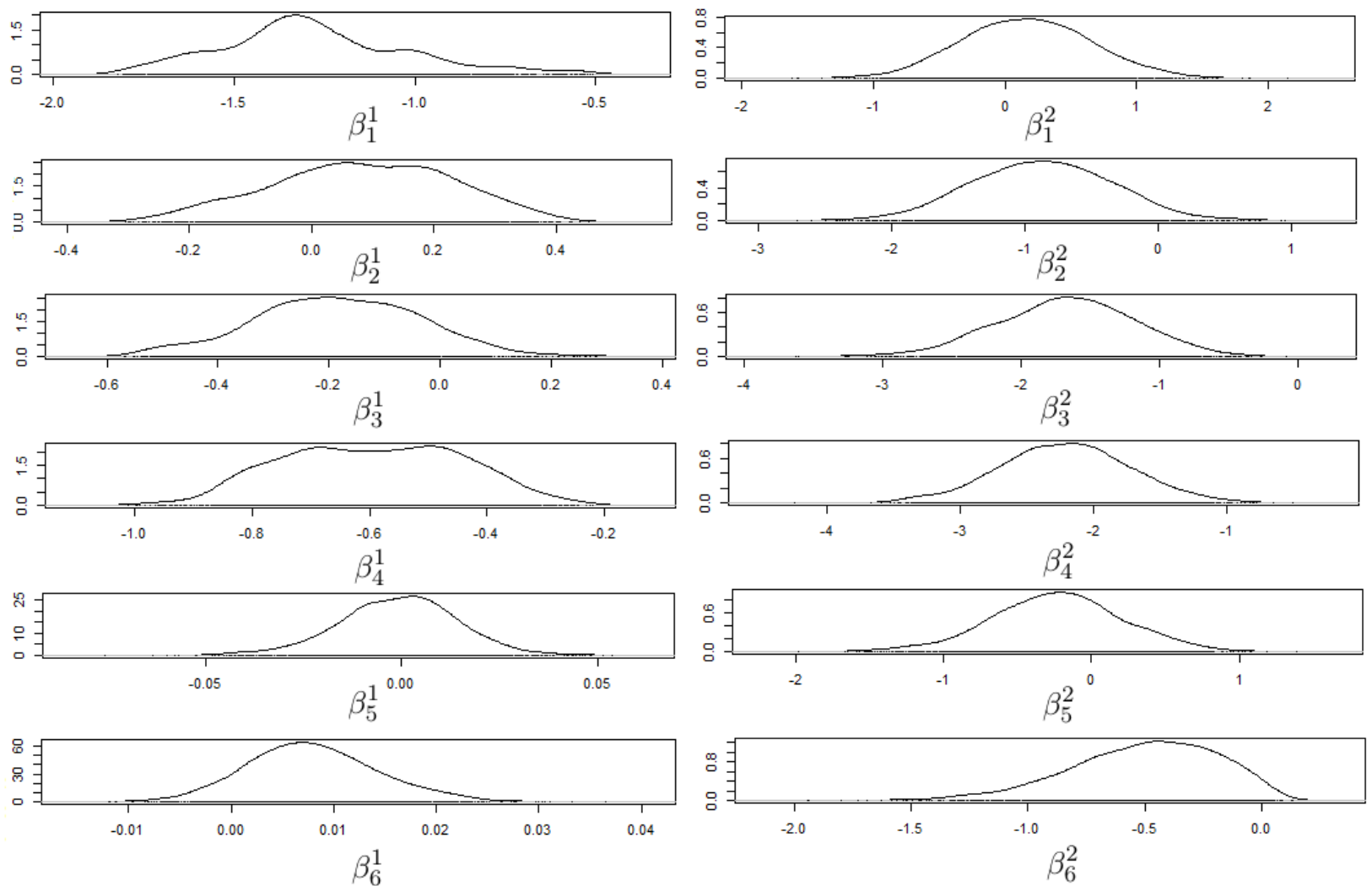

Figura 3.6: Gráfico da função densidade de probabilidade estimada dos parâmetros $\boldsymbol{\beta}^{\boldsymbol{s}}$, $s=1,2$, do modelo final ajustado para a variável anti-ox $\mathbf{L} \boldsymbol{D L}$ dos dados de periodontite. 

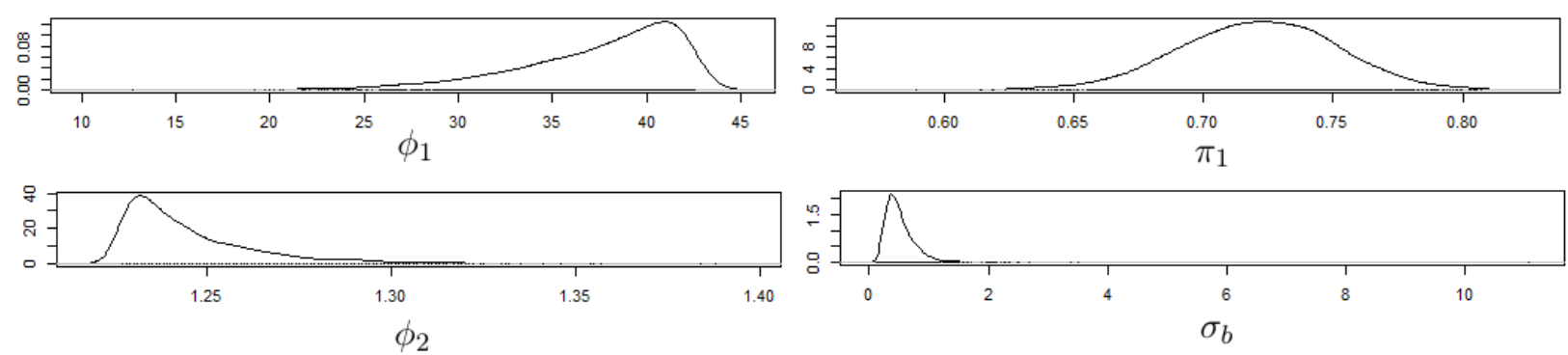

Figura 3.7: Gráfico da função densidade de probabilidade estimada dos parâmetros $\phi_{1}, \phi_{2}, \pi_{1}$ e $\sigma_{b}$ do modelo final ajustado para a variável anti-oxLDL dos dados de periodontite.

Para monitorar a convergência da cadeia analisou-se os gráficos de tendência e estatísticas descritivas dos valores gerados e as medidas de Geweke conforme exemplificado em Robert e Casella (2004) e Gamerman e Lopes (2006); os resultados indicaram a convergência adequada das amostras a posteriori geradas através do algoritmo MCMC.

Tal qual introduzido na Subseção 2.1.3, a avaliação preditiva a posteriori compara as observações da amostra observada com amostras simuladas da distribuição preditiva a posteriori em relação a determinada medida para analisar quão consistentes são os modelos com os dados reais. Essa medida é chamada de medida de discrepância e como resultado dessa análise é obtido o p-valor preditivo a posteriori que é a proporção das réplicas simuladas cuja medida de discrepância assume maior valor que o valor dessa medida quando calculada nos dados reais.

Nessa aplicação a análise preditiva a posteriori foi efetuada considerando como medidas de discrepância a média, a variância e o desvio (deviance) e os respectivos p-valores preditivos a posteriori resultantes são: 0,68, 0,28 e 0,44. Esses valores indicam que os dados condizem com o modelo ajustado em relação à média e ao desvio pois estão próximos de 0,5 . Em relação à variância, o resultado não é tão centrado no intervalo unitário mas ainda assim sugere adequabilidade. Os resíduos quantílicos aleatorizados calculados conforme apresentados em Dunn e Smyth (1996) estão expostos na Figura 3.8 e também indicam que o ajuste é adequado para a aplicação. Nessa figura, os poucos pontos distantes da reta são observações que tiveram valores altos de HDL e altos de oxLDL ou valores altos de LDL e baixos de oxLDL, relações essas que não são expressas pelas estimativas do modelo ajustado.

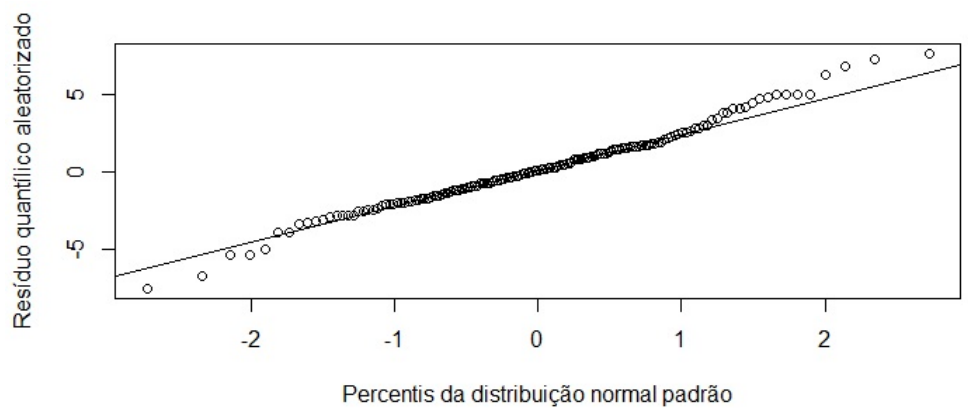

Figura 3.8: Gráfico de probabilidade normal dos resíduos quantílicos aleatorizados do modelo final ajustado aos dados de periodontite.

Veja, na Tabela 3.6 e na Figura 3.6, que, com exceção de $\beta_{2}^{1}$, as estimativas pontuais dos coeficientes relacionados às variáveis indicadoras de tempo pós-tratamento são negativas e suas funções densidade de probabilidade estimadas possuem valor não nulo também nesse intervalo. Isto 
significa que o valor de anti-oxLDL antes do início do tratamento é, em média, maior do que o mesmo após o início do tratamento.

Os valores absolutos destes coeficientes são maiores conforme o número de meses pós-tratamento aumenta; ou seja, ao comparar os valores de anti-oxLDL pré-tratamento com os mesmos após o início do tratamento, em média, a intensidade da diminuição deste indicador é maior conforme o número de meses procedentes ao tratamento aumenta.

Ao comparar esses resultados com os obtidos no ajuste do modelo com $K=1$ (Tabela 3.4), observa-se que há diferenças entre as medianas a posteriori dos coeficientes associados às variáveis de tempo pós-tratamento e HDL e do valor estimado do parâmetro de precisão. Essas mesmas diferenças foram observadas nos resultados do ajuste do modelo de regressão beta linear com efeitos fixos.

Quando é ajustado o modelo de mistura beta linear com efeitos fixos com $K=1,2,3$, há divergência entre o número de elementos da mistura selecionado pelos critérios estudados: o EBIC, que é o critério com maior penalização, seleciona $K=1$ e os demais critérios possuem valores semelhantes para $K=2,3$, o que sinaliza que o modelo apenas com efeitos fixos pode não ser adequado.

Contrastando o modelo de mistura beta linear com dois componentes na mistura e efeitos fixos em relação ao mesmo com efeitos aleatórios (Tabela 3.6), há diferença nos coeficientes estimados associados às concentrações de HDL e LDL, no valor estimado do parâmetro de precisão e na relação dos coeficientes associados estimados das variáveis de tempo pós-tratamento. No modelo com intercepto aleatório ambos os componentes da mistura possuem maiores coeficientes estimados quanto maior o tempo após o tratamento, porém no modelo com apenas efeitos fixos isso não ocorre para o primeiro componente da mistura, fato esse que não condiz com o esperado.

Retomando os objetivos da pesquisa, ao considerar a variável anti-oxLDL como indicadora de risco de doenças cardiovasculares, os resultados obtidos até o presente momento mostram que o fator de risco de doenças cardiovasculares em pacientes com periodontite diminui conforme o tempo pós-tratamento aumenta.

Isto significa também que o tratamento recebido pelos pacientes permanece surtindo efeito e sendo eficaz neste primeiro ano que o sucede.

Como os estudos de simulação da Subseção 3.4.3 mostraram que a identificabilidade do modelo em estudo está vinculada aos valores dos parâmetros, assim como conduzido na mesma, o diagnóstico de identificabilidade desses dados simulados foi averiguado através da análise gráfica do decrescimento dos valores do maior autovalor padronizado da matriz de variâncias e covariâncias a posteriori dos parâmetros para os números de clones cujos algoritmos convergiram, conforme exposto na Figura 3.9. 


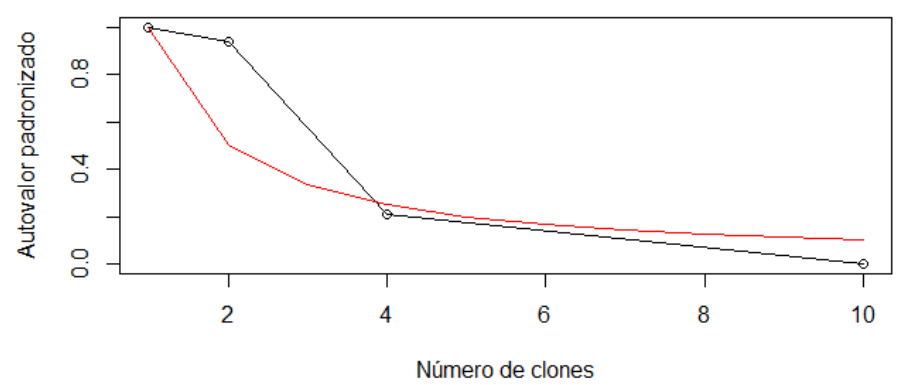

Figura 3.9: Gráfico de linhas do maior autovalor padronizado da matriz de variâncias e covariâncias a posteriori dos parâmetros do modelo de mistura beta misto ajustado aos dados de periodontite.

Os resultados da utilização do método indicam que não há evidências de falta de identificabilidade no modelo já que observa-se o decrescimento do autovalor a medida que o número de clones aumenta e há similaridade do perfil desse declínio com a curva de referência, principalmente a partir de $n_{\text {clone }}=4$.

Por fim, recomenda-se que, conjuntamente ao pesquisador, os interesses de inclusão de covariáveis e identificabilidade sejam ponderados afim de obter um modelo útil aos objetivos do estudo.

\subsubsection{Aglomeração das redes de fast food nas cidades brasileiras}

A expansão das redes e franquias de fast food no território brasileiro vem ocorrendo cada ano com mais intensidade, tanto que, segundo o relatório do desempenho do franchising da Associação Brasileira de Franchising (ABF), em 2015 houve aumento de 10,1\% de unidades franqueadas e o crescimento do faturamento total das empresas desse formato foi de $8,3 \%$, sendo que as redes do setor alimentício foram responsáveis por $20 \%$ deste valor. É de interesse dessas empresas que a definição dos locais de implantação de novas unidades seja efetuada baseando-se em indicadores que influenciam no desenvolvimento comercial das mesmas (Kaufmann et al., 2009). Assim, o objetivo desta aplicação é compreender se existe relação entre a forma como ocorre a aglomeração de redes de fast food nas cidades brasileiras e suas respectivas características.

A ABF efetuou um estudo desbalanceado em 2015 que englobou 7.653 lojas de 270 marcas de redes de fast food brasileiras e estrangeiras localizadas em 542 cidades. Como o objetivo dessa análise é estudar a aglomeração das unidades, esses dados foram utilizados para calcular uma medida que pode ser usada para quantificar essa característica denominada índice de Gini. O índice de Gini é um indicador muito utilizado em estudos de concentração de indústrias que considera tanto a proporção de lojas da mesma rede em cada cidade quanto a proporção de unidades da mesma rede na amostra, como expressa a seguinte equação:

$$
G_{i j}=\left(W_{i j}-W_{j}\right)^{2}
$$

em que $W_{i j}$ é a porcentagem de unidades da $j$-ésima rede na $i$-ésima cidade em relação ao total de unidades de redes de fast food na cidade, $i=1, \ldots, N, j=1,2, \ldots, n_{i}$, e, $W_{j}$ é a porcentagem de unidades da $j$-ésima rede em relação ao total de unidades da amostra. Quando o índice assume valor próximo de um significa que a cidade possui distribuição desigual de marcas ou maior concentração em poucas marcas e, quanto mais próximo de zero é o índice de Gini, mais uniforme é a distribuição da rede na cidade. 
A forma como é calculado o índice de Gini faz com que seja adequada sua análise através da distribuição beta, já que seu uso é indicado para dados restritos a um intervalo definido. Outra característica dos dados é a presença de correlação entre as observações, isso porque as unidades de lojas e de redes em um mesmo município não são independentes; assim, a inclusão de efeitos aleatórios relacionados às cidades é possível por meio dos modelos de regressão beta mistos.

Em virtude do interesse em compreender se existe diferença na forma como as informações institucionais dos municípios influenciam na aglomeração das empresas nacionais e estrangeiras, Giampaoli et al. (2016) e Madeira e Giampaoli (2017) ajustaram modelos beta inflacionados mistos separados para os dados dessas categorias de redes. Na primeira referência a medida de aglomeração utilizada foi o índice de Gini e os autores concluíram que não há diferenças, enquanto que na segunda foi considerada como variável resposta dos modelos o índice de Herfindahl. Porém, ao ajustar modelos separadadamente não existe a possibilidade de incorporar à inferência a questão da concorrência entre todas as empresas, já que os processos de estimação são totalmente independentes; dessa forma, quando existe padrão para expansão das redes, impossibilita-se incluir na análise a existência de empresas nacionais cuja expansão ocorre da mesma forma que as empresas estrangeiras e vice-versa. Pelas suas características, os modelos de mistura são indicados para este tipo de análise.

Portanto, pelos motivos expostos nos dois parágrafos anteriores, é apropriado que a análise dos dados seja efetuada por meio dos modelos de mistura beta mistos.

Com base em estudos anteriores como Glaeser et al. (2004), Kaufmann et al. (2009) e Das e Quirk (2016), foram selecionadas variáveis que possivelmente influenciam na forma como as lojas das redes de fast food se dispersam nos municípios, dentre elas: tamanho populacional, renda per capita, longevidade, índice de escolaridade, acesso à internet, saneamento básico, mobilidade urbana, condições ambientais e habitacionais, infraestrutura urbana, índices de segurança pública, produto interno bruto (PIB), dentre outras. Adicionalmente, como no Brasil existe o programa de alimentação do trabalhador (PAT) que contempla a concessão de crédito periodicamente em cartões eletrônicos destinado à compra de refeições ou produtos de gênero alimentício em estabelecimentos comerciais, a taxa de trabalhadores beneficiados por esse benefício também foi incorporada à base de dados.

Houve dificuldades em obter todas essas informações nos registros de dados dos institutos de pesquisa nacionais e, por fim, as variáveis que foram incorporadas ao banco de dados analisados encontram-se descritas nas páginas 13 à 26 (Seção 2) de Giampaoli et al. (2016). Porém, essa complicação não foi totalmente contornada e algumas observações precisaram ser removidas do banco de dados devido à falta de informações disponíveis dos municípios. No fim, o banco de dados em que se baseou as análises contemplou 40 cidades e 90 marcas.

Inicialmente foi ajustado um modelo de regressão beta com efeitos aleatórios com todas as variáveis explicativas. Em seguida, neste modelo inicial foi aplicado o método de seleção baseado no critério de informação AIC, assim como verificações gerais como multicolinearidade e outras, e as variáveis que permaneceram no modelo foram as seguintes:

- IBEU: índice de bem-estar urbano. Este índice avalia o bem-estar dos habitantes de cada cidade levando em consideração tópicos como mobilidade, condições ambientais, condições habitacionais, qualidade de serviços coletivos e infraestrutura; 
- IDHM-RENDA ou renda: renda municipal per capita é a média da renda dos habitantes das cidades, sendo que os habitantes sem renda também são contabilizados nesse cálculo;

- IDHM-LONGEVIDADE ou longevidade: expectativa de vida ao nascer. Esta variável expressa a quantidade média de anos a partir do nascimento que o indivíduo nascido na cidade viveria;

- IDHM-EDUCAÇÃO: medida resumo da escolaridade da população adulta, jovem e infantil do município;

- matricula_tec: indicador da quantidade de matrículas em cursos profissionais técnicos;

- taxa de homicídios ou homicídios: taxa de homicídios por cem mil habitantes;

- PAT: taxa de trabalhadores beneficiados pelo PAT;

- PIB: PIB per capita municipal;

- estimativa da população das cidades em 2015 categorizada conforme o número de habitantes nas faixas: menos de 5.000, 10.001-20.000, 50.001-100.000, 100.001-500.000 e mais de 500.001. Esta informação foi incluída no modelo através das variáveis indicadoras $I(<5.000)_{p o p, i j}$, $I(10.001-20.000)_{p o p, i j}, I(50.001-100.000)_{p o p, i j}, I(100.001-500.000)_{p o p, i j}, I(>500.001)_{p o p, i j}$, nas quais a primeira categoria foi considerada como referência;

- anos_origem: tempo, em anos, da fundação da empresa.

Após esse procedimento, efetuou-se a análise de diagnóstico e o estudo das estimativas dos parâmetros do modelo beta misto ajustado com as covariáveis listadas anteriormente para seleção das variáveis explicativas do modelo de mistura beta misto. Sejam $\boldsymbol{g}_{\boldsymbol{i}}=\left(g_{i 1}, \ldots, g_{i n_{i}}\right)^{\top}$ e $S_{i}$, respectivamente, o índice de Gini e a variável indicadora discreta do componente da mistura associados à $i$ ésima cidade, $i=1, \ldots, 40$, e, considerando $G_{i j} \mid b_{i} \sim \sum_{S_{i}=1}^{K} \pi_{i S_{i}} \operatorname{Beta}\left(\mu_{S_{i}, i j}, \phi_{S_{i}}\right)$ e $\mu_{S_{i}, i j}=E\left(G_{i j} \mid b_{i}, S_{i}\right)$, este modelo é expresso na Equação 3.13 .

$$
\begin{aligned}
& \log \left(\frac{\mu_{S_{i}, i j}}{1-\mu_{S_{i}, i j}}\right)=\beta_{1}^{S_{i}}+\beta_{2}^{S_{i}} I B E U_{i j}+\beta_{3}^{S_{i}} \text { renda }_{i j}+\beta_{4}^{S_{i}} \text { longevidade }_{i j}+\beta_{5}^{S_{i}} \text { homicídios }_{i j} \\
& +\beta_{6}^{S_{i}} P A T_{i j}+\beta_{7}^{S_{i}} P I B_{i j}+\beta_{8}^{S_{i}} I(10.001-20.000)_{p o p, i j} \\
& +\beta_{9}^{S_{i}} I(50.001-100.000)_{p o p, i j}+\beta_{10}^{S_{i}} I(100.001-500.000)_{p o p, i j} \\
& +\beta_{11}^{S_{i}} I(>500.001)_{p o p, i j}+b_{i} \text {. }
\end{aligned}
$$

O passo seguinte foi ajustar modelos de mistura beta mistos com as covariáveis que foram escolhidas para $K=1,2,3$ e fazer a verificação da convergência dos algoritmos MCMC, a qual foi satisfatória. Então, calculou-se os valores dos critérios de informação EAIC, ECAIC, EBIC, DIC 3 e LPML para esses modelos, os quais estão exibidos na Tabela 3.7. 


\begin{tabular}{c|ccc}
\hline & \multicolumn{3}{|c}{$K$} \\
\hline Critério & 1 & 2 & 3 \\
\hline EAIC & -5928.599 & -6129.149 & -6165.884 \\
ECAIC & -5927.798 & -6126.377 & -6159.873 \\
EBIC & -5871.118 & -6014.187 & -5993.440 \\
$D I C_{3}$ & -6190.544 & -6830.621 & -6640.308 \\
LPML & -2787.588 & -2710.496 & -2901.12 \\
\hline
\end{tabular}

Tabela 3.7: Valores dos critérios de informação EAIC, ECAIC, EBIC, DIC 3 e LPML para o ajuste do modelo (3.13) com $K=1,2,3$ da variável indice de Gini dos dados de redes de fast food no Brasil.

Por fim, com base nos resultados dos critérios de seleção, nos conhecimentos prévios, nas estimativas dos parâmetros e nas análises de diagnóstico, o modelo eleito foi com $K=2$, cujas informações dos parâmetros encontram-se na Tabela 3.8. A análise preditiva a posteriori deste modelo foi efetuada considerando como medidas de discrepância a média, a variância e o desvio (deviance) e os respectivos p-valores preditivos a posteriori resultantes são 0,689, 0,679 e 0,667. Esses valores estão bastante distantes dos extremos e próximos do meio do intervalo unitário, o que evidencia a adequabilidade do modelo para os dados. 


\begin{tabular}{|c|c|c|c|c|}
\hline Parâmetro & Covariável associada & Estimativa & Desvio padrão & IC $90 \%$ \\
\hline$\beta_{1}^{1}$ & - & $-60,460$ & 0,372 & $(-61,15 ;-59,80)$ \\
\hline$\beta_{2}^{1}$ & IBEU & $-1,739$ & 0,419 & $(-2,372 ;-1,003)$ \\
\hline$\beta_{3}^{1}$ & renda & $-7,370$ & 0,454 & $(-8,137 ;-6,677)$ \\
\hline$\beta_{4}^{1}$ & longevidade & 5,531 & 0,452 & $(4,825 ; 6,287)$ \\
\hline$\beta_{5}^{1}$ & homicídios & 0,015 & 0,010 & $(-0,004 ; 0,029)$ \\
\hline$\beta_{6}^{1}$ & PAT & 65,730 & 0,592 & $(64,81 ; 66,71)$ \\
\hline$\beta_{7}^{1}$ & PIB & $-1,647$ & 0,477 & $(-2,478 ;-0,923)$ \\
\hline$\beta_{8}^{1}$ & $I(10.001-20.000)_{p o p}$ & 57,940 & 0,445 & $(57,09 ; 58,62)$ \\
\hline$\beta_{9}^{1}$ & $I(50.001-100.000)_{p o p}$ & 59,770 & 0,370 & $(59,17 ; 60,33)$ \\
\hline$\beta_{10}^{1}$ & $I(100.001-500.000)_{p o p}$ & 59,200 & 0,337 & $(58,74 ; 59,80)$ \\
\hline$\beta_{11}^{1}$ & $I(>500.001)_{p o p}$ & 58,490 & 0,491 & $(57,66 ; 59,23)$ \\
\hline$\phi_{1}$ & - & 9,854 & 0,479 & $(9,49 ; 10,65)$ \\
\hline$\beta_{1}^{2}$ & - & $-1,720$ & 0,404 & $(-2,405 ;-1,096)$ \\
\hline$\beta_{2}^{2}$ & IBEU & 0,045 & 0,407 & $(-0,527 ; 0,815)$ \\
\hline$\beta_{3}^{2}$ & renda & $-1,674$ & 0,401 & $(-2,217 ;-0,928)$ \\
\hline$\beta_{4}^{2}$ & longevidade & $-0,714$ & 0,444 & $(-1,204 ; 0,156)$ \\
\hline$\beta_{5}^{2}$ & homicídios & $-0,028$ & 0,021 & $(-0,069 ;-0,007)$ \\
\hline$\beta_{6}^{2}$ & PAT & $-3,976$ & 0,463 & $(-4,680 ;-3,138)$ \\
\hline$\beta_{7}^{2}$ & PIB & 0,876 & 0,402 & $(0,252 ; 1,546)$ \\
\hline$\beta_{8}^{2}$ & $I(10.001-20.000)_{p o p}$ & $-0,100$ & 0,576 & $(-0,901 ; 0,947)$ \\
\hline$\beta_{9}^{2}$ & $I(50.001-100.000)_{p o p}$ & 3,284 & 0,799 & $(2,234 ; 4,592)$ \\
\hline$\beta_{10}^{2}$ & $I(100.001-500.000)_{p o p}$ & $-0,233$ & 0,328 & $(-0,786 ; 0,290)$ \\
\hline$\beta_{11}^{2}$ & $I(>500.001)_{p o p}$ & $-0,144$ & 0,629 & $(-0,997 ; 1,022)$ \\
\hline$\phi_{2}$ & - & 78,770 & 2,914 & $(74,03 ; 81,27)$ \\
\hline$\pi_{1}$ & - & 0,729 & 0,029 & $(0,683 ; 0,779)$ \\
\hline$\sigma_{b}$ & - & 0,954 & 0,565 & $(0,161 ; 1,768)$ \\
\hline
\end{tabular}

Tabela 3.8: Estimativas pontuais (mediana a posteriori) dos parâmetros, desvios padrões e intervalos de credibilidade de $90 \%$ do modelo final ajustado para a variável indice de Gini dos dados de redes de fast food no Brasil.

O gráfico da Figura 3.10 apresenta os resíduos quantílicos aleatorizados do modelo final ajustado e por ela nota-se que há um grande afastamento de quatro observações em relação à reta nos dois extremos do gráfico. Essas observações são de empresas nacionais e tiveram seu índice de aglomeração subestimados pelo modelo trata-se das cidades em que estão localizadas possuírem alta taxa de homicídios e baixo valor de PIB per capita. 


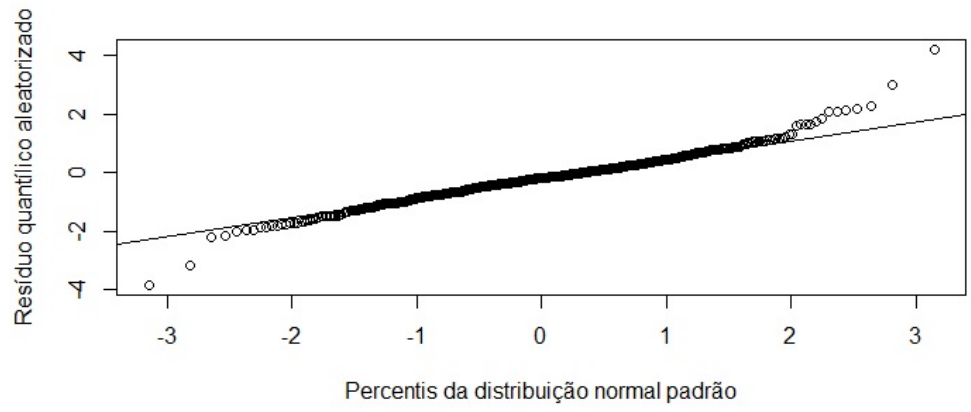

Figura 3.10: Gráfico de probabilidade normal dos resíduos quantilicos aleatorizados do modelo final ajustado aos dados de redes de fast food no Brasil.

Tal qual previamente recomendado, para cada aplicação em dados reais é indicado que seja efetuada a averiguação de identificabilidade do modelo, já que os estudos de simulação mostraram que essa característica está vinculada ao valor dos parâmetros e, consequentemente, à distinção dos componentes da mistura na função densidade de probabilidade da distribuição beta. Na Figura 3.11 estão expostos os resultados para os números de clones cujo algoritmo convergiu. A curva obtida é decrescente com angulação bastante semelhante à curva de referência, do que, baseado no método data cloning, conclui-se que não há evidências de falta de identificabilidade no modelo ajustado.

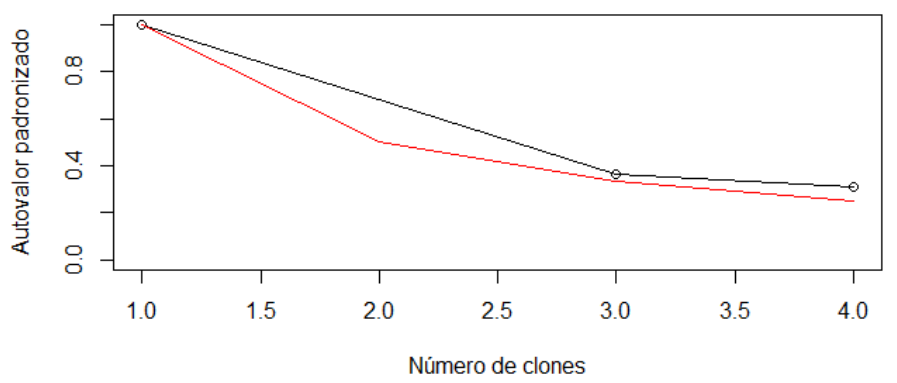

Figura 3.11: Gráfico de linhas do maior autovalor padronizado da matriz de variâncias e covariâncias a posteriori dos parâmetros do modelo de mistura beta misto ajustado aos dados de redes de fast food no Brasil.

Retomando o interesse em compreender as diferenças no padrão de aglomeração das marcas nacionais e estrangeiras que motivou o uso da metodologia de mistura de modelos de regressão, comparando a estimativa de $\pi_{1}(72,9 \%)$ à proporção de empresas nacionais em nível nacional $(73,83 \%)$, nota-se que os parâmetros associados ao primeiro componente da mistura contribuem com maior peso no valor esperado da aglomeração das empresas nacionais e, consequentemente, das empresas estrangeiras com comportamento de aglomeração similar às nacionais. Em caso de interesse de comparação em nível estadual, estas proporções encontram-se ilustradas na Figura 3.12. 


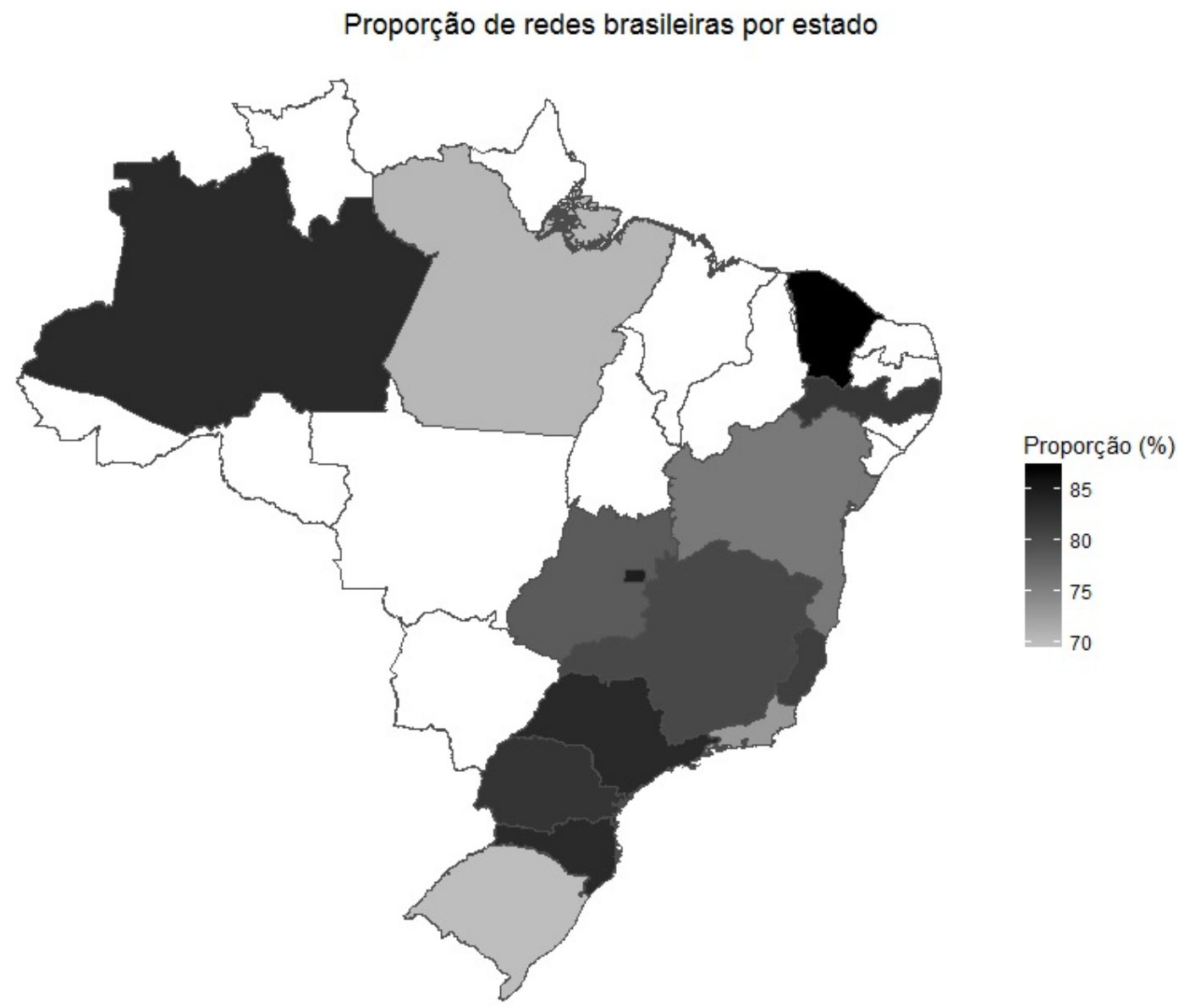

Figura 3.12: Mapa das proporções de redes de fast food nacionais em cada estado brasileiro.

As Figuras 3.13 e 3.14 apresentam as curvas estimadas da função densidade de probabilidade dos parâmetros. 

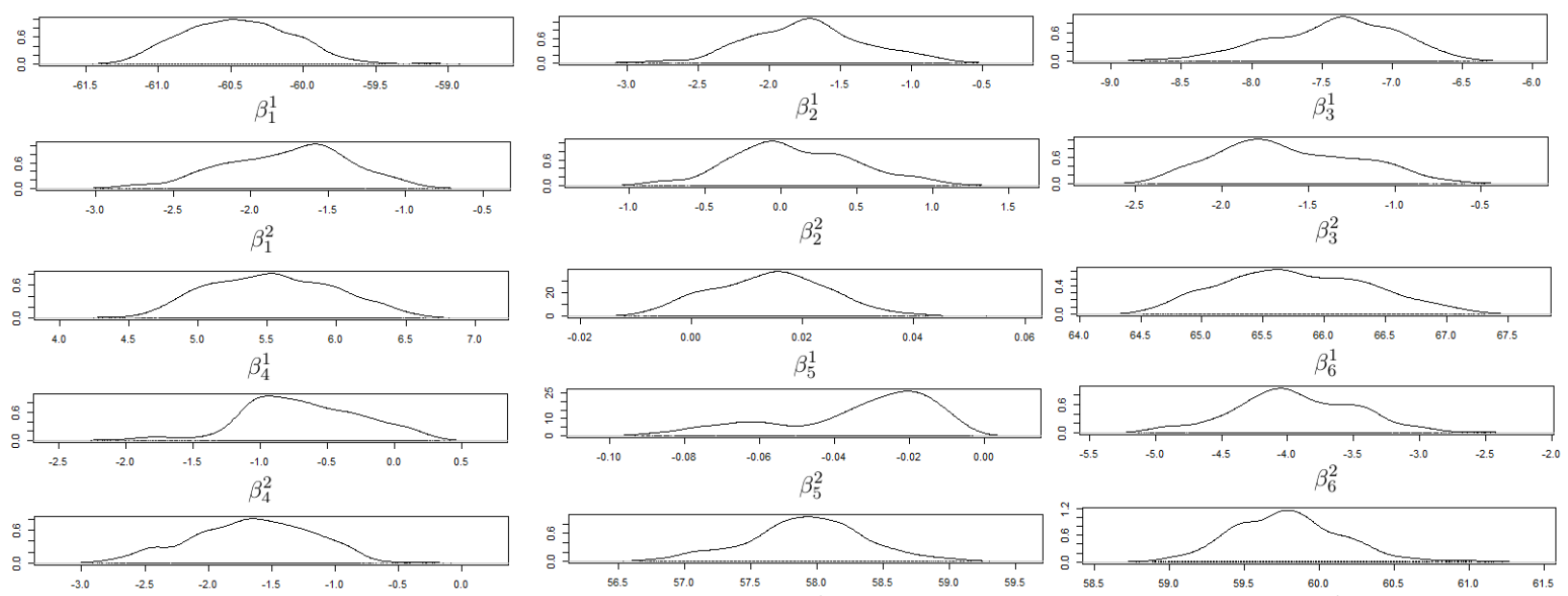

$\beta_{7}^{1}$
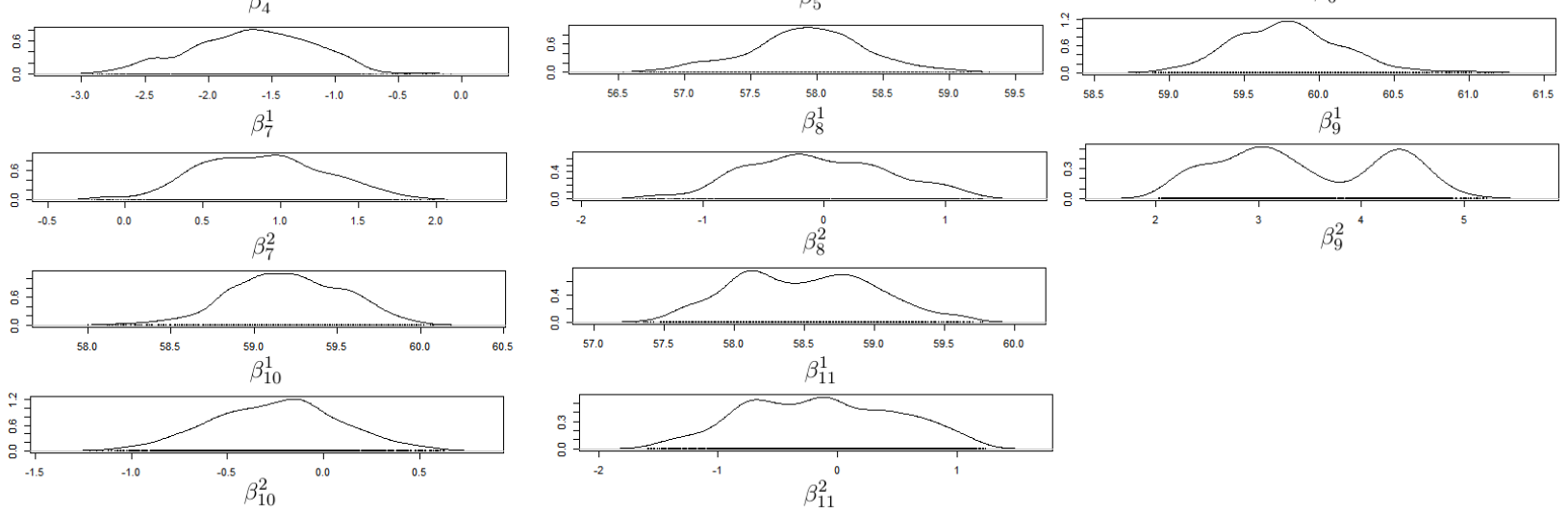

Figura 3.13: Gráfico da função densidade de probabilidade estimada dos parâmetros $\boldsymbol{\beta}^{\boldsymbol{s}}$, $s=1,2$, do modelo final ajustado para a variável indice de Gini dos dados de redes de fast food no Brasil.
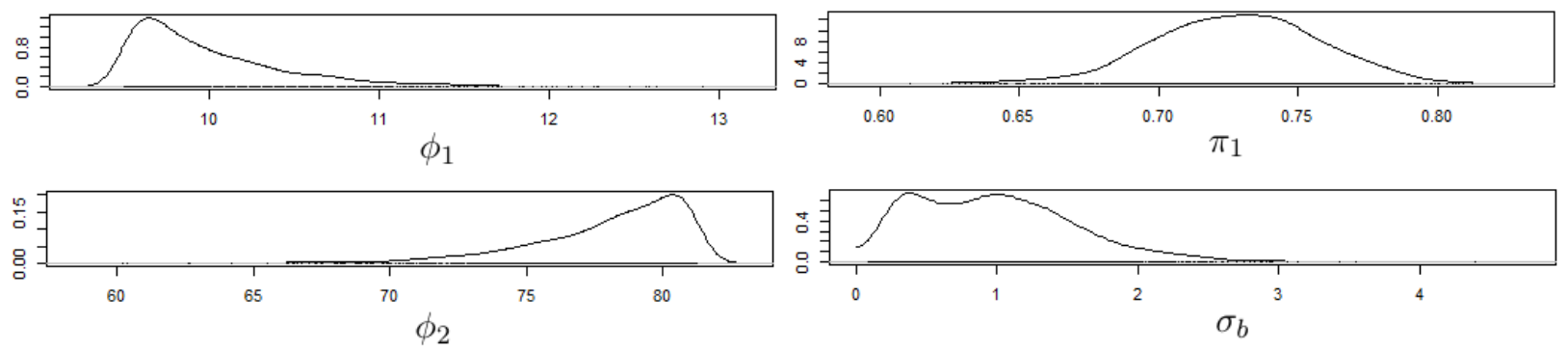

Figura 3.14: Gráfico da função densidade de probabilidade estimada dos parâmetros $\phi_{1}, \phi_{2}, \pi_{1} e \sigma_{b}$ do modelo final ajustado para a variável índice de Gini dos dados de redes de fast food no Brasil.

Observa-se que o valor zero faz parte dos intervalos de credibilidade dos coeficientes $\beta_{5}^{1}, \beta_{2}^{2}, \beta_{4}^{2}, \beta_{8}^{2}$, $\beta_{10}^{2}$ e $\beta_{11}^{2}$, associados respectivamente às variáveis: taxas de homicídios, índice de bem-estar humano, longevidade e tamanho populacional. Adicionalmente, com excessão de $\beta_{5}^{1}$ e $\beta_{4}^{2}$, as curvas estimadas de suas funções densidade de probabilidade também concentram sua massa de probabilidade em torno do valor zero.

As estimativas negativas dos coeficientes das variáveis índice de bem-estar humano e renda indicam que quanto maior os seus valores, há uma menor concentração de marcas, ou seja, maior é a dispersão da rede em termos de marcas. As demais variáveis apresentaram divergência de sinais nos coeficientes estimados para os dois grupos.

Analisando a significância das variáveis com base nos intervalos de credibilidade da Tabela 3.8 e nas curvas estimadas das funções densidade de probabilidade da Figura 3.13 tem-se que a variável longevidade obteve coeficiente estimado positivo no componente um, enquanto o coeficiente para 
taxa de homicídios foi significante e negativo para o segundo componente. Por fim, a taxa de trabalhadores beneficiados pelo PAT teve coeficiente significante positivo para o componente um e negativo para o segundo componente.

Sendo assim, pelo contexto, o uso dos modelos de mistura é mais apropriado pois nestes modelos a aglomeração esperada das empresas nacionais e estrangeiras é explicada pela combinação de componentes, o que é razoável de se considerar já que as redes trabalham em um mesmo mercado e podem se aglomerar de acordo com critérios diferentes dependendo do cenário comercial da localidade.

\subsection{Considerações finais}

O modelo de mistura beta linear com efeitos aleatórios apresentado e estudado nesta seção é inédito pois incorpora efeitos aleatórios ao já explorado modelo de mistura beta linear de efeitos fixos.

Este modelo é vantajoso pois a distribuição de probabilidade de sua variável resposta é indicada para ajustar dados com valores restritos a um intervalo limitado conhecido que apresentam correlação e que derivam de subpopulações heterogêneas com alocação desconhecida.

Sua inferência é de fácil implementação e o software é gratuito. Os resultados dos estudos de simulação efetuados indicam que as performances das estimativas pontuais e do critério de seleção $D I C_{3}$ são satisfatórias. Adicionalmente, por meio da técnica de averiguação de identificabilidade denominada data cloning, foram efetuados estudos de simulação para diferentes cenários e os resultados indicam que não há evidências de falta de identificabilidade do modelo quando há distinção entre os componentes da mistura.

O tempo computacional pode ser considerado um empecilho em sua aplicação, porém os resultados da performance e a capacidade interpretativa dos modelos neste trabalho estudados indicam que sua utilização é vantajosa e, ademais, a disponibilidade de recursos computacionais mais eficientes tem crescido intensamente, o que vai facilitar a contornar essa questão. 


\section{Capítulo 4}

\section{Modelo de Mistura Beta Misto Linear com Dispersão Variável}

Neste capítulo se aborda uma extensão dos modelos apresentados no capítulo anterior ao incorporar um modelo de mistura linear misto também para o parâmetro de precisão. Primeiramente é apresentada uma seção introdutória (4.1) e, em seguida, o modelo é especificado na Seção 4.2 e são sugeridas distribuições a priori para os parâmetros na Seção 4.3. Estudos de simulação foram efetuados para analisar o desempenho inferencial da metodologia bayesiana utilizada na Seção 4.4 e, por fim, um banco de dados é analisado para exemplificar a aplicação da técnica estatística proposta ao longo do capítulo.

\subsection{Introdução}

Inicialmente, os modelos de regressão beta foram definidos assumindo que o valor do parâmetro de precisão é igual para todas as observações, ou seja, ele é constante em toda a amostra. Porém, estudos como Carroll (2003) mostram que ao não atribuir ao parâmetro de precisão uma estrutura de regressão, pode-se obter resultados inferenciais inverossímeis e conclusões errôneas.

Em pesquisas de distintas áreas, o ajuste de modelos de regressão com dispersão variável é adequado e vantajoso pois a precisão da distribuição da variável resposta varia de acordo com o comportamento de uma gama de variáveis explicativas. Na área de saúde, por exemplo, quando se tem o objetivo de estudar dados clínicos de pacientes ao longo do tempo, a dispersão dessa medição pode provir de covariáveis como: tempo após o tratamento, concentração de substâncias no sangue, características físicas do paciente, dentre outras.

Os pioneiros em incorporar a estes modelos um modelo para o parâmetro de precisão foram Smithson e Verkuilen (2006). Essa extensão foi efetuada aos moldes da estrutura dos modelos lineares generalizados com ajuste conjunto para os parâmetros de média e precisão desenvolvidos em Smyth (1989) e, calculou-se as estimativas dos parâmetros da regressão por maximização da verossimilhança. Posteriormente, Simas et al. (2010) possibilitaram que esse modelo tenha estrutura não linear e, em seguida, Zimprich (2010) e Verkuilen e Smithson (2012) adicionaram efeitos aleatórios ao modelo para a média sob as abordagens frequentista e bayesiana, respectivamente.

Em Migliorati et al. (2018) é proposto um modelo de regressão linear de efeitos fixos com base em uma distribuição denominada flexible beta, a qual é um caso especial da mistura de duas distribuições 
beta. Os autores mencionam que essa distribuição possui propriedades de identificabilidade que não são satisfeitas em geral em misturas arbitrárias de duas distribuições beta por exemplo, a distribuição beta retangular que é uma mistura de uma distribuição uniforme e uma distribuição beta e, mesmo possuindo uum parâmetro a menos que a flexible beta, apresenta problemas de identificabilidade quando $\mu=0,5$ e $\phi=2$.

O modelo de regressão beta com dispersão variável é composto por dois modelos, denominados por Smyth (1989) como modelos de locação e dispersão. Por meio das suas funções de ligação, estes modelos associam, respectivamente, aos parâmetros de média e precisão da distribuição beta uma estrutura de regressão com variáveis explicativas e parâmetros, sendo que não há restrições na seleção dessas covariáveis. O modelo do parâmetro de dispersão pode quantificar tanto a variabilidade da variável resposta quanto incertezas e falhas relacionadas ao modelo do parâmetro de locação. Dessa forma, a extensão dos modelos apresentados no capítulo anterior (3) é efetuada neste capítulo ao incorporar um modelo para o parâmetro de dispersão.

\subsection{Modelo Estatístico}

Nesta seção apresenta-se o modelo de mistura beta misto linear com dispersão variável e em sua especificação assume-se que todas as observações de uma mesma unidade amostral $\left(y_{i 1}, \ldots, y_{i n_{i}}\right)$, $i=1, \ldots N$, associam-se ao mesmo componente da mistura.

Estabelecendo que a amostra é composta pelas observações da variável resposta $\boldsymbol{y}_{1}, \ldots, \boldsymbol{y}_{N}$ tais que cada $i$-ésima $(i=1, \ldots, N)$ unidade amostral é composta pelas suas $n_{i}$ aferições, ou seja, $\boldsymbol{y}_{i}=\left(y_{i 1}, \ldots, y_{i n_{i}}\right)^{\top}$, definindo $S_{i}$ como a variável discreta que indica o componente da mistura associado ao $i$-ésimo grupo da amostra, tal que os valores de $S_{i}$ pertencem ao conjunto $\{1, \ldots, K\}$ e considerando $Y_{i j} \mid \boldsymbol{b}_{i}, \boldsymbol{u}_{i} \sim \sum_{S_{i}=1}^{K} \pi_{i S_{i}} \operatorname{Beta}\left(\mu_{S_{i}, i j}, \phi_{S_{i}, i j}\right), \mu_{S_{i}, i j}=E\left(Y_{i j} \mid \boldsymbol{b}_{i}, \boldsymbol{u}_{i}, S_{i}\right)$ e $\phi_{S_{i}, i j}=$ $\frac{\mu_{S_{i}, i j}\left(1-\mu_{S_{i}, i j}\right)}{\operatorname{Var}\left(Y_{i j} \mid \boldsymbol{b}_{i}, \boldsymbol{u}_{i}, S_{i}\right)}-1, j=1, \ldots, n_{i}$, o modelo de mistura beta misto linear com dispersão variável é expresso por:

$$
\begin{gathered}
\eta_{S_{i}, i j}=g_{\mu}\left(\mu_{S_{i}, i j}\right)=\boldsymbol{x}_{i j}^{\top} \boldsymbol{\beta}^{S_{i}}+\boldsymbol{z}_{i j}^{\top} \boldsymbol{b}_{i} \\
\tau_{S_{i}, i j}=g_{\phi}\left(\phi_{S_{i}, i j}\right)=\boldsymbol{w}_{i j}^{\top} \boldsymbol{\delta}^{S_{i}}+\boldsymbol{r}_{i j}^{\top} \boldsymbol{u}_{i}
\end{gathered}
$$

em que:

- $Y_{i j}$ é a variável resposta;

- $K$ é o número de componentes da mistura;

- $N$ é o número de grupos da amostra;

- $n_{i}$ é o número de elementos do $i$-ésimo grupo, $i=1, \ldots, N$;

- $\boldsymbol{\pi}_{i}=\left(\pi_{i 1}, \ldots, \pi_{i K}\right)^{\top}$, em que $\pi_{i s}$ é a probabilidade do $i$-ésimo grupo estar associado ao $s$-ésimo componente da mistura, ou seja, $\pi_{i s}=P\left(S_{i}=s\right), 0<\pi_{i s}<1, \sum_{s=1}^{K} \pi_{i s}=1, i=1, \ldots, N$ e $s=1, \ldots, K ;$ 
- $\boldsymbol{\pi}=\left(\boldsymbol{\pi}_{1}, \ldots, \boldsymbol{\pi}_{N}\right)$

- $g_{\mu}$ é a função de ligação, estritamente monótona e duplamente diferenciável, do modelo para a média;

- $\boldsymbol{\beta}^{s}=\left(\beta_{1}^{s}, \ldots, \beta_{p}^{s}\right)^{\top}$ é o vetor $p$-dimensional de coeficientes fixos relacionado ao $s$-ésimo componente da mistura associada ao modelo para a média, $s=1, \ldots, K$;

- $\boldsymbol{\beta}=\left(\boldsymbol{\beta}^{1}, \ldots, \boldsymbol{\beta}^{K}\right)$ é a matriz de ordem $p \times K$ de coeficientes fixos do modelo para a média;

- $p$ é o número de efeitos fixos do modelo para a média;

- $\boldsymbol{b}_{i}=\left(b_{i 1}, \ldots, b_{i d}\right)^{\top}$ é o vetor de dimensão $d$ de efeitos aleatórios do $i$-ésimo grupo do modelo para a média;

- $d$ é o número de efeitos aleatórios do modelo para a média;

- $\boldsymbol{b}_{i} \mid \boldsymbol{\Sigma}_{b} \stackrel{i i d}{\sim} N_{d}\left(\mathbf{0}, \boldsymbol{\Sigma}_{b}\right)$

- $\boldsymbol{\Sigma}_{b}$ é a matriz de ordem $d \times d$ de variância-covariância dos efeitos aleatórios $\left(\boldsymbol{b}_{1}, \ldots, \boldsymbol{b}_{N}\right)$ do modelo para a média;

- $\boldsymbol{x}_{i j}=\left(x_{i j 1}, \ldots, x_{i j p}\right)^{\top}$ é o vetor $p$-dimensional de covariáveis dos efeitos fixos do $j$-ésimo elemento do $i$-ésimo grupo do modelo para a média, $i=1, \ldots, N$ e $j=1, \ldots, n_{i}$;

- $\boldsymbol{z}_{i j}=\left(z_{i j 1}, \ldots, z_{i j d}\right)^{\top}$ é o vetor $d$-dimensional de covariáveis dos efeitos aleatórios do $j$-ésimo elemento do $i$-ésimo grupo do modelo para a média, $i=1, \ldots, N$ e $j=1, \ldots, n_{i}$;

- $g_{\phi}$ é a função de ligação, estritamente monótona e duplamente diferenciável, do modelo para a precisão;

- $\boldsymbol{\delta}^{s}=\left(\delta_{1}^{s}, \ldots, \delta_{q}^{s}\right)^{\top}$ é o vetor $q$-dimensional de coeficientes fixos relacionado ao $s$-ésimo componente da mistura associada ao modelo para a precisão, $s=1, \ldots, K$;

- $q$ é o número de efeitos fixos do modelo para a precisão;

- $\boldsymbol{u}_{i}=\left(u_{i 1}, \ldots, u_{i d^{*}}\right)^{\top}$ é o vetor de dimensão $d^{*}$ de efeitos aleatórios do $i$-ésimo grupo do modelo para a precisão;

- $d^{*}$ é o número de efeitos aleatórios do modelo para a precisão;

- $\boldsymbol{u}_{i} \mid \boldsymbol{\Sigma}_{u} \stackrel{i i d}{\sim} N_{d^{*}}\left(\mathbf{0}, \boldsymbol{\Sigma}_{u}\right)$

- $\boldsymbol{\Sigma}_{u}$ é a matriz de ordem $d^{*} \times d^{*}$ de variância-covariância dos efeitos aleatórios $\left(\boldsymbol{u}_{1}, \ldots, \boldsymbol{u}_{N}\right)$ do modelo para a precisão;

- $\boldsymbol{w}_{i j}=\left(w_{i j 1}, \ldots, w_{i j q}\right)^{\top}$ é o vetor $q$-dimensional de covariáveis dos efeitos fixos do $j$-ésimo elemento do $i$-ésimo grupo do modelo para a precisão, $i=1, \ldots, N$ e $j=1, \ldots, n_{i}$;

- $\boldsymbol{r}_{i j}=\left(r_{i j 1}, \ldots, r_{i j d^{*}}\right)^{\top}$ é o vetor $d^{*}$-dimensional de covariáveis dos efeitos aleatórios do $j$-ésimo elemento do $i$-ésimo grupo do modelo para a precisão, $i=1, \ldots, N$ e $j=1, \ldots, n_{i}$; 
- $\boldsymbol{\vartheta}=\left\{\boldsymbol{\beta}^{1^{\top}}, \ldots, \boldsymbol{\beta}^{K^{\top}}, \boldsymbol{\delta}^{1^{\top}}, \ldots, \boldsymbol{\delta}^{K^{\top}}, \operatorname{vech}^{\top}\left(\boldsymbol{\Sigma}_{b}\right), \operatorname{vech}^{\top}\left(\boldsymbol{\Sigma}_{u}\right), \boldsymbol{\pi}_{1}^{\top}, \ldots, \boldsymbol{\pi}_{N}^{\top}\right\}$ é o vetor de parâmetros do modelo.

Este modelo também pode ser especificado hierarquicamente como:

$$
\begin{array}{r}
Y_{i j} \mid \boldsymbol{b}_{i}, \boldsymbol{u}_{i}, S_{i}=s \sim \operatorname{Beta}\left(g_{\mu}^{-1}\left(\boldsymbol{x}_{i j}^{\top} \boldsymbol{\beta}^{s}+\boldsymbol{z}_{i j}^{\top} \boldsymbol{b}_{i}\right), g_{\phi}^{-1}\left(\boldsymbol{w}_{i j}^{\top} \boldsymbol{\delta}^{s}+\boldsymbol{r}_{i j}^{\top} \boldsymbol{u}_{i}\right)\right) \\
\boldsymbol{b}_{i} \mid \boldsymbol{\Sigma}_{b} \stackrel{i i d}{\sim} N_{d}\left(\mathbf{0}, \boldsymbol{\Sigma}_{b}\right) \\
\boldsymbol{u}_{i} \mid \boldsymbol{\Sigma}_{u} \stackrel{i i d}{\sim} N_{d^{*}}\left(\mathbf{0}, \boldsymbol{\Sigma}_{u}\right) \\
P\left(S_{i}=s\right)=\pi_{s},
\end{array}
$$

$\operatorname{assim:~} Y_{i j} \mid \boldsymbol{b}_{i}, \boldsymbol{u}_{i} \sim \sum_{s=1}^{K} \pi_{i s} \operatorname{Beta}\left(g_{\mu}^{-1}\left(\boldsymbol{x}_{i j}^{\top} \boldsymbol{\beta}^{s}+\boldsymbol{z}_{i j}^{\top} \boldsymbol{b}_{i}\right), g_{\phi}^{-1}\left(\boldsymbol{w}_{i j}^{\top} \boldsymbol{\delta}^{s}+\boldsymbol{r}_{i j}^{\top} \boldsymbol{u}_{i}\right)\right.$.

De acordo com as particularidades da aplicação destes modelos, é possível considerar outras distribuições para os efeitos aleatórios sem alteração da técnica apresentada, como por exemplo a $t$ de Student, dentre outras. A depender da distribuição considerada para os efeitos aleatórios são adotadas distribuições a priori para os hiperparâmetros dos efeitos aleatórios. Na Seção 2.3 são indicadas referências que discorrem sobre essa atribuição e apresentadas outras considerações.

As verossimilhanças condicional e marginal conjuntas relacionadas ao modelo (4.1) e sem integração nos efeitos aleatórios encontram-se expressas nas Equações (4.3) e (4.4).

$$
\begin{aligned}
L(\boldsymbol{y} \mid \boldsymbol{\vartheta}, \boldsymbol{X}, \boldsymbol{Z}, \boldsymbol{W}, \boldsymbol{R}, \boldsymbol{b}, \boldsymbol{u}, \boldsymbol{S}) & =\prod_{i=1}^{N} \prod_{j=1}^{n_{i}}\left[L_{i j}\left(y_{i j} \mid \boldsymbol{\vartheta}, \boldsymbol{x}_{i j}, \boldsymbol{z}_{i j}, \boldsymbol{w}_{i j}, \boldsymbol{r}_{i j}, \boldsymbol{b}_{i}, \boldsymbol{u}_{i}, S_{i}\right)\right] \\
& =\prod_{i=1}^{N} \prod_{j=1}^{n_{i}} \frac{\Gamma\left(g_{\phi}^{-1}\left(\tau_{i j}\right)\right) y_{i j}^{g_{\mu}^{-1}\left(\eta_{i j}\right) g_{\phi}^{-1}\left(\tau_{i j}\right)-1}\left(1-y_{i j}\right)^{\left(1-g_{\mu}^{-1}\left(\eta_{i j}\right)\right) g_{\phi}^{-1}\left(\tau_{i j}\right)-1}}{\Gamma\left(g_{\mu}^{-1}\left(\eta_{i j}\right) g_{\phi}^{-1}\left(\tau_{i j}\right)\right) \Gamma\left(\left(1-g_{\mu}^{-1}\left(\eta_{i j}\right)\right) g_{\phi}^{-1}\left(\tau_{i j}\right)\right)},
\end{aligned}
$$

em que $g_{\mu}^{-1}\left(\eta_{i j}\right)=g_{\mu}^{-1}\left(\boldsymbol{x}_{i j}^{\top} \boldsymbol{\beta}^{S_{i}}+\boldsymbol{z}_{i j}^{\top} \boldsymbol{b}_{i}\right)$ e $g_{\phi}^{-1}\left(\tau_{i j}\right)=g_{\phi}^{-1}\left(\boldsymbol{w}_{i j}^{\top} \boldsymbol{\delta}^{S_{i}}+\boldsymbol{r}_{i j}^{\top} \boldsymbol{u}_{i}\right)$.

$$
\begin{aligned}
L(\boldsymbol{y} \mid \boldsymbol{\vartheta}, \boldsymbol{X}, \boldsymbol{Z}, \boldsymbol{W}, \boldsymbol{R}, \boldsymbol{b}, \boldsymbol{u}) & =\prod_{i=1}^{N} \prod_{j=1}^{n_{i}}\left[L_{i j}\left(y_{i j} \mid \boldsymbol{\vartheta}, \boldsymbol{x}_{i j}, \boldsymbol{z}_{i j}, \boldsymbol{w}_{i j}, \boldsymbol{r}_{i j}, \boldsymbol{b}_{i}, \boldsymbol{u}_{i}\right)\right] \\
& =\prod_{i=1}^{N} \sum_{S_{i}=1}^{K} \pi_{i S_{i}} \prod_{j=1}^{n_{i}}\left[L_{i j}\left(y_{i j} \mid \boldsymbol{\vartheta}, \boldsymbol{x}_{i j}, \boldsymbol{z}_{i j}, \boldsymbol{w}_{i j}, \boldsymbol{r}_{i j}, \boldsymbol{b}_{i}, \boldsymbol{u}_{i}, S_{i}\right)\right]
\end{aligned}
$$

em que:

$$
\begin{gathered}
L_{i j}\left(y_{i j} \mid \boldsymbol{\vartheta}, \boldsymbol{x}_{i j}, \boldsymbol{z}_{i j}, \boldsymbol{w}_{i j}, \boldsymbol{r}_{i j}, \boldsymbol{b}_{i}, \boldsymbol{u}_{i}, S_{i}\right)= \\
\prod_{i=1}^{N} \prod_{j=1}^{n_{i}} \frac{\Gamma\left(g_{\phi}^{-1}\left(\tau_{i j}\right)\right) y_{i j}^{g_{\mu}^{-1}\left(\eta_{i j}\right) g_{\phi}^{-1}\left(\tau_{i j}\right)-1}\left(1-y_{i j}\right)^{\left(1-g_{\mu}^{-1}\left(\eta_{i j}\right)\right) g_{\phi}^{-1}\left(\tau_{i j}\right)-1}}{\Gamma\left(g_{\mu}^{-1}\left(\eta_{i j}\right) g_{\phi}^{-1}\left(\tau_{i j}\right)\right) \Gamma\left(\left(1-g_{\mu}^{-1}\left(\eta_{i j}\right)\right) g_{\phi}^{-1}\left(\tau_{i j}\right)\right)} .
\end{gathered}
$$

Sejam $p_{b}(\cdot)$ e $p_{u}(\cdot)$, respectivamente, as distribuições de $\boldsymbol{b}_{i}$ e $\boldsymbol{u}_{i}$, incorporando a integração dos efeitos aleatórios, as verossimilhanças condicional e marginal conjuntas sâo expostas nas Equações 
(4.6) e (4.7).

$$
\begin{gathered}
L(\boldsymbol{y} \mid \boldsymbol{\vartheta}, \boldsymbol{X}, \boldsymbol{Z}, \boldsymbol{W}, \boldsymbol{R}, \boldsymbol{S})= \\
\prod_{i=1}^{N} \prod_{j=1}^{n_{i}}\left[\iint L_{i j}\left(y_{i j} \mid \boldsymbol{\vartheta}, \boldsymbol{x}_{i j}, \boldsymbol{z}_{i j}, \boldsymbol{w}_{i j}, \boldsymbol{r}_{i j}, \boldsymbol{b}_{i}, \boldsymbol{u}_{i}, S_{i}\right) p_{b}\left(\boldsymbol{b}_{i}\right) p_{u}\left(\boldsymbol{u}_{i}\right) d \boldsymbol{b}_{i} d \boldsymbol{u}_{i}\right] \\
L(\boldsymbol{y} \mid \boldsymbol{\vartheta}, \boldsymbol{X}, \boldsymbol{Z}, \boldsymbol{W}, \boldsymbol{R})= \\
\prod_{i=1}^{N} \sum_{S_{i}=1}^{K} \pi_{i S_{i}} \prod_{j=1}^{n_{i}}\left[\iint L_{i j}\left(y_{i j} \mid \boldsymbol{\vartheta}, \boldsymbol{x}_{i j}, \boldsymbol{z}_{i j}, \boldsymbol{w}_{i j}, \boldsymbol{r}_{i j}, \boldsymbol{b}_{i}, \boldsymbol{u}_{i}, S_{i}\right) p_{b}\left(\boldsymbol{b}_{i}\right) p_{u}\left(\boldsymbol{u}_{i}\right) d \boldsymbol{b}_{i} d \boldsymbol{u}_{i}\right] .
\end{gathered}
$$

Da mesma forma como foi abordado no capítulo anterior e ao longo de todo esse trabalho, a abordagem bayesiana foi a opção escolhida para fazer inferência nos estudos de simulação e nas aplicações em dados reais. Como previamente descrito, essa escolha foi efetuada tendo por base os relatos e recomendações da literatura especializada, assim como as experências em trabalhos prévios.

\subsection{Distribuições a priori e a posteriori}

Inicialmente, para efetuar a inferência do modelo sob abordagem bayesiana considerando $\boldsymbol{b}_{i} \sim$ $N_{d}\left(\mathbf{0}, \boldsymbol{\Sigma}_{b}\right)$ e $\boldsymbol{u}_{i} \sim N_{d *}\left(\mathbf{0}, \boldsymbol{\Sigma}_{u}\right)$ é necessária a atribuição das distribuições a priori dos parâmetros que compõem o conjunto $\boldsymbol{\vartheta}$, as quais são denotadas por: $p_{\beta}\left(\boldsymbol{\beta}^{s}\right), p_{\delta}\left(\boldsymbol{\delta}^{s}\right), p_{\pi}\left(\boldsymbol{\pi}_{i}\right), p_{\Sigma_{b}}\left(\boldsymbol{\Sigma}_{b}\right), p_{\Sigma_{u}}\left(\boldsymbol{\Sigma}_{u}\right)$, $i=1, \ldots, N$ e $s=1, \ldots, K$.

A Tabela 4.1 apresenta indicações para efetuar essas escolhas com base no conteúdo apresentado sobre atribuição de distribuições a priori no capítulo de contextualizações (2.1.1). Porém, note que são sugestões e que a não aderência não prejudica necessariamente nos resultados, inclusive porque dependendo da pesquisa podem existir distribuições mais apropriadas, desta forma não há perda de generalidade se no modelo proposto e na metodologia para seu ajuste outras distribuições a priori são selecionadas.

\begin{tabular}{c|c|c}
\hline Parâmetro & Notação da distribuição a priori & Distribuição a priori \\
\hline $\boldsymbol{\beta}^{s}$ & $p_{\beta}\left(\boldsymbol{\beta}^{s}\right)$ & $N_{p}\left(\boldsymbol{\mu}_{\boldsymbol{\beta}^{s}}, a_{\beta} \boldsymbol{I}_{p}\right)$ \\
$\boldsymbol{\delta}^{s}$ & $p_{\delta}\left(\boldsymbol{\delta}^{s}\right)$ & $N_{q}\left(\boldsymbol{\mu}_{\boldsymbol{\delta}^{s}}, a_{\delta} \boldsymbol{I}_{q}\right)$ \\
$\boldsymbol{\pi}_{i}$ & $p_{\pi}\left(\boldsymbol{\pi}_{i}\right)$ & $\operatorname{Dir}(\boldsymbol{\alpha})$ \\
$\boldsymbol{\Sigma}_{b}$ & $p_{\Sigma_{b}}\left(\boldsymbol{\Sigma}_{b}\right)$ & $I W\left(\boldsymbol{\Psi}_{b}, \boldsymbol{C}_{b}\right)$ \\
$\boldsymbol{\Sigma}_{u}$ & $p_{\Sigma_{u}}\left(\boldsymbol{\Sigma}_{u}\right)$ & $I W\left(\mathbf{\Psi}_{u}, \boldsymbol{C}_{u}\right)$ \\
\hline
\end{tabular}

Tabela 4.1: Parâmetros e distribuições a priori indicadas para ajuste simultâneo do modelo de mistura beta linear com efeitos aleatórios para a média e precisão, $s=1, \ldots, K$ e $i=1, \ldots, N$.

Assim como no capítulo anterior, para não sobrecarregar a linguagem, tanto vetores de parâmetros quanto componentes de matrizes serão referenciados apenas com a palavra parâmetro e de maneira similar é convencionado em relação aos hiperparâmetros.

A definição dos valores dos hiperparâmetros $\boldsymbol{\mu}_{\boldsymbol{\beta}^{s}}, a_{\beta}, \boldsymbol{\mu}_{\boldsymbol{\delta}^{s}}, a_{\delta}, \boldsymbol{\alpha}, \boldsymbol{\Psi}_{b}, \boldsymbol{C}_{b}, \boldsymbol{\Psi}_{u}$ e $\boldsymbol{C}_{u}$, podem ser efetuada de forma a obter distribuições a priori não informativas ou distribuições a priori que 
agregam informações prévias, podendo considerar também modelos hierárquicos, os quais atribuem distribuições para os hiperparâmetros. Exemplos de valores de hiperparâmetros e seus respectivos desempenhos estão disponíveis nos estudos de simulação.

Sejam $L(\boldsymbol{y} \mid \boldsymbol{\vartheta}, \boldsymbol{X}, \boldsymbol{Z}, \boldsymbol{W}, \boldsymbol{R}, \boldsymbol{b}, \boldsymbol{u})$ a função de verossimilhança $(4.4), p_{b}(\boldsymbol{b} \mid \boldsymbol{\vartheta})$ e $p_{u}(\boldsymbol{u} \mid \boldsymbol{\vartheta})$ as funções densidade de probabilidade dos efeitos aleatórios e $p(\boldsymbol{\vartheta})$ a distribuição a priori do conjunto de parâmetros, aplicando o teorema de Bayes (2.1), a distribuição a posteriori de $\boldsymbol{\vartheta}, p(\boldsymbol{\vartheta}, \boldsymbol{b}, \boldsymbol{u} \mid \boldsymbol{y}, \boldsymbol{X}, \boldsymbol{Z}, \boldsymbol{W}, \boldsymbol{R})$, é especificada na Equação (4.8).

$$
\begin{aligned}
p(\boldsymbol{\vartheta}, \boldsymbol{b}, \boldsymbol{u} \mid \boldsymbol{y}, \boldsymbol{X}, \boldsymbol{Z}, \boldsymbol{W}, \boldsymbol{R}) & \propto L(\boldsymbol{y} \mid \boldsymbol{\vartheta}, \boldsymbol{X}, \boldsymbol{Z}, \boldsymbol{W}, \boldsymbol{R}, \boldsymbol{b}, \boldsymbol{u}) p_{b}(\boldsymbol{b} \mid \boldsymbol{\vartheta}) p_{u}(\boldsymbol{u} \mid \boldsymbol{\vartheta}) p(\boldsymbol{\vartheta}) \\
& =p_{b}\left(\boldsymbol{b} \mid \boldsymbol{\Sigma}_{b}\right) p_{\Sigma_{b}}\left(\boldsymbol{\Sigma}_{b}\right) p_{u}\left(\boldsymbol{u} \mid \boldsymbol{\Sigma}_{u}\right) p_{\Sigma_{u}}\left(\boldsymbol{\Sigma}_{u}\right) \prod_{i=1}^{N} \sum_{s=1}^{K} \pi_{i s} p_{\pi}\left(\boldsymbol{\pi}_{i}\right) p_{\beta}\left(\boldsymbol{\beta}^{s}\right) \\
& \times p_{\delta}\left(\boldsymbol{\delta}^{s}\right) \prod_{j=1}^{n_{i}}\left[L_{i j}\left(y_{i j} \mid \boldsymbol{\vartheta}, \boldsymbol{x}_{i j}, \boldsymbol{z}_{i j}, \boldsymbol{w}_{i j}, \boldsymbol{r}_{i j}, \boldsymbol{b}_{i}, \boldsymbol{u}_{i}, s\right)\right]
\end{aligned}
$$

\subsection{Estudos de simulação}

Esta seção tem como objetivo apresentar estudos de simulação planejados de forma a explorar a performance da inferência do ajuste do modelo (4.1) considerando um caso particular com duas componentes de mistura. Os aspectos analisados são:

1. precisão das estimativas pontuais obtidas pela média e mediana da distribuição a posteriori;

2. performance das medidas de seleção de modelos para determinação do número de componentes da mistura e,

3. averiguação de falhas de identificabilidade.

Considere o modelo a seguir.

$$
\begin{array}{r}
\eta_{i j}=\log \left(\frac{\mu_{i j}}{1-\mu_{i j}}\right)=\beta_{1}^{S_{i}}+\beta_{2}^{S_{i}} x_{i j 2}+b_{i 1} \\
\tau_{i j}=\log \left(\phi_{i j}\right)=\delta_{1}+\delta_{2} w_{i j 2},
\end{array}
$$

em que $i=1,2, \ldots, N, j=1,2, \ldots, n_{i}, S_{i} \in\{1, \ldots, K\}, x_{i j 2} \sim \operatorname{Unif}(0,1)$ e $w_{i j 2} \sim \operatorname{Unif}(0,1)$.

Inicialmente, nesta introdução, são descritos os passos efetuados para definir os cenários estudados e as distribuições a priori dos parâmetros e seus hiperparâmetros. Posteriormente, nas Subseções 4.4.1, 4.4.2 e 4.4.3 são esclarecidos os preceitos e expostos os resultados dos estudos de simulação delineados para os aspectos enumerados previamente.

Com o intuito de definir os valores dos parâmetros dos cenários abordados nos estudos de simulação com réplicas foi efetuada uma análise exploratória do diagnóstico de convergência e do desempenho da estimação de parâmetros de modelos com valores: $N=100,400, n_{i}=5, K=2$, $\boldsymbol{\pi}_{i}^{\top}=(0,7 ; 0,3), \Sigma_{b}=\sigma_{b}=1, \boldsymbol{\delta}^{\top}=(3 ; 1,5),(2 ; 2),(4 ; 0,5), n_{i t}=30000,50000,100000, n_{b}=$ $10000,20000,50000, n_{t}=5,10$ e $n_{c}=1$. Essa análise inicial é necessária para selecionar cenários de interesse pois o custo computacional da execução dos algoritmos é alto, o que torna inviável explorar muitos cenários. 
O diagnóstico de convergência nessa primeira análise foi efetuado por meio da análise dos gráficos da trajetória das cadeias, de autocorrelação, da mediana ao longo das iterações e histograma das amostras resultantes e, das estatísticas de Geweke (Geweke, 1992), de Gelman-Rubin (Gelman e Rubin, 1992) e de Raftery-Lewis (Raftery e Lewis, 1992) em virtude da não existência de réplicas. Após a verificação da convergência, avaliou-se os gráficos da amostra resultante, os intervalos de credibilidade e as estimativas pontuais para então estabelecer os valores.

Sejam $\pi_{i s}=P\left(S_{i}=s\right), s=1,2$, a probabilidade da $i$-ésima unidade amostral estar associada ao $s$-ésimo componente da mistura, $S_{1}, S_{2}, \ldots, S_{N}$ são variáveis aleatórias independentes Assim, sendo $\boldsymbol{\pi}_{i}^{\top}=\left(\pi_{i 1} ; \pi_{i 2}\right) \sim \operatorname{Dir}(\boldsymbol{\alpha})$, estabeleceu-se $\boldsymbol{\pi}_{i}^{\top}=(0,7 ; 0,3)$ e foi efetuado um estudo prévio para valores de $\boldsymbol{\alpha}$ iguais a $(160 ; 40),(50 ; 50)$ e $\boldsymbol{\alpha} \sim \operatorname{Gama}(1 ; 0,001)$. Então, à título dos estudos com réplicas, optou-se por utilizar $\boldsymbol{\alpha}=(160 ; 40)$.

\begin{tabular}{c|c}
\hline Cenário & $\left\{\boldsymbol{\beta}^{1} ; \boldsymbol{\beta}^{2}\right\}$ \\
\hline 1 & $\{(-0,75 ; 2) ;(-1,5 ; 0,5)\}$ \\
2 & $\{(-1,5 ; 1) ;(-0,5 ; 3)\}$ \\
3 & $\{(-2 ; 0,5) ;(-0,5 ; 3)\}$ \\
4 & $\{(-1 ; 3) ;(-3 ; 1)\}$ \\
\hline
\end{tabular}

Tabela 4.2: Definição dos cenários estudados por simulação para os parâmetros $\boldsymbol{\beta}^{s}, s=1,2$.

\begin{tabular}{c|c}
\hline Cenário & $\left\{\boldsymbol{\delta}^{\mid}\right\}$ \\
\hline 1 & $\{(3 ; 1.5)\}$ \\
2 & $\{(4 ; 0.5)\}$ \\
3 & $\{(2 ; 2)\}$ \\
\hline
\end{tabular}

Tabela 4.3: Definição dos cenários estudados por simulação para os parâmetros $\boldsymbol{\delta}$.
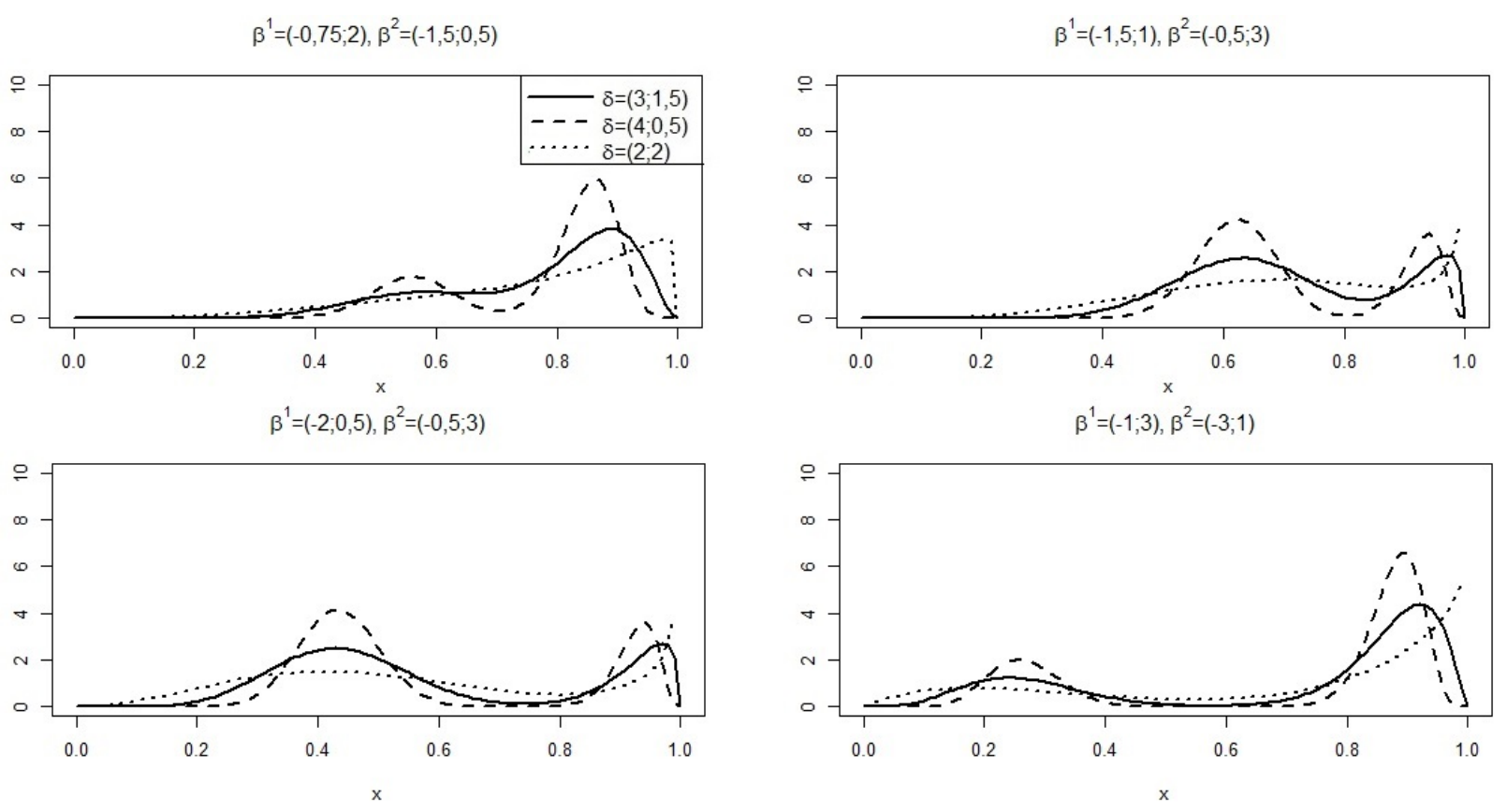

Figura 4.1: Comparação entre as densidades da distribuição beta para diferentes valores dos parâmetros $\beta$ e $\delta$ nos cenários englobados pelos estudos de simulação.

As Tabelas 4.2 e 4.3 contêm os valores dos parâmetros $\boldsymbol{\beta}^{s}, s=1,2$ e $\boldsymbol{\delta}$ analisados neste estudo 
de simulação. As funções densidade de probabilidade geradas com esses valores estão esboçadas na Figura 4.1.

Nesses gráficos observa-se que os cenários (de $\boldsymbol{\delta}$ ) 1 e 2 apresentam bimodalidade, sendo o segundo o que concentra mais massa de probabilidade em torno das modas. O cenário 3 de $\boldsymbol{\delta}$ gerou densidades com fraca ou nenhuma distinção entre os componentes da mistura para todos os cenários de $\boldsymbol{\beta}^{s}, s=1,2$, característica que pode interferir na qualidade da inferência dos modelos, mas sobre a qual também há interesse em ser analisada.

Diferentemente do hiperparâmetro $\boldsymbol{\mu}_{\delta}$, que foi considerado igual a $\mathbf{0}$, os estudos efetuados na Seção 3.4 indicaram que ao atribuir à distribuição a priori uma distribuição normal não informativa $\left(N_{2}\left(\mathbf{0}, \alpha_{\beta} \boldsymbol{I}_{2}\right), \alpha_{\beta} \in\{10,20\}\right)$ para $\boldsymbol{\beta}^{s}, s=1,2$, os resultados não justificam o aumento substancial no custo computacional comparado com a distribuição a priori informativa $N_{2}\left(\boldsymbol{\mu}_{\boldsymbol{\beta}^{s}}, 10 \boldsymbol{I}_{2}\right)$. Portanto, os valores de $\boldsymbol{\mu}_{\boldsymbol{\beta}^{s}}$ foram definidos em dois passos:

1. categorizar as unidades amostrais em dois grupos por meio do método de agrupamento Kmédias (K-means) para dados longitudinais implementado no pacote kml (Genolini et al. , 2015) do software $R$;

2. ajustar um modelo beta misto (2.18) para cada um dos dois grupos compostos no passo 1 através do pacote gamlss do software R, obtendo suas respectivas estimativas dos coeficientes dos efeitos fixos $\widehat{\boldsymbol{\beta}}^{1}, \widehat{\boldsymbol{\beta}}^{2}$;

3. por fim, estabelecer $\boldsymbol{\mu}_{\boldsymbol{\beta}^{s}}=\widehat{\boldsymbol{\beta}}^{s}, s=1,2$.

Da mesma forma que anteriormente efetuado, para o parâmetro $\boldsymbol{\Sigma}_{b}$, nesse caso denotado por $\sigma_{b}$ pois $d=1$, foram estudadas distribuições a priori $\operatorname{Gama}(\epsilon ; \epsilon), \epsilon \in\{0,05 ; 0,1 ; 0,3 ; 0,5 ; 1 ; 3 ; 5 ; 7\}$, para $\psi=\frac{1}{\sigma_{b}}$ e optou-se por $\epsilon=1$. Essa quantidade de opções foram testadas para minimizar a subestimação de $\sigma_{b}$ que é frequentemente observada na literatura (Gelman, 2006) e suas respectivas funções densidade de probabilidade encontram-se graficadas na Figura 3.2.

\subsubsection{Acurácia das estimativas pontuais a posteriori}

Os $R=50$ bancos de dados compostos por observações de $N=100$ grupos com $n=5$ observações por grupo foram gerados do modelo (4.9). Este número de réplicas simuladas demanda que a análise dos estudos seja efetuada com relação às estimativas pontuais dos parâmetros, ainda que se tenha informação de uma amostra significante da distribuição a posteriori. Ambas estimativas da média e da mediana amostrais a posteriori foram calculadas e analisadas, todavia, apenas os resultados da mediana serão apresentados e comentados pois os valores obtidos foram extremamente semelhantes.

Erro relativo médio (ER), erro absoluto relativo médio (EAR) e raíz do erro quadrático médio (REQM) são as medidas escolhidas para avaliar a acurácia dessas estimativas pontuais. Adicionalmente, foi calculada a porcentagem das réplicas cujo verdadeiro valor dos parâmetros está contido nos seus respectivos intervalos de credibilidade de 95\%, também denominada probabilidade de cobertura frequentista.

A estimação bayesiana via algoritmos MCMC foi efetuada com $n_{i t}=50000$ iterações, $n_{b}=10000$ iterações de aquecimento, $n_{t}=10$ iterações de intervalos e $n_{c}=1$ cadeia, totalizando amostras de tamanho 4000 da distribuição a posteriori de cada conjunto de dados. 
Os resultados do estudo planejado para os cenários das Tabelas 4.2 e 4.3 encontram-se nas tabelas do Apêndice A.4 e nos gráficos das estimativas pontuais dos parâmetros ao longo das réplicas dispostos no Apêndice B.7.

Assim como nos estudos de simulação do capítulo anterior, na totalidade das réplicas os verdadeiros valores dos parâmetros estavam inclusos nos seus respectivos intervalos de credibilidade de 95\%, resultado esse muito satisfatório dada a complexidade dos modelos de regressão em estudo.

$\mathrm{Na}$ estimação pontual por meio da mediana a posteriori, foi observada alta acurácia e leve tendência de superestimação para o parâmetro $\pi_{1}$. Nos cenários 1 e 2 a estimação de $\sigma_{b}$ foi eficiente e precisa, enquanto para o cenáro 4 houve superestimação.

Com relação aos coeficientes fixos do modelo para a média $(\mu)$, o cenário 1 foi o único que apresentou casos de confundimento dos componentes da mistura, fato esse observado na densidade esboçada na Figura 4.1. Nos outros cenários observou-se a correta distinção entre os elementos da mistura e estimativas precisas para os parâmetros do primeiro componente $\left(\beta_{1}^{1} \mathrm{e} \beta_{2}^{1}\right)$. Porém, exceto pelo cenário 2, o desempenho da mediana a posteriori na estimação pontual de $\beta_{1}^{2}$ e $\beta_{2}^{2}$ não foi tão satisfatório por apresentar viés.

Quanto aos coeficientes fixos do modelo para a precisão $(\phi)$, todos os cenários apresentaram bons resultados para o parâmetro $\delta_{1}$, sendo o cenário 1 o que apresentou maior precisão. Com relação às estimativas do parâmetro $\delta_{2}$, o cenário 1 apresentou boa performance enquanto os resultados para os cenários 2 e 3 apresentaram tendências de superestimação e subestimação, respectivamente.

Logo, os resultados observados foram bastante satisfatórios para $\pi_{1}$ e $\sigma_{b}$ e, no geral, a acurácia das estimativas pontuais dos outros parâmetros é maior nos cenários que se tem maior distinção das curvas dos componentes da mistura, como esperado.

\subsubsection{Performance dos critérios de seleção de modelos}

Com o intuito de analisar a performance de critérios de informação para seleção do número de componentes da mistura e de seus respectivos modelos ajustados efetuou-se um estudo de simulação. Os critérios escolhidos para serem analisados são EAIC, ECAIC, EBIC, $D I C_{3}$ e LPML, pelos motivos expostos na Subseção 2.1.3, na qual também encontram-se suas definições.

Para tal análise, a técnica considerada foi gerar bancos de dados de acordo com diferentes valores de parâmetros e um determinado número de componentes da mistura fixo $(K=2)$ e a esses dados ajustar os modelos em estudo com diferentes valores de $K$. Após isso, calcula-se os valores dos critérios de informação para os modelos ajustados e contabiliza-se a proporção de réplicas que cada critério, por si só, seleciona o modelo com o verdadeiro valor de $K$ para cada cenário.

O número de bancos de dados (réplicas) gerados para cada cenário foi $R=10$ em virtude da complexidade computacional e a estes dados foram ajustados os modelos (4.9) com $K=1,2, \ldots, 5$ componentes na mistura.

Considerou-se os valores dos parâmetros $\boldsymbol{\beta}^{s}, s=1, \ldots, K$, apresentados na Tabela 4.2 e $\boldsymbol{\delta}$ definidos na Tabela 4.3, tal qual estabelecido na introdução desta seção (4.4), com suas respectivas distribuições a priori e métodos de estimação. Nesse caso, utilizou-se $N=100$ grupos com $n=5$ observações por grupo, $n_{i t}=30000, n_{b}=10000, n_{t}=10$ e $n_{c}=1$, totalizando 2000 amostras da distribuição a posteriori.

Como os dados foram gerados com $K=2$, quanto maior a porcentagem para este valor, melhor é o desempenho do critério para seleção dos modelos; se o critério na maioria das réplicas seleciona 
valores mais altos de $K$, isso é um indício de que este critério superestima o número de componentes quando utilizado como único método de seleção do modelo, o que pode ocasionar problemas inferenciais e interpretações errôneas. Os resultados encontram-se na Tabela 4.4.

\begin{tabular}{|c|c|c|c|c|c|c|c|c|c|c|c|}
\hline \multirow[b]{3}{*}{ Critério } & \multirow[b]{3}{*}{$K$} & \multicolumn{3}{|c|}{ Cenário 1} & \multicolumn{2}{|c|}{ Cenário 2} & \multicolumn{2}{|c|}{ Cenário 3} & \multicolumn{3}{|c|}{ Cenário 4} \\
\hline & & \multicolumn{10}{|c|}{ Cenário $\delta$} \\
\hline & & 1 & 2 & 3 & 1 & 2 & 1 & 2 & 1 & 2 & 3 \\
\hline \multirow[t]{5}{*}{ EAIC } & 1 & 0 & 0 & 0 & 0 & 0 & 0 & 0 & 0 & 0 & 0 \\
\hline & 2 & 20 & 10 & 30 & 0 & 10 & 0 & 0 & 0 & 0 & 0 \\
\hline & 3 & 40 & 20 & 70 & 20 & 0 & 0 & 0 & 0 & 0 & 10 \\
\hline & 4 & 30 & 70 & 0 & 50 & 60 & 40 & 50 & 30 & 40 & 60 \\
\hline & 5 & 10 & 0 & 0 & 30 & 30 & 60 & 50 & 70 & 60 & 30 \\
\hline \multirow[t]{5}{*}{ ECAIC } & 1 & 0 & 10 & 0 & 10 & 10 & 10 & 0 & 0 & 10 & 10 \\
\hline & 2 & 20 & 10 & 30 & 0 & 10 & 0 & 0 & 0 & 0 & 0 \\
\hline & 3 & 40 & 10 & 70 & 20 & 0 & 0 & 0 & 10 & 0 & 10 \\
\hline & 4 & 30 & 70 & 0 & 40 & 50 & 30 & 50 & 20 & 40 & 50 \\
\hline & 5 & 10 & 0 & 0 & 30 & 30 & 60 & 50 & 70 & 50 & 30 \\
\hline \multirow[t]{5}{*}{ EBIC } & 1 & 0 & 0 & 30 & 0 & 0 & 0 & 0 & 30 & 0 & 0 \\
\hline & 2 & 30 & 20 & 40 & 0 & 10 & 0 & 0 & 0 & 0 & 20 \\
\hline & 3 & 70 & 20 & 30 & 40 & 0 & 20 & 0 & 0 & 10 & 30 \\
\hline & 4 & 0 & 60 & 0 & 30 & 80 & 70 & 60 & 30 & 40 & 40 \\
\hline & 5 & 0 & 0 & 0 & 30 & 10 & 10 & 40 & 40 & 50 & 10 \\
\hline \multirow[t]{5}{*}{$\mathrm{DIC}_{3}$} & 1 & 0 & 0 & 20 & 0 & 0 & 0 & 0 & 70 & 0 & 0 \\
\hline & 2 & 20 & 20 & 30 & 0 & 0 & 0 & 0 & 0 & 30 & 80 \\
\hline & 3 & 20 & 10 & 0 & 40 & 0 & 0 & 0 & 0 & 0 & 10 \\
\hline & 4 & 30 & 40 & 40 & 10 & 40 & 30 & 20 & 0 & 30 & 10 \\
\hline & 5 & 30 & 30 & 10 & 50 & 60 & 70 & 80 & 30 & 40 & 0 \\
\hline \multirow[t]{5}{*}{$L P M L$} & 1 & 0 & 0 & 0 & 0 & 0 & 0 & 0 & 0 & 0 & 0 \\
\hline & 2 & 0 & 0 & 0 & 0 & 0 & 0 & 0 & 0 & 0 & 0 \\
\hline & 3 & 40 & 30 & 20 & 0 & 0 & 0 & 0 & 0 & 0 & 0 \\
\hline & 4 & 30 & 60 & 30 & 60 & 60 & 50 & 30 & 0 & 10 & 40 \\
\hline & 5 & 30 & 10 & 50 & 40 & 40 & 50 & 70 & 100 & 90 & 60 \\
\hline
\end{tabular}

Tabela 4.4: Porcentagem das 10 réplicas simuladas do modelo de mistura beta linear com efeitos aleatórios para a média e precisão cujos critérios EAIC, ECAIC, EBIC, DIC $C_{3}$ e LPML selecionaram cada um dos 5 possiveis valores de números de componentes da mistura $(K)$.

Claramente nota-se tendência de superestimação de $K$ para todos os critérios em estudo, sendo que os resultados mais satisfatórios destes foram observados para o cenário 1 de $\boldsymbol{\beta}$. Por pouca diferença de performance, o EBIC e o $D I C_{3}$ foram os critérios que mais vezes indicaram a escolha do modelo que gerou os dados, sendo que, por definição, o EBIC tem maior penalização para o número de parâmetros.

Diferentemente dos resultados observados nos estudos de simulação para o modelo com o parâmetro de precisão $(\phi)$ constante, os resultados não são satisfatórios para serem considerados como opção de seleção automática do número de componentes da mistura, sendo necessários, adicional- 
mente, análises descritivas e conhecimento sobre a pesquisa em análise.

\subsubsection{Diagnóstico de identificabilidade}

Tal como previamente mencionado, estudar a identificabilidade desses modelos é importante e ao mesmo tempo muito complexo tanto pela não trivialidade de se averiguar identificabilidade, em geral, quanto pela complexidade do modelo. Uma metodologia parcimoniosa para esse caso é o data cloning pelas considerações apresentadas na Seção 2.7 .

Considere os modelos de mistura beta mistos com dispersão variável com valores dos parâmetros $\boldsymbol{\beta}^{s}, s=1, \ldots, K$, e $\boldsymbol{\delta}$ respectivamente apresentados nas Tabelas 4.2 e $4.3, K=2, \pi_{1}=0,7, N=100$ e $n=5$. Na introdução desta seção (4.4) estão especificados as distribuições a priori e métodos de estimação.

Os números de clones selecionados foram os que obtiveram convergência adequada das cadeias MCMC no algoritmo de acordo com o critério de Gelman \& Rubin, assim como recomendado em Sólymos (2010).

Após a execução do algoritmo foi obtido o maior autovalor padronizado da matriz de variâncias e covariâncias a posteriori dos parâmetros para cada banco de dados construído de acordo com um determinado número de clones. Esses resultados encontram-se na Figura 4.2.

De acordo com o método, a presença de decrescimento desses autovalores para o valor zero com o aumento do número de clones e a similaridade dessa convergência com a taxa de convergência do inverso do número de clones são evidências de que não há falhas de identificabilidade no caso em estudo.
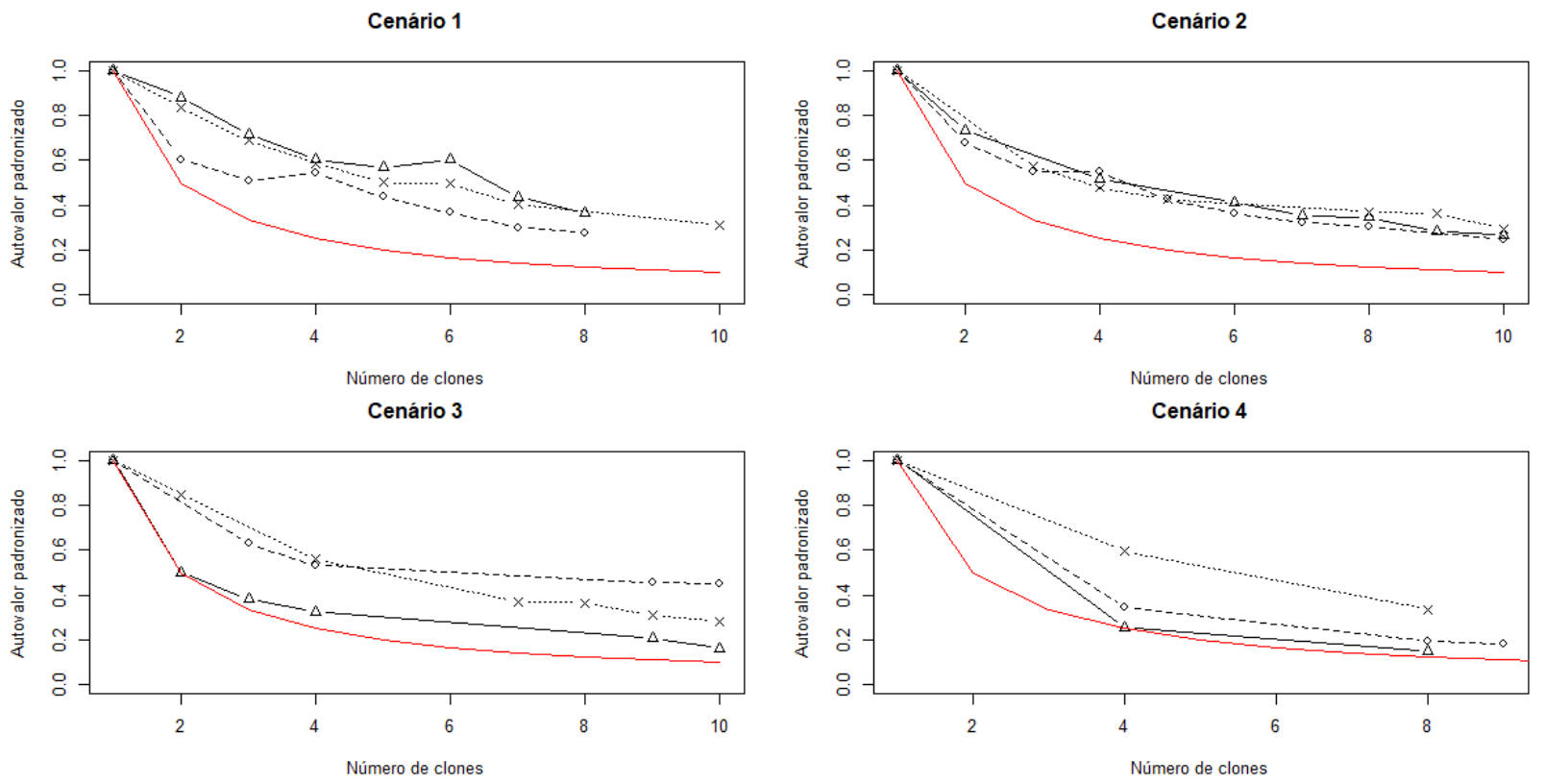

Figura 4.2: Gráfico de linhas do inverso do número de clones (traço vermelho) e do maior autovalor padronizado da matriz de variâncias e covariâncias a posteriori dos parâmetros do modelo de mistura beta misto linear com dispersão variável com cenários $1,2,3,4$ para os coeficientes fixos do modelo para a média e cenários $1(\triangle), 2(\bigcirc)$ e $3(\times)$ para os coeficientes fixos do modelo para o parâmetro de precisão.

Os resultados obtidos são concordantes com o que foi previamente apresentado nos gráficos das densidades (Figura 4.1) e observado nos estudos de simulação da acurácia da estimação pontual (Subseção 4.4.1). 
O primeiro gráfico da Figura 4.2 indica que pode haver falhas de identificabilidade para o respectivo cenário pois, respectivamente, nos cenários 1 e 2 de $\boldsymbol{\delta}$ o autovalor não seguiu a tendência de decrescimento. Essas conclusões são condinzentes com os casos de confundimento entre os componentes da mistura expostos nas Figuras B.67, B.68 e B.69.

Nos outros três gráficos é observado decrescimento em todos os casos, exceto pelo autovalor de $n_{\text {clone }}=4$ do cenário 2 do segundo gráfico. O perfil do decrescimento das curvas foi semelhante à forma do declínio da função inversa do número de clones e juntamente com o fato de que em nenhuma das cinquenta réplicas foi observado confundimento entre os componentes da mistura nos estudos de simulação da Subseção 4.4.1, pode-se dizer que não há evidências de falhas de identificabilidade.

Dessas análises conclui-se que, exceto para $\left\{\boldsymbol{\beta}^{1^{\top}} ; \boldsymbol{\beta}^{2^{\top}}\right\}=\{(-0,75 ; 2) ;(-1,5 ; 0,5)\}$ (cenário 1 ), sob as condições analisados não há evidências de falta de identificabilidade dos modelos simulados. $\mathrm{O}$ que reforça a ideia de que a identificabilidade dos modelos estudados neste capítulo está diretamente vinculada à aplicação, pois quanto mais distinguíveis são as densidades estimadas dos componentes da mistura do modelo ajustado mais evidências o data cloning apresenta sobre a inexistência de falhas de identificabilidade no modelo. Por conseguinte, é indicado que estudo análogo ao descrito nesta subseção seja efetuado quando se deseja aplicar este modelo.

\subsection{Aplicação a dados de pacientes diagnosticados com periodontite}

O propósito dessa aplicação é estudar se há ganhos em incorporar o ajuste de um modelo de regressão para o parâmetro de precisão na análise dos dados de periodontite desenvolvida na Subseção 3.5.1 do capítulo anterior e, em caso positivo, obter um modelo mais útil para estudo dos pesquisadores da área.

Por se tratar de um problema já abordado neste trabalho, a contextualização do estudo não será novamente apresentada já que ela encontra-se disponível na Subseção 3.5.1.

Inicialmente, foi ajustado o modelo apresentado no presente capítulo (4.1) com todas as variáveis explicativas previamente selecionadas pelos especialistas (sexo, idade, raça, imc, $H D L, L D L, P C R$, $P B>4 m m$, mes.3, mes.6 e mes.12) e um efeito aleatório $\left(d=d^{*}=1\right)$. Então, com base na viabilidade dos ajustes, na análise dos resultados inferenciais, da análise de diagnóstico, dentre outros aspectos do ajuste com $K=1$, serão analisados os modelos de mistura sem as variáveis sexo, idade, raça e imc.

Considerando:

- $Y_{i j} \mid b_{i}, u_{i} \sim \sum_{S_{i}=1}^{K} \pi_{i S_{i}} \operatorname{Beta}\left(g_{\mu}^{-1}\left(\eta_{S_{i}, i j}\right), g_{\phi}^{-1}\left(\tau_{S_{i}, i j}\right)\right)$;

- $\mu_{S_{i}, i j}=E\left(Y_{i j} \mid b_{i}, u_{i}, S_{i}\right)$ e,

- $\phi_{S_{i}, i j}=\frac{\mu_{i j}\left(1-\mu_{i j}\right)}{\operatorname{Var}\left(Y_{i j} \mid b_{i}, u_{i}, S_{i}\right)}-1$, 
para $i=1, \ldots, 40$ e $j=1, \ldots, 4$, o modelo resultante é expresso na Equação (4.10).

$$
\begin{array}{r}
\eta_{S_{i}, i j}=g_{\mu}\left(\mu_{S_{i}, i j}\right)=\beta_{0}^{S_{i}}+\beta_{1}^{S_{i}} m e s .3_{i j}+\beta_{2}^{S_{i}} m e s .6_{i j}+\beta_{3}^{S_{i}} m e s .12_{i j}+\beta_{4}^{S_{i}} H D L_{i j}+\beta_{5}^{S_{i}} L D L_{i j}+b_{i} \\
\tau_{S_{i}, i j}=g_{\phi}\left(\phi_{S_{i}, i j}\right)=\delta_{0}^{S_{i}}+\delta_{1}^{S_{i}} H D L_{i j}+u_{i}, \\
\boldsymbol{b}_{i} \mid \boldsymbol{\Sigma}_{b} \stackrel{i i d}{\sim} N_{d}\left(\mathbf{0}, \boldsymbol{\Sigma}_{b}\right), \\
\boldsymbol{u}_{i} \mid \boldsymbol{\Sigma}_{u} \stackrel{i i d}{\sim} N_{d^{*}}\left(\mathbf{0}, \boldsymbol{\Sigma}_{u}\right) .
\end{array}
$$

em que $\boldsymbol{y}_{1}, \ldots, \boldsymbol{y}_{40}$ é a amostra composta pelas observações $\boldsymbol{y}_{i}=\left(y_{i 1}, \ldots, y_{i 4}\right)^{\top}$ da variável resposta tais que cada $i$-ésima $(i=1, \ldots, 40)$ unidade amostral é composta pelas suas $n_{i}=4$ aferições, $S_{i}$ é a variável indicadora discreta do componente da mistura associada ao $i$-ésimo grupo da amostra, $S_{i} \in\{1, \ldots, K\}$ e, $g_{\mu}$ e $g_{\phi}$ são, respectivamente, as funções de ligação logito e logarítmica.

Os ajustes dos modelos foram efetuados através do software OpenBUGS com $n_{i t}=50000$, $n_{b}=10000, n_{t}=10$ e $n_{c}=1$ e as distribuições a priori foram especificadas conforme as indicações da Seção $4.3 \operatorname{com} \alpha_{\beta}=\alpha_{\delta}=4$.

Os resultados inferenciais (mediana a posteriori, desvio padrão e intervalo de credibilidade de 95\%) do ajuste do modelo (3.11) para $K=1$ encontram-se na Tabela 4.5.

\begin{tabular}{c|c|c|c|c}
\hline Parâmetro & Covariável associada & Estimativa pontual & Desvio padrão & IC 95\% \\
\hline$\beta_{1}^{1}$ & - & $-1,255$ & 0,493 & $(-2,211 ;-0,274)$ \\
$\beta_{2}^{1}$ & mes.3 & $-0,162$ & 0,485 & $(-1,161 ; 0,778)$ \\
$\beta_{3}^{1}$ & mes.6 & $-0,289$ & 0,509 & $(-1,299 ; 0,707)$ \\
$\beta_{4}^{1}$ & mes.12 & $-0,669$ & 0,518 & $(-1,664 ; 0,335)$ \\
$\beta_{5}^{1}$ & HDL & $-0,209$ & 0,435 & $(-1,115 ; 0,608)$ \\
$\beta_{6}^{1}$ & LDL & $-0,452$ & 0,324 & $(-1,214 ; 0,009)$ \\
$\delta_{1}^{1}$ & - & $-3,204$ & 0,004 & $(-3,209 ;-3,199)$ \\
$\delta_{2}^{1}$ & HDL & $-0,231$ & 0,003 & $(-0,236 ;-0,226)$ \\
$\sigma_{b}$ & - & 0,650 & 0,108 & $(0,526 ; 0,834)$ \\
$\sigma_{u}$ & - & 1,782 & 0,763 & $(1,455 ; 2,267)$ \\
\hline
\end{tabular}

Tabela 4.5: Estimativas pontuais (mediana a posteriori) dos parâmetros, desvios padrões e intervalos de credibilidade (IC) de $95 \%$ do modelo final com $K=1$ ajustado para a variável anti-oxLDL dos dados de periodontite.

Comparando esse ajuste com as informações inferenciais do ajuste do modelo beta misto com precisão constante (Tabela 3.4), observa-se que as estimativas pontuais dos coeficientes relacionados ao tempo pós-tratamento $\left(\beta_{2}^{1}, \beta_{3}^{1}\right.$ e $\left.\beta_{4}^{1}\right)$ possuem o mesmo decrescimento, que os coeficientes estimados das variáveis HDL e LDL $\left(\beta_{5}^{1}\right.$ e $\left.\beta_{6}^{1}\right)$ continuaram tendo intervalos de credibilidade com o valor zero incluído e que a estimativa pontual do desvio padrão do intercepto aleatório $\left(\sigma_{b}\right)$ foi muito semelhante, sendo que no modelo com dispersão variável o desvio padrão de $\sigma_{b}$ teve metade do desvio padrão do outro.

Em seguida, ajustou-se o modelo (4.10) para $K=2,3$ e no caso $K=3$ não foi possível gerar as amostras a posteriori dos parâmetros em função de erros de execução do algoritmo, sendo esse 
caso então desconsiderado por esses erros indicarem a inadequabilidade do modelo. Os valores dos critérios EAIC, ECAIC, EBIC, $D I C_{3}$ e LPML dos outros dois casos foram calculados e encontram-se na Tabela 4.6.

\begin{tabular}{c|cc}
\hline & \multicolumn{2}{|c}{$K$} \\
\hline Critério & 1 & 2 \\
\hline EAIC & $-1946,00$ & $-2116,93$ \\
ECAIC & $-1943,82$ & $-2110,04$ \\
EBIC & $-1915,50$ & $-2058,98$ \\
$D I C_{3}$ & $-15282,15$ & $-12747,63$ \\
LPML & $-41633,23$ & $-40661,67$ \\
\hline
\end{tabular}

Tabela 4.6: Valores dos critérios de informação EAIC, ECAIC, EBIC, DIC $C_{3}$ e LPML para o ajuste do modelo (4.10) com $K=1,2$ para os dados de periodontite.

Lembrando que nos estudos de simulação do desempenho desses critérios para tal finalidade (Subseção 4.4.2) não foi obtida performance geral satisfatória de nenhum dos critérios em isolado e foram observados dois casos em que o $D I C_{3}$ subestimou $K$. Dessa forma, considerando que dos cinco critérios analisados apenas um $\left(D I C_{3}\right)$ não selecionou $K=2$ e a análise da adequabilidade do ajuste em relação ao resultados inferenciais, o modelo de mistura beta misto linear com dispersão variável com $K=2$ foi o selecionado.

A Tabela 4.7 apresenta as informações relacionadas aos parâmetros do modelo de mistura beta misto linear com dispersão variável selecionado (3.11) e as funções densidade de probabilidade estimadas desses parâmetros a posteriori encontram-se nas Figuras 4.3 e 4.4 . 


\begin{tabular}{c|c|c|c|c}
\hline Parâmetro & Covariável associada & Estimativa pontual & Desvio padrão & IC 95\% \\
\hline$\beta_{1}^{1}$ & - & $-1,347$ & 0,491 & $(-2,292 ;-0,387)$ \\
$\beta_{2}^{1}$ & mes.3 & $-0,004$ & 0,495 & $(-1,015 ; 0,965)$ \\
$\beta_{3}^{1}$ & mes.6 & $-0,042$ & 0,492 & $(-1,004 ; 0,923)$ \\
$\beta_{4}^{1}$ & mes.12 & $-0,332$ & 0,503 & $(-1,340 ; 0,650)$ \\
$\beta_{5}^{1}$ & HDL & $-0,213$ & 0,438 & $(-1,091 ; 0,617)$ \\
$\beta_{6}^{1}$ & LDL & $-0,442$ & 0,317 & $(-1,205 ;-0,001)$ \\
$\delta_{1}^{1}$ & - & $-1,025$ & 0,001 & $(-1,030 ;-1,020)$ \\
$\delta_{2}^{1}$ & HDL & $-0,297$ & 0,001 & $(-0,302 ;-0,292)$ \\
$\beta_{1}^{2}$ & - & $-0,185$ & 0,489 & $(-1,107 ; 0,814)$ \\
$\beta_{2}^{2}$ & mes.3 & $-0,809$ & 0,506 & $(-1,801 ; 0,191)$ \\
$\beta_{3}^{2}$ & mes.6 & $-1,283$ & 0,501 & $(-2,309 ;-0,311)$ \\
$\beta_{4}^{2}$ & mes.12 & $-1,933$ & 0,506 & $(-2,909 ;-0,907)$ \\
$\beta_{5}^{2}$ & HDL & 0,382 & 0,424 & $(-0,449 ; 1,215)$ \\
$\beta_{6}^{2}$ & LDL & 0,591 & 0,298 & $(0,132 ; 1,271)$ \\
$\delta_{1}^{2}$ & - & $-3,247$ & 0,001 & $(-3,252 ;-3,242)$ \\
$\delta_{2}^{2}$ & HDL & $-0,211$ & 0,001 & $(-0,216 ;-0,206)$ \\
$\pi_{1}$ & - & 0,704 & 0,029 & $(0,643 ; 0,761)$ \\
$\sigma_{b}$ & - & 1,373 & 0,506 & $(1,096 ; 1,775)$ \\
$\sigma_{u}$ & - & 1,468 & 0,539 & $(1,187 ; 1,877)$ \\
\hline
\end{tabular}

Tabela 4.7: Estimativas pontuais (média a posteriori) dos parâmetros, desvios padrões e intervalos de credibilidade de $95 \%$ do modelo final ajustado para a variável anti-oxLDL dos dados de periodontite.
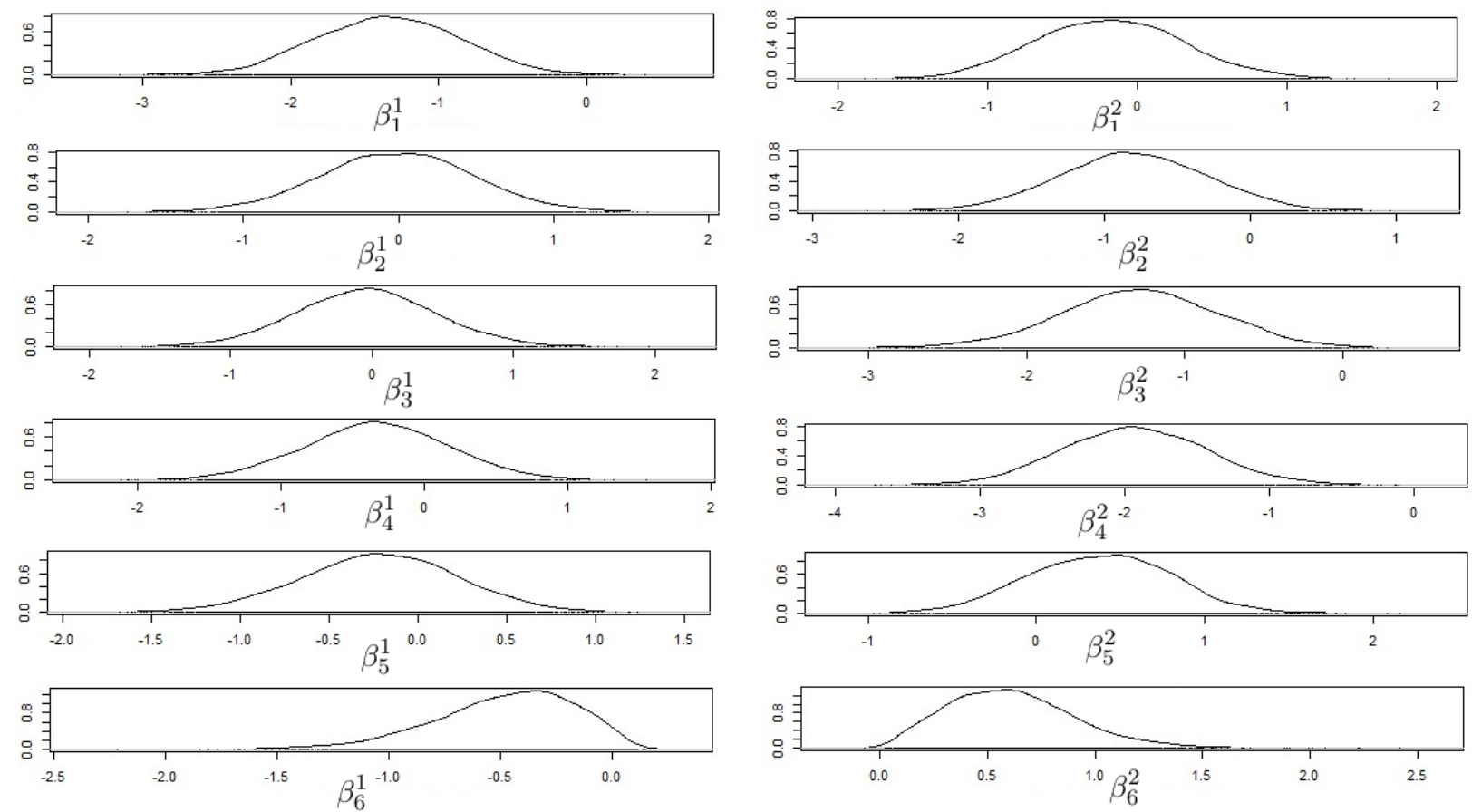

Figura 4.3: Gráfico da função densidade de probabilidade estimada dos parâmetros $\boldsymbol{\beta}^{\boldsymbol{s}}, s=1,2$, do modelo final ajustado para a variável anti-oxLDL dos dados de periodontite. 

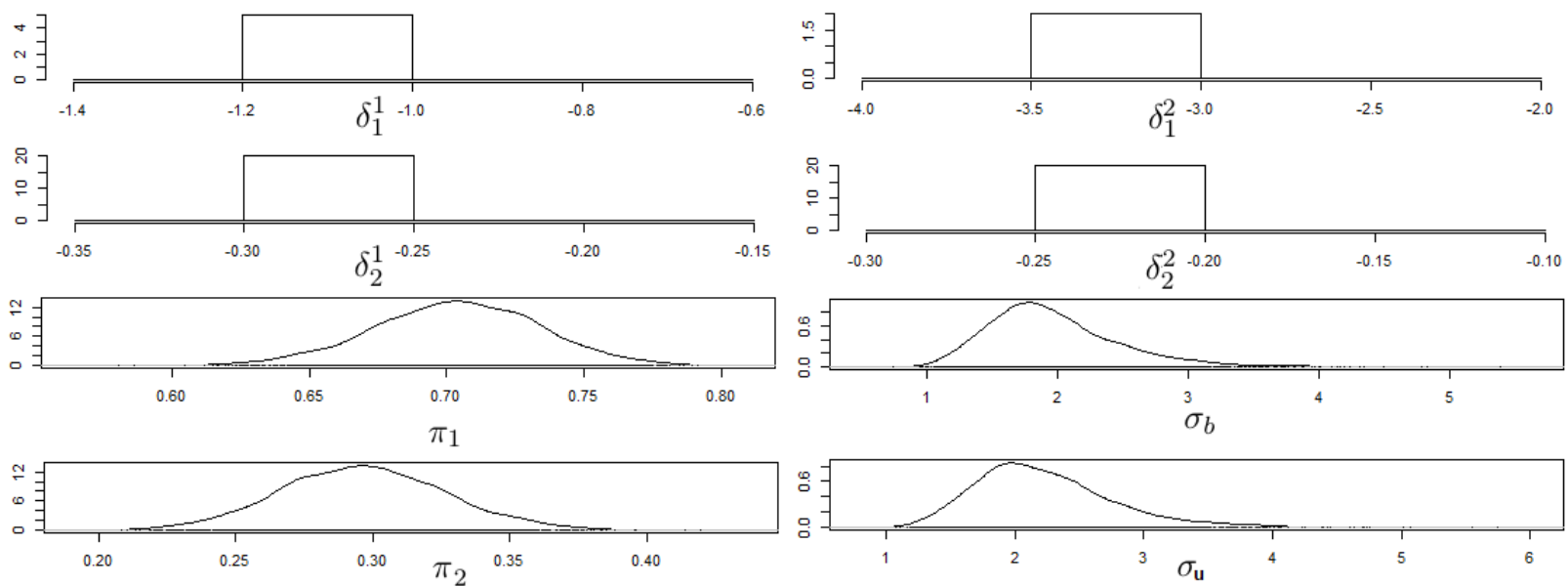

Figura 4.4: Gráfico da funçẫo densidade de probabilidade estimada dos parâmetros $\boldsymbol{\delta}^{\boldsymbol{s}}, s=1,2, \pi_{1}, \pi_{2}, \sigma_{b}$ e $\sigma_{u}$ do modelo final ajustado para a variável anti-oxL $\boldsymbol{D L}$ dos dados de periodontite.

Assim como explicado na Subseção 2.1.3, a avaliação preditiva a posteriori averigua a adequabilidade de modelos ajustados analisando quão consistentes são estes modelos com os dados observados compararando as observações da amostra observada com amostras simuladas da distribuição preditiva a posteriori (dados replicados) em relação a uma determinada medida de discrepância. Para essa aplicação a análise preditiva a posteriori foi efetuada considerando como medidas de discrepância a média e o desvio (deviance) e os respectivos p-valores preditivos a posteriori resultantes são: 0,553 e 0,438. Segundo o método, quanto mais próximo de 0,5 são esses p-valores preditivos mais condinzentes aos dados observados são as especificações do modelo, do que se conclui que o modelo em análise é muito adequado aos dados com relação às medidas de discrepância média e desvio.

Os resíduos quantílicos aleatorizados (normalizados) apresentados em Dunn e Smyth (1996) são úteis para verificação da adequabilidade de ajustes pois, de acordo com a sua definição, eles são normalmente distribuídos quando as especificações do modelo são satisfeitas. A Figura 4.5 exibe o gráfico desses resíduos dessa aplicação. Exceto por quatro casos, os pontos estão próximos da reta indicando que adequabilidade do ajuste.

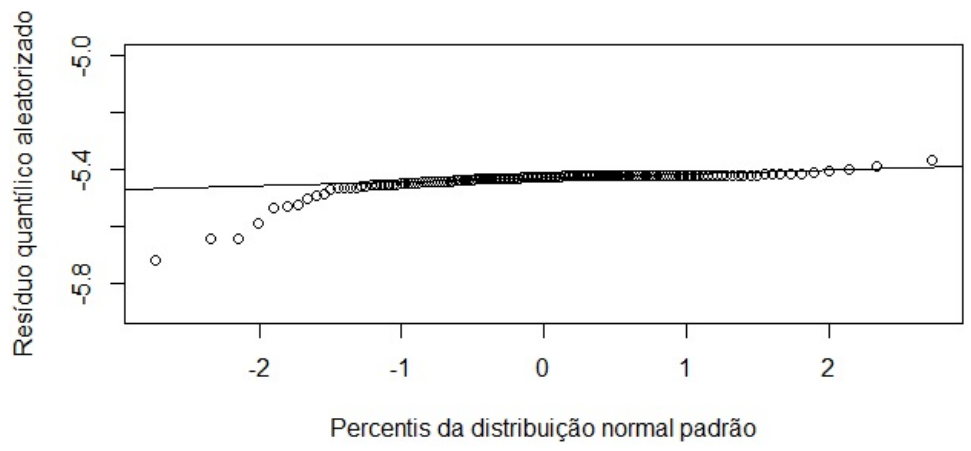

Figura 4.5: Gráfico de probabilidade normal dos resíduos quantílicos aleatorizados do modelo final ajustado aos dados de periodontite.

Analisando os resultados inferenciais da Tabela 4.7 e os gráficos das Figuras 4.3 e 4.4 conclui-se que as estimativas pontuais dos coeficientes relacionados às variáveis indicadoras de tempo póstratamento são negativas e quanto maior esse tempo maiores são os valores absolutos desses coefici- 
entes, ou seja, em média, o valor de anti-oxLDL (variável resposta) decresce a medida que o tempo pós-tratamento aumenta. Nota-se também que os valores absolutos desses coeficientes estimados são maiores no segundo componente da mistura $\left(\beta_{2}^{2}, \beta_{3}^{2}\right.$ e $\left.\beta_{4}^{2}\right)$.

O parâmetro $\pi_{1}$ teve estimativa deveras semelhante ao do modelo com precisão constante. Neste, a mediana a posteriori de $\sigma_{b}$ é metade do valor da mesma no modelo com dispersão variável.

O coeficiente associado à covariável LDL foi negativo no primeiro componente da mistura e positivo no segundo, indicando que a LDL oxidada média diminui em $s=1$ e aumenta em $s=2$ conforme o valor desta variável aumenta.

No modelo para ajuste do parâmetro de precisão as estimativas de $\delta_{2}^{1}$ e $\delta_{2}^{2}$ foram próximas e ambas negativas, apontando que a quanto maior o valor de HDL menor é o parâmetro de precisão. O desvio padrão do intercepto aleatório $\left(\sigma_{u}\right)$ tem intervalo de credibilidade de $95 \%$ igual a $(1,187 ; 1,877)$ e sua função densidade de probabilidade a posteriori é bastante semelhante à observada para sigma . $_{\text {. }}$

Portanto, retomando o propósito da aplicação de avaliar o risco de doenças cardiovasculares através da variável anti-oxLDL, a avaliação dos dados por meio do modelo apresentado neste capítulo, no geral, indica que o fator de risco de doenças cardiovasculares em pacientes com periodontite diminui com o passar do tempo pós-tratamento, o que indica também que o tratamento ocasiona melhoras ao longo de todo o primeiro ano que o sucede.

Devido às características da metodologia considerada neste trabalho para averiguação de identificabilidade recomenda-se que para cada aplicação em dados reais a análise de data cloning seja efetuada. Diferentemente das aplicações dos modelos lineares com precisão constante (Subseções 3.5.1 e 3.5.2) e de todos os estudos de simulação anteriores, neste caso o método não foi adequado pois não foi possível sua realização em função de erros na execução do algoritmo.

No software OpenBUGS a identificação e detalhamento dos erros de execução não são claros e, por isso, o que foi identificado foi uma falha na atualização dos parâmetros da distribuição beta. Isso pode ter ocorrido pois ao clonar os dados o algoritmo diminui a influência das distribuições a priori no processo e a atualização dos valores é efetuada de forma não coerente com o modelo ajustado, ocasionando erros nos cálculos das verossimilhanças. Dessa forma, o estudo de outras opções de métodos de averiguação de identificabilidade é uma proposta de trabalho futuro para analisar apropriadamente essa questão.

Por fim, ao comparar o ajuste dos modelos selecionados na Subseção 3.5.1 e nesta seção com base nos critérios de seleção considerados (respectivamente, Tabelas 3.5 e 4.6), tem-se que quatro dos cinco critérios (EAIC, ECAIC, EBIC e $D I C_{3}$ ) indicam que o modelo com dispersão variável é mais apropriado. Porém, nesse modelo não foi possível averiguar a identificabilidade do modelo, o que pode ser um agravante para a pesquisa. Dessa forma, recomenda-se que o grupo de pesquisadores avalie as questões apontadas e todo o restante do material apresentado para então efetuar a escolha que mais lhe convém.

\subsection{Considerações finais}

Inédito por agregar um modelo linear explicativo para o parâmetro de precisão aos modelos de mistura beta mistos lineares com dispersão constante, o modelo apresentado neste capítulo foi especificado e, por meio de estudos de simulação, sua inferência foi avaliada para alguns cenários sob a abordagem bayesiana. 
Os resultados desses estudos indicaram boa acurácia na estimação pontual dos parâmetros, principalmente quando se tem distinguibilidade razoável entre as densidades dos elementos da mistura. Ademais, também nessa condição, o algoritmo data cloning apresentou resultados que indicam que não há falta de identificabilidade do modelo e esse fato é especialmente positivo em vista de ser um modelo com maior número de parâmetros.

A análise de dados reais longitudinais de pacientes com periodontite que passaram por tratamento foi efetuada e o modelo ajustado foi validado por meio do diagnóstico dos critérios de seleção do número de componentes da mistura, das inferências dos parâmetros e dos resíduos quantílicos aleatorizados, do que concluiu-se ser a aplicação satisfatória embora houve problemas na confirmação da identificabilidade segundo o método considerado.

O software empregado é gratuito e de fácil utilização, porém o tempo computacional nas execuções efetuadas neste capítulo foi um pouco mais custoso que o modelo com dispersão constante, como esperado devido ao aumento no número de parâmetros. Contudo, a efetiva performance inferencial obtida e os resultados favoráveis do diagnóstico de identificabilidade favorecem a aplicação do modelo obstante essa questão computacional, que cada vez mais está sendo facilitada com os avanços tecnológicos.

A aplicação dos modelos de mistura beta mistos lineares com dispersão variável em dados com as características especificadas neste capítulo e exemplificadas tanto nos estudos de simulação quanto no estudo de um banco de dados reais é sugerida não só por apresentar bons resultados inferencias, mas também pois ao não atribuir um modelo de regressão ao parâmetro de precisão pode-se obter erros inferenciais e conclusões inexatas. 


\section{Capítulo 5}

\section{Modelo de Mistura Beta Não Linear com Efeitos Aleatórios}

Este capítulo primeiramente apresenta uma breve contextualização e motivação do modelo abordado, assim como revisão bibliográfica, na Seção 5.1. Em seguida é efetuada a especificação do modelo de mistura beta misto não linear (Seção 5.2), distribuições a priori são sugeridas para os parâmetros (Seção 5.3) e analisada por meio de estudos de simulação a performance inferencial da metodologia bayesiana utilizada (Seção 5.4). Por fim, para ilustrar a utilização da técnica estatística proposta, um banco de dados é analisado na Seção 5.5.

\subsection{Introdução}

Modelos de regressão estudam e quantificam a relação de variáveis explicativas ou independentes com variáveis resposta ou dependentes por meio de um modelo matemático. Esse modelo é dito ser não linear quando a dependência se dá de forma não linear em relação aos parâmetros.

Comparando os modelos lineares com os não lineares de forma geral tem-se que o primeiro expressa uma relação empírica enquanto, no geral, o segundo é desenvolvido com base em conhecimentos prévios e os parâmetros são interpretáveis no contexto da aplicação (Lindstrom e Bates, 1990).

Em muitas áreas de estudo o conhecimento de especialistas ou a análise exploratória dos dados indicam que a utilização de funções não lineares na análise é mais adequada, como é o caso de dados de expressões gênicas (Zhou, 2004; Wakefield et al., 2003) e farmacocinéticos. Um estudo completo sobre modelos farmacocinéticos é apresentado em Tatarinova e Schumitzky (2014), modelos esses que descrevem a trajetória e os efeitos de medicamentos administrados de diferentes formas em doses variadas por meio de modelos de mistura não lineares com efeitos aleatórios. Segundo os autores, esses modelos são apropriados pois a resposta dos organismos às drogas é não linear por natureza e os componentes da mistura são necessários para a segregação da população em subpopulações que apresentam reações diferenciadas em função de características diversas como metabolismo, por exemplo.

Adicionalmente, o comportamento dos dados ao longo do tempo pode ser caracterizado por heterogeneidade proveniente de fontes não observadas, casos esses em que pode ser conveniente utilizar a metodologia de modelos de mistura finita. Apesar de assumir serem fixas as distribuições 
e as funções sistemáticas, esses modelos são vantajosos por permitirem a variação dos parâmetros para cada subpopulação ao considerá-las como variáveis latentes.

A combinação de modelos mistos não lineares com os modelos de mistura finita proporcionam ajustar as trajetórias de cada subpopulação por funções não lineares de forma que as unidades amostrais de mesma subpopulação tenham similaridades representadas por efeitos fixos e diferenças expressas por efeitos aleatórios.

Segundo Pinheiro e Bates (1995), a extensão dos modelos não lineares de efeitos fixos para os modelos mistos não lineares foi efetuada em diferentes versões em Sheiner e Beal (1980), Mallet et al. (1988), Lindstrom e Bates (1990), Vonesh e Carter (1992), Davidian e Gallant (1992), Wakefield et al. (1994).

Juntamente a flexibilidade dos modelos de mistura mistos de efeitos fixos não lineares, também denominados em inglês por Growth Mixture Model, existe a complexidade da estimação dos parâmetros desse modelo. Dentre as referências que abordam essa questão estão: Kelley (2008), Grimm et al. (2010), Harring (2012), Codd e Cudeck (2014), Lu e Huang (2014) e Kohli et al. (2016), que propõem e estudam métodos de estimação baseados em, por exemplo, linearização por séries de Taylor, máxima verossimilhança utilizando algoritmo EM e aproximação por quadratura de GaussHermite, além de abordagens bayesianas. Em Serang et al. (2015) é apresentado um estudo de simulação para o caso dos modelos normais comparando as performances dos métodos bayesiano e de equação estrutural, respectivamente, dos softwares OpenBUGS e Mplus e os resultados obtidos indicam a validação da utilização do primeiro software que é livre pois a eficiência de ambos foi similar.

Os modelos de regressão beta não lineares de efeitos fixos foram inicialmente abordados em Simas et al. (2010) e Ferrari e Pinheiro (2011) sob abordagem frequentista. Em Espinheira et al. (2017a) e Espinheira et al. (2017b) são apresentadas, respectivamente, técnicas de seleção e de análise de diagnóstico desses modelos. Mais recentemente, o modelo de regressão não linear beta truncado misto bayesiano foi estudado em Paraíba et al. (2018).

Assim sendo, o modelo de mistura beta não linear com efeitos aleatórios é especificado e estudado sob a abordagem bayesiana neste capítulo de forma inédita.

\subsection{Modelo Estatístico}

Como contextualizado anteriormente, o modelo de mistura beta não linear com efeitos aleatórios é indicado para análise de dados que apresentam heterogeneidade intra e entre subpopulações como, por exemplo, dados correlacionados com heterogeneidade não observada.

O modelo apresentado nesta seção foi desenvolvido assumindo-se que todas as observações de uma mesma unidade amostral $\left(y_{i 1}, \ldots, y_{i n_{i}}\right), i=1, \ldots N$, se associam a um mesmo componente da mistura.

Estudos sobre a performance da estimação dos parâmetros e dos demais aspectos inferenciais do modelo estão expostos na Seção 5.4.

Sendo as observações da variável resposta $\boldsymbol{y}_{1}, \ldots, \boldsymbol{y}_{N}$ tais que cada $i$-ésima $(i=1, \ldots, N)$ unidade amostral é composta pelas suas $n_{i}$ aferições, ou seja, $\boldsymbol{y}_{i}=\left(y_{i 1}, \ldots, y_{i n_{i}}\right)^{\top}, S_{i}$ a variável discreta que indica o componente da mistura associado ao $i$-ésimo grupo da amostra, tal que os valores de 
$S_{i}$ pertencem ao conjunto $\{1, \ldots, K\}$ e, assumindo que $Y_{i j} \mid \boldsymbol{b}_{i} \sim \sum_{S_{i}=1}^{K} \pi_{i S_{i}} \operatorname{Beta}\left(\mu_{S_{i}, i j}, \phi_{S_{i}}\right)$ e $\mu_{S_{i}, i j}=$ $E\left(Y_{i j} \mid \boldsymbol{b}_{i}, S_{i}\right)$, o modelo de mistura beta não linear com efeitos aleatórios para a média tem sua parte sistemática dada por:

$$
\eta_{S_{i}, i j}=g_{\mu}\left(\mu_{S_{i}, i j}\right)=h_{\mu}\left(\boldsymbol{x}_{i j}, \boldsymbol{\beta}^{S_{i}}\right)+\boldsymbol{z}_{i j}^{\top} \boldsymbol{b}_{i}
$$

em que:

- $Y_{i j}$ é a variável resposta;

- $K$ é o número de componentes da mistura;

- $N$ é o número de grupos da amostra;

- $n_{i}$ é o número de elementos do $i$-ésimo grupo, $i=1, \ldots, N$;

- $\phi=\left(\phi_{1}, \ldots, \phi_{K}\right)$;

- $\boldsymbol{\pi}_{i}=\left(\pi_{i 1}, \ldots, \pi_{i K}\right)^{\top}$, em que $\pi_{i s}$ é a probabilidade do $i$-ésimo grupo estar associado ao $s$-ésimo componente da mistura, ou seja, $\pi_{i s}=P\left(S_{i}=s\right), 0<\pi_{i s}<1, \sum_{s=1}^{K} \pi_{i s}=1, i=1, \ldots, N$ e $s=1, \ldots, K$

- $\boldsymbol{\pi}=\left(\boldsymbol{\pi}_{1}, \ldots, \boldsymbol{\pi}_{N}\right)$

- $g_{\mu}$ é a função de ligação, estritamente monótona e duplamente diferenciável, do modelo para a média;

- $h_{\mu}$ é a função sistemática não linear e conhecida do modelo para a média;

- $\boldsymbol{\beta}^{s}=\left(\beta_{1}^{s}, \ldots, \beta_{p}^{s}\right)^{\top}$ é o vetor $p$-dimensional de coeficientes fixos relacionado ao $s$-ésimo componente da mistura associada ao modelo para a média, $s=1, \ldots, K$;

- $\boldsymbol{\beta}=\left(\boldsymbol{\beta}^{1}, \ldots, \boldsymbol{\beta}^{K}\right)$ é a matriz de ordem $p \times K$ de coeficientes fixos do modelo para a média;

- $p$ é o número de efeitos fixos do modelo para a média;

- $\boldsymbol{b}_{i}=\left(b_{i 1}, \ldots, b_{i d}\right)^{\top}$ é o vetor de dimensão $d$ de efeitos aleatórios do $i$-ésimo grupo do modelo para a média;

- $d$ é o número de efeitos aleatórios do modelo para a média;

- $\boldsymbol{b}_{i} \mid \boldsymbol{\Sigma}_{b} \stackrel{i i d}{\sim} N_{d}\left(\mathbf{0}, \boldsymbol{\Sigma}_{b}\right)$

- $\boldsymbol{\Sigma}_{b}$ é a matriz de ordem $d \times d$ de variância-covariância dos efeitos aleatórios $\left(\boldsymbol{b}_{1}, \ldots, \boldsymbol{b}_{N}\right)$ do modelo para a média;

- $\boldsymbol{x}_{i j}=\left(x_{i j 1}, \ldots, x_{i j p}\right)^{\top}$ é o vetor $p$-dimensional de covariáveis dos efeitos fixos do $j$-ésimo elemento do $i$-ésimo grupo do modelo para a média, $i=1, \ldots, N$ e $j=1, \ldots, n_{i}$;

- $\boldsymbol{z}_{i j}=\left(z_{i j 1}, \ldots, z_{i j d}\right)^{\top}$ é o vetor $d$-dimensional de covariáveis dos efeitos aleatórios do $j$-ésimo elemento do $i$-ésimo grupo do modelo para a média, $i=1, \ldots, N$ e $j=1, \ldots, n_{i}$; 
- $\boldsymbol{\vartheta}=\left\{\boldsymbol{\beta}^{1^{\top}}, \ldots, \boldsymbol{\beta}^{K^{\top}}, \operatorname{vech}^{\top}\left(\boldsymbol{\Sigma}_{b}\right), \boldsymbol{\pi}_{1}^{\top}, \ldots, \boldsymbol{\pi}_{N}^{\top}, \boldsymbol{\phi}\right\}$ é o vetor de parâmetros do modelo.

As notações acima descritas encontram-se também disponibilizadas na lista de símbolos do início do texto para consultas.

Adicionalmente, a especificação deste modelo pode ser efetuada de forma hierárquica do seguinte modo:

$$
\begin{array}{r}
Y_{i j} \mid \boldsymbol{b}_{i}, S_{i}=s \sim \operatorname{Beta}\left(g_{\mu}^{-1}\left(h\left(\boldsymbol{x}_{i j}, \boldsymbol{\beta}^{s}\right)+\boldsymbol{z}_{i j}^{\top} \boldsymbol{b}_{i}\right), \phi_{s}\right) \\
\boldsymbol{b}_{i} \mid \boldsymbol{\Sigma}_{b} \stackrel{i i d}{\sim} N_{d}\left(\mathbf{0}, \boldsymbol{\Sigma}_{b}\right) \\
P\left(S_{i}=s\right)=\pi_{s},
\end{array}
$$

então: $Y_{i j} \mid \boldsymbol{b}_{i} \sim \sum_{s=1}^{K} \pi_{i s} \operatorname{Beta}\left(g_{\mu}^{-1}\left(h\left(\boldsymbol{x}_{i j}, \boldsymbol{\beta}^{s}\right)+\boldsymbol{z}_{i j}^{\top} \boldsymbol{b}_{i}\right), \phi_{s}\right)$.

Em modelos não lineares, a forma funcional $\left(h_{\mu}\right)$ está diretamente relacionada à aplicação, pois retrata as relações existentes no contexto estudado. A escolha dessa forma funcional pode ser efetuada com base na análise exploratória dos dados, experiência dos pesquisadores da área, referências anteriores sobre o tópico analisado, dentre outros.

Para as finalidades desse trabalho foram considerados efeitos aleatórios com distribuição normal, porém pode-se considerar outras distribuições que mais se adequam, como por exemplo a distribuição $t$ de Student. A utilização de outras distribuições para os efeitos aleatórios apenas acarreta a necessidade da atribuição de distribuições a priori para os hiperparâmetros dos efeitos aleatórios pois toda a metodologia discorrida permanece válida. Outra possibilidade é considerar o parâmetro de precisão igual para todos os componentes da mistura (constante), ou seja, $\phi_{1}=\phi_{2}=\ldots=\phi_{K}=\phi$, o que simplifica o processo de inferência do modelo por reduzir o número de parâmetros (dimensão de $\boldsymbol{\vartheta})$.

Em seguida, respectivamente nas Expressões (5.3) e (5.4), são apresentadas condicionadas aos efeitos aleatórios as verossimilhanças condicional conjunta e marginal conjunta quando o modelo em questão é o (5.1).

$$
\begin{aligned}
L(\boldsymbol{y} \mid \boldsymbol{\vartheta}, \boldsymbol{X}, \boldsymbol{Z}, \boldsymbol{b}, \boldsymbol{S}) & =\prod_{i=1}^{N} \prod_{j=1}^{n_{i}}\left[L_{i j}\left(y_{i j} \mid \boldsymbol{\vartheta}, \boldsymbol{x}_{i j}, \boldsymbol{z}_{i j}, \boldsymbol{b}_{i}, S_{i}\right)\right] \\
& =\prod_{i=1}^{N} \prod_{j=1}^{n_{i}} \frac{\Gamma\left(\phi_{S_{i}}\right) y_{i j}^{g_{\mu}^{-1}\left(\eta_{i j}\right) \phi_{S_{i}}-1}\left(1-y_{i j}\right)^{\left(1-g_{\mu}^{-1}\left(\eta_{i j}\right)\right) \phi_{S_{i}}-1}}{\Gamma\left(g_{\mu}^{-1}\left(\eta_{i j}\right) \phi_{S_{i}}\right) \Gamma\left(\left(1-g_{\mu}^{-1}\left(\eta_{i j}\right)\right) \phi_{S_{i}}\right)} \\
& =\prod_{i=1}^{N} \prod_{j=1}^{n_{i}} \frac{\Gamma\left(\phi_{S_{i}}\right) y_{i j}^{g_{\mu}^{-1}\left(h_{\mu}\left(\boldsymbol{x}_{i j}, \boldsymbol{\beta}^{S_{i}}\right)+\boldsymbol{z}_{i j}^{\top} \boldsymbol{b}_{i}\right) \phi_{S_{i}}-1}\left(1-y_{i j}\right)^{\left(1-g_{\mu}^{-1}\left(h_{\mu}\left(\boldsymbol{x}_{i j}, \boldsymbol{\beta}^{S_{i}}\right)+\boldsymbol{z}_{i j}^{\top} \boldsymbol{b}_{i}\right)\right) \phi_{S_{i}}-1}}{\Gamma\left(g_{\mu}^{-1}\left(h_{\mu}\left(\boldsymbol{x}_{i j}, \boldsymbol{\beta}^{S_{i}}\right)+\boldsymbol{z}_{i j}^{\top} \boldsymbol{b}_{i}\right) \phi_{S_{i}}\right) \Gamma\left(\left(1-g_{\mu}^{-1}\left(h_{\mu}\left(\boldsymbol{x}_{i j}, \boldsymbol{\beta}^{S_{i}}\right)+\boldsymbol{z}_{i j}^{\top} \boldsymbol{b}_{i}\right)\right) \phi_{S_{i}}\right)} .
\end{aligned}
$$




$$
\begin{aligned}
L(\boldsymbol{y} \mid \boldsymbol{\vartheta}, \boldsymbol{X}, \boldsymbol{Z}, \boldsymbol{b}) & =\prod_{i=1}^{N} \prod_{j=1}^{n_{i}}\left[L_{i j}\left(y_{i j} \mid \boldsymbol{\vartheta}, \boldsymbol{x}_{i j}, \boldsymbol{z}_{i j}, \boldsymbol{b}_{i}\right)\right] \\
& =\prod_{i=1}^{N} \prod_{j=1}^{n_{i}} \sum_{S_{i}=1}^{K} \pi_{i S_{i}}\left[L_{i j}\left(y_{i j} \mid \boldsymbol{\vartheta}, \boldsymbol{x}_{i j}, \boldsymbol{z}_{i j}, \boldsymbol{b}_{i}, S_{i}\right)\right] \\
& =\prod_{i=1}^{N} \sum_{S_{i}=1}^{K} \pi_{i S_{i}} \prod_{j=1}^{n_{i}}\left[L_{i j}\left(y_{i j} \mid \boldsymbol{\vartheta}, \boldsymbol{x}_{i j}, \boldsymbol{z}_{i j}, \boldsymbol{b}_{i}, S_{i}\right)\right]
\end{aligned}
$$

em que:

$L_{i j}\left(y_{i j} \mid \boldsymbol{\vartheta}, \boldsymbol{x}_{i j}, \boldsymbol{z}_{i j}, \boldsymbol{b}_{i}, S_{i}\right)=\frac{\Gamma\left(\phi_{S_{i}}\right) y_{i j}^{g_{\mu}^{-1}\left(h_{\mu}\left(\boldsymbol{x}_{i j}, \boldsymbol{\beta}^{S_{i}}\right)+\boldsymbol{z}_{i j}^{\top} \boldsymbol{b}_{i}\right) \phi_{S_{i}}-1}\left(1-y_{i j}\right)^{\left(1-g_{\mu}^{-1}\left(h_{\mu}\left(\boldsymbol{x}_{i j}, \boldsymbol{\beta}^{S_{i}}\right)+\boldsymbol{z}_{i j}^{\top} \boldsymbol{b}_{i}\right)\right) \phi_{S_{i}}-1}}{\Gamma\left(g_{\mu}^{-1}\left(h_{\mu}\left(\boldsymbol{x}_{i j}, \boldsymbol{\beta}^{S_{i}}\right)+\boldsymbol{z}_{i j}^{\top} \boldsymbol{b}_{i}\right) \phi_{S_{i}}\right) \Gamma\left(\left(1-g_{\mu}^{-1}\left(h_{\mu}\left(\boldsymbol{x}_{i j}, \boldsymbol{\beta}^{S_{i}}\right)+\boldsymbol{z}_{i j}^{\top} \boldsymbol{b}_{i}\right)\right) \phi_{S_{i}}\right)}$.

Considerando a integração em relação aos efetios aleatórios, seja $p_{b}(\cdot)$ a distribuição de $\boldsymbol{b}_{i}$, as verossimilhanças condicional e marginal conjuntas sâo expostas nas Equações (5.6) e (5.7).

$$
\begin{array}{r}
L(\boldsymbol{y} \mid \boldsymbol{\vartheta}, \boldsymbol{X}, \boldsymbol{Z}, \boldsymbol{S})=\prod_{i=1}^{N} \prod_{j=1}^{n_{i}}\left[\int L_{i j}\left(y_{i j} \mid \boldsymbol{\vartheta}, \boldsymbol{x}_{i j}, \boldsymbol{z}_{i j}, \boldsymbol{b}_{i}, S_{i}\right) p_{b}\left(\boldsymbol{b}_{i}\right) d \boldsymbol{b}_{i}\right] \\
L(\boldsymbol{y} \mid \boldsymbol{\vartheta}, \boldsymbol{X}, \boldsymbol{Z})=\prod_{i=1}^{N} \prod_{j=1}^{n_{i}}\left[\int L_{i j}\left(y_{i j} \mid \boldsymbol{\vartheta}, \boldsymbol{x}_{i j}, \boldsymbol{z}_{i j}, \boldsymbol{b}_{i}\right) p_{b}\left(\boldsymbol{b}_{i}\right) d \boldsymbol{b}_{i}\right] \\
=\prod_{i=1}^{N} \prod_{j=1}^{n_{i}} \sum_{S_{i}=1}^{K} \pi_{i S_{i}}\left[\int L_{i j}\left(y_{i j} \mid \boldsymbol{\vartheta}, \boldsymbol{x}_{i j}, \boldsymbol{z}_{i j}, \boldsymbol{b}_{i}, S_{i}\right) p_{b}\left(\boldsymbol{b}_{i}\right) d \boldsymbol{b}_{i}\right] \\
=\prod_{i=1}^{N} \sum_{S_{i}=1}^{K} \pi_{i S_{i}} \prod_{j=1}^{n_{i}}\left[\int L_{i j}\left(y_{i j} \mid \boldsymbol{\vartheta}, \boldsymbol{x}_{i j}, \boldsymbol{z}_{i j}, \boldsymbol{b}_{i}, S_{i}\right) p_{b}\left(\boldsymbol{b}_{i}\right) d \boldsymbol{b}_{i}\right],
\end{array}
$$

em que $L_{i j}\left(y_{i j} \mid \boldsymbol{\vartheta}, \boldsymbol{x}_{i j}, \boldsymbol{z}_{i j}, \boldsymbol{b}_{i}, S_{i}\right)$ é expresso na Equação (5.5).

Em contrapartida à adequabilidade da utilização destes modelos para os casos indicados, existe a complexidade do processo inferencial. Considerando o caso mais simples do modelo de regressão não linear com efeitos aleatórios $(K=1)$, sob abordagem clássica existe a dificuldade da falta de solução de forma fechada da integração sob a distribuição dos efeitos aleatórios para obter a distribuição marginal dos dados. Davidian e Giltinan (1993) descrevem algumas soluções para essa questão como efetuar estimações separadamente para cada grupo, aproximar a verossimilhança por meio de linearização das funções não lineares, dentre outras. Um estudo de simulação interessante sobre o desempenho desses métodos de estimação e do software bayesiano WinBUGS é apresentado em Harring e Liu (2016), no qual os resultados indicaram que, ponderando o custo computacional com as falhas de convergência e a acurácia das estimativas dos parâmetros, o método bayesiano obteve performance muito satisfatória.

Para valores maiores de componentes da mistura, a grande maioria das metodologias com base 
na abordagem frequentista utilizam-se do algoritmo EM. Uma exceção é a proposta de Serang et al. (2015) que além de linearizar o modelo por expansão em série de Taylor antes de efetuar a estimação por máxima verossilhança, utiliza-se de equações estruturais; processo esse que obteve desempenho inferior ao disponibilizado pelo software bayesiano OpenBUGS.

Por esses e outros inúmeros resultados descritos na literatura, neste trabalho a inferência dos modelos será efetuada sob abordagem bayesiana através do software livre OpenBUGS.

\subsection{Distribuições a priori e a posteriori}

O primeiro passo para ajustar o modelo é definir as distribuições a priori para os parâmetros que compõem $\boldsymbol{\vartheta}$, as quais são denotadas respectivamente por: $p_{\beta}\left(\boldsymbol{\beta}^{s}\right), p_{\phi}\left(\phi_{s}\right), p_{\pi}\left(\boldsymbol{\pi}_{i}\right), p_{\Sigma_{b}}\left(\boldsymbol{\Sigma}_{b}\right)$, $i=1, \ldots, N$ e $s=1, \ldots, K$.

$\mathrm{Na}$ Tabela 5.1 encontram-se sugestões de atribuições para essas distribuições a priori. Essas indicações são baseadas no conteúdo do capítulo de contextualizações (2.1.1), porém essas escolhas podem ser efetuadas de forma a serem mais adequados ao contexto dos dados.

\begin{tabular}{c|c|c}
\hline Parâmetro & Notação da distribuição a priori & Distribuição a priori \\
\hline $\boldsymbol{\beta}^{s}$ & $p_{\beta}\left(\boldsymbol{\beta}^{s}\right)$ & $N_{p}\left(\boldsymbol{\mu}_{\boldsymbol{\beta}^{s}}, a_{\beta} \boldsymbol{I}_{p}\right)$ \\
$\boldsymbol{\pi}_{i}$ & $p_{\pi}\left(\boldsymbol{\pi}_{i}\right)$ & $\operatorname{Dir}(\boldsymbol{\alpha})$ \\
$\phi_{s}$ & $p_{\phi}\left(\phi_{s}\right)$ & $\operatorname{Unif}(a, b)$ \\
$\boldsymbol{\Sigma}_{b}$ & $p_{\Sigma_{b}}\left(\boldsymbol{\Sigma}_{b}\right)$ & $I W(\boldsymbol{\Psi}, \boldsymbol{C})$ \\
\hline
\end{tabular}

Tabela 5.1: Parâmetros e distribuições a priori indicadas para ajuste do modelo de mistura beta não linear com efeitos aleatórios para a média, $s=1, \ldots, K$ e $i=1, \ldots, N$.

Os elementos $\boldsymbol{\mu}_{\boldsymbol{\beta}^{s}}, a_{\beta}, \boldsymbol{\alpha}, a, b, \boldsymbol{\Psi}$ e $\boldsymbol{C}$ são denominados hiperparâmetros e os seus valores podem ser escolhidos tanto de forma a obter distribuições a priori não informativas como distribuições a priori que agregam informações prévias. Outra opção é atribuir distribuições para esses hiperparâmetros. Exemplos de valores de hiperparâmetros são descritos no estudos de simulação.

Sejam $L(\boldsymbol{y} \mid \boldsymbol{\vartheta}, \boldsymbol{X}, \boldsymbol{Z}, \boldsymbol{b})$ a função de verossimilhança $(5.4), p_{b}(\boldsymbol{b} \mid \boldsymbol{\vartheta})$ a função densidade de probabilidade dos efeitos aleatórios e $p(\boldsymbol{\vartheta})$ a distribuição a priori do vetor de parâmetros, aplicando o teorema de Bayes (2.1), a distribuição a posteriori de $\boldsymbol{\vartheta}, p(\boldsymbol{\vartheta}, \boldsymbol{b} \mid \boldsymbol{y}, \boldsymbol{X}, \boldsymbol{Z})$, é especificada na Equação (5.8).

$$
\begin{aligned}
p(\boldsymbol{\vartheta}, \boldsymbol{b} \mid \boldsymbol{y}, \boldsymbol{X}, \boldsymbol{Z}) & \propto L(\boldsymbol{y} \mid \boldsymbol{\vartheta}, \boldsymbol{X}, \boldsymbol{Z}, \boldsymbol{b}) p_{b}(\boldsymbol{b} \mid \boldsymbol{\vartheta}) p(\boldsymbol{\vartheta}) \\
& =p_{b}\left(\boldsymbol{b} \mid \boldsymbol{\Sigma}_{b}\right) p_{\Sigma_{b}}\left(\boldsymbol{\Sigma}_{b}\right) \prod_{i=1}^{N} \sum_{s=1}^{K} \pi_{i s} p_{\pi}\left(\boldsymbol{\pi}_{i}\right) p_{\phi}\left(\phi_{s}\right) p_{\beta}\left(\boldsymbol{\beta}^{s}\right) \prod_{j=1}^{n_{i}}\left[L_{i j}\left(y_{i j} \mid \boldsymbol{\vartheta}, \boldsymbol{x}_{i j}, \boldsymbol{z}_{i j}, \boldsymbol{b}_{i}, s\right)\right] .
\end{aligned}
$$

\subsection{Estudos de simulação}

Os estudos de simulação desta seção têm como objetivo explorar o desempenho inferencial dos modelos de mistura beta não lineares com interceptos aleatórios $(d=1)$. A função de ligação utilizada foi a logito, $g_{\mu}\left(\mu_{i j}\right)=\log \left(\frac{\mu_{i j}}{1-\mu_{i j}}\right)$, e se estabeleceu que $\phi_{1}=\phi_{2}=\ldots=\phi_{K}=\phi$. Para delinear os estudos de simulação, são definidos nesta introdução da seção: a função não linear, os 
valores dos parâmetros dos cenários estudados e suas distribuições a priori e hiperparâmetros. Em seguida, são explicados passo a passo os procedimentos dos estudos de simulação para cada um dos seguintes aspectos estudados:

1. precisão das estimativas pontuais obtidas pela média e mediana da distribuição a posteriori;

2. performance das medidas de seleção de modelos para determinação de $K$ e,

3. averiguação de não identificabilidade,

e os correspondentes resultados obtidos são apresentados nas Subseções 5.4.1, 5.4.2 e 5.4.3.

Como a função não linear $h_{\mu}$ e os valores dos parâmetros são elementos muito específicos de cada aplicação no caso de modelos não lineares, eles foram escolhidos com base nos estudos de simulação efetuados em Espinheira et al. (2017b). Assim, seja $h_{\mu}\left(x_{i j}, \boldsymbol{\beta}^{s}\right)=\beta_{1}^{s}+x_{i j}^{\beta_{2}^{s}}, s=1,2 \mathrm{e}$ $x_{i j} \sim \operatorname{Unif}(0,1)$, os valores dos parâmetros $\boldsymbol{\beta}^{1}, \boldsymbol{\beta}^{2}$ dos dois cenários estão expressos na Tabela 5.2 e o esboço da forma funcional é apresentado no gráfico da Figura 5.1.

\begin{tabular}{c|c}
\hline Cenário & $\left\{\boldsymbol{\beta}^{1^{\mid}} ; \boldsymbol{\beta}^{\mathbf{}^{\dagger}}\right\}$ \\
\hline 1 & $\{(-2,4 ; 0,8) ;(-0,5 ;-0,6)\}$ \\
2 & $\{(-2,4 ; 0,8) ;(-1 ; 2,8)\}$ \\
\hline
\end{tabular}

Tabela 5.2: Definição dos cenários estudados por simulação para os parâmetros $\boldsymbol{\beta}^{s}, s=1,2$.

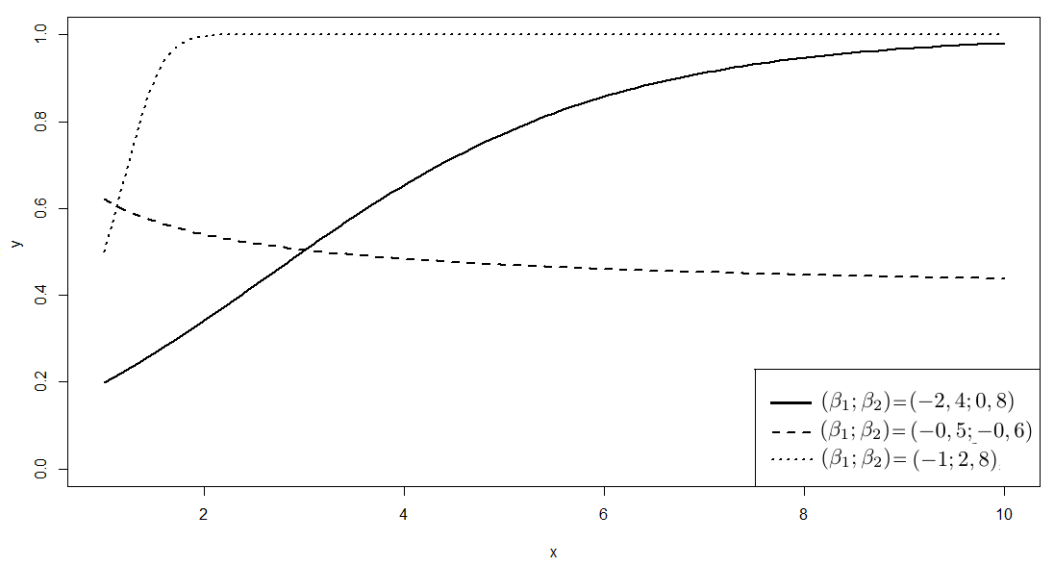

Figura 5.1: Gráfico das observações geradas com $h_{\mu}(x, \boldsymbol{\beta})=\beta_{1}+x_{i j}^{\beta_{2}}$ e ligação logito para valores de $\left(\beta_{1} ; \beta_{2}\right)$ que compõem os cenários dos estudos de simulação.

Adicionalmente, foram considerados $\boldsymbol{\pi}_{i}^{\top}=\left(\pi_{i 1} ; \pi_{i 2}\right)=(0,5 ; 0,5), \phi \in\{30,50,70\}, \sigma_{b}=1 \mathrm{e}$ distribuições a priori tais quais exposto na Tabela 5.1. Os hiperparâmetros foram definidos aos moldes do efetuado na Seção 3.4: $\boldsymbol{\alpha}=(100 ; 100), a=\phi-20, b=\phi+20, \psi=2, C=2$ e $\alpha_{\beta}=4$. Para determinar $\boldsymbol{\mu}_{\boldsymbol{\beta}^{s}}, s=1,2$, primeiramente foi utilizado o pacote kml (Genolini et al., 2015) do software $R$ para classificar as unidades amostrais em $K=2$ grupos e, em seguida, ajustados modelos beta mistos não lineares para cada um desses grupos através do pacote gamlss.nl (Stasinopoulos e Rigby, 2012), cujas estimativas dos coeficientes fixos são os valores dos hiperparâmetros.

A Figura 3.1 esboça as funções densidade de probabilidade geradas com os valores anteriormente definidos. 

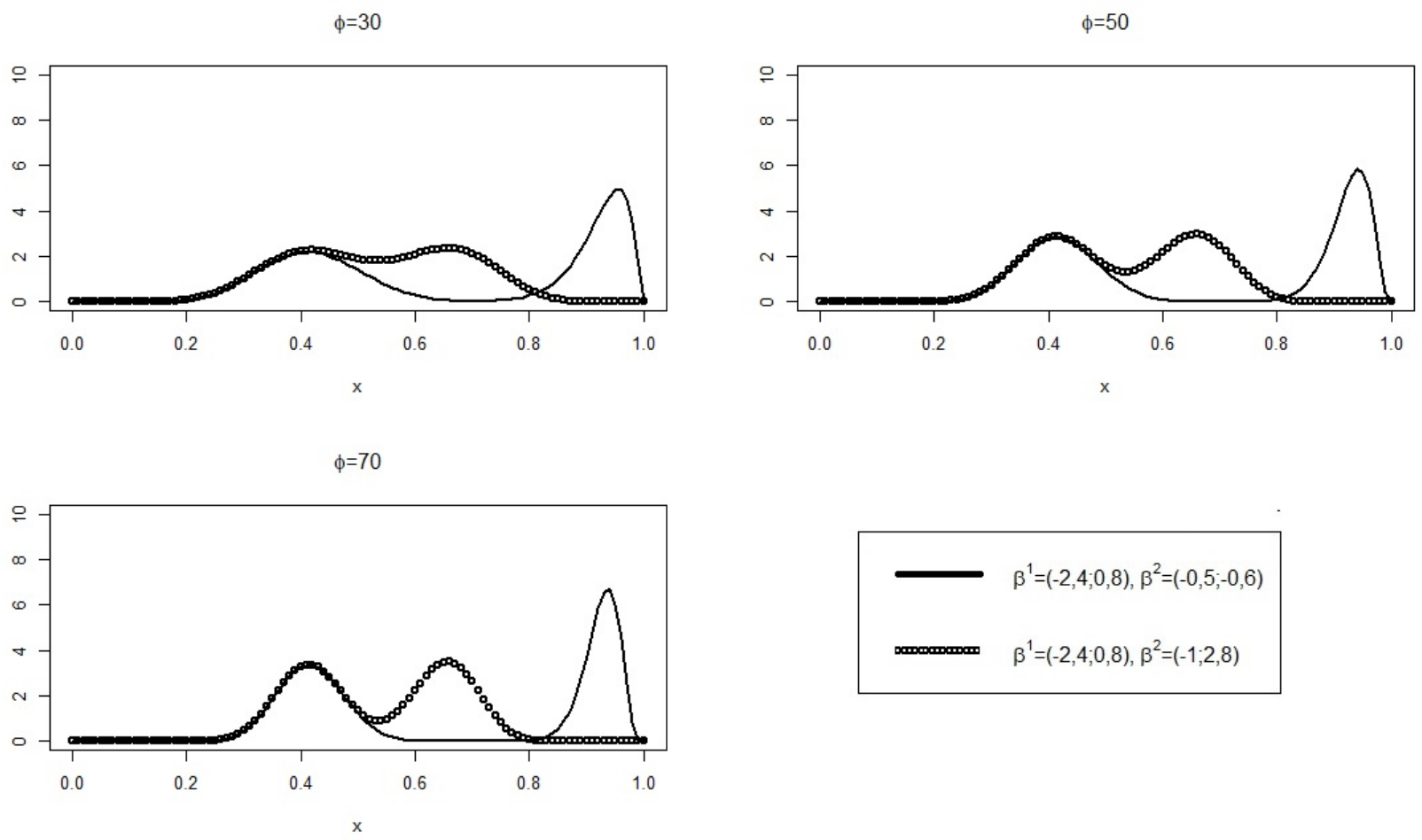

Figura 5.2: Comparação entre as densidades da distribuição beta para diferentes valores dos parâmetros $\beta$ e $\phi$ nos cenários englobados pelos estudos de simulação.

Os dois cenários apresentam bimodalidade e maiores valores do parâmetro de precisão $\phi$ consecutem em maior concentração da massa de probabilidade em torno dessas modas. $O$ primeiro cenário possui maior distinção dos componentes da mistura, enquanto o segundo contém inclusive intervalos de confundimento como é observado para $\phi=30$.

Ademais, afim de analisar o desempenho da acurácia da estimação pontual a posteriori no cenário da aplicação deste capítulo (Seção 5.5), o cenário 3 abrange o modelo da Equação (5.9) com valores de parâmetros iguais às estimativas pontuais expostos na Tabela 5.5.

\subsubsection{Acurácia das estimativas pontuais a posteriori}

Foram gerados $R=50$ bancos de dados (réplicas) contendo observações de $N=100$ grupos com $n=n_{1}=\ldots=n_{N}=5$ observações por grupo. Devido à inviabilização de analisar as amostras a posteriori dos 300 ajustes, os resultados foram analisados com base na mediana amostrais a posteriori.

Adicionalmente, para estudar o desempenho da acurácia da estimação pontual a posteriori no cenário da aplicação deste capítulo (Seção 5.5), foram gerados $R=25$ bancos de dados contendo observações de $N=100$ grupos com $n=n_{1}=\ldots=n_{N}=5$ observações por grupo do modelo da Equação (5.9) com valores de parâmetros iguais às estimativas pontuais expostos na Tabela 5.5.

As medidas de avaliação do desempenho das estimativas pontuais utilizadas foram o erro relativo médio (ER), o erro absoluto relativo médio (EAR) e a raíz do erro quadrático médio (REQM). Além disso, também foi calculada a probabilidade de cobertura frequentista dos intervalos de credibilidade de $95 \%$, ou seja, a porcentagem das réplicas nas quais o verdadeiro valor dos parâmetros está contido no seu respectivo intervalo de credibilidade de $95 \%$.

Os algoritmos MCMC foram executados com $n_{i t}=50000$ iterações, $n_{b}=10000, n_{t}=10 \mathrm{e}$ 
$n_{c}=1$, totalizando amostras de tamanho 4000 da distribuição a posteriori de cada conjunto de dados.

$\mathrm{Na}$ totalidade das réplicas todos os verdadeiros valores dos parâmetros estão inclusos aos seus respectivos intervalos de credibilidade de $95 \%$ resultando em uma probabilidade de cobertura frequentista de $100 \%$, exceto para os parâmetros $\beta_{i_{p}}^{1}, i_{p} \in\{1,2,3\}$ do cenário 3 que obtiveram valores respectivamente iguais a $0,88,0,84$ e 0,88 . Resultados esses que validam a eficiência da utilização dessa estimação pontual

Os resultados das medidas de acurácia das estimativas pontuais encontram-se dispostos nas tabelas da Seção A.5 do Apêndice A e os gráficos dessas estimativas ao longo das réplicas encontramse na Seção B.8.

A estimação pontual dos parâmetros $\pi$ e $\sigma_{b}$ dos cenários 1 e 2 apresentou grande precisão em todas as réplicas de todos os cenários estudados, conforme observa-se nos gráficos da Seção B.8.

No caso do parâmetro de precisão $\phi$ nos cenários 1 e 2, a performance da estimação através da mediana a posteriori foi satisfatória, sendo que a acurácia aumenta e a tendência de superestimação diminui a medida que o valor de $\phi$ aumenta.

Considerando os cenários 1 e 2, o desempenho das estimação dos coeficientes fixos $\left(\beta_{1}^{1}, \beta_{2}^{1}\right.$, $\beta_{1}^{2}$ e $\beta_{2}^{2}$ ) nos estudos de simulação foram promissores por apresentar boa acurácia e também no sentido de não ter havido nenhum caso de confundimento dos elementos da mistura apesar das densidades do cenário 2, expostos na Figura 5.2, apresentarem intervalos de confundimento entre os componentes da mistura, principalmente quando $\phi=30$. Sendo que, inclusive, o cenário 2 foi o que obteve melhor desempenho na estimação dos coeficientes fixos. Esse resultado contribui para a análise de identificabilidade do modelo, indicando que ao utilizar a estimação pontual da mediana a posteriori para cenários tais quais os retratados nesse estudo os resultados são empiricamente identificáveis.

Com relação ao cenário 3, nas Figuras B.85 e B.87 observa-se que as medianas a posteriori dos parâmetros $\beta_{i_{p}}^{s}, i_{p} \in\{1,2,3\}, s=1,2, \pi_{1}$ e $\phi_{2}$ são muito próximas aos verdadeiros valores e que o parâmetro $\phi_{1}$ teve tendência de superestimação, assim como nos demais cenários. Os resultados obtidos com relação aos parâmetros associados aos efeitos aleatórios foram satisfatórios mas não tão precisos quanto nos cenários 1 e 2, o que é esperado por ser um modelo com três efeitos aleteatórios e, portanto, seis parâmetros relacionados a serem estimados.

Desse modo, os resultados obtidos evidenciam que o desempenho da estimação pontual por meio da mediana a posteriori é eficiente.

\subsubsection{Performance dos critérios de seleção de modelos}

Tal qual apresentado na Seção 2.5, a seleção do número de componentes da mistura $(K)$ é um tópico que pode ser abordado de diferentes formas. Neste trabalho esse valor é primeiramente fixado para efetuar a estimação dos parâmetros e seleciona-se o modelo de acordo com a sua adequabilidade, a qual é avaliada através de critérios de informação, medidas de qualidade e diagnóstico do ajuste, análise das estimativas, dentre outros.

Afim de verificar a eficiência dos critérios de informação EAIC, ECAIC, EBIC, $D I C_{3}$ e LPML, definidos na Subseção 2.1.3, para escolha de $K$, foram efetuados os estudos de simulação aqui apresentados. Primeiramente foram ajustados os modelos para diferentes números de componentes da mistura aos dados simulados de acordo com os cenários estabelecidos, depois calculou-se os 
valores desses critérios de informação para os modelos ajustados e contabilizadas a proporção de vezes que o modelo que gerou os dados foi selecionado ao utilizar apenas essas medidas como critério de escolha.

Os valores de $\boldsymbol{\beta}^{s}, s=1, \ldots, K$ são os apresentados na Tabela $5.2, \phi \in\{30 ; 50 ; 70\}, K=2 \mathrm{e}$ $\pi_{1}=0,7$. As respectivas distribuições a priori e métodos de estimação estão descritos no início da seção, as amostras são compostas por $N=100$ grupos com $n=5$ observações por grupo e $n_{i t}=50000, n_{b}=15000, n_{t}=10$ e $n_{c}=1$, totalizando 3500 amostras da distribuição a posteriori.

Com base no custo computacional, estabeleceu-se o número de réplicas simuladas igual a 10 para cada cenário e aos dados foram ajustados modelos com $K=1,2, \ldots, 5$ componentes na mistura. Exclusivamente com base nos valores dos critérios de informação desses ajustes seleciona-se o valor de $K$ para cada uma das réplicas de cada um dos cenários e calcula-se a porcentagen de réplicas em que determinado valor de $K$ foi selecionado em cada cenário. Esses resultados encontram-se na Tabela 5.3 e, como os dados foram gerados com $K=2$, quanto maior a porcentagem para este valor, melhor é o desempenho do critério para seleção dos modelos. 


\begin{tabular}{|c|c|c|c|c|c|c|c|}
\hline \multirow[b]{3}{*}{ Critério } & \multirow[b]{3}{*}{$K$} & \multicolumn{3}{|c|}{ Cenário 1} & \multicolumn{3}{|c|}{ Cenário 2} \\
\hline & & & & $c$ & & & \\
\hline & & 30 & 50 & 70 & 30 & 50 & 70 \\
\hline \multirow[t]{5}{*}{ EAIC } & 1 & 0 & 0 & 0 & 0 & 0 & 0 \\
\hline & 2 & 20 & 10 & 0 & 80 & 30 & 0 \\
\hline & 3 & 70 & 80 & 60 & 20 & 60 & 80 \\
\hline & 4 & 10 & 10 & 10 & 0 & 10 & 20 \\
\hline & 5 & 0 & 0 & 30 & 0 & 0 & 0 \\
\hline \multirow[t]{5}{*}{ ECAIC } & 1 & 0 & 0 & 0 & 0 & 0 & 0 \\
\hline & 2 & 20 & 10 & 0 & 80 & 30 & 0 \\
\hline & 3 & 70 & 80 & 60 & 20 & 70 & 80 \\
\hline & 4 & 10 & 10 & 10 & 0 & 0 & 20 \\
\hline & 5 & 0 & 0 & 30 & 0 & 0 & 0 \\
\hline \multirow[t]{5}{*}{ EBIC } & 1 & 0 & 0 & 0 & 0 & 0 & 0 \\
\hline & 2 & 30 & 10 & 10 & 100 & 40 & 20 \\
\hline & 3 & 70 & 80 & 70 & 0 & 60 & 80 \\
\hline & 4 & 0 & 10 & 20 & 0 & 0 & 0 \\
\hline & 5 & 0 & 0 & 0 & 0 & 0 & 0 \\
\hline \multirow[t]{5}{*}{$\mathrm{DIC}_{3}$} & 1 & 100 & 100 & 100 & 90 & 80 & 60 \\
\hline & 2 & 0 & 0 & 0 & 10 & 0 & 30 \\
\hline & 3 & 0 & 0 & 0 & 0 & 20 & 10 \\
\hline & 4 & 0 & 0 & 0 & 0 & 0 & 0 \\
\hline & 5 & 0 & 0 & 0 & 0 & 0 & 0 \\
\hline \multirow[t]{5}{*}{$L P M L$} & 1 & 0 & 0 & 0 & 0 & 0 & 0 \\
\hline & 2 & 0 & 0 & 0 & 0 & 0 & 0 \\
\hline & 3 & 30 & 10 & 0 & 30 & 20 & 0 \\
\hline & 4 & 10 & 20 & 40 & 0 & 30 & 10 \\
\hline & 5 & 60 & 70 & 60 & 70 & 50 & 90 \\
\hline
\end{tabular}

Tabela 5.3: Porcentagem das 10 réplicas simuladas do modelo de mistura beta não linear com efeitos aleatórios para a média cujos critérios EAIC, ECAIC, EBIC, DIC 3 e LPML selecionaram cada um dos 5 possiveis valores de números de componentes da mistura $(K)$.

Os resultados do EBIC foram os mais precisos, com destaque para o cenário 2 e $\phi=30$, seguido do ECAIC e EAIC, o que pode ser explicado pelo EBIC ter maior penalização para o número de parâmetros.

Quanto menores os valores do parâmetros de precisão, mais precisos foram os desempenhos observados de todos os critérios. Na maioria dos casos o cenário 2 teve melhores resultados que o 1 , o que pode ser decorrente da melhor acurácia da estimação pontual dos coeficientes fixos do modelo (tabelas da Seção A.5).

No geral, observa-se que o desempenho dos critérios estudados não foi satisfatório e que as medidas estudadas selecionaram valores maiores de $K$ que o verdadeiro, com exceção do $D I C_{3}$ que subestimou o número de elementos da mistura. Em comparação com os estudos análogos dos outros capítulos, o LPML também foi o que escolheu modelos com mais elementos de componentes 
da mistura, pois esse critério baseia-se na função de verossimilhança sem penalizar o número de parâmetros.

\subsubsection{Diagnóstico de identificabilidade}

Nessa seção o interesse é estudar a identificabilidade dos modelos de mistura beta mistos não lineares. Sejam $\boldsymbol{\beta}^{s}, s=1, \ldots, K$, tal qual apresentados na Tabela $5.2, \phi \in\{30 ; 50 ; 70\}, K=2$, $\pi_{1}=0,5, N=100$ e $n=5$. A escolha das distribuições a priori e dos métodos de estimação previamente definidos foi efetuada tal como explicado na introdução desta seção (5.4).

A técnica aplicada é o data cloning e na Seção 2.7 são esclarecidas as razões da adequabilidade de sua utilização. Os números de clones foram adotados de acordo com a convergência obtida, isso porque, como advertido em Lele et al. (2010), podem ocorrer problemas de convergência das cadeias MCMC na aplicação do método.

Na Figura 3.3 estão graficados os valores do maior autovalor padronizado da matriz de variâncias e covariâncias a posteriori dos parâmetros para os números de clones considerados.

Cenário 1

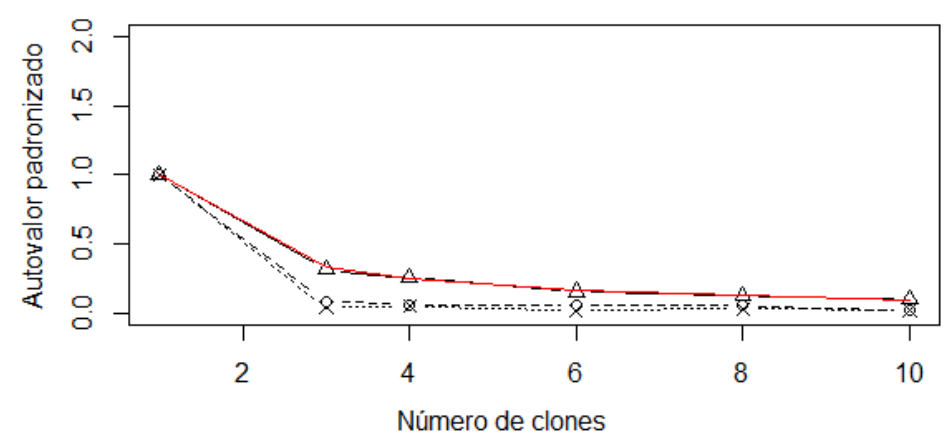

Cenário 2

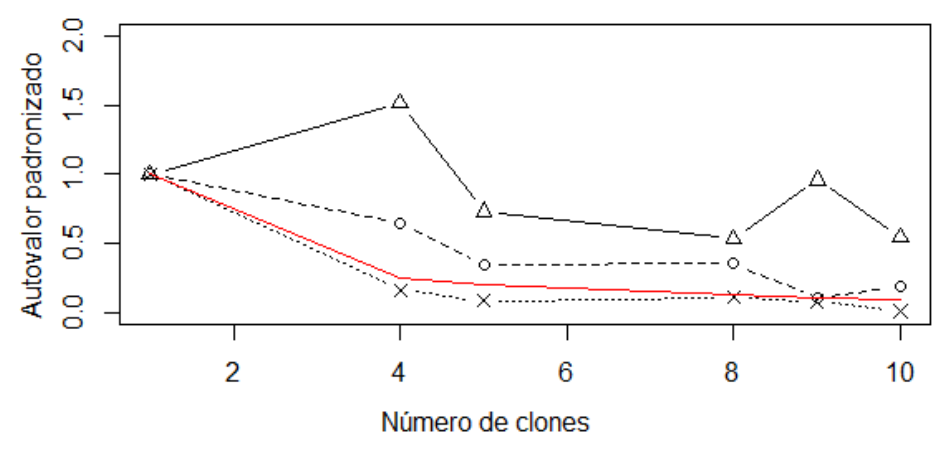

Figura 5.3: Gráfico de linhas do inverso do número de clones (traço vermelho) e do maior autovalor padronizado da matriz de variâncias e covariâncias a posteriori dos parâmetros do modelo de mistura beta misto não linear com cenários 1,2 e parâmetro de precisãa assumindo valores $30(\triangle), 50(\bigcirc), 70(\times)$.

A convergência desses autovalores para o limite zero com o crescimento do número de clones, principalmente se essa taxa de convergência for próxima a observada no inverso do número de clones, indica que que não existem evidências de falta de identificabilidade.

Esse decrescimento próximo à curva de referência é nítido para o cenário 1, o que era esperado pelo distanciamento dos componentes da mistura observado na densidade desse cenário ilustrada na Figura 5.2. 
Os resultados também representaram essa distinção dos componentes da mistura para o cenário 2, indicando falhas de identificabilidade para o modelo com menor valor do parâmetro de precisão $(\phi=30)$. Quando $\phi=70$ os resultados foram satisfatórios e pode-se concluir que não há problemas de identificabilidade no modelo.

Em vista disso, constata-se que, sob as condições descritas, quanto mais distinguíveis são os elementos da mistura do modelo menos evidências de falta de identificabilidade do modelo existem.

\subsection{Aplicação a dados de desenvolvimento de habilidades de leitura no ensino básico}

Nesta aplicação o banco de dados considerado provém de um estudo longitudinal desenvolvido nos Estados Unidos do inglês denominado Early Childhood Longitudinal Program, Kindergarten Class of 1998-99 (ECLS-K). Esse estudo acompanhou a trajetória de 21.409 estudantes desde o ingresso na pré-escola no segundo semestre de 1998 até a oitava série do ensino básico, coletando dados sobre seus desenvolvimentos cognitivo, social, emocional e físico, além de informações sobre suas famílias, professores e escolas. As informações foram aferidas em sete momentos: primeiro e segundo semestres da pré-escola (segundo semestre de 1998 e primeiro semestre de 1999), primeiro e segundo semestres da primeira série (segundo semestre de 1999 e primeiro semestre de 2000) e segundos semestres da terceira, quinta e oitava série (primeiros semestres de 2002, 2004 e 2007).

Inúmeros estudos comportamentais afirmam que, em geral, o desenvolvimento da habilidade de leitura é não linear (Kaplan, 2002): na pré-escola esse desenvolvimento é lento, cresce rapidamente à medida que os alunos entram em contato com novos conhecimentos e, então, diminui o ritmo de crescimento, que nessa fase é alavancado pelo aperfeiçoamento das capacidades previamente adquiridas e conhecimentos provindos de influências externas ao ambiente escolar. Estudos empíricos como Grimm e Ram (2009), Grimm et al. (2010), Serang et al. (2015) e Serang et al. (2016) sugerem que os dados de habilidade de leitura coletados no ECLS-K apresentam não linearidade. Isso pode ser claramente observado na Figura 5.4, a qual ilustra o desenvolvimento do desempenho de leitura de 100 alunos da amostra. Para a grande maioria da amostra, o crescimento do escore é mais intenso nos dois primeiros anos e segue crescendo com cada vez menos intensidade a partir da terceira série, confirmando o que os estudos teóricos descrevem. 


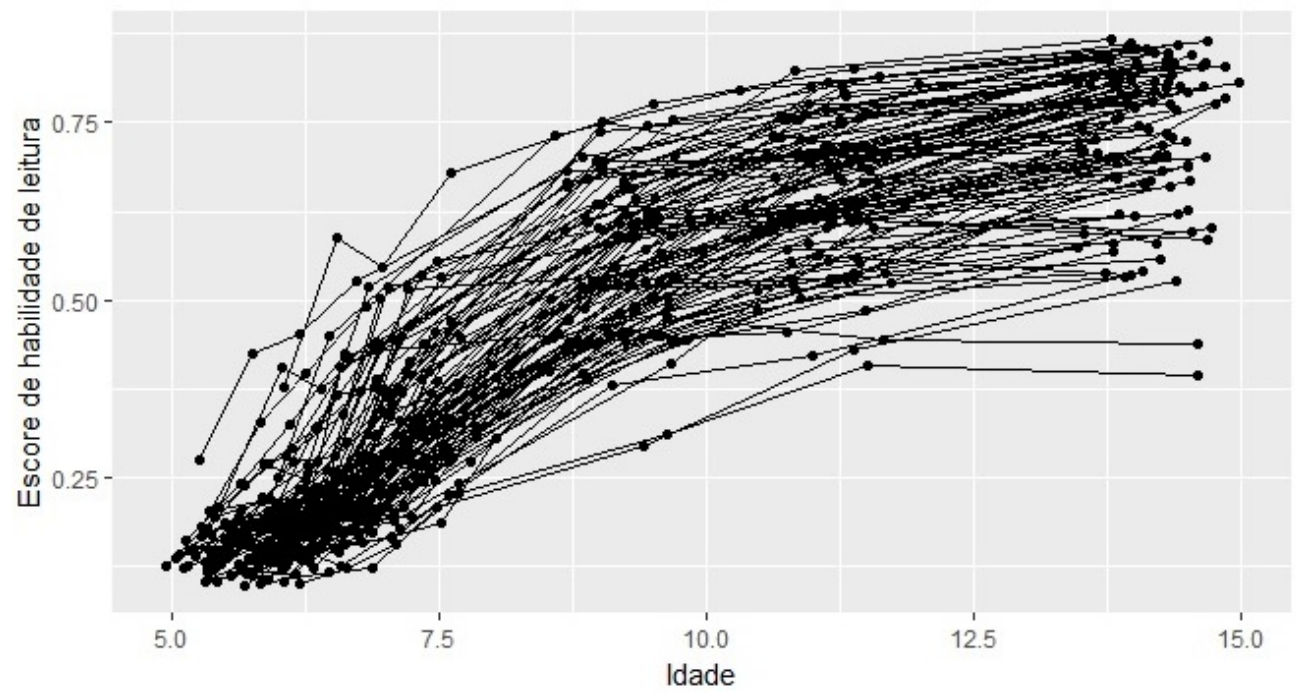

Figura 5.4: Gráfico de perfis do desenvolvimento da habilidade de leitura de 100 alunos participantes do estudo ECLS-K.

Outro atributo observado na Figura 5.4 é a heterogeneidade da trajetória dos alunos ao longo do tempo. Kaplan (2002), Grimm et al. (2010) e Serang et al. (2016) são referências que afirmam existir no mínimo dois padrões interpretáveis de desenvolvimento.
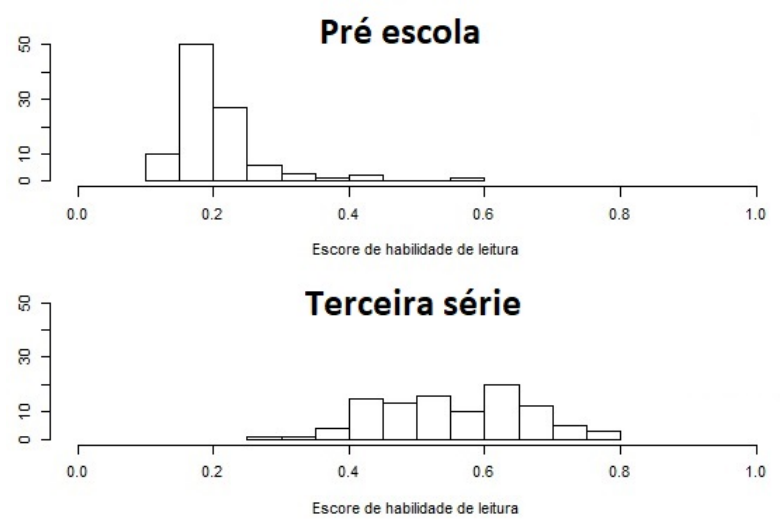

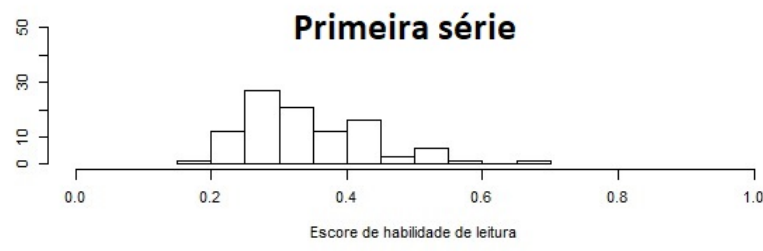

Quinta série

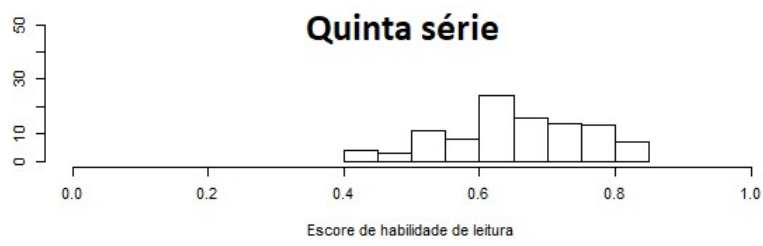

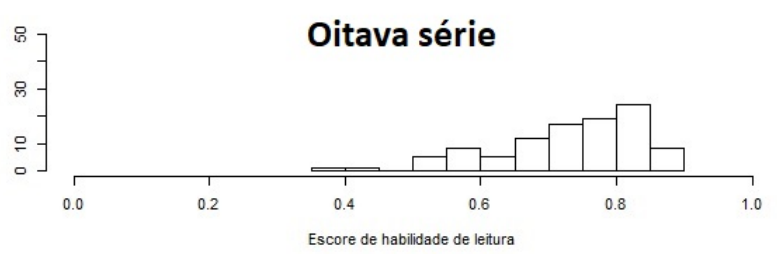

Figura 5.5: Histograma do escore de habilidade de leitura de 100 alunos participantes do estudo ECLS-K nos diferentes momentos de aferição.

Na Figura 5.5 encontram-se os histogramas do escore de habilidade de leitura mensurados em diferentes momentos do estudo, nos quais é notável que há diferença nos escores observados ao longo do tempo. Além disso, também se constata a presença de assimetria principalmente nas primeiras e últimas coletas.

Essas três características, não linearidade, heterogeneidade e assimetria, indicam a adequabilidade de utilização do método desenvolvido no presente capítulo para análise dos dados. Assim, com o propósito de exemplificar a aplicação desse método, o modelo de mistura beta não linear com efeitos aleatórios foi ajustado ao escore de habilidade de leitura coletado no estudo ECLS-K. 
Amostras dos dados de escore de habilidade de leitura do ECLS-K já foram analisadas em estudos anteriores por meio de modelos de mistura não lineares normais com efeitos aleatórios. Em Grimm et al. (2010) eles são ajustados por meio de equações estruturais e conclui-se que cada componente da mistura caracteriza um perfil de desenvolvimento da habilidade de leitura dos estudantes: o primeiro componente está associado a um desenvolvimento considerado padrão e o segundo componente caracteriza o perfil de desenvolvimento precoce. Em seguida, em Serang et al. (2015) e Serang et al. (2016), a estimação é efetuada com expansão em séries de Taylor e sob abordagem bayesiana no software OpenBUGS e também foram obtidos resultados que evidenciam a associação de componentes da mistura à perfis de desenvolvimento da habilidade de leitura dos estudantes.

Dos 21.409 estudantes que participaram da pesquisa apenas 2.053 não apresentaram dados faltantes nas variáveis em análise. Destes, foi selecionada uma amostra aleatória para compor a base de dados considerada nesta aplicação, procedimento esse utilizado em Grimm e Ram (2009), Grimm et al. (2010) e Serang et al. (2015). Devido ao tempo custo computacional, nesta aplicação foram ajustadas as observações de $N=100$ alunos.

O escore de habilidade de leitura $(y)$ é uma pontuação calculada através da teoria de resposta ao item e sintetiza inúmeras competências relacionadas à habilidade de leitura, como por exemplo reconhecimento de letras e palavras comuns, conhecimento de vocabulários, compreensão de gêneros literários e muitos outros. Para atender às restrições da distribuição beta, esse escore, que já é originalmente positivo, foi padronizado através de sua divisão pelo valor máximo delimitado no estudo. A idade $(x)$, em anos, dos estudantes no momento das aferições foi utilizada como variável explicativa e ela foi reescalonada subtraindo-se seu valor mínimo. Essa transformação foi efetuada para facilitar a interpretação dos parâmetros do modelo com relação à idade mínima observada na amostra.

Devido à recomendação das referências da área de que a escolha dos modelos não lineares ajustados deve se basear na aplicação, a função sistemática não linear utilizada é a mesma aplicada em Serang et al. (2015) e Serang et al. (2016). Do mesmo modo como efetuado em Serang et al.

(2015) e pelo embasamento dos especialistas, a estratégia empregada foi ajustar modelos com $K=1,2,3$ e função sistemática não linear $h_{\mu}$ conforme especificado na Equação (5.9).

Sejam $\boldsymbol{y}_{\boldsymbol{i}}=\left(y_{i 1}, \ldots, y_{i n_{i}}\right)^{\top}$ e $S_{i}$, respectivamente, o vetor de observações da variável resposta e a variável indicadora discreta do componente da mistura associados ao $i$-ésimo aluno, $i=1, \ldots, 100$, e considerando $Y_{i j} \mid \boldsymbol{b}_{i} \sim \sum_{S_{i}=1}^{K} \pi_{i S_{i}} \operatorname{Beta}\left(\mu_{S_{i}, i j}, \phi_{S_{i}}\right)$ e $\mu_{S_{i}, i j}=E\left(Y_{i j} \mid \boldsymbol{b}_{i}, S_{i}\right)$, o modelo de mistura beta não linear com efeitos aleatórios ajustado é expresso por:

$$
\log \left(\frac{\mu_{S_{i}, i j}}{1-\mu_{S_{i}, i j}}\right)=\left(\beta_{1}^{S_{i}}+b_{i 1}\right)+\left(\beta_{2}^{S_{i}}+b_{i 2}\right)\left(1-\exp \left\{-\left(\beta_{3}^{S_{i}}+b_{i 3}\right) x_{i j}\right\}\right)
$$

em que:

- $\boldsymbol{b}_{i}=\left(b_{i 1}, b_{i 2}, b_{i 3}\right)^{\top}$ é o vetor de efeitos aleatórios associado ao $i$-ésimo aluno;

- $\boldsymbol{b}_{1}, \boldsymbol{b}_{2}, \ldots, \boldsymbol{b}_{100}$ são independentes e identicamente distribuídos $N\left(0, \Sigma_{b}\right)$;

- $\Sigma_{b}=\left(\begin{array}{lll}\sigma_{\boldsymbol{b}, 11} & \sigma_{\boldsymbol{b}, 12} & \sigma_{\boldsymbol{b}, 13} \\ \sigma_{\boldsymbol{b}, 12} & \sigma_{\boldsymbol{b}, 22} & \sigma_{\boldsymbol{b}, 23} \\ \sigma_{\boldsymbol{b}, 13} & \sigma_{\boldsymbol{b}, 23} & \sigma_{\boldsymbol{b}, 33}\end{array}\right) \mathrm{e}$ 
- $\beta_{1}^{s}, \beta_{2}^{s}, \beta_{3}^{s}, \phi_{s}, \pi_{s}, s=1, \ldots, K$, e os elementos de $\Sigma_{b}$ são parâmetros do modelo.

Os modelos foram ajustados através do software OpenBUGS com $n_{i t}=100000, n_{b}=20000$, $n_{t}=20$ e $n_{c}=1$.

As distribuições a priori foram atribuídas de acordo com a Tabela 5.1. Dado que em Serang et al. (2016) são relatados problemas de convergência com a utilização do software OpenBUGS, os hiperparâmetros $\boldsymbol{\mu}_{\boldsymbol{\beta}^{s}}, s=1, \ldots, K$, foram definidos da seguinte forma: primeiramente as unidades amostrais foram classificadas com o uso do pacote $\mathrm{kml}$ (Genolini et al., 2015) do software $R$ (método de agrupamento K-médias para dados longitudinais) e para cada um desses $K$ grupos foram ajustados os modelos (5.9) com $K=1$. Por fim, as medianas a posteriori dos parâmetros nesses ajustes resultantes são os valores estabelecidos para $\boldsymbol{\mu}_{\boldsymbol{\beta}^{s}}, s=1, \ldots, K$.

Os critérios de seleção calculados para essa aplicação foram o EAIC, ECAIC e EBIC, pois são critérios análogos aos utilizados em Grimm et al. (2010) e Serang et al. (2016) e também devido ao cálculo das medidas $D I C_{3}$ e LPML ser computacionalmente intensivo e custoso devido à presença de integrais multivariadas. Os valores resultantes encontram-se na Tabela 5.4.

\begin{tabular}{c|ccc}
\hline & \multicolumn{3}{|c}{$K$} \\
\hline Critério & 1 & 2 & 3 \\
\hline EAIC & $-546,6976$ & $-2228,969$ & $-1572,718$ \\
ECAIC & $-546,2434$ & $-2228,072$ & $-1571,223$ \\
EBIC & $-501,1868$ & $-2160,703$ & $-1481,697$ \\
\hline
\end{tabular}

Tabela 5.4: Valores dos critérios de informação EAIC, ECAIC, EBIC para o ajuste do modelo (5.9) com $K=1,2,3$ para os dados de habilidade de leitura do estudo ECLS-K.

O modelo com $K=2$ é o mais adequado segundo os três critérios de seleção considerados. Esse valor de $K$ também foi o selecionado em Serang et al. (2016), no qual foi ajustado o modelo de mistura normal não linear com efeitos aleatórios para $K=1,2$. Os resultados inferenciais dos parâmetros do ajuste desse modelo selecionado encontram-se na Tabela 5.5 e estes valores foram utilizados para compor um dos cenários do estudo de simulação efetuado na Subseção 5.4.1. A análise preditiva a posteriori deste modelo considerando como medida de discrepância a média teve p-valor preditivo a posteriori resultante igual a 0,619 . Esse valor está próximo do valor 0,5 e, por isso, há evidências de que as especificações do modelo são condizentes com os dados. 


\begin{tabular}{c|c|c|c}
\hline Parâmetro & Estimativa & Desvio padrão & IC 95\% \\
\hline$\beta_{1}^{1}$ & $-1,590$ & 0,069 & $(-1,745 ;-1,476)$ \\
$\beta_{2}^{1}$ & 3,058 & 0,069 & $(2,930 ; 3,192)$ \\
$\beta_{3}^{1}$ & 0,943 & 0,001 & $(0,941 ; 0,944)$ \\
$\phi_{1}$ & 56,610 & 0,445 & $(55,300 ; 56,930)$ \\
$\beta_{1}^{2}$ & $-0,076$ & 0,313 & $(-0,667 ; 0,548)$ \\
$\beta_{2}^{2}$ & 0,224 & 0,319 & $(-0,412 ; 0,838)$ \\
$\beta_{3}^{2}$ & 1,497 & 0,316 & $(0,875 ; 2,122)$ \\
$\phi_{2}$ & 6,257 & 2,405 & $(1,996 ; 9,970)$ \\
$\pi_{1}$ & 0,899 & 0,021 & $(0,854 ; 0,937)$ \\
$\sigma_{b, 11}$ & 1,304 & 0,229 & $(0,934 ; 1,847)$ \\
$\sigma_{b, 12}$ & $-1,164$ & 0,211 & $(-1,657 ;-0,828)$ \\
$\sigma_{b, 13}$ & 0,997 & 0,158 & $(0,743 ; 1,353)$ \\
$\sigma_{b, 22}$ & 1,253 & 0,222 & $(0,902 ; 1,776)$ \\
$\sigma_{b, 23}$ & $-0,891$ & 0,150 & $(-1,239 ;-0,649)$ \\
$\sigma_{b, 33}$ & 0,848 & 0,123 & $(0,653 ; 1,128)$ \\
\hline
\end{tabular}

Tabela 5.5: Estimativas pontuais (mediana a posteriori) dos parâmetros, desvios padrões e intervalos de credibilidade (IC) de $95 \%$ do modelo final ajustado para os dados de habilidade de leitura do estudo ECLS-K.

As Figuras 5.6 e 5.7 apresentam as funções densidade de probabilidade a posteriori estimadas dos parâmetros.
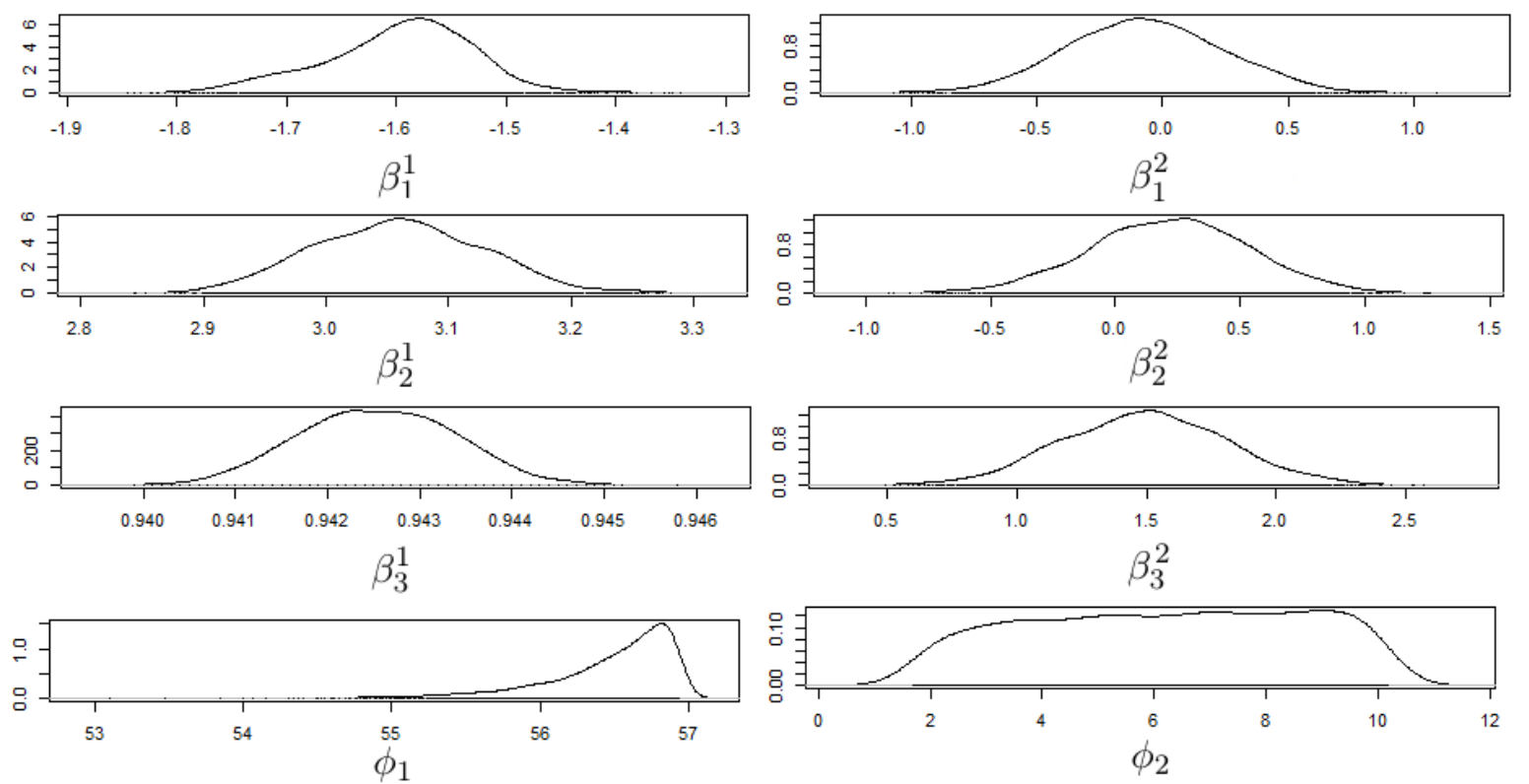

Figura 5.6: Gráfico da função densidade de probabilidade estimada dos parâmetros $\boldsymbol{\beta}^{\boldsymbol{s}}$ e $\phi_{s}, s=1,2$, do modelo final ajustado para os dados de habilidade de leitura do estudo ECLS-K. 

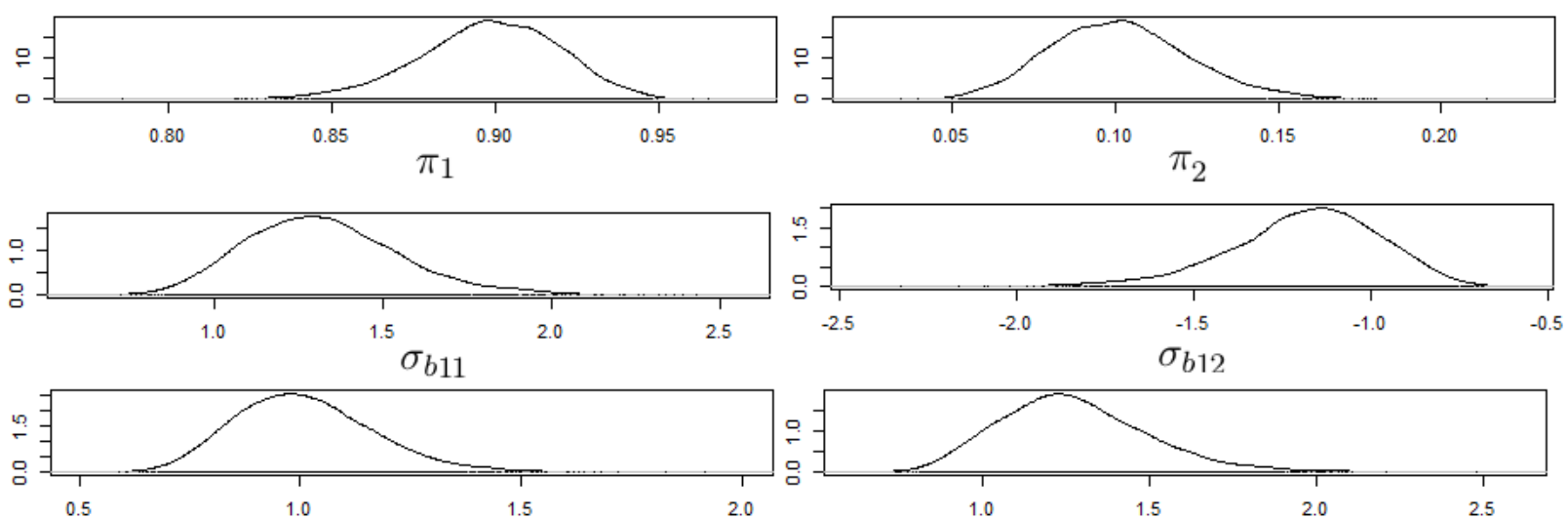

$\sigma_{b 13}$
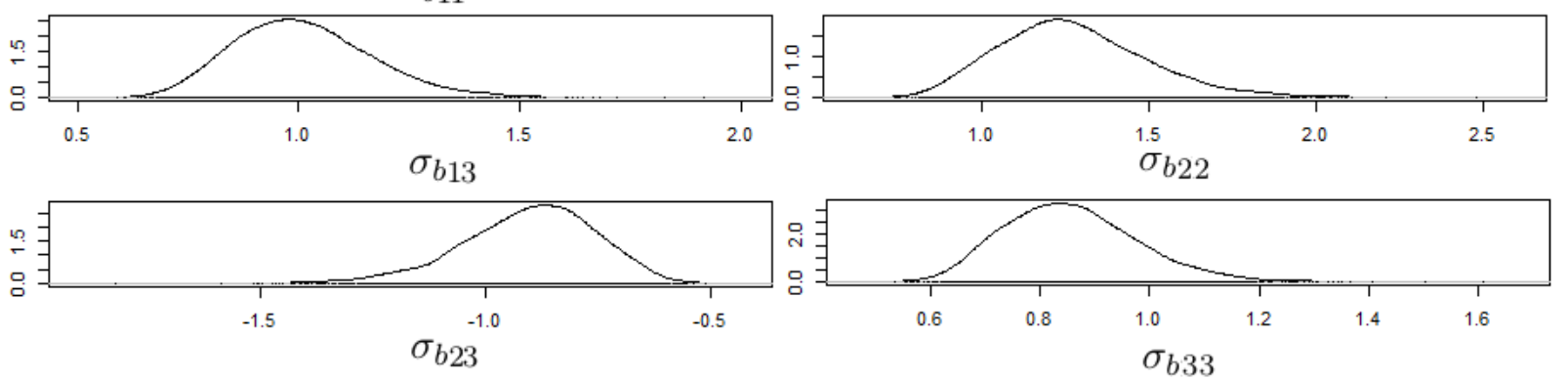

Figura 5.7: Gráfico da função densidade de probabilidade estimada dos parâmetros $\pi_{1}$ e $\Sigma_{b}$ do modelo final ajustado para os dados de habilidade de leitura do estudo ECLS-K.

As estimativas pontuais obtidas são concordantes com os resultados obtidos em análises anteriores desse estudo como Pianta et al. (2008), Grimm et al. (2010) e Serang et al. (2016) que ajustaram modelos de mistura não lineares normais com efeitos aleatórios por meio de diferentes abordagens inferenciais. Porém, o modelo com a variável resposta condicionalmente distribuída por misturas de distribuições beta é mais adequado para ajustar o escore de habilidade de leitura pois esta é uma variável que assume valores em um intervalo limitado conhecido e, dessa forma, os resultados inferenciais são mais robustos.

O modelo final obtido nesta seção contém dois componentes de mistura e, inclusive, a mediana a posteriori dos parâmetros $\pi_{1}$ e $\pi_{2}$ são iguais a 0,899 e 0,101 que são valores exatamente iguais aos obtidos em Serang et al. (2016) que ajustou um modelo com mesma função $h_{\mu}$ por meio do WinBUGS com tamanho de amostra maior. O primeiro componente está associado à estudantes que desenvolvem a habilidade de leitura de forma típica/padrão e o segundo se relaciona à estudantes que desenvolvem precocemente a habilidade de leitura, assim como observado em Grimm et al. (2010).

A averiguação de identificabilidade do modelo, característica essa que, segundo os estudos de simulação, está vinculada à distinção dos componentes da mistura na função densidade de probabilidade, foi efetuada por meio do data cloning. Os resultados para os números de clones cujo algoritmo convergiu encontram-se na Figura 5.8. 


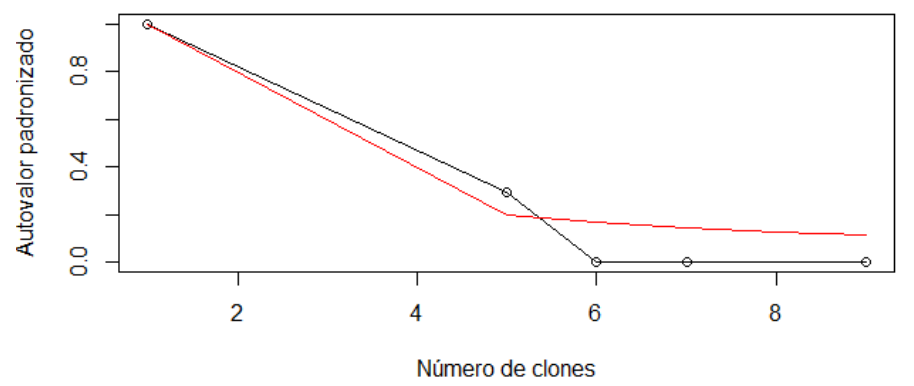

Figura 5.8: Gráfico de linhas do maior autovalor padronizado da matriz de variâncias e covariâncias a posteriori dos parâmetros do modelo de mistura beta misto não linear ajustado aos dados de habilidade de leitura do estudo ECLS-K.

Nesse caso o data cloning indica que não há evidências de falta de identificabilidade pois com o aumento do número de clones foi obtido decrescimento do autovalor padronizado de forma similar ao da curva de referência.

Portanto, por tudo o que foi apresentado nesta seção, a análise dos dados de desenvolvimento de habilidades de leitura por meio do ajuste de modelos de mistura beta não lineares mistos é muito favorável. Os resultados obtidos são concordantes com os alcançados em estudos anteriores como Serang et al. (2016) e perfis de desenvolvimento padrão e precoce foram identificados. Porém, os modelos com distribuição beta são mais oportunos e indicados pois a distribuição beta é mais adequada para variáveis resposta contínuas e limitadas, e as análises provenientes da inferência do modelo ajustado são mais robustas devido a essa adequabilidade da distribuição beta.

\subsection{Considerações finais}

O conteúdo deste capítulo discorre sobre os modelos de mistura beta não lineares mistos que são inéditos, tanto por abranger não linearidade dos parâmetros nos modelos de mistura beta quanto por incluirem efeitos aleatórios a estes modelos e, por fim, pelo seu aspecto inferencial sob a abordagem bayesiana.

Ressaltando que tanto na etapa de escolha da função não linear quanto no processo de atribuição das distribuições a priori, conhecimentos específicos de pesquisadores da área dos estudos são incorporados na análise dos dados e isso agrega ainda interpretabilidade no contexto da aplicação.

Com o intuito de avaliar o desempenho inferencial sob abordagem bayesiana foram delineados estudos de simulação com uma função não linear e dois cenários de valores dos parâmetros. Em vista da vasta gama de possíveis funções não lineares, esta foi selecionada com base na literatura de modelos de regressão beta não lineares de efeitos fixos, assim como os cenários estudados.

Os resultados apresentaram evidências para concluir que a estimação pontual dos parâmetros por meio da mediana a posteriori é eficiente, que os critérios de seleção do número de componentes de mistura possuem desempenhos diferentes dependendo da forma da função densidade da distribuição da variável resposta do modelo e que a averiguação de identificabilidade por meio do algoritmo data cloning indica que não há falhas de identificabilidade no modelo quando os os elementos da mistura são distinguíveis.

Conquanto os estudos de simulação tenham abrangido apenas duas funções não lineares e três cenários, as conclusões obtidas sinalizam que, em termos gerais, há grande viabilidade da aplicação da metodologia. 
Os modelos aqui especificados foram aplicados em dados reais de desenvolvimento de escore de habilidades de leitura ao longo de um período escolar que foram previamente analisados em outras pesquisas com outras metodologias que não levaram em conta que os dados são limitados. A função não linear empregada foi selecionada com base nessas referências prévias e os resultados foram compatíveis com as mesmas com um tamanho de amostra consideravelmente menor.

A execução dos algoritmos MCMC nos estudos de simulação e na aplicação a dados reais teve tempo computacional similar ao do modelo linear discorrido no Capítulo 3. Assim pode-se concluir, em vista da performance inferencial eficiente evidenciada tanto nos estudos de simulação quanto na aplicação a dados reais, da pertinência do emprego da distribuição beta e da relevância da utilização de funções não lineares, os modelos de mistura beta mistos não lineares com efeitos aleatórios possuem emprego vantajoso e uma ampla potencialidade de uso. 


\section{Capítulo 6}

\section{Conclusão}

O escopo deste capítulo é expor ao leitor, na Seção 6.1, um panorama de todo o conteúdo desenvolvido ao longo desta tese. Destas considerações, são identificados, na Seção 6.2, temas adicionais que se tem intenção de estudar em trabalhos futuros para aperfeiçoar e estender o conteúdo já apresentado e que de alguma maneira mostram a potencialidade da proposta abordada neste texto.

\subsection{Considerações Finais}

Nesta tese foi apresentada e explorada a metodologia original de modelos de mistura beta mistos que é efetivamente conveniente para a análise de dados com essas especificidades. Toda a metodologia exposta foi explorada sob abordagem bayesiana, como recomendado em inúmeras referências da temática, em função da complexidade de sua inferência.

Para considerar uma ampla abrangência com a máxima adequabilidade, foram propostos os modelos linear, com dispersão constante e variável, e não linear em relação aos parâmetros. A performance da aplicação destes modelos foi avaliada por meio de estudos de simulação e aplicações em dados reais.

Os estudos de simulação avaliaram três aspectos da inferência dos modelos especificados: desempenho da estimação pontual dos parâmetros por meio da mediana a posteriori, performance dos critérios de informação EAIC, ECAIC, EBIC, DIC 3 e LPML na seleção do número de componentes da mistura e diagnóstico de não identificabilidade por meio do algoritmo data cloning.

Os resultados desses estudos indicaram boa acurácia na estimação pontual dos parâmetros, sendo que em todas as réplicas os intervalos de credibilidade de $95 \%$ continham os verdadeiros valores dos parâmetros. $\mathrm{O} D I C_{3}$ e o EBIC foram, respectivamente, os critérios com melhor desempenho na seleção do número de elementos da mistura dos modelos linear com dispersão constante e não linear, enquanto que no modelo linear com dispersão variável nenhum dos critérios avaliados teve boa performance.

Ao utilizar o algoritmo data cloning concluiu-se que não há indícios de falta de identificabilidade de nenhum dos modelos e esse fato é muito favorável tendo em vista a complexidade dos modelos apresentados. Ademais, todos esses resultados são mais satisfatórios a medida que as densidades dos elementos da mistura são mais distinguíveis.

Especificamente para o modelo não linear, em que a gama de possíveis funções não lineares é vasta por essa função expressar características e padrões muito particulares das áreas das pesquisas, 
os estudos de simulação abrangeram uma função não linear com dois cenários. Porém, as conclusões obtidas sinalizam que, em termos gerais, há grande viabilidade da aplicação da metodologia.

Para ilustrar a aplicação da metodologia inédita desta tese, ao todo foram analisadas três bases de dados reais. A primeira aplicação trata de um estudo longitudinal relacionado ao risco de doenças cardiovasculares de pacientes diagnosticados com periodontite.

Foi ajustado o modelo de mistura beta misto linear com dispersão não variável para estudar a aglomeração esperada das empresas nacionais e estrangeiras de redes de fast food. Isso é razoável de se considerar pois as redes trabalham em um mesmo mercado e podem se aglomerar de acordo com critérios diferentes dependendo do cenário comercial da localidade e o uso de mistura resulta pertinente.

Por fim, uma base de dados de escore de habilidade de leitura ao longo da trajetória escolar de alunos foi estudada com o ajuste de um modelo de mistura beta misto não linear. Resumidamente, os resultados indicam a existência de duas subpopulações de estudantes, as quais se diferenciam pelos momentos de maior crecimento do escore avaliado.

Um tópico que pode dificultar a aplicação dessas técnicas é o tempo computacional da execução dos algoritmos MCMC para ajuste dos modelos. Entretanto, a performance inferencial eficiente evidenciada tanto nos estudos de simulação quanto na aplicação a dados reais, a pertinência do emprego da distribuição beta e, no caso do modelo não linear, a relevância de utilizar funções não lineares para expressar a relação entre a variável resposta e as variáveis explicativas, encorajam o uso dos modelos de mistura beta mistos com efeitos aleatórios. Inclusive porque essa questão de tempo computacional tende a ser cada vez menos custosa com o intenso progresso dos recursos computacionais.

Pode-se afirmar que neste trabalho foi apresentada uma metodologia que pode ser aplicada e estendida a outros modelos de mistura.

\subsection{Trabalhos futuros}

Os esforços empregados na especificação dos modelos de mistura beta mistos, na avaliação do seu desempenho inferencial e na ilustração de suas aplicações revelaram resultados promissores. Após a obtenção destes resultados, dentre os passos subsequentes para dar continuidade e enriquecer a temática desta tese podem-se citar, entre outros, como trabalhos futuros:

- Implementação e estudo do desempenho de outras técnicas de seleção do número de elementos da mistura dos modelos de mistura beta mistos;

- implementação e estudo do desempenho do ajuste de modelos de mistura beta mistos por meio de algoritmos MCMC que considerem o número de elementos da mistura como parâmetro no processo inferencial;

- estudo do desempenho inferencial dos modelos de mistura beta mistos para fins de classificação;

- estudo do desempenho inferencial dos modelos de mistura beta mistos com distribuições não normais para os efeitos aleatórios;

- desenvolvimento de resíduos e técnicas de diagnóstico específicas para este tipo de modelos; 
- desenvolvimento e implementação de técnicas alternativas de avaliação de identificabilidade dos modelos de mistura beta mistos;

- estudo de desempenho de softwares de implemtentação automatizada de inferência bayesiana como alternativa ao OpenBUGS e,

- implementação e estudo do desempenho inferencial de outras possíveis técnnicas de ajuste dos modelos tanto sob abordagem bayesiana como abordagem frequentista.

Portanto, pelos resultados obtidos e considerando os desafios da pesquisa, acredita-se que a proposta tem perspectivas muito promissoras de utilização e desenvolvimento futuro. 
104 CONCLUSÃO 


\section{Apêndice A}

\section{Tabelas com os Resultados de Medidas de Desempenho}

\section{A.1 Modelo linear com parâmetro de precisão constante}

\begin{tabular}{c|cccccccc}
\hline Parâmetro & $\beta_{1}^{1}$ & $\beta_{2}^{1}$ & $\beta_{1}^{2}$ & $\beta_{2}^{2}$ & $\phi$ & $\pi_{1}$ & $\pi_{2}$ & $\sigma_{b}$ \\
\hline Valor verdadeiro & -0.750 & 2 & -1.500 & 0.500 & 30 & 0.700 & 0.300 & 1 \\
\hline \hline \multicolumn{7}{c}{$\mathrm{N}=100, \mathrm{n}=5, \mathrm{R}=50$} \\
\hline Mediana a posteriori média & -0.623 & 1.810 & -1.430 & 0.891 & 44.100 & 0.771 & 0.229 & 0.948 \\
ER da mediana a posteriori & 0.035 & -0.152 & -0.293 & 1.530 & 0.462 & 0.100 & -0.234 & -0.044 \\
EAR da mediana a posteriori & 0.367 & 0.155 & 0.372 & 1.580 & 0.462 & 0.100 & 0.234 & 0.078 \\
REQM da mediana a posteriori & 0.305 & 0.428 & 0.795 & 1.050 & 14.000 & 0.071 & 0.071 & 0.095 \\
\hline \multicolumn{7}{c}{$\mathrm{N}=100, \mathrm{n}=10, \mathrm{R}=50$} \\
\hline Mediana a posteriori média & -0.593 & 1.850 & -1.500 & 1.110 & 41.400 & 0.756 & 0.244 & 0.949 \\
ER da mediana a posteriori & -0.018 & -0.160 & -0.167 & 1.490 & 0.381 & 0.074 & -0.172 & -0.038 \\
EAR da mediana a posteriori & 0.380 & 0.168 & 0.256 & 1.500 & 0.381 & 0.078 & 0.182 & 0.064 \\
REQM da mediana a posteriori $i$ & 0.329 & 0.498 & 0.582 & 0.921 & 11.800 & 0.060 & 0.060 & 0.074 \\
\hline \multicolumn{7}{c}{$\mathrm{N}=500, \mathrm{n}=5, \mathrm{R}=25$} \\
\hline Mediana a posteriori média & -0.665 & 1.960 & -1.520 & 0.696 & 30.900 & 0.720 & 0.280 & 1.020 \\
ER da mediana a posteriori & -0.114 & -0.020 & 0.025 & 0.340 & 0.030 & 0.034 & -0.080 & 0.025 \\
EAR da mediana a posteriori & 0.115 & 0.040 & 0.047 & 0.374 & 0.037 & 0.038 & 0.090 & 0.035 \\
REQM da mediana a posteriori $i$ & 0.097 & 0.104 & 0.095 & 0.219 & 1.320 & 0.035 & 0.035 & 0.047 \\
\hline \multicolumn{7}{c}{$\mathrm{N}=500, \mathrm{n}=10, \mathrm{R}=25$} \\
\hline Mediana a posteriori média & -0.508 & 1.780 & -1.680 & 1.320 & 27.300 & 0.664 & 0.336 & 1.010 \\
ER da mediana a posteriori & -0.238 & -0.121 & 0.045 & 1.680 & -0.094 & -0.043 & 0.100 & 0.015 \\
EAR da mediana a posteriori & 0.369 & 0.121 & 0.174 & 1.680 & 0.094 & 0.046 & 0.106 & 0.028 \\
REQM da mediana a posteriori & 0.298 & 0.276 & 0.382 & 0.858 & 2.890 & 0.039 & 0.039 & 0.037 \\
\hline
\end{tabular}

Tabela A.1: Resultados descritivos: ER, EAR e REQM, da mediana a posteriori dos parâmetros do modelo de mistura beta linear com efeitos aleatórios para a média dos estudos de simulação com cenário 1 e $\phi=30$ de $R$ bancos de dados com tamanhos amostrais $N=100,500$ e $n=5,10$. 


\begin{tabular}{|c|c|c|c|c|c|c|c|c|}
\hline Parâmetro & $\beta_{1}^{1}$ & $\beta_{2}^{1}$ & $\beta_{1}^{2}$ & $\beta_{2}^{2}$ & $\phi$ & $\pi_{1}$ & $\pi_{2}$ & $\sigma_{b}$ \\
\hline Valor verdadeiro & -0.750 & 2 & -1.500 & 0.500 & 50 & 0.700 & 0.300 & 1 \\
\hline \multicolumn{9}{|c|}{$\mathrm{N}=100, \mathrm{n}=5, \mathrm{R}=50$} \\
\hline Mediana a posteriori média & -0.617 & 1.770 & -1.410 & 1.000 & 65.800 & 0.764 & 0.236 & 0.948 \\
\hline ER da mediana a posteriori & 0.028 & -0.168 & -0.266 & 1.610 & 0.291 & 0.089 & -0.208 & -0.039 \\
\hline EAR da mediana a posteriori & 0.383 & 0.173 & 0.356 & 1.610 & 0.291 & 0.091 & 0.212 & 0.080 \\
\hline REQM da mediana a posteriori & 0.325 & 0.458 & 0.753 & 1.020 & 15.100 & 0.067 & 0.067 & 0.094 \\
\hline \multicolumn{9}{|c|}{$\mathrm{N}=100, \mathrm{n}=10, \mathrm{R}=50$} \\
\hline Mediana a posteriori média & -0.616 & 1.760 & -1.410 & 1.340 & 61.200 & 0.746 & 0.254 & 0.966 \\
\hline ER da mediana a posteriori & 0.027 & -0.207 & -0.216 & 1.790 & 0.234 & 0.058 & -0.135 & -0.020 \\
\hline EAR da mediana a posteriori & 0.401 & 0.211 & 0.279 & 1.790 & 0.235 & 0.072 & 0.169 & 0.060 \\
\hline REQM da mediana a posteriori & 0.346 & 0.582 & 0.602 & 1.030 & 13.000 & 0.055 & 0.055 & 0.072 \\
\hline \multicolumn{9}{|c|}{$\mathrm{N}=500, \mathrm{n}=5, \mathrm{R}=25$} \\
\hline Mediana a posteriori média & -0.614 & 1.920 & -1.580 & 0.973 & 46.700 & 0.695 & 0.305 & 1.010 \\
\hline ER da mediana a posteriori & -0.196 & -0.050 & 0.045 & 0.929 & -0.061 & -0.009 & 0.022 & 0.036 \\
\hline EAR da mediana a posteriori & 0.196 & 0.054 & 0.065 & 0.929 & 0.068 & 0.023 & 0.054 & 0.047 \\
\hline REQM da mediana a posteriori & 0.157 & 0.141 & 0.123 & 0.498 & 4.230 & 0.021 & 0.021 & 0.067 \\
\hline \multicolumn{9}{|c|}{$\mathrm{N}=500, \mathrm{n}=10, \mathrm{R}=25$} \\
\hline Mediana a posteriori média & -0.521 & 1.790 & -1.590 & 1.400 & 42.100 & 0.649 & 0.351 & 1.060 \\
\hline ER da mediana a posteriori & -0.299 & -0.106 & 0.064 & 1.760 & -0.161 & -0.073 & 0.171 & 0.064 \\
\hline EAR da mediana a posteriori & 0.299 & 0.106 & 0.066 & 1.760 & 0.161 & 0.074 & 0.172 & 0.064 \\
\hline REQM da mediana a posteriori & 0.231 & 0.218 & 0.123 & 0.884 & 8.160 & 0.060 & 0.060 & 0.068 \\
\hline
\end{tabular}

Tabela A.2: Resultados descritivos: ER, EAR e REQM, da mediana a posteriori dos parâmetros do modelo de mistura beta linear com efeitos aleatórios para a média dos estudos de simulação com cenário 1 e $\phi=50$ de $R$ bancos de dados com tamanhos amostrais $N=100,500$ e $n=5,10$.

\begin{tabular}{c|cccccccc}
\hline Parâmetro & $\beta_{1}^{1}$ & $\beta_{2}^{1}$ & $\beta_{1}^{2}$ & $\beta_{2}^{2}$ & $\phi$ & $\pi_{1}$ & $\pi_{2}$ & $\sigma_{b}$ \\
\hline Valor verdadeiro & -0.750 & 2 & -1.500 & 0.500 & 70 & 0.700 & 0.300 & 1 \\
\hline \hline \multicolumn{7}{c}{$\mathrm{N}=100, \mathrm{n}=5, \mathrm{R}=50$} \\
\hline Mediana a posteriori média & -0.606 & 1.780 & -1.430 & 1.130 & 84.700 & 0.752 & 0.248 & 0.959 \\
ER da mediana a posteriori & 0.046 & -0.189 & -0.240 & 1.640 & 0.175 & 0.069 & -0.160 & -0.035 \\
EAR da mediana a posteriori & 0.418 & 0.190 & 0.318 & 1.650 & 0.186 & 0.082 & 0.191 & 0.075 \\
REQM da mediana a posteriori & 0.359 & 0.527 & 0.695 & 0.988 & 14.000 & 0.061 & 0.061 & 0.089 \\
\hline \multicolumn{7}{c}{$\mathrm{N}=100, \mathrm{n}=10, \mathrm{R}=50$} \\
\hline Mediana a posteriori média & -0.523 & 1.750 & -1.480 & 1.350 & 75.700 & 0.736 & 0.264 & 0.961 \\
ER da mediana a posteriori & -0.069 & -0.216 & -0.162 & 1.920 & 0.099 & 0.039 & -0.091 & -0.027 \\
EAR da mediana a posteriori & 0.442 & 0.217 & 0.232 & 1.920 & 0.126 & 0.060 & 0.139 & 0.067 \\
REQM da mediana a posteriori & 0.372 & 0.604 & 0.534 & 1.030 & 10.800 & 0.047 & 0.047 & 0.080 \\
\hline \multicolumn{7}{c}{$\mathrm{N}=500, \mathrm{n}=5, \mathrm{R}=25$} \\
\hline Mediana a posteriori média & -0.526 & 1.810 & -1.640 & 1.240 & 56.400 & 0.680 & 0.320 & 1.020 \\
ER da mediana a posteriori & -0.279 & -0.092 & 0.095 & 1.400 & -0.186 & -0.032 & 0.074 & 0.027 \\
EAR da mediana a posteriori & 0.279 & 0.092 & 0.099 & 1.400 & 0.189 & 0.038 & 0.088 & 0.041 \\
REQM da mediana a posteriori & 0.222 & 0.199 & 0.173 & 0.720 & 14.200 & 0.031 & 0.031 & 0.056 \\
\hline \multicolumn{7}{c}{$\mathrm{N}=500, \mathrm{n}=10, \mathrm{R}=25$} \\
\hline Mediana a posteriori média & -0.522 & 1.750 & -1.610 & 1.440 & 54.000 & 0.653 & 0.347 & 1.070 \\
ER da mediana a posteriori & -0.298 & -0.125 & 0.075 & 1.880 & -0.231 & -0.066 & 0.154 & 0.068 \\
EAR da mediana a posteriori & 0.298 & 0.125 & 0.075 & 1.880 & 0.231 & 0.066 & 0.155 & 0.068 \\
REQM da mediana a posteriori & 0.228 & 0.252 & 0.127 & 0.939 & 16.400 & 0.049 & 0.049 & 0.075 \\
\hline
\end{tabular}

Tabela A.3: Resultados descritivos: ER, EAR e REQM, da mediana a posteriori dos parâmetros do modelo de mistura beta linear com efeitos aleatórios para a média dos estudos de simulação com cenário 1 e $\phi=70$ de $R$ bancos de dados com tamanhos amostrais $N=100,500$ e $n=5,10$. 


\begin{tabular}{|c|c|c|c|c|c|c|c|c|}
\hline Parâmetro & $\beta_{1}^{1}$ & $\beta_{2}^{1}$ & $\beta_{1}^{2}$ & $\beta_{2}^{2}$ & $\phi$ & $\pi_{1}$ & $\pi_{2}$ & $\sigma_{b}$ \\
\hline Valor verdadeiro & -1.500 & 1 & -0.500 & 3 & 30 & 0.700 & 0.300 & 1 \\
\hline \multicolumn{9}{|c|}{$\mathrm{N}=100, \mathrm{n}=5, \mathrm{R}=50$} \\
\hline Mediana a posteriori média & -1.470 & 1.120 & -0.303 & 2.550 & 40.900 & 0.760 & 0.240 & 0.967 \\
\hline ER da mediana a posteriori & -0.018 & 0.134 & -0.406 & -0.161 & 0.328 & 0.087 & -0.204 & -0.021 \\
\hline EAR da mediana a posteriori & 0.057 & 0.147 & 0.407 & 0.162 & 0.328 & 0.087 & 0.204 & 0.081 \\
\hline REQM da mediana a posteriori & 0.104 & 0.183 & 0.234 & 0.561 & 10.500 & 0.064 & 0.064 & 0.108 \\
\hline \multicolumn{9}{|c|}{$\mathrm{N}=100, \mathrm{n}=10, \mathrm{R}=50$} \\
\hline Mediana a posteriori média & -1.540 & 1.220 & -0.394 & 2.190 & 34.300 & 0.742 & 0.258 & 1.020 \\
\hline ER da mediana a posteriori & 0.023 & 0.242 & -0.195 & -0.276 & 0.162 & 0.052 & -0.122 & 0.007 \\
\hline EAR da mediana a posteriori & 0.068 & 0.244 & 0.280 & 0.276 & 0.163 & 0.057 & 0.134 & 0.062 \\
\hline REQM da mediana a posteriori & 0.142 & 0.343 & 0.180 & 0.879 & 5.940 & 0.044 & 0.044 & 0.077 \\
\hline \multicolumn{9}{|c|}{$\mathrm{N}=500, \mathrm{n}=5, \mathrm{R}=25$} \\
\hline Mediana a posteriori média & -1.580 & 1.070 & -0.422 & 2.500 & 26.800 & 0.706 & 0.294 & 1.020 \\
\hline ER da mediana a posteriori & 0.046 & 0.090 & -0.194 & -0.146 & -0.102 & 0.005 & -0.012 & 0.029 \\
\hline EAR da mediana a posteriori & 0.049 & 0.100 & 0.194 & 0.146 & 0.102 & 0.027 & 0.064 & 0.040 \\
\hline REQM da mediana a posteriori & 0.088 & 0.128 & 0.116 & 0.487 & 3.350 & 0.022 & 0.022 & 0.056 \\
\hline \multicolumn{9}{|c|}{$\mathrm{N}=500, \mathrm{n}=10, \mathrm{R}=25$} \\
\hline Mediana a posteriori média & -1.700 & 1.230 & -0.414 & 2.060 & 23.800 & 0.667 & 0.333 & 1.040 \\
\hline ER da mediana a posteriori & 0.124 & 0.241 & -0.169 & -0.315 & -0.221 & -0.052 & 0.122 & 0.041 \\
\hline EAR da mediana a posteriori & 0.124 & 0.241 & 0.169 & 0.315 & 0.221 & 0.052 & 0.122 & 0.041 \\
\hline REQM da mediana a posteriori & 0.195 & 0.246 & 0.106 & 0.957 & 6.740 & 0.041 & 0.041 & 0.054 \\
\hline
\end{tabular}

Tabela A.4: Resultados descritivos: ER, EAR e REQM, da mediana a posteriori dos parâmetros do modelo de mistura beta linear com efeitos aleatórios para a média dos estudos de simulação com cenário 2 e $\phi=30$ de $R$ bancos de dados com tamanhos amostrais $N=100,500$ e $n=5,10$.

\begin{tabular}{c|cccccccc}
\hline Parâmetro & $\beta_{1}^{1}$ & $\beta_{2}^{1}$ & $\beta_{1}^{2}$ & $\beta_{2}^{2}$ & $\phi$ & $\pi_{1}$ & $\pi_{2}$ & $\sigma_{b}$ \\
\hline Valor verdadeiro & -1.500 & 1 & -0.500 & 3 & 50 & 0.700 & 0.300 & 1 \\
\hline \hline \multicolumn{7}{c}{$\mathrm{N}=100, \mathrm{n}=5, \mathrm{R}=50$} \\
\hline Mediana a posteriori média & -1.530 & 1.150 & -0.297 & 2.260 & 54.100 & 0.747 & 0.253 & 0.968 \\
ER da mediana a posteriori & 0.013 & 0.181 & -0.376 & -0.243 & 0.100 & 0.070 & -0.162 & -0.015 \\
EAR da mediana a posteriori & 0.063 & 0.188 & 0.390 & 0.243 & 0.143 & 0.070 & 0.164 & 0.081 \\
REQM da mediana a posteriori & 0.110 & 0.222 & 0.221 & 0.774 & 8.760 & 0.053 & 0.053 & 0.107 \\
\hline \multicolumn{7}{c}{$\mathrm{N}=100, \mathrm{n}=10, \mathrm{R}=50$} \\
\hline Mediana a posteriori média & -1.560 & 1.250 & -0.425 & 2.030 & 47.700 & 0.728 & 0.272 & 1.000 \\
ER da mediana a posteriori & 0.040 & 0.231 & -0.180 & -0.313 & -0.020 & 0.042 & -0.098 & 0.014 \\
EAR da mediana a posteriori & 0.054 & 0.233 & 0.233 & 0.313 & 0.100 & 0.044 & 0.102 & 0.059 \\
REQM da mediana a posteriori & 0.099 & 0.265 & 0.146 & 0.969 & 6.220 & 0.034 & 0.034 & 0.078 \\
\hline \multicolumn{7}{c}{$\mathrm{N}=500, \mathrm{n}=5, \mathrm{R}=25$} \\
\hline Mediana a posteriori média & -1.650 & 1.200 & -0.343 & 2.170 & 38.500 & 0.688 & 0.312 & 0.994 \\
ER da mediana a posteriori & 0.088 & 0.200 & -0.221 & -0.269 & -0.231 & -0.028 & 0.066 & 0.0004 \\
EAR da mediana a posteriori & 0.090 & 0.200 & 0.277 & 0.269 & 0.231 & 0.028 & 0.066 & 0.047 \\
REQM da mediana a posteriori $i$ & 0.147 & 0.210 & 0.153 & 0.824 & 12.300 & 0.026 & 0.026 & 0.064 \\
\hline \multicolumn{7}{c}{$\mathrm{N}=500, \mathrm{n}=10, \mathrm{R}=25$} \\
\hline Mediana a posteriori média & -1.680 & 1.290 & -0.448 & 1.930 & 34.600 & 0.646 & 0.354 & 1.060 \\
ER da mediana a posteriori & 0.132 & 0.301 & -0.101 & -0.363 & -0.298 & -0.078 & 0.182 & 0.082 \\
EAR da mediana a posteriori & 0.132 & 0.301 & 0.104 & 0.363 & 0.298 & 0.078 & 0.182 & 0.082 \\
REQM da mediana a posteriori & 0.202 & 0.309 & 0.059 & 1.090 & 15.100 & 0.055 & 0.055 & 0.096 \\
\hline
\end{tabular}

Tabela A.5: Resultados descritivos: ER, EAR e REQM, da mediana a posteriori dos parâmetros do modelo de mistura beta linear com efeitos aleatórios para a média dos estudos de simulação com cenário 2 e $\phi=50$ de $R$ bancos de dados com tamanhos amostrais $N=100,500$ e $n=5,10$. 


\begin{tabular}{|c|c|c|c|c|c|c|c|c|}
\hline Parâmetro & $\beta_{1}^{1}$ & $\beta_{2}^{1}$ & $\beta_{1}^{2}$ & $\beta_{2}^{2}$ & $\phi$ & $\pi_{1}$ & $\pi_{2}$ & $\sigma_{b}$ \\
\hline Valor verdadeiro & -1.500 & 1 & -0.500 & 3 & 70 & 0.700 & 0.300 & 1 \\
\hline \multicolumn{9}{|c|}{$\mathrm{N}=100, \mathrm{n}=5, \mathrm{R}=50$} \\
\hline Mediana a posteriori média & -1.550 & 1.240 & -0.336 & 2.100 & 68.300 & 0.742 & 0.258 & 0.987 \\
\hline ER da mediana a posteriori & 0.029 & 0.208 & -0.342 & -0.284 & -0.048 & 0.059 & -0.137 & -0.005 \\
\hline EAR da mediana a posteriori & 0.057 & 0.210 & 0.349 & 0.284 & 0.130 & 0.059 & 0.137 & 0.083 \\
\hline REQM da mediana a posteriori & 0.106 & 0.239 & 0.201 & 0.887 & 10.700 & 0.045 & 0.045 & 0.107 \\
\hline \multicolumn{9}{|c|}{$\mathrm{N}=100, \mathrm{n}=10, \mathrm{R}=50$} \\
\hline Mediana a posteriori média & -1.580 & 1.270 & -0.402 & 1.960 & 56.400 & 0.730 & 0.270 & 1.020 \\
\hline ER da mediana a posteriori & 0.039 & 0.254 & -0.159 & -0.320 & -0.132 & 0.041 & -0.095 & 0.029 \\
\hline EAR da mediana a posteriori & 0.065 & 0.259 & 0.217 & 0.320 & 0.181 & 0.043 & 0.100 & 0.065 \\
\hline REQM da mediana a posteriori & 0.115 & 0.293 & 0.128 & 1.000 & 13.900 & 0.036 & 0.036 & 0.082 \\
\hline \multicolumn{9}{|c|}{$\mathrm{N}=500, \mathrm{n}=5, \mathrm{R}=25$} \\
\hline Mediana a posteriori média & -1.670 & 1.240 & -0.448 & 1.980 & 50.400 & 0.646 & 0.354 & 1.050 \\
\hline ER da mediana a posteriori & 0.119 & 0.247 & -0.100 & -0.342 & -0.252 & -0.071 & 0.165 & 0.066 \\
\hline EAR da mediana a posteriori & 0.119 & 0.247 & 0.133 & 0.342 & 0.252 & 0.071 & 0.165 & 0.079 \\
\hline REQM da mediana a posteriori & 0.188 & 0.254 & 0.075 & 1.030 & 18.200 & 0.053 & 0.053 & 0.100 \\
\hline \multicolumn{9}{|c|}{$\mathrm{N}=500, \mathrm{n}=10, \mathrm{R}=25$} \\
\hline Mediana a posteriori média & -1.690 & 1.310 & -0.478 & 1.920 & 50.100 & 0.644 & 0.356 & 1.100 \\
\hline ER da mediana a posteriori & 0.128 & 0.315 & -0.021 & -0.363 & -0.282 & -0.081 & 0.189 & 0.108 \\
\hline EAR da mediana a posteriori & 0.128 & 0.315 & 0.096 & 0.363 & 0.282 & 0.081 & 0.189 & 0.108 \\
\hline REQM da mediana a posteriori & 0.198 & 0.320 & 0.054 & 1.090 & 19.800 & 0.059 & 0.059 & 0.116 \\
\hline
\end{tabular}

Tabela A.6: Resultados descritivos: ER, EAR e REQM, da mediana a posteriori dos parâmetros do modelo de mistura beta linear com efeitos aleatórios para a média dos estudos de simulação com cenário 2 e $\phi=70$ de $R$ bancos de dados com tamanhos amostrais $N=100,500$ e $n=5,10$.

\begin{tabular}{|c|c|c|c|c|c|c|c|c|}
\hline Parâmetro & $\beta_{1}^{1}$ & $\beta_{2}^{1}$ & $\beta_{1}^{2}$ & $\beta_{2}^{2}$ & $\phi$ & $\pi_{1}$ & $\pi_{2}$ & $\sigma_{b}$ \\
\hline Valor verdadeiro & -2 & 0.500 & -0.500 & 3 & 30 & 0.700 & 0.300 & 1 \\
\hline \multicolumn{9}{|c|}{$\mathrm{N}=100, \mathrm{n}=5, \mathrm{R}=50$} \\
\hline Mediana a posteriori média & -2.010 & 0.745 & -0.381 & 2.270 & 32.800 & 0.753 & 0.247 & 1.030 \\
\hline ER da mediana a posteriori & 0.005 & 0.539 & -0.181 & -0.247 & 0.113 & 0.076 & -0.177 & 0.069 \\
\hline EAR da mediana a posteriori & 0.033 & 0.543 & 0.296 & 0.247 & 0.144 & 0.076 & 0.177 & 0.115 \\
\hline REQM da mediana a posteriori & 0.081 & 0.323 & 0.186 & 0.773 & 5.550 & 0.055 & 0.055 & 0.165 \\
\hline \multicolumn{9}{|c|}{$\mathrm{N}=100, \mathrm{n}=10, \mathrm{R}=50$} \\
\hline Mediana a posteriori média & -2.010 & 0.787 & -0.554 & 2.070 & 30.200 & 0.737 & 0.263 & 1.160 \\
\hline ER da mediana a posteriori & 0.009 & 0.622 & 0.127 & -0.313 & 0.008 & 0.054 & -0.126 & 0.150 \\
\hline EAR da mediana a posteriori & 0.031 & 0.622 & 0.199 & 0.313 & 0.070 & 0.054 & 0.126 & 0.153 \\
\hline REQM da mediana a posteriori & 0.076 & 0.346 & 0.127 & 0.964 & 2.650 & 0.041 & 0.041 & 0.185 \\
\hline \multicolumn{9}{|c|}{$\mathrm{N}=500, \mathrm{n}=5, \mathrm{R}=25$} \\
\hline Mediana a posteriori média & -2.120 & 0.770 & -0.404 & 2.270 & 22.500 & 0.693 & 0.307 & 1.070 \\
\hline ER da mediana a posteriori & 0.059 & 0.525 & -0.128 & -0.240 & -0.214 & -0.0001 & 0.0001 & 0.084 \\
\hline EAR da mediana a posteriori & 0.059 & 0.525 & 0.155 & 0.240 & 0.214 & 0.021 & 0.049 & 0.092 \\
\hline REQM da mediana a posteriori & 0.130 & 0.267 & 0.094 & 0.740 & 7.090 & 0.015 & 0.015 & 0.122 \\
\hline \multicolumn{9}{|c|}{$\mathrm{N}=500, \mathrm{n}=10, \mathrm{R}=25$} \\
\hline Mediana a posteriori média & -2.100 & 0.856 & -0.574 & 2.080 & 21.200 & 0.683 & 0.317 & 1.160 \\
\hline ER da mediana a posteriori & 0.052 & 0.694 & 0.138 & -0.319 & -0.292 & -0.027 & 0.064 & 0.147 \\
\hline EAR da mediana a posteriori & 0.052 & 0.694 & 0.166 & 0.319 & 0.292 & 0.028 & 0.066 & 0.147 \\
\hline REQM da mediana a posteriori & 0.117 & 0.351 & 0.093 & 0.968 & 8.920 & 0.023 & 0.023 & 0.157 \\
\hline
\end{tabular}

Tabela A.7: Resultados descritivos: ER, EAR e REQM, da mediana a posteriori dos parâmetros do modelo de mistura beta linear com efeitos aleatórios para a média dos estudos de simulação com cenário 3 e $\phi=30$ de $R$ bancos de dados com tamanhos amostrais $N=100,500$ e $n=5,10$. 


\begin{tabular}{|c|c|c|c|c|c|c|c|c|}
\hline Parâmetro & $\beta_{1}^{1}$ & $\beta_{2}^{1}$ & $\beta_{1}^{2}$ & $\beta_{2}^{2}$ & $\phi$ & $\pi_{1}$ & $\pi_{2}$ & $\sigma_{b}$ \\
\hline Valor verdadeiro & -2 & 0.500 & -0.500 & 3 & 50 & 0.700 & 0.300 & 1 \\
\hline \multicolumn{9}{|c|}{$\mathrm{N}=100, \mathrm{n}=5, \mathrm{R}=50$} \\
\hline Mediana a posteriori média & -2.010 & 0.780 & -0.434 & 2.190 & 45.100 & 0.750 & 0.250 & 1.060 \\
\hline ER da mediana a posteriori & 0.009 & 0.584 & -0.121 & -0.286 & -0.064 & 0.068 & -0.159 & 0.087 \\
\hline EAR da mediana a posteriori & 0.035 & 0.584 & 0.246 & 0.286 & 0.158 & 0.068 & 0.159 & 0.121 \\
\hline REQM da mediana a posteriori & 0.086 & 0.327 & 0.161 & 0.886 & 9.160 & 0.051 & 0.051 & 0.159 \\
\hline \multicolumn{9}{|c|}{$\mathrm{N}=100, \mathrm{n}=10, \mathrm{R}=50$} \\
\hline Mediana a posteriori média & -2.020 & 0.822 & -0.589 & 2.030 & 41.800 & 0.734 & 0.266 & 1.140 \\
\hline ER da mediana a posteriori & 0.005 & 0.636 & 0.151 & -0.328 & -0.149 & 0.051 & -0.120 & 0.158 \\
\hline EAR da mediana a posteriori & 0.032 & 0.636 & 0.258 & 0.328 & 0.168 & 0.051 & 0.120 & 0.158 \\
\hline REQM da mediana a posteriori & 0.080 & 0.350 & 0.156 & 1.010 & 9.350 & 0.039 & 0.039 & 0.185 \\
\hline \multicolumn{9}{|c|}{$\mathrm{N}=500, \mathrm{n}=5, \mathrm{R}=25$} \\
\hline Mediana a posteriori média & -2.140 & 0.824 & -0.465 & 2.060 & 33.200 & 0.689 & 0.311 & 1.120 \\
\hline ER da mediana a posteriori & 0.072 & 0.642 & -0.065 & -0.307 & -0.303 & -0.017 & 0.040 & 0.136 \\
\hline EAR da mediana a posteriori & 0.072 & 0.642 & 0.126 & 0.307 & 0.303 & 0.025 & 0.059 & 0.136 \\
\hline REQM da mediana a posteriori & 0.151 & 0.325 & 0.081 & 0.932 & 16.100 & 0.021 & 0.021 & 0.157 \\
\hline \multicolumn{9}{|c|}{$\mathrm{N}=500, \mathrm{n}=10, \mathrm{R}=25$} \\
\hline Mediana a posteriori média & -2.100 & 0.874 & -0.563 & 2.070 & 31.300 & 0.685 & 0.315 & 1.160 \\
\hline ER da mediana a posteriori & 0.058 & 0.725 & 0.158 & -0.329 & -0.349 & -0.021 & 0.049 & 0.182 \\
\hline EAR da mediana a posteriori & 0.058 & 0.725 & 0.158 & 0.329 & 0.349 & 0.025 & 0.058 & 0.182 \\
\hline REQM da mediana a posteriori & 0.121 & 0.368 & 0.090 & 0.998 & 17.600 & 0.019 & 0.019 & 0.192 \\
\hline
\end{tabular}

Tabela A.8: Resultados descritivos: ER, EAR e REQM, da mediana a posteriori dos parâmetros do modelo de mistura beta linear com efeitos aleatórios para a média dos estudos de simulação com cenário 3 e $\phi=50$ de $R$ bancos de dados com tamanhos amostrais $N=100,500$ e $n=5,10$.

\begin{tabular}{|c|c|c|c|c|c|c|c|c|}
\hline Parâmetro & $\beta_{1}^{1}$ & $\beta_{2}^{1}$ & $\beta_{1}^{2}$ & $\beta_{2}^{2}$ & $\phi$ & $\pi_{1}$ & $\pi_{2}$ & $\sigma_{b}$ \\
\hline Valor verdadeiro & -2 & 0.500 & -0.500 & 3 & 70 & 0.700 & 0.300 & 1 \\
\hline \multicolumn{9}{|c|}{$\mathrm{N}=100, \mathrm{n}=5, \mathrm{R}=50$} \\
\hline Mediana a posteriori média & -2.020 & 0.823 & -0.485 & 2.060 & 54.300 & 0.741 & 0.259 & 1.110 \\
\hline ER da mediana a posteriori & 0.012 & 0.626 & -0.019 & -0.311 & -0.151 & 0.060 & -0.140 & 0.125 \\
\hline EAR da mediana a posteriori & 0.032 & 0.626 & 0.232 & 0.311 & 0.201 & 0.060 & 0.140 & 0.129 \\
\hline REQM da mediana a posteriori & 0.078 & 0.343 & 0.151 & 0.964 & 15.100 & 0.045 & 0.045 & 0.166 \\
\hline \multicolumn{9}{|c|}{$\mathrm{N}=100, \mathrm{n}=10, \mathrm{R}=50$} \\
\hline Mediana a posteriori média & -1.990 & 0.858 & -0.610 & 2.030 & 51.200 & 0.736 & 0.264 & 1.190 \\
\hline ER da mediana a posteriori & 0.001 & 0.718 & 0.187 & -0.338 & -0.234 & 0.053 & -0.123 & 0.184 \\
\hline EAR da mediana a posteriori & 0.029 & 0.718 & 0.278 & 0.338 & 0.238 & 0.053 & 0.124 & 0.184 \\
\hline REQM da mediana a posteriori & 0.074 & 0.387 & 0.164 & 1.040 & 17.200 & 0.040 & 0.040 & 0.206 \\
\hline \multicolumn{9}{|c|}{$\mathrm{N}=500, \mathrm{n}=5, \mathrm{R}=25$} \\
\hline Mediana a posteriori média & -2.130 & 0.861 & -0.544 & 2.030 & 50.100 & 0.684 & 0.316 & 1.180 \\
\hline ER da mediana a posteriori & 0.076 & 0.712 & 0.053 & -0.323 & -0.256 & -0.025 & 0.058 & 0.187 \\
\hline EAR da mediana a posteriori & 0.076 & 0.712 & 0.135 & 0.323 & 0.256 & 0.029 & 0.068 & 0.187 \\
\hline REQM da mediana a posteriori & 0.160 & 0.363 & 0.077 & 0.982 & 18.400 & 0.025 & 0.025 & 0.198 \\
\hline \multicolumn{9}{|c|}{$\mathrm{N}=500, \mathrm{n}=10, \mathrm{R}=25$} \\
\hline Mediana a posteriori média & -2.130 & 0.893 & -0.626 & 2.020 & 50.100 & 0.681 & 0.319 & 1.210 \\
\hline ER da mediana a posteriori & 0.070 & 0.736 & 0.243 & -0.334 & -0.284 & -0.030 & 0.070 & 0.222 \\
\hline EAR da mediana a posteriori & 0.070 & 0.736 & 0.243 & 0.334 & 0.284 & 0.030 & 0.070 & 0.222 \\
\hline REQM da mediana a posteriori & 0.150 & 0.373 & 0.142 & 1.010 & 19.900 & 0.024 & 0.024 & 0.232 \\
\hline
\end{tabular}

Tabela A.9: Resultados descritivos: ER, EAR e REQM, da mediana a posteriori dos parâmetros do modelo de mistura beta linear com efeitos aleatórios para a média dos estudos de simulação com cenário 3 e $\phi=70$ de $R$ bancos de dados com tamanhos amostrais $N=100,500$ e $n=5,10$. 


\begin{tabular}{|c|c|c|c|c|c|c|c|c|}
\hline Parâmetro & $\beta_{1}^{1}$ & $\beta_{2}^{1}$ & $\beta_{1}^{2}$ & $\beta_{2}^{2}$ & $\phi$ & $\pi_{1}$ & $\pi_{2}$ & $\sigma_{b}$ \\
\hline Valor verdadeiro & -1 & 3 & -3 & 1 & 30 & 0.700 & 0.300 & 1 \\
\hline \multicolumn{9}{|c|}{$\mathrm{N}=100, \mathrm{n}=5, \mathrm{R}=50$} \\
\hline Mediana a posteriori média & -0.890 & 2.880 & -2.800 & 2.080 & 40.100 & 0.749 & 0.251 & 1.290 \\
\hline ER da mediana a posteriori & -0.093 & -0.043 & -0.086 & 1.020 & 0.329 & 0.070 & -0.164 & 0.288 \\
\hline EAR da mediana a posteriori & 0.157 & 0.048 & 0.092 & 1.020 & 0.329 & 0.070 & 0.164 & 0.288 \\
\hline REQM da mediana a posteriori & 0.233 & 0.174 & 0.454 & 1.060 & 10.300 & 0.052 & 0.052 & 0.329 \\
\hline \multicolumn{9}{|c|}{$\mathrm{N}=100, \mathrm{n}=10, \mathrm{R}=50$} \\
\hline Mediana a posteriori média & -0.915 & 2.860 & -2.790 & 2.120 & 38.200 & 0.751 & 0.249 & 1.320 \\
\hline ER da mediana a posteriori & -0.068 & -0.048 & -0.069 & 1.120 & 0.271 & 0.072 & -0.168 & 0.335 \\
\hline EAR da mediana a posteriori & 0.102 & 0.048 & 0.077 & 1.120 & 0.271 & 0.072 & 0.168 & 0.335 \\
\hline REQM da mediana a posteriori & 0.122 & 0.163 & 0.281 & 1.140 & 8.440 & 0.053 & 0.053 & 0.349 \\
\hline \multicolumn{9}{|c|}{$\mathrm{N}=500, \mathrm{n}=5, \mathrm{R}=25$} \\
\hline Mediana a posteriori média & -0.885 & 2.880 & -2.820 & 2.110 & 28.300 & 0.663 & 0.337 & 1.280 \\
\hline ER da mediana a posteriori & -0.122 & -0.038 & -0.053 & 1.110 & -0.070 & -0.053 & 0.124 & 0.277 \\
\hline EAR da mediana a posteriori & 0.122 & 0.039 & 0.061 & 1.110 & 0.071 & 0.053 & 0.124 & 0.277 \\
\hline REQM da mediana a posteriori & 0.131 & 0.137 & 0.222 & 1.110 & 2.540 & 0.043 & 0.043 & 0.284 \\
\hline \multicolumn{9}{|c|}{$\mathrm{N}=500, \mathrm{n}=10, \mathrm{R}=25$} \\
\hline Mediana a posteriori média & -0.967 & 2.840 & -2.860 & 2.070 & 26.700 & 0.678 & 0.322 & 1.270 \\
\hline ER da mediana a posteriori & -0.055 & -0.051 & -0.037 & 1.070 & -0.102 & -0.025 & 0.059 & 0.301 \\
\hline EAR da mediana a posteriori & 0.056 & 0.051 & 0.046 & 1.070 & 0.102 & 0.033 & 0.078 & 0.301 \\
\hline REQM da mediana a posteriori & 0.077 & 0.156 & 0.160 & 1.080 & 3.190 & 0.028 & 0.028 & 0.305 \\
\hline
\end{tabular}

Tabela A.10: Resultados descritivos: ER, EAR e REQM, da mediana a posteriori dos parâmetros do modelo de mistura beta linear com efeitos aleatórios para a média dos estudos de simulação com cenário 4 e $\phi=30$ de $R$ bancos de dados com tamanhos amostrais $N=100,500$ e $n=5,10$.

\begin{tabular}{|c|c|c|c|c|c|c|c|c|}
\hline Parâmetro & $\beta_{1}^{1}$ & $\beta_{2}^{1}$ & $\beta_{1}^{2}$ & $\beta_{2}^{2}$ & $\phi$ & $\pi_{1}$ & $\pi_{2}$ & $\sigma_{b}$ \\
\hline Valor verdadeiro & -1 & 3 & -3 & 1 & 50 & 0.700 & 0.300 & 1 \\
\hline \multicolumn{9}{|c|}{$\mathrm{N}=100, \mathrm{n}=5, \mathrm{R}=50$} \\
\hline Mediana a posteriori média & -0.855 & 2.880 & -2.770 & 2.160 & 62.000 & 0.744 & 0.256 & 1.260 \\
\hline ER da mediana a posteriori & -0.100 & -0.049 & -0.086 & 1.080 & 0.213 & 0.063 & -0.147 & 0.285 \\
\hline EAR da mediana a posteriori & 0.172 & 0.050 & 0.091 & 1.080 & 0.219 & 0.063 & 0.147 & 0.285 \\
\hline REQM da mediana a posteriori & 0.261 & 0.189 & 0.437 & 1.120 & 12.100 & 0.047 & 0.047 & 0.314 \\
\hline \multicolumn{9}{|c|}{$\mathrm{N}=100, \mathrm{n}=10, \mathrm{R}=50$} \\
\hline Mediana a posteriori média & -0.922 & 2.850 & -2.810 & 2.190 & 59.800 & 0.750 & 0.250 & 1.330 \\
\hline ER da mediana a posteriori & -0.051 & -0.061 & -0.071 & 1.160 & 0.181 & 0.068 & -0.158 & 0.333 \\
\hline EAR da mediana a posteriori & 0.122 & 0.061 & 0.077 & 1.160 & 0.182 & 0.073 & 0.170 & 0.333 \\
\hline REQM da mediana a posteriori & 0.180 & 0.316 & 0.318 & 1.200 & 10.100 & 0.054 & 0.054 & 0.348 \\
\hline \multicolumn{9}{|c|}{$\mathrm{N}=500, \mathrm{n}=5, \mathrm{R}=25$} \\
\hline Mediana a posteriori média & -0.892 & 2.880 & -2.860 & 2.090 & 44.000 & 0.664 & 0.336 & 1.310 \\
\hline ER da mediana a posteriori & -0.106 & -0.044 & -0.044 & 1.110 & -0.131 & -0.056 & 0.131 & 0.312 \\
\hline EAR da mediana a posteriori & 0.108 & 0.044 & 0.050 & 1.110 & 0.132 & 0.056 & 0.131 & 0.312 \\
\hline REQM da mediana a posteriori & 0.122 & 0.140 & 0.179 & 1.120 & 7.220 & 0.046 & 0.046 & 0.318 \\
\hline \multicolumn{9}{|c|}{$\mathrm{N}=500, \mathrm{n}=10, \mathrm{R}=25$} \\
\hline Mediana a posteriori média & -0.954 & 2.850 & -2.910 & 2.130 & 43.600 & 0.674 & 0.326 & 1.320 \\
\hline ER da mediana a posteriori & -0.053 & -0.047 & -0.035 & 1.130 & -0.143 & -0.035 & 0.082 & 0.323 \\
\hline EAR da mediana a posteriori & 0.059 & 0.047 & 0.041 & 1.130 & 0.143 & 0.038 & 0.088 & 0.323 \\
\hline REQM da mediana a posteriori & 0.072 & 0.145 & 0.164 & 1.130 & 7.370 & 0.032 & 0.032 & 0.327 \\
\hline
\end{tabular}

Tabela A.11: Resultados descritivos: ER, EAR e REQM, da mediana a posteriori dos parâmetros do modelo de mistura beta linear com efeitos aleatórios para a média dos estudos de simulação com cenário 4 e $\phi=50$ de $R$ bancos de dados com tamanhos amostrais $N=100,500$ e $n=5,10$. 


\begin{tabular}{|c|c|c|c|c|c|c|c|c|}
\hline Parâmetro & $\beta_{1}^{1}$ & $\beta_{2}^{1}$ & $\beta_{1}^{2}$ & $\beta_{2}^{2}$ & $\phi$ & $\pi_{1}$ & $\pi_{2}$ & $\sigma_{b}$ \\
\hline Valor verdadeiro & -1 & 3 & -3 & 1 & 70 & 0.700 & 0.300 & 1 \\
\hline \multicolumn{9}{|c|}{$\mathrm{N}=100, \mathrm{n}=5, \mathrm{R}=50$} \\
\hline Mediana a posteriori média & -0.896 & 2.840 & -2.770 & 2.190 & 80.500 & 0.749 & 0.251 & 1.290 \\
\hline ER da mediana a posteriori & -0.060 & -0.055 & -0.082 & 1.110 & 0.111 & 0.069 & -0.162 & 0.307 \\
\hline EAR da mediana a posteriori & 0.147 & 0.055 & 0.087 & 1.110 & 0.155 & 0.069 & 0.162 & 0.307 \\
\hline REQM da mediana a posteriori & 0.258 & 0.198 & 0.423 & 1.160 & 11.700 & 0.052 & 0.052 & 0.335 \\
\hline \multicolumn{9}{|c|}{$\mathrm{N}=100, \mathrm{n}=10, \mathrm{R}=50$} \\
\hline Mediana a posteriori média & -0.913 & 2.850 & -2.800 & 2.180 & 76.700 & 0.747 & 0.253 & 1.330 \\
\hline ER da mediana a posteriori & -0.090 & -0.049 & -0.072 & 1.180 & 0.081 & 0.065 & -0.153 & 0.324 \\
\hline EAR da mediana a posteriori & 0.116 & 0.049 & 0.080 & 1.180 & 0.101 & 0.066 & 0.153 & 0.324 \\
\hline REQM da mediana a posteriori & 0.138 & 0.164 & 0.295 & 1.210 & 8.450 & 0.049 & 0.049 & 0.337 \\
\hline \multicolumn{9}{|c|}{$\mathrm{N}=500, \mathrm{n}=5, \mathrm{R}=25$} \\
\hline Mediana a posteriori média & -0.922 & 2.860 & -2.930 & 2.080 & 58.800 & 0.679 & 0.321 & 1.320 \\
\hline ER da mediana a posteriori & -0.075 & -0.046 & -0.025 & 1.080 & -0.162 & -0.031 & 0.072 & 0.320 \\
\hline EAR da mediana a posteriori & 0.077 & 0.046 & 0.038 & 1.080 & 0.162 & 0.032 & 0.076 & 0.320 \\
\hline REQM da mediana a posteriori & 0.086 & 0.151 & 0.158 & 1.080 & 12.400 & 0.027 & 0.027 & 0.322 \\
\hline \multicolumn{9}{|c|}{$\mathrm{N}=500, \mathrm{n}=10, \mathrm{R}=25$} \\
\hline Mediana a posteriori média & -0.944 & 2.870 & -2.840 & 2.200 & 56.600 & 0.658 & 0.342 & 1.340 \\
\hline ER da mediana a posteriori & -0.051 & -0.043 & -0.056 & 1.200 & -0.206 & -0.054 & 0.126 & 0.355 \\
\hline EAR da mediana a posteriori & 0.066 & 0.043 & 0.057 & 1.200 & 0.206 & 0.054 & 0.126 & 0.355 \\
\hline REQM da mediana a posteriori & 0.082 & 0.133 & 0.198 & 1.200 & 14.700 & 0.042 & 0.042 & 0.360 \\
\hline
\end{tabular}

Tabela A.12: Resultados descritivos: ER, EAR e REQM, da mediana a posteriori dos parâmetros do modelo de mistura beta linear com efeitos aleatórios para a média dos estudos de simulação com cenário 4 e $\phi=70$ de $R$ bancos de dados com tamanhos amostrais $N=100,500$ e $n=5,10$.

\section{A.2 Modelo linear com parâmetro de precisão constante - $\mathrm{N}=100$,}

$$
\mathbf{n}=\mathbf{5}, \Sigma_{\beta^{s}}=40 I_{2}
$$

\begin{tabular}{c|cccccccc}
\hline & \multicolumn{7}{|c}{ Parâmetro } \\
\cline { 2 - 9 } & $\beta_{1}^{1}$ & $\beta_{2}^{1}$ & $\beta_{1}^{2}$ & $\beta_{2}^{2}$ & $\phi$ & $\pi_{1}$ & $\pi_{2}$ & $\sigma_{b}$ \\
\hline \hline Valor verdadeiro & -0.750 & 2 & -1.500 & 0.500 & 30 & 0.700 & 0.300 & 1 \\
Mediana a posteriori média & -0.481 & 1.730 & -1.560 & 1.240 & 41.200 & 0.762 & 0.238 & 0.938 \\
ER da mediana a posteriori & -0.008 & -0.170 & -0.271 & 1.760 & 0.371 & 0.088 & -0.206 & -0.062 \\
EAR da mediana a posteriori & 0.562 & 0.170 & 0.414 & 1.760 & 0.371 & 0.088 & 0.206 & 0.081 \\
REQM da mediana a posteriori & 0.450 & 0.415 & 0.870 & 1.000 & 11.400 & 0.064 & 0.064 & 0.098 \\
\hline
\end{tabular}

Tabela A.13: Resultados descritivos: ER, EAR e REQM, da mediana a posteriori dos parâmetros do modelo de mistura beta linear com efeitos aleatórios para a média dos estudos de simulação com cenário 1 e $\phi=30$ de $R=50$ bancos de dados e com $N=100, n=5$ e $\Sigma_{\beta^{s}}=40 I_{2}$.

\begin{tabular}{c|cccccccc}
\hline & \multicolumn{7}{|c}{ Parâmetro } \\
\cline { 2 - 9 } & $\beta_{1}^{1}$ & $\beta_{2}^{1}$ & $\beta_{1}^{2}$ & $\beta_{2}^{2}$ & $\phi$ & $\pi_{1}$ & $\pi_{2}$ & $\sigma_{b}$ \\
\hline \hline Valor verdadeiro & -0.750 & 2 & -1.500 & 0.500 & 50 & 0.700 & 0.300 & 1 \\
Mediana a posteriori média & -0.492 & 1.680 & -1.550 & 1.360 & 60.700 & 0.754 & 0.246 & 0.963 \\
ER da mediana a posteriori & 0.012 & -0.181 & -0.269 & 1.880 & 0.199 & 0.074 & -0.173 & -0.029 \\
EAR da mediana a posteriori & 0.577 & 0.181 & 0.390 & 1.880 & 0.203 & 0.074 & 0.174 & 0.078 \\
REQM da mediana a posteriori & 0.463 & 0.415 & 0.832 & 1.010 & 11.200 & 0.055 & 0.055 & 0.094 \\
\hline
\end{tabular}

Tabela A.14: Resultados descritivos: ER, EAR e REQM, da mediana a posteriori dos parâmetros do modelo de mistura beta linear com efeitos aleatórios para a média dos estudos de simulação com cenário 1 e $\phi=50$ de $R=50$ bancos de dados e com $N=100, n=5$ e $\Sigma_{\beta^{s}}=40 I_{2}$. 


\begin{tabular}{c|cccccccc}
\hline & \multicolumn{9}{|c}{ Parâmetro } & \multicolumn{1}{c}{} \\
\cline { 2 - 9 } & $\beta_{1}^{1}$ & $\beta_{2}^{1}$ & $\beta_{1}^{2}$ & $\beta_{2}^{2}$ & $\phi$ & $\pi_{1}$ & $\pi_{2}$ & $\sigma_{b}$ \\
\hline \hline Valor verdadeiro & -0.750 & 2 & -1.500 & 0.500 & 70 & 0.700 & 0.300 & 1 \\
Mediana a posteriori média & -0.497 & 1.670 & -1.550 & 1.370 & 77.200 & 0.751 & 0.249 & 0.976 \\
ER da mediana a posteriori & 0.001 & -0.185 & -0.248 & 1.920 & 0.084 & 0.063 & -0.148 & -0.009 \\
EAR da mediana a posteriori & 0.571 & 0.185 & 0.358 & 1.920 & 0.125 & 0.065 & 0.151 & 0.080 \\
REQM da mediana a posteriori & 0.461 & 0.420 & 0.780 & 1.010 & 9.920 & 0.049 & 0.049 & 0.095 \\
\hline
\end{tabular}

Tabela A.15: Resultados descritivos: ER, EAR e REQM, da mediana a posteriori dos parâmetros do modelo de mistura beta linear com efeitos aleatórios para a média dos estudos de simulação com cenário 1 e $\phi=70$ de $R=50$ bancos de dados e com $N=100, n=5$ e $\Sigma_{\beta}=40 I_{2}$.

\begin{tabular}{c|cccccccc}
\hline & \multicolumn{9}{|c}{ Parâmetro } & \multicolumn{1}{c}{$\pi^{2}$} & $\sigma_{b}$ \\
\cline { 2 - 9 } & $\beta_{1}^{1}$ & $\beta_{2}^{1}$ & $\beta_{1}^{2}$ & $\beta_{2}^{2}$ & $\phi$ & $\pi_{1}$ & $\pi_{2}$ & 1 \\
\hline \hline Valor verdadeiro & -1.500 & 1 & -0.500 & 3 & 30 & 0.700 & 0.300 & 0.960 \\
Mediana a posteriori & -1.580 & 1.180 & -0.231 & 2.270 & 36.700 & 0.748 & 0.252 & -0.017 \\
ER da mediana a posteriori & 0.051 & 0.182 & -0.530 & -0.247 & 0.228 & 0.071 & -0.166 & 0.089 \\
EAR da mediana a posteriori & 0.064 & 0.189 & 0.530 & 0.247 & 0.228 & 0.072 & 0.168 & 0.089 \\
REQM da mediana a posteriori & 0.115 & 0.217 & 0.279 & 0.775 & 7.580 & 0.053 & 0.053 & 0.107 \\
\hline
\end{tabular}

Tabela A.16: Resultados descritivos: ER, EAR e REQM, da mediana a posteriori dos parâmetros do modelo de mistura beta linear com efeitos aleatórios para a média dos estudos de simulação com cenário 2 e $\phi=30$ de $R=50$ bancos de dados e com $N=100, n=5$ e $\Sigma_{\beta^{s}}=40 I_{2}$.

\begin{tabular}{c|ccccccccc}
\hline & \multicolumn{7}{c}{ Parâmetro } \\
\cline { 2 - 7 } & $\beta_{1}^{1}$ & $\beta_{2}^{1}$ & $\beta_{1}^{2}$ & $\beta_{2}^{2}$ & $\phi$ & $\pi_{1}$ & $\pi_{2}$ & $\sigma_{b}$ \\
\hline \hline Valor verdadeiro & -1.500 & 1 & -0.500 & 3 & 50 & 0.700 & 0.300 \\
Mediana a posteriori & -1.600 & 1.240 & -0.265 & 2.120 & 50.000 & 0.737 & 0.263 & 0.991 \\
ER da mediana a posteriori & 0.061 & 0.227 & -0.460 & -0.294 & 0.026 & 0.057 & -0.133 & 0.010 \\
EAR da mediana a posteriori & 0.068 & 0.227 & 0.460 & 0.294 & 0.114 & 0.057 & 0.133 & 0.079 \\
REQM da mediana a posteriori & 0.118 & 0.251 & 0.248 & 0.906 & 7.050 & 0.043 & 0.043 & 0.103 \\
\hline
\end{tabular}

Tabela A.17: Resultados descritivos: ER, EAR e REQM, da mediana a posteriori dos parâmetros do modelo de mistura beta linear com efeitos aleatórios para a média dos estudos de simulação com cenário 2 e $\phi=50$ de $R=50$ bancos de dados e com $N=100, n=5$ e $\Sigma_{\beta^{s}}=40 I_{2}$.

\begin{tabular}{c|ccccccccc}
\hline & \multicolumn{7}{c}{ Parâmetro } \\
\cline { 2 - 7 } & $\beta_{1}^{1}$ & $\beta_{2}^{1}$ & $\beta_{1}^{2}$ & $\beta_{2}^{2}$ & $\phi$ & $\pi_{1}$ & $\pi_{2}$ & $\sigma_{b}$ \\
\hline \hline Valor verdadeiro & -1.500 & 1 & -0.500 & 3 & 70 & 0.700 & 0.300 \\
Mediana a posteriori & -1.620 & 1.260 & -0.286 & 2.040 & 59.300 & 0.737 & 0.263 & 1.010 \\
ER da mediana a posteriori & 0.065 & 0.258 & -0.442 & -0.321 & -0.105 & 0.052 & -0.120 & 0.020 \\
EAR da mediana a posteriori & 0.071 & 0.258 & 0.442 & 0.321 & 0.166 & 0.052 & 0.121 & 0.070 \\
REQM da mediana a posteriori & 0.120 & 0.276 & 0.240 & 0.978 & 12.800 & 0.040 & 0.040 & 0.094 \\
\hline
\end{tabular}

Tabela A.18: Resultados descritivos: ER, EAR e REQM, da mediana a posteriori dos parâmetros do modelo de mistura beta linear com efeitos aleatórios para a média dos estudos de simulação com cenário 2 e $\phi=70$ de $R=50$ bancos de dados e com $N=100, n=5$ e $\Sigma_{\beta^{s}}=40 I_{2}$. 


\section{A.3 Modelo linear com parâmetro de precisão constante - $\mathrm{N}=40$, $\mathrm{n}=5$}

\begin{tabular}{c|cccccccc}
\hline & \multicolumn{7}{|c}{ Parâmetro } \\
\cline { 2 - 9 } & $\beta_{1}^{1}$ & $\beta_{2}^{1}$ & $\beta_{1}^{2}$ & $\beta_{2}^{2}$ & $\phi$ & $\pi_{1}$ & $\pi_{2}$ & $\sigma_{b}$ \\
\hline \hline Valor verdadeiro & -0.750 & 2 & -1.500 & 0.500 & 30 & 0.700 & 0.300 & 1 \\
Mediana a posteriori média & -0.658 & 1.730 & -1.440 & 0.901 & 45.600 & 0.789 & 0.211 & 0.839 \\
ER da mediana a posteriori & 0.048 & -0.197 & -0.283 & 1.350 & 0.515 & 0.129 & -0.301 & -0.142 \\
EAR da mediana a posteriori & 0.373 & 0.203 & 0.408 & 1.450 & 0.515 & 0.129 & 0.301 & 0.158 \\
REQM da mediana a posteriori & 0.321 & 0.512 & 0.844 & 0.929 & 15.500 & 0.091 & 0.091 & 0.179 \\
\hline
\end{tabular}

Tabela A.19: Resultados descritivos: ER, EAR e REQM, da mediana a posteriori dos parâmetros do modelo de mistura beta linear com efeitos aleatórios para a média dos estudos de simulação com cenário 1 e $\phi=30$ de $R=50$ bancos de dados e com $N=40, n=5$.

\begin{tabular}{c|cccccccc}
\hline & \multicolumn{9}{|c}{ Parâmetro } \\
\cline { 2 - 9 } & $\beta_{1}^{1}$ & $\beta_{2}^{1}$ & $\beta_{1}^{2}$ & $\beta_{2}^{2}$ & $\phi$ & $\pi_{1}$ & $\pi_{2}$ & $\sigma_{b}$ \\
\hline \hline Valor verdadeiro & -0.750 & 2 & -1.500 & 0.500 & 50 & 0.700 & 0.300 & 1 \\
Mediana a posteriori média & -0.673 & 1.720 & -1.350 & 0.908 & 65.700 & 0.785 & 0.215 & 0.837 \\
ER da mediana a posteriori & 0.119 & -0.198 & -0.323 & 1.360 & 0.315 & 0.123 & -0.286 & -0.145 \\
EAR da mediana a posteriori & 0.402 & 0.200 & 0.438 & 1.410 & 0.315 & 0.123 & 0.286 & 0.162 \\
REQM da mediana a posteriori & 0.354 & 0.510 & 0.866 & 0.939 & 15.900 & 0.087 & 0.087 & 0.177 \\
\hline
\end{tabular}

Tabela A.20: Resultados descritivos: ER, EAR e REQM, da mediana a posteriori dos parâmetros do modelo de mistura beta linear com efeitos aleatórios para a média dos estudos de simulação com cenário 1 e $\phi=50$ de $R=50$ bancos de dados e com $N=40, n=5$.

\begin{tabular}{c|cccccccc}
\hline & \multicolumn{7}{|c}{ Parâmetro } & \multicolumn{1}{c}{$\pi^{2}$} \\
\cline { 2 - 9 } & $\beta_{1}^{1}$ & $\beta_{2}^{1}$ & $\beta_{1}^{2}$ & $\beta_{2}^{2}$ & $\phi$ & $\pi_{1}$ & $\pi_{2}$ \\
\hline \hline Valor verdadeiro & -0.750 & 2 & -1.500 & 0.500 & 70 & 0.700 & 0.300 & 1 \\
Mediana a posteriori média & -0.690 & 1.650 & -1.260 & 1.100 & 85.400 & 0.785 & 0.215 & 0.863 \\
ER da mediana a posteriori & 0.135 & -0.225 & -0.342 & 1.560 & 0.208 & 0.114 & -0.266 & -0.129 \\
EAR da mediana a posteriori & 0.450 & 0.226 & 0.427 & 1.570 & 0.208 & 0.114 & 0.266 & 0.139 \\
REQM da mediana a posteriori & 0.379 & 0.601 & 0.834 & 0.995 & 14.800 & 0.082 & 0.082 & 0.161 \\
\hline
\end{tabular}

Tabela A.21: Resultados descritivos: ER, EAR e REQM, da mediana a posteriori dos parâmetros do modelo de mistura beta linear com efeitos aleatórios para a média dos estudos de simulação com cenário 1 e $\phi=70$ de $R=50$ bancos de dados e com $N=40, n=5$.

\begin{tabular}{c|ccccccccc}
\hline & \multicolumn{7}{|c}{ Parâmetro } \\
\cline { 2 - 8 } & $\beta_{1}^{1}$ & $\beta_{2}^{1}$ & $\beta_{1}^{2}$ & $\beta_{2}^{2}$ & $\phi$ & $\pi_{1}$ & $\pi_{2}$ \\
\hline \hline Valor verdadeiro & -1.500 & 1 & -0.500 & 3 & 30 & 0.700 & 0.300 \\
Mediana a posteriori média & -1.450 & 1.060 & -0.291 & 2.600 & 46.100 & 0.781 & 0.219 & 0.863 \\
ER da mediana a posteriori & -0.046 & 0.111 & -0.319 & -0.139 & 0.506 & 0.118 & -0.276 & -0.104 \\
EAR da mediana a posteriori & 0.091 & 0.177 & 0.473 & 0.141 & 0.506 & 0.118 & 0.276 & 0.150 \\
REQM da mediana a posteriori & 0.222 & 0.268 & 0.300 & 0.546 & 15.400 & 0.084 & 0.084 & 0.173 \\
\hline
\end{tabular}

Tabela A.22: Resultados descritivos: ER, EAR e REQM, da mediana a posteriori dos parâmetros do modelo de mistura beta linear com efeitos aleatórios para a média dos estudos de simulação com cenário 2 e $\phi=30$ de $R=50$ bancos de dados e com $N=40, n=5$. 


\begin{tabular}{|c|c|c|c|c|c|c|c|c|}
\hline & \multicolumn{8}{|c|}{ Parâmetro } \\
\hline & $\beta_{1}^{1}$ & $\beta_{2}^{1}$ & $\beta_{1}^{2}$ & $\beta_{2}^{2}$ & $\phi$ & $\pi_{1}$ & $\pi_{2}$ & $\sigma_{b}$ \\
\hline Valor verdadeiro & -1.500 & 1 & -0.500 & 3 & 50 & 0.700 & 0.300 & 1 \\
\hline Mediana a posteriori média & -1.470 & 1.100 & -0.325 & 2.570 & 65.300 & 0.781 & 0.219 & 0.883 \\
\hline ER da mediana a posteriori & -0.056 & 0.195 & -0.229 & -0.182 & 0.279 & 0.112 & -0.262 & -0.089 \\
\hline EAR da mediana a posteriori & 0.102 & 0.237 & 0.495 & 0.183 & 0.279 & 0.112 & 0.262 & 0.137 \\
\hline REQM da mediana a posteriori & 0.242 & 0.445 & 0.308 & 0.705 & 14.500 & 0.080 & 0.080 & 0.158 \\
\hline
\end{tabular}

Tabela A.23: Resultados descritivos: ER, EAR e REQM, da mediana a posteriori dos parâmetros do modelo de mistura beta linear com efeitos aleatórios para a média dos estudos de simulação com cenário 2 e $\phi=50$ de $R=50$ bancos de dados e com $N=40, n=5$.

\begin{tabular}{c|cccccccc}
\hline & \multicolumn{9}{|c}{ Parâmetro } & \multicolumn{1}{c}{$\pi^{2}$} & $\sigma_{b}$ \\
\cline { 2 - 10 } & $\beta_{1}^{1}$ & $\beta_{2}^{1}$ & $\beta_{1}^{2}$ & $\beta_{2}^{2}$ & $\phi$ & $\pi_{1}$ & $\pi_{2}$ & 1 \\
\hline \hline Valor verdadeiro & -1.500 & 1 & -0.500 & 3 & 70 & 0.700 & 0.300 & 1 \\
Mediana a posteriori média & -1.450 & 1.120 & -0.371 & 2.530 & 82.900 & 0.777 & 0.223 & 0.874 \\
ER da mediana a posteriori & -0.050 & 0.220 & -0.157 & -0.204 & 0.145 & 0.107 & -0.250 & -0.090 \\
EAR da mediana a posteriori & 0.099 & 0.260 & 0.492 & 0.204 & 0.167 & 0.107 & 0.250 & 0.142 \\
REQM da mediana a posteriori & 0.239 & 0.482 & 0.320 & 0.775 & 12.800 & 0.077 & 0.077 & 0.164 \\
\hline
\end{tabular}

Tabela A.24: Resultados descritivos: ER, EAR e REQM, da mediana a posteriori dos parâmetros do modelo de mistura beta linear com efeitos aleatórios para a média dos estudos de simulação com cenário 2 e $\phi=70$ de $R=50$ bancos de dados e com $N=40, n=5$.

\begin{tabular}{c|cccccccccc}
\hline & \multicolumn{9}{c}{ Parâmetro } \\
\cline { 2 - 8 } & $\beta_{1}^{1}$ & $\beta_{2}^{1}$ & $\beta_{1}^{2}$ & $\beta_{2}^{2}$ & $\phi$ & $\pi_{1}$ & $\pi_{2}$ \\
\hline \hline Valor verdadeiro & -1.500 & 1 & -0.500 & 3 & 30 & 0.700 & 0.300 \\
Mediana a posteriori média & -1.970 & 0.664 & -0.399 & 2.470 & 41.600 & 0.779 & 0.221 & 0.966 \\
ER da mediana a posteriori & -0.012 & 0.401 & -0.183 & -0.180 & 0.370 & 0.112 & -0.261 & -0.010 \\
EAR da mediana a posteriori & 0.049 & 0.452 & 0.286 & 0.181 & 0.372 & 0.112 & 0.261 & 0.133 \\
REQM da mediana a posteriori & 0.123 & 0.287 & 0.176 & 0.604 & 12.000 & 0.079 & 0.079 & 0.159 \\
\hline
\end{tabular}

Tabela A.25: Resultados descritivos: ER, EAR e REQM, da mediana a posteriori dos parâmetros do modelo de mistura beta linear com efeitos aleatórios para a média dos estudos de simulação com cenário 3 e $\phi=30$ de $R=50$ bancos de dados e com $N=40, n=5$.

\begin{tabular}{c|cccccccccc}
\hline & \multicolumn{7}{|c}{ Parâmetro } \\
\cline { 2 - 8 } & $\beta_{1}^{1}$ & $\beta_{2}^{1}$ & $\beta_{1}^{2}$ & $\beta_{2}^{2}$ & $\phi$ & $\pi_{1}$ & $\pi_{2}$ \\
\hline \hline Valor verdadeiro & -1.500 & 1 & -0.500 & 3 & 50 & 0.700 & 0.300 \\
Mediana a posteriori média & -1.980 & 0.732 & -0.399 & 2.350 & 57.100 & 0.778 & 0.222 & 0.993 \\
ER da mediana a posteriori & -0.025 & 0.503 & -0.114 & -0.217 & 0.128 & 0.109 & -0.254 & 0.007 \\
EAR da mediana a posteriori & 0.056 & 0.559 & 0.384 & 0.220 & 0.190 & 0.109 & 0.254 & 0.129 \\
REQM da mediana a posteriori & 0.197 & 0.420 & 0.254 & 0.769 & 11.200 & 0.078 & 0.078 & 0.155 \\
\hline
\end{tabular}

Tabela A.26: Resultados descritivos: ER, EAR e REQM, da mediana a posteriori dos parâmetros do modelo de mistura beta linear com efeitos aleatórios para a média dos estudos de simulação com cenário 3 e $\phi=50$ de $R=50$ bancos de dados e com $N=40, n=5$. 


\begin{tabular}{|c|c|c|c|c|c|c|c|c|}
\hline & \multicolumn{8}{|c|}{ Parâmetro } \\
\hline & $\beta_{1}^{1}$ & $\beta_{2}^{1}$ & $\beta_{1}^{2}$ & $\beta_{2}^{2}$ & $\phi$ & $\pi_{1}$ & $\pi_{2}$ & $\sigma_{b}$ \\
\hline Valor verdadeiro & -1.500 & 1 & -0.500 & 3 & 70 & 0.700 & 0.300 & 1 \\
\hline Mediana a posteriori média & -1.980 & 0.804 & -0.400 & 2.330 & 67.400 & 0.776 & 0.224 & 1.020 \\
\hline ER da mediana a posteriori & -0.041 & 0.729 & -0.031 & -0.259 & -0.007 & 0.108 & -0.252 & 0.035 \\
\hline EAR da mediana a posteriori & 0.070 & 0.773 & 0.407 & 0.260 & 0.168 & 0.108 & 0.252 & 0.142 \\
\hline REQM da mediana a posteriori & 0.260 & 0.618 & 0.289 & 0.929 & 12.800 & 0.077 & 0.077 & 0.176 \\
\hline
\end{tabular}

Tabela A.27: Resultados descritivos: ER, EAR e REQM, da mediana a posteriori dos parâmetros do modelo de mistura beta linear com efeitos aleatórios para a média dos estudos de simulação com cenário 3 e $\phi=70$ de $R=50$ bancos de dados e com $N=40, n=5$.

\begin{tabular}{c|cccccccc}
\hline & \multicolumn{9}{|c}{ Parâmetro } \\
\cline { 2 - 9 } & $\beta_{1}^{1}$ & $\beta_{2}^{1}$ & $\beta_{1}^{2}$ & $\beta_{2}^{2}$ & $\phi$ & $\pi_{1}$ & $\pi_{2}$ & $\sigma_{b}$ \\
\hline \hline Valor verdadeiro & -1.500 & 1 & -0.500 & 3 & 30 & 0.700 & 0.300 & 1 \\
Mediana a posteriori média & -0.870 & 2.820 & -2.770 & 1.840 & 45.000 & 0.776 & 0.224 & 1.120 \\
ER da mediana a posteriori & -0.093 & -0.062 & -0.072 & 0.794 & 0.487 & 0.107 & -0.251 & 0.162 \\
EAR da mediana a posteriori & 0.180 & 0.068 & 0.100 & 0.797 & 0.487 & 0.107 & 0.251 & 0.188 \\
REQM da mediana a posteriori & 0.290 & 0.267 & 0.448 & 0.866 & 14.800 & 0.076 & 0.076 & 0.266 \\
\hline
\end{tabular}

Tabela A.28: Resultados descritivos: ER, EAR e REQM, da mediana a posteriori dos parâmetros do modelo de mistura beta linear com efeitos aleatórios para a média dos estudos de simulação com cenário 4 e $\phi=30$ de $R=50$ bancos de dados e com $N=40, n=5$.

\begin{tabular}{c|ccccccccc}
\hline & \multicolumn{7}{c}{ Parâmetro } \\
\cline { 2 - 8 } & $\beta_{1}^{1}$ & $\beta_{2}^{1}$ & $\beta_{1}^{2}$ & $\beta_{2}^{2}$ & $\phi$ & $\pi_{1}$ & $\pi_{2}$ & $\sigma_{b}$ \\
\hline \hline Valor verdadeiro & -1.500 & 1 & -0.500 & 3 & 50 & 0.700 & 0.300 & 1 \\
Mediana a posteriori média & -0.873 & 2.800 & -2.810 & 1.880 & 65.000 & 0.772 & 0.228 & 1.170 \\
ER da mediana a posteriori & -0.092 & -0.067 & -0.075 & 0.850 & 0.274 & 0.105 & -0.245 & 0.158 \\
EAR da mediana a posteriori & 0.183 & 0.071 & 0.095 & 0.850 & 0.275 & 0.105 & 0.245 & 0.183 \\
REQM da mediana a posteriori & 0.282 & 0.268 & 0.431 & 0.915 & 14.300 & 0.075 & 0.075 & 0.232 \\
\hline
\end{tabular}

Tabela A.29: Resultados descritivos: ER, EAR e REQM, da mediana a posteriori dos parâmetros do modelo de mistura beta linear com efeitos aleatórios para a média dos estudos de simulação com cenário 4 e $\phi=50$ de $R=50$ bancos de dados e com $N=40, n=5$.

\begin{tabular}{c|ccccccccc}
\hline & \multicolumn{7}{c}{ Parâmetro } \\
\cline { 2 - 8 } & $\beta_{1}^{1}$ & $\beta_{2}^{1}$ & $\beta_{1}^{2}$ & $\beta_{2}^{2}$ & $\phi$ & $\pi_{1}$ & $\pi_{2}$ \\
\hline \hline Valor verdadeiro & -1.500 & 1 & -0.500 & 3 & 70 & 0.700 & 0.300 \\
Mediana a posteriori média & -0.887 & 2.820 & -2.800 & 1.890 & 83.000 & 0.773 & 0.227 & 1.170 \\
ER da mediana a posteriori & -0.082 & -0.066 & -0.076 & 0.885 & 0.157 & 0.106 & -0.246 & 0.182 \\
EAR da mediana a posteriori & 0.173 & 0.068 & 0.096 & 0.885 & 0.171 & 0.106 & 0.246 & 0.204 \\
REQM da mediana a posteriori & 0.270 & 0.253 & 0.434 & 0.943 & 12.800 & 0.075 & 0.075 & 0.250 \\
\hline
\end{tabular}

Tabela A.30: Resultados descritivos: ER, EAR e REQM, da mediana a posteriori dos parâmetros do modelo de mistura beta linear com efeitos aleatórios para a média dos estudos de simulação com cenário 4 e $\phi=70$ de $R=50$ bancos de dados e com $N=40, n=5$. 


\section{A.4 Modelo linear com dispersão variável}

\begin{tabular}{c|ccccccccc}
\hline & \multicolumn{10}{c}{ Parâmetro } \\
\cline { 2 - 10 } & $\beta_{1}^{1}$ & $\beta_{2}^{1}$ & $\beta_{1}^{2}$ & $\beta_{2}^{2}$ & $\delta_{11}$ & $\delta_{12}$ & $\pi_{1}$ & $\pi_{2}$ & $\sigma_{b}$ \\
\hline \hline Valor verdadeiro & -0.750 & 2 & -1.500 & 0.500 & 3 & 1.500 & 0.700 & 0.300 & 1 \\
Mediana a posteriori média & -0.609 & 1.810 & -1.470 & 0.907 & 3.140 & 1.530 & 0.773 & 0.227 & 0.938 \\
ER da mediana a posteriori & 0.001 & -0.145 & -0.241 & 1.290 & 0.041 & -0.022 & 0.099 & -0.231 & -0.048 \\
EAR da mediana a posteriori & 0.347 & 0.146 & 0.327 & 1.300 & 0.057 & 0.158 & 0.103 & 0.239 & 0.076 \\
REQM da mediana a posteriori & 0.302 & 0.411 & 0.733 & 0.876 & 0.194 & 0.293 & 0.074 & 0.074 & 0.091 \\
\hline
\end{tabular}

Tabela A.31: Resultados descritivos: ER, EAR e REQM, da mediana a posteriori dos parâmetros do modelo de mistura beta linear com efeitos aleatórios para a média e precisão dos estudos de simulação com respectivos cenários para os parâmetros fixos: 1 e 1 de $R=50$ bancos de dados.

\begin{tabular}{c|ccccccccc}
\hline & \multicolumn{7}{|c}{ Parâmetro } \\
\cline { 2 - 10 } & $\beta_{1}^{1}$ & $\beta_{2}^{1}$ & $\beta_{1}^{2}$ & $\beta_{2}^{2}$ & $\delta_{11}$ & $\delta_{12}$ & $\pi_{1}$ & $\pi_{2}$ & $\sigma_{b}$ \\
\hline \hline Valor verdadeiro & -0.750 & 2 & -1.500 & 0.500 & 4 & 0.500 & 0.700 & 0.300 & 1 \\
Mediana a posteriori média & -0.588 & 1.790 & -1.470 & 1.090 & 3.630 & 1.250 & 0.759 & 0.241 & 0.949 \\
ER da mediana a posteriori & -0.001 & -0.162 & -0.252 & 1.600 & -0.106 & 1.400 & 0.082 & -0.191 & -0.040 \\
EAR da mediana a posteriori & 0.387 & 0.163 & 0.318 & 1.620 & 0.108 & 1.410 & 0.083 & 0.193 & 0.082 \\
REQM da mediana a posteriori & 0.324 & 0.433 & 0.714 & 0.983 & 0.515 & 0.812 & 0.062 & 0.062 & 0.097 \\
\hline
\end{tabular}

Tabela A.32: Resultados descritivos: ER, EAR e REQM, da mediana a posteriori dos parâmetros do modelo de mistura beta linear com efeitos aleatórios para a média e precisão dos estudos de simulação com respectivos cenários para os parâmetros fixos: 1 e 2 de $R=50$ bancos de dados.

\begin{tabular}{c|ccccccccc}
\hline & \multicolumn{9}{c}{ Parâmetro } \\
\cline { 2 - 10 } & $\beta_{1}^{1}$ & $\beta_{2}^{1}$ & $\beta_{1}^{2}$ & $\beta_{2}^{2}$ & $\delta_{11}$ & $\delta_{12}$ & $\pi_{1}$ & $\pi_{2}$ & $\sigma_{b}$ \\
\hline \hline Valor verdadeiro & -0.750 & 2 & -1.500 & 0.500 & 2 & 2 & 0.700 & 0.300 & 1 \\
Mediana a posteriori média & -0.594 & 1.780 & -1.570 & 0.919 & 2.400 & 1.600 & 0.785 & 0.215 & 0.922 \\
ER da mediana a posteriori & -0.006 & -0.156 & -0.248 & 1.340 & 0.211 & -0.232 & 0.114 & -0.267 & -0.076 \\
EAR da mediana a posteriori & 0.380 & 0.158 & 0.409 & 1.360 & 0.211 & 0.232 & 0.114 & 0.267 & 0.098 \\
REQM da mediana a posteriori & 0.313 & 0.402 & 0.844 & 0.911 & 0.431 & 0.510 & 0.082 & 0.082 & 0.119 \\
\hline
\end{tabular}

Tabela A.33: Resultados descritivos: ER, EAR e REQM, da mediana a posteriori dos parâmetros do modelo de mistura beta linear com efeitos aleatórios para a média e precisão dos estudos de simulação com respectivos cenários para os parâmetros fixos: 1 e 3 de $R=50$ bancos de dados.

\begin{tabular}{c|ccccccccc}
\hline & \multicolumn{9}{|c}{ Parâmetro } \\
\cline { 2 - 10 } & $\beta_{1}^{1}$ & $\beta_{2}^{1}$ & $\beta_{1}^{2}$ & $\beta_{2}^{2}$ & $\delta_{11}$ & $\delta_{12}$ & $\pi_{1}$ & $\pi_{2}$ & $\sigma_{b}$ \\
\hline \hline Valor verdadeiro & -1.500 & 1 & -0.500 & 3 & 3 & 1.500 & 0.700 & 0.300 & 1 \\
Mediana a posteriori média & -1.440 & 1.160 & -0.254 & 2.430 & 3.080 & 1.360 & 0.768 & 0.232 & 0.957 \\
ER da mediana a posteriori & -0.030 & 0.175 & -0.478 & -0.188 & 0.012 & -0.094 & 0.095 & -0.221 & -0.013 \\
EAR da mediana a posteriori & 0.054 & 0.178 & 0.480 & 0.188 & 0.049 & 0.185 & 0.095 & 0.221 & 0.078 \\
REQM da mediana a posteriori & 0.094 & 0.217 & 0.274 & 0.623 & 0.178 & 0.337 & 0.069 & 0.069 & 0.102 \\
\hline
\end{tabular}

Tabela A.34: Resultados descritivos: ER, EAR e REQM, da mediana a posteriori dos parâmetros do modelo de mistura beta linear com efeitos aleatórios para a média e precisão dos estudos de simulação com respectivos cenários para os parâmetros fixos: 2 e 1 de $R=50$ bancos de dados. 


\begin{tabular}{c|ccccccccc}
\hline & \multicolumn{1}{|c}{ Parâmetro } & \multicolumn{1}{c}{$\pi^{2}$} & $\sigma_{b}$ \\
\cline { 2 - 10 } & $\beta_{1}^{1}$ & $\beta_{2}^{1}$ & $\beta_{1}^{2}$ & $\beta_{2}^{2}$ & $\delta_{11}$ & $\delta_{12}$ & $\pi_{1}$ & $\pi_{2}$ \\
\hline \hline Valor verdadeiro & -1.500 & 1 & -0.500 & 3 & 4 & 0.500 & 0.700 & 0.300 & 1 \\
Mediana a posteriori média & -1.450 & 1.200 & -0.279 & 2.330 & 3.500 & 1.070 & 0.764 & 0.236 & 0.991 \\
ER da mediana a posteriori & -0.030 & 0.210 & -0.414 & -0.227 & -0.145 & 1.220 & 0.089 & -0.208 & 0.006 \\
EAR da mediana a posteriori & 0.048 & 0.215 & 0.435 & 0.227 & 0.146 & 1.270 & 0.089 & 0.208 & 0.087 \\
REQM da mediana a posteriori & 0.086 & 0.246 & 0.241 & 0.728 & 0.679 & 0.762 & 0.064 & 0.064 & 0.113 \\
\hline
\end{tabular}

Tabela A.35: Resultados descritivos: ER, EAR e REQM, da mediana a posteriori dos parâmetros do modelo de mistura beta linear com efeitos aleatórios para a média e precisão dos estudos de simulação com respectivos cenários para os parâmetros fixos: 2 e 2 de $R=50$ bancos de dados.

\begin{tabular}{c|ccccccccc}
\hline & \multicolumn{9}{|c}{ Parâmetro } \\
\cline { 2 - 9 } & $\beta_{1}^{1}$ & $\beta_{2}^{1}$ & $\beta_{1}^{2}$ & $\beta_{2}^{2}$ & $\delta_{11}$ & $\delta_{12}$ & $\pi_{1}$ & $\pi_{2}$ & $\sigma_{b}$ \\
\hline \hline Valor verdadeiro & -2 & 0.500 & -0.500 & 3 & 3 & 1.500 & 0.700 & 0.300 & 1 \\
Mediana a posteriori média & -1.950 & 0.845 & -0.369 & 2.260 & 2.920 & 1.200 & 0.767 & 0.233 & 1.080 \\
ER da mediana a posteriori & -0.032 & 0.680 & -0.276 & -0.249 & -0.032 & -0.187 & 0.095 & -0.221 & 0.110 \\
EAR da mediana a posteriori & 0.042 & 0.680 & 0.354 & 0.249 & 0.064 & 0.268 & 0.095 & 0.221 & 0.137 \\
REQM da mediana a posteriori & 0.108 & 0.366 & 0.220 & 0.782 & 0.251 & 0.488 & 0.068 & 0.068 & 0.190 \\
\hline
\end{tabular}

Tabela A.36: Resultados descritivos: ER, EAR e REQM, da mediana a posteriori dos parâmetros do modelo de mistura beta linear com efeitos aleatórios para a média e precisão dos estudos de simulação com respectivos cenários para os parâmetros fixos: 3 e 1 de $R=50$ bancos de dados.

\begin{tabular}{|c|c|c|c|c|c|c|c|c|c|}
\hline & \multicolumn{9}{|c|}{ Parâmetro } \\
\hline & $\beta_{1}^{1}$ & $\beta_{2}^{1}$ & $\beta_{1}^{2}$ & $\beta_{2}^{2}$ & $\delta_{11}$ & $\delta_{12}$ & $\pi_{1}$ & $\pi_{2}$ & $\sigma_{b}$ \\
\hline Valor verdadeiro & -2 & 0.500 & -0.500 & 3 & 4 & 0.500 & 0.700 & 0.300 & 1 \\
\hline Mediana a posteriori média & -1.940 & 0.866 & -0.390 & 2.150 & 3.330 & 1.030 & 0.760 & 0.240 & 1.110 \\
\hline ER da mediana a posteriori & -0.026 & 0.766 & -0.178 & -0.294 & -0.184 & 0.972 & 0.086 & -0.202 & 0.134 \\
\hline EAR da mediana a posteriori & 0.037 & 0.766 & 0.282 & 0.294 & 0.184 & 1.200 & 0.086 & 0.202 & 0.154 \\
\hline REQM da mediana a posteriori & 0.088 & 0.407 & 0.170 & 0.912 & 0.824 & 0.734 & 0.063 & 0.063 & 0.208 \\
\hline
\end{tabular}

Tabela A.37: Resultados descritivos: ER, EAR e REQM, da mediana a posteriori dos parâmetros do modelo de mistura beta linear com efeitos aleatórios para a média e precisão dos estudos de simulação com respectivos cenários para os parâmetros fixos: 3 e 2 de $R=50$ bancos de dados.

\begin{tabular}{c|ccccccccc}
\hline & \multicolumn{10}{c}{ Parâmetro } \\
\cline { 2 - 10 } & $\beta_{1}^{1}$ & $\beta_{2}^{1}$ & $\beta_{1}^{2}$ & $\beta_{2}^{2}$ & $\delta_{11}$ & $\delta_{12}$ & $\pi_{1}$ & $\pi_{2}$ \\
\hline \hline Valor verdadeiro & -1 & 3 & -3 & 1 & 3 & 1.500 & 0.700 & 0.300 & 1 \\
Mediana a posteriori média & -0.852 & 2.880 & -2.730 & 2.100 & 3.120 & 1.400 & 0.742 & 0.258 & 1.300 \\
ER da mediana a posteriori & -0.117 & -0.051 & -0.105 & 1.090 & 0.022 & -0.086 & 0.060 & -0.141 & 0.307 \\
EAR da mediana a posteriori & 0.174 & 0.051 & 0.107 & 1.090 & 0.055 & 0.188 & 0.060 & 0.141 & 0.307 \\
REQM da mediana a posteriori & 0.231 & 0.196 & 0.476 & 1.120 & 0.194 & 0.352 & 0.045 & 0.045 & 0.336 \\
\hline
\end{tabular}

Tabela A.38: Resultados descritivos: ER, EAR e REQM, da mediana a posteriori dos parâmetros do modelo de mistura beta linear com efeitos aleatórios para a média e precisão dos estudos de simulação com respectivos cenários para os parâmetros fixos: 4 e 1 de $R=50$ bancos de dados. 


\begin{tabular}{|c|c|c|c|c|c|c|c|c|c|}
\hline & \multicolumn{9}{|c|}{ Parâmetro } \\
\hline & $\beta_{1}^{1}$ & $\beta_{2}^{1}$ & $\beta_{1}^{2}$ & $\beta_{2}^{2}$ & $\delta_{11}$ & $\delta_{12}$ & $\pi_{1}$ & $\pi_{2}$ & $\sigma_{b}$ \\
\hline Valor verdadeiro & -1 & 3 & -3 & 1 & 4 & 0.500 & 0.700 & 0.300 & 1 \\
\hline Mediana a posteriori média & -0.871 & 2.860 & -2.700 & 2.180 & 3.590 & 1.130 & 0.737 & 0.263 & 1.350 \\
\hline ER da mediana a posteriori & -0.080 & -0.055 & -0.107 & 1.170 & -0.120 & 1.260 & 0.058 & -0.136 & 0.342 \\
\hline EAR da mediana a posteriori & 0.161 & 0.055 & 0.110 & 1.170 & 0.122 & 1.280 & 0.058 & 0.136 & 0.342 \\
\hline REQM da mediana a posteriori & 0.244 & 0.200 & 0.471 & 1.190 & 0.575 & 0.772 & 0.044 & 0.044 & 0.366 \\
\hline
\end{tabular}

Tabela A.39: Resultados descritivos: ER, EAR e REQM, da mediana a posteriori dos parâmetros do modelo de mistura beta linear com efeitos aleatórios para a média e precisão dos estudos de simulação com respectivos cenários para os parâmetros fixos: 4 e 2 de $R=50$ bancos de dados.

\begin{tabular}{c|ccccccccc}
\hline & \multicolumn{10}{c}{ Parâmetro } & \multicolumn{1}{c}{$\pi_{2}$} & $\sigma_{b}$ \\
\cline { 2 - 10 } & $\beta_{1}^{1}$ & $\beta_{2}^{1}$ & $\beta_{1}^{2}$ & $\beta_{2}^{2}$ & $\delta_{11}$ & $\delta_{12}$ & $\pi_{1}$ & $\pi_{2}$ \\
\hline \hline Valor verdadeiro & -1 & 3 & -3 & 1 & 2 & 2 & 0.700 & 0.300 & 1 \\
Mediana a posteriori média & -0.816 & 2.900 & -2.780 & 1.930 & 2.390 & 1.500 & 0.746 & 0.254 & 1.200 \\
ER da mediana a posteriori & -0.174 & -0.040 & -0.071 & 0.886 & 0.185 & -0.238 & 0.066 & -0.155 & 0.208 \\
EAR da mediana a posteriori & 0.186 & 0.046 & 0.078 & 0.892 & 0.185 & 0.241 & 0.066 & 0.155 & 0.219 \\
REQM da mediana a posteriori & 0.207 & 0.182 & 0.284 & 0.939 & 0.385 & 0.526 & 0.050 & 0.050 & 0.260 \\
\hline
\end{tabular}

Tabela A.40: Resultados descritivos: ER, EAR e REQM, da mediana a posteriori dos parâmetros do modelo de mistura beta linear com efeitos aleatórios para a média e precisão dos estudos de simulação com respectivos cenários para os parâmetros fixos: 4 e 3 de $R=50$ bancos de dados.

\section{A.5 Modelo não linear com parâmetro de precisão constante}

\begin{tabular}{c|cccccccc}
\hline & \multicolumn{9}{|c}{ Parâmetro } & \multicolumn{1}{c}{$\pi_{1}$} & $\pi_{b}$ \\
\cline { 2 - 9 } & $\beta_{1}^{1}$ & $\beta_{2}^{1}$ & $\beta_{1}^{2}$ & $\beta_{2}^{2}$ & $\phi$ & 0.500 & 1 \\
\hline Valor verdadeiro & -2.400 & 0.800 & -0.500 & -0.600 & 30 & 0.500 & 0.500 & 0.995 \\
Mediana a posteriori média & -2.240 & 0.593 & -0.421 & -0.522 & 39.200 & 0.498 & 0.502 & 0.004 \\
ER da mediana a posteriori & -0.072 & -0.292 & -0.125 & -0.160 & 0.310 & 0.002 & -0.002 & 0.002 \\
EAR da mediana a posteriori & 0.076 & 0.308 & 0.219 & 0.183 & 0.310 & 0.028 & 0.028 & 0.061 \\
REQM da mediana a posteriori & 0.213 & 0.290 & 0.133 & 0.139 & 9.700 & 0.017 & 0.017 & 0.080 \\
\hline
\end{tabular}

Tabela A.41: Resultados descritivos: ER, EAR e REQM, da mediana a posteriori dos parâmetros do modelo de mistura beta não linear com efeitos aleatórios para a média dos estudos de simulação com cenário 1 e $\phi=30$ de $R=50$ bancos de dados e com $N=100, n=5$.

\begin{tabular}{c|cccccccc}
\hline & \multicolumn{9}{|c}{ Parâmetro } \\
\cline { 2 - 9 } & $\beta_{1}^{1}$ & $\beta_{2}^{1}$ & $\beta_{1}^{2}$ & $\beta_{2}^{2}$ & $\phi$ & $\pi_{1}$ & $\pi_{2}$ & $\sigma_{b}$ \\
\hline \hline Valor verdadeiro & -2.400 & 0.800 & -0.500 & -0.600 & 50 & 0.500 & 0.500 & 1 \\
Mediana a posteriori média & -2.150 & 0.427 & -0.494 & -0.444 & 55.800 & 0.499 & 0.501 & 1.060 \\
ER da mediana a posteriori & -0.097 & -0.432 & -0.037 & -0.261 & 0.136 & 0.002 & -0.002 & 0.051 \\
EAR da mediana a posteriori & 0.099 & 0.443 & 0.210 & 0.266 & 0.147 & 0.029 & 0.029 & 0.068 \\
REQM da mediana a posteriori & 0.277 & 0.402 & 0.137 & 0.191 & 9.130 & 0.019 & 0.019 & 0.080 \\
\hline
\end{tabular}

Tabela A.42: Resultados descritivos: ER, EAR e REQM, da mediana a posteriori dos parâmetros do modelo de mistura beta não linear com efeitos aleatórios para a média dos estudos de simulação com cenário 1 e $\phi=50$ de $R=50$ bancos de dados e com $N=100, n=5$. 


\begin{tabular}{c|cccccccc}
\hline & \multicolumn{9}{|c}{ Parâmetro } \\
\cline { 2 - 9 } & $\beta_{1}^{1}$ & $\beta_{2}^{1}$ & $\beta_{1}^{2}$ & $\beta_{2}^{2}$ & $\phi$ & $\pi_{1}$ & $\pi_{2}$ & $\sigma_{b}$ \\
\hline \hline Valor verdadeiro & -2.400 & 0.800 & -0.500 & -0.600 & 70 & 0.500 & 0.500 & 1 \\
Mediana a posteriori média & -2.180 & 0.404 & -0.551 & -0.408 & 67.300 & 0.493 & 0.507 & 1.060 \\
ER da mediana a posteriori & -0.089 & -0.477 & 0.084 & -0.333 & -0.014 & -0.015 & 0.015 & 0.073 \\
EAR da mediana a posteriori & 0.093 & 0.477 & 0.206 & 0.336 & 0.098 & 0.032 & 0.032 & 0.079 \\
REQM da mediana a posteriori & 0.262 & 0.422 & 0.128 & 0.230 & 9.050 & 0.020 & 0.020 & 0.098 \\
\hline
\end{tabular}

Tabela A.43: Resultados descritivos: ER, EAR e REQM, da mediana a posteriori dos parâmetros do modelo de mistura beta não linear com efeitos aleatórios para a média dos estudos de simulação com cenário 1 e $\phi=70$ de $R=50$ bancos de dados e com $N=100, n=5$.

\begin{tabular}{c|cccccccc}
\hline & \multicolumn{7}{|c}{ Parâmetro } & \multicolumn{1}{c}{$\pi_{1}$} \\
\cline { 2 - 9 } & $\beta_{1}^{1}$ & $\beta_{2}^{1}$ & $\beta_{1}^{2}$ & $\beta_{2}^{2}$ & $\phi$ & $\pi_{b}$ & \multicolumn{1}{c}{$\pi_{2}$} \\
\hline \hline Valor verdadeiro & -2.400 & 0.800 & -1 & 2.800 & 30 & 0.500 & 0.500 & 1 \\
Mediana a posteriori média & -2.290 & 0.890 & -0.878 & 2.770 & 42.200 & 0.497 & 0.503 & 0.909 \\
ER da mediana a posteriori & -0.043 & 0.133 & -0.112 & -0.009 & 0.395 & -0.004 & 0.004 & -0.087 \\
EAR da mediana a posteriori & 0.053 & 0.204 & 0.147 & 0.037 & 0.395 & 0.023 & 0.023 & 0.099 \\
REQM da mediana a posteriori & 0.154 & 0.216 & 0.176 & 0.133 & 12.000 & 0.015 & 0.015 & 0.115 \\
\hline
\end{tabular}

Tabela A.44: Resultados descritivos: ER, EAR e REQM, da mediana a posteriori dos parâmetros do modelo de mistura beta não linear com efeitos aleatórios para a média dos estudos de simulação com cenário 2 e $\phi=30$ de $R=50$ bancos de dados e com $N=100, n=5$.

\begin{tabular}{c|cccccccccc}
\hline & \multicolumn{7}{c}{ Parâmetro } \\
\cline { 2 - 8 } & $\beta_{1}^{1}$ & $\beta_{2}^{1}$ & $\beta_{1}^{2}$ & $\beta_{2}^{2}$ & $\phi$ & $\pi_{1}$ & $\pi_{2}$ \\
\hline \hline Valor verdadeiro & -2.400 & 0.800 & -1 & 2.800 & 50 & 0.500 & 0.500 \\
Mediana a posteriori média & -2.260 & 0.970 & -0.879 & 2.710 & 65.900 & 0.501 & 0.499 & 0.923 \\
ER da mediana a posteriori & -0.054 & 0.257 & -0.109 & -0.034 & 0.284 & 0.002 & -0.002 & -0.073 \\
EAR da mediana a posteriori & 0.056 & 0.269 & 0.139 & 0.050 & 0.286 & 0.025 & 0.025 & 0.084 \\
REQM da mediana a posteriori & 0.159 & 0.290 & 0.167 & 0.179 & 15.400 & 0.016 & 0.016 & 0.098 \\
\hline
\end{tabular}

Tabela A.45: Resultados descritivos: ER, EAR e REQM, da mediana a posteriori dos parâmetros do modelo de mistura beta não linear com efeitos aleatórios para a média dos estudos de simulação com cenário 2 e $\phi=50$ de $R=50$ bancos de dados e com $N=100, n=5$.

\begin{tabular}{c|cccccccc}
\hline & \multicolumn{7}{|c}{ Parâmetro } \\
\cline { 2 - 9 } & $\beta_{1}^{1}$ & $\beta_{2}^{1}$ & $\beta_{1}^{2}$ & $\beta_{2}^{2}$ & $\phi$ & $\pi_{1}$ & $\pi_{2}$ & $\sigma_{b}$ \\
\hline \hline Valor verdadeiro & -2.400 & 0.800 & -1 & 2.800 & 70 & 0.500 & 0.500 & 1 \\
Mediana a posteriori média & -2.250 & 0.997 & -0.895 & 2.690 & 81.700 & 0.500 & 0.500 & 0.924 \\
ER da mediana a posteriori & -0.051 & 0.316 & -0.110 & -0.055 & 0.154 & -0.0001 & 0.0001 & -0.077 \\
EAR da mediana a posteriori & 0.058 & 0.326 & 0.124 & 0.062 & 0.200 & 0.027 & 0.027 & 0.084 \\
REQM da mediana a posteriori & 0.164 & 0.334 & 0.154 & 0.237 & 16.800 & 0.016 & 0.016 & 0.097 \\
\hline
\end{tabular}

Tabela A.46: Resultados descritivos: ER, EAR e REQM, da mediana a posteriori dos parâmetros do modelo de mistura beta não linear com efeitos aleatórios para a média dos estudos de simulação com cenário 2 e $\phi=70$ de $R=50$ bancos de dados e com $N=100, n=5$. 


\begin{tabular}{c|cccccccccccc}
\hline & \multicolumn{9}{c}{ Parâmetro } \\
\cline { 2 - 8 } & $\beta_{1}^{1}$ & $\beta_{2}^{1}$ & $\beta_{3}^{1}$ & $\beta_{1}^{2}$ & $\beta_{2}^{2}$ & $\beta_{3}^{2}$ & $\phi_{1}$ \\
\hline \hline Valor verdadeiro & -1.590 & 3.058 & 0.943 & -0.076 & 0.224 & 1.497 & 56.6 & 6.26 & 0.899 \\
Mediana a posteriori média & -1.850 & 3.170 & 1.240 & -0.123 & 0.157 & 1.610 & 66.000 & 5.810 & 0.885 & -0.15 \\
ER da mediana a posteriori & 0.127 & 0.030 & 0.307 & -0.863 & -0.315 & 0.119 & 0.144 & -0.106 & -0.020 \\
EAR da mediana a posteriori & 0.183 & 0.074 & 0.375 & 2.490 & 0.540 & 0.137 & 0.149 & 0.154 & 0.048 \\
REQM da mediana a posteriori & 0.353 & 0.298 & 0.465 & 0.374 & 0.157 & 0.338 & 10.400 & 1.560 & 0.052 \\
\hline
\end{tabular}

Tabela A.47: Resultados descritivos: ER, EAR e REQM, da mediana a posteriori dos parâmetros $\beta_{i_{p}}^{s}, \phi_{s}, i_{p}=1,2,3, s=1,2$ e $\pi_{1}$ do modelo de mistura beta não linear com efeitos aleatórios para a média dos estudos de simulação com cenário 3 de $R=25$ bancos de dados.

\begin{tabular}{c|cccccc}
\hline & \multicolumn{7}{|c}{ Parâmetro } \\
\cline { 2 - 7 } & $\sigma_{b, 11}$ & $\sigma_{b, 12}$ & $\sigma_{b, 13}$ & $\sigma_{b, 22}$ & $\sigma_{b, 23}$ & $\sigma_{b, 33}$ \\
\hline \hline Valor verdadeiro & 1.304 & -1.164 & 0.997 & 1.253 & -0.891 & 0.848 \\
Mediana a posteriori média & 0.709 & -0.351 & 0.403 & 0.587 & -0.313 & 0.825 \\
ER da mediana a posteriori & 2.000 & -1.370 & 0.174 & 3.460 & -1.260 & 0.319 \\
EAR da mediana a posteriori & 2.830 & 2.050 & 1.230 & 4.320 & 1.430 & 0.665 \\
REQM da mediana a posteriori $i$ & 14.300 & 7.320 & 3.250 & 19.800 & 2.770 & 1.000 \\
\hline
\end{tabular}

Tabela A.48: Resultados descritivos: ER, EAR e REQM, da mediana a posteriori dos parâmetros $\sigma_{b, i_{d 1} i_{d 2}}, i_{d 1}, i_{d 2} \in\{1,2,3\}$ do modelo de mistura beta não linear com efeitos aleatórios para a média dos estudos de simulaçấo com cenário 3 de $R=25$ bancos de dados. 


\section{Apêndice B}

\section{Gráficos de Dispersão correspondentes aos Estudos de Simulação}

B.1 Modelo linear com parâmetro de precisão constante - $\mathrm{N}=100$, $\mathrm{n}=\mathbf{5}$
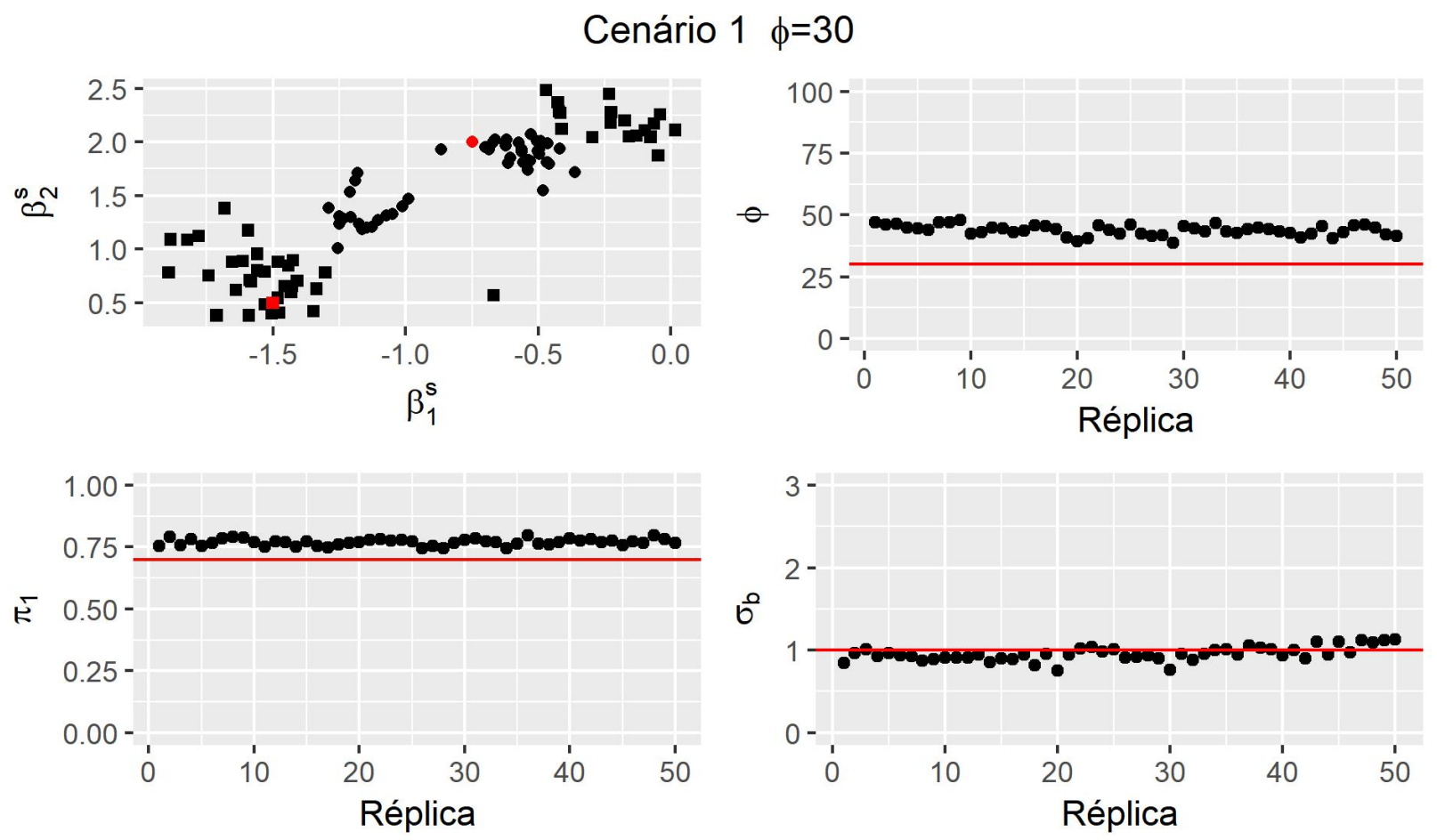

Figura B.1: Gráfico de dispersão das estimativas pontuais - mediana a posteriori - dos estudos de simulação de $R=50$ bancos de dados do modelo de mistura beta linear com efeitos aleatórios para a média com cenário 1, $\phi=30, N=100$ e $n=5$, em que $s=1(\bullet), 2(\bullet)$, e dos verdadeiros valores dos parâmetros (em vermelho). 

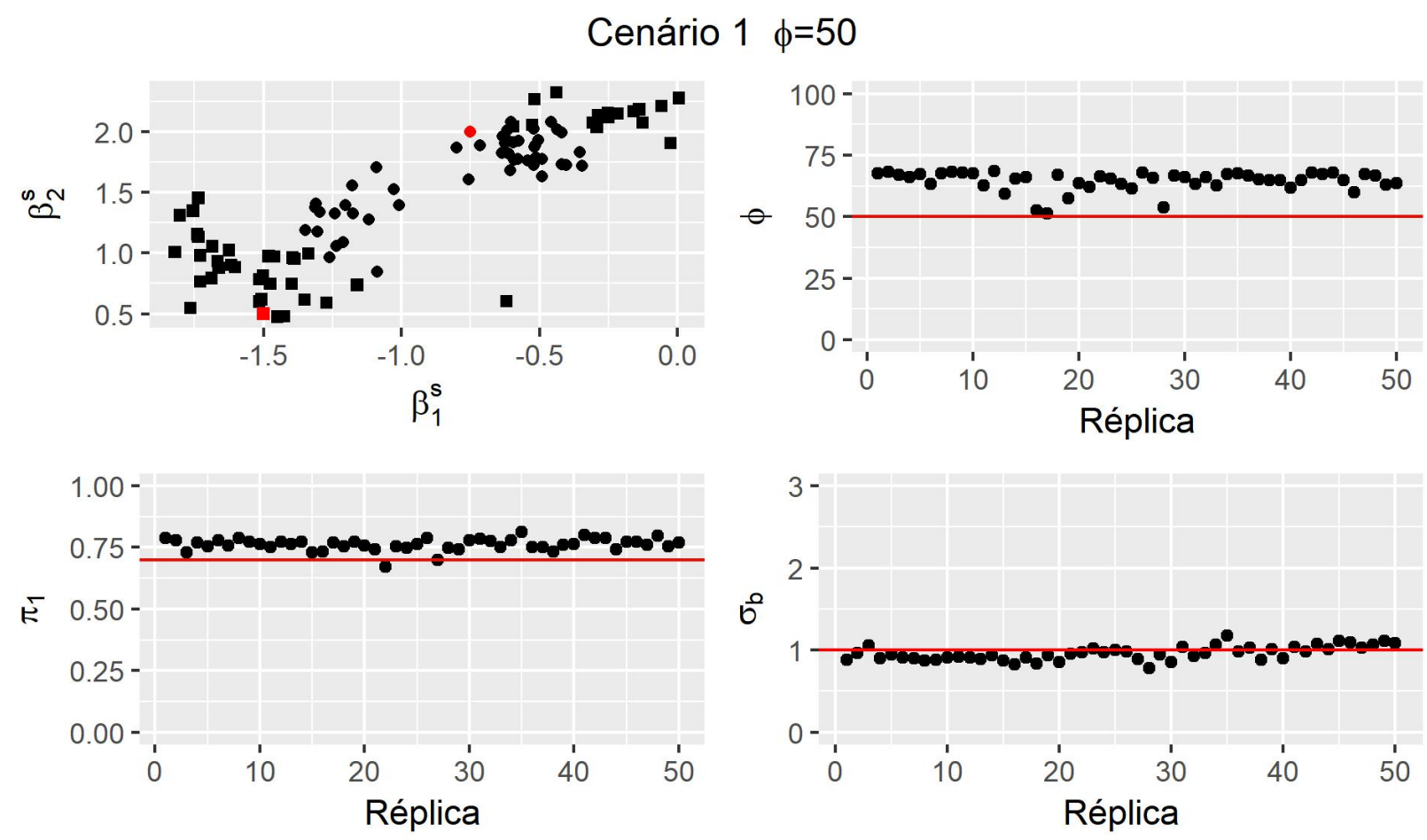

Figura B.2: Gráfico de dispersão das estimativas pontuais - mediana a posteriori - dos estudos de simulação de $R=50$ bancos de dados do modelo de mistura beta linear com efeitos aleatórios para a média com cenário 1, $\phi=50, N=100$ e $n=5$, em que $s=1(\bullet), 2(\mathbf{\square})$, e dos verdadeiros valores dos parâmetros (em vermelho).
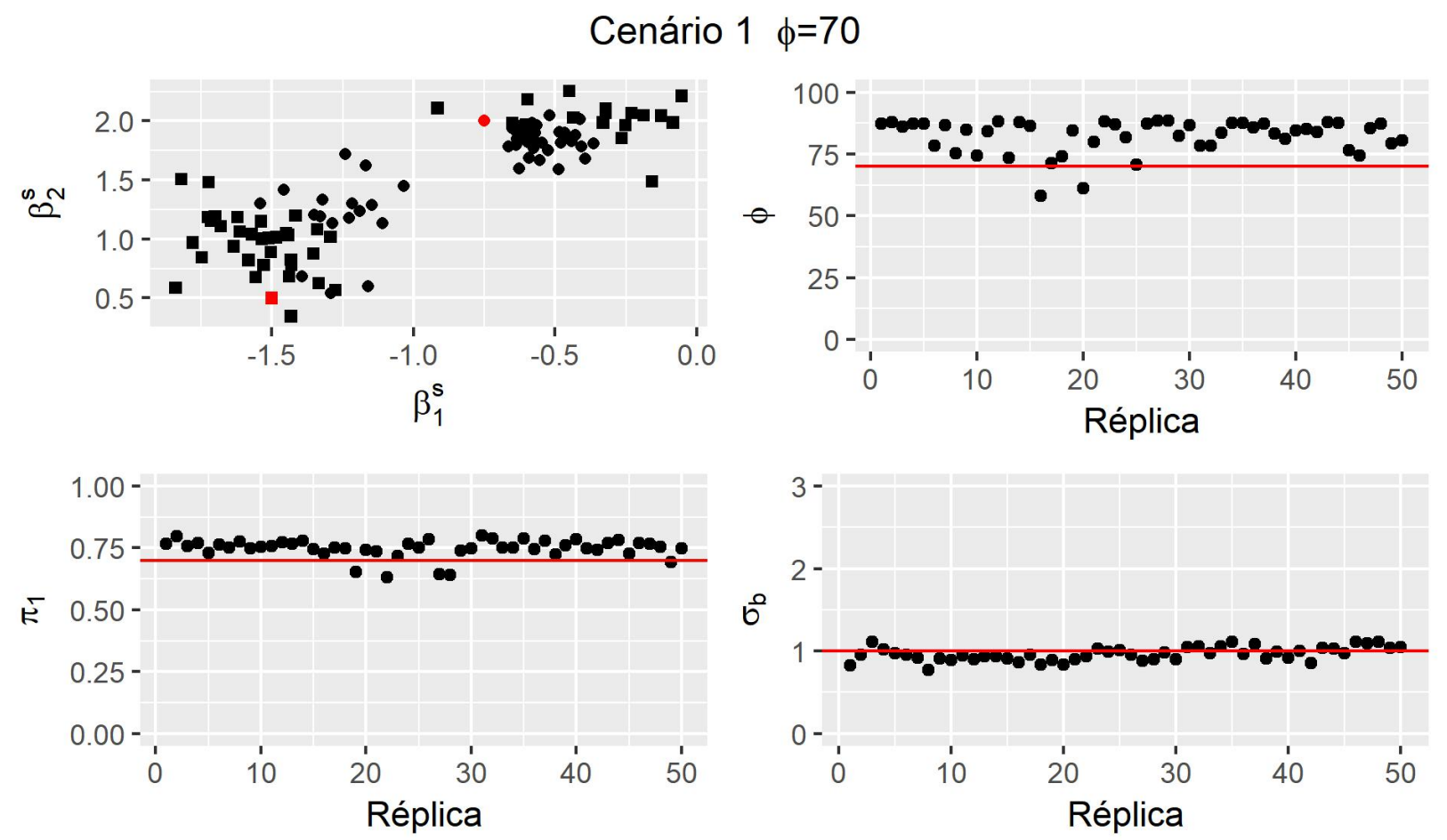

Figura B.3: Gráfico de dispersão das estimativas pontuais - mediana a posteriori - dos estudos de simulação de $R=50$ bancos de dados do modelo de mistura beta linear com efeitos aleatórios para a média com cenário 1, $\phi=70, N=100$ e $n=5$, em que $s=1(\bullet), 2(\mathbf{\bullet})$, e dos verdadeiros valores dos parâmetros (em vermelho). 

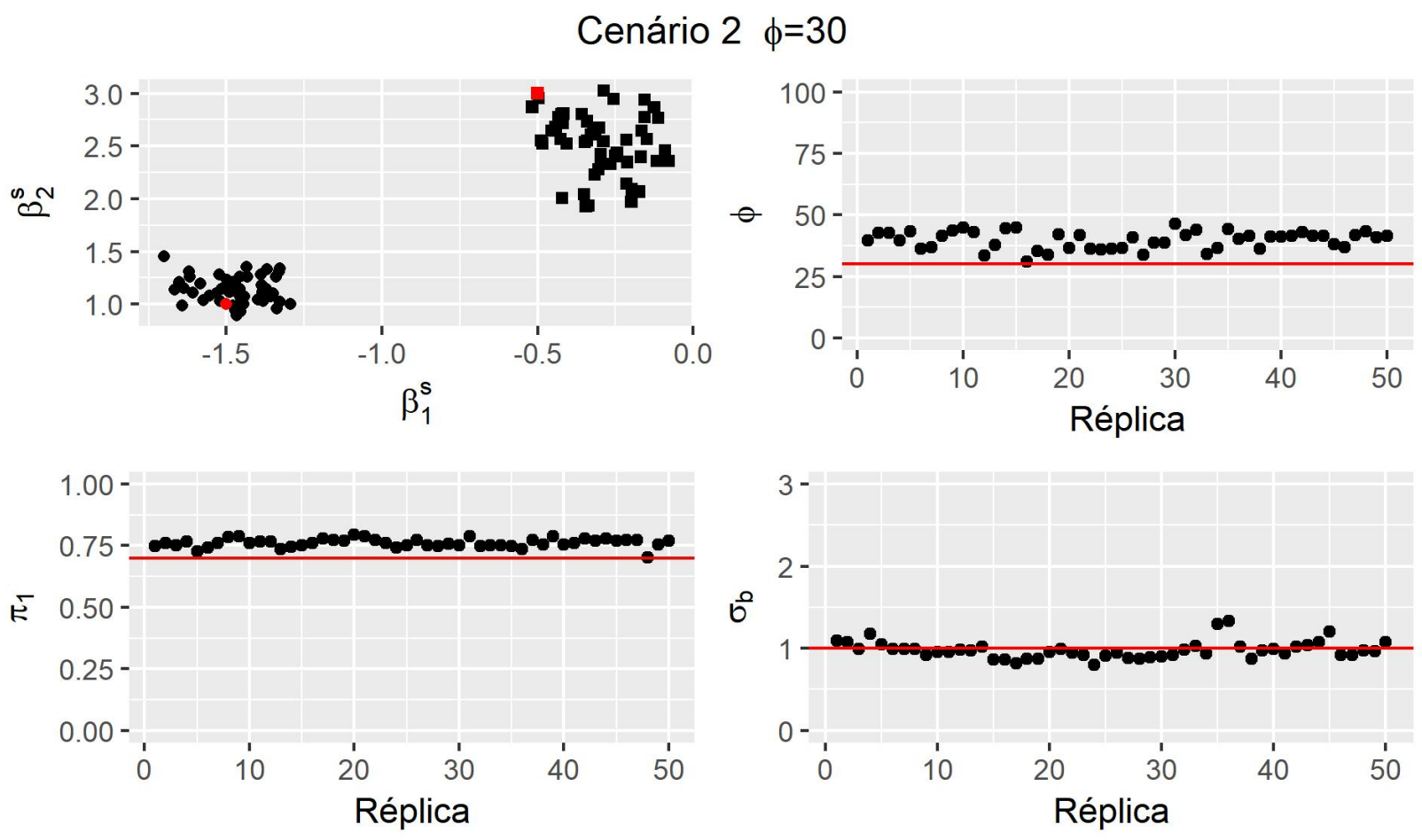

Figura B.4: Gráfico de dispersão das estimativas pontuais - mediana a posteriori - dos estudos de simulação de $R=50$ bancos de dados do modelo de mistura beta linear com efeitos aleatórios para a média com cenário $2, \phi=30, N=100$ e $n=5$, em que $s=1(\bullet), 2(\mathbf{\bullet})$, e dos verdadeiros valores dos parâmetros (em vermelho).

\section{Cenário $2 \phi=50$}
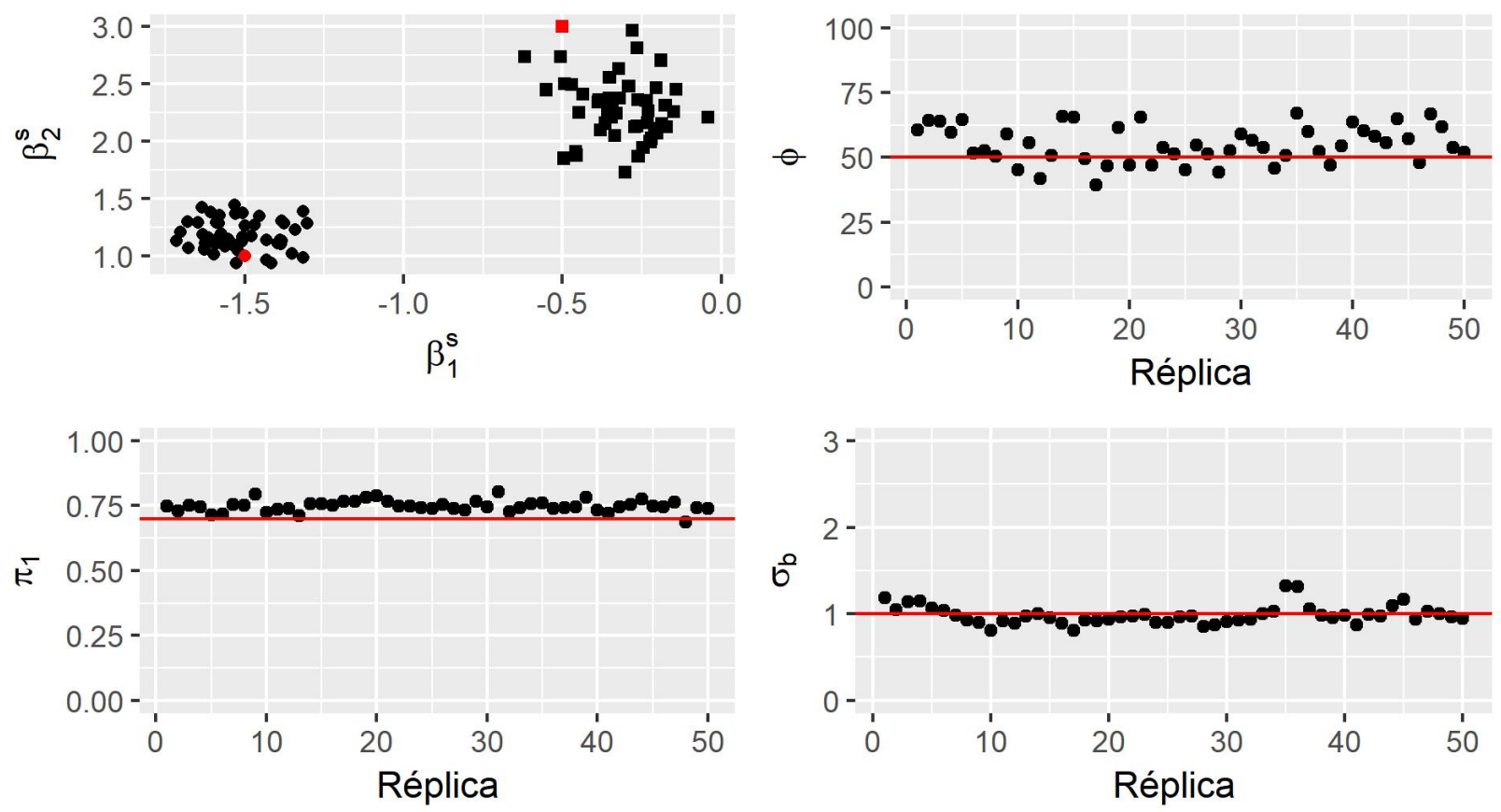

Figura B.5: Gráfico de dispersão das estimativas pontuais - mediana a posteriori - dos estudos de simulação de $R=50$ bancos de dados do modelo de mistura beta linear com efeitos aleatórios para a média com cenário $2, \phi=50, N=100$ e $n=5$, em que $s=1(\bullet), 2(\bullet)$, e dos verdadeiros valores dos parâmetros (em vermelho). 
Cenário $2 \phi=70$
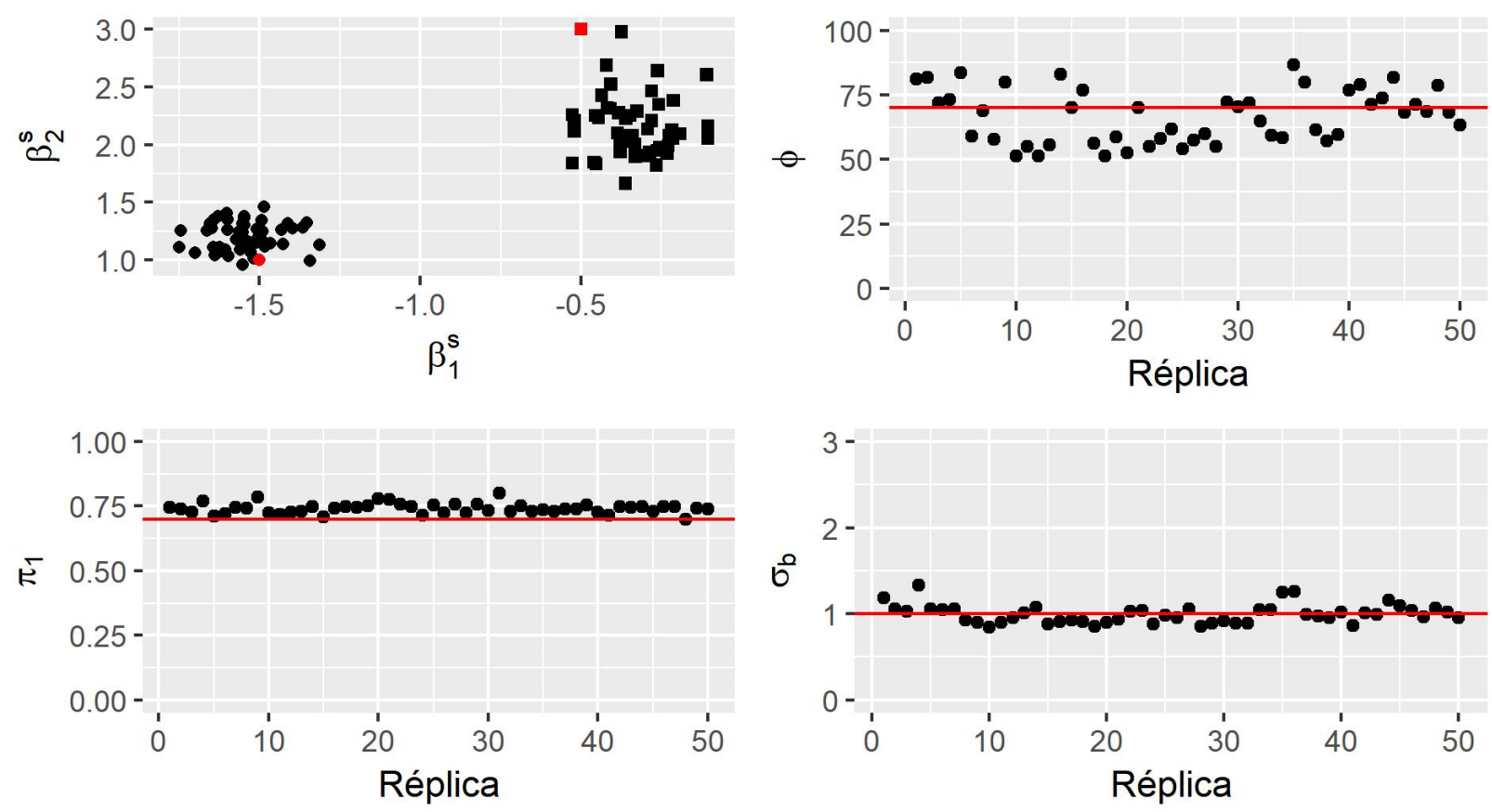

Figura B.6: Gráfico de dispersão das estimativas pontuais - mediana a posteriori - dos estudos de simulação de $R=50$ bancos de dados do modelo de mistura beta linear com efeitos aleatórios para a média com cenário 2, $\phi=70, N=100$ e $n=5$, em que $s=1(\bullet), 2(\mathbf{\square})$, e dos verdadeiros valores dos parâmetros (em vermelho).

\section{Cenário $3 \phi=30$}
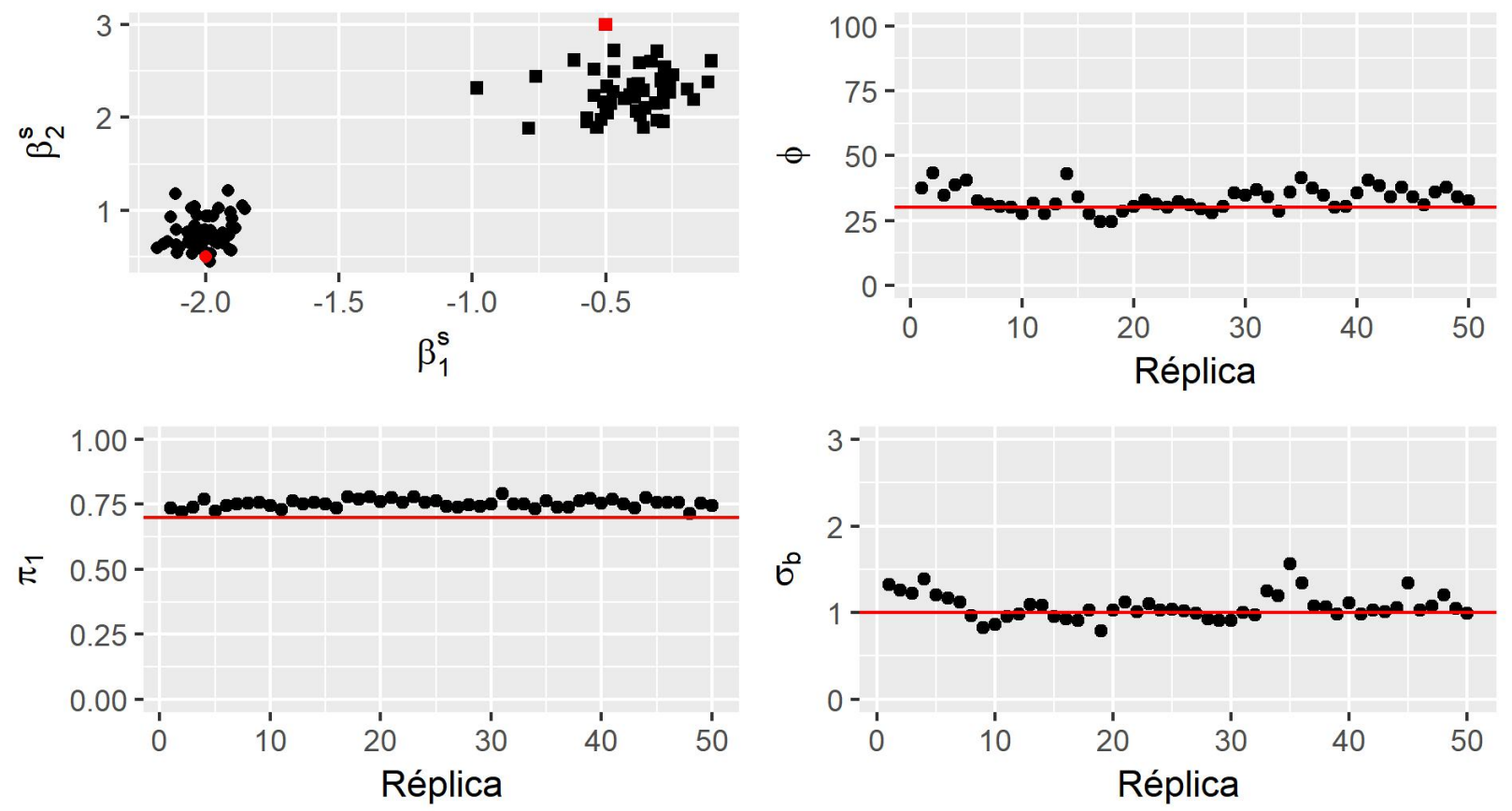

Figura B.7: Gráfico de dispersão das estimativas pontuais - mediana a posteriori - dos estudos de simulação de $R=50$ bancos de dados do modelo de mistura beta linear com efeitos aleatórios para a média com cenário 3, $\phi=30, N=100$ e $n=5$, em que $s=1(\bullet), 2(\mathbf{\bullet})$, e dos verdadeiros valores dos parâmetros (em vermelho). 


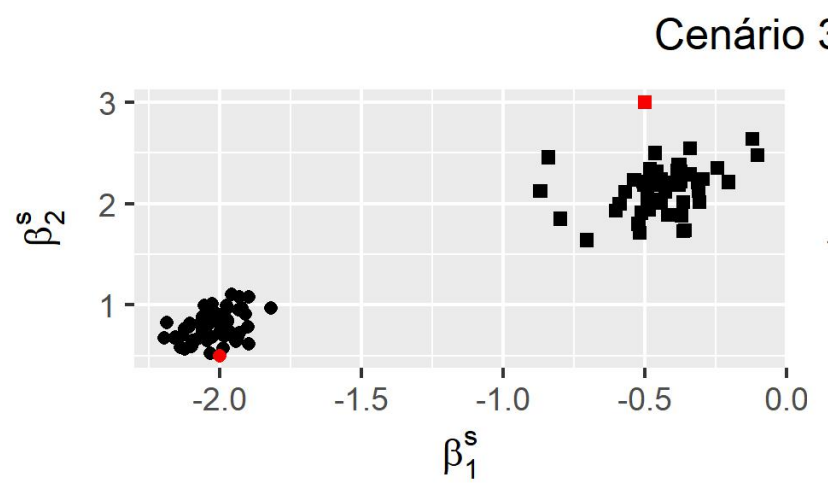

$3 \phi=50$
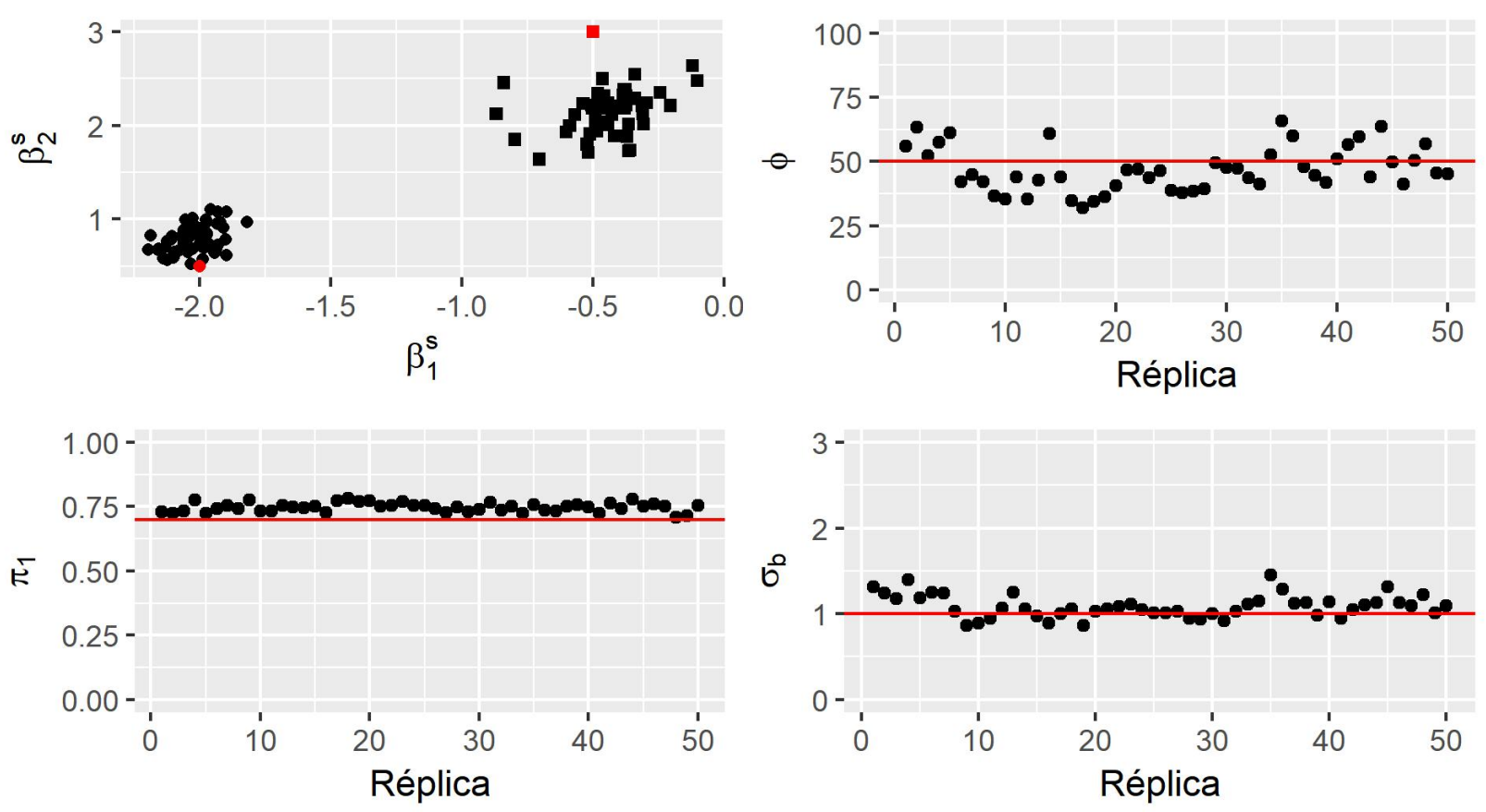

Figura B.8: Gráfico de dispersão das estimativas pontuais - mediana a posteriori - dos estudos de simulação de $R=50$ bancos de dados do modelo de mistura beta linear com efeitos aleatórios para a média com cenário 3, $\phi=50, N=100$ e $n=5$, em que $s=1(\bullet), 2(\mathbf{\bullet})$, e dos verdadeiros valores dos parâmetros (em vermelho).
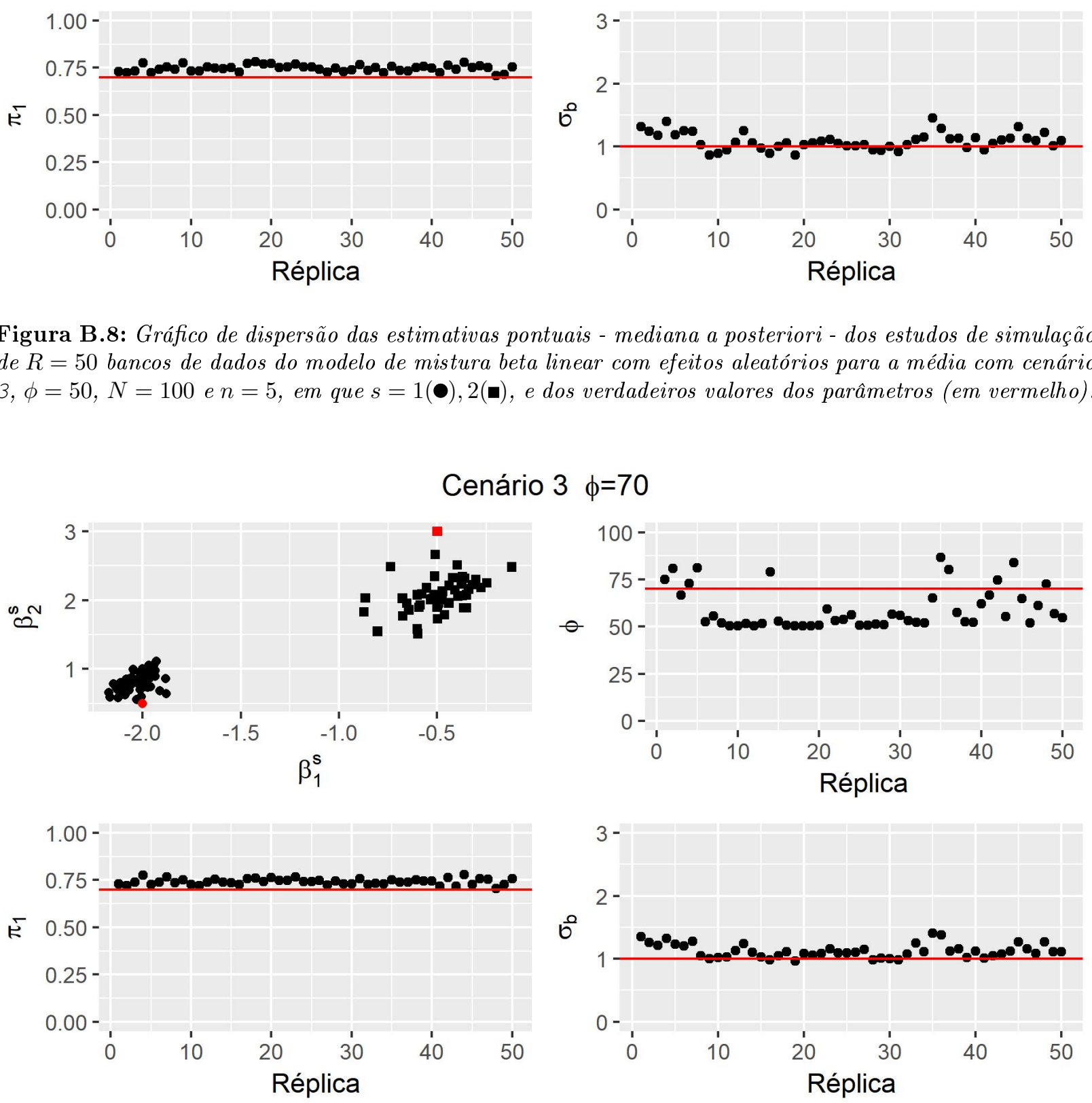

Figura B.9: Gráfico de dispersão das estimativas pontuais - mediana a posteriori - dos estudos de simulação de $R=50$ bancos de dados do modelo de mistura beta linear com efeitos aleatórios para a média com cenário 3, $\phi=70, N=100$ e $n=5$, em que $s=1(\bullet), 2(\bullet)$, e dos verdadeiros valores dos parâmetros (em vermelho). 


\section{Cenário $4 \phi=30$}
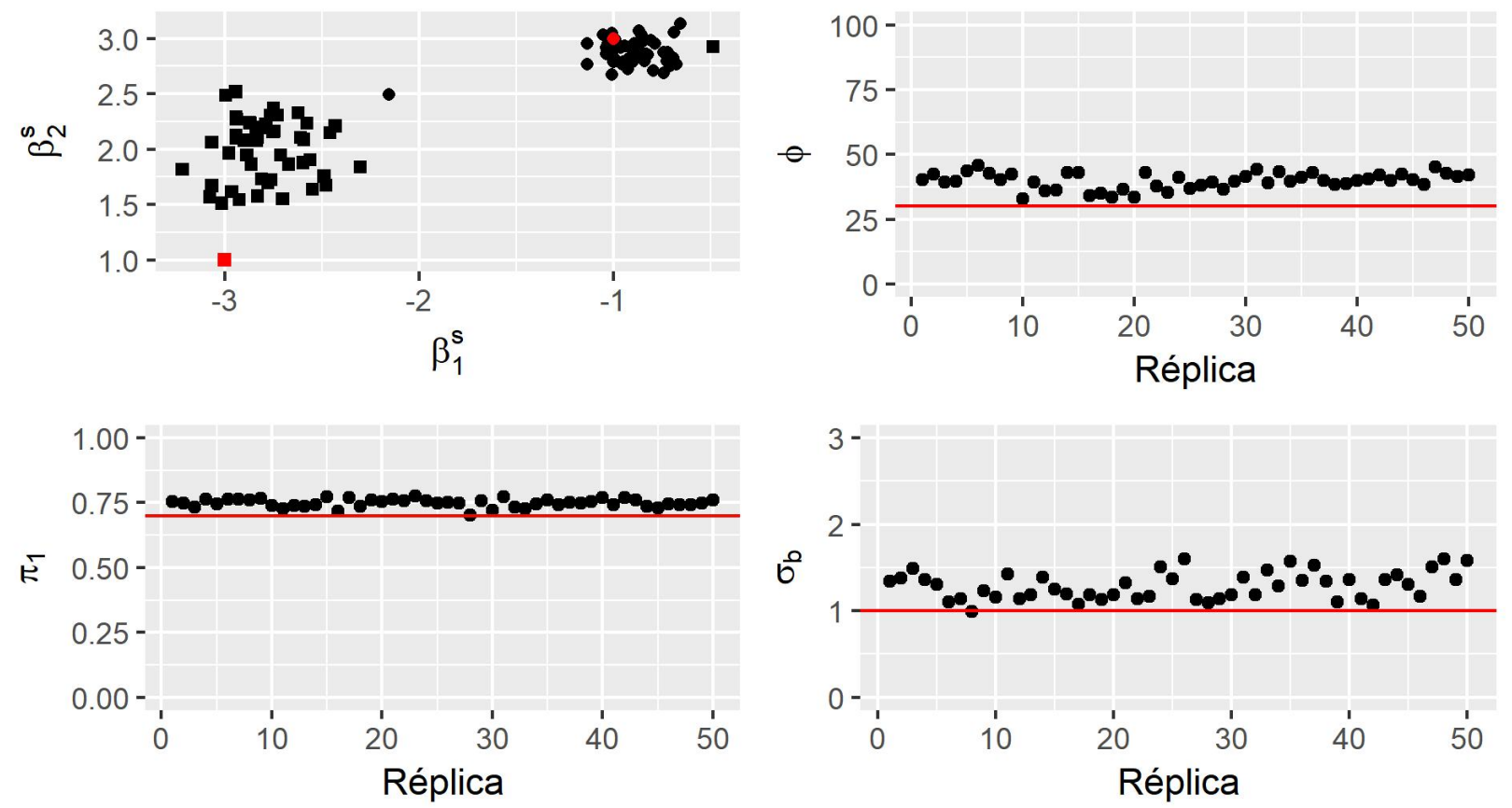

Figura B.10: Gráfico de dispersão das estimativas pontuais - mediana a posteriori - dos estudos de simulação de $R=50$ bancos de dados do modelo de mistura beta linear com efeitos aleatórios para a média com cenário 4, $\phi=30, N=100$ e $n=5$, em que $s=1(\bullet), 2(\mathbf{\bullet})$, e dos verdadeiros valores dos parâmetros (em vermelho).
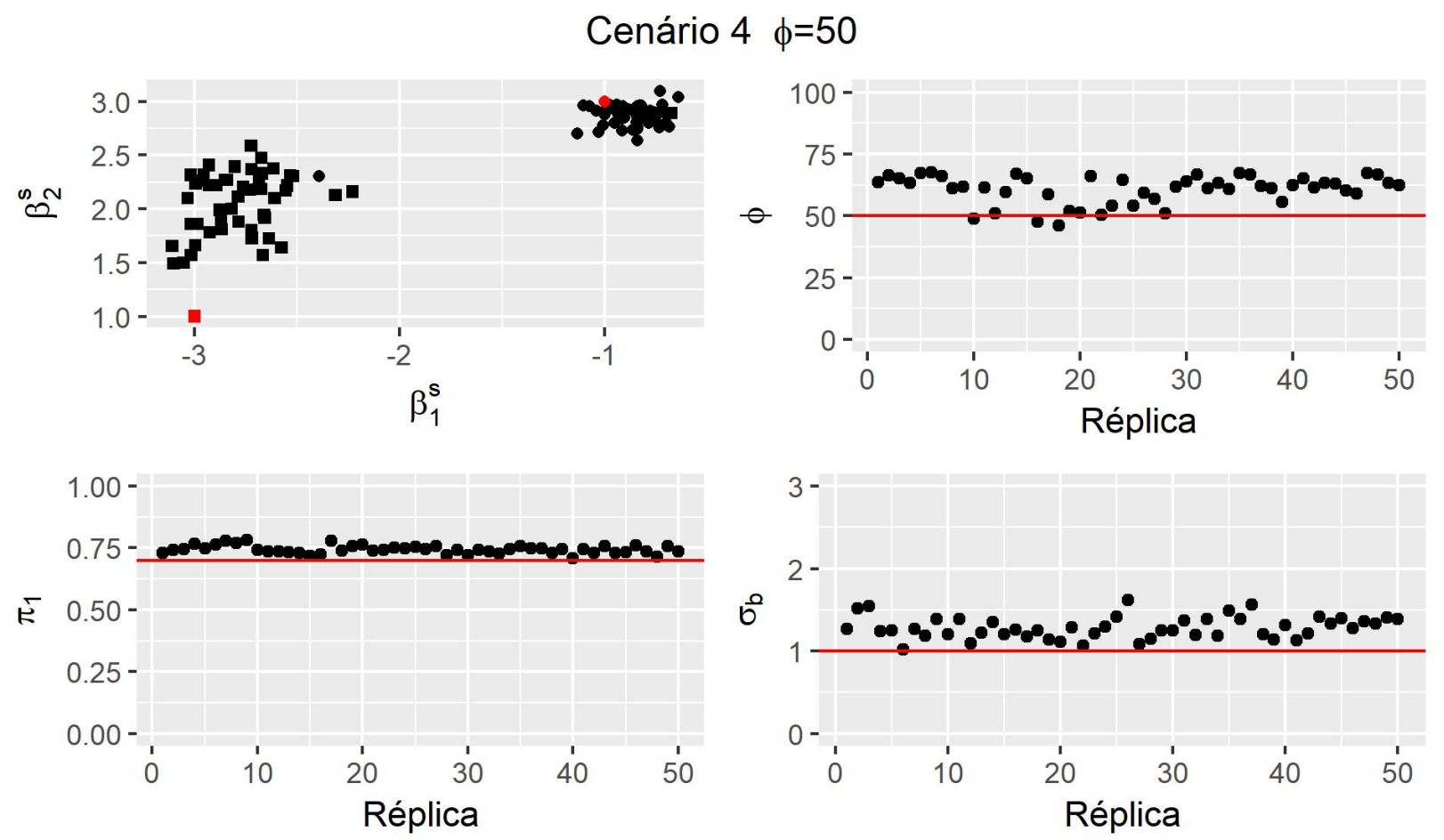

Figura B.11: Gráfico de dispersão das estimativas pontuais - mediana a posteriori - dos estudos de simulação de $R=50$ bancos de dados do modelo de mistura beta linear com efeitos aleatórios para a média com cenário 4, $\phi=50, N=100$ e $n=5$, em que $s=1(\bullet), 2(\boldsymbol{\bullet})$, e dos verdadeiros valores dos parâmetros (em vermelho). 


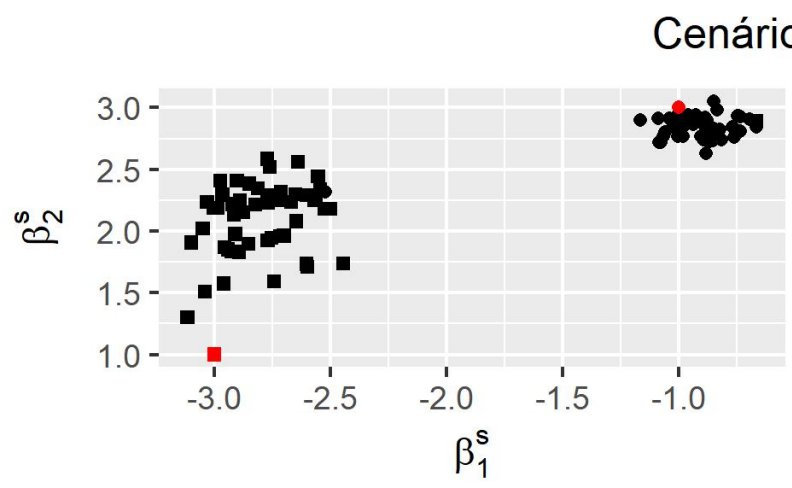

$4 \phi=70$
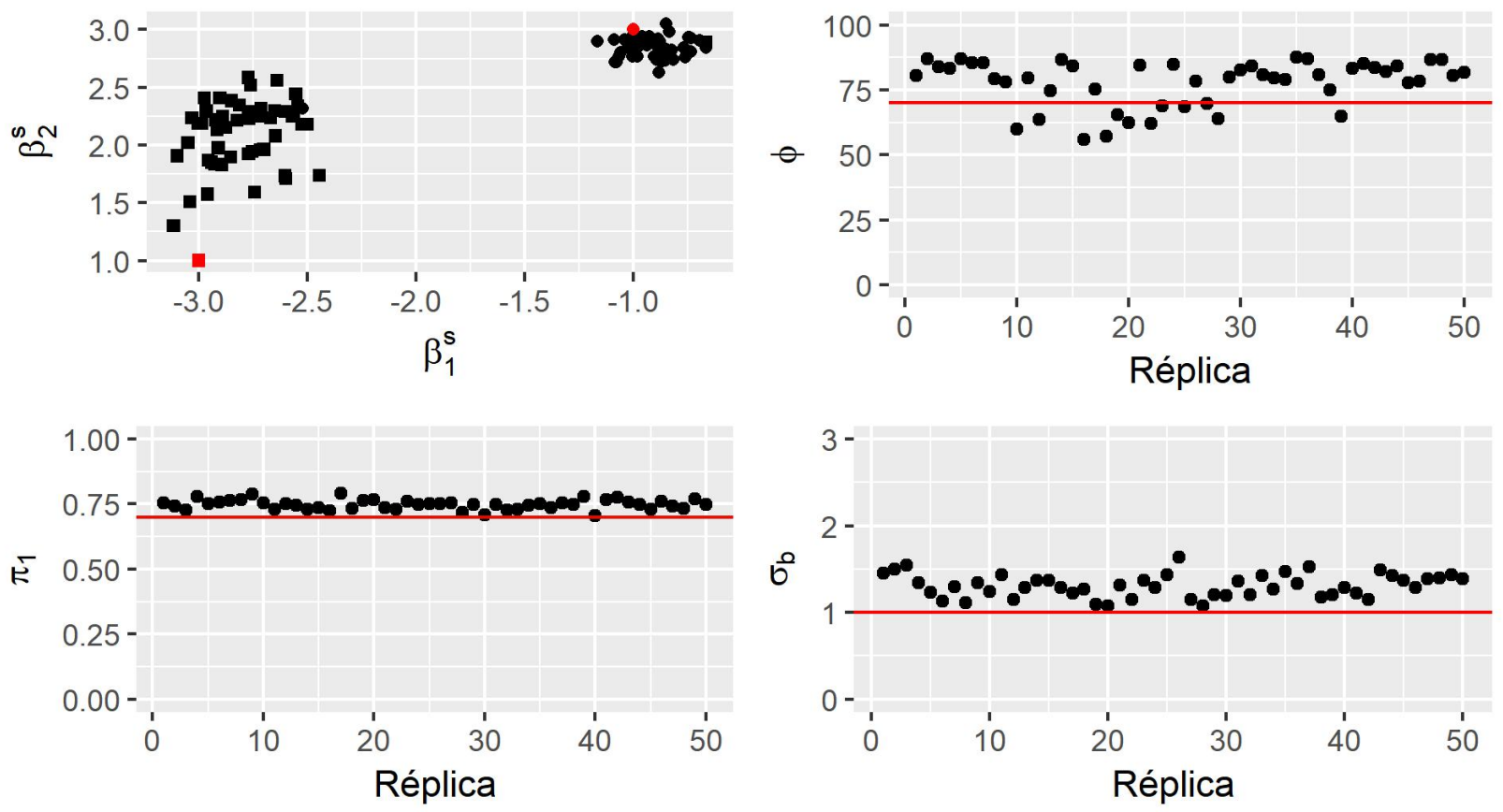

Figura B.12: Gráfico de dispersão das estimativas pontuais - mediana a posteriori - dos estudos de simulação de $R=50$ bancos de dados do modelo de mistura beta linear com efeitos aleatórios para a média com cenário $4, \phi=70, N=100$ e $n=5$, em que $s=1(\bullet), 2(\boldsymbol{\bullet})$, e dos verdadeiros valores dos parâmetros (em vermelho). 


\section{B.2 Modelo linear com parâmetro de precisão constante - $\mathrm{N}=\mathbf{1 0 0 \text { , }}$} $\mathrm{n}=\mathbf{1 0}$
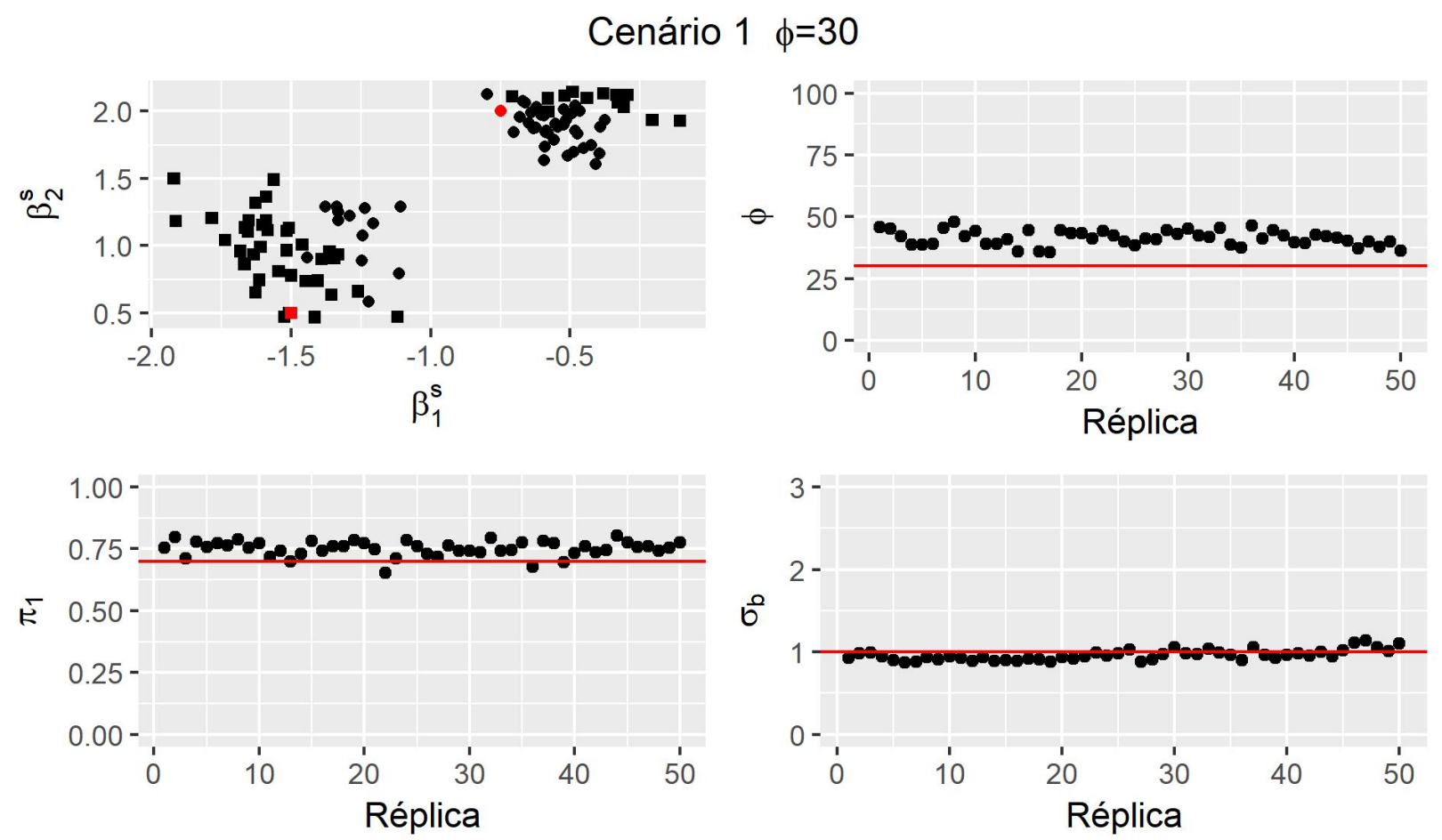

Figura B.13: Gráfico de dispersão das estimativas pontuais - mediana a posteriori - dos estudos de simulação de $R=50$ bancos de dados do modelo de mistura beta linear com efeitos aleatórios para a média com cenário 1, $\phi=30, N=100$ e $n=10$, em que $s=1(\bullet), 2(\mathbf{\bullet})$, e dos verdadeiros valores dos parâmetros (em vermelho). 


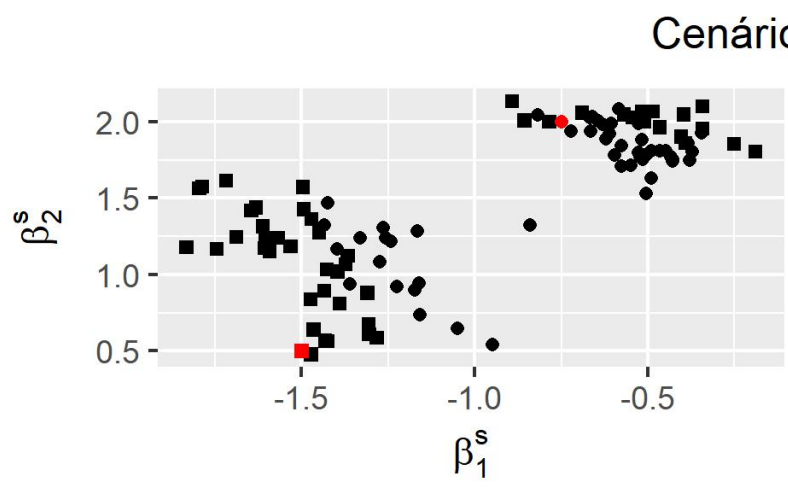

$1 \phi=50$
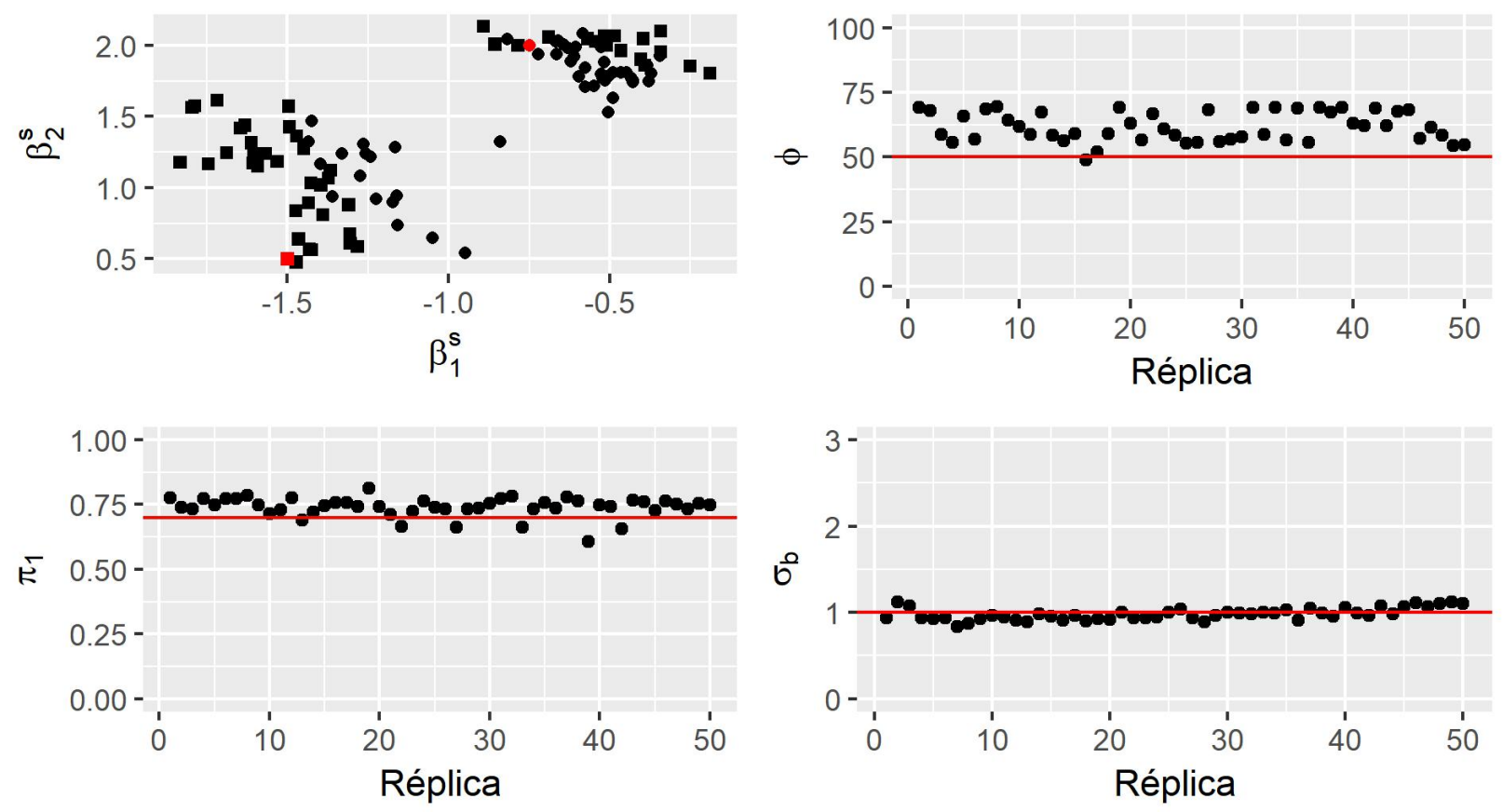

Figura B.14: Gráfico de dispersão das estimativas pontuais - mediana a posteriori - dos estudos de simulação de $R=50$ bancos de dados do modelo de mistura beta linear com efeitos aleatórios para a média com cenário 1, $\phi=50, N=100$ e $n=10$, em que $s=1(\bullet), 2(\mathbf{\square})$, e dos verdadeiros valores dos parâmetros (em vermelho).
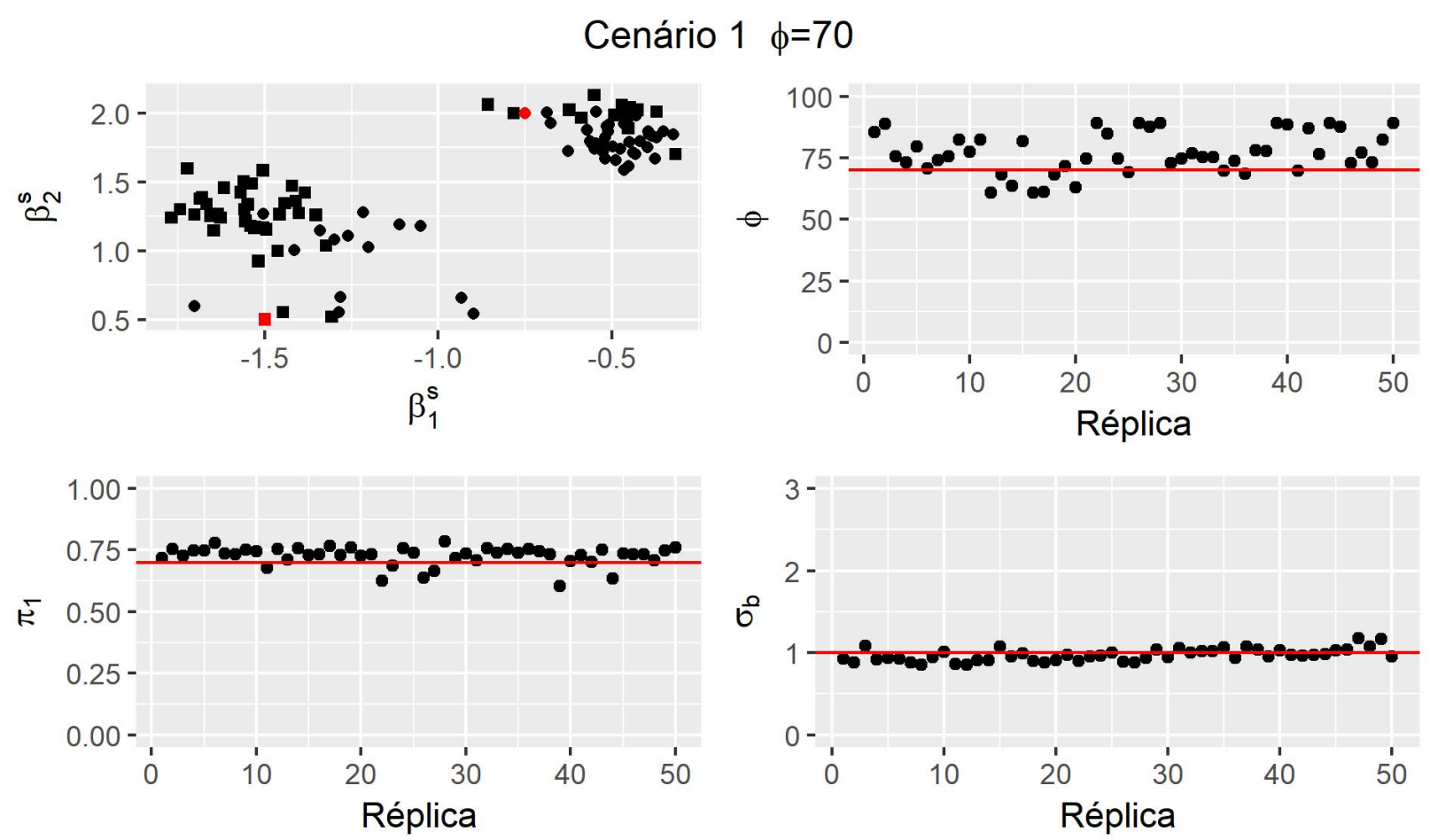

Figura B.15: Gráfico de dispersão das estimativas pontuais - mediana a posteriori - dos estudos de simulação de $R=50$ bancos de dados do modelo de mistura beta linear com efeitos aleatórios para a média com cenário 1, $\phi=70, N=100$ e $n=10$, em que $s=1(\bullet), 2(\square)$, e dos verdadeiros valores dos parâmetros (em vermelho). 
Cenário $2 \phi=30$
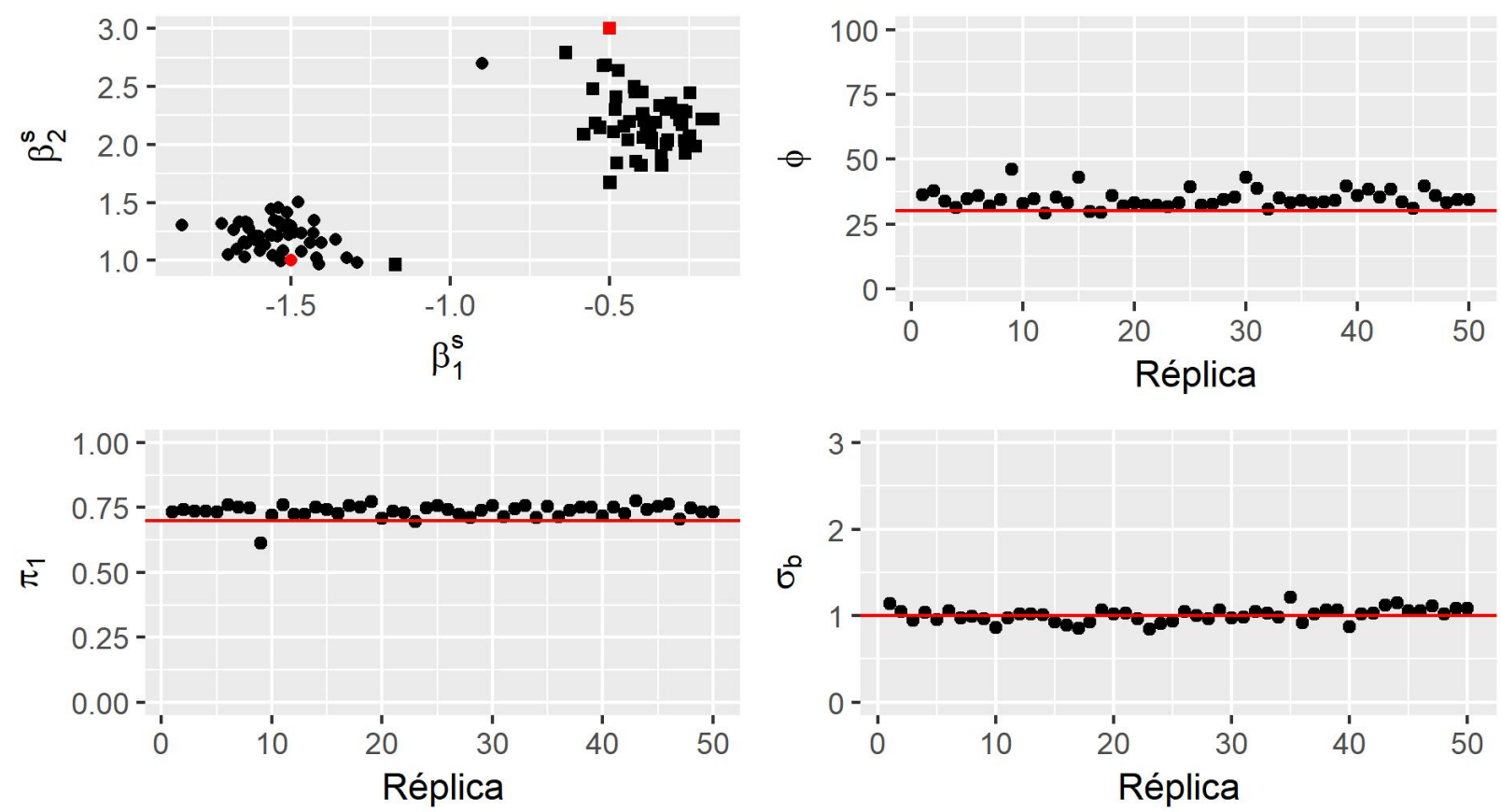

Figura B.16: Gráfico de dispersão das estimativas pontuais - mediana a posteriori - dos estudos de simulação de $R=50$ bancos de dados do modelo de mistura beta linear com efeitos aleatórios para a média com cenário 2, $\phi=30, N=100$ e $n=10$, em que $s=1(\bullet), 2(\mathbf{\bullet})$, e dos verdadeiros valores dos parâmetros (em vermelho).

\section{Cenário $2 \phi=50$}
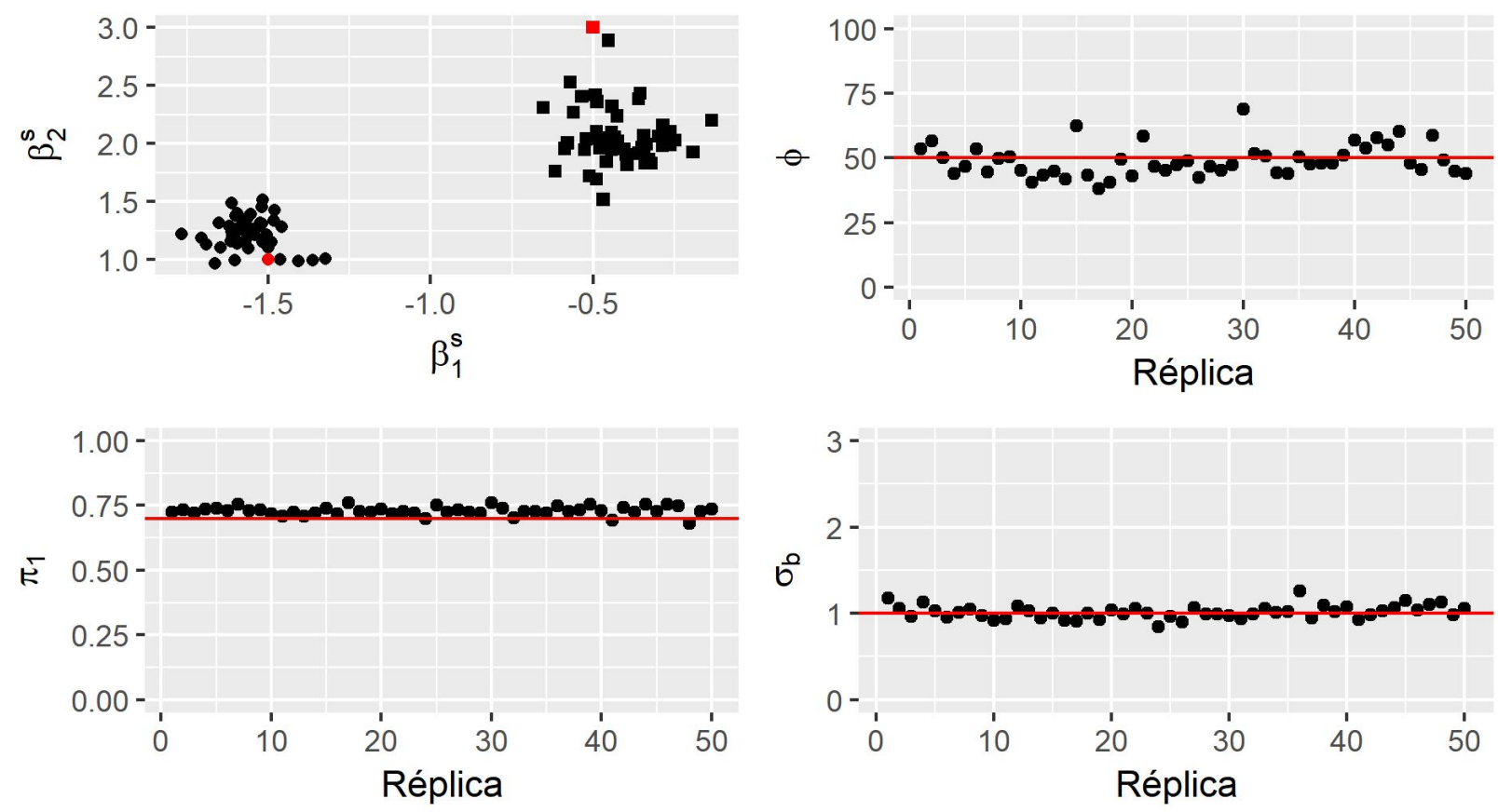

Figura B.17: Gráfico de dispersão das estimativas pontuais - mediana a posteriori - dos estudos de simulação de $R=50$ bancos de dados do modelo de mistura beta linear com efeitos aleatórios para a média com cenário 2, $\phi=50, N=100$ e $n=10$, em que $s=1(\bullet), 2(\mathbf{\bullet})$, e dos verdadeiros valores dos parâmetros (em vermelho). 

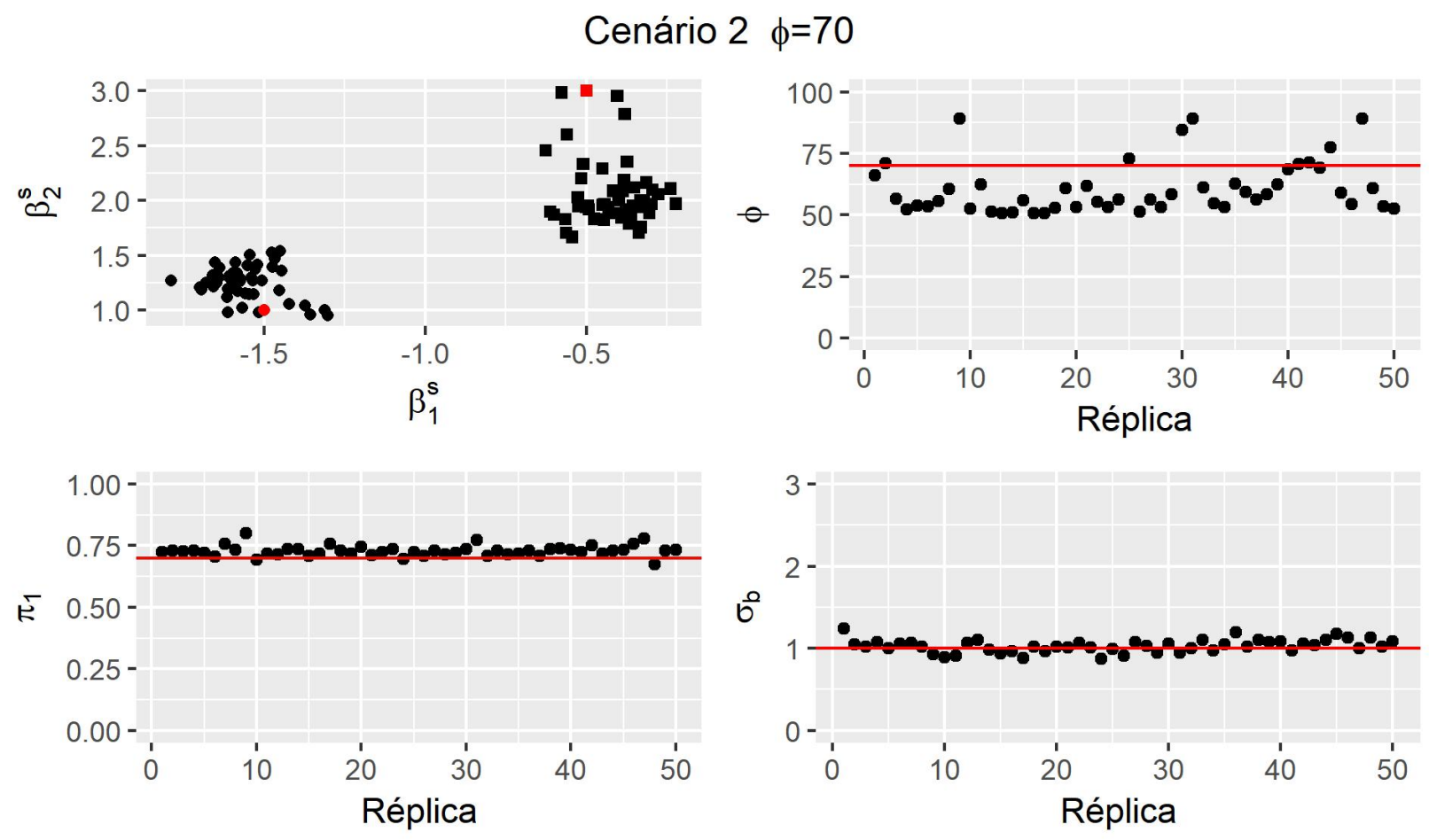

Figura B.18: Gráfico de dispersão das estimativas pontuais - mediana a posteriori - dos estudos de simulação de $R=50$ bancos de dados do modelo de mistura beta linear com efeitos aleatórios para a média com cenário 2, $\phi=70, N=100$ e $n=10$, em que $s=1(\bullet), 2(\mathbf{\square})$, e dos verdadeiros valores dos parâmetros (em vermelho).

Cenário $3 \phi=30$
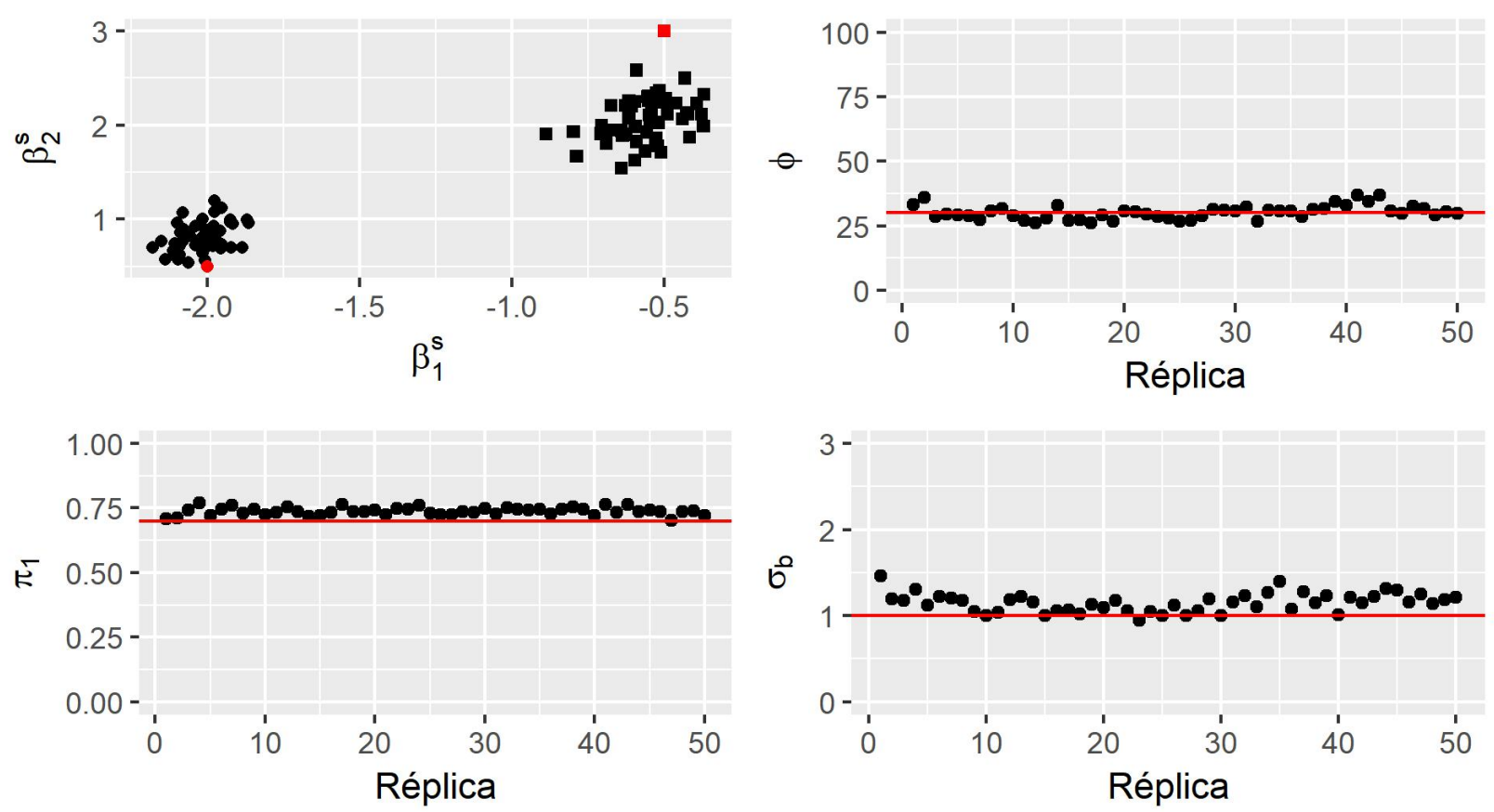

Figura B.19: Gráfico de dispersão das estimativas pontuais - mediana a posteriori - dos estudos de simulação de $R=50$ bancos de dados do modelo de mistura beta linear com efeitos aleatórios para a média com cenário 3, $\phi=30, N=100$ e $n=10$, em que $s=1(\bullet), 2(\mathbf{\square})$, e dos verdadeiros valores dos parâmetros (em vermelho). 

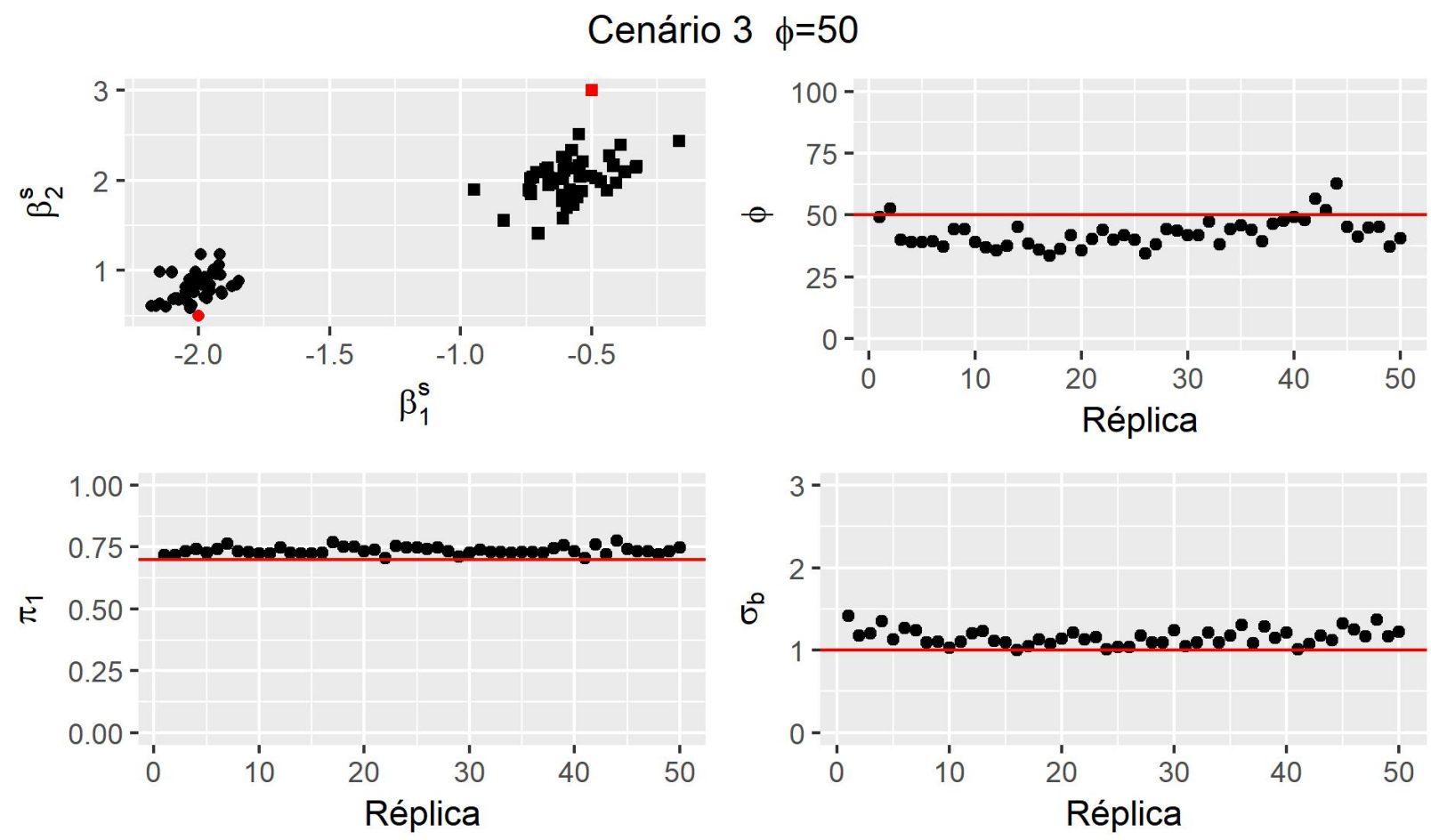

Figura B.20: Gráfico de dispersão das estimativas pontuais - mediana a posteriori - dos estudos de simulação de $R=50$ bancos de dados do modelo de mistura beta linear com efeitos aleatórios para a média com cenário 3, $\phi=50, N=100$ e $n=10$, em que $s=1(\bullet), 2(\mathbf{\bullet})$, e dos verdadeiros valores dos parâmetros (em vermelho).

\section{Cenário $3 \phi=70$}
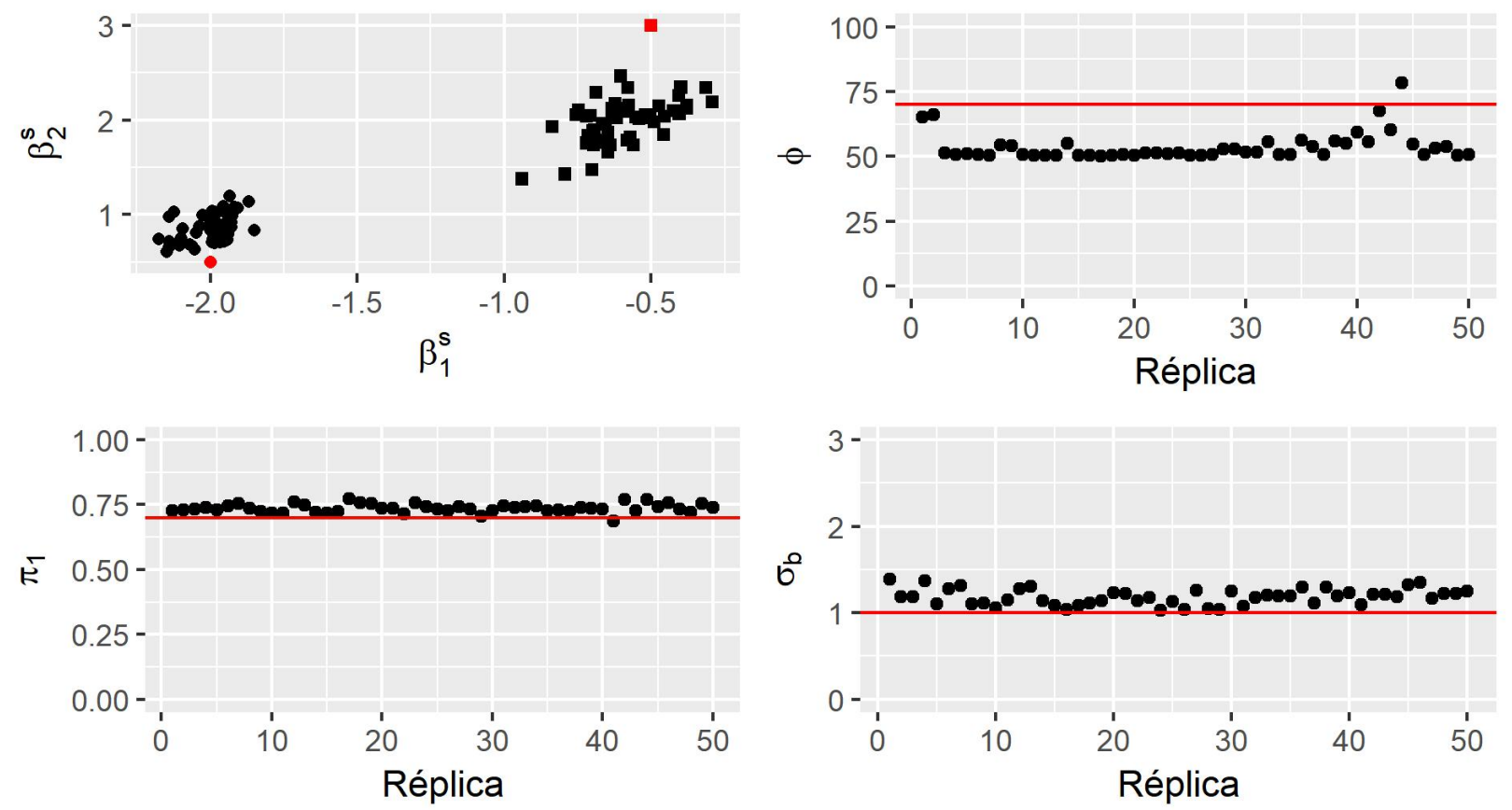

Figura B.21: Gráfico de dispersão das estimativas pontuais - mediana a posteriori - dos estudos de simulação de $R=50$ bancos de dados do modelo de mistura beta linear com efeitos aleatórios para a média com cenário 3, $\phi=70, N=100$ e $n=10$, em que $s=1(\bullet), 2(\mathbf{\bullet})$, e dos verdadeiros valores dos parâmetros (em vermelho). 


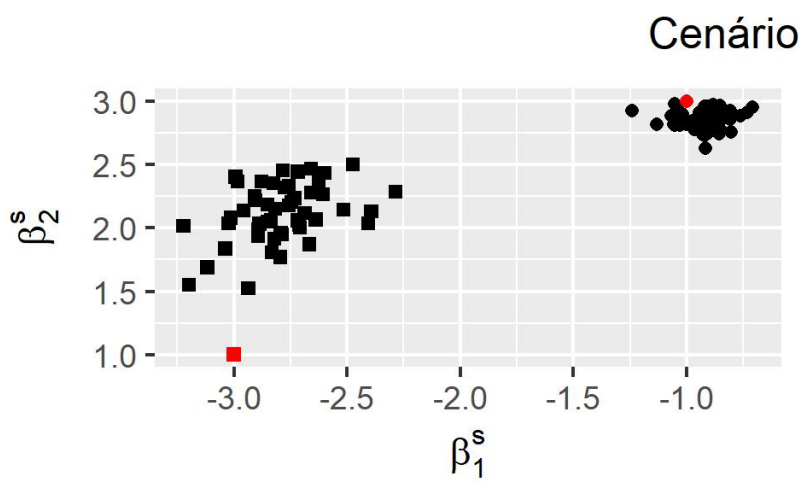

$4 \phi=30$
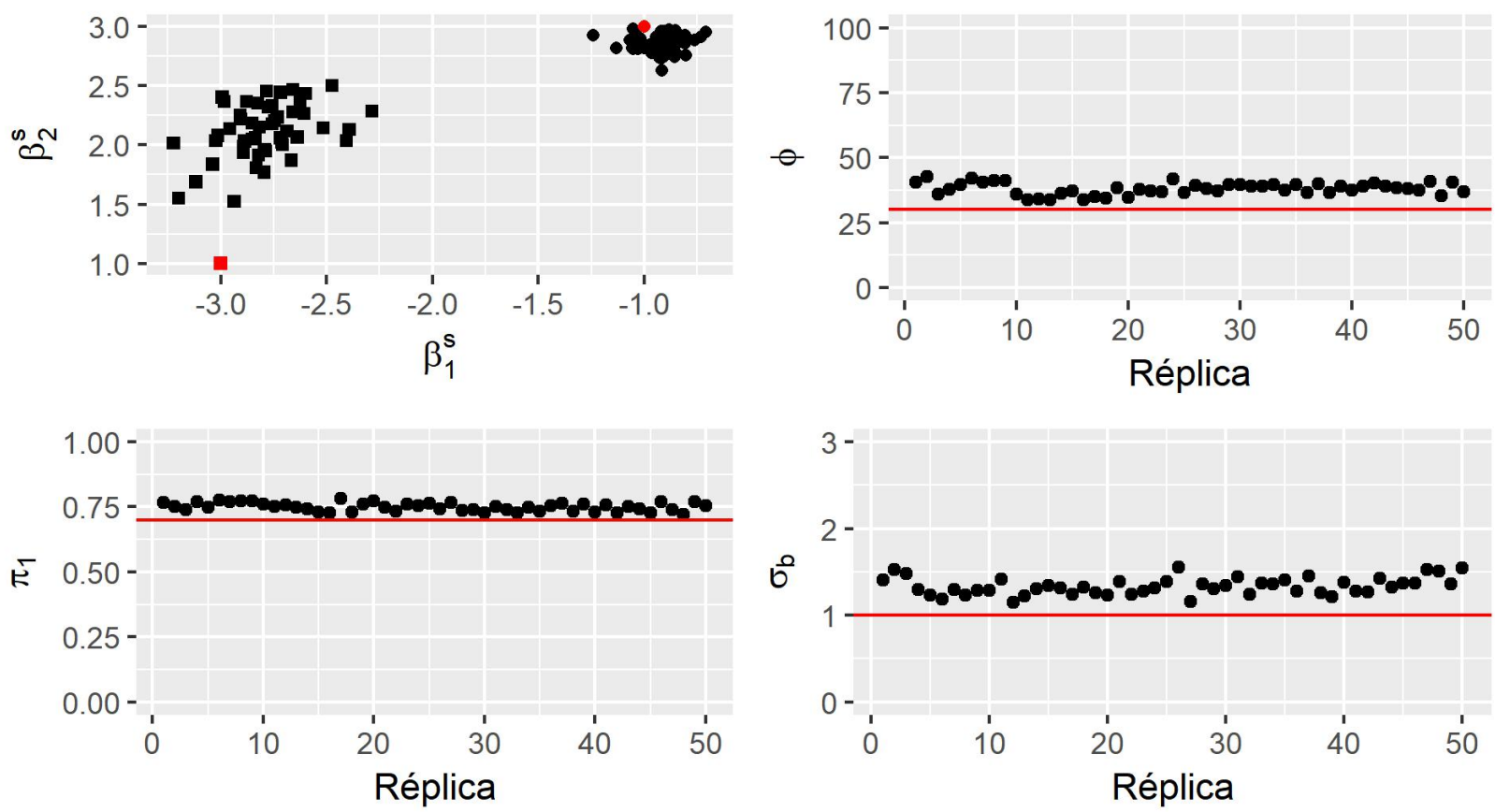

Figura B.22: Gráfico de dispersão das estimativas pontuais - mediana a posteriori - dos estudos de simulação de $R=50$ bancos de dados do modelo de mistura beta linear com efeitos aleatórios para a média com cenário 4, $\phi=30, N=100$ e $n=10$, em que $s=1(\bullet), 2(\mathbf{\square})$, e dos verdadeiros valores dos parâmetros (em vermelho).
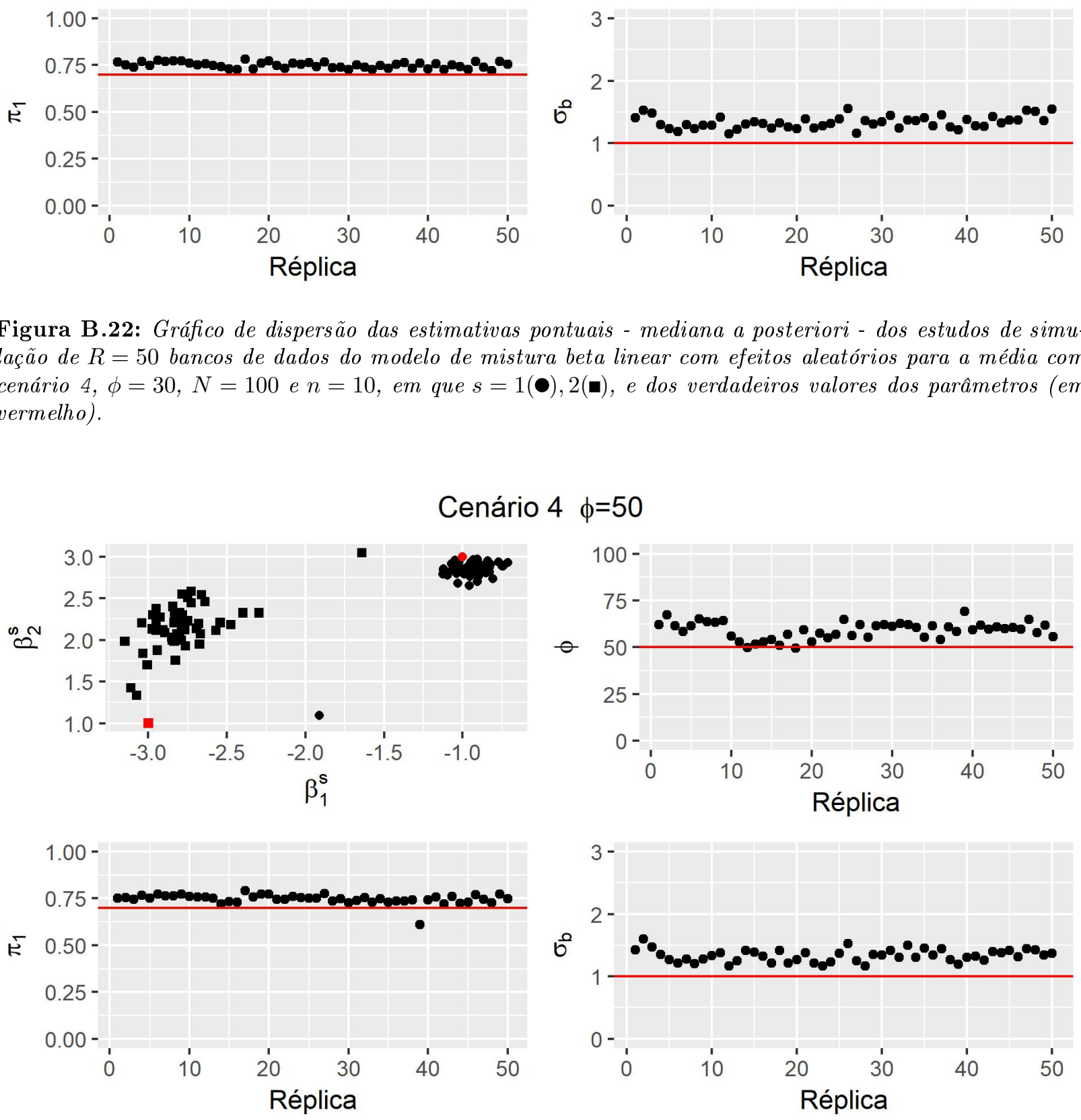

Figura B.23: Gráfico de dispersão das estimativas pontuais - mediana a posteriori - dos estudos de simulação de $R=50$ bancos de dados do modelo de mistura beta linear com efeitos aleatórios para a média com cenário $4, \phi=50, N=100$ e $n=10$, em que $s=1(\bullet), 2(\mathbf{\square})$, e dos verdadeiros valores dos parâmetros (em vermelho). 


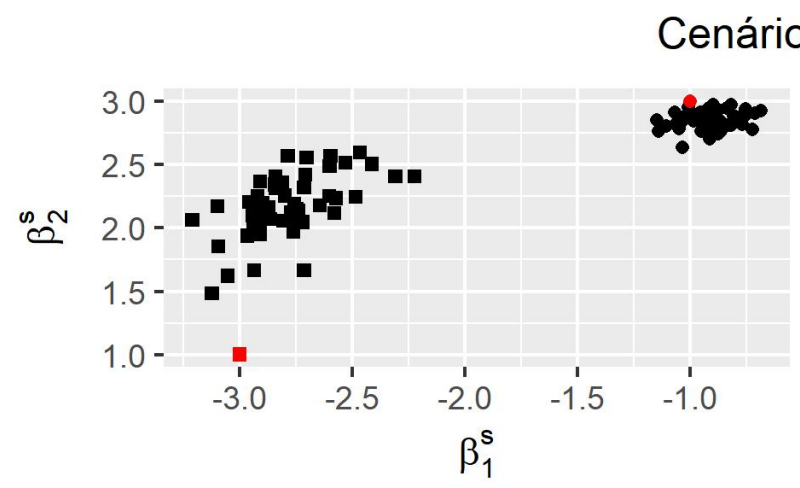

$4 \phi=70$
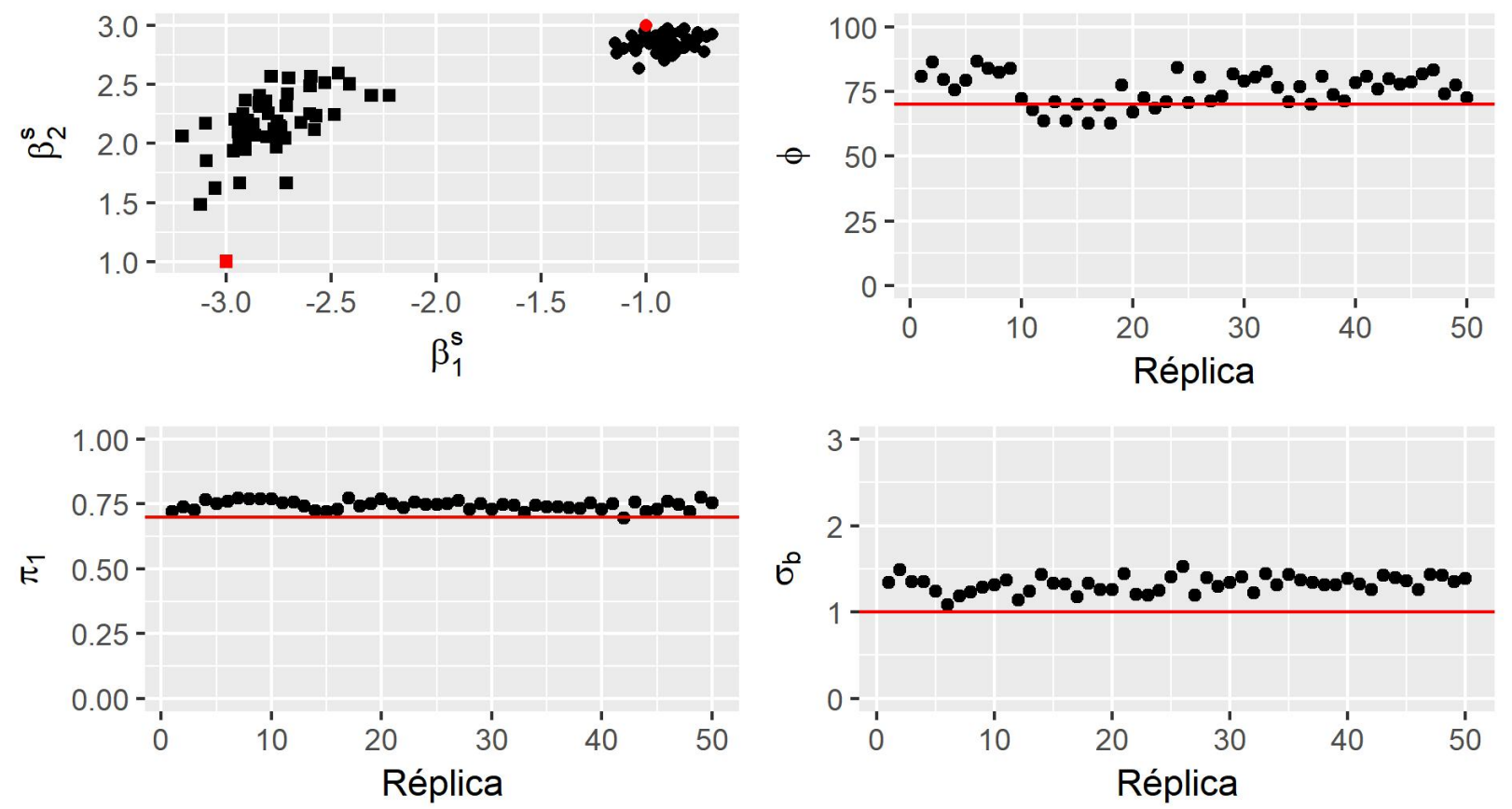

Figura B.24: Gráfico de dispersão das estimativas pontuais - mediana a posteriori - dos estudos de simulação de $R=50$ bancos de dados do modelo de mistura beta linear com efeitos aleatórios para a média com cenário 4, $\phi=70, N=100$ e $n=10$, em que $s=1(\bullet), 2(\mathbf{\bullet})$, e dos verdadeiros valores dos parâmetros (em vermelho). 
B.3 Modelo linear com parâmetro de precisão constante - $\mathrm{N}=500$, $\mathrm{n}=5$
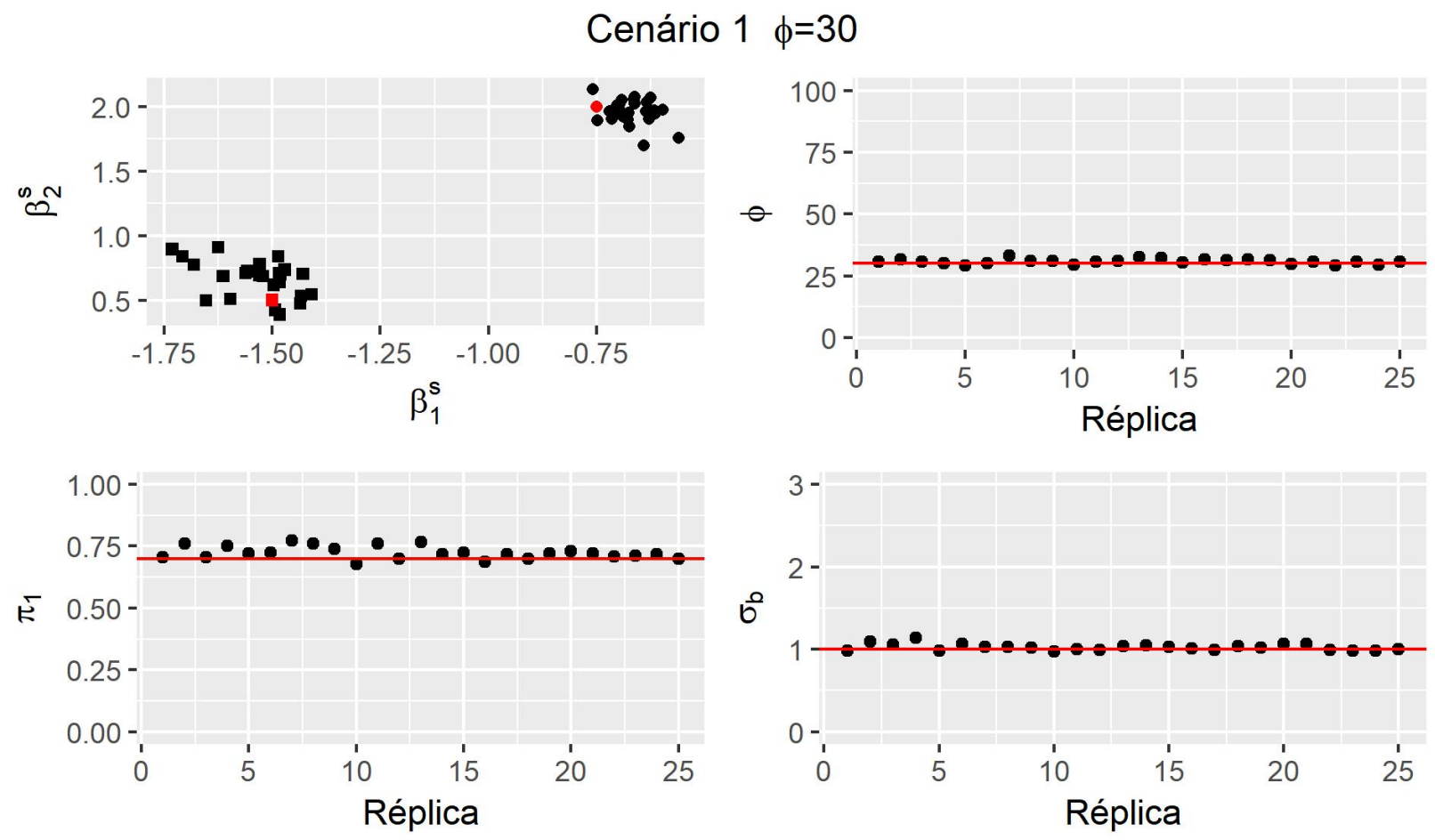

Figura B.25: Gráfico de dispersão das estimativas pontuais - mediana a posteriori - dos estudos de simulação de $R=25$ bancos de dados do modelo de mistura beta linear com efeitos aleatórios para a média com cenário $1, \phi=30, N=500$ e $n=5$, em que $s=1(\bullet), 2(\bullet)$, e dos verdadeiros valores dos parâmetros (em vermelho). 

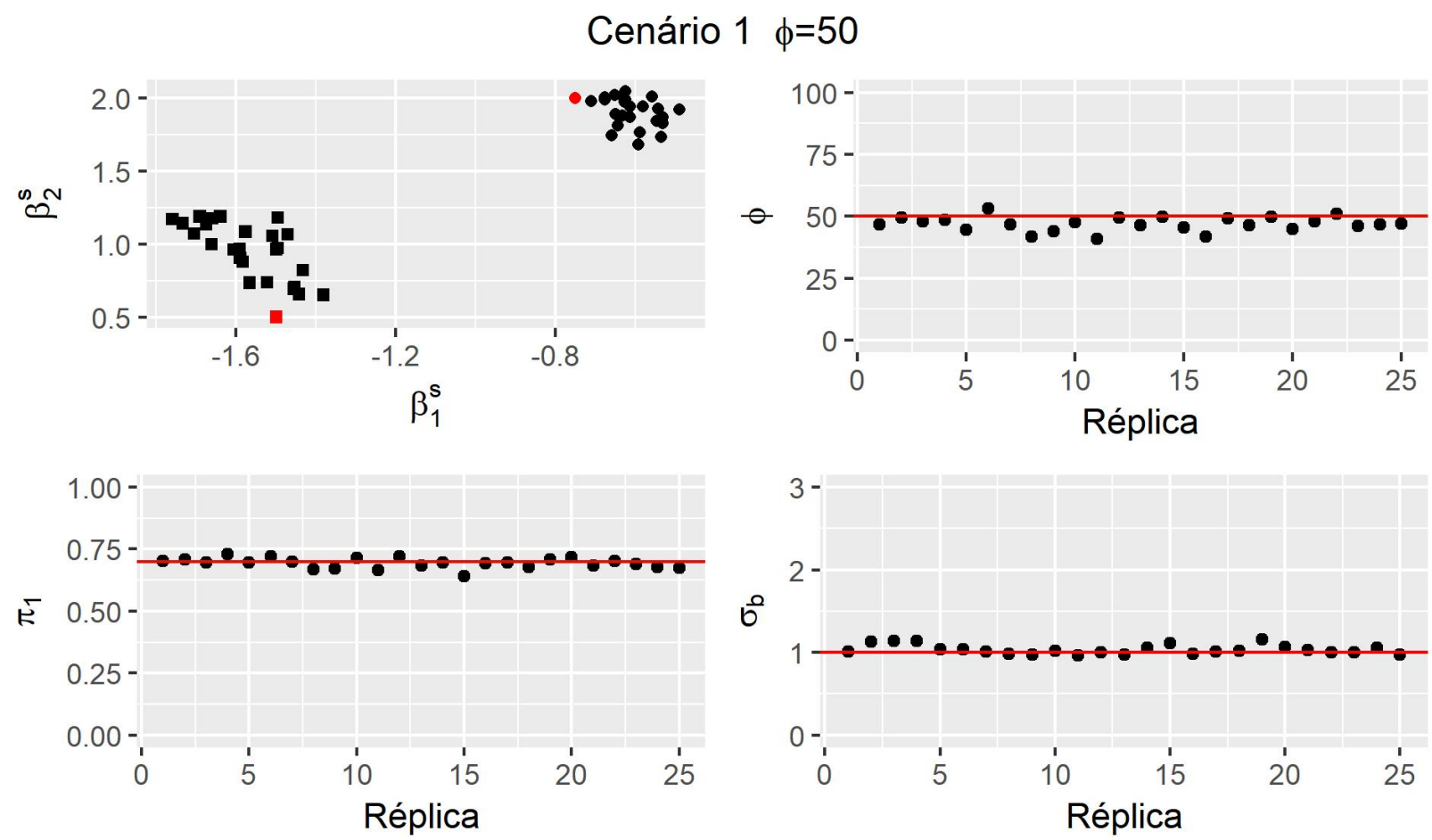

Figura B.26: Gráfico de dispersão das estimativas pontuais - mediana a posteriori - dos estudos de simulação de $R=25$ bancos de dados do modelo de mistura beta linear com efeitos aleatórios para a média com cenário 1, $\phi=50, N=500$ e $n=5$, em que $s=1(\bullet), 2(\mathbf{\bullet})$, e dos verdadeiros valores dos parâmetros (em vermelho).
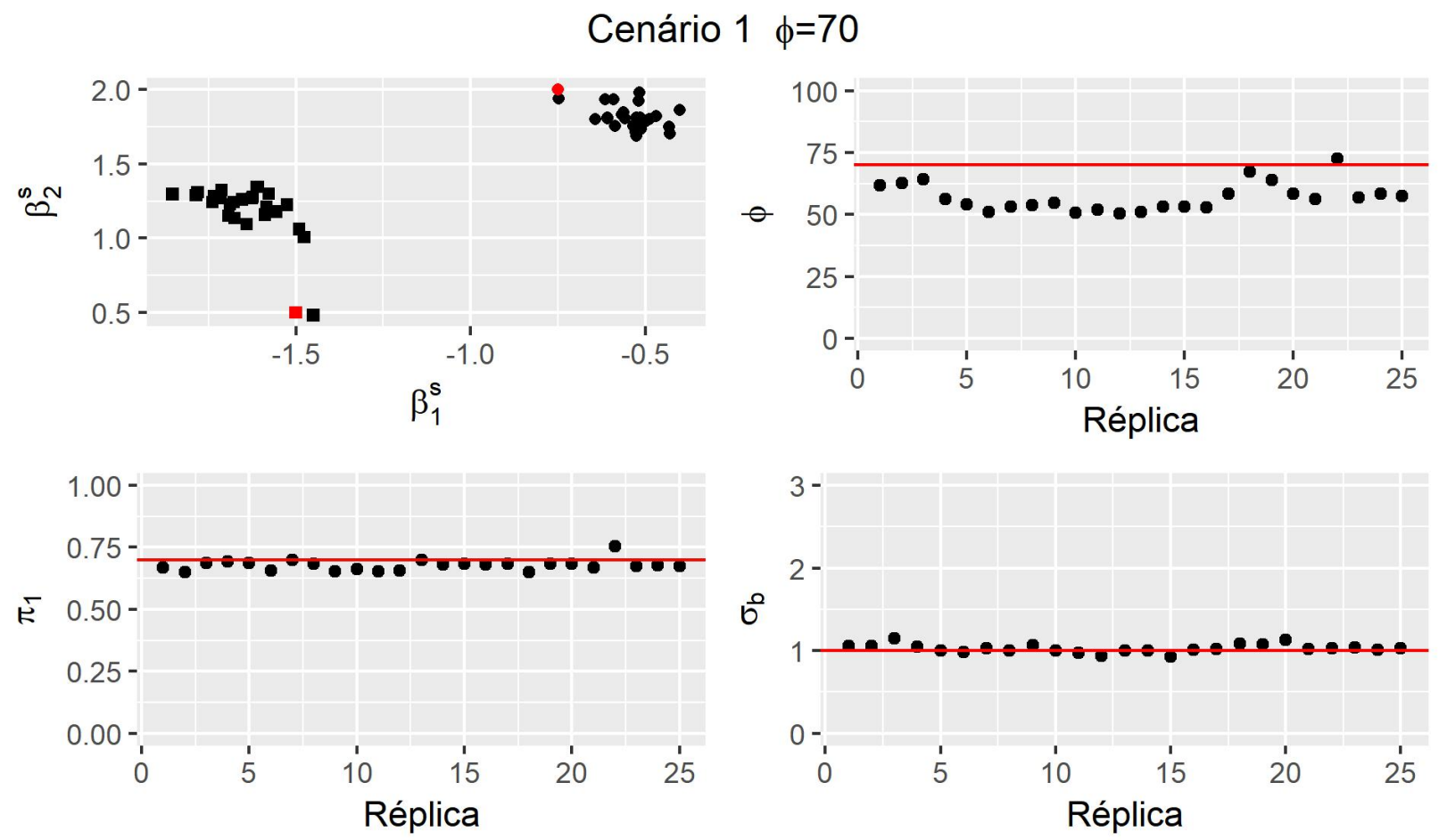

Figura B.27: Gráfico de dispersão das estimativas pontuais - mediana a posteriori - dos estudos de simulação de $R=25$ bancos de dados do modelo de mistura beta linear com efeitos aleatórios para a média com cenário 1, $\phi=70, N=500$ e $n=5$, em que $s=1(\bullet), 2(\boldsymbol{\bullet})$, e dos verdadeiros valores dos parâmetros (em vermelho). 

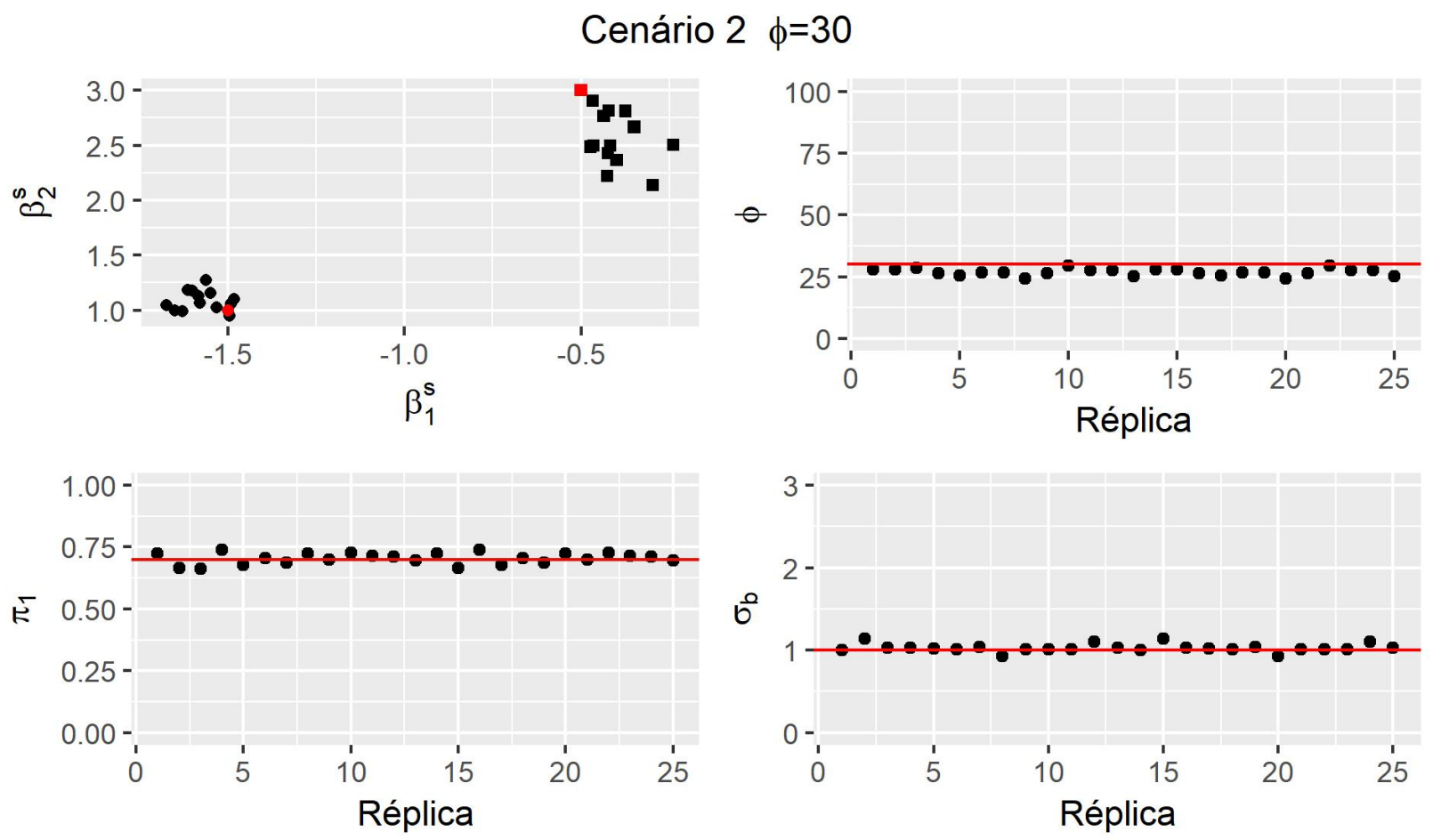

Figura B.28: Gráfico de dispersão das estimativas pontuais - mediana a posteriori - dos estudos de simulação de $R=25$ bancos de dados do modelo de mistura beta linear com efeitos aleatórios para a média com cenário 2, $\phi=30, N=500$ e $n=5$, em que $s=1(\bullet), 2(\mathbf{\bullet})$, e dos verdadeiros valores dos parâmetros (em vermelho).
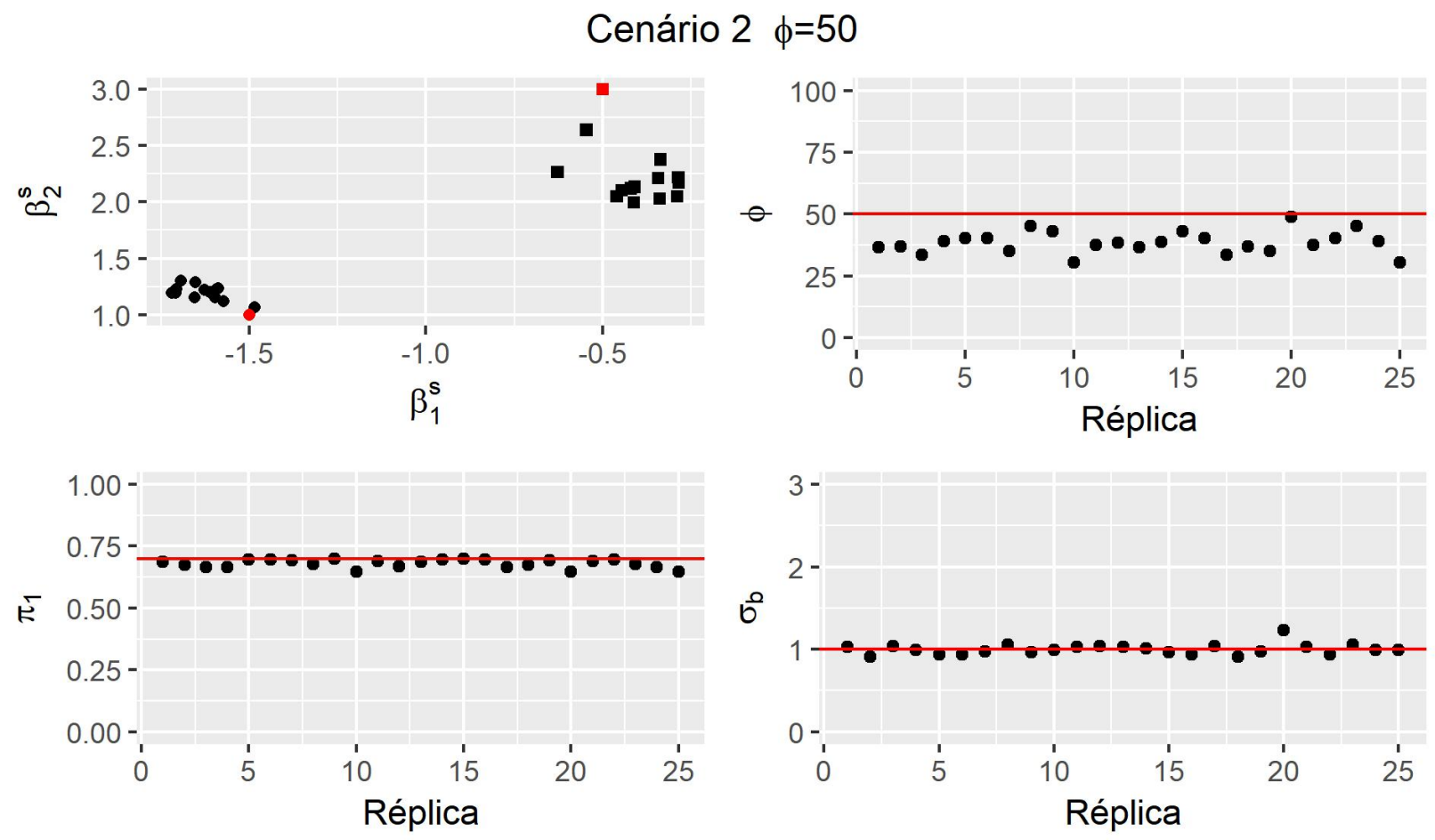

Figura B.29: Gráfico de dispersão das estimativas pontuais - mediana a posteriori - dos estudos de simulação de $R=25$ bancos de dados do modelo de mistura beta linear com efeitos aleatórios para a média com cenário 2, $\phi=50, N=500$ e $n=5$, em que $s=1(\bullet), 2(\boldsymbol{\bullet})$, e dos verdadeiros valores dos parâmetros (em vermelho). 


\section{Cenário $2 \phi=70$}
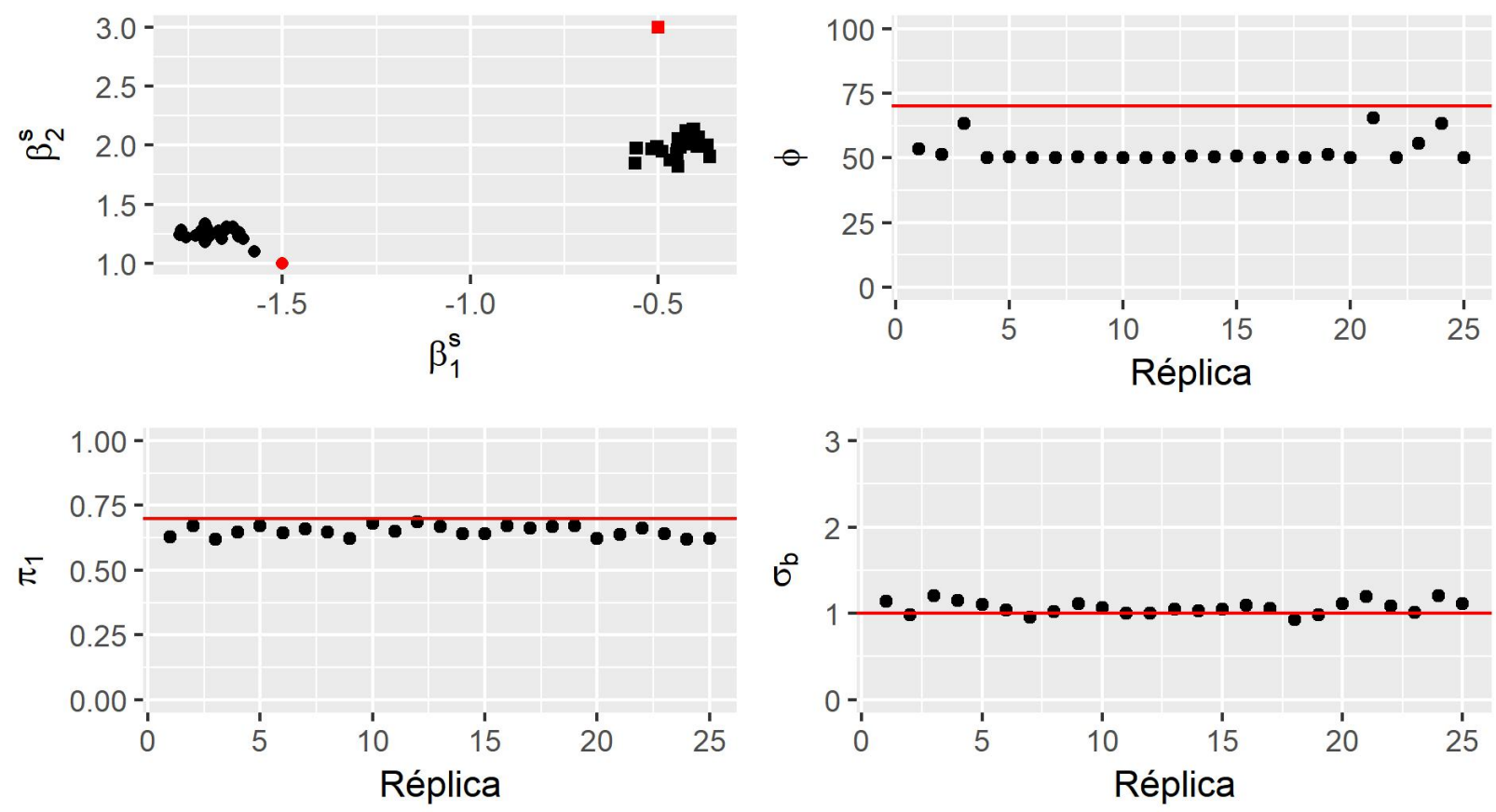

Figura B.30: Gráfico de dispersão das estimativas pontuais - mediana a posteriori - dos estudos de simulação de $R=25$ bancos de dados do modelo de mistura beta linear com efeitos aleatórios para a média com cenário 2, $\phi=70, N=500$ e $n=5$, em que $s=1(\bullet), 2(\boldsymbol{\bullet})$, e dos verdadeiros valores dos parâmetros (em vermelho).

\section{Cenário $3 \phi=30$}
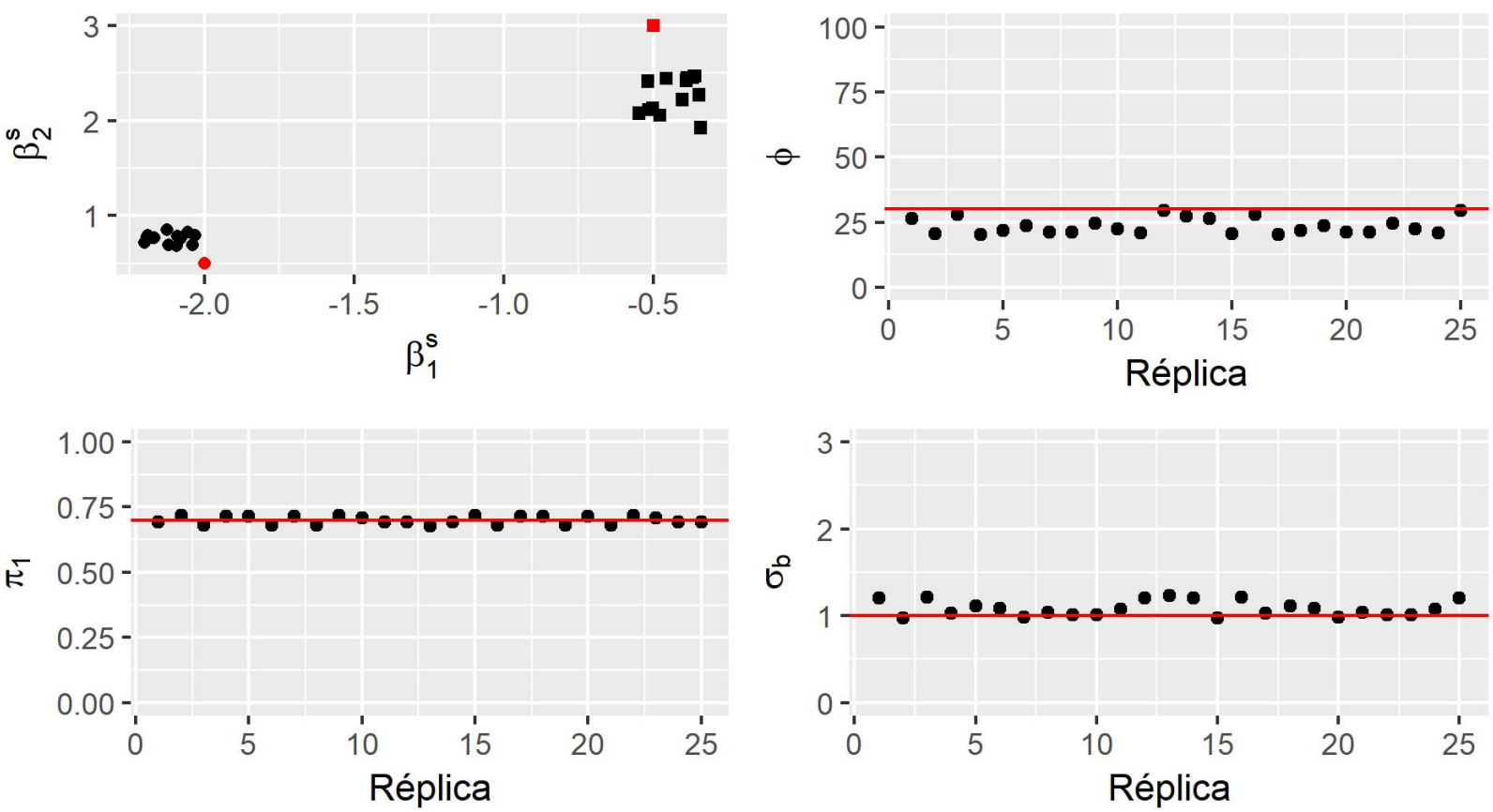

Figura B.31: Gráfico de dispersão das estimativas pontuais - mediana a posteriori - dos estudos de simulação de $R=25$ bancos de dados do modelo de mistura beta linear com efeitos aleatórios para a média com cenário 3, $\phi=30, N=500$ e $n=5$, em que $s=1(\bullet), 2(\mathbf{\bullet})$, e dos verdadeiros valores dos parâmetros (em vermelho). 
Cenário $3 \phi=50$
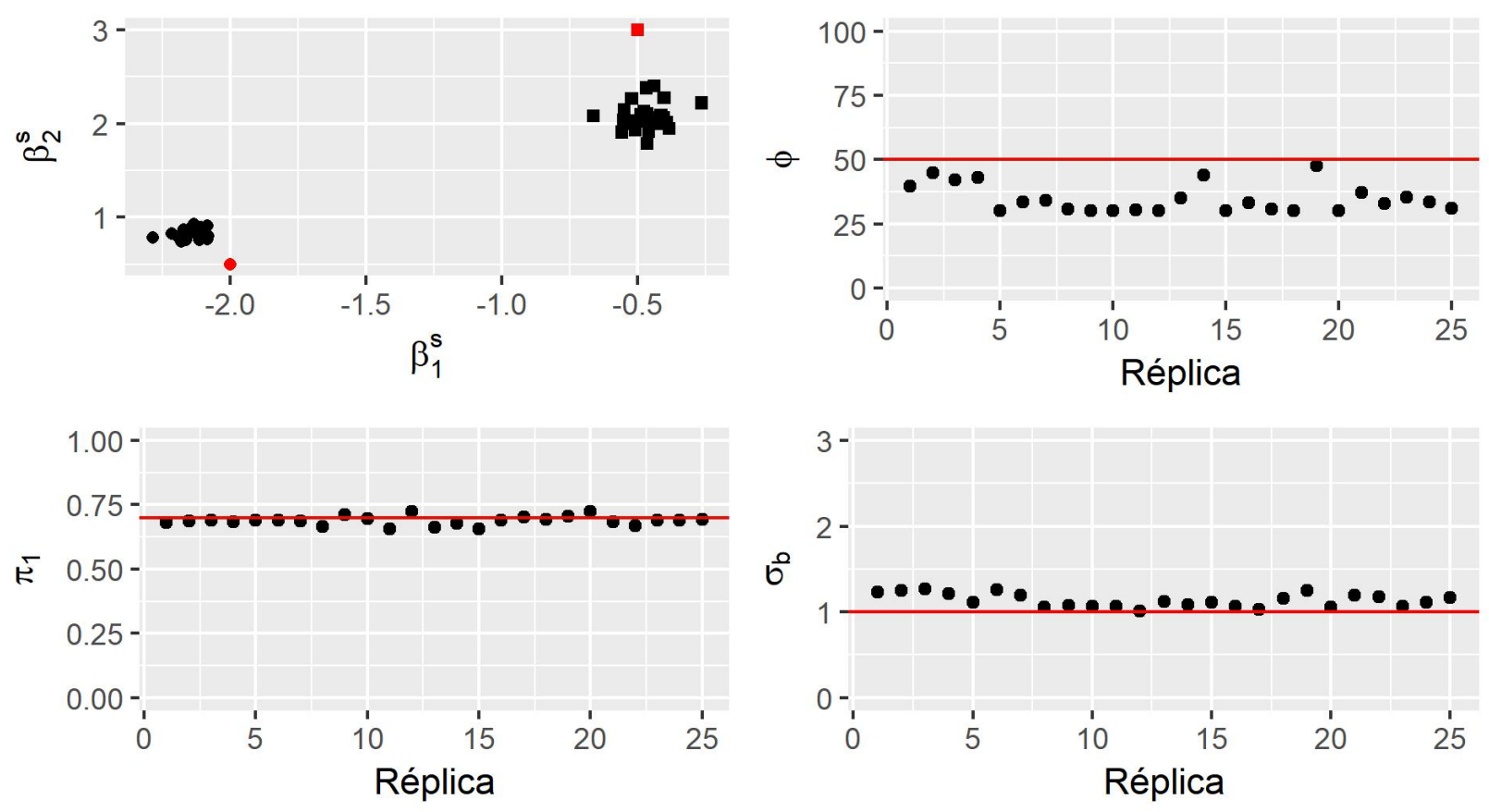

Figura B.32: Gráfico de dispersão das estimativas pontuais - mediana a posteriori - dos estudos de simulação de $R=25$ bancos de dados do modelo de mistura beta linear com efeitos aleatórios para a média com cenário 3, $\phi=50, N=500$ e $n=5$, em que $s=1(\bullet), 2(\mathbf{\bullet})$, e dos verdadeiros valores dos parâmetros (em vermelho).

Cenário $3 \phi=70$
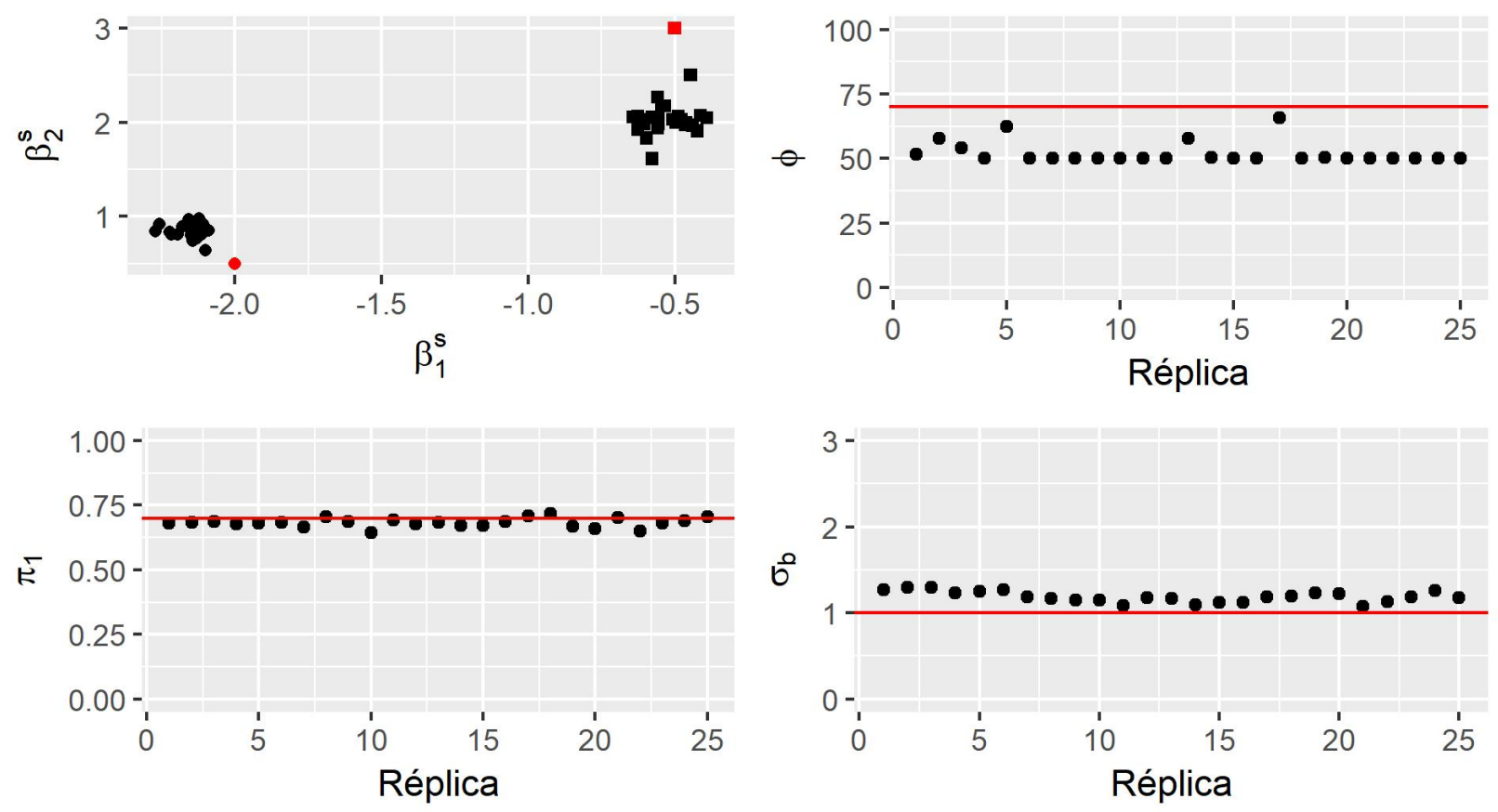

Figura B.33: Gráfico de dispersão das estimativas pontuais - mediana a posteriori - dos estudos de simulação de $R=25$ bancos de dados do modelo de mistura beta linear com efeitos aleatórios para a média com cenário 3, $\phi=70, N=500$ e $n=5$, em que $s=1(\bullet), 2(\boldsymbol{\bullet})$, e dos verdadeiros valores dos parâmetros (em vermelho). 
Cenário $4 \phi=30$
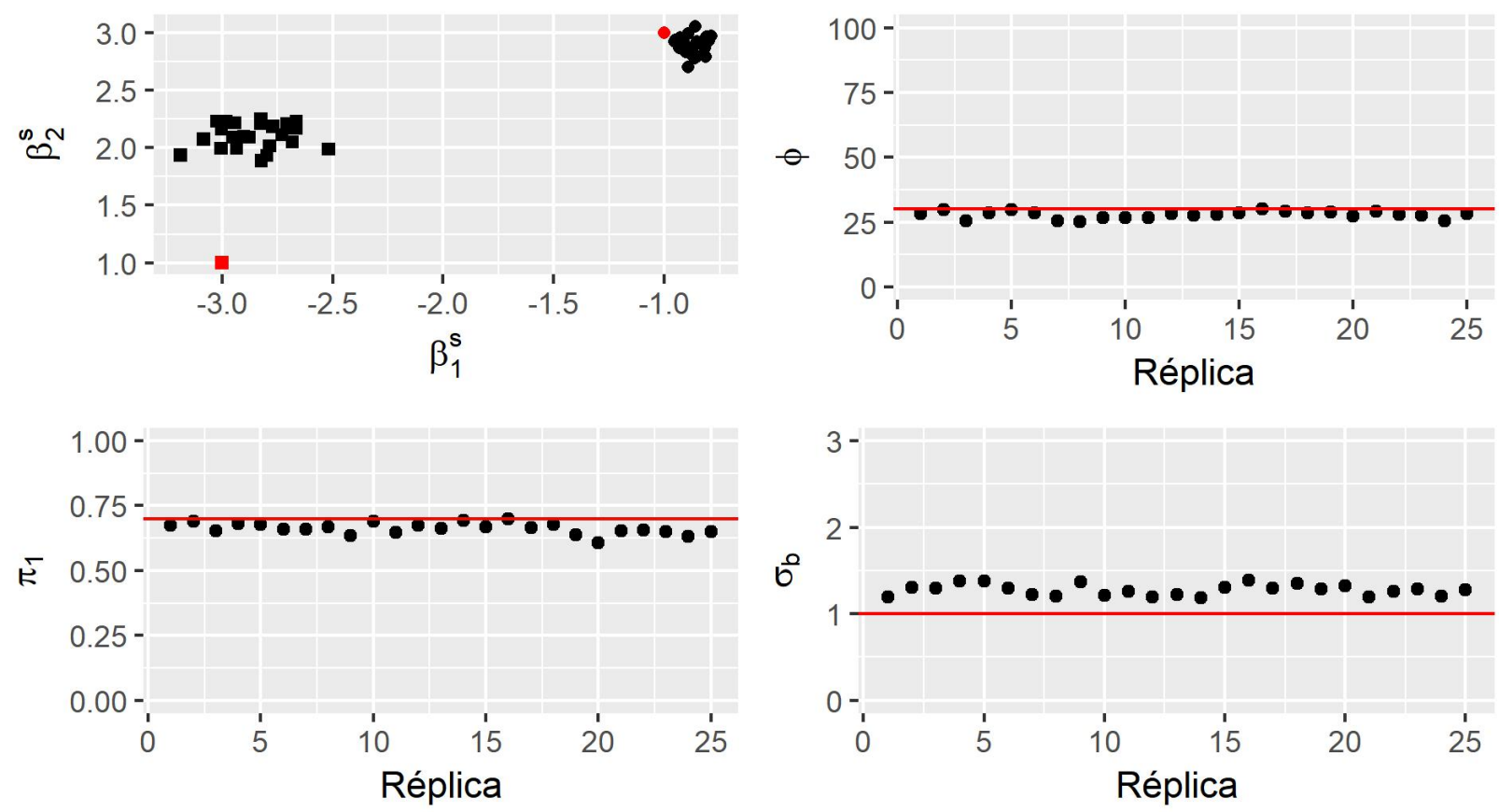

Figura B.34: Gráfico de dispersão das estimativas pontuais - mediana a posteriori - dos estudos de simulação de $R=25$ bancos de dados do modelo de mistura beta linear com efeitos aleatórios para a média com cenário 4, $\phi=30, N=500$ e $n=5$, em que $s=1(\bullet), 2(\mathbf{\bullet})$, e dos verdadeiros valores dos parâmetros (em vermelho).

\section{Cenário $4 \phi=50$}
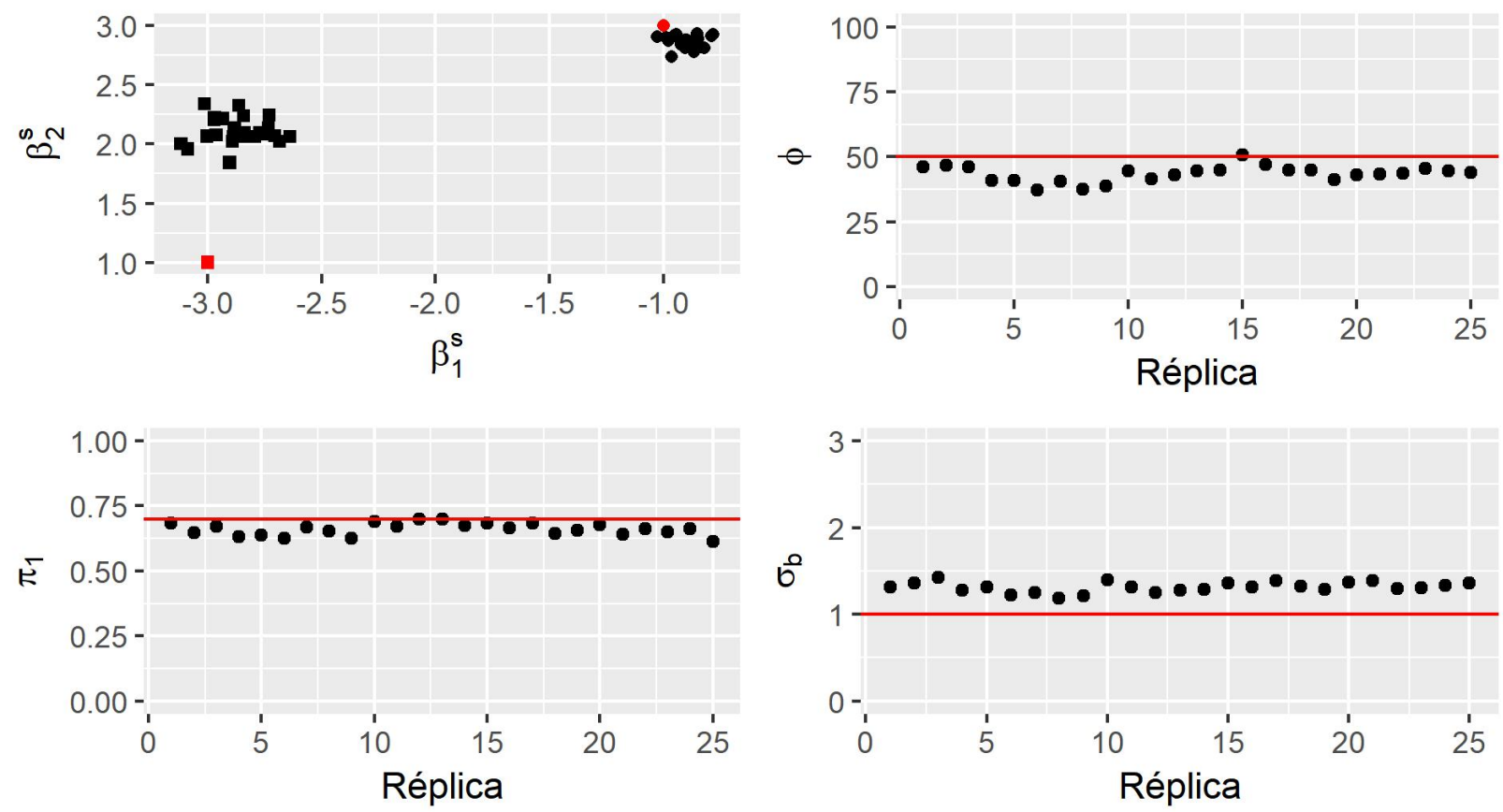

Figura B.35: Gráfico de dispersão das estimativas pontuais - mediana a posteriori - dos estudos de simulação de $R=25$ bancos de dados do modelo de mistura beta linear com efeitos aleatórios para a média com cenário 4, $\phi=50, N=500$ e $n=5$, em que $s=1(\bullet), 2(\boldsymbol{\bullet})$, e dos verdadeiros valores dos parâmetros (em vermelho). 

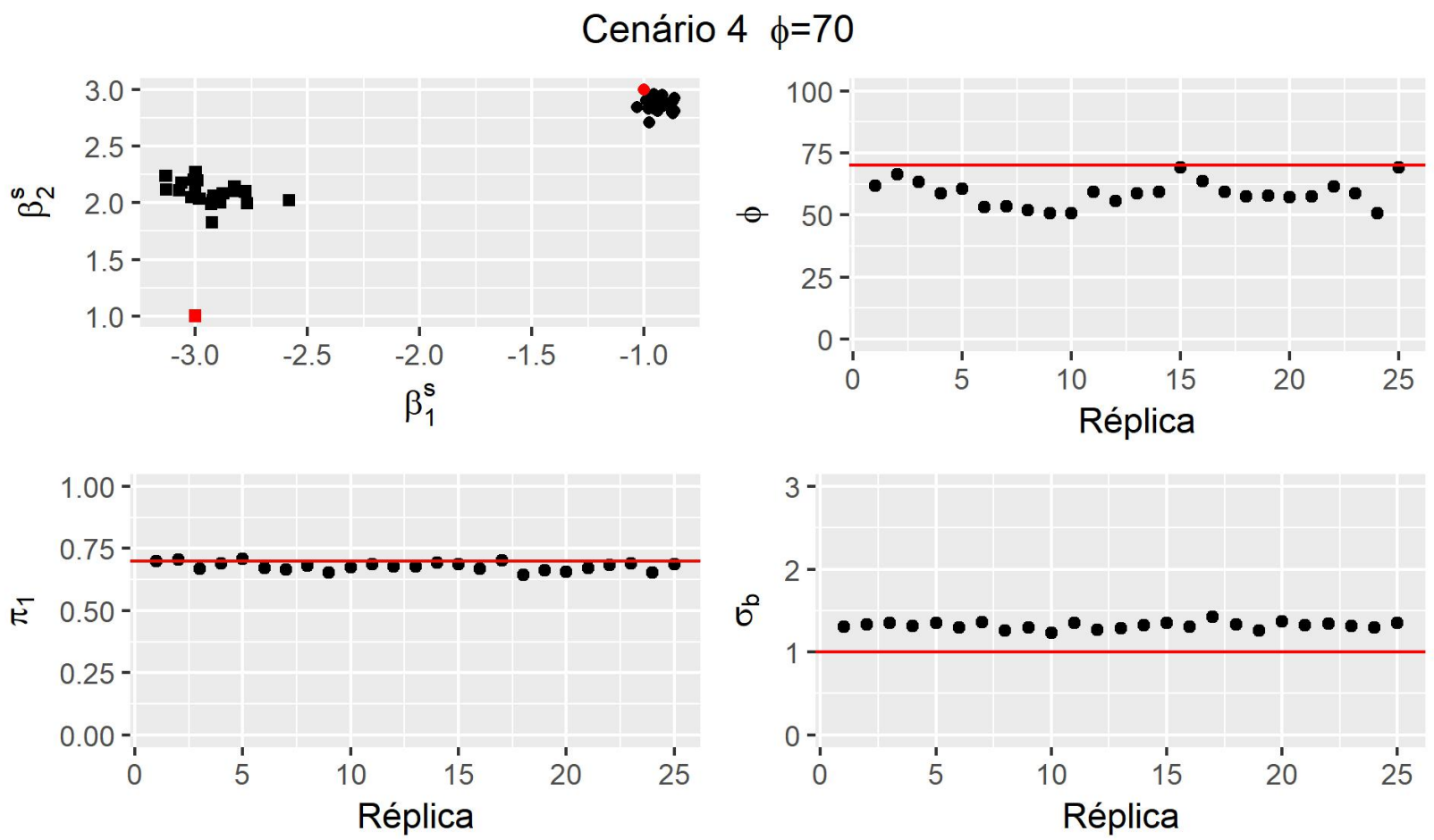

Figura B.36: Gráfico de dispersão das estimativas pontuais - mediana a posteriori - dos estudos de simulação de $R=25$ bancos de dados do modelo de mistura beta linear com efeitos aleatórios para a média com cenário $4, \phi=70, N=500$ e $n=5$, em que $s=1(\bullet), 2(\boldsymbol{\bullet})$, e dos verdadeiros valores dos parâmetros (em vermelho). 
B.4 Modelo linear com parâmetro de precisão constante - $\mathrm{N}=500$, $\mathrm{n}=\mathbf{1 0}$
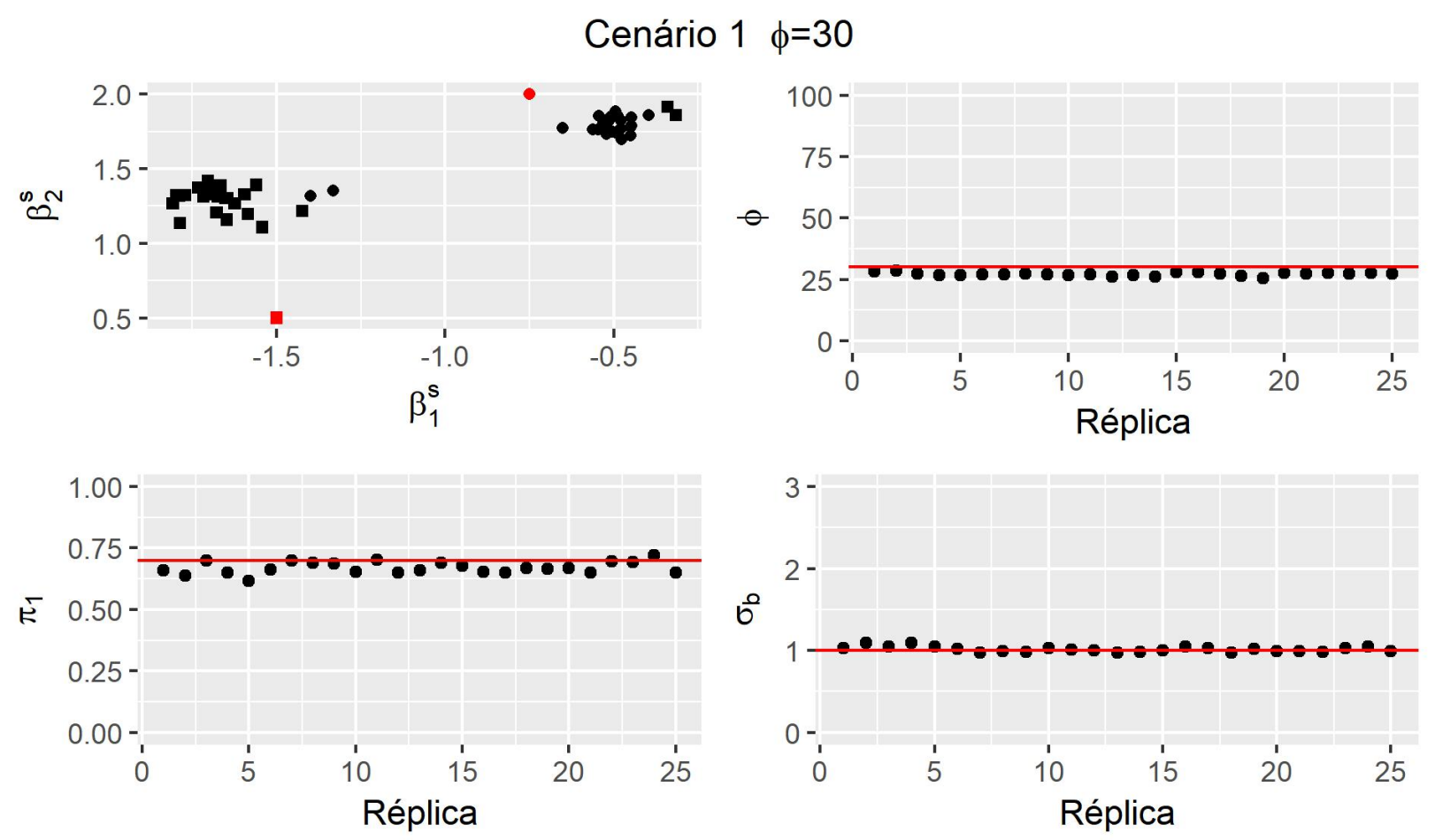

Figura B.37: Gráfico de dispersão das estimativas pontuais - mediana a posteriori - dos estudos de simulação de $R=25$ bancos de dados do modelo de mistura beta linear com efeitos aleatórios para a média com cenário $1, \phi=30, N=500$ e $n=10$, em que $s=1(\bullet), 2(\mathbf{\bullet})$, e dos verdadeiros valores dos parâmetros (em vermelho). 

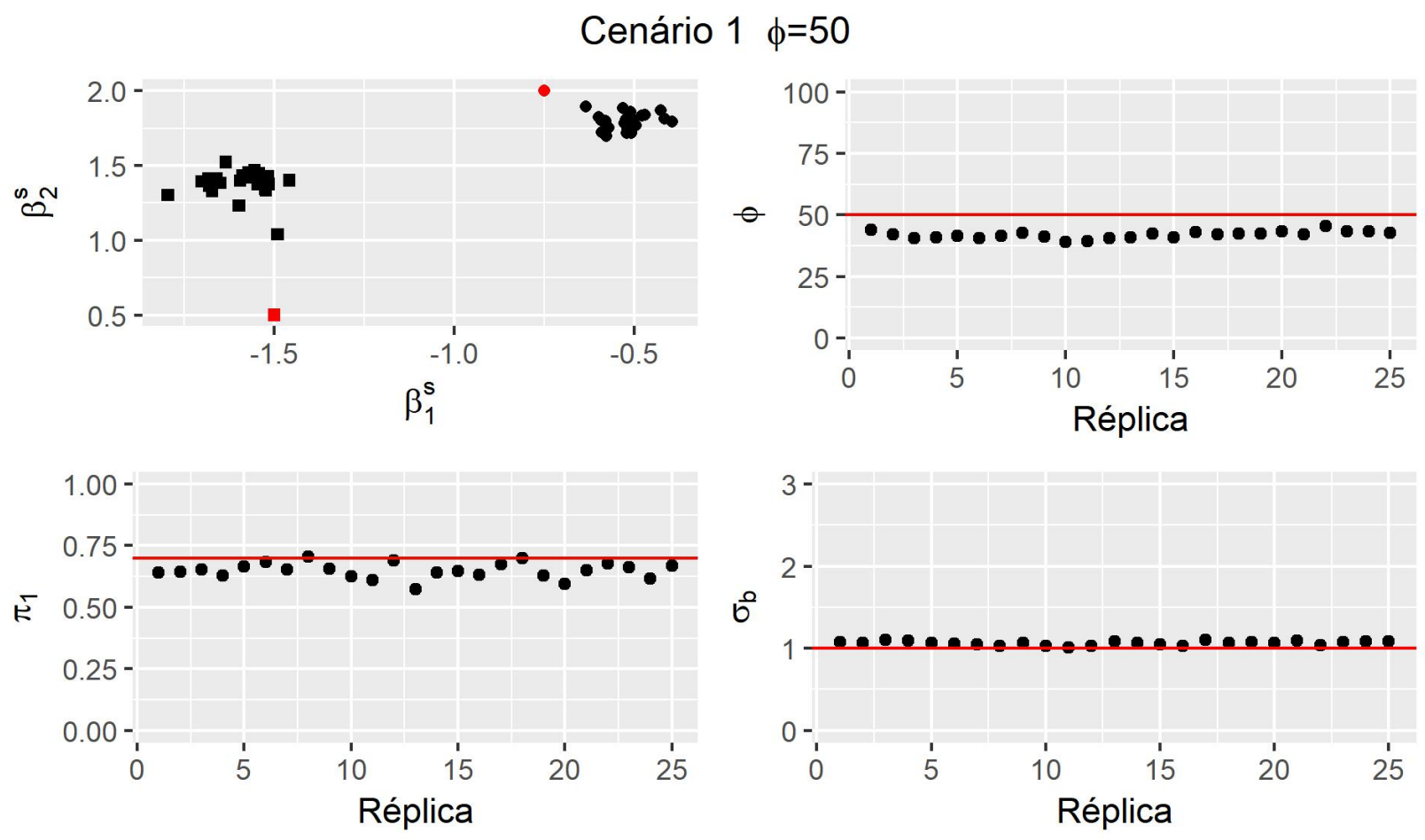

Figura B.38: Gráfico de dispersão das estimativas pontuais - mediana a posteriori - dos estudos de simulação de $R=25$ bancos de dados do modelo de mistura beta linear com efeitos aleatórios para a média com cenário 1, $\phi=50, N=500$ e $n=10$, em que $s=1(\bullet), 2(\mathbf{\bullet ) , ~ e ~ d o s ~ v e r d a d e i r o s ~ v a l o r e s ~ d o s ~ p a r a ̂ m e t r o s ~ ( e m ~}$ vermelho).
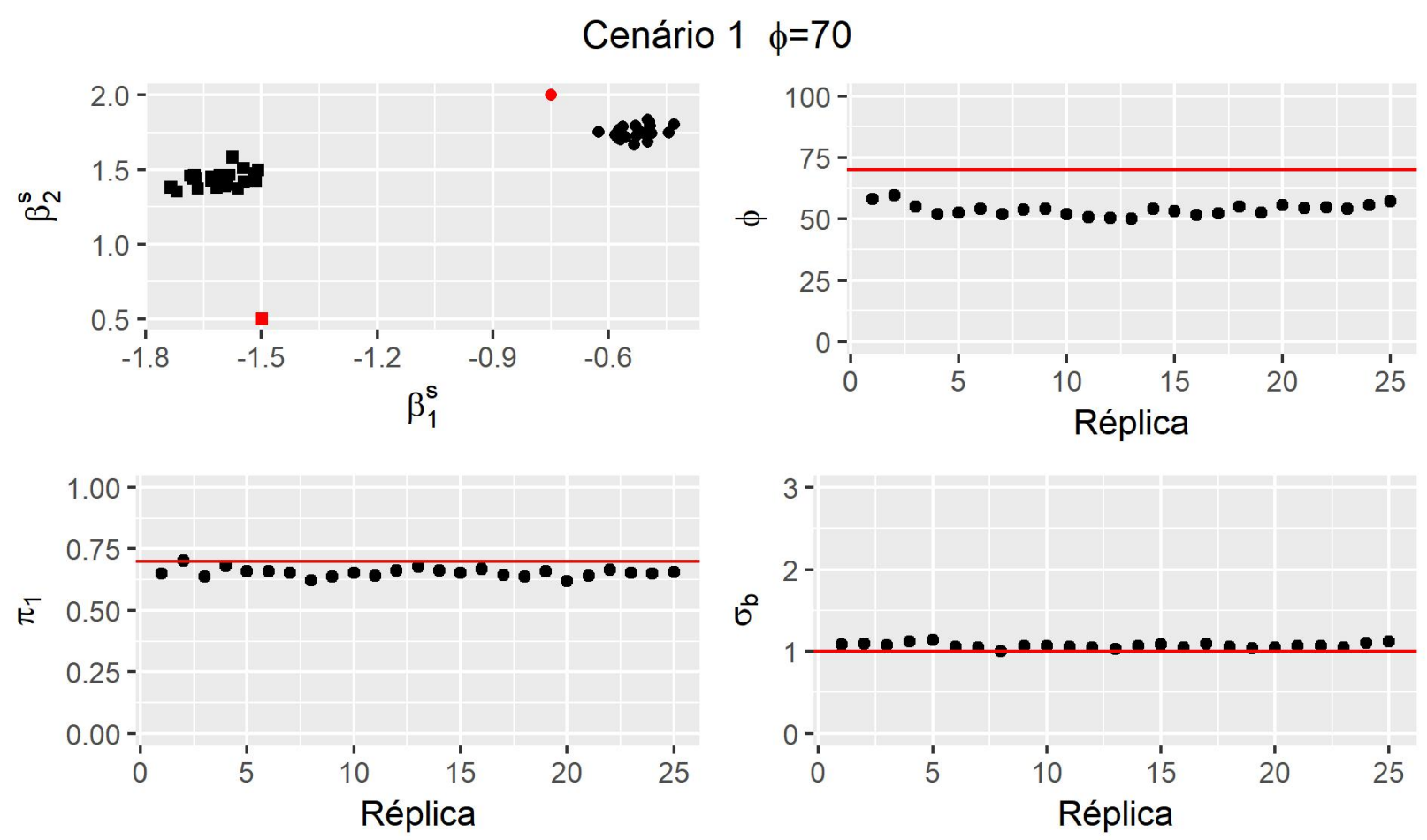

Figura B.39: Gráfico de dispersão das estimativas pontuais - mediana a posteriori - dos estudos de simulação de $R=25$ bancos de dados do modelo de mistura beta linear com efeitos aleatórios para a média com cenário 1, $\phi=70, N=500$ e $n=10$, em que $s=1(\bullet), 2(\mathbf{\bullet ) , ~ e ~ d o s ~ v e r d a d e i r o s ~ v a l o r e s ~ d o s ~ p a r a ̂ m e t r o s ~ ( e m ~}$ vermelho). 


\section{Cenário $2 \phi=30$}
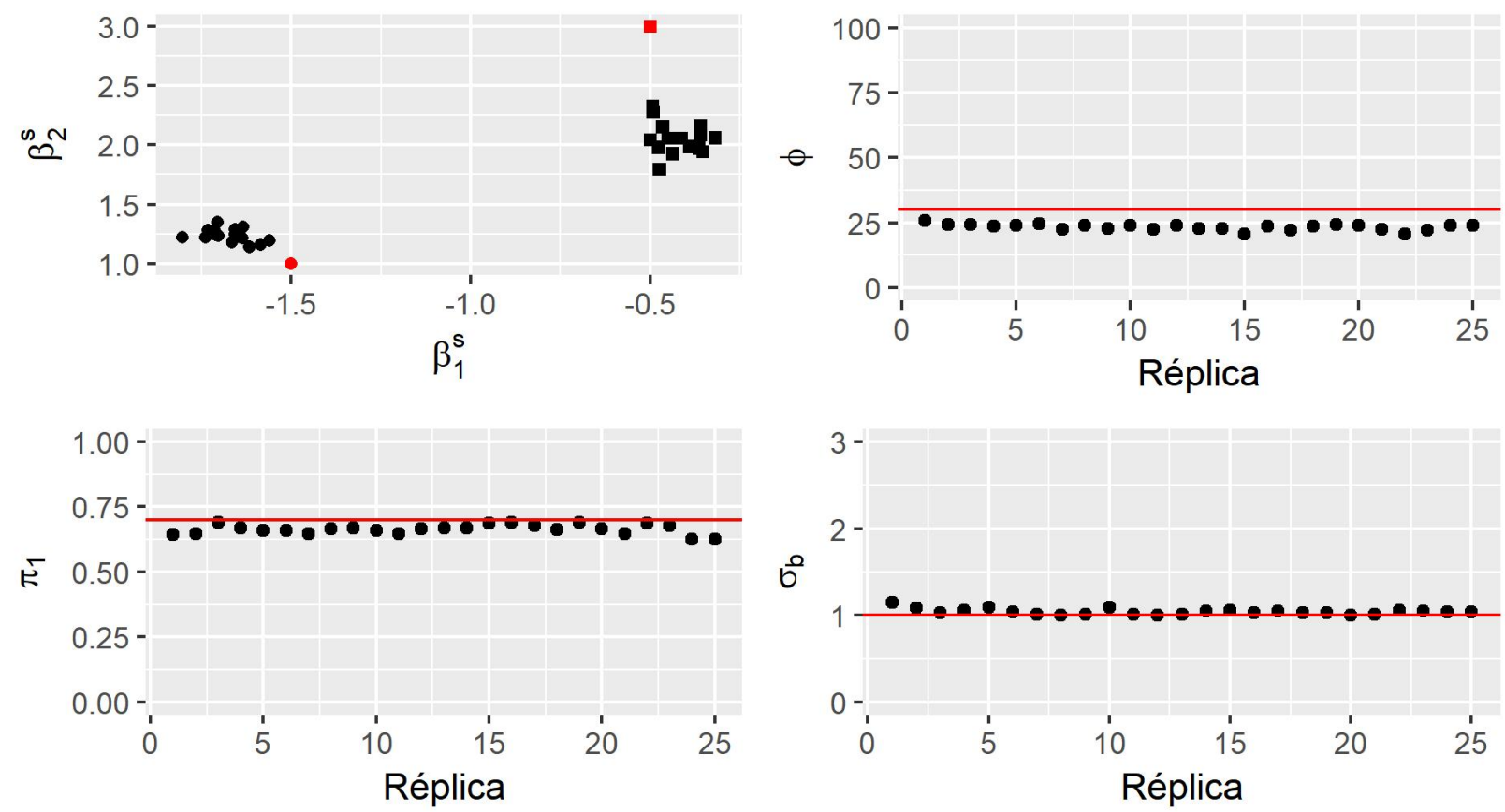

Figura B.40: Gráfico de dispersão das estimativas pontuais - mediana a posteriori - dos estudos de simulação de $R=25$ bancos de dados do modelo de mistura beta linear com efeitos aleatórios para a média com cenário $2, \phi=30, N=500$ e $n=10$, em que $s=1(\bullet), 2(\mathbf{\bullet})$, e dos verdadeiros valores dos parâmetros (em vermelho).

\section{Cenário $2 \phi=50$}
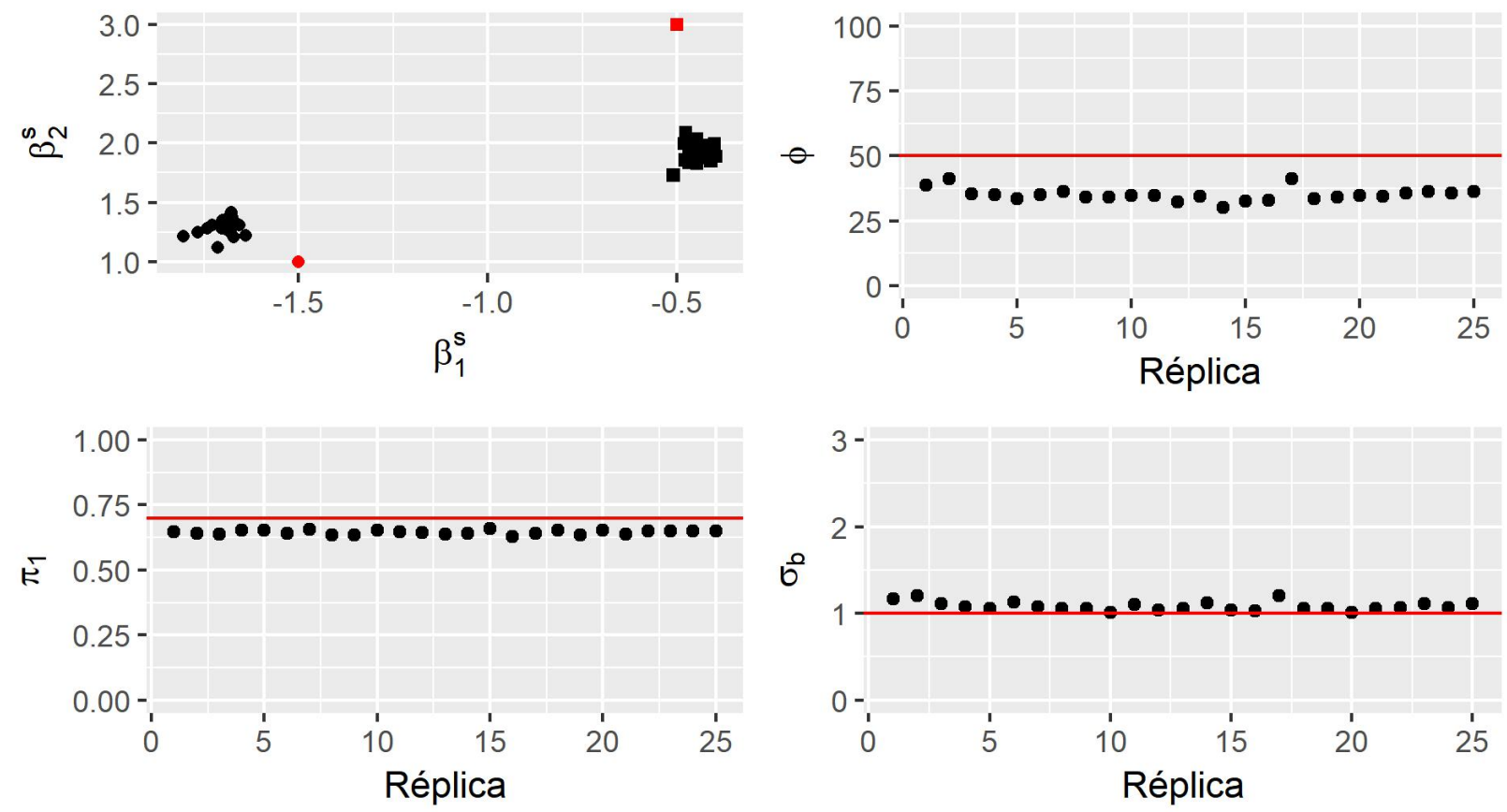

Figura B.41: Gráfico de dispersão das estimativas pontuais - mediana a posteriori - dos estudos de simulação de $R=25$ bancos de dados do modelo de mistura beta linear com efeitos aleatórios para a média com cenário 2, $\phi=50, N=500$ e $n=10$, em que $s=1(\bullet), 2(\mathbf{\bullet})$, e dos verdadeiros valores dos parâmetros (em vermelho). 

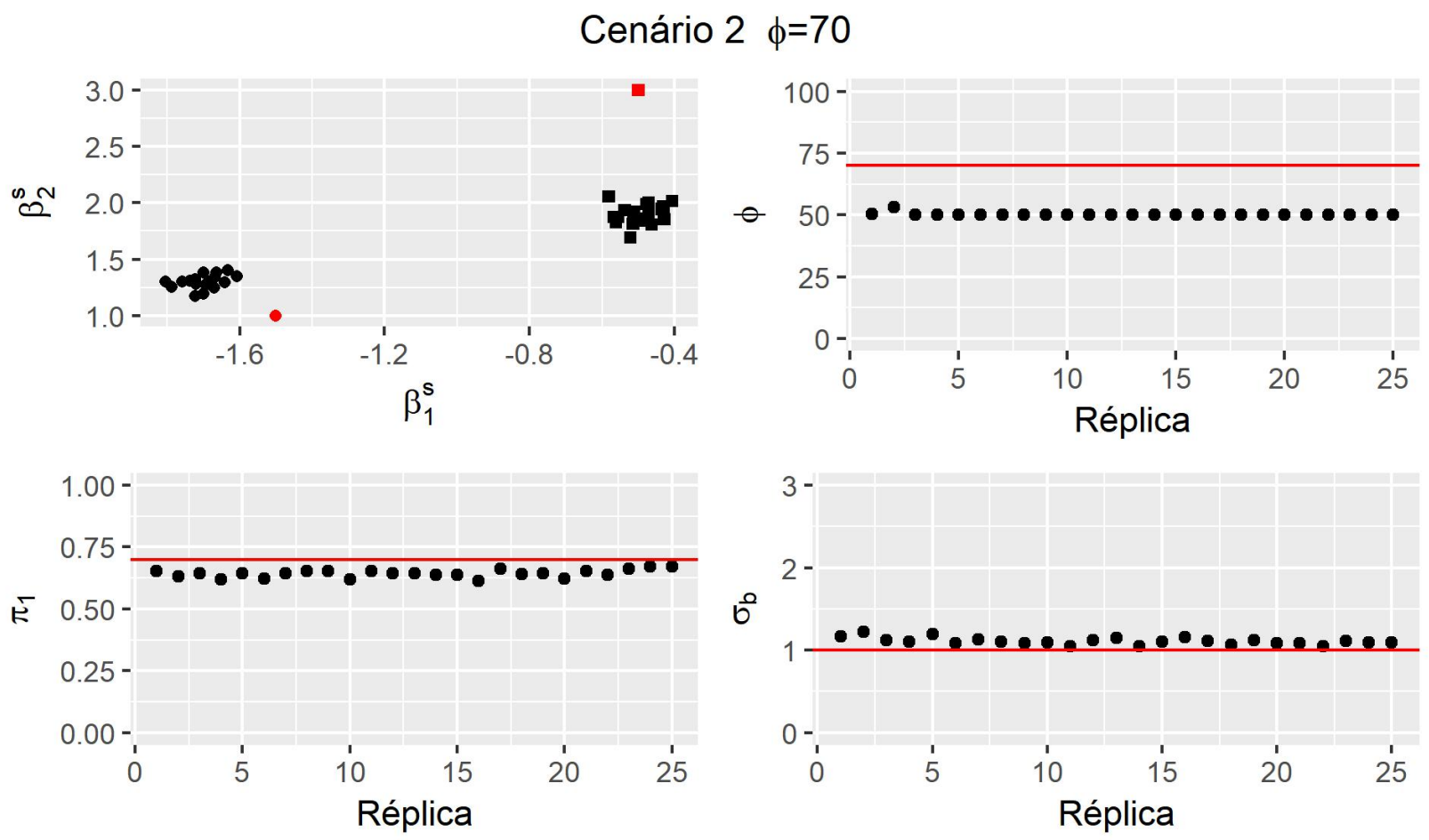

Figura B.42: Gráfico de dispersão das estimativas pontuais - mediana a posteriori - dos estudos de simulação de $R=25$ bancos de dados do modelo de mistura beta linear com efeitos aleatórios para a média com cenário 2, $\phi=70, N=500$ e $n=10$, em que $s=1(\bullet), 2(\mathbf{\square})$, e dos verdadeiros valores dos parâmetros (em vermelho).

Cenário $3 \phi=30$
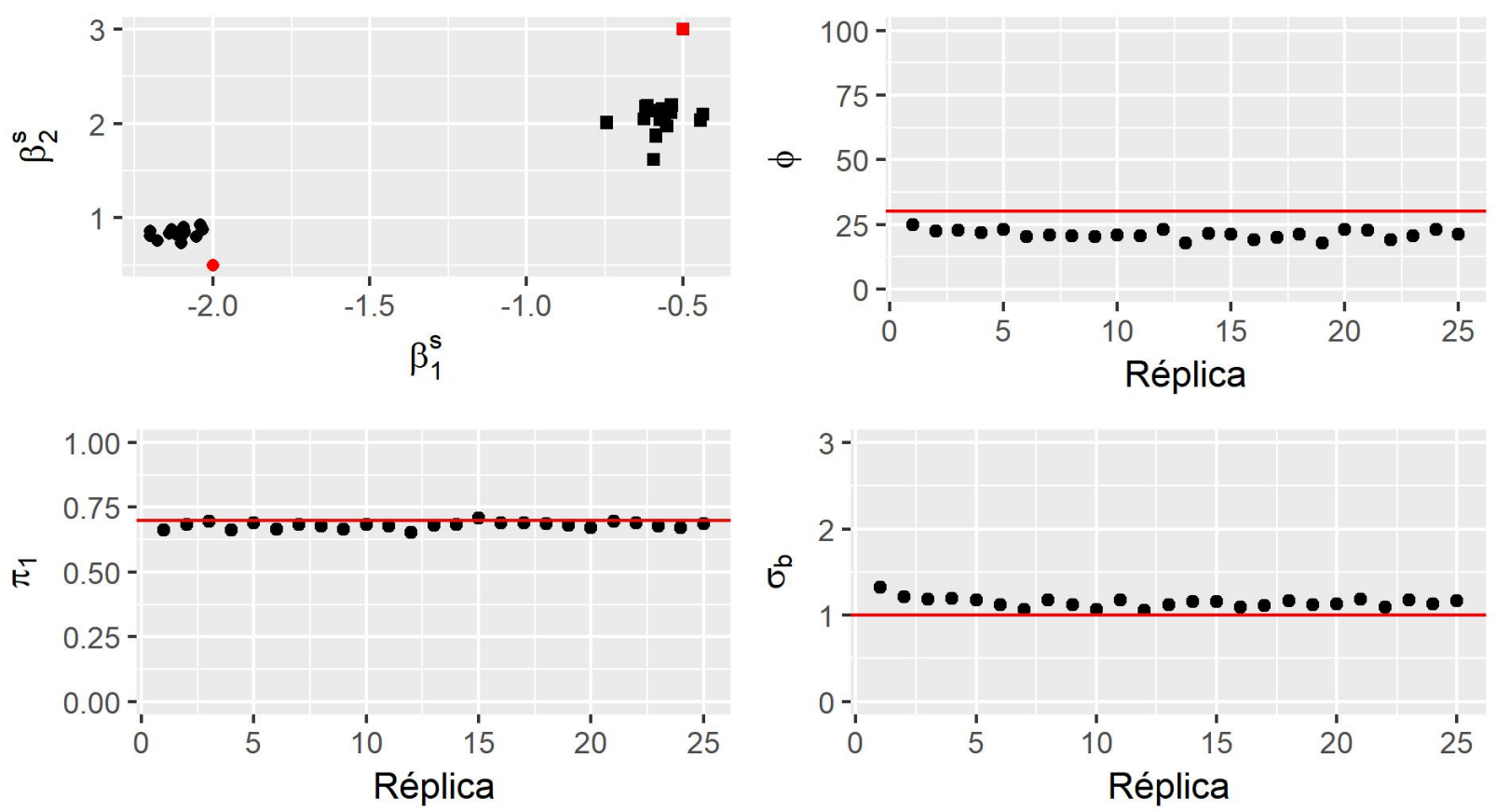

Figura B.43: Gráfico de dispersão das estimativas pontuais - mediana a posteriori - dos estudos de simulação de $R=25$ bancos de dados do modelo de mistura beta linear com efeitos aleatórios para a média com cenário 3, $\phi=30, N=500$ e $n=10$, em que $s=1(\bullet), 2(\mathbf{\square})$, e dos verdadeiros valores dos parâmetros (em vermelho). 

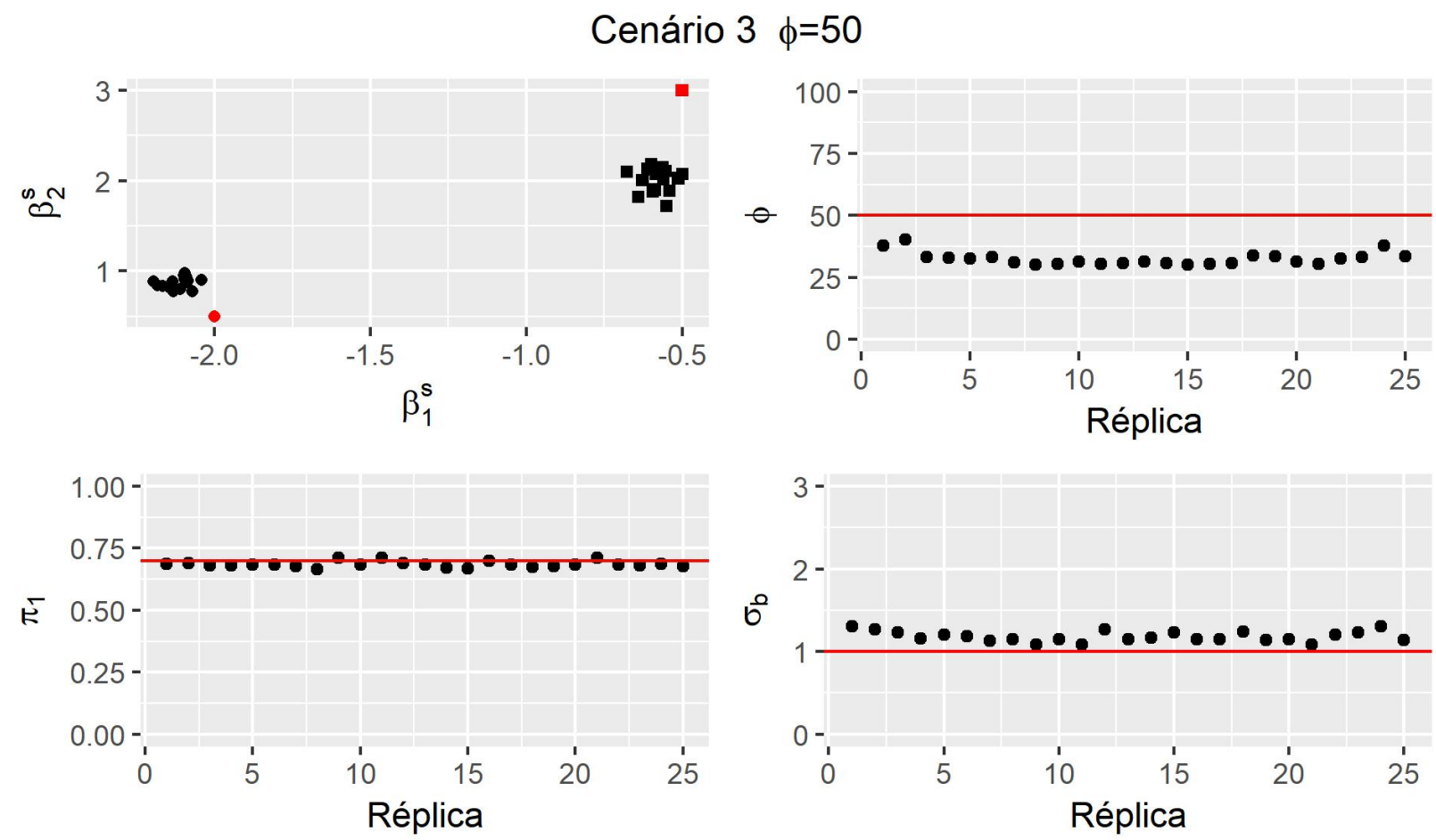

Figura B.44: Gráfico de dispersão das estimativas pontuais - mediana a posteriori - dos estudos de simulação de $R=25$ bancos de dados do modelo de mistura beta linear com efeitos aleatórios para a média com cenário 3, $\phi=50, N=500$ e $n=10$, em que $s=1(\bullet), 2(\bullet)$, e dos verdadeiros valores dos parâmetros (em vermelho).

\section{Cenário $3 \phi=70$}
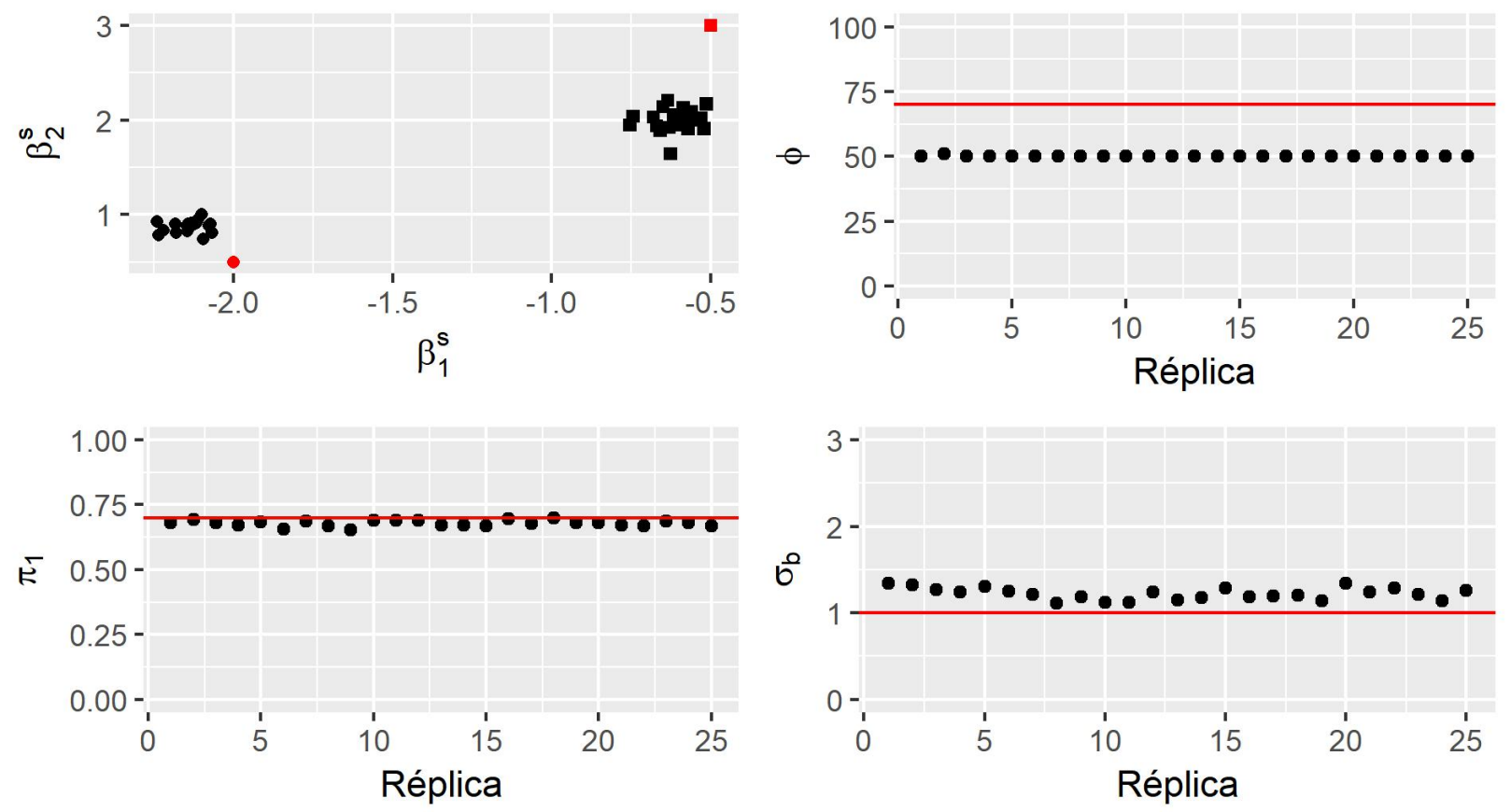

Figura B.45: Gráfico de dispersão das estimativas pontuais - mediana a posteriori - dos estudos de simulação de $R=25$ bancos de dados do modelo de mistura beta linear com efeitos aleatórios para a média com cenário 3, $\phi=70, N=500$ e $n=10$, em que $s=1(\bullet), 2(\mathbf{\bullet})$, e dos verdadeiros valores dos parâmetros (em vermelho). 


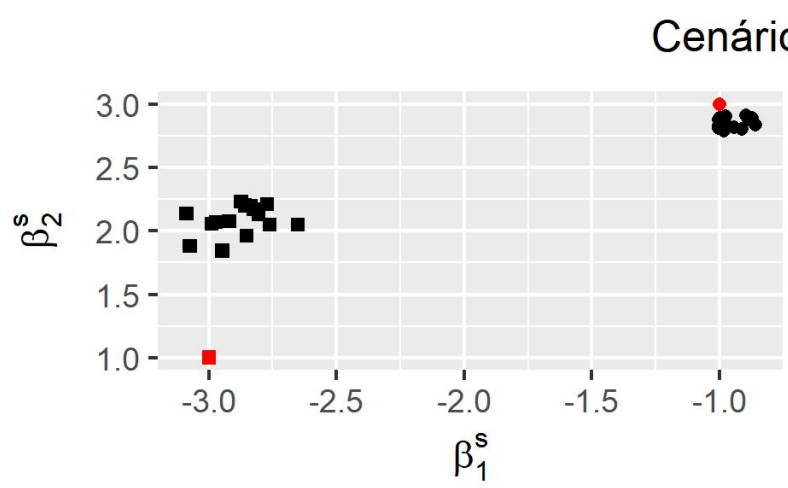

$4=30$
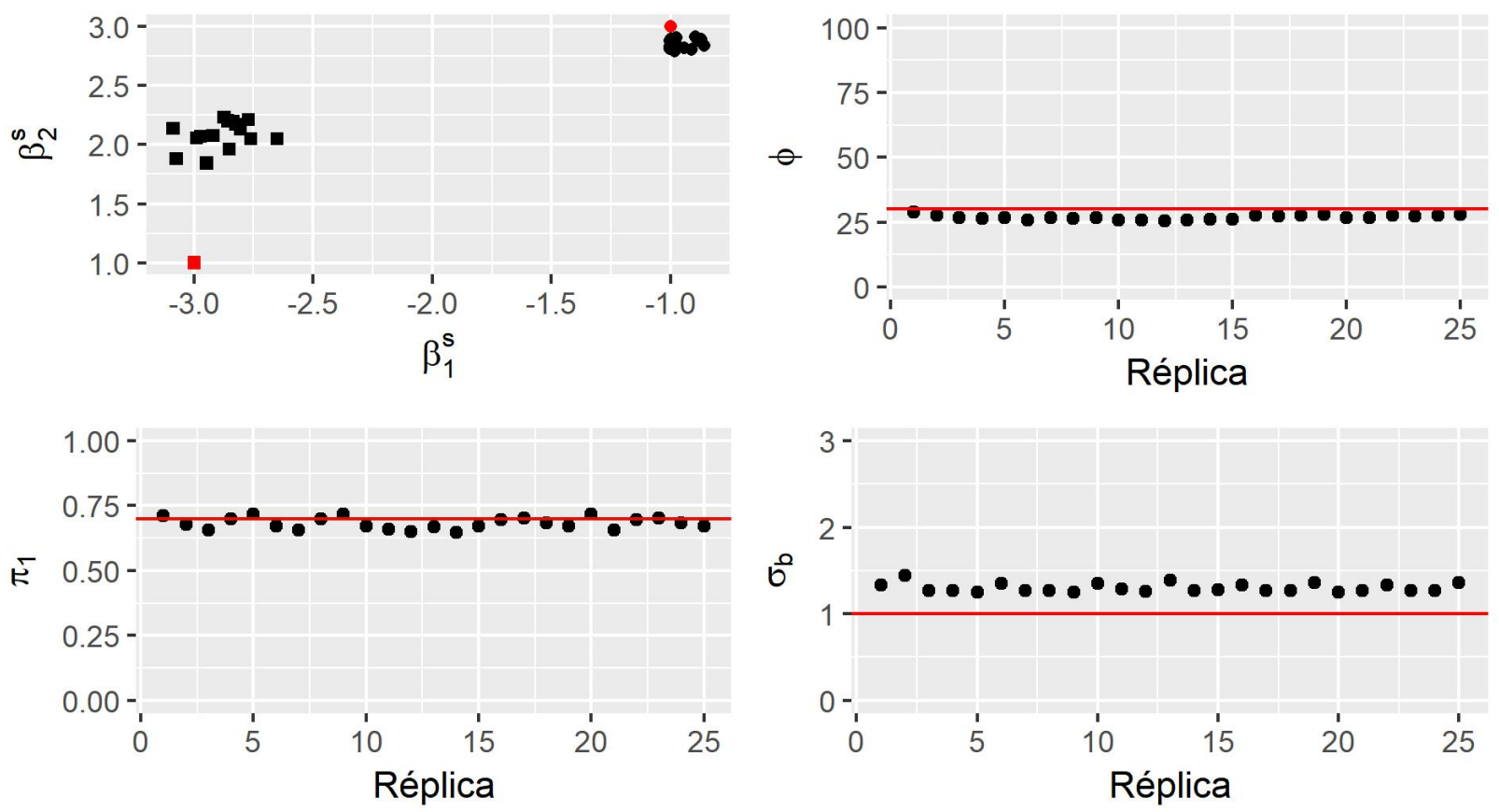

Figura B.46: Gráfico de dispersão das estimativas pontuais - mediana a posteriori - dos estudos de simulação de $R=25$ bancos de dados do modelo de mistura beta linear com efeitos aleatórios para a média com cenário $4, \phi=30, N=500$ e $n=10$, em que $s=1(\bullet), 2(\mathbf{\square})$, e dos verdadeiros valores dos parâmetros (em vermelho).
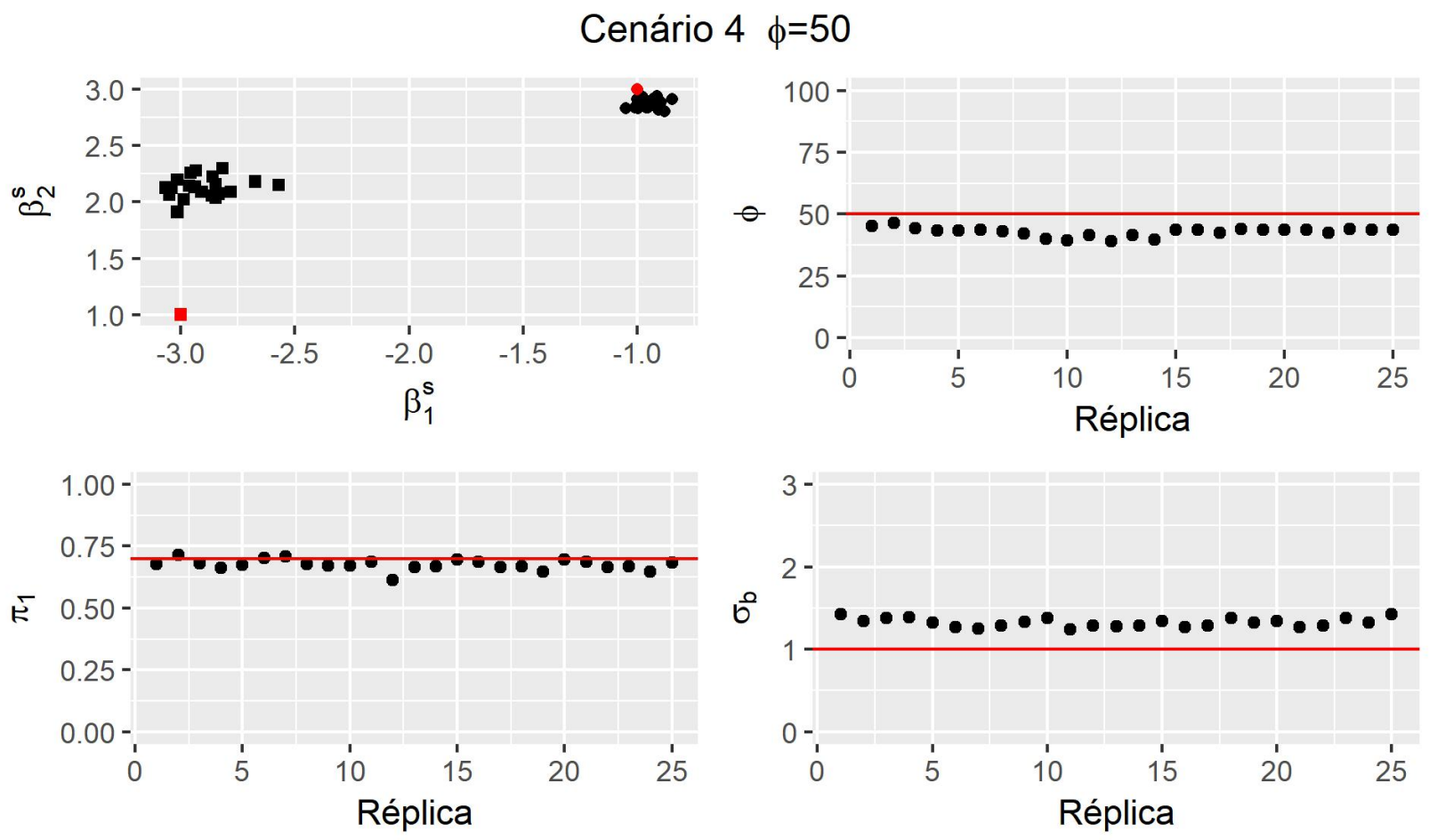

Figura B.47: Gráfico de dispersão das estimativas pontuais - mediana a posteriori - dos estudos de simulação de $R=25$ bancos de dados do modelo de mistura beta linear com efeitos aleatórios para a média com cenário $4, \phi=50, N=500$ e $n=10$, em que $s=1(\bullet), 2(\mathbf{\square})$, e dos verdadeiros valores dos parâmetros (em vermelho). 

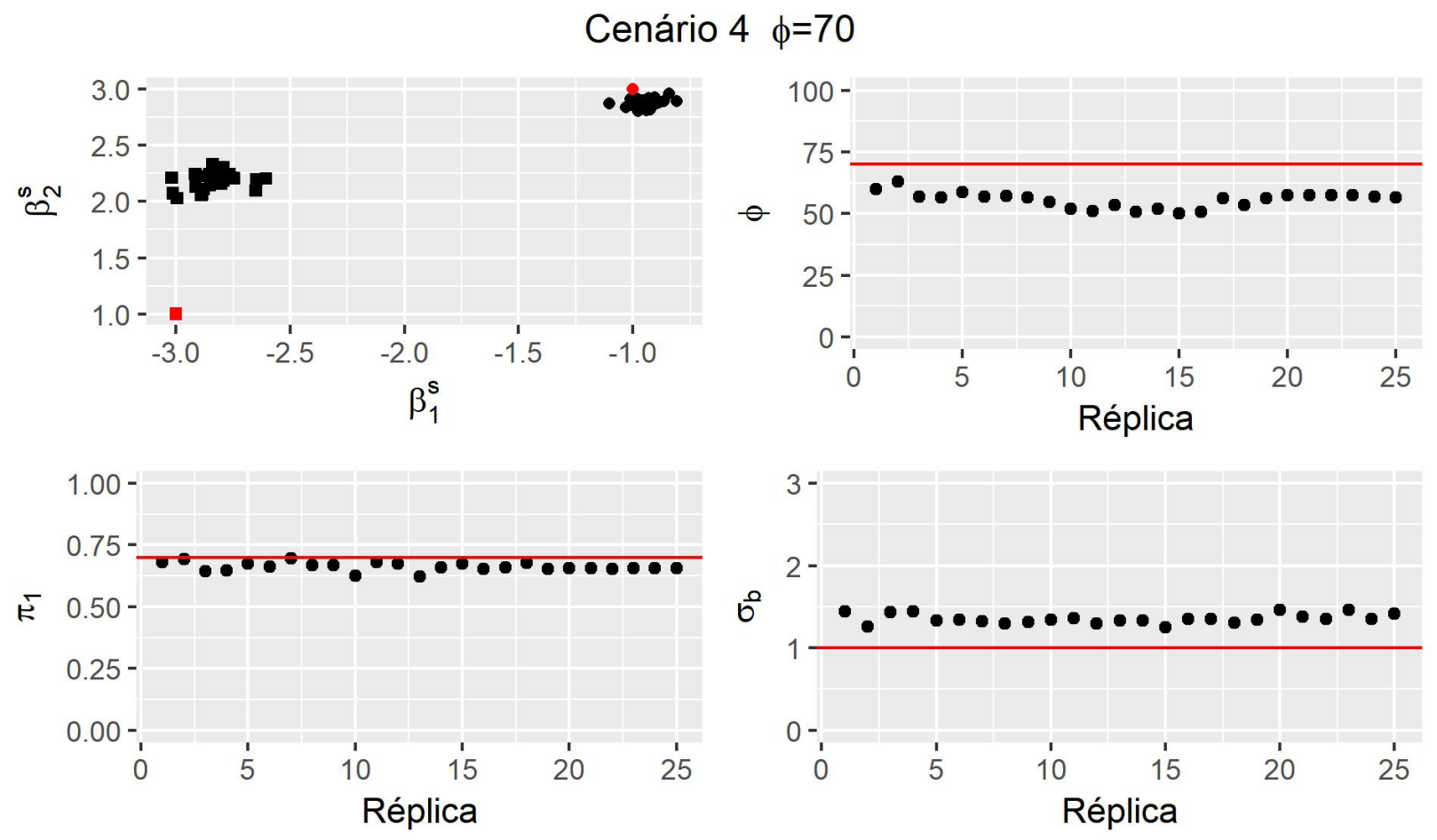

Figura B.48: Gráfico de dispersão das estimativas pontuais - mediana a posteriori - dos estudos de simulação de $R=25$ bancos de dados do modelo de mistura beta linear com efeitos aleatórios para a média com cenário 4, $\phi=70, N=500$ e $n=10$, em que $s=1(\bullet), 2(\mathbf{\bullet})$, e dos verdadeiros valores dos parâmetros (em vermelho). 


\section{B.5 Modelo linear com parâmetro de precisão constante - $\mathrm{N}=40$, $\mathrm{n}=5$}
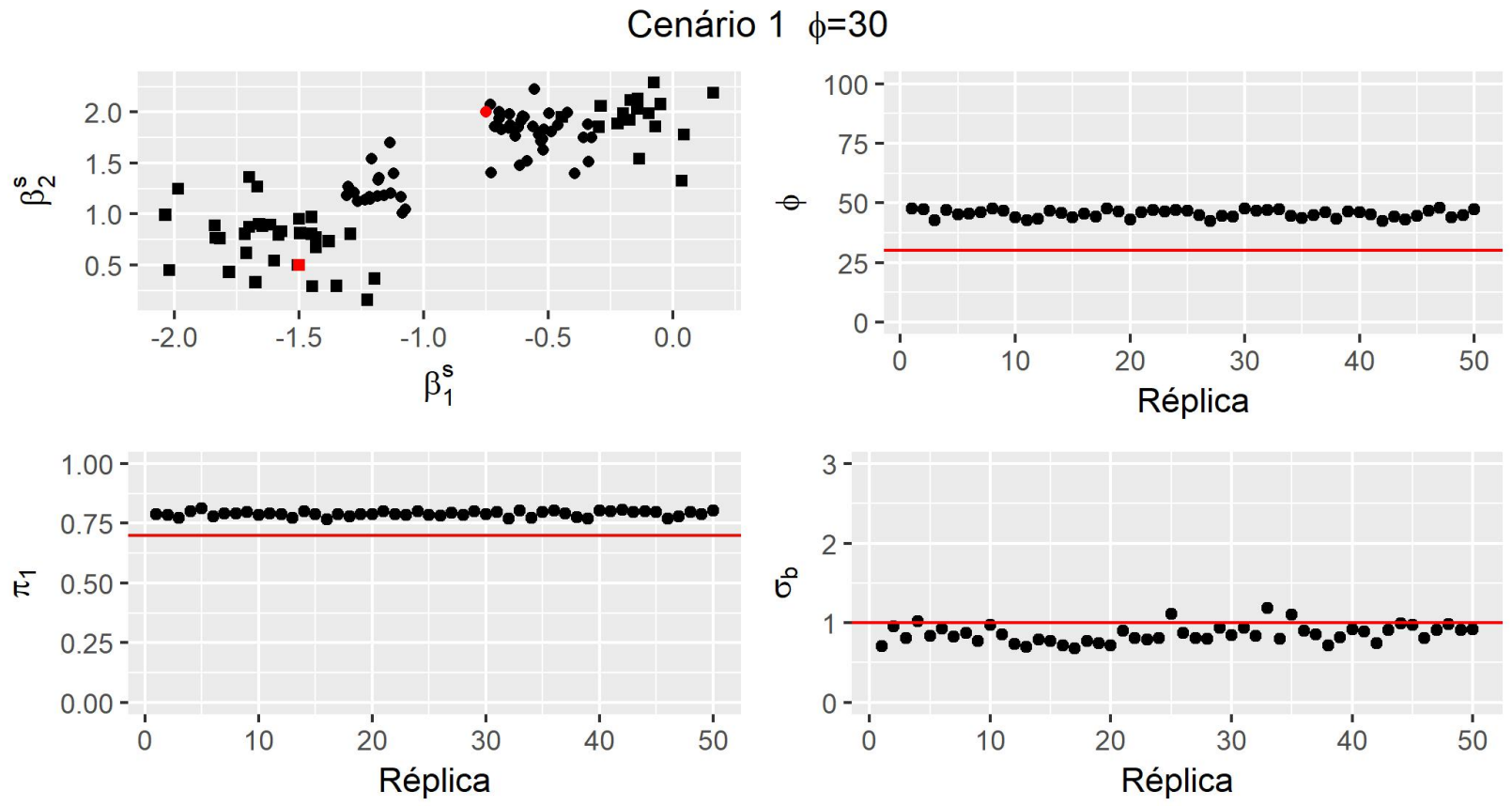

Figura B.49: Gráfico de dispersão das estimativas pontuais - mediana a posteriori - dos estudos de simulação de $R=50$ bancos de dados do modelo de mistura beta linear com efeitos aleatórios para a média com cenário $1, \phi=30, N=40$ e $n=5$, em que $s=1(\bullet), 2(\mathbf{\bullet})$, e dos verdadeiros valores dos parâmetros (em vermelho).
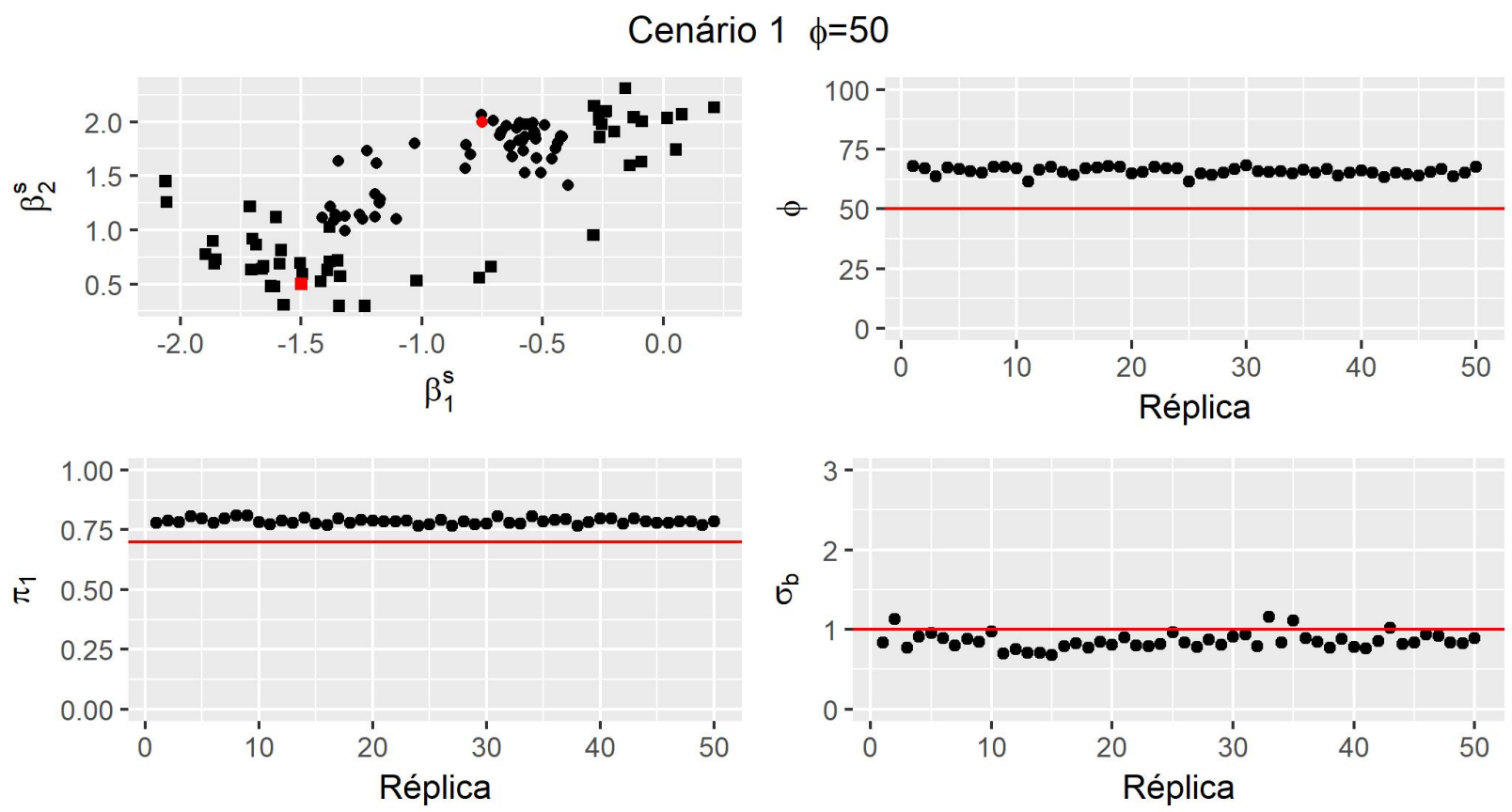

Figura B.50: Gráfico de dispersão das estimativas pontuais - mediana a posteriori - dos estudos de simulação de $R=50$ bancos de dados do modelo de mistura beta linear com efeitos aleatórios para a média com cenário $1, \phi=50, N=40$ e $n=5$, em que $s=1(\bullet), 2(\mathbf{\bullet})$, e dos verdadeiros valores dos parâmetros (em vermelho). 

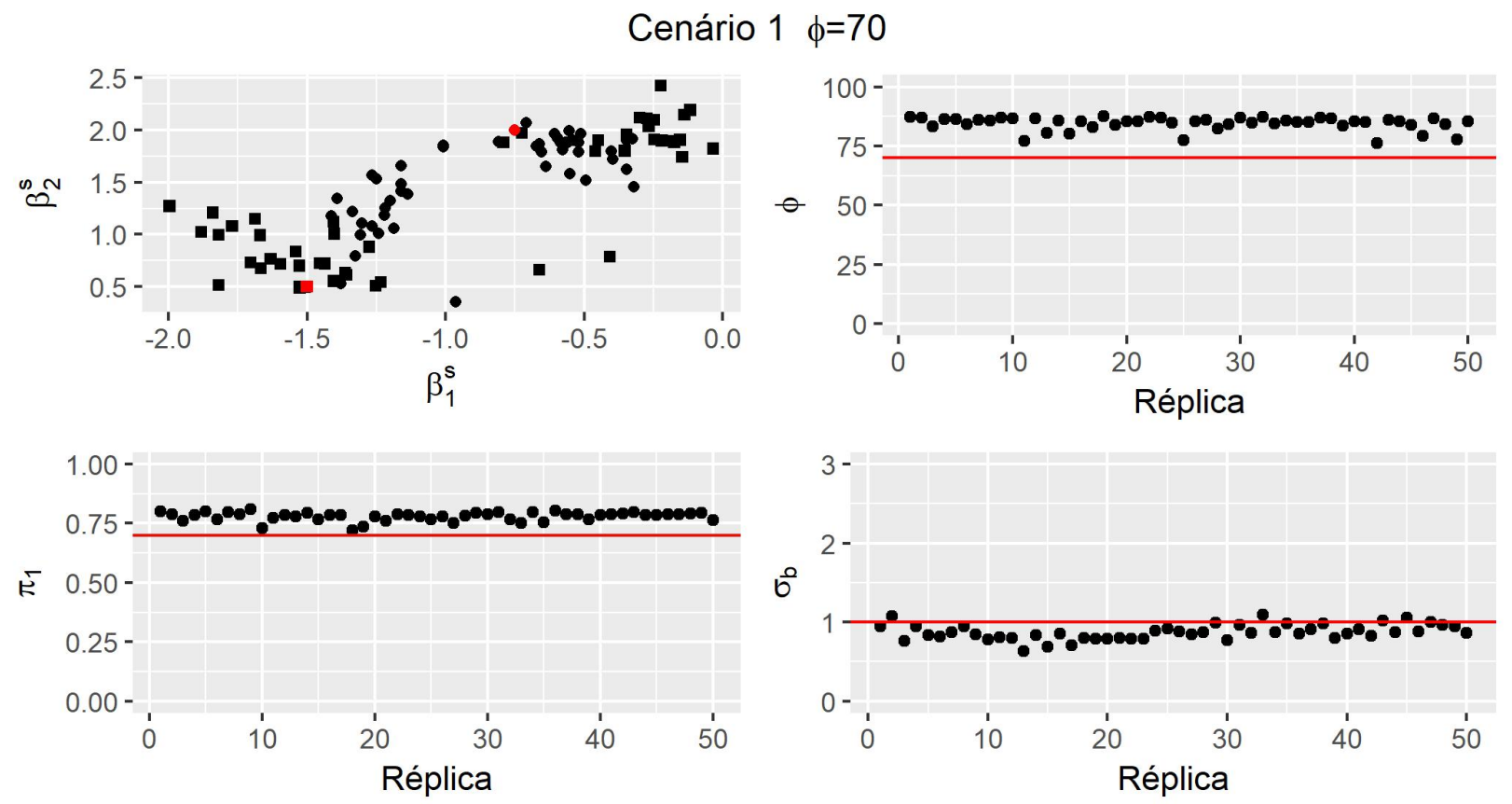

Figura B.51: Gráfico de dispersão das estimativas pontuais - mediana a posteriori - dos estudos de simulação de $R=50$ bancos de dados do modelo de mistura beta linear com efeitos aleatórios para a média com cenário 1, $\phi=70, N=40$ e $n=5$, em que $s=1(\bullet), 2(\mathbf{\bullet})$, e dos verdadeiros valores dos parâmetros (em vermelho).
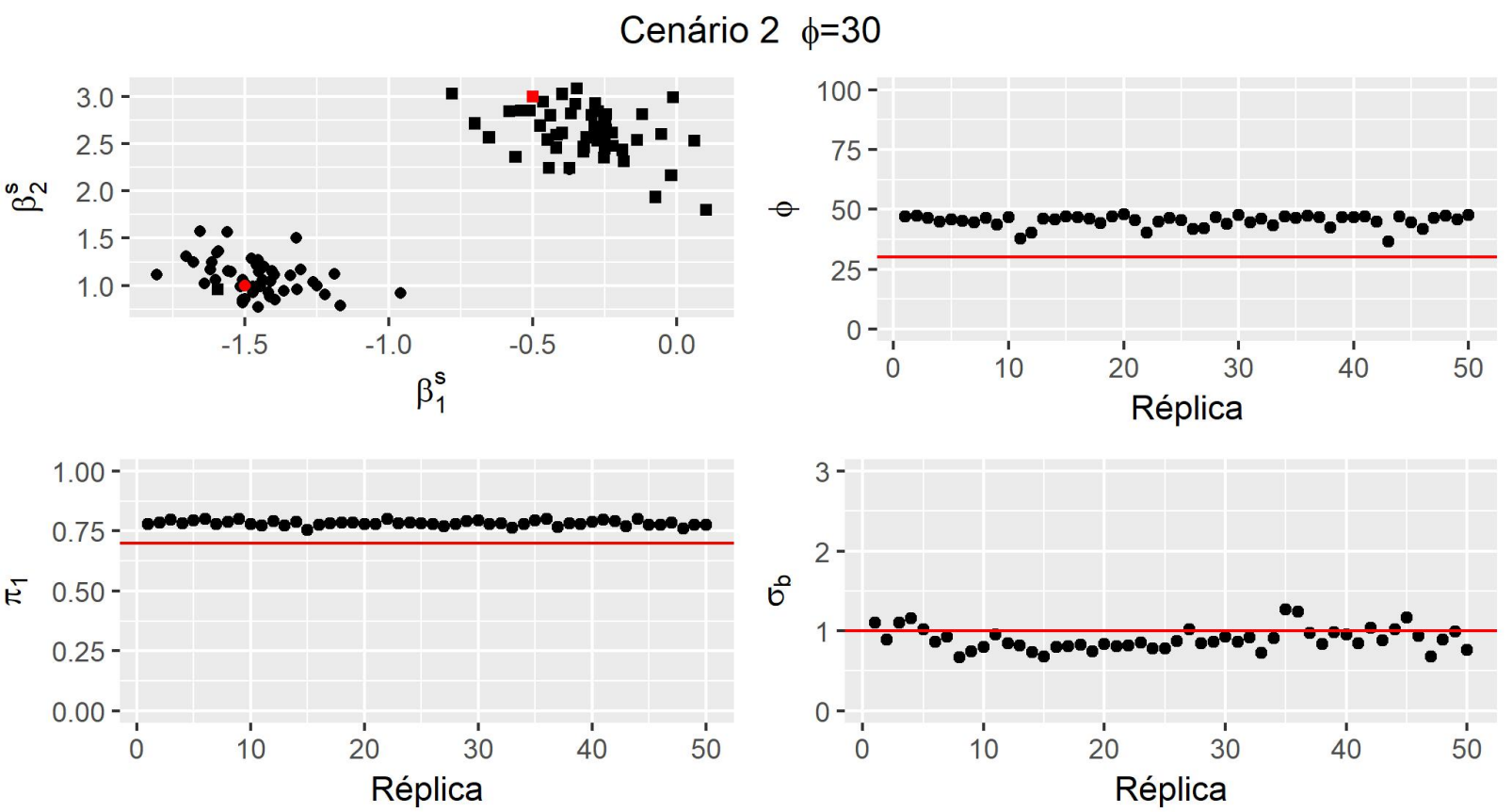

Figura B.52: Gráfico de dispersão das estimativas pontuais - mediana a posteriori - dos estudos de simulação de $R=50$ bancos de dados do modelo de mistura beta linear com efeitos aleatórios para a média com cenário $2, \phi=30, N=40$ e $n=5$, em que $s=1(\bullet), 2(\bullet)$, e dos verdadeiros valores dos parâmetros (em vermelho). 

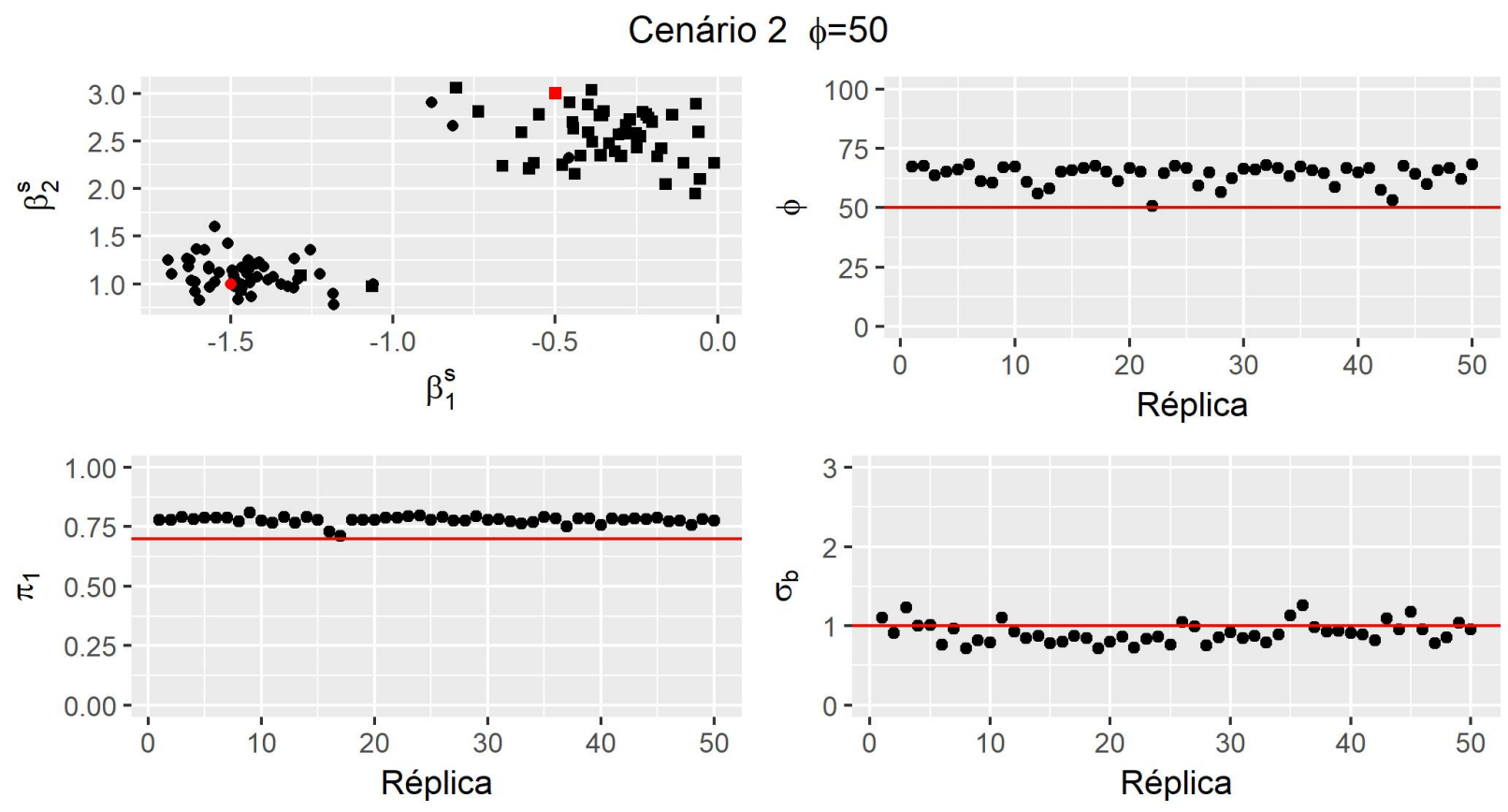

Figura B.53: Gráfico de dispersão das estimativas pontuais - mediana a posteriori - dos estudos de simulação de $R=50$ bancos de dados do modelo de mistura beta linear com efeitos aleatórios para a média com cenário $2, \phi=50, N=40$ e $n=5$, em que $s=1(\bullet), 2(\bullet)$, e dos verdadeiros valores dos parâmetros (em vermelho).
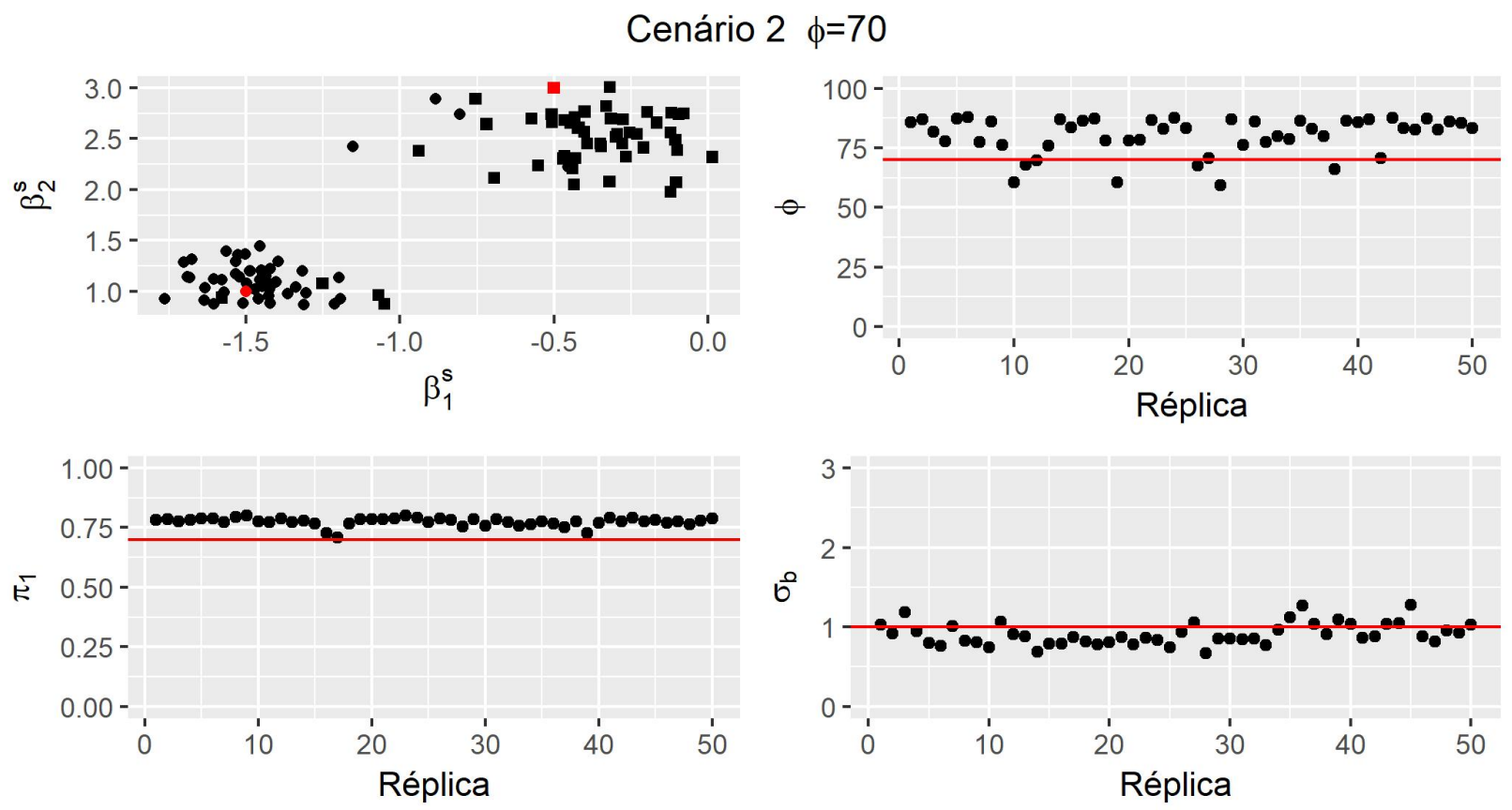

Figura B.54: Gráfico de dispersão das estimativas pontuais - mediana a posteriori - dos estudos de simulação de $R=50$ bancos de dados do modelo de mistura beta linear com efeitos aleatórios para a média com cenário 2, $\phi=70, N=40$ e $n=5$, em que $s=1(\bullet), 2(\mathbf{\bullet})$, e dos verdadeiros valores dos parâmetros (em vermelho). 

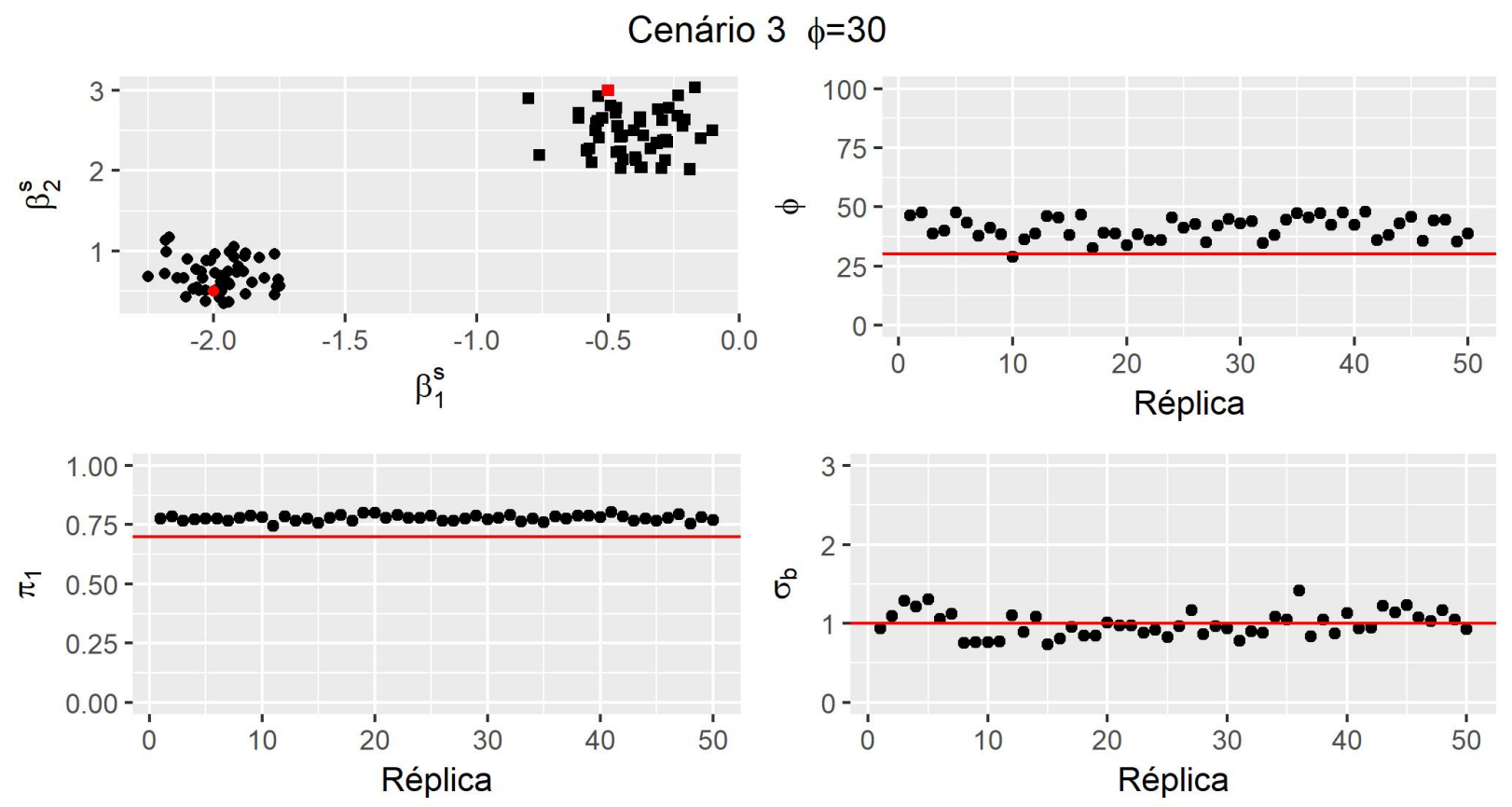

Figura B.55: Gráfico de dispersão das estimativas pontuais - mediana a posteriori - dos estudos de simulação de $R=50$ bancos de dados do modelo de mistura beta linear com efeitos aleatórios para a média com cenário 3, $\phi=30, N=40$ e $n=5$, em que $s=1(\bullet), 2(\mathbf{\bullet})$, e dos verdadeiros valores dos parâmetros (em vermelho).
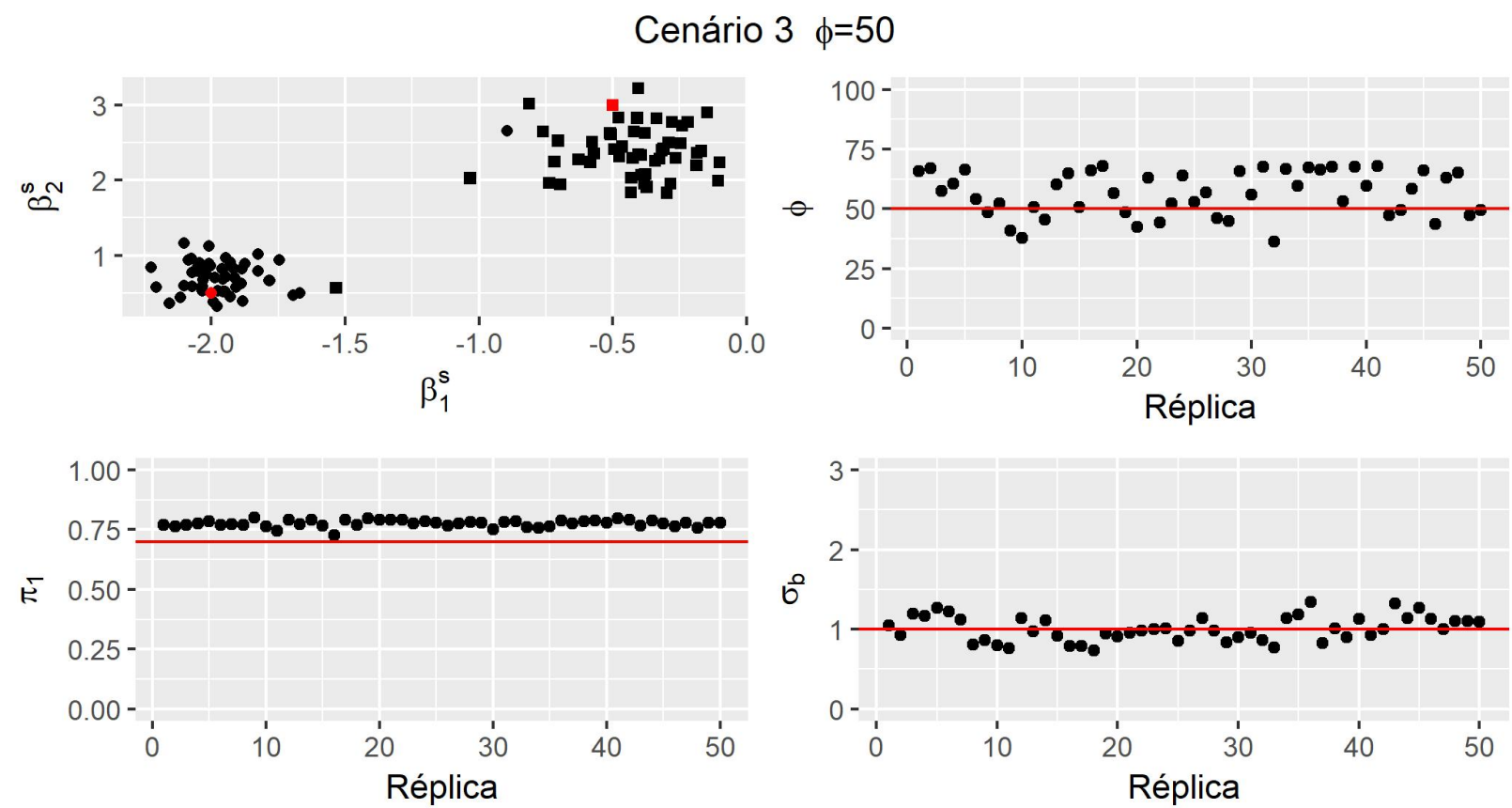

Figura B.56: Gráfico de dispersão das estimativas pontuais - mediana a posteriori - dos estudos de simulação de $R=50$ bancos de dados do modelo de mistura beta linear com efeitos aleatórios para a média com cenário 3, $\phi=50, N=40$ e $n=5$, em que $s=1(\bullet), 2(\mathbf{\bullet})$, e dos verdadeiros valores dos parâmetros (em vermelho). 


\section{Cenário $3 \phi=70$}
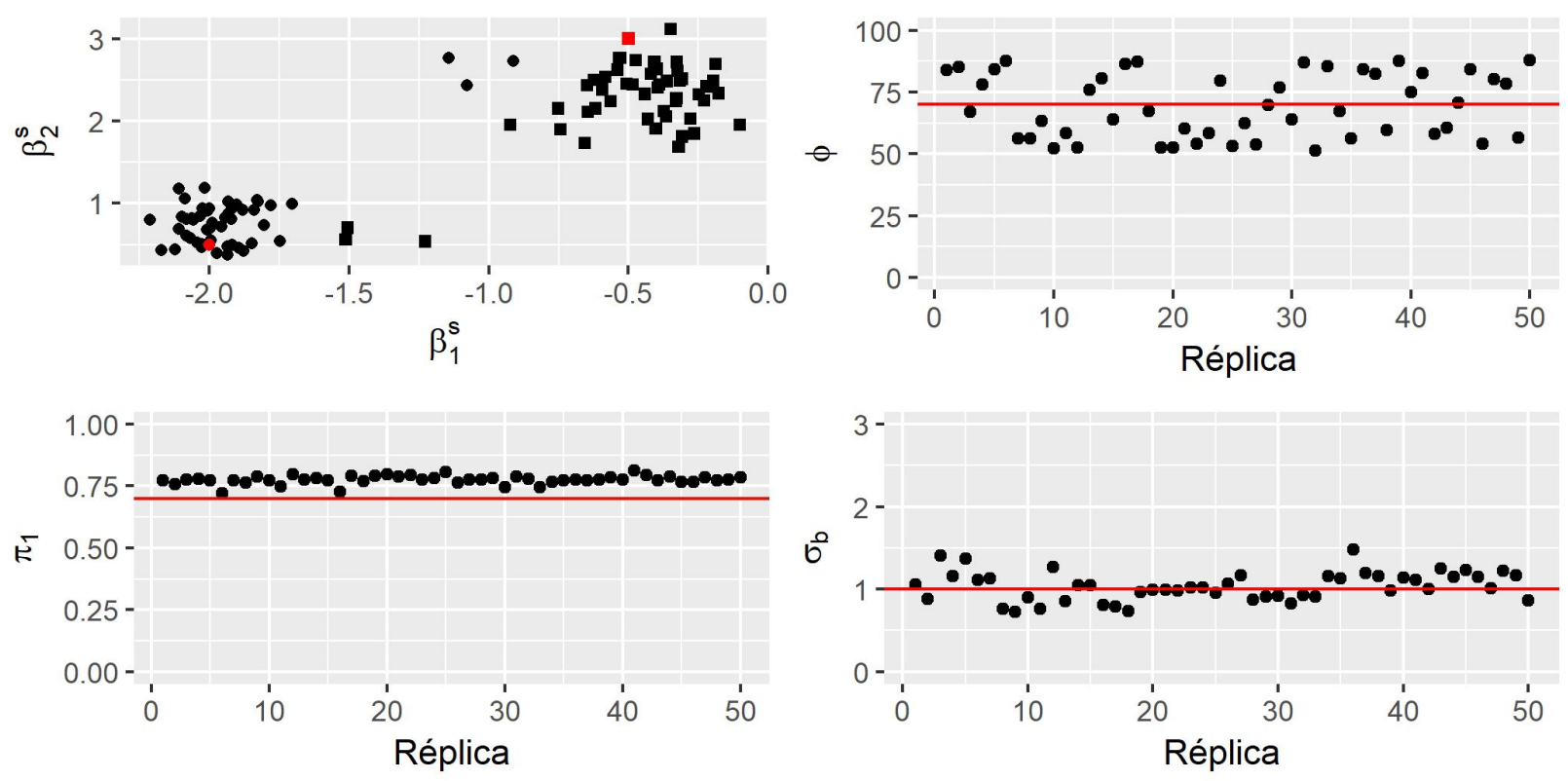

Figura B.57: Gráfico de dispersão das estimativas pontuais - mediana a posteriori - dos estudos de simulação de $R=50$ bancos de dados do modelo de mistura beta linear com efeitos aleatórios para a média com cenário $3, \phi=70, N=40$ e $n=5$, em que $s=1(\bullet), 2(\bullet)$, e dos verdadeiros valores dos parâmetros (em vermelho).
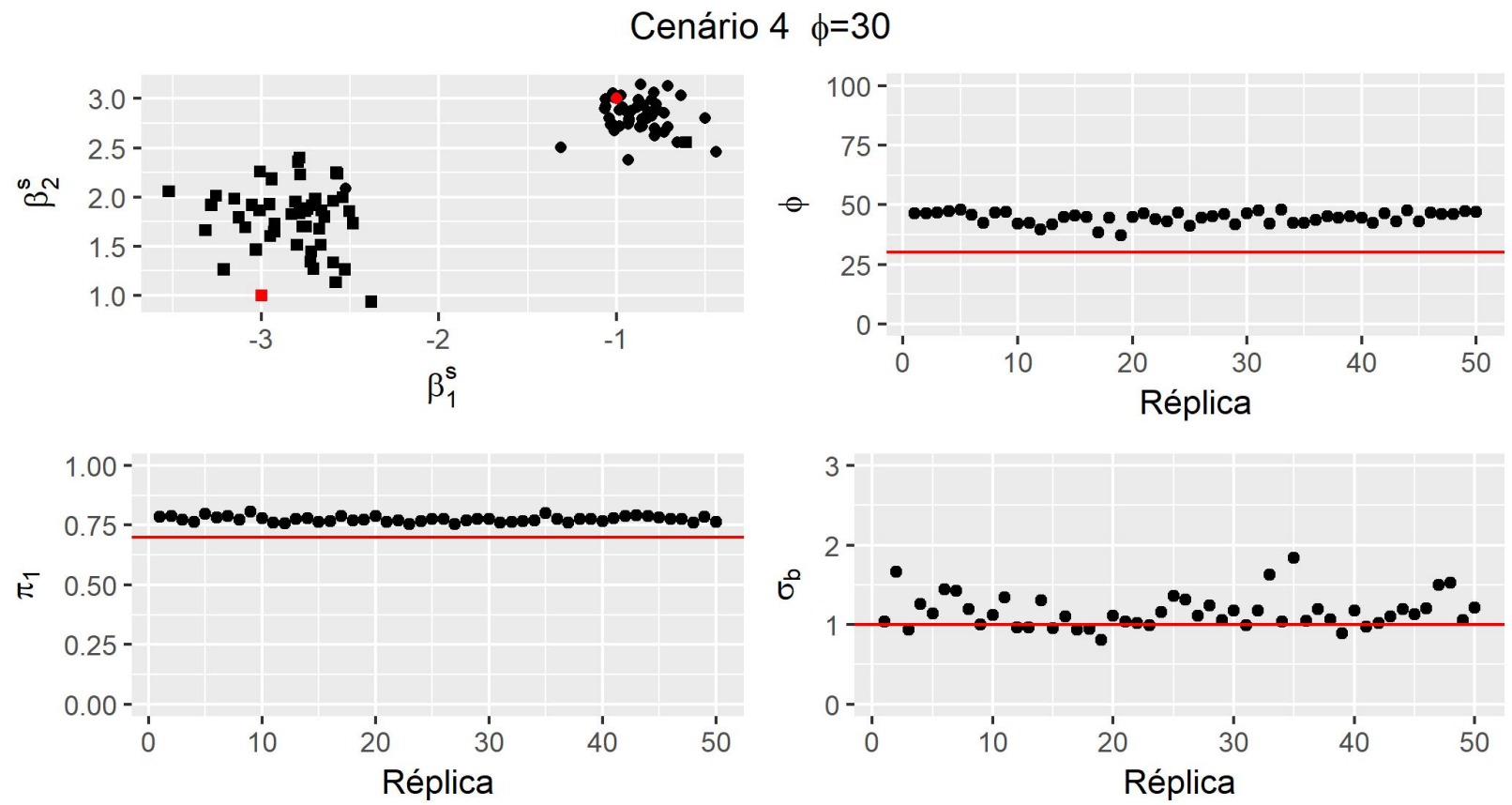

Figura B.58: Gráfico de dispersão das estimativas pontuais - mediana a posteriori - dos estudos de simulação de $R=50$ bancos de dados do modelo de mistura beta linear com efeitos aleatórios para a média com cenário $4, \phi=30, N=40$ e $n=5$, em que $s=1(\bullet), 2(\mathbf{\bullet})$, e dos verdadeiros valores dos parâmetros (em vermelho). 

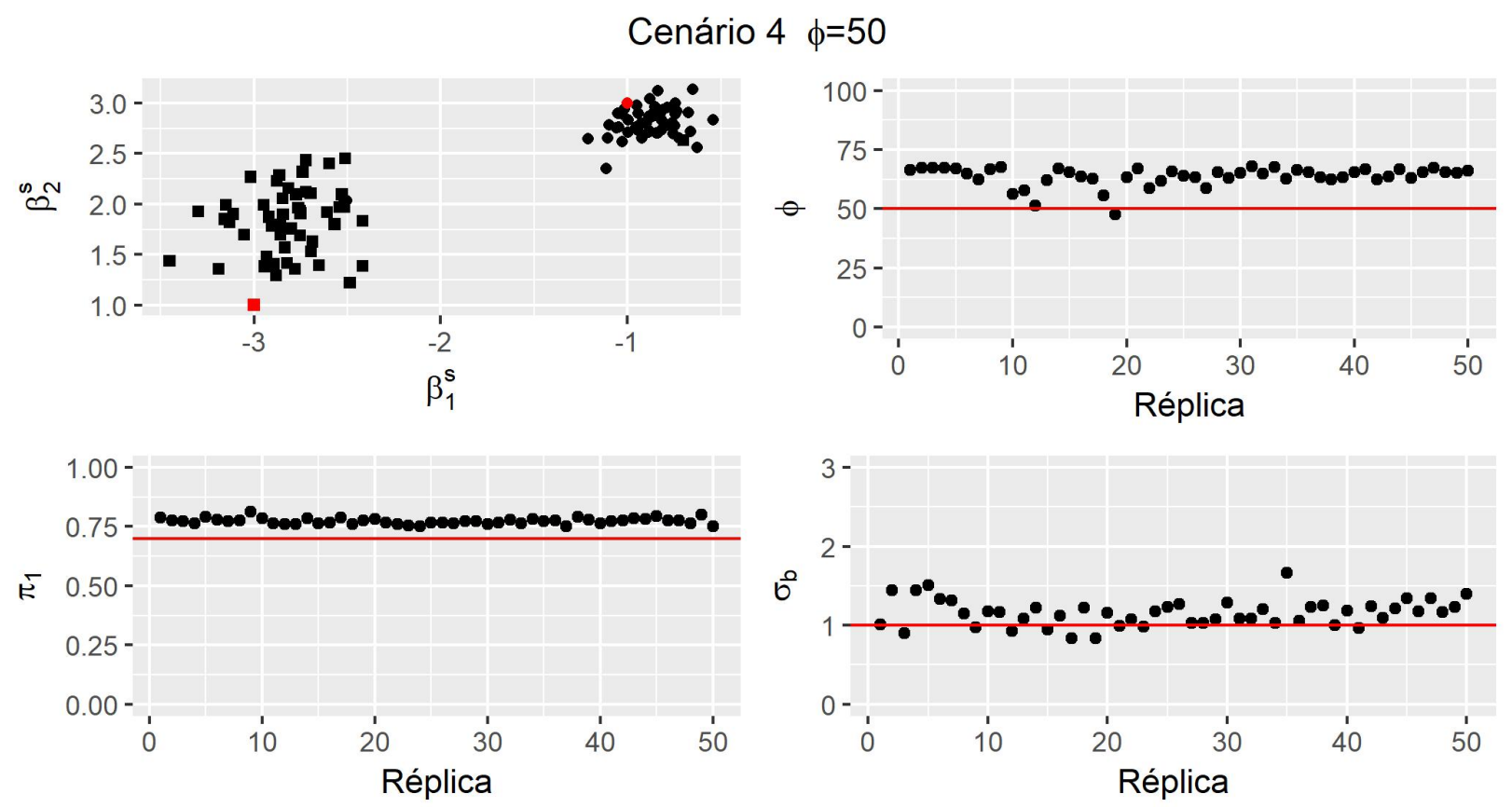

Figura B.59: Gráfico de dispersão das estimativas pontuais - mediana a posteriori - dos estudos de simulação de $R=50$ bancos de dados do modelo de mistura beta linear com efeitos aleatórios para a média com cenário $4, \phi=50, N=40$ e $n=5$, em que $s=1(\bullet), 2(\mathbf{\bullet})$, e dos verdadeiros valores dos parâmetros (em vermelho).
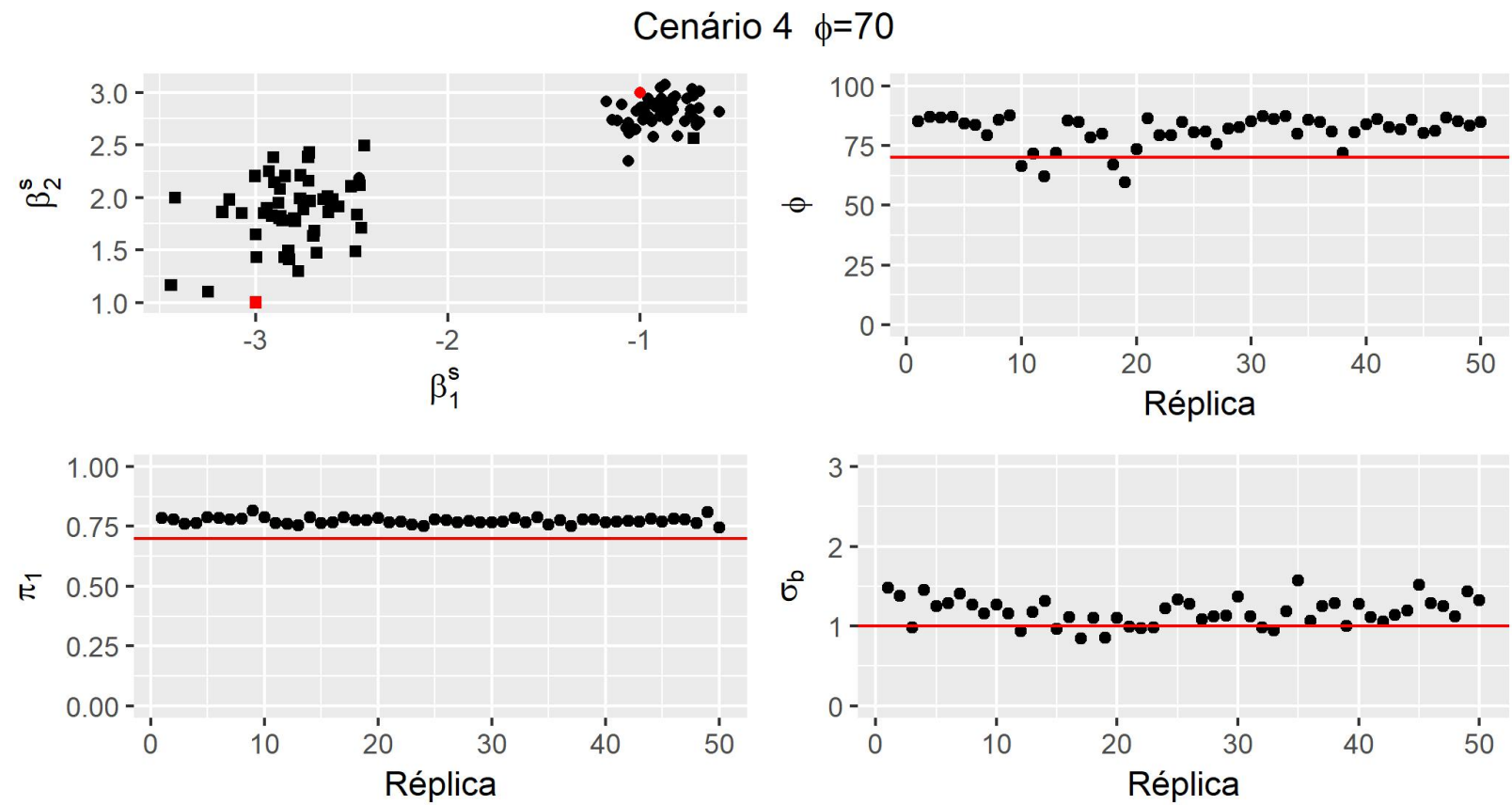

Figura B.60: Gráfico de dispersão das estimativas pontuais - mediana a posteriori - dos estudos de simulação de $R=50$ bancos de dados do modelo de mistura beta linear com efeitos aleatórios para a média com cenário $4, \phi=70, N=40$ e $n=5$, em que $s=1(\bullet), 2(\bullet)$, e dos verdadeiros valores dos parâmetros (em vermelho). 
B.6 Modelo linear com parâmetro de precisão constante - $\mathrm{N}=100$, $\mathbf{n}=\mathbf{5}, \Sigma_{\beta^{s}}=40 I_{2}$
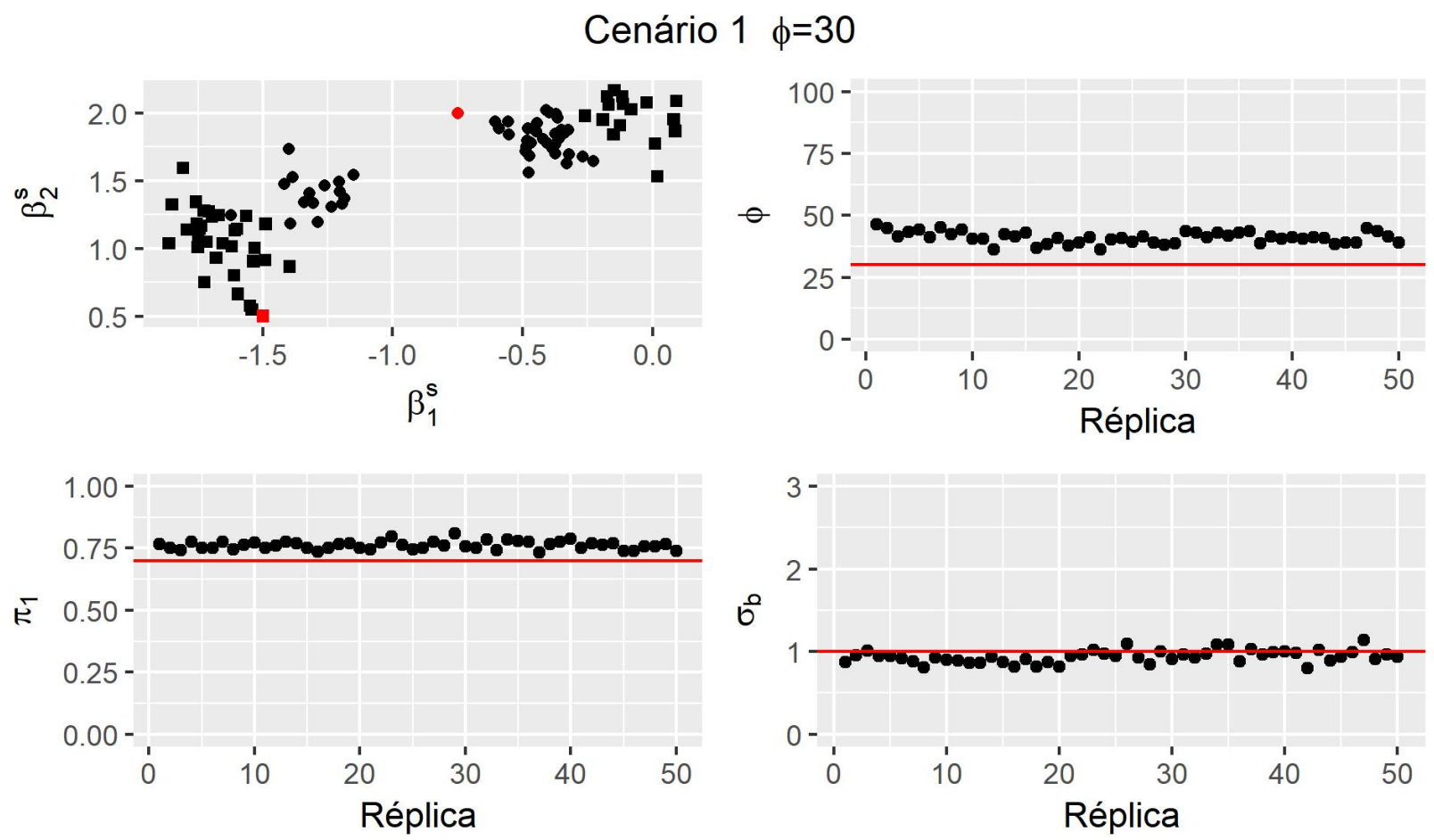

Figura B.61: Gráfico de dispersão das estimativas pontuais - mediana a posteriori - dos estudos de simulação de $R=50$ bancos de dados do modelo de mistura beta linear com efeitos aleatórios para a média com cenário 1, $\phi=30, N=100, n=5$ e $\Sigma_{\beta^{s}}=40 I_{2}$, em que $s=1(\bullet), 2(\mathbf{\square})$, e dos verdadeiros valores dos parâmetros (em vermelho). 

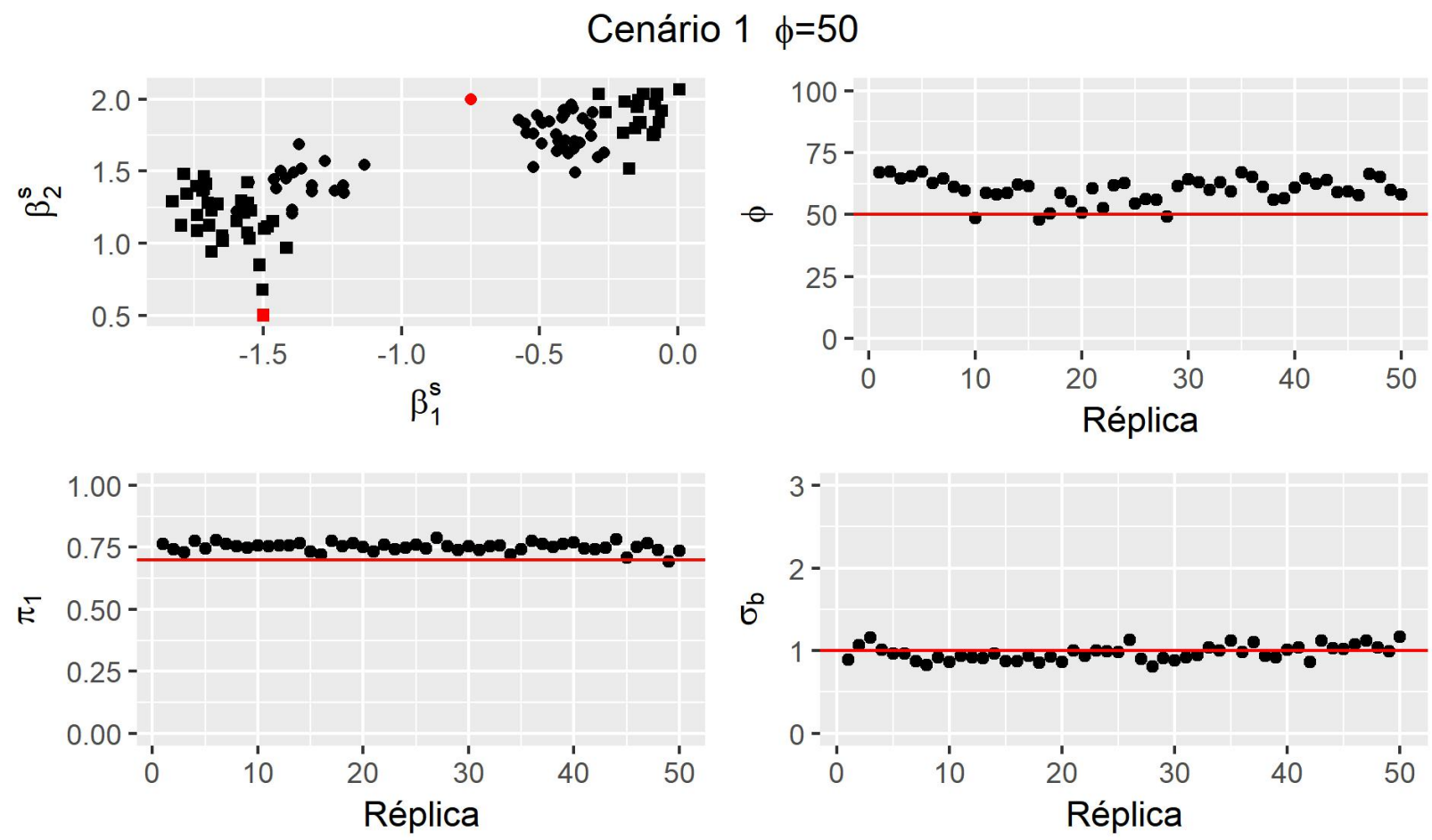

Figura B.62: Gráfico de dispersão das estimativas pontuais - mediana a posteriori - dos estudos de simulação de $R=50$ bancos de dados do modelo de mistura beta linear com efeitos aleatórios para a média com cenário 1, $\phi=50, N=100, n=5$ e $\Sigma_{\beta^{s}}=40 I_{2}$, em que $s=1(\bullet), 2(\mathbf{\square})$, e dos verdadeiros valores dos parâmetros (em vermelho).
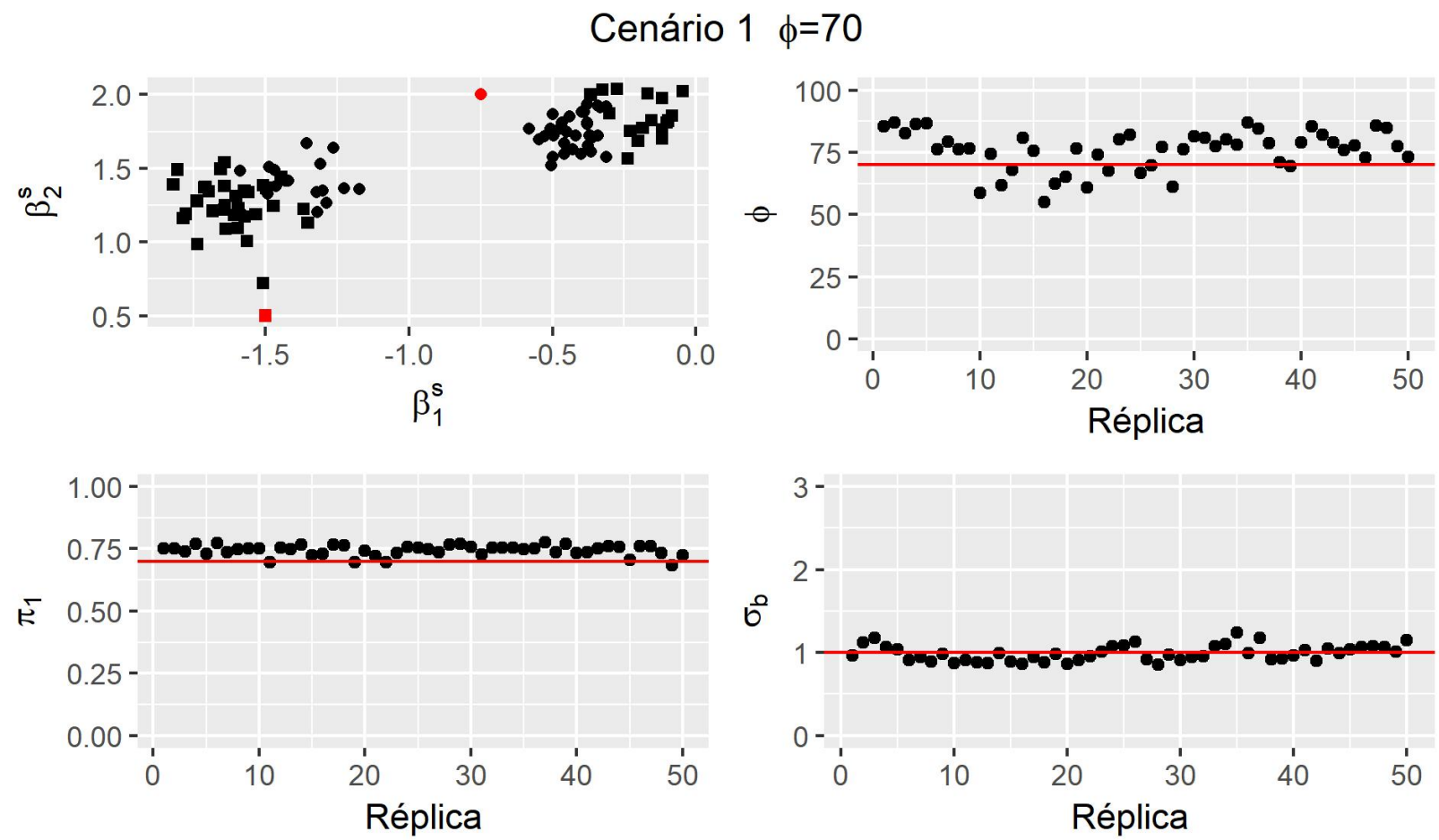

Figura B.63: Gráfico de dispersão das estimativas pontuais - mediana a posteriori - dos estudos de simulação de $R=50$ bancos de dados do modelo de mistura beta linear com efeitos aleatórios para a média com cenário 1, $\phi=70, N=100, n=5$ e $\Sigma_{\beta^{s}}=40 I_{2}$, em que $s=1(\bullet), 2(\mathbf{\bullet})$, e dos verdadeiros valores dos parâmetros (em vermelho). 

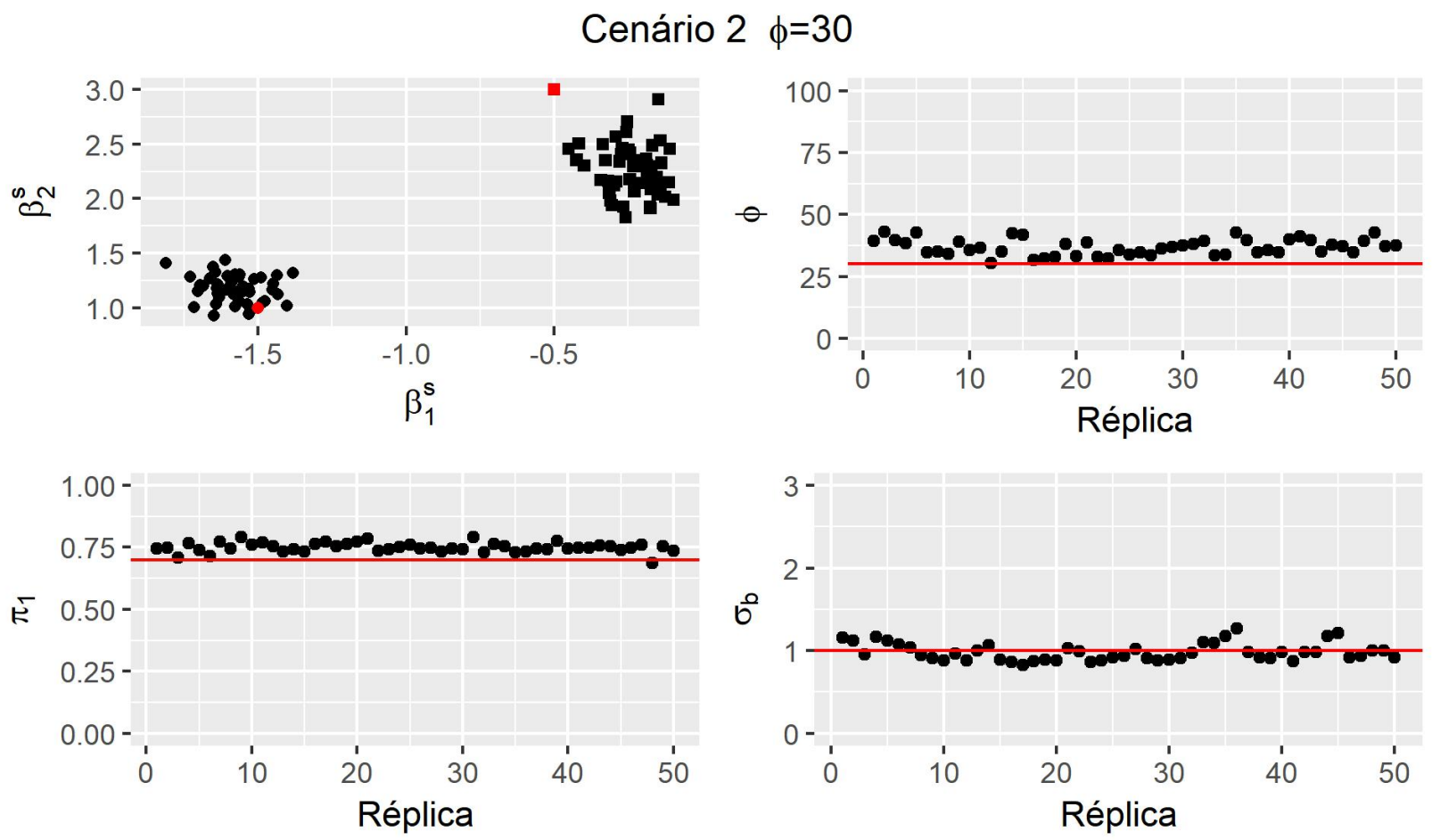

Figura B.64: Gráfico de dispersão das estimativas pontuais - mediana a posteriori - dos estudos de simulação de $R=50$ bancos de dados do modelo de mistura beta linear com efeitos aleatórios para a média com cenário 2, $\phi=30, N=100, n=5$ e $\Sigma_{\beta^{s}}=40 I_{2}$, em que $s=1(\bullet), 2(\mathbf{\square})$, e dos verdadeiros valores dos parâmetros (em vermelho).
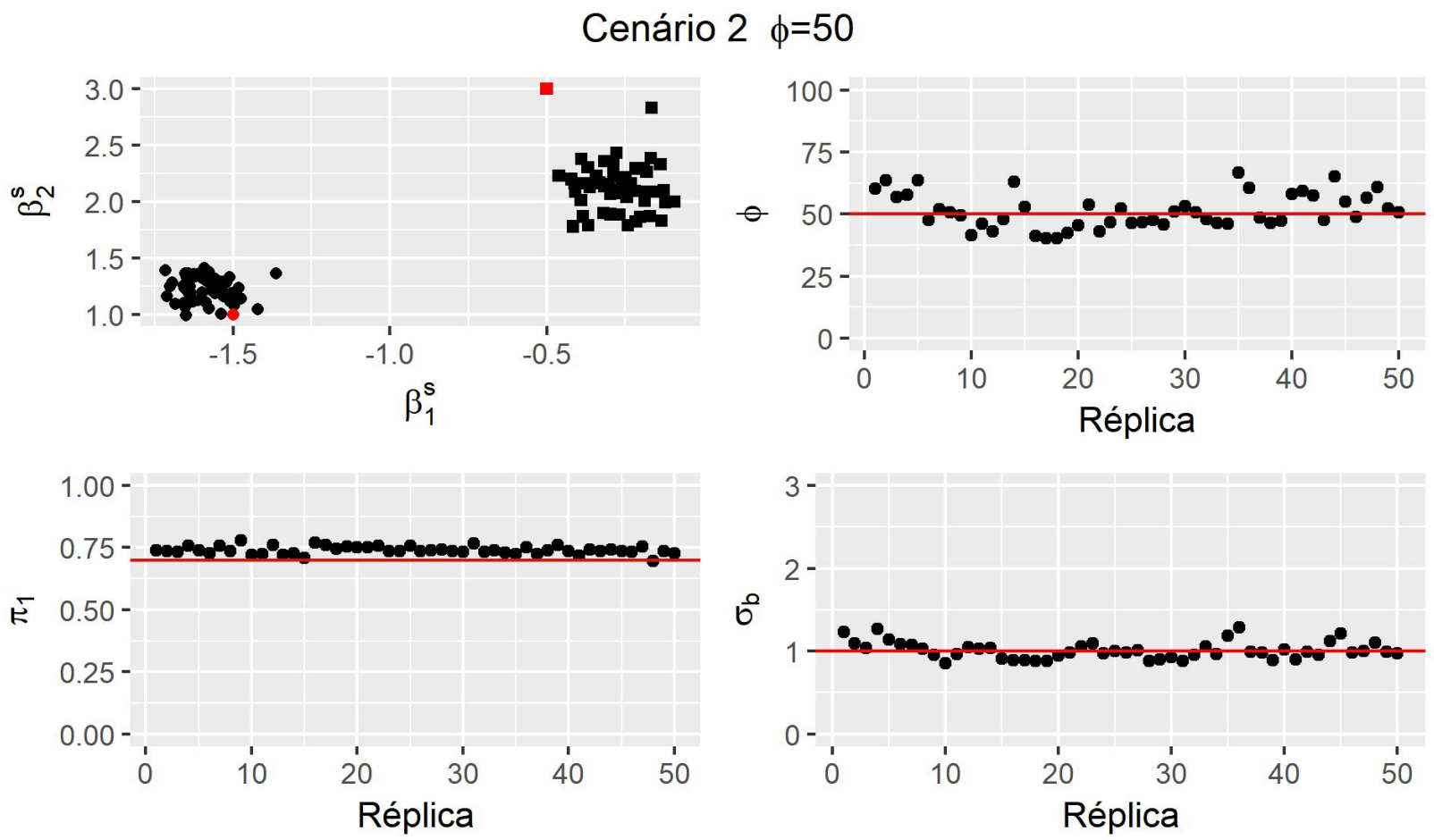

Figura B.65: Gráfico de dispersão das estimativas pontuais - mediana a posteriori - dos estudos de simulação de $R=50$ bancos de dados do modelo de mistura beta linear com efeitos aleatórios para a média com cenário $2, \phi=50, N=100, n=5$ e $\Sigma_{\beta^{s}}=40 I_{2}$, em que $s=1(\bullet), 2(\mathbf{\square})$, e dos verdadeiros valores dos parâmetros (em vermelho). 


\section{Cenário $2 \phi=70$}
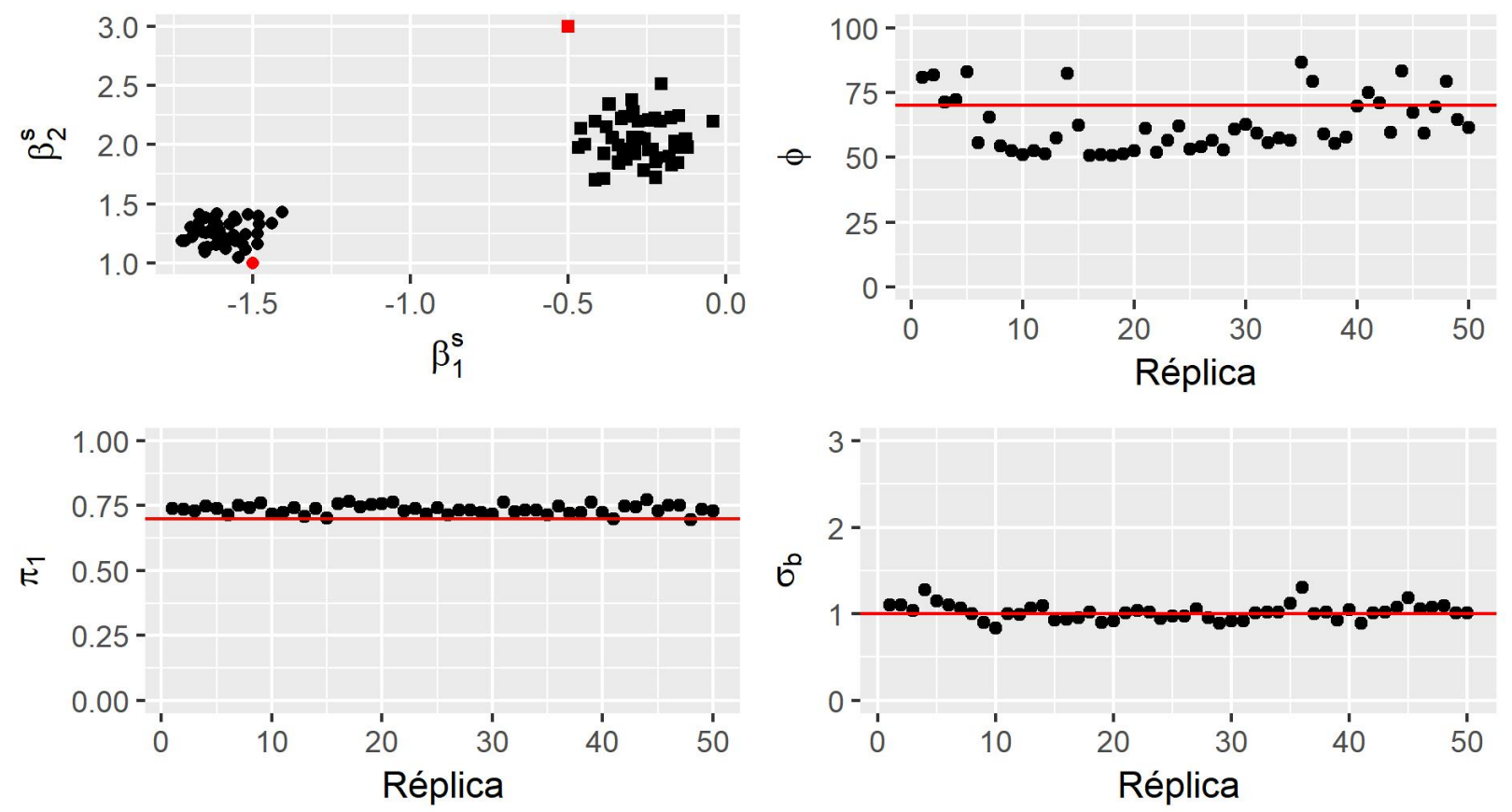

Figura B.66: Gráfico de dispersão das estimativas pontuais - mediana a posteriori - dos estudos de simulação de $R=50$ bancos de dados do modelo de mistura beta linear com efeitos aleatórios para a média com cenário $2, \phi=70, N=100, n=5$ e $\Sigma_{\beta^{s}}=40 I_{2}$, em que $s=1(\bullet), 2(\mathbf{\square})$, e dos verdadeiros valores dos parâmetros (em vermelho). 


\section{B.7 Modelo linear com dispersão variável}
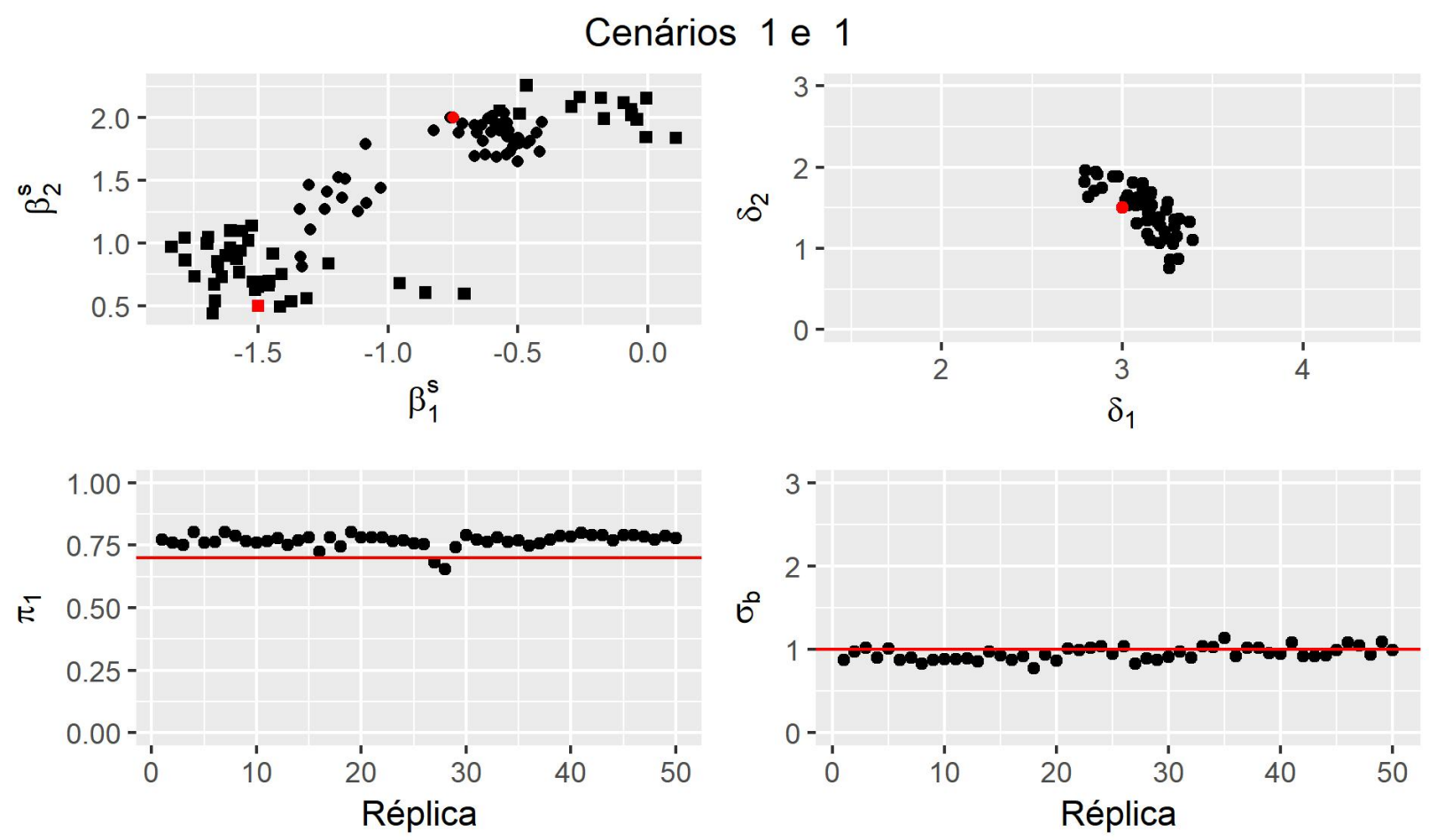

Figura B.67: Gráfico de dispersão das estimativas pontuais - mediana a posteriori - dos estudos de simulação de $R=50$ bancos de dados do modelo de mistura beta linear com efeitos aleatórios para a média e precisão com respectivos cenários para os parâmetros fixos: 1 e 1, em que $s=1(\bullet), 2(\bullet)$, e dos verdadeiros valores dos parâmetros (em vermelho). 


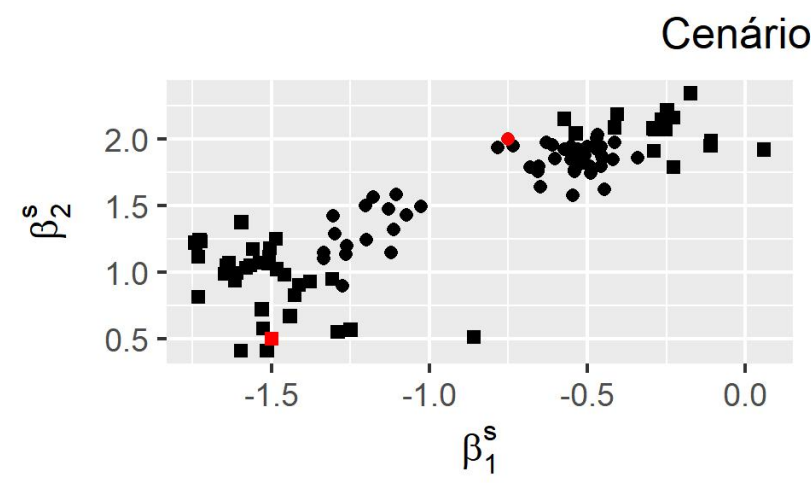

1 e 2
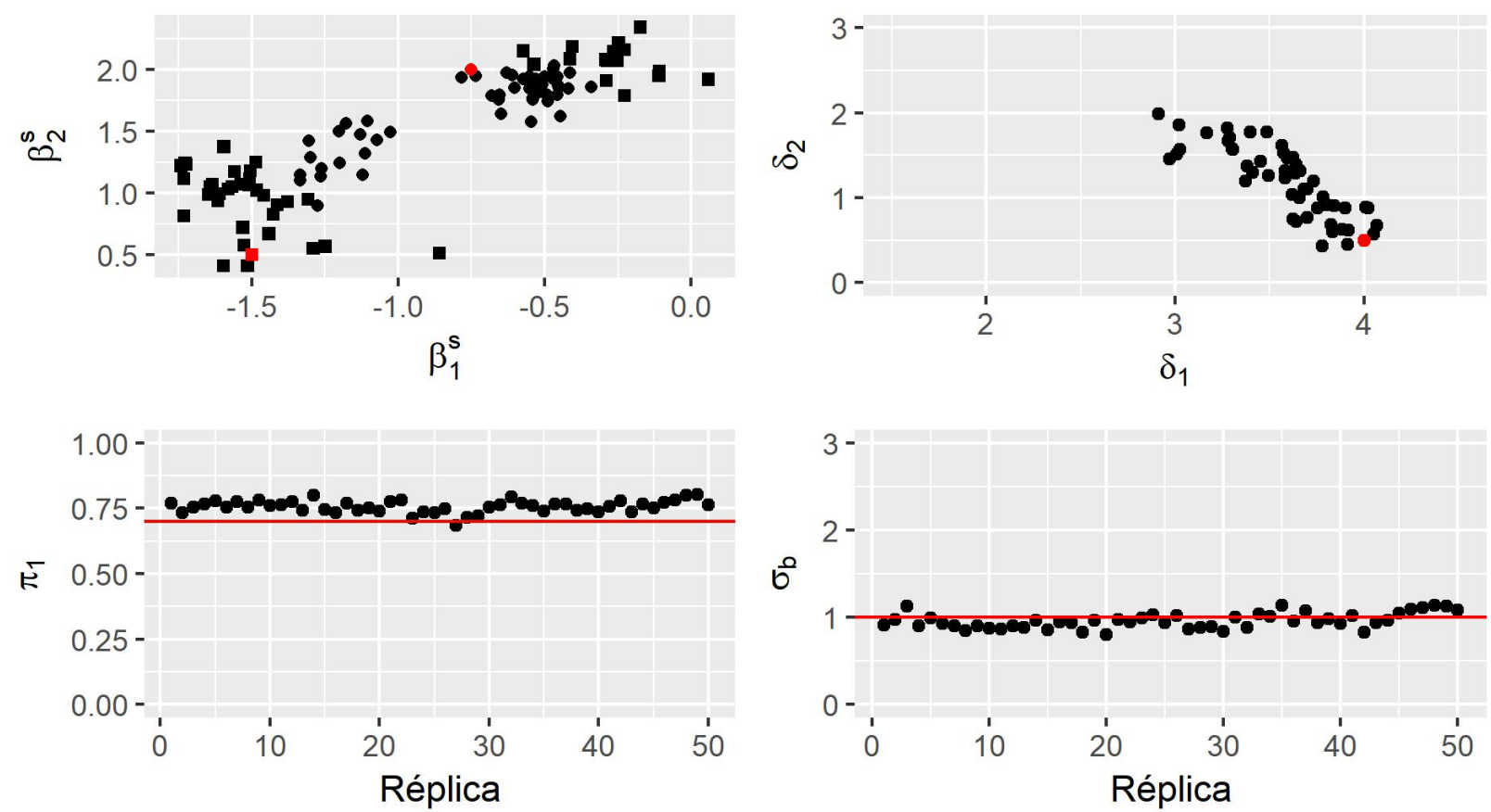

Figura B.68: Gráfico de dispersão das estimativas pontuais - mediana a posteriori - dos estudos de simulação de $R=50$ bancos de dados do modelo de mistura beta linear com efeitos aleatórios para a média e precisão com respectivos cenários para os parâmetros fixos: 1 e 2, em que $s=1(\bullet), 2(\mathbf{\bullet})$, e dos verdadeiros valores dos parâmetros (em vermelho).
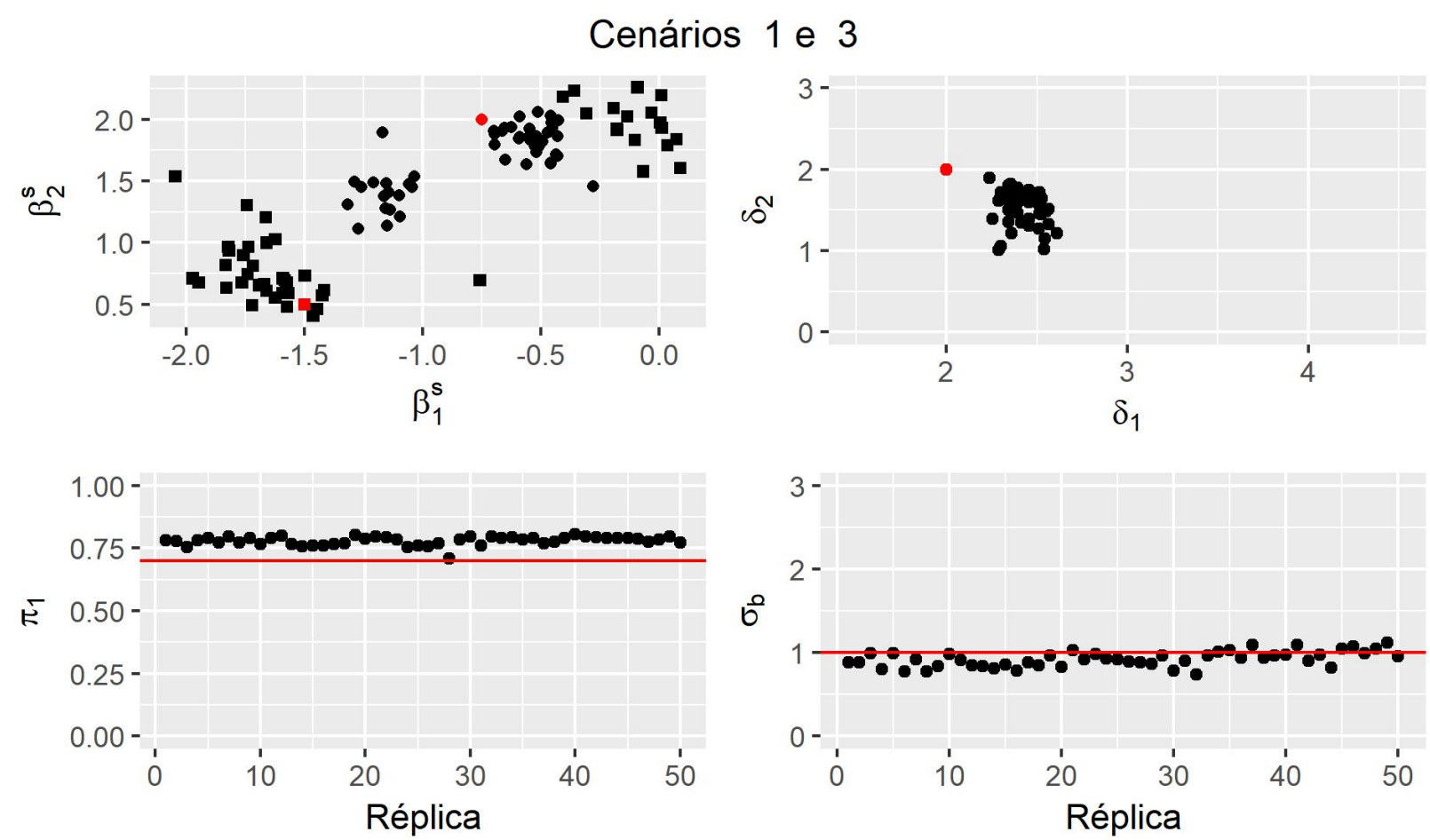

Figura B.69: Gráfico de dispersão das estimativas pontuais - mediana a posteriori - dos estudos de simulação de $R=50$ bancos de dados do modelo de mistura beta linear com efeitos aleatórios para a média e precisão com respectivos cenários para os parâmetros fixos: 1 e 3, em que $s=1(\bullet), 2(\square)$, e dos verdadeiros valores dos parâmetros (em vermelho). 


\section{Cenários 2 e 1}
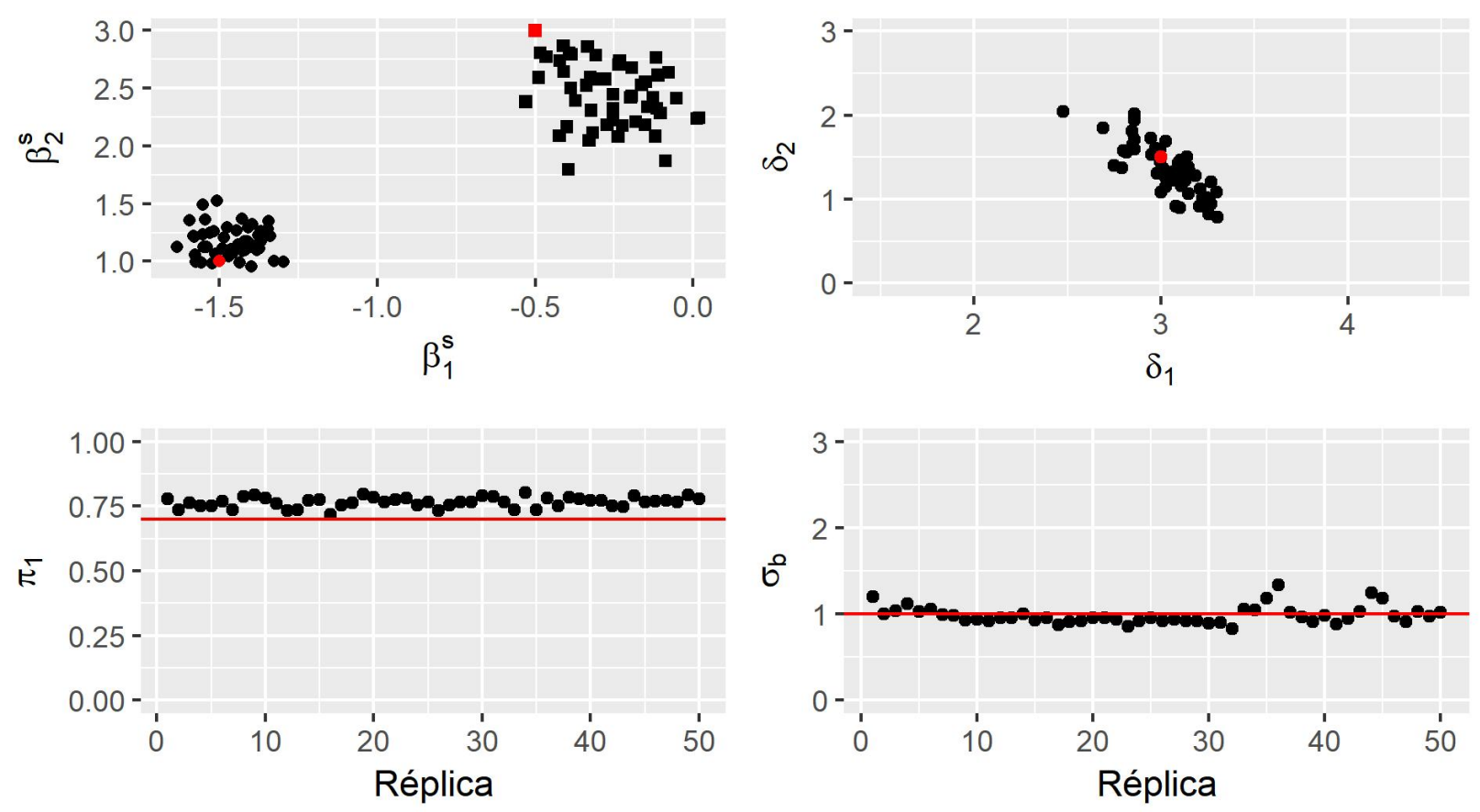

Figura B.70: Gráfico de dispersão das estimativas pontuais - mediana a posteriori - dos estudos de simulação de $R=50$ bancos de dados do modelo de mistura beta linear com efeitos aleatórios para a média e precisão com respectivos cenários para os parâmetros fixos: 2 e 1, em que $s=1(\bullet), 2(\mathbf{\square})$, e dos verdadeiros valores dos parâmetros (em vermelho).

Cenários 2 e 2
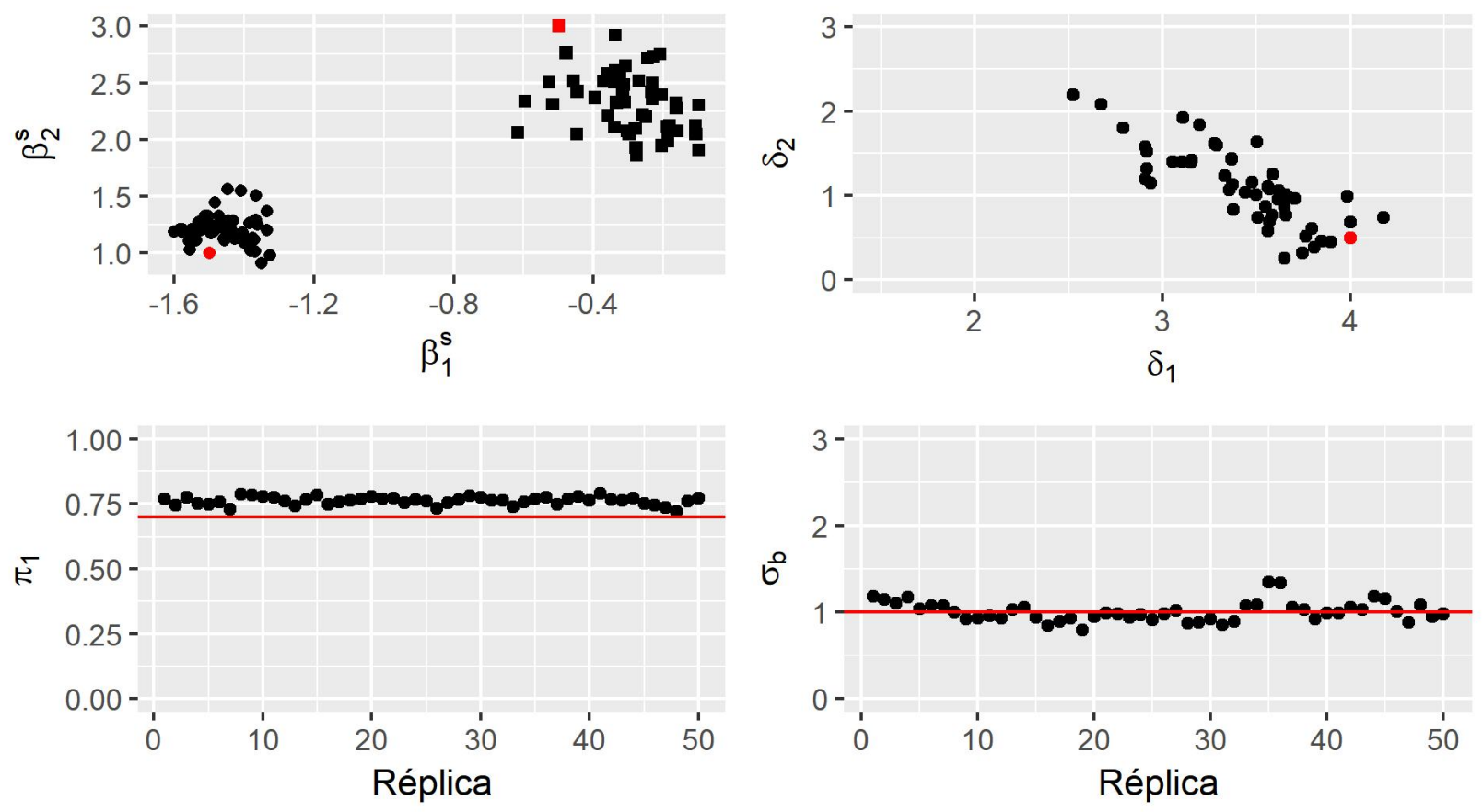

Figura B.71: Gráfico de dispersão das estimativas pontuais - mediana a posteriori - dos estudos de simulação de $R=50$ bancos de dados do modelo de mistura beta linear com efeitos aleatórios para a média e precisão com respectivos cenários para os parâmetros fixos: 2 e 2, em que $s=1(\bullet), 2(\mathbf{\bullet})$, e dos verdadeiros valores dos parâmetros (em vermelho). 


\section{Cenários 2 e 3}
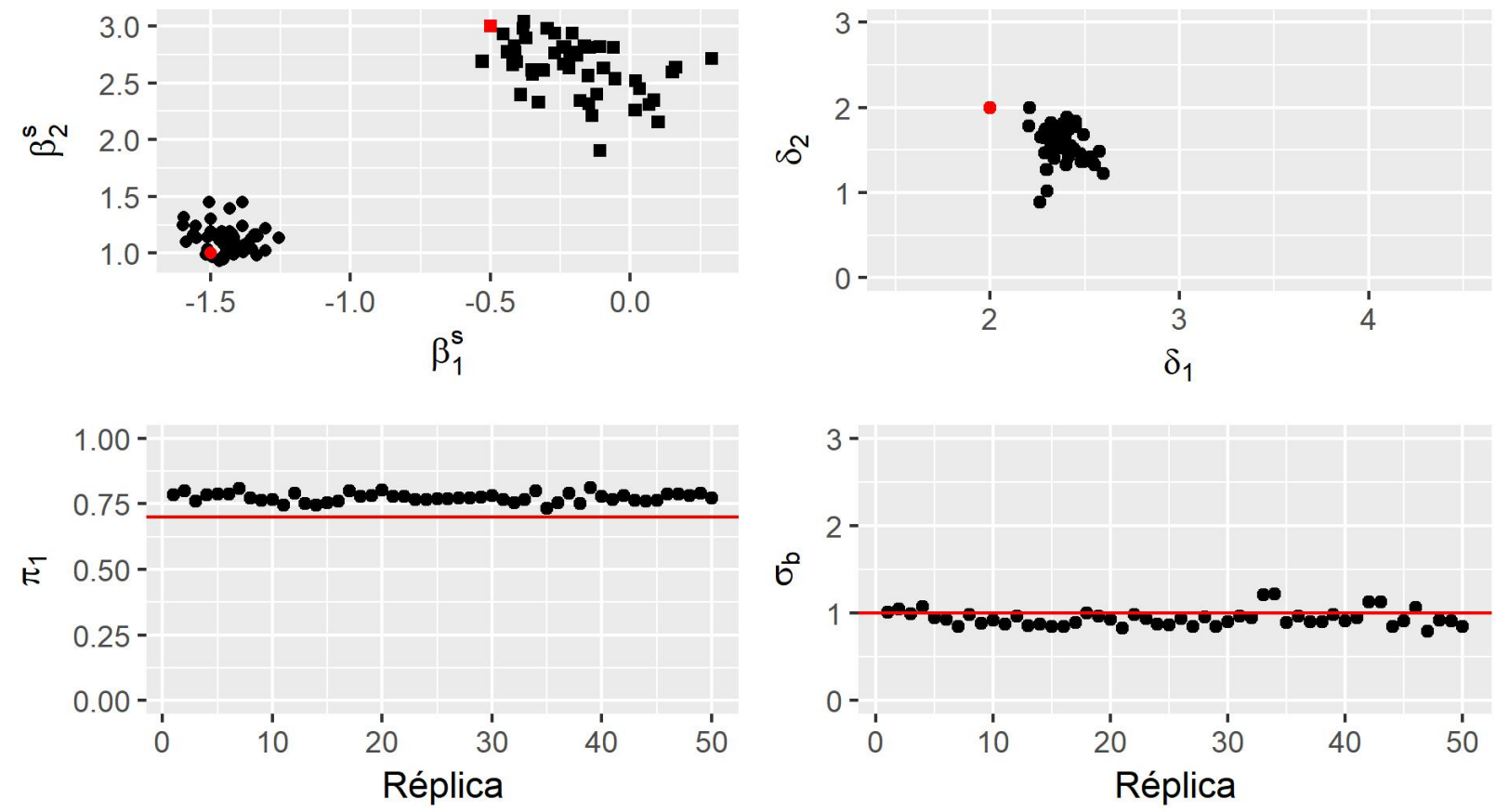

Figura B.72: Gráfico de dispersão das estimativas pontuais - mediana a posteriori - dos estudos de simulação de $R=50$ bancos de dados do modelo de mistura beta linear com efeitos aleatórios para a média e precisão com respectivos cenários para os parâmetros fixos: 2 e 3, em que $s=1(\bullet), 2(\mathbf{\bullet})$, e dos verdadeiros valores dos parâmetros (em vermelho).
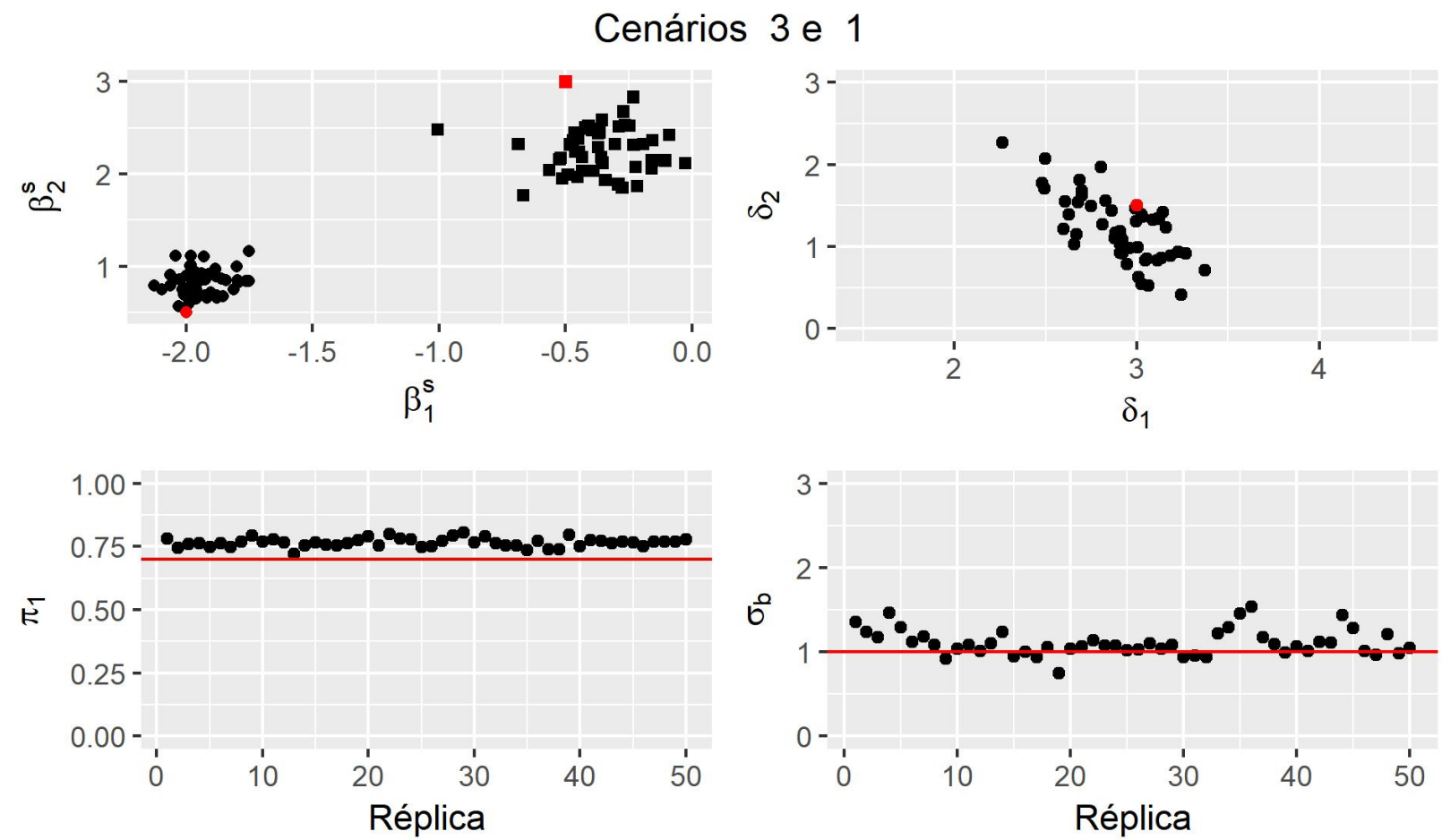

Figura B.73: Gráfico de dispersão das estimativas pontuais - mediana a posteriori - dos estudos de simulação de $R=50$ bancos de dados do modelo de mistura beta linear com efeitos aleatórios para a média e precisão com respectivos cenários para os parâmetros fixos: 3 e 1, em que $s=1(\bullet), 2(\square)$, e dos verdadeiros valores dos parâmetros (em vermelho). 


\section{Cenários 3 e 2}
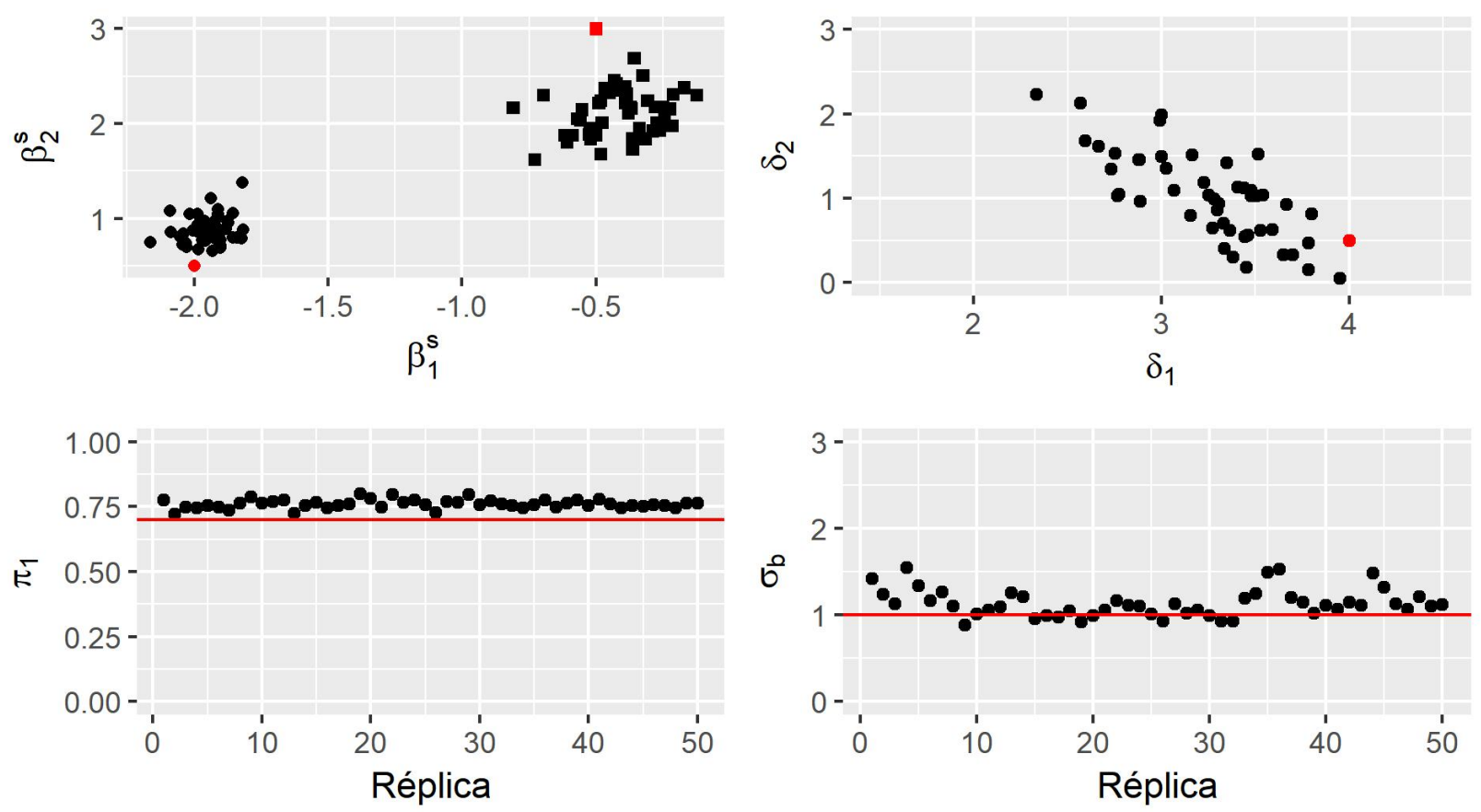

Figura B.74: Gráfico de dispersão das estimativas pontuais - mediana a posteriori - dos estudos de simulação de $R=50$ bancos de dados do modelo de mistura beta linear com efeitos aleatórios para a média e precisão com respectivos cenários para os parâmetros fixos: 3 e 2, em que $s=1(\bullet), 2(\mathbf{\square})$, e dos verdadeiros valores dos parâmetros (em vermelho).
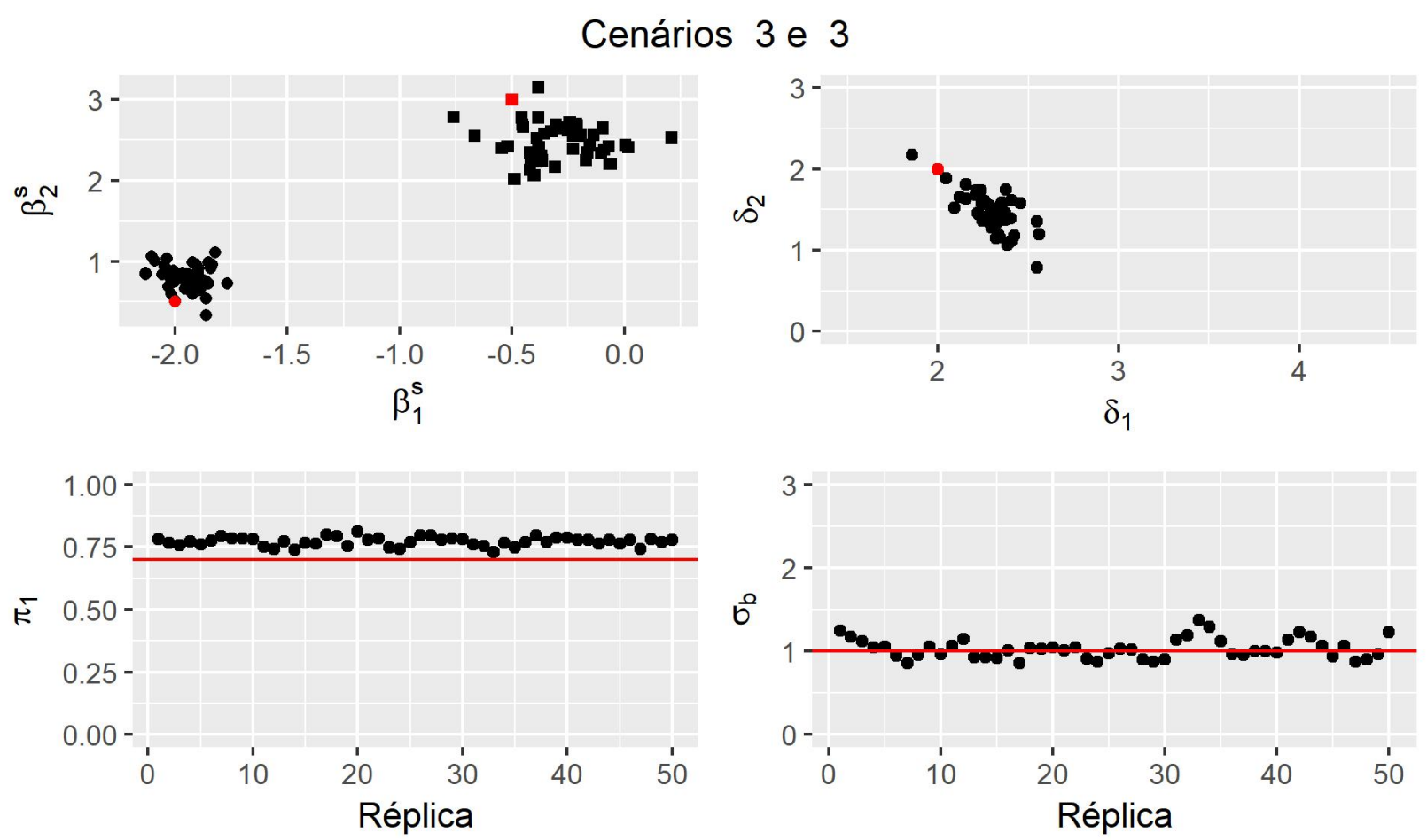

Figura B.75: Gráfico de dispersão das estimativas pontuais - mediana a posteriori - dos estudos de simulação de $R=50$ bancos de dados do modelo de mistura beta linear com efeitos aleatórios para a média e precisão com respectivos cenários para os parâmetros fixos: 3 e 3 , em que $s=1(\bullet), 2(\mathbf{\bullet})$, e dos verdadeiros valores dos parâmetros (em vermelho). 

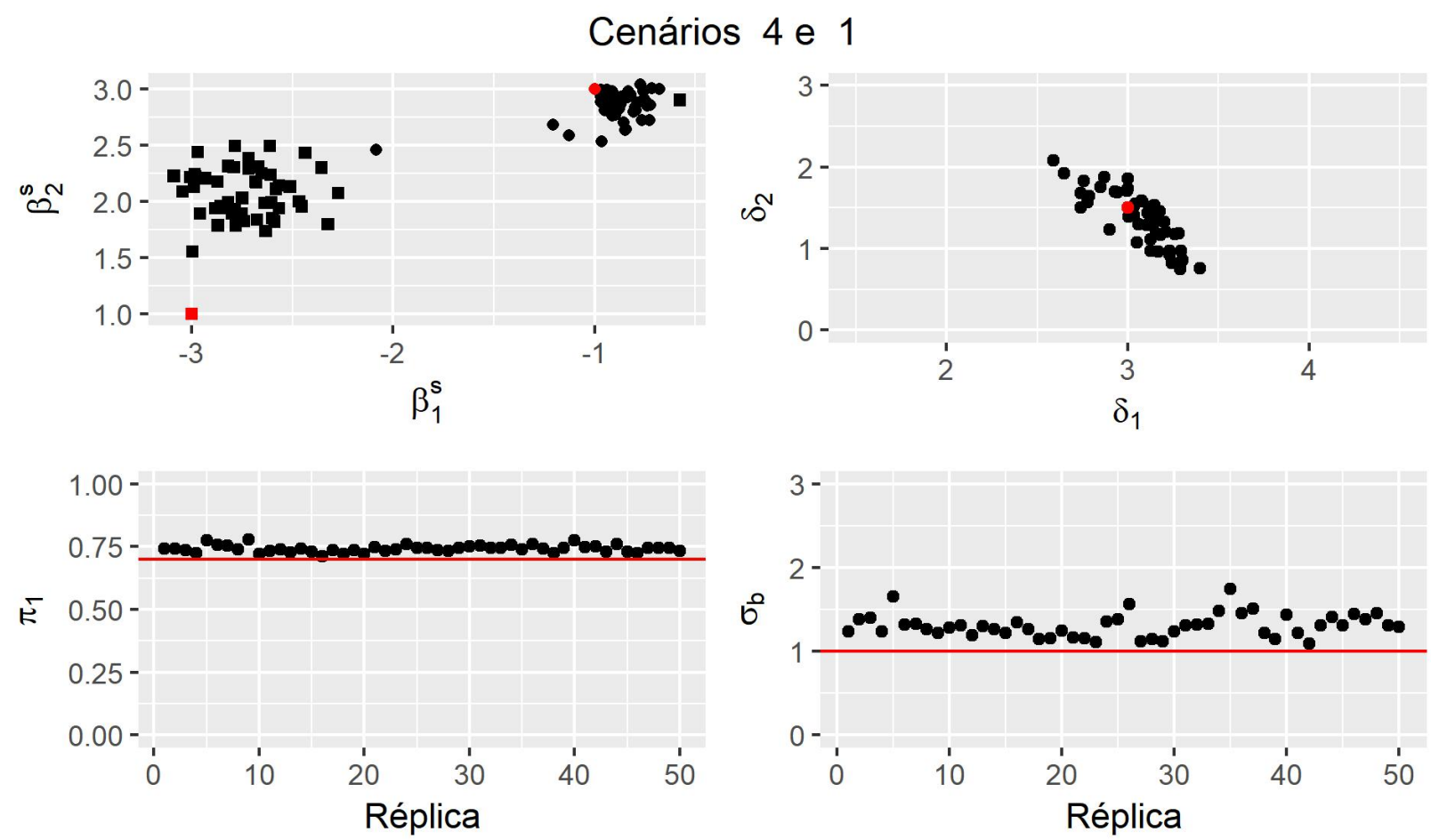

Figura B.76: Gráfico de dispersão das estimativas pontuais - mediana a posteriori - dos estudos de simulação de $R=50$ bancos de dados do modelo de mistura beta linear com efeitos aleatórios para a média e precisão com respectivos cenários para os parâmetros fixos: 4 e 1 , em que $s=1(\bullet), 2(\mathbf{\bullet})$, e dos verdadeiros valores dos parâmetros (em vermelho).

\section{Cenários 4 e 2}
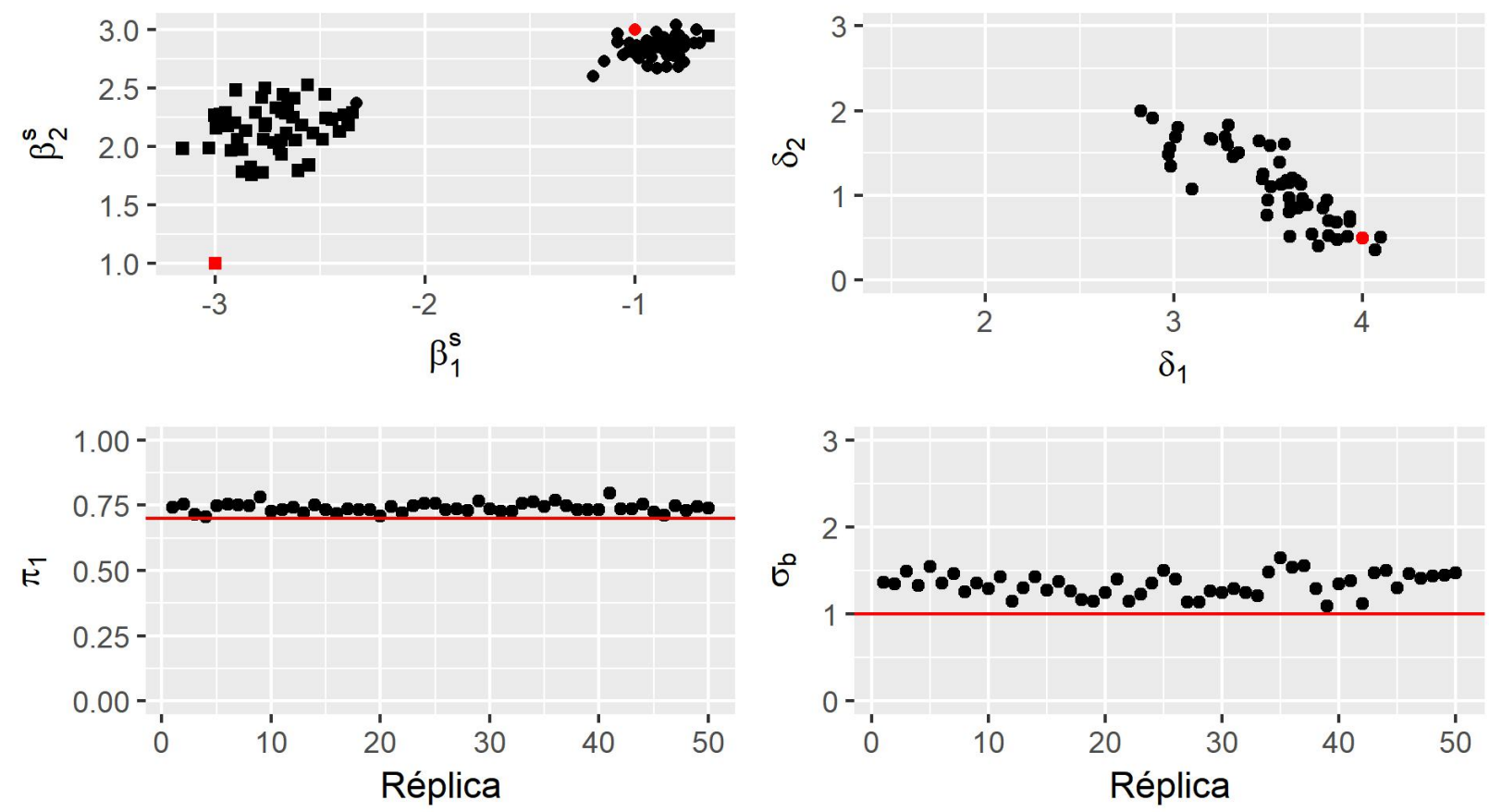

Figura B.77: Gráfico de dispersão das estimativas pontuais - mediana a posteriori - dos estudos de simulação de $R=50$ bancos de dados do modelo de mistura beta linear com efeitos aleatórios para a média e precisão com respectivos cenários para os parâmetros fixos: 4 e 2, em que $s=1(\bullet), 2(\bullet)$, e dos verdadeiros valores dos parâmetros (em vermelho). 
Cenários 4 e 3
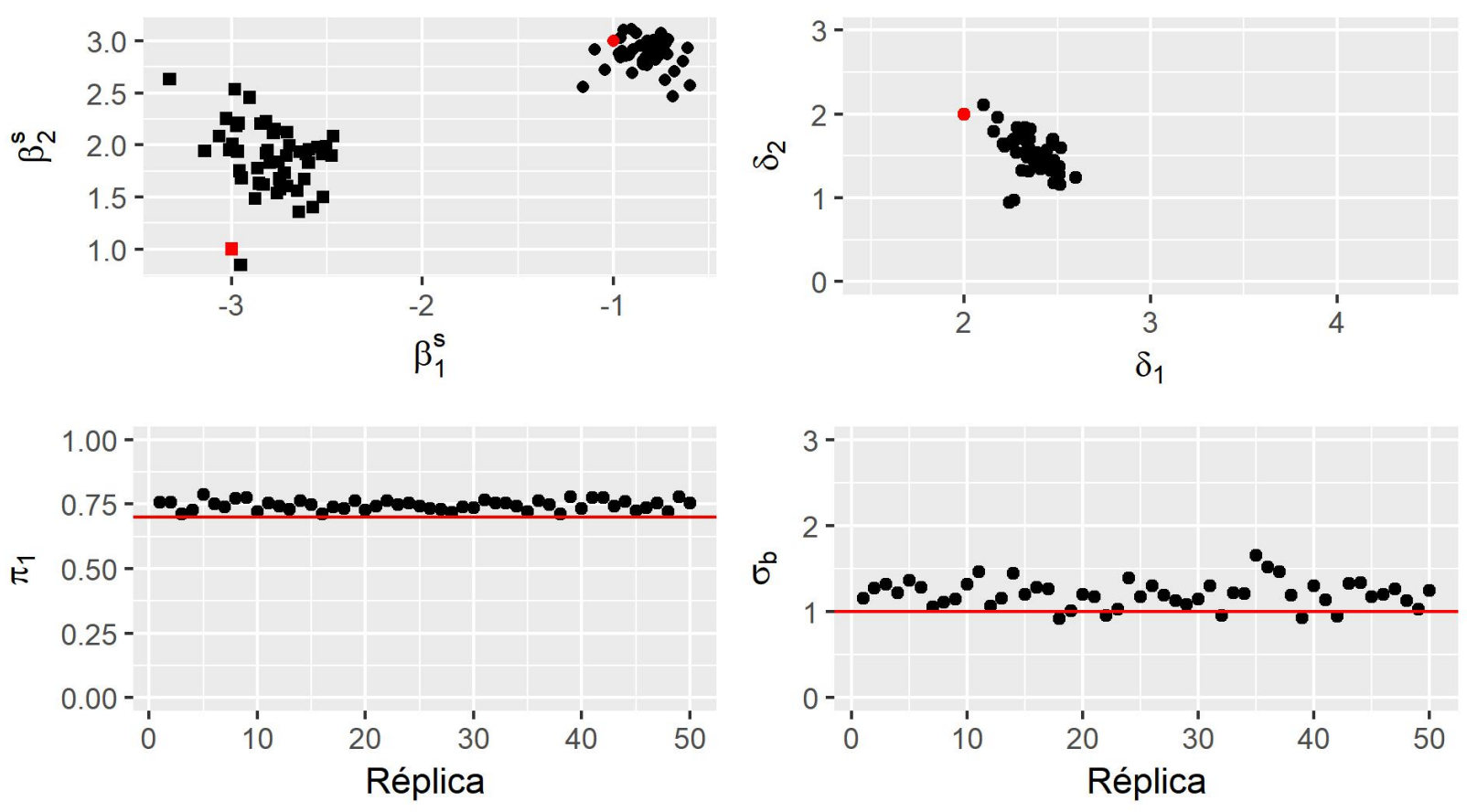

Figura B.78: Gráfico de dispersão das estimativas pontuais - mediana a posteriori - dos estudos de simulação de $R=50$ bancos de dados do modelo de mistura beta linear com efeitos aleatórios para a média e precisão com respectivos cenários para os parâmetros fixos: 4 e 3, em que $s=1(\bullet), 2(\mathbf{\square})$, e dos verdadeiros valores dos parâmetros (em vermelho). 


\section{B.8 Modelo não linear com parâmetro de precisão constante}
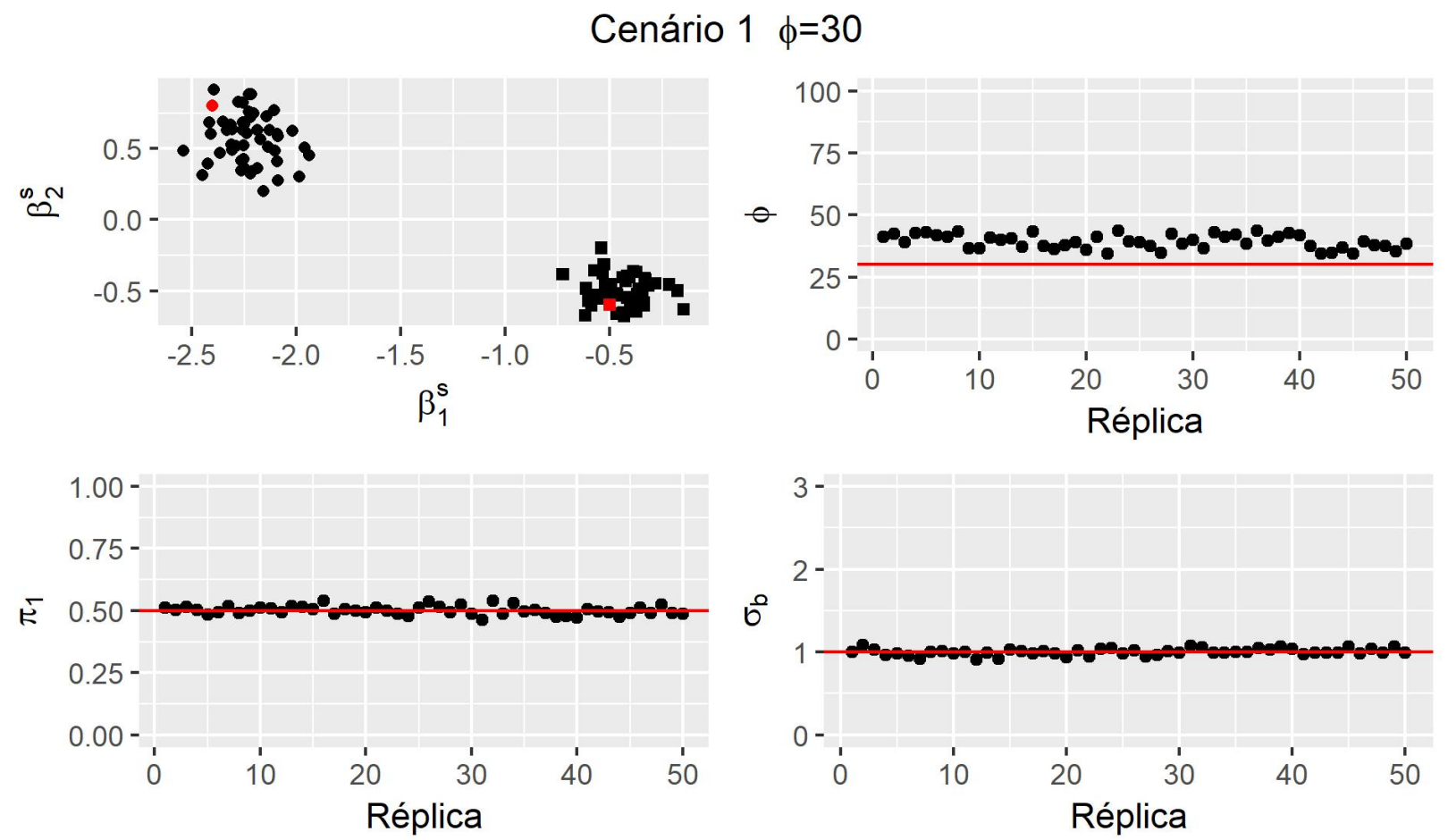

Figura B.79: Gráfico de dispersão das estimativas pontuais - mediana a posteriori - dos estudos de simulação de $R=50$ bancos de dados do modelo de mistura beta não linear com efeitos aleatórios para a média com cenário $1, \phi=30, N=100$ e $n=5$, em que $s=1(\bullet), 2(\mathbf{\bullet})$, e dos verdadeiros valores dos parâmetros (em vermelho). 


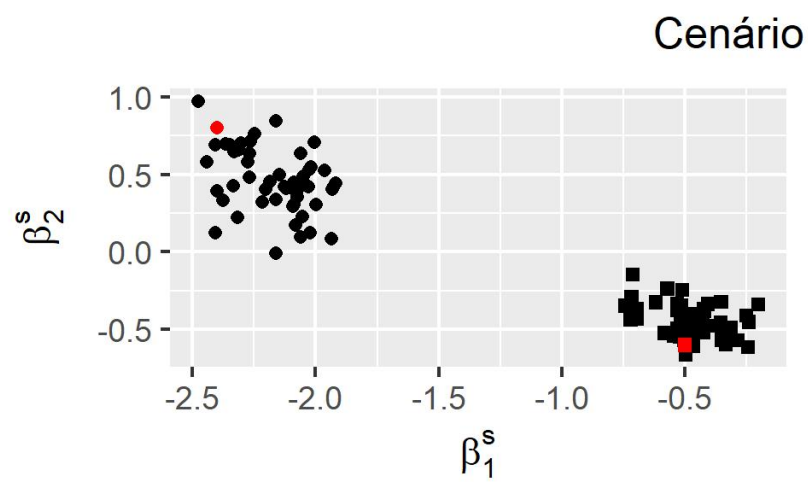

$1 \phi=50$
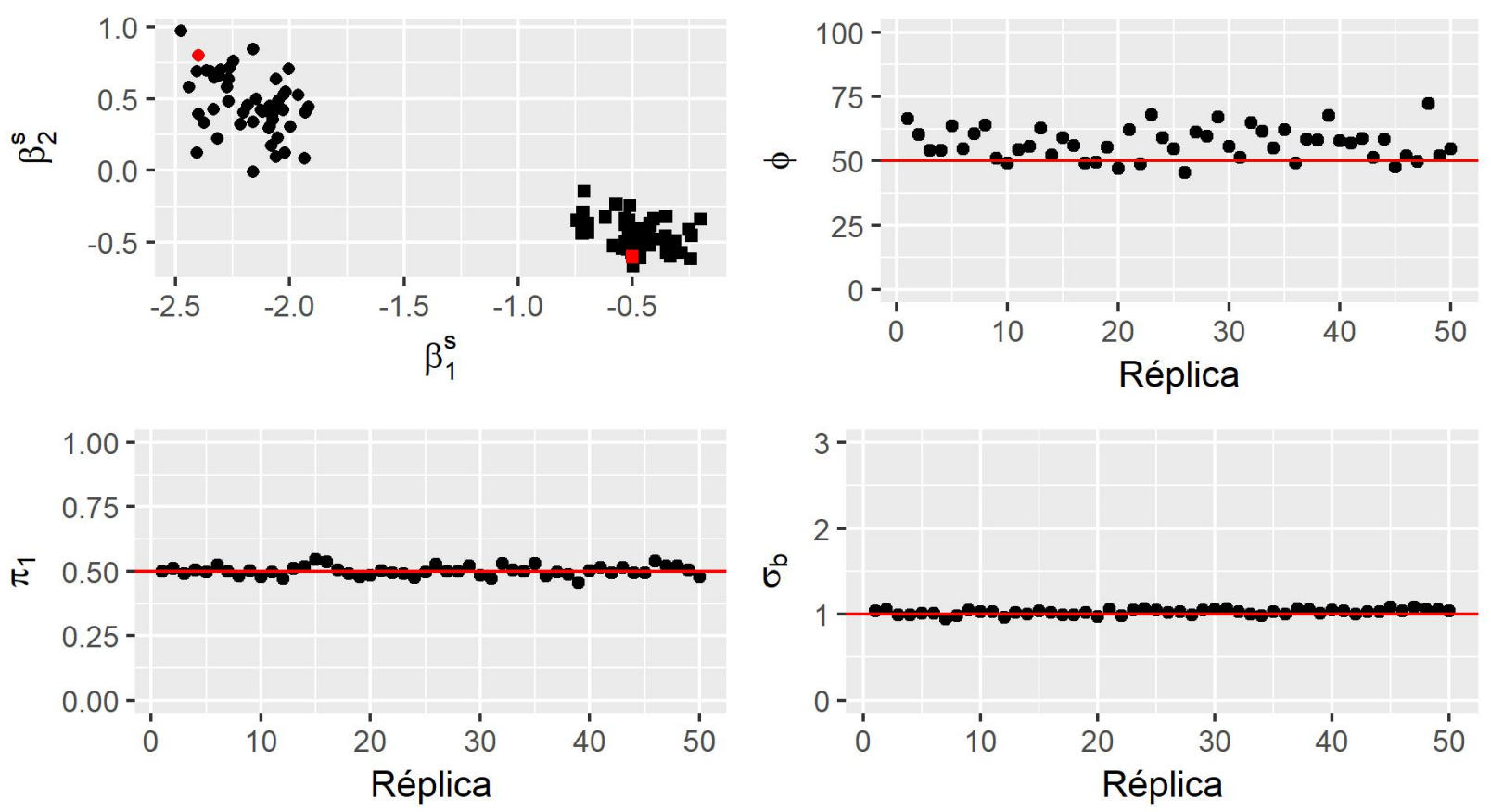

Figura B.80: Gráfico de dispersão das estimativas pontuais - mediana a posteriori - dos estudos de simulação de $R=50$ bancos de dados do modelo de mistura beta não linear com efeitos aleatórios para a média com cenário $1, \phi=50, N=100$ e $n=5$, em que $s=1(\bullet), 2(\mathbf{\bullet})$, e dos verdadeiros valores dos parâmetros (em vermelho).

\section{Cenário $1 \phi=70$}
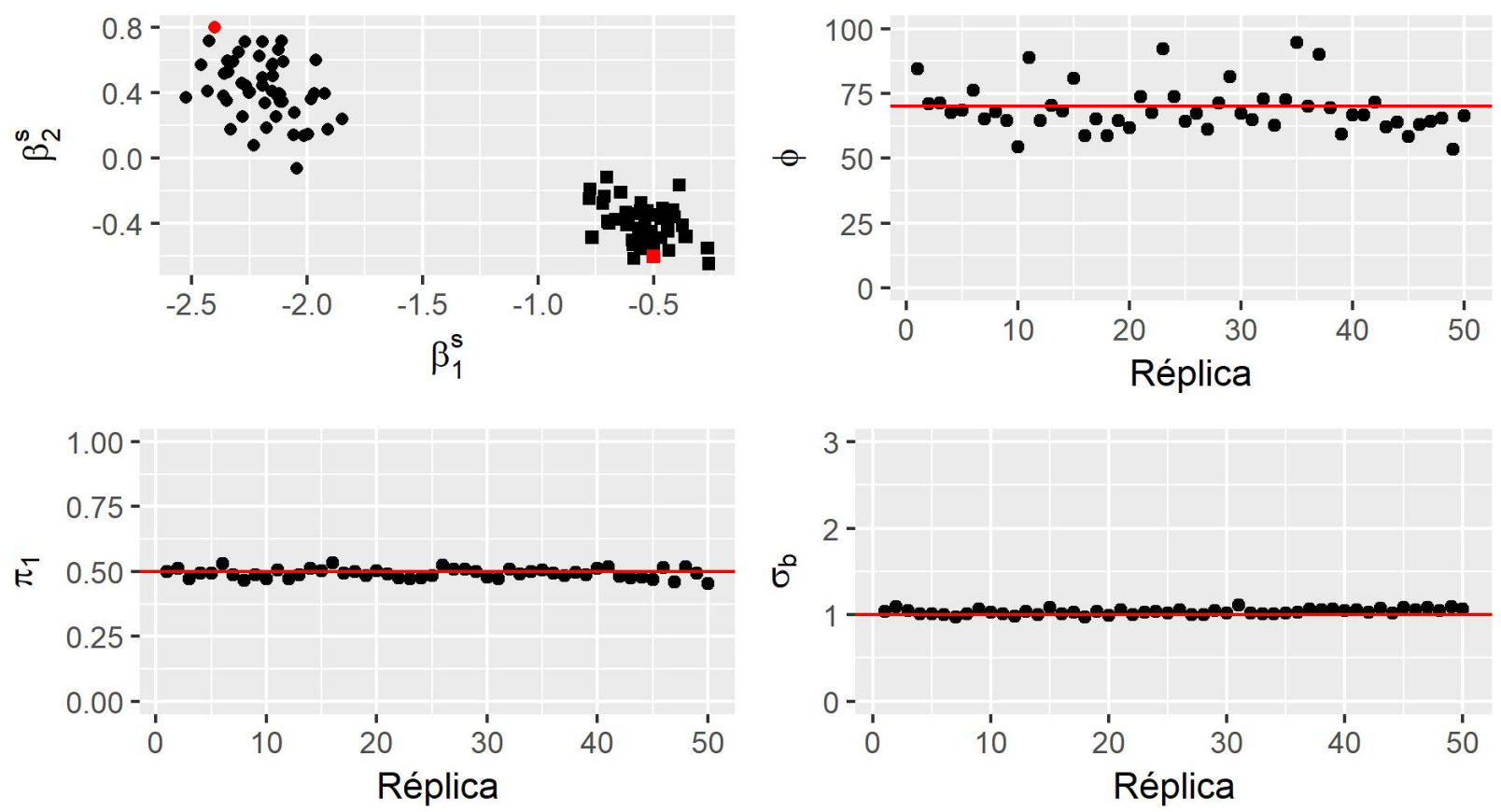

Figura B.81: Gráfico de dispersão das estimativas pontuais - mediana a posteriori - dos estudos de simulação de $R=50$ bancos de dados do modelo de mistura beta não linear com efeitos aleatórios para a média com cenário $1, \phi=70, N=100$ e $n=5$, em que $s=1(\bullet), 2(\mathbf{\bullet})$, e dos verdadeiros valores dos parâmetros (em vermelho). 

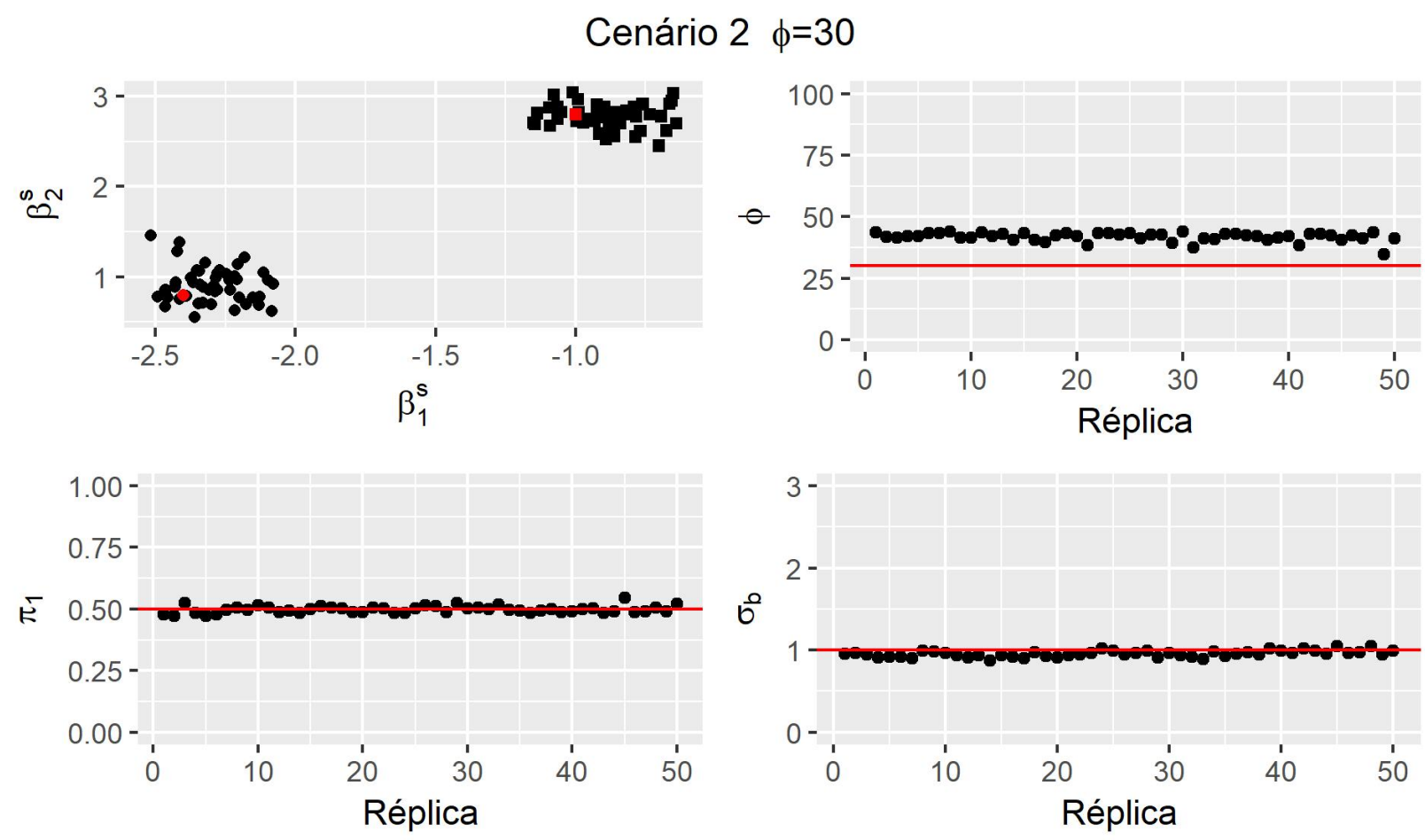

Figura B.82: Gráfico de dispersão das estimativas pontuais - mediana a posteriori - dos estudos de simulação de $R=50$ bancos de dados do modelo de mistura beta não linear com efeitos aleatórios para a média com cenário $2, \phi=30, N=100$ e $n=5$, em que $s=1(\bullet), 2(\mathbf{\bullet})$, e dos verdadeiros valores dos parâmetros (em vermelho).

\section{Cenário $2 \phi=50$}
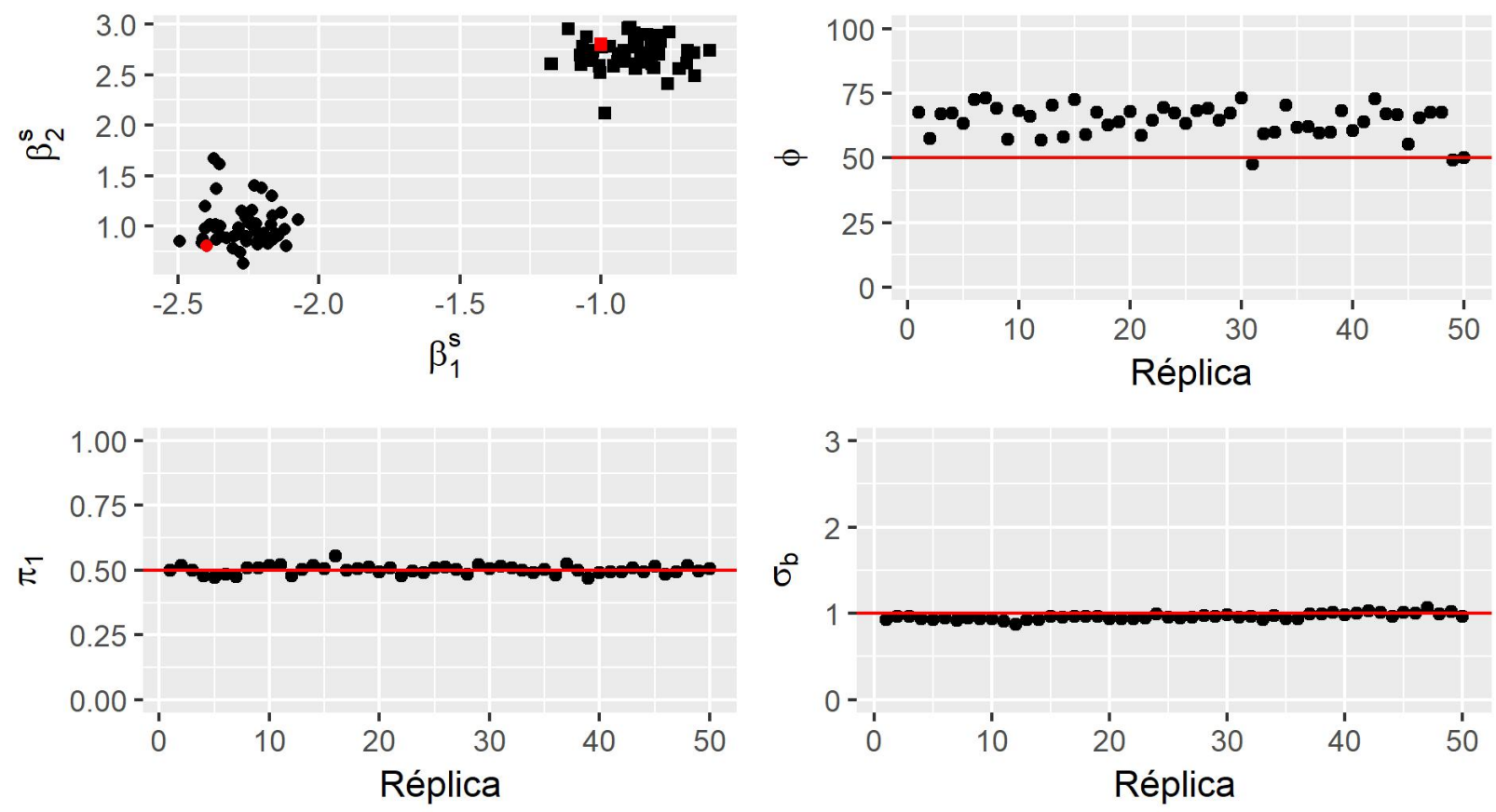

Figura B.83: Gráfico de dispersão das estimativas pontuais - mediana a posteriori - dos estudos de simulação de $R=50$ bancos de dados do modelo de mistura beta não linear com efeitos aleatórios para a média com cenário $2, \phi=50, N=100$ e $n=5$, em que $s=1(\bullet), 2(\mathbf{\bullet})$, e dos verdadeiros valores dos parâmetros (em vermelho). 

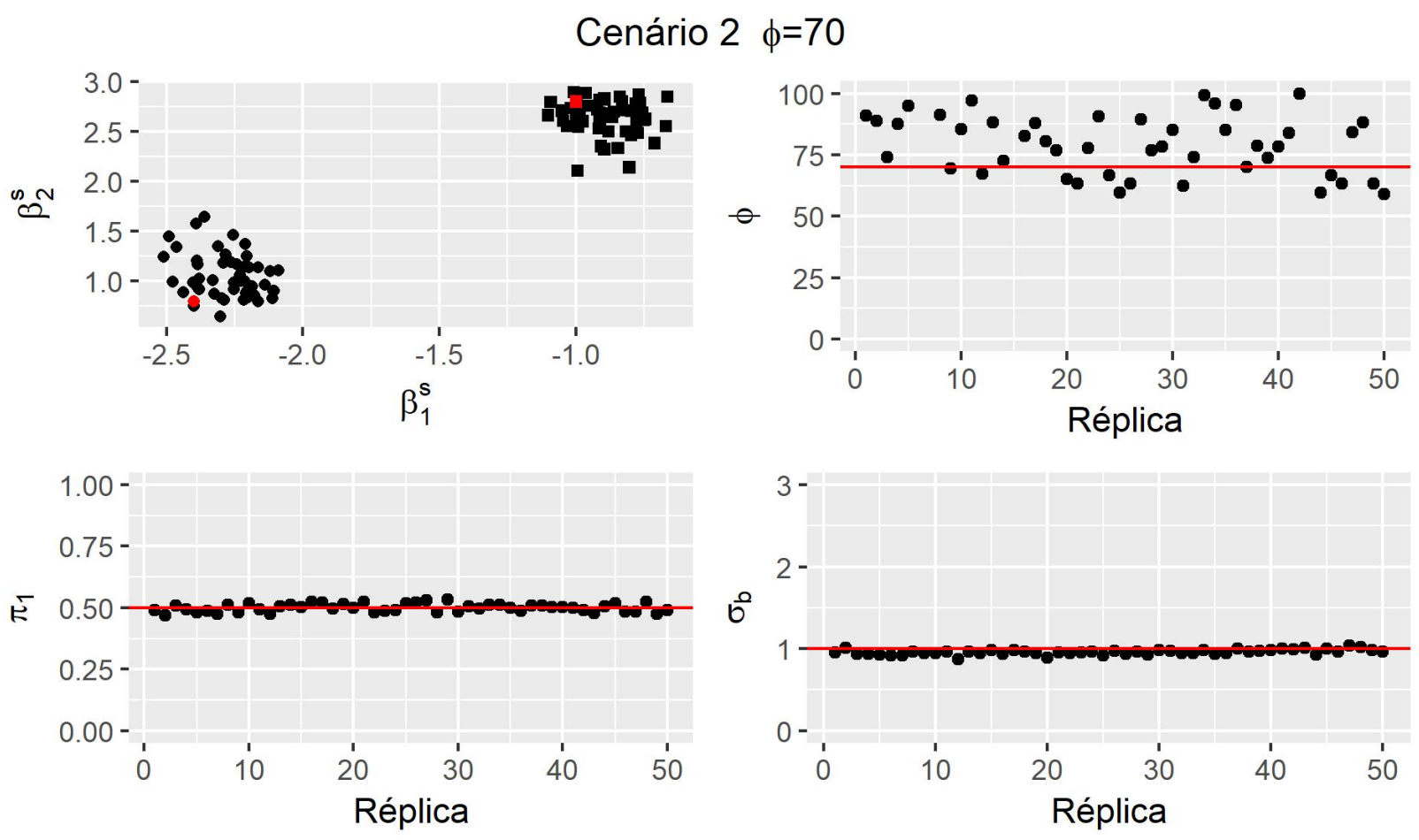

Figura B.84: Gráfico de dispersão das estimativas pontuais - mediana a posteriori - dos estudos de simulação de $R=50$ bancos de dados do modelo de mistura beta não linear com efeitos aleatórios para a média com cenário 2, $\phi=70, N=100$ e $n=5$, em que $s=1(\bullet), 2(\mathbf{\bullet})$.

\section{Cenário 3}
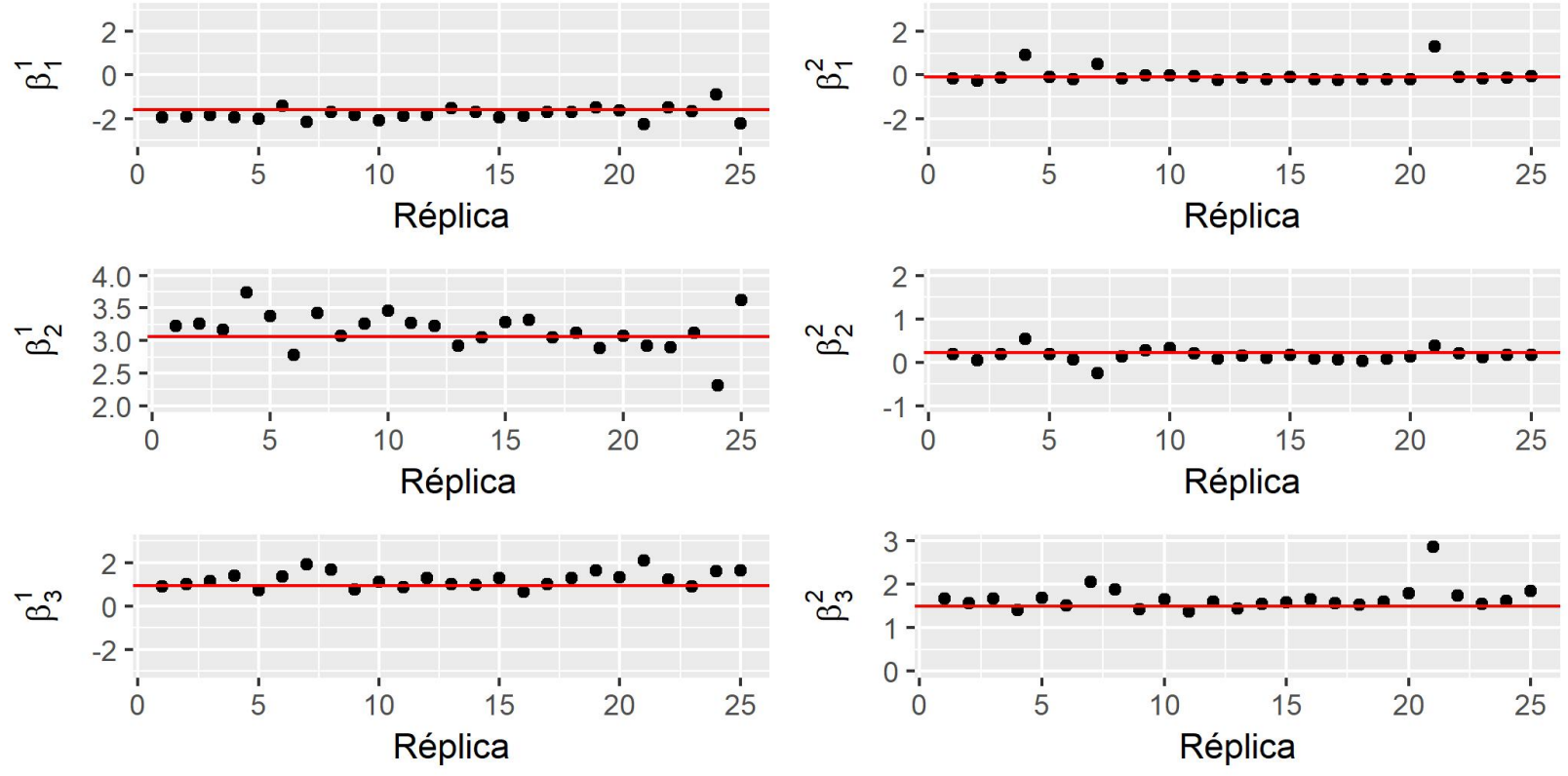

Figura B.85: Gráfico de dispersão das estimativas pontuais - mediana a posteriori - dos parâmetros $\beta_{i_{p}}^{s}, i_{p}=$ $1,2,3 s=1,2$, dos estudos de simulação de $R=25$ bancos de dados do modelo de mistura beta não linear com efeitos aleatórios para a média com cenário 3. 


\section{Cenário 3}
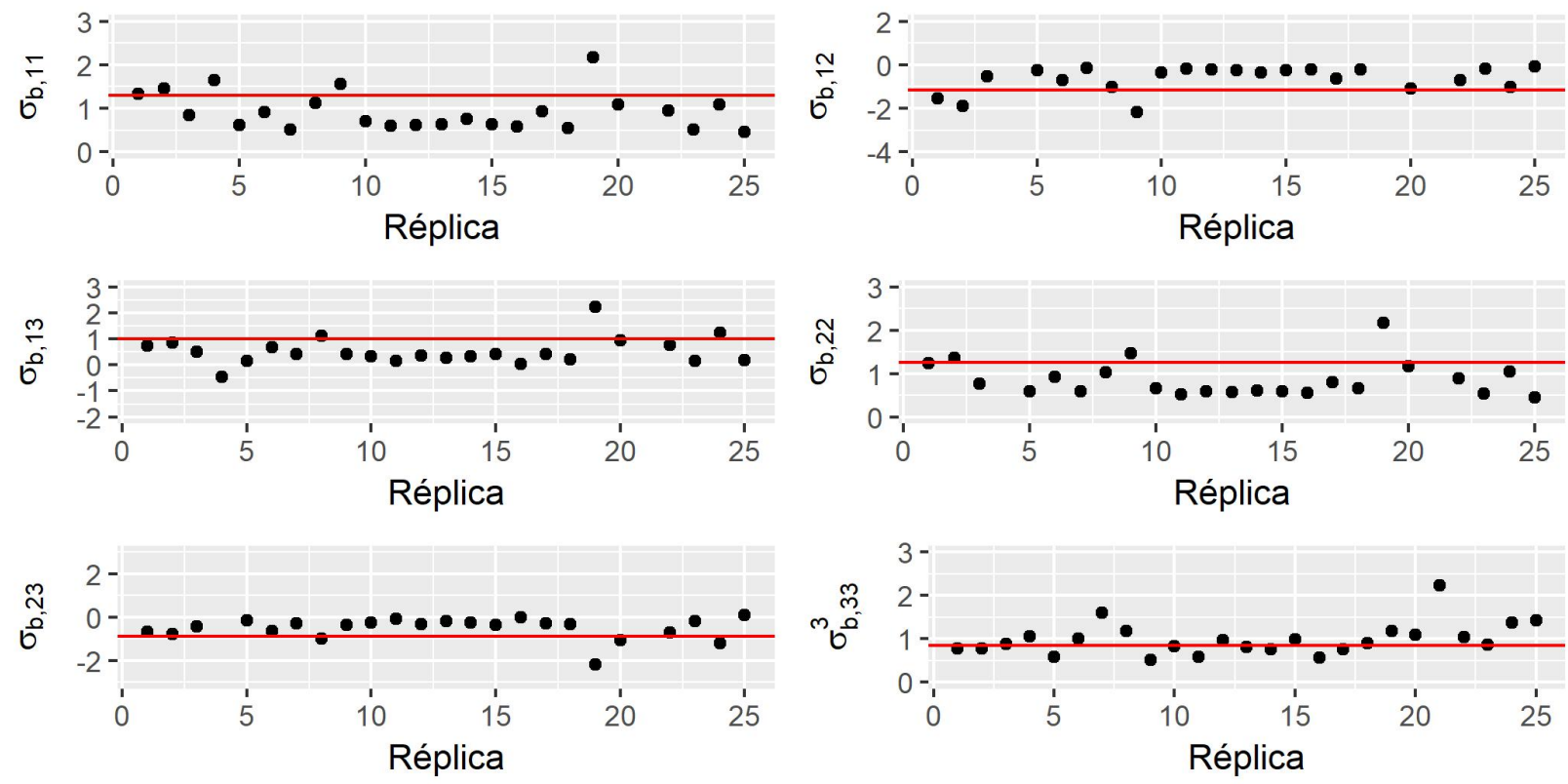

Figura B.86: Gráfico de dispersão das estimativas pontuais - mediana a posteriori - dos parâmetros $\sigma_{b, i_{d 1} i_{d 2}}, i_{d 1}, i_{d 2} \in\{1,2,3\}$ dos estudos de simulação de $R=25$ bancos de dados do modelo de mistura beta não linear com efeitos aleatórios para a média com cenário 3.

\section{Cenário 3}
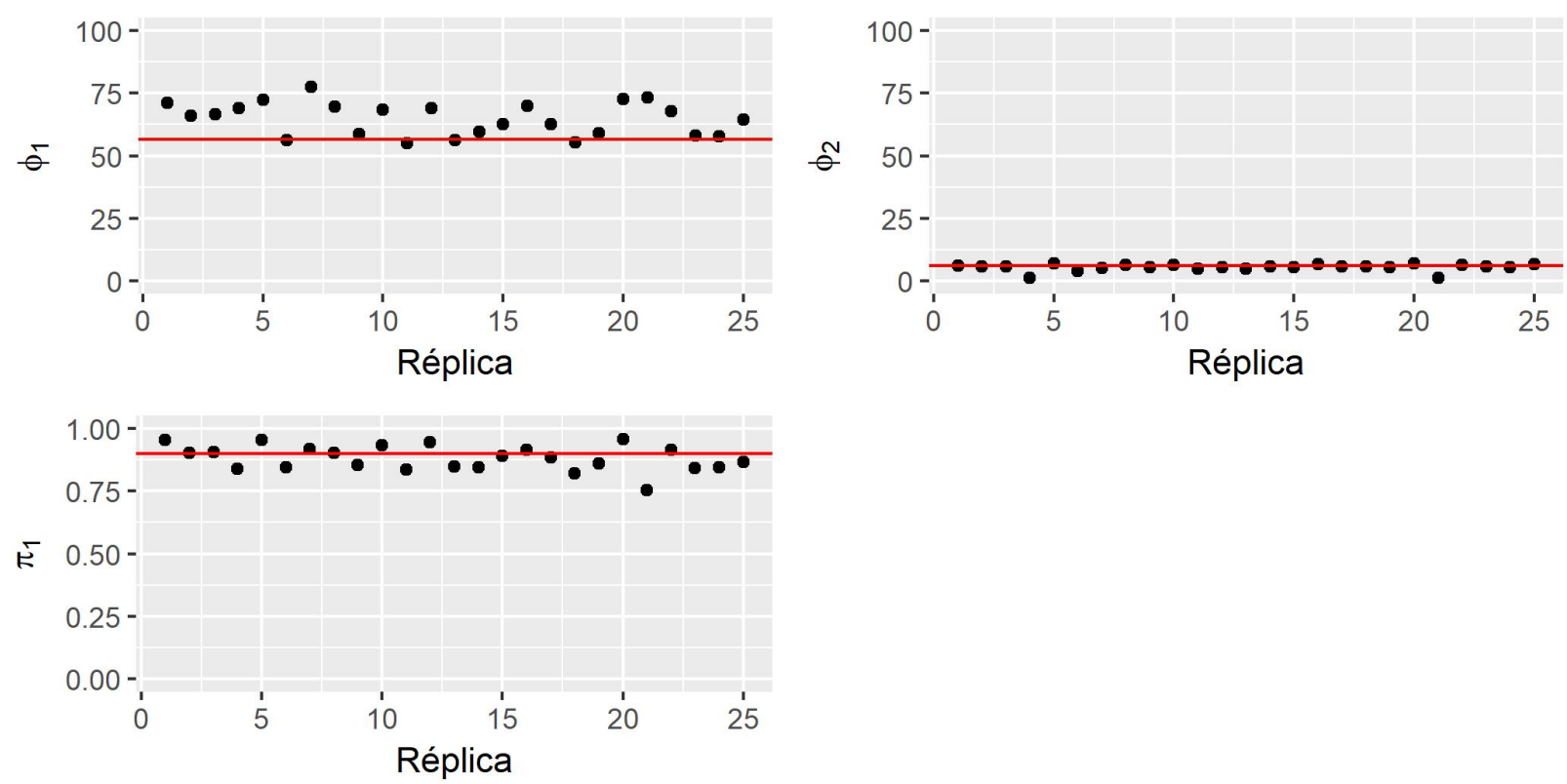

Figura B.87: Gráfico de dispersão das estimativas pontuais - mediana a posteriori - dos parâmetros $\phi_{1}$, $\phi_{2}$ e $\pi_{1}$ dos estudos de simulação de $R=25$ bancos de dados do modelo de mistura beta não linear com efeitos aleatórios para a média com cenário 3. 


\section{Referências Bibliográficas}

Adhikari et al. (2016) P.R. Adhikari, A. Vavpetič, J. Kralj, N. Lavrač e J. Hollmén. Explaining mixture models through semantic pattern mining and banded matrix visualization. Machine Learning, 105(1):3-39. Citado na pág. 28

Aitkin e Rubin (1985) M. Aitkin e D.B. Rubin. Estimation and hypothesis testing in finite mixture models. Journal of the Royal Statistical Society. Series B, 47:67-75. Citado na pág. 23

Albert (2009) J. Albert. Bayesian computation with R. Springer Science \& Business Media. Citado na pág. 5

Anuradha e Satyanarayana (2017) S. Anuradha e Ch. Satyanarayana. A methodology for tumor identification from mri images using beta mixture models and noise elimination filters. International Journal of Computer $\& 3$ Mathematical Sciences, 6(9):37-40. Citado na pág. 28

Astuti et al. (2016) A.B. Astuti, N. Iriawan, H.K. Irhamah e L. Sasiarini. Blood sugar levels of diabetes mellitus patients modeling with bayesian mixture model averaging. Global Journal of Pure and Applied Mathematics, 12(4):3143-3158. Citado na pág. 27

Belin e Rubin (1995) T.R. Belin e D.B. Rubin. The analysis of repeated-measures data on schizophrenic reaction times using mixture models. Statistics in Medicine, 14(8):747-768. Citado na pág. 32

Bell et al. (2016) A. Bell, M. Fairbrother e K. Jones. Fixed and random effects: making an informed choice. Trabalho em andamento, 2016. Citado na pág. 17

Bernardo e Smith (1994) J.M. Bernardo e A.F.M. Smith. Bayesian Theory. John Wiley \& Sons. Citado na pág. 5

Bonat et al. (2014) W.H. Bonat, P.J. Ribeiro Jr. e S.E. Shimakura. Bayesian analysis for a class of beta mixed models. arXiv preprint arXiv:1401.2957v2. Citado na pág. 18, 19

Bonat et al. (2015) W.H. Bonat, P.J. Ribeiro Jr e W.M. Zeviani. Likelihood analysis for a class of beta mixed models. Journal of Applied Statistics, 42(2):252-266. Citado na pág. 18

Bouguila et al. (2006) N. Bouguila, D. Ziou e E. Monga. Practical bayesian estimation of a finite beta mixture through gibbs sampling and its applications. Statistics and Computing, 16 (2):215-225. Citado na pág. 27, 28, 36

Brooks et al. (2011) S. Brooks, A. Gelman, G.L. Jones e X.L. Meng. Handbook of Markov Chain Monte Carlo. Chapman \& Hall/CRC. Citado na pág. 5, 9

Brooks (2002) S.P. Brooks. Discussion on the paper by spiegelhalter, best, carlin and van der linde (2002). Journal of the Royal Statistical Society: Series B, 64(3):616-618. Citado na pág. 12

Buhlin et al. (2003) K. Buhlin, A. Gustafsson, A.G. Pockley, J. Frostegard e B. Klinge. Risk factors for cardiovascular disease in patients with periodontitis. European Heart Journal, 24(23): 2099-2107. Citado na pág. 46 
Cairo et al. (2008) F. Cairo, S. Castellani, A.M. Gori, M. Nieri, G. Baldelli, R. Abbate e G.P. Pini-Prato. Severe periodontitis in young adults is associated with sub-clinical atherosclerosis. Journal of Clinical Periodontology, 35(6):465-472. Citado na pág. 46

Carlin e Louis (2000) B.P. Carlin e T.A. Louis. Bayes and empirical Bayes methods for data analysis. Boca Raton: Chapman \& Hall-CRC Press. Citado na pág. 7, 12

Carrasco et al. (2014) J.M.F. Carrasco, S.L.P. Ferrari e R.B. Arellano-Valle. Errors-in-variables beta regression models. Journal of Applied Statistics, 41(7):1530-1547. Citado na pág. 46

Carroll et al. (2015) R. Carroll, A.B. Lawson, C. Faes, R.S. Kirby, M. Aregay e K. Watjou. Comparing INLA and OpenBUGS for hierarchical Poisson modeling in disease mapping. Spatial and Spatio-Temporal Epidemiology, 14-15:45-54. Citado na pág. 19

Carroll (2003) R.J. Carroll. Variances are not always nuisance parameters. Biometrics, 59(2): 211-220. Citado na pág. 63

Casella e George (1992) G. Casella e E.I. George. Explaining the gibbs sampler. The American Statistician, 46(3):167-174. Citado na pág. 8

Celeux et al. (2006) G. Celeux, F. Forbes, C.P. Robert e D.M. Titterington. Deviance information criteria for missing data models. Bayesian Analysis, 1(4):651-674. Citado na pág. 11

Cepeda-Cuervo (2001) E. Cepeda-Cuervo. Modelagem da variabilidade em modelos lineares generalizados. Tese de Doutorado, Universidade Federal do Rio de Janeiro. Citado na pág. 16

Cepeda-Cuervo et al. (2016) E. Cepeda-Cuervo, D. Jaimes, M. Marín e J. Rojas. Bayesian beta regression with bayesianbetareg r-package. Computational Statistics, 31(1):165-187. Citado na pág. 16

Chen et al. (2000) M-H. Chen, Q-M. Shao e J.G. Ibrahim. Monte Carlo methods in Bayesian computation. New York: Springer Verlag. Citado na pág. 5

Cnaan et al. (1997) A. Cnaan, N.M. Laird e P. Slasor. Tutorial in biostatistics: using the general linear mixed model to analyse unbalanced repeated measures and longitudinal data. Statistics in Medicine, 16:2349-2380. Citado na pág. 48

Codd e Cudeck (2014) C.L. Codd e R. Cudeck. Nonlinear random-effects mixture models for repeated measures. Psychometrika, 79(1):60-83. Citado na pág. 82

Cribari-Neto e Souza (2012) F. Cribari-Neto e T.C. Souza. Testing inference in variable dispersion beta regressions. Journal of Statistical Computation and Simulation, 82(12):1827-1843. Citado na pág. 14

Das e Quirk (2016) K. Das e T. Quirk. Which institutions promote growth? revisiting the evidence. Economic Papers: A Journal of Applied Economics and Policy, 35(1):37-58. Citado na pág. 54

David Jr (1974) D.W. David Jr. Maximum likelihood estimates of the parameters of a mixture of two regression lines. Communications in Statistics, 3(10):995-1006. Citado na pág. 26

Davidian e Gallant (1992) M. Davidian e A.R. Gallant. Smooth nonparametric maximum likelihood estimation for population pharmacokinetics, with application to quinidine. Journal of Pharmacokinetics and Biopharmaceutics, 20(5):529-556. Citado na pág. 82

Davidian e Giltinan (1993) M. Davidian e D.M. Giltinan. Some general estimation methods for nonlinear mixed-effects model. Journal of Biopharmaceutical Statistics, 3(1):23-55. Citado na pág. 19,85 
DeIorio e Robert (2002) M. DeIorio e C.P. Robert. Discussion of spiegelhalter et al. Journal of the Royal Statistical Society, Series B, 64:629-630. Citado na pág. 11

DeSarbo e Cron (1988) Wayne S DeSarbo e William L Cron. A maximum likelihood methodology for clusterwise linear regression. Journal of Classification, 5(2):249-282. Citado na pág. 26

Diebolt e Robert (1990) J. Diebolt e C.P. Robert. Bayesian estimation of finite mixture distributions, part ii: Sampling implementation. Technical Report, LSTA, Universite Paris VI, 111. Citado na pág. 27

Diebolt e Robert (1994) J. Diebolt e C.P. Robert. Estimation of finite mixture distributions through bayesian sampling. Journal of the Royal Statistical Society. Series B (Methodological), 56(2):363-375. Citado na pág. 27

Dunn e Smyth (1996) P.K. Dunn e G.K. Smyth. Randomized quantile residuals. Journal of Computational and Graphical Statistics, 5(3):236-244. Citado na pág. 51, 78

Escobar e West (1995) M.D. Escobar e M. West. Bayesian density estimation and inference using mixtures. Journal of the American Statistical Association, 90(430):577-588. Citado na pág. 27

Espinheira et al. (2017a) P.L. Espinheira, L.C.M. da Silva, A.D.O. Silva e R. Ospina. Prediction measures in nonlinear beta regression models. arXiv preprint arXiv:1705.07941. Citado na pág. 82

Espinheira et al. (2017b) P.L. Espinheira, E.G. Santos e F. Cribari-Neto. On nonlinear beta regression residuals. Biometrical Journal, 59(3):445-461. Citado na pág. 82, 87

Evans et al. (1992) M. Evans, I. Guttman e I. Olkin. Numerical aspects in estimating the parameters of a mixture of normal distributions. Journal of Computational and Graphical Statistics, 1(4):351-365. Citado na pág. 27

Everitt e Hand (1981) B.S. Everitt e O.J. Hand. Finite mixture distributions, 1981. Citado na pág. 21

Feller (1943) W. Feller. On a general class of "contagious"distributions. The Annals of Mathematical Statistics, 14(4):389-400. Citado na pág. 21

Ferrari e Cribari-Neto (2004) S. Ferrari e F. Cribari-Neto. Beta regression for modelling rates and proportions. Journal of Applied Statistics, 31(7):799-815. Citado na pág. 13, 15

Ferrari e Pinheiro (2011) S.L.P. Ferrari e E.L. Pinheiro. Improved likelihood inference in beta regression. Journal of Statistical Computation and Simulation, 81(4):431-443. Citado na pág. 82

Figueroa-Zúñiga et al. (2013) J.I. Figueroa-Zúñiga, R.B. Arellano-Valle e S.L.P. Ferrari. Mixed beta regression: A bayesian perspective. Computational Statistics \& Data Analysis, 61(0):137147. Citado na pág. $16,17,18,19,39$

Frühwirth-Schnatter (2006) S. Frühwirth-Schnatter. Finite mixture and Markov switching models. New York: Springer. Citado na pág. 21, 23, 32, 36

Fu et al. (2011) R. Fu, D.K. Dey e K.E. Holsinger. A beta-mixture model for assessing genetic population structure. Biometrics, 67(3):1073-1082. Citado na pág. 25

Gamerman e Lopes (2006) D. Gamerman e H.F. Lopes. Markov Chain Monte Carlo: Stochastic Simulation for Bayesian Inference. Chapman \& Hall/CRC. Citado na pág. 5, 6, 8, 51

Geisser e Eddy (1979) S. Geisser e W.F. Eddy. A predictive approach to model selection. Journal of the American Statistical Association, 74(365):153-160. Citado na pág. 12 
Gelman (2006) A. Gelman. Prior distributions for variance parameters in hierarchical models. Bayesian Analysis, 1(3):515-534. Citado na pág. 18, 39, 70

Gelman (2009) A. Gelman. Bayes, jeffreys, prior distributions and the philosophy of statistics. Statistical Science, 24(2):176-178. Citado na pág. 7

Gelman e King (1990) A. Gelman e G. King. Estimating the electoral consequences of legislative redistricting. Journal of the American Statistical Association, 85(410):274-282. Citado na pág. 27

Gelman e Rubin (1992) A. Gelman e D.B. Rubin. Inference from iterative simulation using multiple sequences. Statistical Science, 7(4):457-472. Citado na pág. 38, 69

Gelman et al. (2013) A. Gelman, J.B. Carlin, H.S. Stern, D.B. Dunson, A. Vehtari e D.B. Rubin. Bayesian data analysis, volume 3. Chapman \& Hall/CRC. Citado na pág. 9, 13

Genolini et al. (2015) C. Genolini, X. Alacoque, M. Sentenac e C. Arnaud. kml and kml3d: R packages to cluster longitudinal data. Journal of Statistical Software, 65(4):1-34. Citado na pág. 39, $70,87,96$

Geweke (1992) J. Geweke. Evaluating the accuracy of sampling-based approaches to the calculation of posterior moments. Em Bayesian Statistics 4, páginas 169-193. Oxford University Press, Oxford. Citado na pág. 38, 69

Gheorghe et al. (2016) M. Gheorghe, S. Picavet, M. Verschuren, W.B.F. Brouwer e P.H.M. van Baal. Health losses at the end of life: a bayesian mixed beta regression approach. Journal of the Royal Statistical Society: Series A (Statistics in Society), 180(3):723-749. Citado na pág. 18

Giampaoli et al. (2016) V. Giampaoli, A.N. Ferraz Junior e I.A.C. Castro. Relatório de análise estatística sobre o projeto: Aglomeração das redes de fast food no brasil. Relatório técnico. Instituto de Matemática e Estatística, Universidade de São Paulo, Brasil (RAE-CEA-16P12). Citado na pág. 54

Glaeser et al. (2004) E.L. Glaeser, R. La Porta, F. Lopez de Silanes e A. Shleifer. Do institutions cause growth? Journal of Economic Growth, 9(3):271-303. Citado na pág. 54

Green (2017) P.J. Green. Introduction to finite mixtures. ArXiv e-prints. Citado na pág. 23

Grimm e Ram (2009) K.J. Grimm e N. Ram. Nonlinear growth models in Mplus and SAS. Structural Equation Modeling, 16(4):676-701. Citado na pág. 93, 95

Grimm et al. (2010) K.J. Grimm, N. Ram e R. Estabrook. Nonlinear structured growth mixture models in Mplus and OpenMx. Multivariate Behavioral Research, 45(6):887-909. Citado na pág. 82, 93, 94, 95, 96, 98

Grün e Leisch (2008) B. Grün e F. Leisch. Identifiability of finite mixtures of multinomial logit models with varying and fixed effects. Journal of Classification, 25(2):225-247. Citado na pág. 27

Hall e Wang (2005) D.B. Hall e L. Wang. Two-component mixtures of generalized linear mixed effects models for cluster correlated data. Statistical Modelling, 5(1):21-37. Citado na pág. 32

Harring (2012) J.R. Harring. Finite mixtures of nonlinear mixed effects models. Em J.R. Harring e G.R. Hancock, editors, Advances in longitudinal methods in the social and behavioral sciences, páginas 159-192. Charlotte, NC: Information Age. Citado na pág. 82

Harring e Liu (2016) J.R. Harring e J. Liu. A comparison of estimation methods for nonlinear mixed-effects models under model misspecification and data sparseness: A simulation study. Journal of Modern Applied Statistical Methods, 15(1):539-569. Citado na pág. 85 
Hastings (1970) W.K. Hastings. Monte carlo sampling methods using markov chains and their applications. Biometrika, 57(1):97-109. Citado na pág. 8

Heisterkamp e Pennings (2004) S.H. Heisterkamp e J.L.A. Pennings. The use of a finite mixture of beta distributions in the analysis of microarray data. Kwantitatieve Methoden, 71:1-24. Citado na pág. 28

Hennig (2000) C. Hennig. Identifiablity of models for clusterwise linear regression. Journal of Classification, 17(2):273-296. Citado na pág. 27

Hernández et al. (submetido a) F. Hernández, O. Usuga e V. Giampaoli. Improving the adaptive gaussian quadrature. Journal of Statistical Software. Citado na pág. 17

Holzmann et al. (2006) H. Holzmann, A. Munk e T. Gneiting. Identifiability of finite mixtures of elliptical distributions. Scandinavian journal of statistics, 33(4):753-763. Citado na pág. 23

Jansen e Nijs (1993) R.C. Jansen e A.P.M. Den Nijs. A statistical mixture model for estimating the proportion of unreduced pollen grains in perennial ryegrass (lolium perenne 1.) via the size of pollen grains. Euphytica, 70(3):205-215. Citado na pág. 26

Jasra et al. (2005) A. Jasra, C.C. Holmes e D.A. Stephens. Markov chain monte carlo methods and the label switching problem in bayesian mixture modeling. Statistical Science, 20(1):50-67. Citado na pág. 23, 26

Ji et al. (2005) Y. Ji, C. Wu, P. Liu, J. Wang e K.R. Coombes. Applications of beta-mixture models in bioinformatics. Bioinformatics, 21(9):2118-2122. Citado na pág. 1, 26, 32

Johnson et al. (2007) W.E. Johnson, C. Li e A. Rabinovic. Adjusting batch effects in microarray expression data using empirical bayes methods. Biostatistics, 8(1):118-127. Citado na pág. 7

Kaplan (2002) D. Kaplan. Methodological advances in the analysis of individual growth with relevance to education policy. Peabody Journal of Education, 77(4):189-215. Citado na pág. 93, 94

Kaufmann et al. (2009) D. Kaufmann, A. Kraay e M. Mastruzzi. Governance matters VIII: aggregate and individual governance indicators, 1996-2008. Policy Research Working Paper 4978. Citado na pág. 53, 54

Kelley (2008) K. Kelley. Nonlinear change models in populations with unobserved heterogeneity. Methodology: European Journal of Research Methods for the Behavioral and Social Sciences, 4 (3):97-112. Citado na pág. 82

Kieschnick e McCullough (2003) R. Kieschnick e B.D. McCullough. Regression analysis of variates observed on $(0,1)$ : percentages, proportions and fractions. Statistical Modelling, 3(3): 193-213. Citado na pág. 15

Kohli et al. (2016) N. Kohli, J.R. Harring e C. Zopluoglu. A finite mixture of nonlinear random coefficient models for continuous repeated measures data. Psychometrika, 81(3):851-880. Citado na pág. 82

Laurila et al. (2011) K. Laurila, B. Oster, C.L. Andersen, P. Lamy, T. Orntoft, O. Yli-Harja e C. Wiuf. A beta-mixture model for dimensionality reduction, sample classification and analysis. BMC Bioinformatics, 12:215. Citado na pág. 28

Lavine e West (1992) M. Lavine e M. West. A bayesian method for classification and discrimination. Canadian Journal of Statistics, 20(4):451-461. Citado na pág. 27

Lele et al. (2007) S.R. Lele, B. Dennis e F. Lutscher. Data cloning: easy maximum likelihood estimation for complex ecological models using bayesian markov chain monte carlo methods. Ecology Letters, 10(7):551-563. Citado na pág. 18, 29 
Lele et al. (2010) S.R. Lele, K. Nadeem e B. Schmuland. Estimability and likelihood inference for generalized linear mixed models using data cloning. Journal of the American Statistical Association, 105(492):1617-1625. Citado na pág. 28, 29, 44, 92

Lenk e DeSarbo (2000) P.J Lenk e W.S. DeSarbo. Bayesian inference for finite mixtures of generalized linear models with random effects. Psychometrika, 65(1):93-119. Citado na pág. 41

Liang e Zou (2008) H. Liang e G. Zou. Improved AIC selection strategy for survival analysis. Computational Statistics \& Data Analysis, 52(5):2538-2548. Citado na pág. 12

Liang e Zeger (1986) K.Y. Liang e S.L. Zeger. Longitudinal data analysis using generalized linear models. Biometrika, 73(1):13-22. Citado na pág. 19

Lin e Lee (2008) T.I. Lin e J.C. Lee. Estimation and prediction in linear mixed models with skew-normal random effects for longitudinal data. Statistics in Medicine, 27(9):1490-1507. Citado na pág. 48

Lindstrom e Bates (1990) M.J. Lindstrom e D.M. Bates. Nonlinear mixed effects models for repeated measures data. Biometrics, 46(3):673-687. Citado na pág. 19, 20, 81, 82

Liu e Eugenio (2016) F. Liu e E.C. Eugenio. A review and comparison of bayesian and likelihoodbased inferences in beta regression and zero-or-one-inflated beta regression. Statistical Methods in Medical Research, 0(0):1-21. Citado na pág. 17

Liu e Li (2014) F. Liu e Q. Li. A bayesian model for joint analysis of multivariate repeated measures and time to event data in crossover trials. Statistical Methods in Medical Research, 25 (5):2180-2192. Citado na pág. 40

Liu e Pierce (1994) Q. Liu e D.A. Pierce. A note on Gauss-Hermite quadrature. Biometrika, 81 (3):624-629. Citado na pág. 17

Lu e Huang (2014) X. Lu e Y. Huang. Bayesian analysis of nonlinear mixed-effects mixture models for longitudinal data with heterogeneity and skewness. Statistics in Medicine, 33(16): 2830-2849. Citado na pág. 82

Lunn et al. (2009) D. Lunn, D. Spiegelhalter, A. Thomas e N. Best. The BUGS project: Evolution, critique and future directions. Statistics in Medicine, 28(25):3049-3067. Citado na pág. 9, 18

Lunn et al. (2012) D. Lunn, C. Jackson, N. Best, A. Thomas e D. Spiegelhalter. The BUGS book: A practical introduction to Bayesian analysis. Chapman \& Hall/CRC. Citado na pág. 9

Ma e Leijon (2011) Z. Ma e A. Leijon. Bayesian estimation of beta mixture models with variational inference. IEEE Transactions on Pattern Analysis and Machine Intelligence, 33(11): 2160-2173. Citado na pág. 28

Madeira e Giampaoli (2017) A.B. Madeira e V. Giampaoli. Agglomeration of fast food companies in brazil. Management Research: Journal of the Iberoamerican Academy of Management, 15 (3):292-312. Citado na pág. 54

Mallet et al. (1988) A. Mallet, F. Mentré, J.-L. Steimer e F. Lokiec. Nonparametric maximum likelihood estimation for population pharmacokinetics, with application to cyclosporine. Journal of Pharmacokinetics and Biopharmaceutics, 16(3):311-327. Citado na pág. 82

Martins et al. (2013) T.G. Martins, D. Simpson, F. Lindgren e H. Rue. Bayesian computing with INLA: new features. Computational Statistics \&3 Data Analysis, 67:68-83. Citado na pág. 10

McCulloch et al. (2008) C.E. McCulloch, S.R. Searle e J.M. Neuhaus. Generalized, Linear, and Mixed Models. Wiley. Second Edition. Citado na pág. 48 
McLachlan e Peel (2000) G. McLachlan e D. Peel. Finite Mixture Models. Wiley Series in Probability and Mathematical Statistics. John Wiley \& Sons, Inc. Citado na pág. 21, 23

McLachlan e Basford (1988) G.J. McLachlan e K.E. Basford. Mixture models: Inference and applications to clustering. Statistics: Textbooks and Monographs, New York: Dekker, 1988. Citado na pág. 21

Metropolis et al. (1953) N. Metropolis, A.W. Rosenbluth, M.N. Rosenbluth, A.H. Teller e E. Teller. Equation of state calculations by fast computing machines. The Journal of Chemical Physics, 21(6):1087-1092. Citado na pág. 8

Migliorati et al. (2018) S. Migliorati, A.M. Di Brisco e A. Ongaro. A new regression model for bounded responses. Bayesian Analysis, 13(3):845-872. Citado na pág. 63

Monteiro et al. (2012) A.M. Monteiro, M.A.N. Jardini, V. Giampaoli, S.Alves, A.M.F. Neto e M. Gidlund. Measurement of the nonlinear optical response of low-density lipoprotein solutions from patients with periodontitis before and after periodontal treatment: evaluation of cardiovascular risk markers. Journal of Biomedical Optics, 17(11):115004-115004. Citado na pág. 46

Ng et al. (2006) S.K Ng, G.J. McLachlan, K. Wang, L. Ben-Tovim Jones e S.-W. Ng. A mixture model with random-effects components for clustering correlated gene-expression profiles. Bioinformatics, 22(14):1745-1752. Citado na pág. 32

Paolino (2001) P. Paolino. Maximum likelihood estimation of models with beta-distributed dependent variables. Political Analysis, 9(4):325-346. Citado na pág. 15

Paraíba et al. (2018) C.C.M. Paraíba, N. Bochkina e C.A.R. Diniz. Bayesian truncated beta nonlinear mixed-effects models. Journal of Applied Statistics, 45(2):320-346. Citado na pág. 82

Pearson (1894) K. Pearson. Contributions to the mathematical theory of evolution. Philosophical Transactions of the Royal Society of London. A, 185:71-110. Citado na pág. 21

Pianta et al. (2008) R.C. Pianta, J. Belsky, N. Vandergrift, R. Houts e F.J. Morrison. Classroom effects on childrens achievement trajectories in elementary school. American Educational Research Journal, 45(2):365-397. Citado na pág. 98

Pinheiro e Bates (1995) J.C. Pinheiro e D.M. Bates. Model building for nonlinear mixed-effects models. Technical Report 91. Department of Biostatistics, University of Wisconsin - Madison. Citado na pág. 82

Pinheiro e Bates (2000) J.C. Pinheiro e D.M. Bates. Mixed-Effects Models in S and S-PLUS. New York: Springer-Verlag. Citado na pág. 19

Plummer et al. (2006) M. Plummer, N. Best, K. Cowles e K. Vines. Coda: Convergence diagnosis and output analysis for mcmc. $R$ News, 6(1):7-11. Citado na pág. 9

Ponciano et al. (2012) J.M. Ponciano, J.G. Burleigh, E.L. Braun e M.L. Taper. Assessing parameter identifiability in phylogenetic models using data cloning. Systematic Biology, 61(6):955-972. Citado na pág. 29

Qi e Charnigo (2017) Y. Qi e R. Charnigo. Large-scale hypothesis testing with a three-component beta contamination model. Trabalho apresentado no 2017 Joint Statistical Meetings, Baltimore, MD, Estados Unidos, 2017. Citado na pág. 25, 26

Quandt (1972) R.E. Quandt. A new approach to estimating switching regressions. Journal of the American Statistical Association, 67(338):306-310. Citado na pág. 26 
Quandt e Ramsey (1978) R.E. Quandt e J.B. Ramsey. Estimating mixtures of normal distributions and switching regressions. Journal of the American statistical Association, 73(364):730-738. Citado na pág. 26

R Core Team (2016) R Core Team. R: A Language and Environment for Statistical Computing. $\mathrm{R}$ Foundation for Statistical Computing, Vienna, Austria, 2016. URL https://www.R-project.org/. Citado na pág. 3,17

Raftery e Lewis (1992) A.E. Raftery e S.M. Lewis. One long run with diagnostics: Implementation strategies for markov chain monte carlo. Statistical Science, 7(4):493-497. Citado na pág. 38, 69

Resende et al. (2001) M.D.V. Resende, L.L. Duda, P.R.B. Guimarães e J.S.C. Fernandes. Análise de modelos lineares mistos via inferência bayesiana. Rev. Mat. Estat., 19:41-70. Citado na pág. 17

Richardson e Green (1997) S. Richardson e P.J. Green. On bayesian analysis of mixtures with an unknown number of components (with discussion). Journal of the Royal Statistical Society: series B (statistical methodology), 59(4):731-792. Citado na pág. 22

Rigby e Stasinopoulos (2005) R.A. Rigby e D.M. Stasinopoulos. Generalized additive models for location, scale and shape. Applied Statistics, 54(3):507-554. Citado na pág. 16

Robbins (1956) H. Robbins. An empirical bayes approach to statistics. Proceedings of the Third Berkeley Symposium on Mathematical Statistics and Probability, Volume 1: Contributions to the Theory of Statistics, páginas 157-163. Citado na pág. 7

Robert e Casella (2004) C.P. Robert e G. Casella. Monte Carlo Statistical Methods. New York: Springer. Citado na pág. $5,8,51$

Robert e Casella (2009) C.P. Robert e G. Casella. Introducing Monte Carlo Methods with R. New York: Springer. Citado na pág. 8

Rue et al. (2009) H. Rue, S. Martino e N. Chopin. Approximate bayesian inference for latent gaussian models by using integrated nested laplace approximations (with discussion). Journal of the Royal Statistical Society: Series B, 71(2):319-392. Citado na pág. 10

Sarstedt (2008) M. Sarstedt. Market segmentation with mixture regression models: Understanding measures that guide model selection. Journal of Targeting, Measurement and Analysis for Marketing, 16(3):228-246. Citado na pág. 27

Schlattmann (2009) P. Schlattmann. Medical applications of finite mixture models. SpringerVerlag; Berlin. Citado na pág. 27

Serang et al. (2015) S. Serang, Z. Zhang, J. Helm, J.S. Steele e K.J.Grimm. Evaluation of a bayesian approach to estimating nonlinear mixed-effects mixture models. Structural Equation Modeling: A Multidisciplinary Journal, 22(2):202-215. Citado na pág. 82, 86, 93,95

Serang et al. (2016) S. Serang, K.J. Grimm e J.J. McArdle. Estimation of time-unstructured nonlinear mixed-effects mixture models. Structural Equation Modeling: A Multidisciplinary Journal, 23(6):856-869. Citado na pág. 93, 94, 95, 96, 98, 99

Sheiner e Beal (1980) L.B. Sheiner e S.L. Beal. Evaluation of methods for estimating population pharmacokinetic parameters. I. Michaelis-Menten model: routine clinical pharmacokinetic data. Journal of Pharmacokinetics and Biopharmaceutics, 8(6):553-571. Citado na pág. 82

Sheiner e Beal (1983) L.B. Sheiner e S.L. Beal. Evaluation of methods for estimating population pharmacokinetic parameters. iii. monoexponential model: routine clinical pharmacokinetic data. Journal of Pharmacokinetics and Biopharmaceutics, 11(3):303-319. Citado na pág. 19 
Simas et al. (2010) A.B. Simas, W. Barreto-Souza e A.V. Rocha. Improved estimators for a general class of beta regression models. Computational Statistics 83 Data Analysis, 54(2):348-366. Citado na pág. $15,19,63,82$

Smithson e Verkuilen (2006) M. Smithson e J. Verkuilen. A better lemon squeezer? maximumlikelihood regression with beta-distributed dependent variables. Psychological Methods, 11(1): 54-71. Citado na pág. 16, 63

Smithson et al. (2011) M. Smithson, E.C. Merkle e J.J. Verkuilen. Beta regression finite mixture models of polarization and priming. Journal of Educational and Behavioral Statistics, 36(6): 804-831. Citado na pág. $18,25,28,32$

Smyth (1989) G.K. Smyth. Generalized linear models with varying dispersion. Journal of the Royal Statistical Society, 51(1):47-60. Citado na pág. 63, 64

Sólymos (2010) P. Sólymos. dclone: Data cloning in R. The $R$ Journal, 2(2):29-37. Citado na pág. $29,30,73$

Sorensen (1996) D. Sorensen. Gibbs sampling in quantitative genetics. Internal Report no. 82, Danish Institute of Animal Science, Tjele, Denmark. Citado na pág. 17

Spiegelhalter et al. (1999) D.J. Spiegelhalter, A. Thomas e N.G. Best. Winbugs version 1.2 user manual. MRC Biostatistics Unit, 83. Citado na pág. 3, 9

Spiegelhalter et al. (2002) D.J. Spiegelhalter, N.G. Best, B.P. Carlin e A. Van Der Linde. Bayesian measures of model complexity and fit. Journal of the Royal Statistical Society: Series B (Statistical Methodology), 64(4):583-639. Citado na pág. 11

Spiegelhalter et al. (2014) D.J. Spiegelhalter, N.G. Best, B.P. Carlin e A. Van Der Linde. The deviance information criterion: 12 years on. Journal of the Royal Statistical Society: Series B (Statistical Methodology), 76(3):485-493. Citado na pág. 11

Stasinopoulos et al. (2008) D.M. Stasinopoulos, R.A. Rigby e C. Akantziliotou. Instructions on how to use the gamlss package in $R, 2008$. Citado na pág. 17

Stasinopoulos e Rigby (2012) M. Stasinopoulos e B. Rigby. gamlss.nl: Fitting non linear parametric GAMLSS models, 2012. URL https://CRAN.R-project.org/package=gamlss.nl. R package version 4.1-0. Citado na pág. 87

Stephens (2000) M. Stephens. Dealing with label switching in mixture models. Journal of the Royal Statistical Society: Series B (Statistical Methodology), 62(4):795-809. Citado na pág. 23

Sturtz et al. (2005) S. Sturtz, U. Ligges e A. Gelman. R2winbugs: A package for running WinBUGS from R. Journal of Statistical Software, 12(3):1-16. Citado na pág. 9, 20

Su e Yajima (2015) Y.-S. Su e M. Yajima. R2jags: Using $R$ to Run 'JAGS', 2015. URL https://CRAN.R-project.org/package=R2jags. R package version 0.5-7. Citado na pág. 20

Swihart e Lindsey (2017) B. Swihart e J. Lindsey. repeated: Non-Normal Repeated Measurements Models, 2017. URL https://CRAN.R-project.org/package=repeated. R package version 1.1.0. Citado na pág. 20

Tatarinova e Schumitzky (2014) T. Tatarinova e A. Schumitzky. Nonlinear mixture models: a bayesian approach. World Scientific. Citado na pág. 81

Teicher (1963) H. Teicher. Identifiability of finite mixtures. The Annals of Mathematical Statistics, 34(4):1265-1269. Citado na pág. 22 
Theobald et al. (2012) C. Theobald, A. Chatterjee e G. Horgan. A hierarchical bayesian mixture model for repeated dietary records. Food and Chemical Toxicology, 50(2):320-327. Citado na pág. 27,32

Titterington (1990) D.M. Titterington. Some recent research in the analysis of mixture distributions. Statistics, 21(4):619-641. Citado na pág. 21

Titterington et al. (1985) D.M. Titterington, A.F.M. Smith e U.E. Makov. Statistical analysis of finite mixture distributions. New York: Wiley. Citado na pág. 21, 23

Tutz e Oelker (2017) G. Tutz e M.-R. Oelker. Modelling clustered heterogeneity: Fixed effects, random effects and mixtures. International Statistical Review, 85(2):204-227. Citado na pág. 32

Usuga (2013) O.C. Usuga. Modelos de regressão beta com efeitos aleatórios normais e não normais para dados longitudinais. Tese de Doutorado, Instituto de Matemática e Estatística, Universidade de São Paulo, Brasil. Citado na pág. 16, 17

Verdinelli e Wasserman (1991) I. Verdinelli e L. Wasserman. Bayesian analysis of outlier problems using the gibbs sampler. Statistics and Computing, 1(2):105-117. Citado na pág. 27

Verkuilen e Smithson (2012) J. Verkuilen e M. Smithson. Mixed and mixture regression models for continuous bounded responses using the beta distribution. Journal of Educational and Behavioral Statistics, 37(1):82-113. Citado na pág. 12, 16, 18, 28, 32, 63

Vermunt (2008) J.K. Vermunt. Latent class and finite mixture models for multilevel data sets. Statistical Methods in Medical Research, 17(1):33-51. Citado na pág. 32

Vonesh e Carter (1992) E.F. Vonesh e R.L. Carter. Mixed-effects nonlinear regression for unbalanced repeated measures. Biometrics, 48(1):1-17. Citado na pág. 19, 82

Vonesh e Chinchilli (1997) E.F. Vonesh e V.M. Chinchilli. Linear and nonlinear models for the analysis of repeated measurements. New York: Marcel Dekker. Citado na pág. 19

Wakefield et al. (1994) J.C. Wakefield, A.F.M. Smith, A. Racine-Poon e A.E. Gelfand. Bayesian analysis of linear and non-linear population models by using the gibbs sampler. Applied Statistics, páginas 201-221. Citado na pág. 82

Wakefield et al. (2003) J.C. Wakefield, C. Zhou e S. Self. Modelling gene expression over time: curve clustering with informative prior distributions. Bayesian Statistics, 7:721-732. Citado na pág. 81

Wang et al. (2002) K. Wang, K.K.W. Yau e A.H. Lee. A hierarchical poisson mixture regression model to analyse maternity length of hospital stay. Statistics in Medicine, 21(23):3639-3654. Citado na pág. 32

Wedel e DeSarbo (1994) M. Wedel e W.S. DeSarbo. A review of recent developments in latent class regression models. Em Advanced methods of marketing research, páginas 352-388. Citado na pág. 26

World Health Organization, WHO and World Heart Federation and World Stroke Organization (2011) World Health Organization, WHO and World Heart Federation and World Stroke Organization. Global atlas on cardiovascular disease prevention and control. Citado na pág. 45

Xu e Hedeker (2001) W. Xu e D. Hedeker. A random-effects mixture model for classifying treatment response in longitudinal clinical trials. Journal of Biopharmaceutical Statistics, 11(4): 253-273. Citado na pág. 1, 21, 26 
Yakowitz e Spragins (1968) S.J. Yakowitz e J.D. Spragins. On the identifiability of finite mixtures. The Annals of Mathematical Statistics, páginas 209-214. Citado na pág. 23

Yu e Yau (2013) D. Yu e K.W. Yau. Robust REML estimation for k-component Poisson mixture with random effects: application to the epilepsy seizure count data and urinary tract infections data. Statistics in Medicine, 32(14):2479-2499. Citado na pág. 32

Zeger et al. (1988) S.L. Zeger, K.Y. Liang e P.S. Albert. Models for longitudinal data: A generalized estimating equation approach. Biometrics, 44(4):1049-1060. Citado na pág. 19

Zerbeto (2014) A.P. Zerbeto. Melhor preditor empírico aplicado aos modelos beta mistos. Dissertação de Mestrado, Universidade de São Paulo. Citado na pág. 46

Zhou (2004) C. Zhou. A bayesian model for curve clustering with application to gene expression data analysis. Tese de Doutorado, University of Washington. Citado na pág. 81

Zimprich (2010) D. Zimprich. Modeling change in skewed variables using mixed beta regression models. Research in Human Development, 7(1):9-26. Citado na pág. 63 